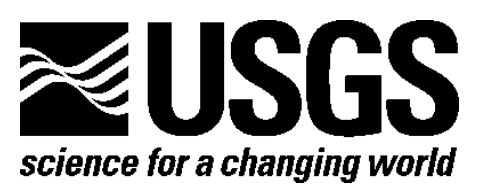

\title{
Efficacy of Pseudomonas fluorescens (Pf-CL145A) Spray Dried Powder for Controlling Zebra Mussels Adhering to Test Substrates
}

By James A. Luoma, Todd J. Severson, Kerry L. Weber, and Denise A. Mayer

Open-File Report 2015-1050

U.S. Department of the Interior

U.S. Geological Survey 


\section{U.S. Department of the Interior \\ SALLY JEWELL, Secretary}

\section{U.S. Geological Survey \\ Suzette M. Kimball, Acting Director}

U.S. Geological Survey, Reston, Virginia: 2015

For more information on the USGS—-the Federal source for science about the Earth, its natural and living resources, natural hazards, and the environment-visit http://www.usgs.gov or call 1-888-ASK-USGS (1-888-275-8747)

For an overview of USGS information products, including maps, imagery, and publications, visit http://www.usgs.gov/pubprod

To order this and other USGS information products, visit http://store.usgs.gov

Any use of trade, firm, or product names is for descriptive purposes only and does not imply endorsement by the U.S. Government.

Although this information product, for the most part, is in the public domain, it also may contain copyrighted materials as noted in the text. Permission to reproduce copyrighted items must be secured from the copyright owner.

Suggested citation:

Luoma, J.A., Severson, T.J., Weber, K.L., and Mayer, D.A., 2015, Efficacy of Pseudomonas fluorescens (PfCL145A) spray dried powder for controlling zebra mussels adhering to test substrates: U.S. Geological Survey Open-File Report 2015-1050, 519 p., http://dx.doi.org/10.3133/ofr20151050. 


\section{Acknowledgments}

This study was funded through a combination of a Great Lakes Restoration Initiative Grant and U.S. Geological Survey appropriated funds. The authors thank Jeremy K. Wise (Upper Midwest Environmental Science Center) for his technical assistance, which made this study possible; Mark P. Gaikowski (Upper Midwest Environmental Science Center), who assisted with study design, data analysis, and report preparation; Jon Motquin, (Shawano County Invasive Species Coordinator), who assisted with test animal collection and technical support; and Nathan Olson and Elizabeth Murray (Minnesota Department of Natural Resources) and Keith Marquardt (Wisconsin Department of Natural Resources), who assisted with field logistics, test site location, and permitting. 


\section{Contents}

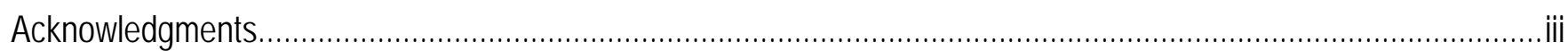

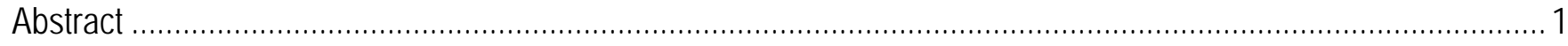

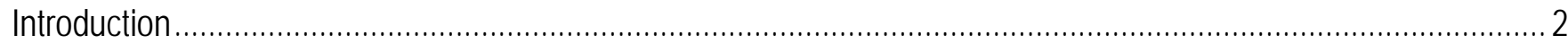

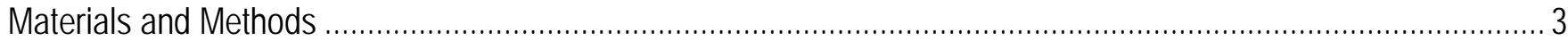

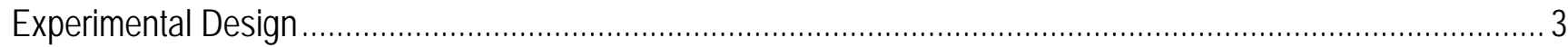

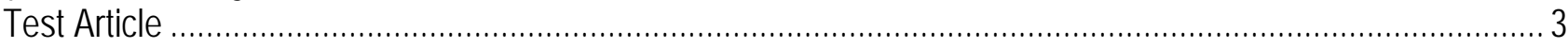

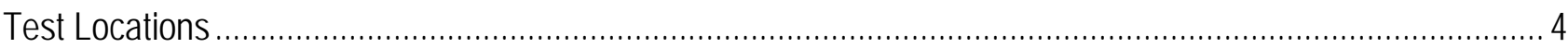

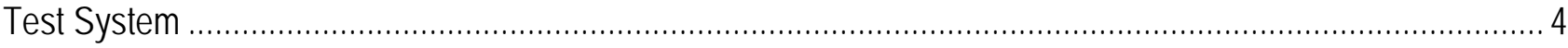

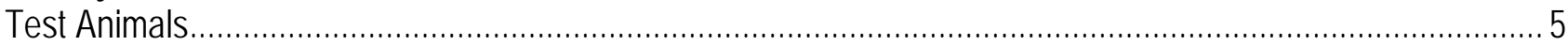

Test Animal Collection and Initial Placement ...................................................................................... 5

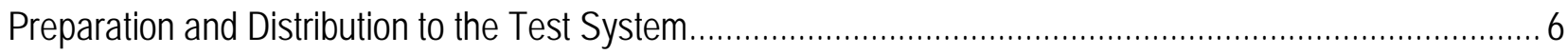

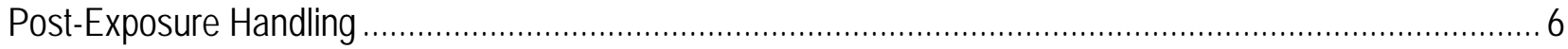

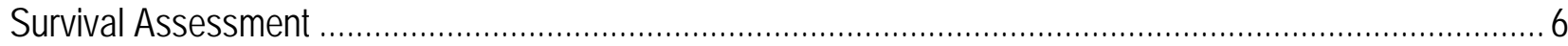

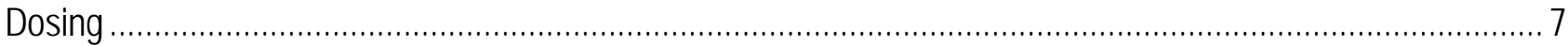

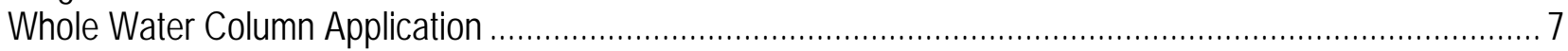

Benthic Injection Application...........................................................................................................

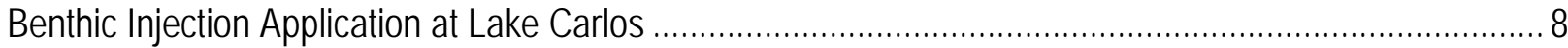

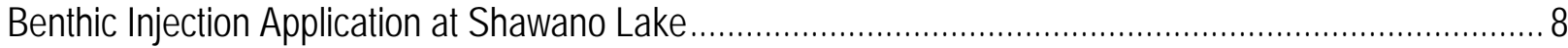

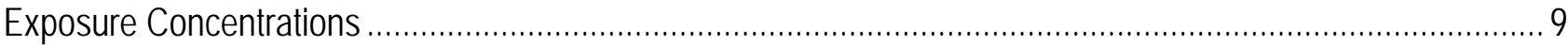

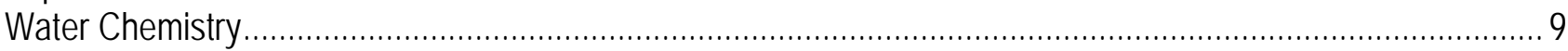

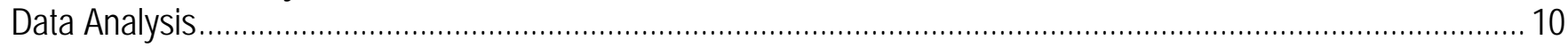

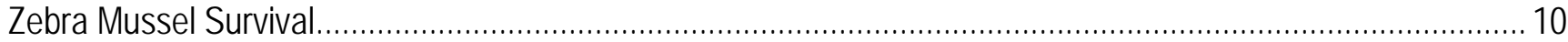

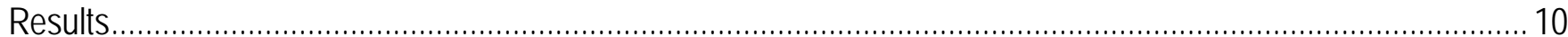

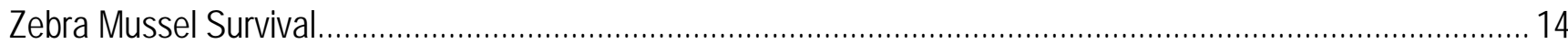

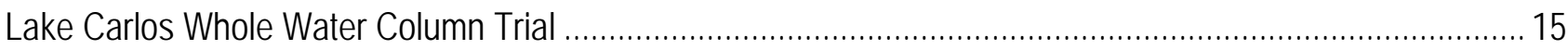

Shawano Lake Whole Water Column Trial ......................................................................................... 15

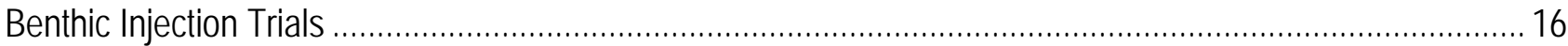

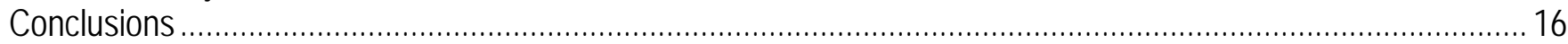

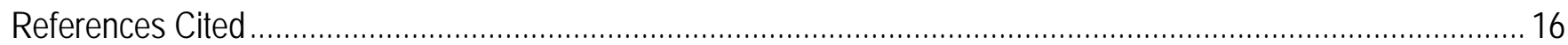

Appendix 1.Study Protocol, Amendments, and Datasheets.............................................................................. 18

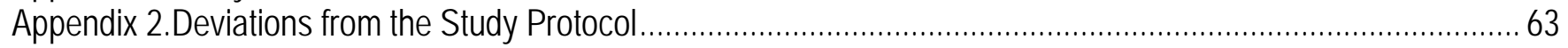

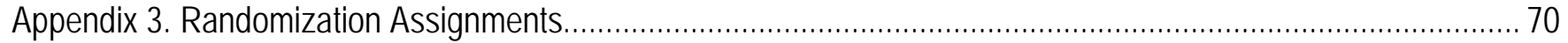

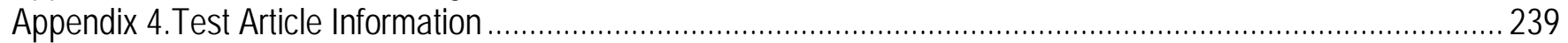

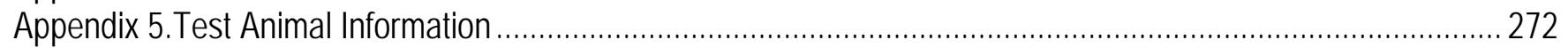

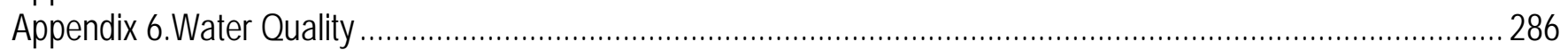

Appendix 7.Spectrophotometric Summary, SAS Program, Output and Log ...................................................... 319

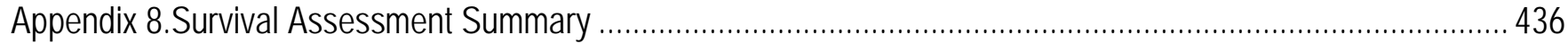

Appendix 9.Statistical Analysis including SAS Programs, Outputs and Logs for Survival Data ............................ 451 


\section{Figures}

Figure 1. Schematic of mobile bioassay laboratory.

4

Figure 2. Example of test substrate with zebra mussels during initial placement (left), after adherence (center), and prepared for exposure in a semirigid plastic mesh containment bag (right)............... 5

Figure 3. Example of stacked test substrates (left) and wire mesh cage (right). ......................................... 6

Figure 4. Schematic of benthic injection application system. .............................................................. 8

Figure 5. Mean (standard deviation) SDP active ingredient concentration of water samples collected during the exposure period. WWC graphs are from the surface samples; BI graphs are from bottom samples (Lake Carlos) and suspended samples (Shawano Lake).

\section{Tables}

Table 1. Mean (standard deviation) water chemistry parameters (dissolved oxygen and temperature) and $\mathrm{pH}$ range of each treatment group during the study period.

Table 2. Mean (standard deviation) hardness, alkalinity, and conductivity of filtered (200 micrometers) source water collected from the delivery system headboxes prior to exposure.

Table 3. Mean (standard deviation) total ammonia nitrogen (TAN) and un-ionized ammonia $\left(\mathrm{NH}_{3}\right)$ of each treatment group by lake, application type, and exposure duration.

Table 4. Mean (standard deviation) percent zebra mussel survival for each lake, application type, and exposure duration. 


\section{Conversion Factors}

International System of Units to Inch/Pound

\begin{tabular}{|c|c|c|}
\hline Multiply & By & To obtain \\
\hline \multicolumn{3}{|c|}{ Length } \\
\hline centimeter (cm) & 0.3937 & inch (in.) \\
\hline micrometer $(\mu \mathrm{m})$ & $3.937 \times 10^{-5}$ & inch (in.) \\
\hline millimeter (mm) & 0.03937 & inch (in.) \\
\hline meter (m) & 1.094 & yard (yd) \\
\hline nanometer (nm) & $3.937 \times 10^{-8}$ & inch (in.) \\
\hline \multicolumn{3}{|c|}{ Area } \\
\hline hectare (ha) & 2.471 & acre \\
\hline \multicolumn{3}{|c|}{ Volume } \\
\hline liter (L) & 1.057 & quart (qt) \\
\hline milliliter (mL) & 0.03382 & ounce, fluid (fl. oz) \\
\hline \multicolumn{3}{|c|}{ Flow rate } \\
\hline liter per minute (L/min) & 0.2642 & gallon per minute (gal/min) \\
\hline milliliter per minute (mL/min) & 0.0002642 & gallon per minute (gal/min) \\
\hline \multicolumn{3}{|c|}{ Mass } \\
\hline $\operatorname{gram}(\mathrm{g})$ & 0.03527 & ounce, avoirdupois (oz) \\
\hline milligram (mg) & $3.527 \times 10^{-5}$ & ounce, avoirdupois (oz) \\
\hline
\end{tabular}

Conductivity is given in microsiemens per centimeter at 25 degrees Celsius $\left(\mu \mathrm{S} / \mathrm{cm}\right.$ at $\left.25^{\circ} \mathrm{C}\right)$.

Concentrations of chemical constituents in water are given in milligrams per liter (mg/L).

Temperature in degrees Celsius $\left({ }^{\circ} \mathrm{C}\right)$ may be converted to degrees Fahrenheit $\left({ }^{\circ} \mathrm{F}\right)$ as ${ }^{\circ} \mathrm{F}=\left(1.8 \times{ }^{\circ} \mathrm{C}\right)+32$. 


$\begin{array}{ll}\text { Abbreviations } \\ \text { AEH } & \text { Aquatic Ecosystem Health } \\ \text { ASTM } & \text { American Society for Testing and Material } \\ \mathrm{BI} & \text { benthic injection } \\ \mathrm{CaCO}_{3} & \text { calcium carbonate } \\ \text { d } & \text { day(s) } \\ \text { DNR } & \text { Department of Natural Resources } \\ \text { DO } & \text { dissolved oxygen } \\ \text { EPA } & \text { U.S. Environmental Protection Agency } \\ \text { h } & \text { hour(s) } \\ \text { ID } & \text { inside diameter } \\ \text { MBI } & \text { Marrone Bio Innovations } \\ \text { NH } 3 & \text { un-ionized ammonia } \\ \text { SAS } & \text { Statistical Analysis Software } \\ \text { SDP } & \text { spray dried powder } \\ \text { TAN } & \text { total ammonia nitrogen } \\ \text { Pf-CL145A } & \text { Pseudomonas fluorescens strain CL145A } \\ \text { UMESC } & \text { Upper Midwest Environmental Sciences Center } \\ \text { USGS } & \text { U.S. Geological Survey } \\ \text { WWC } & \text { whole water column } \\ & \end{array}$




\title{
Efficacy of Pseudomonas fluorescens (Pf-CL145A) Spray Dried Powder for Controlling Zebra Mussels Adhering to Test Substrates
}

\author{
By James A. Luoma, ${ }^{1}$ Todd J. Severson, ${ }^{1}$ Kerry L. Weber, ${ }^{1}$ and Denise A. Mayer ${ }^{2}$
}

\begin{abstract}
A mobile bioassay trailer was used to assess the efficacy of Pseudomonas fluorescens (PfCL145A) spray dried powder (SDP) formulation for controlling zebra mussels (Dreissena polymorpha) from two midwestern lakes: Lake Carlos (Alexandria, Minnesota) and Shawano Lake (Shawano, Wisconsin). The effects of SDP exposure concentration and exposure duration on zebra mussel survival were evaluated along with the evaluation of a benthic injection application technique to reduce the amount of SDP required to induce zebra mortality.

Groups of zebra mussels were collected from each lake and allowed to adhere to test substrates for at least 15 days before exposure to SDP. Two independent trials were completed at each lake: (1) a whole water column (WWC) application trial was used to evaluate the effects of SDP exposure concentration and exposure duration on zebra mussel survival; and (2) a benthic injection (BI) application trial in which the SDP was injected into the test tanks to determine the efficacy of a benthic injection application technique to reduce the amount of SDP required to induced zebra mussel mortality. Three exposure durations (6, 9, and 12 hours) were evaluated in the WWC trials and a 12-hour exposure duration was evaluated in the BI trials. All trials contained zebra mussels which were removed at the completion of each exposure duration, consolidated into wire mesh cages, and held in the lake for approximately 30 days before being assessed for survival.

For all trials, treatment was assigned to each test tank according to a randomized block design ( $n=3$ test tanks per treatment). The treatment groups included (1) an untreated control group, (2) a group that received an application of 50 milligrams of SDP per liter (mg SDP/L), and (3) a group that received an application of $100 \mathrm{mg}$ SDP/L. During the BI trials, SDP was administered to achieve the desired exposure concentration in the bottom 50 percent $(175 \mathrm{~L})$ of the test tank. All exposure concentrations are reported as active ingredient.

Approximately 30 days after exposure, zebra mussels were sorted into live and dead, and enumerated. Mean survival of zebra mussels in control treatments exceeded 95 percent. Mean survival of zebra mussels in the Lake Carlos WWC SDP-treated groups ranged from 0.5 to 2.1 percent and when compared at the same exposure duration, no difference was detected in survival between the 50 and 100 milligrams per liter (mg/L) treatment groups. Similarly, mean survival of zebra mussels in the Shawano Lake WWC SDP-treated groups ranged from 2.0 to 12.6 percent and when compared at the same exposure duration, no difference was detected in survival between the 50 - and $100-\mathrm{mg} / \mathrm{L}$ treatment

\footnotetext{
${ }^{1}$ U.S. Geological Survey

${ }^{2}$ New York State Education Department
} 
groups. Mean survival of zebra mussels in the Lake Carlos BI trial SDP-treated groups did not differ ( $p=0.93$ ) and was 18.1 and 18.0 percent in the 50- and 100-mg/L treatment groups, respectively. Mean survival of zebra mussels in the Shawano Lake BI trial SDP-treated groups differed $(p<0.01)$ and was 2.9 and 0.9 percent in the 50 - and $100-\mathrm{mg} / \mathrm{L}$ treatment groups, respectively. Survival of zebra mussels assigned to the SDP-treated groups in the Lake Carlos WWC trial (12-hour exposure duration) differed from the survival of zebra mussels assigned to the SDP-treated groups in the Lake Carlos BI trial; however, after modification of the BI application technique, no difference $(p=0.22)$ was detected between the survival of zebra mussel in the Shawano Lake WWC (12-hour exposure duration) and BI trials.

\section{Introduction}

Freshwater mussels native to North America (order Unionoida) are considered the most diverse in the world, consisting of approximately 297 recognized taxa, but they have been declining at an alarming rate due to anthropogenic activities such as pollution, habitat alteration, and over harvest (Williams and others, 1993; Neves and others, 1997). A survey completed by the Nature Conservancy revealed 55 percent of North America's mussels are either extinct or imperiled (Master, 1990). The North American mussel extinction rate is predicted to be 6.4 percent per decade, which equates to the extinction of 127 species in the next 100 years (Ricciardi and Rasmussen, 1999). This prediction may be conservative, as it did not account for the invasion of North American waterways by dreissenid mussels (zebra mussel, Dreissena polymorpha and quagga mussel, Dreissena bugensis).

Dreissenid mussels are ideal invaders due to their high fecundity and their planktonic larvae, which are capable of dispersal over vast areas (Birnbaum, 2011). Dreissenid mussels pose a serious ecological threat and negatively affect many native aquatic species, particularly freshwater mussels. Dreissenid mussels can quickly inundate freshwater mussels and cause obstruction of valve movement as well as inhibition of feeding and respiration (Burlakova and others, 2000) resulting in an increased cost of metabolism, decreased fitness, and ultimately death (Baker and Hornbach, 1997).

Natural resource managers lack readily available, environmentally safe, and effective tools for controlling dreissenid mussels in open-water environments. One potential tool for limited open-water control of dreissenid mussels is a commercially formulated spray dried powder (SDP) formulation of Pseudomonas fluorescens (Zequanox ${ }^{\circledR}$ ), produced by Marrone Bio Innovations, Inc. (MBI; Davis, California), which contains nonviable cells of a specific strain (CL145A) of the common soil bacterium Pseudomonas fluorescens. The SDP formulation was developed by MBI and registered by the U.S. Environmental Protection Agency for control non-native dreissenid (zebra and quagga) mussels in raw water conduit systems (that is, industrial cooling and irrigation systems, and so forth) and it has recently been approved for use in limited open-water environments.

The objectives of this study were (1) to evaluate the potential use of Pseudomonas fluorescens spray dried powder (SDP) formulation for controlling zebra mussels (Dreissena polymorpha) in limited open-water environments; and (2) to evaluate the use of a benthic injection (BI) application technique to reduce the amount of SDP required to induce zebra mussel mortality.

The applications for this study were completed in the Upper Midwest Environmental Science Center's (UMESC) mobile bioassay laboratory, which used water and test animals from two midwestern lakes: Lake Carlos (Alexandria, Minnesota) and Shawano Lake (Shawano, Wisconsin). This final study report summarizes four separate field trials with activities carried out from October, 2011 to November, 2013. Applications of SDP were completed on August 15 and 17, 2012 at Lake Carlos and on September 6 and 8, 2012 at Shawano Lake. 


\section{Materials and Methods}

All methods and materials followed the written protocol and its amendments, except those instances that were identified as deviations (appendix 2, items 1-6). The study protocol and amendments for this study are contained in appendix 1 (items 1-2).

\section{Experimental Design}

The study SDP applications were completed within the UMESC mobile bioassay laboratory, which used water and test animals from two midwestern lakes: Lake Carlos (Alexandria, Minn.) and Shawano Lake (Shawano, Wis.). Survival of zebra mussels was assessed approximately 30 days after exposure to SDP. Groups of zebra mussels were collected from each lake and allowed to adhere to perforated aluminum test substrates for at least 15 days before exposure to SDP. Zebra mussels adhering to the test substrates were exposed to SDP in a series of nine 350-liter (L) test. Two independent trials were completed at each test location (1) a whole water column (WWC) application trial, which evaluated the effects of SDP exposure concentration and exposure duration on zebra mussel survival, and (2) a BI application trial, which evaluated the use of a BI application technique to reduce the amount of SDP required to induce zebra mussel mortality.

Treatments were administered in triplicate according to a randomized block design (appendix 3, items 1, 4, 7, and 10) and included (1) an untreated control group, (2) a group that received an application of 50 milligrams SDP per liter (mg SDP/L), and (3) a group that received an application of $100 \mathrm{mg}$ SDP/L. The experimental unit was the individual test tank. Test substrates with adhering zebra mussels were distributed to test tanks according to a random distribution scheme (appendix 3, items 2 , 5, 8, and 11).

Each WWC test tank contained nine test substrates with adhering zebra mussels and each BI test tank contained either three (Lake Carlos) or four (Shawano Lake) test substrates with adhering zebra mussels. Upon exposure termination during the WWC trials (6, 9, and 12 hours), three randomly selected test substrates were removed from each test tank. Upon exposure termination during the BI trials (12 hours), all test substrates were removed from each test tank. After exposure, the test substrates with adhering zebra mussels were consolidated into wire mesh cages, which were placed in approximately 2.5 meters (m) of water for the post-exposure period. Approximately 30 days after SDP exposure, zebra mussels were sorted into live and dead, and enumerated. Zebra mussels from one test substrate of each treatment level and exposure duration were retained in 70 percent isopropyl alcohol for length measurement.

\section{Test Article}

The test article was a commercially prepared SDP formulation of Pseudomonas fluorescens, strain CL145A containing 50 percent active ingredient (weight to weight ratio [w/w] $P$. fluorescens, strain CL145A). The test article was provided by the manufacturer as a mixed lot (401P12163C and 401P12164C; Certificates of Analysis, appendix 4, items 3 and 4). Test article use was documented in the test chemical log books (appendix 4, items 9-13). Concentrations of the test article are reported as active ingredient. Retention of test article biological activity was assessed after exposure by New York State Museum Field Research Laboratory (Cambridge, New York) using their standard dreissenid mussel bioassay (appendix 4, item 8). Results of the biological activity bioassay demonstrated a mean (standard deviation) mortality of 70.7 percent (4.6) at $200 \mathrm{mg} / \mathrm{L}$, which was similar to mean mortality exhibited by the cell fraction positive control which was 73.3 percent (8.3), confirming the biological activity of the test article. 


\section{Test Locations}

Two midwestern lakes with different water-quality characteristics were the test locations and the source of the test water and test animals. Lake Carlos is a 1,020-hectare (ha) mesotrophic lake located near Alexandria, Minn., and it is the deepest natural lake in Minnesota (excluding Lake Superior), with a maximum depth of $49.7 \mathrm{~m}$. Shawano Lake is a 2,515-ha eutrophic lake located in Shawano, Wis., with a maximum depth of $12 \mathrm{~m}$. Zebra mussels were first reported in Shawano Lake in 2002 and in Lake Carlos in 2009 (Turyk and others, 2008; Engel and others, 2010).

\section{Test System}

The test system was a series of nine independent circular test tanks (76 x 95 centimeters (cm), diameter $x$ height; 350 L capacity) contained within the UMESC mobile bioassay laboratory. The test tanks were positioned in two rows with four test tanks in one row and five test tanks in the other (fig. 1). Test substrates were used as the medium to facilitate zebra mussel handling during the study period. The test substrates were constructed of perforated aluminum (4.8 millimeter (mm) hole, 51 percent open area, $1.6 \mathrm{~mm}$ thick) folded into trays (15.2 x $15.2 \times 2.5 \mathrm{~cm}$, length $\mathrm{x}$ width $\mathrm{x}$ height [fig. 2]).

Test water was supplied to the test system from a 3-horsepower submersible well pump (ITT Goulds Pumps, Seneca Falls, N.Y., model 18GS30). The water was filtered (200 micrometers [ $\mu \mathrm{m}])$ using a microscreen filtration system (Forstra Filter Inc., Los Angeles, California; model M1-90), delivered to two headboxes (30.5 x 55.9 x 114.3 x $30.5 \mathrm{~cm}$, width x length x height; one headbox per test tank row), and gravity fed to each test tank at approximately 6 liters per minute, providing approximately one tank-exchange per hour. Water flow was interrupted during the exposure period. Untreated water was discharged to the lake; SDP-treated water was collected in frame tanks and removed by a state-licensed septic hauler and disposed of by land application (Minnesota) or discharge to a sanitary sewer system (Wisconsin).

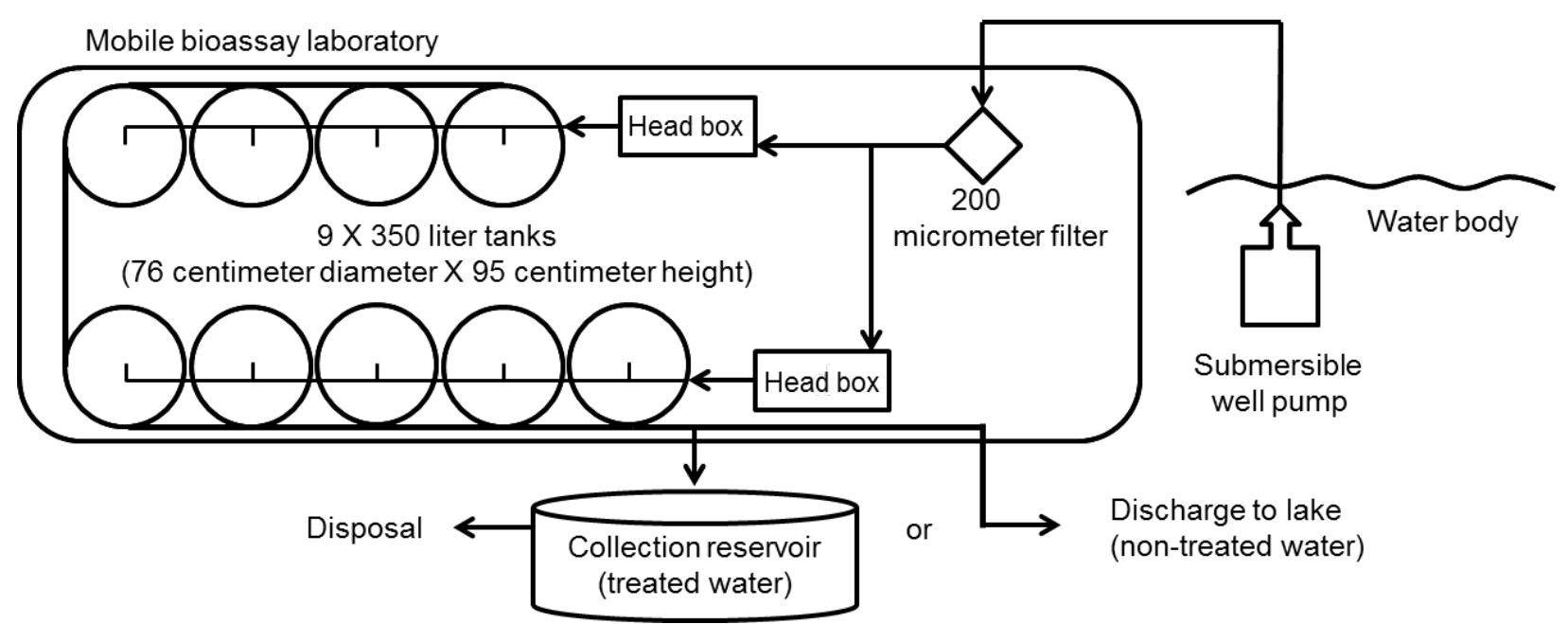

Figure 1. Schematic of mobile bioassay laboratory. 

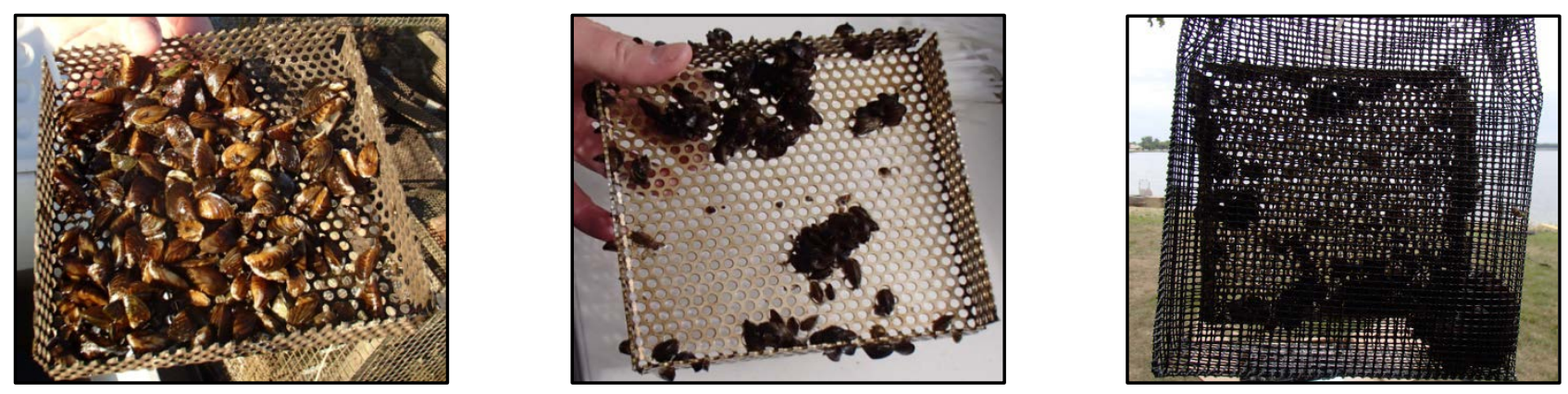

Figure 2. Example of test substrate with zebra mussels during initial placement (left), after adherence (center), and prepared for exposure in a semirigid plastic mesh containment bag (right).

\section{Test Animals}

Zebra mussels were collected from existing colonies within each lake and placed on test substrates. Zebra mussels adhering to the test substrates immediately prior to allocation to the test tanks were used as the test animals. Mean shell length for the zebra mussels used in the Lake Carlos trials ranged from 11.26 to $11.85 \mathrm{~mm}$ and the mean shell length for the zebra mussels used in the Shawano Lake trials ranged from 18.27 to $18.74 \mathrm{~mm}$ (appendix 5, items 2-5).

Test Animal Collection and Initial Placement

In October and November 2011, natural substrates (that is, rocks, sticks, and native mussels) with adhering zebra mussels were collected from each lake and the zebra mussels were removed by severing the byssus with a scalpel. Zebra mussels were held in coolers containing lake water until placed onto the test substrates. Approximately 200 to 300 zebra mussels were indiscriminately selected and placed on each test substrate. After zebra mussel placement, wood spacers $(\approx 2 \mathrm{~cm}$ thick $)$ were used to separate the test substrates (fig. 3 ) before they were secured in vertical stacks $(\approx 10$ substrates per stack). Six stacks were placed in each of three wire mesh cages at each lake ( $\approx 180$ substrates per lake; fig. 3). The wire mesh cages were placed in $\approx 2 \mathrm{~m}$ of water to allow for zebra mussel adherence through winter. Due to poor overwinter survival, zebra mussels on the Shawano Lake test substrates were replaced in August of 2012 following the procedures previously described. The zebra mussels used in the Shawano Lake trial were allowed to adhere to the test substrates $\approx 16$ days prior to exposure. 

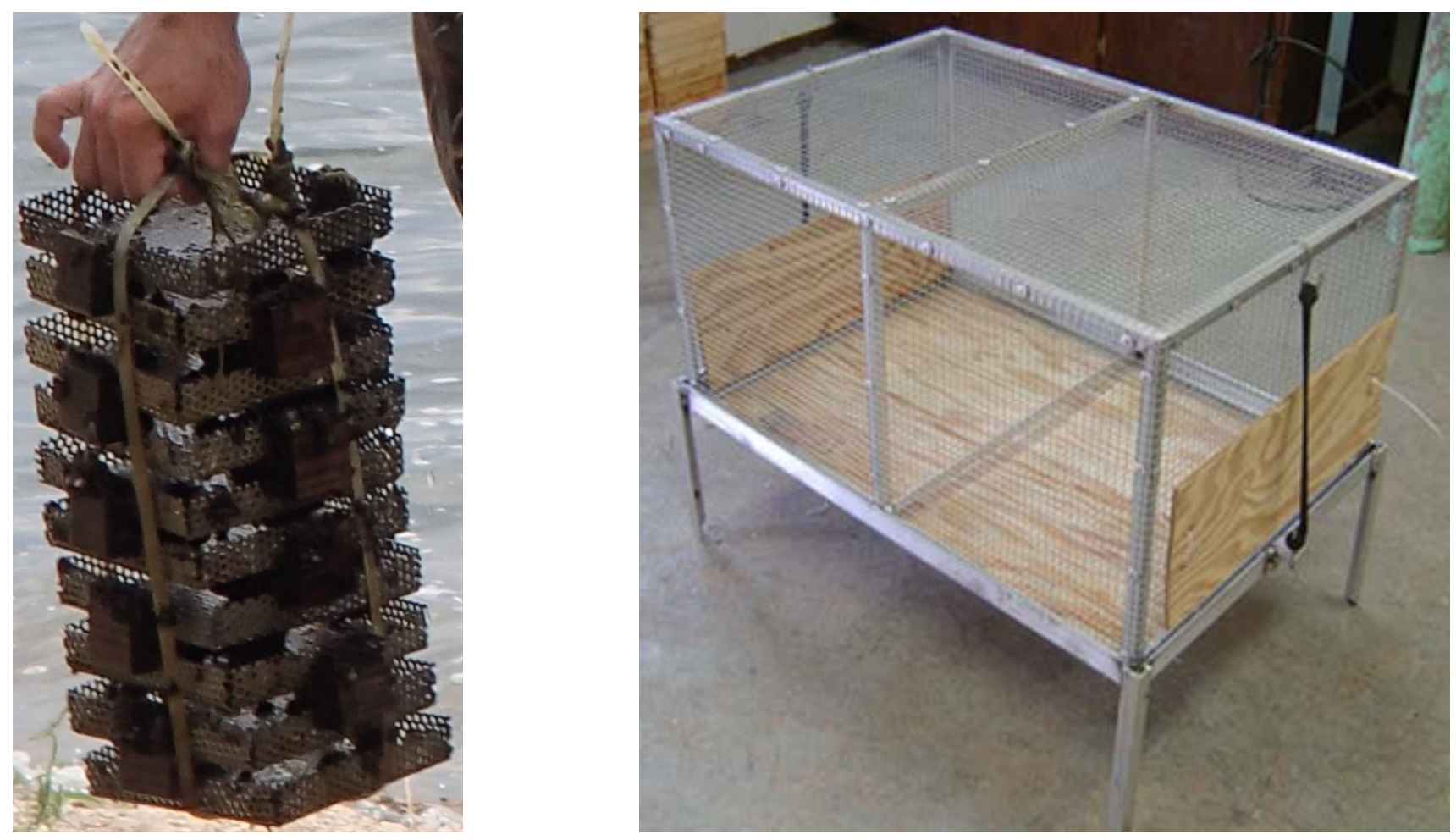

Figure 3. Example of stacked test substrates (left) and wire mesh cage (right).

Preparation and Distribution to the Test System

One day prior to SDP exposure, test substrates were inverted to dislodge non-adhering zebra mussels. Moribund zebra mussels and shell fragments were removed from each test substrate with forceps. Test substrates with adhering zebra mussels were placed into a uniquely identified semirigid plastic mesh containment bags ( $20.3 \times 25.4 \times 5.1 \mathrm{~cm} ; 0.32$ x $0.42 \mathrm{~cm}$ openings) and randomly allocated to test tanks (appendix 3, items 2, 5, 8, and 11). The WWC trials at both lakes received nine test substrates per test tank (three per exposure duration), whereas the BI trials received three (Lake Carlos) or four (Shawano Lake) test substrates per test tank.

\section{Post-Exposure Handling}

Upon exposure termination (that is, 6, 9, and 12 hours [h] for WWC; $12 \mathrm{~h}$ for BI), test substrates were removed from the test tanks according to a randomization scheme (appendix 3, items 3, 6, 9, and 12) and consolidated in wire mesh cages. Test substrates removed from all test tanks at each exposure duration were indiscriminately placed into a single wire mesh cage (for example, all test substrates removed at $6 \mathrm{~h}$ were placed into the same wire mesh cage). The wire mesh cages were placed in $\approx 2.5 \mathrm{~m}$ of water, in the respective lake, for the $\approx 30$-day post-exposure period.

\section{Survival Assessment}

Zebra mussel survival was assessed 26-27 days after SDP exposure at Lake Carlos and 32-34 days after SDP exposure at Shawano Lake. Survival was determined by examining valve movement in response to mechanical stimuli or resistance to valve pressure by adductor muscle contraction. Zebra mussels $<6 \mathrm{~mm}$ were excluded from the analyses because they could readily pass through the plastic 
mesh containment bags and because of the potential for inconsistent survival assessment of small mussels (that is, inconsistent assessment of adductor muscle response). Zebra mussels from each test substrate were sorted into groups of live or dead and enumerated. Zebra mussels from one test substrate at each treatment level and exposure duration were indiscriminately selected and retained in 70 percent isopropyl alcohol to measure for shell length with digital calipers.

\section{Dosing}

Stock solutions used to administer treatments were prepared by adding pre-weighed aliquots of SDP (appendix 4, items 5 and 6) into known volumes of filtered $(200 \mu \mathrm{m})$ lake water and mechanically mixing with a paint mixer attached to an electric drill for 3-5 minutes. The solution was then immediately poured through a mesh colander and any clumps of SDP were pulverized with a pestle and rinsed into the stock solution with filtered lake water. During all trials, control treatments were completed using the same methods as the SDP-treated groups with the exception that no SDP was applied. For all trials, concentrations of SDP are reported as active ingredient.

\section{Whole Water Column Application}

Separate stock solutions were prepared for each test tank replicate in the WWC trials. Stock solutions for the WWC were prepared by adding SDP (35 grams [g] for the 50 milligrams per liter [mg/L] treatment; $70 \mathrm{~g}$ for the $100-\mathrm{mg} / \mathrm{L}$ treatment) to $\approx 8 \mathrm{~L}$ of water removed from each test tank. Immediately after preparation, each stock was poured into the respective test tank and gently mixed with the test tank water.

\section{Benthic Injection Application}

An injection system was constructed to treat the bottom 50 percent $(175 \mathrm{~L})$ of each test tank during the BI trials (fig. 4). A peristaltic pump (Masterflex Digi-staltic drive, model 77310; ColeParmer, Vernon Hills, Illinois) was used to inject the SDP stock at the selected depth in each test tank through a delivery apparatus consisting of four peristaltic tubing lines (Masterflex L/S 14 tubing; $1.6 \mathrm{~mm}$ Inside Diameter [ID]), which terminated with dispersion nozzles designed to disperse test article horizontally in the water column. Each dispersion nozzle was constructed from a modified plastic cylindrical check valve (Penn-Plax, Inc.; model CV1 check-valve/air-filter; Hauppauge, N.Y.) with four 2-mm holes drilled around the circumference (fig. 4). At Shawano Lake, the SDP stock concentrations were diluted, the stock delivery rate was increased, and the injection height was raised to decrease SDP settling. 


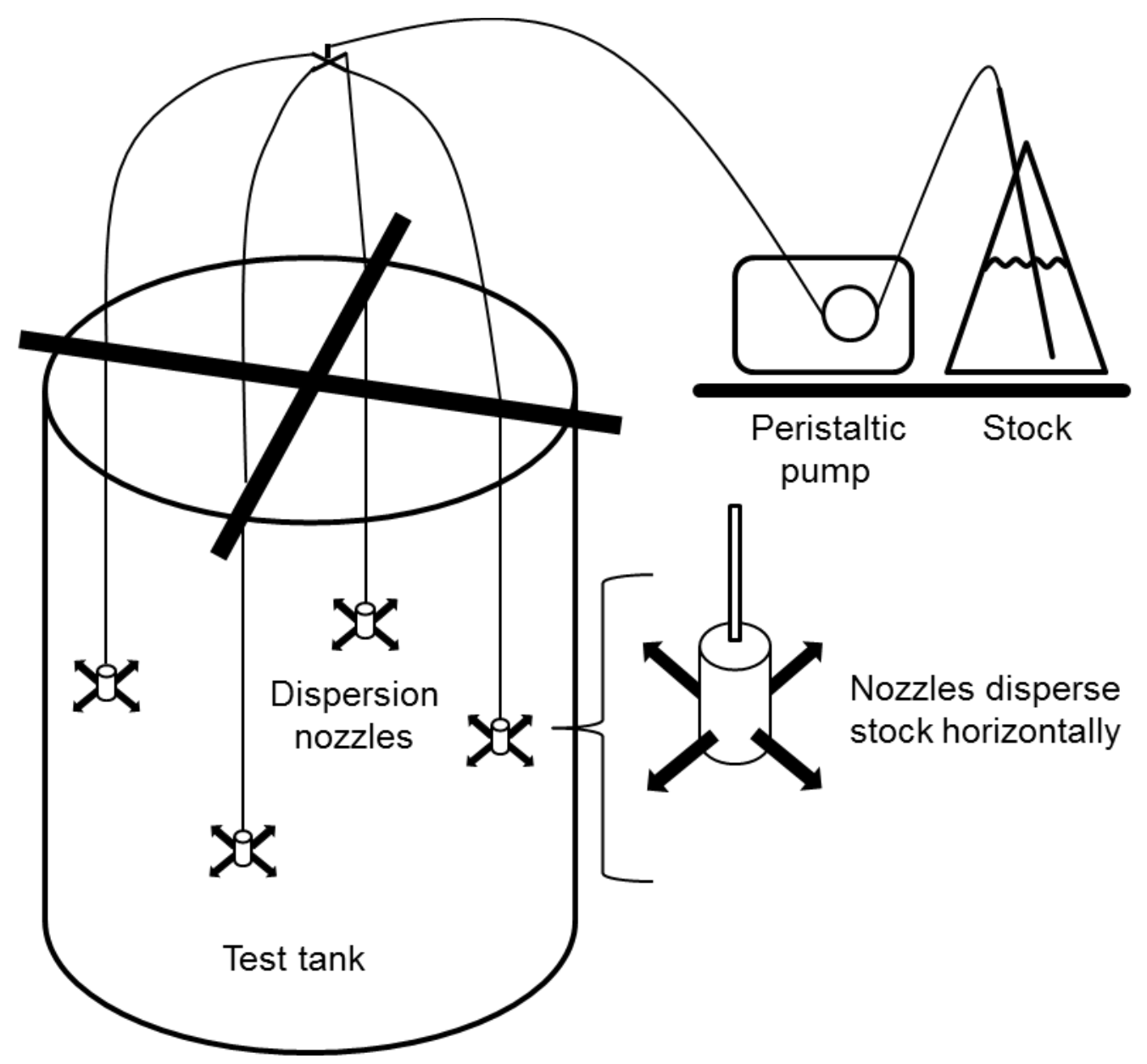

Figure 4. Schematic of benthic injection application system.

Benthic Injection Application at Lake Carlos

Separate 15,000 and 30,000 mg SDP/L stock solutions were prepared for the 50- and 100-mg/L Lake Carlos BI treatment applications, respectively. Approximately 583 milliliters (mL) of the appropriate stock solution was delivered to each test tank at $62 \mathrm{~mL} /$ minute, resulting in delivery of $\approx 8,750$ and $17,500 \mathrm{mg}$ of SDP to each 50 and $100 \mathrm{mg} / \mathrm{L}$ test tank replicate, respectively. The amount of stock solution injected was the volume required to achieve the target SDP exposure concentration in the bottom 50 percent ( $175 \mathrm{~L}$ ) of the test tank. The stock solution was delivered $\approx 19 \mathrm{~cm}$ from the bottom of each test tank.

\section{Benthic Injection Application at Shawano Lake}

Separate 2,500 and 5,000 $\mathrm{mg} \mathrm{SDP/L} \mathrm{stock} \mathrm{solutions} \mathrm{were} \mathrm{prepared} \mathrm{for} \mathrm{the} \mathrm{50-} \mathrm{and} \mathrm{100-mg/L}$ Shawano Lake BI treatment applications, respectively. Approximately 3,500 $\mathrm{mL}$ of the appropriate stock solution was delivered to each test tank at $350 \mathrm{~mL} /$ minute, resulting in delivery of $\approx 8,750$ and $17,500 \mathrm{mg}$ of SDP to each 50 and $100 \mathrm{mg} / \mathrm{L}$ test tank replicate, respectively. The amount of stock solution injected was the volume required to achieve the target SDP exposure concentration in the bottom 50 percent $(175 \mathrm{~L}$ ) of the test tank. The stock solution was delivered $\approx 38 \mathrm{~cm}$ from the bottom of each test tank. 


\section{Exposure Concentrations}

Water samples were collected for SDP exposure concentration determination from different locations within test tanks depending on application type (WWC or BI) and trial location. Surface samples were collected by submersing a 50 -mL beaker below the water surface. Suspended $(\approx 15$ or $19 \mathrm{~cm}$ from the test tank bottom) and bottom samples (Lake Carlos BI trial only) were collected from test tanks using a peristaltic pump (Masterflex Digi-staltic drive, model 77310; Cole-Parmer, Vernon Hills, Ill.) fitted with four 1.6-mm ID collection lines. Approximately $200 \mathrm{~mL}$ of exposure water was purged through peristaltic tubing and discarded before sample collection.

Exposure concentrations were determined by comparing the test tank water sample absorbance to a linear regression curve created from known active ingredient concentrations of SDP (25, 50, 100, and $200 \mathrm{mg} / \mathrm{L}$ ). Absorbance was measured using a Barnstead/Turner SP-830 Plus spectrophotometer (model SM110215) at 660 nanometers (nm). Linear regression equations were fit using the Statistical Analysis Software Proc Reg procedure (SAS ${ }^{\circledR}$ Version 9.3, SAS Institute, Inc., Cary, North Carolina). The exposure concentrations were predicted from the regression analysis (appendix 7, items 2-4, 6-8, 10-12, 14-16). The known concentrations of SDP used to create the linear regression were maintained at approximately 4 degrees Celsius $\left({ }^{\circ} \mathrm{C}\right)$ and measured for absorbance at 6,9 , and $12 \mathrm{~h}$ after treatment administration to ensure proper spectrophotometer function with the exception of the Lake Carlos WWC trial, in which the known concentrations were measured at 9 and $12 \mathrm{~h}$.

The SDP exposure concentrations in the Lake Carlos WWC trial were determined from surface water samples at $1,3,6,9$, and $12 \mathrm{~h}$ and suspended $(\approx 15 \mathrm{~cm})$ water samples at 3, 6, 9, and $12 \mathrm{~h}$. The SDP exposure concentrations in the Lake Carlos BI trial were determined from suspended $(\approx 19 \mathrm{~cm})$ water samples at 1, 3, 6, 9, and $12 \mathrm{~h}$ and from bottom water samples at 6, 9, and $12 \mathrm{~h}$. Because sample absorbance was below the detection limit, samples from control test tanks were not analyzed for SDP concentration during the Lake Carlos trials. Control water samples were analyzed for SDP in the Shawano Lake trials, and were below the detection limit. The SDP exposure concentrations in the Shawano Lake WWC trial were determined from surface water and suspended $(\approx 15 \mathrm{~cm})$ water samples at 1, 3, 6, 9, and $12 \mathrm{~h}$. The SDP exposure concentrations in the Shawano Lake BI trial were determined from surface water and suspended $(\approx 15 \mathrm{~cm})$ water samples at $1,3,6,9$, and $12 \mathrm{~h}$.

\section{Water Chemistry}

Dissolved oxygen, $\mathrm{pH}$, temperature, hardness, alkalinity, and conductivity were measured $\approx 1 \mathrm{~h}$ prior to treatment administration from filtered lake water samples collected from the distribution headboxes. Dissolved oxygen, $\mathrm{pH}$, and temperature were measured in each test tank within $1 \mathrm{~h}$ and $\approx 3,6,9$, and $12 \mathrm{~h}$ after treatment administration during the WWC and BI trials.

Water samples for total ammonia nitrogen (TAN) analysis were collected $12 \mathrm{~h}$ after SDP application during all trials and at 6 and $9 \mathrm{~h}$ after SDP application during the Lake Carlos WWC trial. Water samples were filtered $(0.45 \mu \mathrm{m})$, acidified with 10 percent sulfuric acid to $\leq \mathrm{pH} 2.5$, and stored at $\approx 4{ }^{\circ} \mathrm{C}$ until analyzed for TAN by the UMESC water-quality laboratory using the automated phenate method (Standard Method 4500G; American Public Health Association, 2012). Un-ionized ammonia concentrations were calculated from TAN, $\mathrm{pH}$, and temperature from time of sample collection using the formula identified by Emerson and others (1975).

Four data loggers (Onset Inc, Bourne, Massachusetts; HOBO ${ }^{\circledR}$ Pendent Temperature/Light Data Logger, model UA-002064) were attached to the wire mesh cages at each lake and used to measure water temperature four times daily during the post-exposure period. 


\section{Data Analysis}

Data analyses for water chemistry parameters were limited to simple summary statistics; comparative statistics were not generated. Exposure concentration means were determined using SAS ${ }^{\circledR}$ software version 9.3 (SAS Institute, Inc., Cary, N.C.). The SAS ${ }^{\circledR}$ software Proc Means procedure was used to determine the mean exposure concentration by individual test tank, treatment group, and exposure duration (appendix 7, items 2-4, 6-8, 10-12, 14-16). Because of observations of SDP settling and non-detectable levels of SDP in the suspended water samples, the bottom water sample exposure concentrations for the Lake Carlos BI trial are reported. The suspended water exposure concentrations for Shawano Lake BI trials are reported due to negligible $(\leq 3 \mathrm{mg} / \mathrm{L})$ SDP exposure concentrations measured in the surface water samples.

\section{Zebra Mussel Survival}

Statistical comparisons of zebra mussel survival were completed using SAS ${ }^{\circledR}$ software version 9.3. Significance was declared at $\alpha \leq 0.05$. A generalized linear mixed model was used to analyze the survival of zebra mussels in each treatment group (appendix 9, items 1-3). The proportion of mortalities (number of dead zebra mussels compared to the total number of zebra mussels in the sample) was modeled using the SAS ${ }^{\circledR}$ software Proc GLIMMIX procedure with a binomial distribution and a logit link function. A scale parameter was added to the model using the SAS ${ }^{\circledR}$ software random_residual_ statement. Zebra mussel survival in each treatment group was individually compared to the zebra mussel survival in the untreated control group using a two-sided means comparison test.

\section{Results}

Water chemistry parameters (dissolved oxygen, $\mathrm{pH}$, and temperature) collected from the test tanks are summarized in table 1 and the water chemistry data are in appendix 6 (items 1-14). Water hardness, alkalinity, and conductivity are summarized in table 2. Dissolved oxygen levels remained above the minimum threshold recommended for freshwater mussels (4.0 mg/L, ASTM International, 2013) and the mean TAN remained below the criterion for acute exposure and below 4-day maximum criterion for chronic exposure during the exposure period (U.S. Environmental Protection Agency, 2013; table 3). Mean daily temperatures ranged from 20.6 to $24.4^{\circ} \mathrm{C}$ (Lake Carlos) and from 10.1 to $21.5^{\circ} \mathrm{C}$ (Shawano Lake) during the post-exposure period. 
Table 1. Mean (standard deviation) water chemistry parameters (dissolved oxygen and temperature) and $\mathrm{pH}$ range of each treatment group during the study period.

[mg/L, milligrams per liter; DO, dissolved oxygen; ${ }^{\circ} \mathrm{C}$, degrees Celsius]

\begin{tabular}{|c|c|c|c|c|c|c|c|}
\hline $\begin{array}{l}\text { Water chemistry } \\
\text { parameter }\end{array}$ & $\begin{array}{c}\text { Treatment } \\
\text { group }(\mathrm{mg} / \mathrm{L})\end{array}$ & $\begin{array}{c}\text { Pre- } \\
\text { exposure }^{1}\end{array}$ & $\leq 1 \mathrm{~h}$ & $3 \mathrm{~h}$ & $6 \mathrm{~h}$ & $9 \mathrm{~h}$ & $12 \mathrm{~h}$ \\
\hline \multicolumn{8}{|c|}{ Lake Carlos whole water column application } \\
\hline \multirow[t]{3}{*}{$\mathrm{DO}(\mathrm{mg} / \mathrm{L})$} & 0 & $8.77(0.02)$ & $8.72(0.04)$ & $8.58(0.09)$ & $8.51(0.04)$ & $8.25(0.03)$ & $8.11(0.01)$ \\
\hline & 50 & $8.76(0.01)$ & $8.66(0.02)$ & $8.42(0.01)$ & $8.21(0.04)$ & $7.80(0.09)$ & $6.62(0.13)$ \\
\hline & 100 & $8.76(0.01)$ & $8.64(0.01)$ & $8.38(0.04)$ & $8.06(0.10)$ & $7.74(0.07)$ & $6.84(0.07)$ \\
\hline \multirow[t]{3}{*}{$\mathrm{pH}$} & 0 & 8.62-8.64 & $8.60-8.63$ & $8.56-8.58$ & $8.54-8.58$ & $8.25-8.31$ & $8.55-8.58$ \\
\hline & 50 & $8.61-8.65$ & 8.57 & $8.47-8.49$ & $8.47-8.49$ & $8.20-8.21$ & 8.33-8.35 \\
\hline & 100 & $8.60-8.63$ & 8.53 & 8.37-8.39 & 8.37-8.39 & 8.12 & $8.21-8.24$ \\
\hline \multirow[t]{3}{*}{ Temperature $\left({ }^{\circ} \mathrm{C}\right)$} & 0 & $22.2(<0.1)$ & $22.3(<0.1)$ & $22.4(<0.1)$ & $22.5(<0.1)$ & $22.5(<0.1)$ & $22.5(<0.1)$ \\
\hline & 50 & $22.1(<0.1)$ & $22.3(<0.1)$ & $22.4(<0.1)$ & $22.5(0.0)$ & $22.4(0.1)$ & $22.4(<0.1)$ \\
\hline & 100 & $22.2(0.0)$ & $22.3(0.0)$ & $22.4(<0.1)$ & $22.5(<0.1)$ & $22.4(0.1)$ & $22.4(0.1)$ \\
\hline \multicolumn{8}{|c|}{ Shawano Lake whole water column application } \\
\hline \multirow[t]{3}{*}{$\mathrm{DO}(\mathrm{mg} / \mathrm{L})$} & 0 & $7.34(0.02)$ & $7.24(0.07)$ & $7.07(0.03)$ & $6.85(0.06)$ & $6.63(0.02)$ & $6.43(0.10)$ \\
\hline & 50 & $7.36(0.02)$ & $7.24(0.04)$ & $6.97(0.10)$ & $6.57(0.08)$ & $5.86(0.19)$ & $4.22(0.64)$ \\
\hline & 100 & $7.36(0.01)$ & $7.25(0.07)$ & $7.01(0.03)$ & $6.56(0.08)$ & $6.03(0.18)$ & $4.79(0.55)$ \\
\hline \multirow[t]{3}{*}{$\mathrm{pH}$} & 0 & $9.31-9.34$ & $9.27-9.28$ & $9.23-9.24$ & $9.14-9.15$ & $9.12-9.16$ & $9.02-9.08$ \\
\hline & 50 & $9.33-9.34$ & 9.22 & $9.11-9.14$ & $9.06-9.08$ & 8.99-9.02 & 8.73-8.85 \\
\hline & 100 & 9.34 & $9.14-9.16$ & $9.07-9.08$ & $8.97-9.00$ & 8.87-8.93 & $8.62-8.73$ \\
\hline \multirow[t]{3}{*}{ Temperature $\left({ }^{\circ} \mathrm{C}\right)$} & 0 & $22.6(0.0)$ & $22.0(<0.1)$ & $22.0(0.2)$ & $22.1(0.3)$ & $22.2(0.4)$ & $22.0(0.4)$ \\
\hline & 50 & $22.6(<0.1)$ & $21.9(0.1)$ & $21.9(0.1)$ & $21.9(0.2)$ & $21.9(0.2)$ & $21.8(0.2)$ \\
\hline & 100 & $22.6(0.0)$ & $21.9(<0.1)$ & $21.9(0.1)$ & $22.0(0.1)$ & $22.0(0.1)$ & $21.8(0.1)$ \\
\hline \multicolumn{8}{|c|}{ Lake Carlos benthic injection application } \\
\hline \multirow[t]{3}{*}{$\overline{\mathrm{DO}(\mathrm{mg} / \mathrm{L})}$} & 0 & $8.42(0.01)$ & $7.97(0.02)$ & $7.97(0.02)$ & $7.95(0.03)$ & $7.84(0.05)$ & $\overline{7.79(0.05)}$ \\
\hline & 50 & $8.40(0.02)$ & $7.97(0.02)$ & $7.97(0.02)$ & $7.83(0.05)$ & $7.81(0.01)$ & $7.16(0.26)$ \\
\hline & 100 & $8.40(0.03)$ & $7.90(0.08)$ & $7.95(0.02)$ & $7.91(0.02)$ & $7.81(0.05)$ & $7.24(0.14)$ \\
\hline \multirow[t]{3}{*}{$\mathrm{pH}$} & 0 & 8.70 & $8.52-8.59$ & $8.51-8.55$ & $8.47-8.50$ & 8.38-8.39 & $8.55-8.58$ \\
\hline & 50 & 8.70 & $8.60-8.61$ & 8.55 & 8.48 & 8.38-8.41 & $8.12-8.20$ \\
\hline & 100 & $8.69-8.70$ & $8.57-8.60$ & $8.52-8.55$ & $8.44-8.48$ & 8.39-8.41 & 7.18-7.39 \\
\hline \multirow[t]{3}{*}{ Temperature $\left({ }^{\circ} \mathrm{C}\right)$} & 0 & $21.2(0.0)$ & $21.3(0.0)$ & $21.2(0.0)$ & $21.5(0.1)$ & $21.4(0.2)$ & $21.0(0.1)$ \\
\hline & 50 & $21.2(<0.1)$ & $21.3(0.0)$ & $21.2(<0.1)$ & $21.7(0.3)$ & $21.6(0.3)$ & $21.1(0.2)$ \\
\hline & 100 & $21.2(0.0)$ & $21.3(0.1)$ & $21.3(<0.1)$ & $21.6(0.1)$ & $21.5(0.1)$ & $21.1(0.1)$ \\
\hline
\end{tabular}




\begin{tabular}{lccccccc}
\hline $\begin{array}{c}\text { Water chemistry } \\
\text { parameter }\end{array}$ & $\begin{array}{c}\text { Treatment } \\
\text { group (mg/L) }\end{array}$ & $\begin{array}{c}\text { Pre- } \\
\text { exposure }\end{array}$ & $\mathbf{s} \mathbf{h}$ & $\mathbf{3 h}$ & $\mathbf{6 h}$ & $\mathbf{9 h}$ & $\mathbf{1 2 ~} \mathbf{~ h}$ \\
\hline \multicolumn{7}{c}{ Shawano Lake benthic injection application } \\
\hline DO (mg/L) & 0 & $7.41(0.01)$ & $7.26(0.01)$ & $7.15(0.07)$ & $7.02(0.01)$ & $6.98(0.04)$ & $6.63(0.02)$ \\
& 50 & $7.43(<0.1)$ & $7.22(0.03)$ & $7.23(0.01)$ & $7.28(0.02)$ & $7.14(0.07)$ & $5.11(0.67)$ \\
& 100 & $7.44(<0.1)$ & $7.17(0.03)$ & $7.23(0.03)$ & $7.29(0.03)$ & $7.24(0.01)$ & $6.21(0.34)$ \\
$\mathrm{pH}$ & 0 & $9.10-9.12$ & $9.05-9.06$ & $9.02-9.03$ & $8.91-8.94$ & $8.64-8.68$ & $8.92-8.94$ \\
& 50 & $9.13-9.14$ & $9.02-9.04$ & $9.02-9.04$ & $8.93-8.95$ & $8.69-8.71$ & $8.69-8.88$ \\
& 100 & $9.13-9.14$ & $8.99-9.01$ & 9.03 & $8.95-8.96$ & $8.69-8.71$ & $8.58-8.68$ \\
Temperature $\left({ }^{\circ} \mathrm{C}\right)$ & 0 & $19.5(0.0)$ & $18.2(0.1)$ & $18.4(0.3)$ & $18.6(0.3)$ & $18.5(0.2)$ & $18.2(0.2)$ \\
& 50 & $19.5(0.0)$ & $18.2(<0.1)$ & $18.4(0.1)$ & $18.5(0.2)$ & $18.4(0.2)$ & $18.2(0.1)$ \\
& 100 & $19.5(0.0)$ & $18.0(0.1)$ & $18.1(0.1)$ & $18.2(0.1)$ & $18.2(0.1)$ & $17.9(0.1)$ \\
\hline
\end{tabular}

${ }^{1}$ Pre-exposure time points were measured approximately $1 \mathrm{~h}$ prior to test article application.

Table 2. Mean (standard deviation) hardness, alkalinity, and conductivity of filtered (200 micrometers) source water collected from the delivery system headboxes prior to exposure.

[mg/L, milligrams per liter; $\mu \mathrm{S} / \mathrm{cm}$, microsiemens per centimeter; <, less-than; $\mathrm{CaCO}$, calcium carbonate; ${ }^{\circ} \mathrm{C}$, degrees Celsius]

\begin{tabular}{llcc}
\hline \multicolumn{1}{c}{ Application type } & Hardness $(\mathrm{mg} / \mathrm{L})^{1}$ & Alkalinity $(\mathrm{mg} / \mathrm{L})^{1}$ & $\begin{array}{c}\text { Conductivity } \\
(\boldsymbol{\mu S} / \mathrm{cm})^{2}\end{array}$ \\
\hline Whole water column & $177(1)$ & Lake Carlos \\
Benthic injection & $177(1)$ & $163(1)$ & $395(1)$ \\
\hline & \multicolumn{5}{c}{$164(<1)$} & $363(3)$ \\
\hline Whole water column & $118(1)$ & Shawano Lake \\
Benthic injection & $125(1)$ & $105(<1)$ & $248(2)$ \\
\hline
\end{tabular}

${ }^{1}$ Reported as milligrams per liter $\mathrm{CaCO}_{3}$.

${ }^{2}$ Temperature compensated to $25^{\circ} \mathrm{C}$. 
Table 3. Mean (standard deviation) total ammonia nitrogen (TAN) and un-ionized ammonia $\left(\mathrm{NH}_{3}\right)$ of each treatment group by lake, application type, and exposure duration.

[mg/L, milligrams per liter; h, hours; WWC, whole water column application; BI, benthic injection application; TAN, total ammonia nitrogen; $\mathrm{SD}$, standard deviation; $\mathrm{NH}_{3}$, un-ionized ammonia; $\mathrm{mg} \mathrm{NH}_{3}-\mathrm{N} / \mathrm{L}$, milligrams un-ionized ammonia nitrogen per liter; -, no sample]

\begin{tabular}{|c|c|c|c|c|c|}
\hline $\begin{array}{c}\text { Water } \\
\text { chemistry } \\
\text { parameter }\end{array}$ & $\begin{array}{l}\text { Treatment } \\
\text { group } \\
\text { (mg/L) }\end{array}$ & $6 \mathrm{~h} \mathrm{WWC}$ & $9 \mathrm{~h} \mathrm{WWC}$ & $12 \mathrm{~h}$ WWC & $12 \mathrm{~h} \mathrm{BI}$ \\
\hline \multicolumn{6}{|c|}{ Lake Carlos } \\
\hline \multirow[t]{3}{*}{ TAN $^{1}(\mathrm{SD})$} & 0 & $0.17(<0.01)$ & $0.17(<0.01)$ & $0.24(0.01)$ & $0.22(0.01)$ \\
\hline & 50 & $0.22(0.01)$ & $0.23(<0.01)$ & $0.33(0.01)$ & $0.40(0.01)$ \\
\hline & 100 & $0.27(<0.01)$ & $0.27(0.00)$ & $0.34(0.01)$ & $1.37(0.18)$ \\
\hline \multirow[t]{3}{*}{$\mathrm{NH}_{3}(\mathrm{SD})$} & 0 & $0.03(<0.01)$ & $0.01(<0.01)$ & $0.04(<0.01)$ & $0.03(<0.01)$ \\
\hline & 50 & $0.03(<0.01)$ & $0.02(0.00)$ & $0.03(<0.01)$ & $0.02(<0.01)$ \\
\hline & 100 & $0.03(<0.01)$ & $0.02(0.00)$ & $0.03(0.00)$ & $0.01(<0.01)$ \\
\hline \multicolumn{6}{|c|}{ Shawano Lake } \\
\hline \multirow[t]{3}{*}{$\operatorname{TAN}^{1}(\mathrm{SD})$} & 0 & - & - & $0.13(<0.01)$ & $0.06(0.01)$ \\
\hline & 50 & - & - & $0.19(0.01)$ & $0.11(0.01)$ \\
\hline & 100 & - & - & $0.23(0.01)$ & $0.16(0.01)$ \\
\hline \multirow[t]{3}{*}{$\mathrm{NH}_{3}(\mathrm{SD})$} & 0 & - & - & $0.04(<0.01)$ & $0.01(<0.01)$ \\
\hline & 50 & - & - & $0.04(<0.01)$ & $0.02(<0.01)$ \\
\hline & 100 & - & - & $0.04(<0.01)$ & $0.02(<0.01)$ \\
\hline
\end{tabular}

${ }^{1}$ Total ammonia nitrogen reported as mg $\mathrm{NH}_{3}-\mathrm{N} / \mathrm{L}$.

Mean exposure concentrations during the exposure period are shown in figure 5 . The SAS ${ }^{\circledR}$ software linear regression, $\mathrm{SAS}^{\circledR}$ software predicted exposure concentrations, and data are in appendix 7 (items 1-16). In the Lake Carlos and Shawano Lake WWC trials, the surface water exposure concentrations are reported due to negligible differences between the measured exposure concentrations in the surface water and suspended water samples (mean difference $\leq 0.46$ and $0.93 \mathrm{mg} / \mathrm{L}$ for the 50 and 100-mg/L treatment groups, respectively). Mean test tank SDP exposure concentrations in surface water samples during the Lake Carlos WWC trial (that is, 6-, 9-, and 12-h exposure durations) ranged from 43.9 to $47.3 \mathrm{mg} / \mathrm{L}$ and 90.2 to $95.3 \mathrm{mg} / \mathrm{L}$ in the 50 - and 100-mg/L treatment groups, respectively 
(fig. 5). Mean test tank SDP concentrations in surface water samples during the Shawano Lake WWC trial (that is, 6-, 9-, and 12-h exposure durations) ranged from 43.8 to $45.9 \mathrm{mg} / \mathrm{L}$ and 93.8 to $99.3 \mathrm{mg} / \mathrm{L}$ in the 50- and 100-mg/L treatment groups, respectively.

The SDP concentrations were below the detection limit in the initial suspended samples collected during the Lake Carlos BI trial; therefore, bottom sampling was initiated at 6 hours and continued throughout the duration of the exposure period. The mean SDP concentrations in the bottom samples were $100.3 \mathrm{mg} / \mathrm{L}$ for the $50-\mathrm{mg} / \mathrm{L}$ treatment group and $234.7 \mathrm{mg} / \mathrm{L}$ for the $100-\mathrm{mg} / \mathrm{L}$ treatment group. Mean SDP concentrations in suspended samples collected during the Shawano Lake BI trial were $38.8 \mathrm{mg} / \mathrm{L}$ for the $50-\mathrm{mg} / \mathrm{L}$ treatment group and $92.9 \mathrm{mg} / \mathrm{L}$ for the $100-\mathrm{mg} / \mathrm{L}$ treatment group.

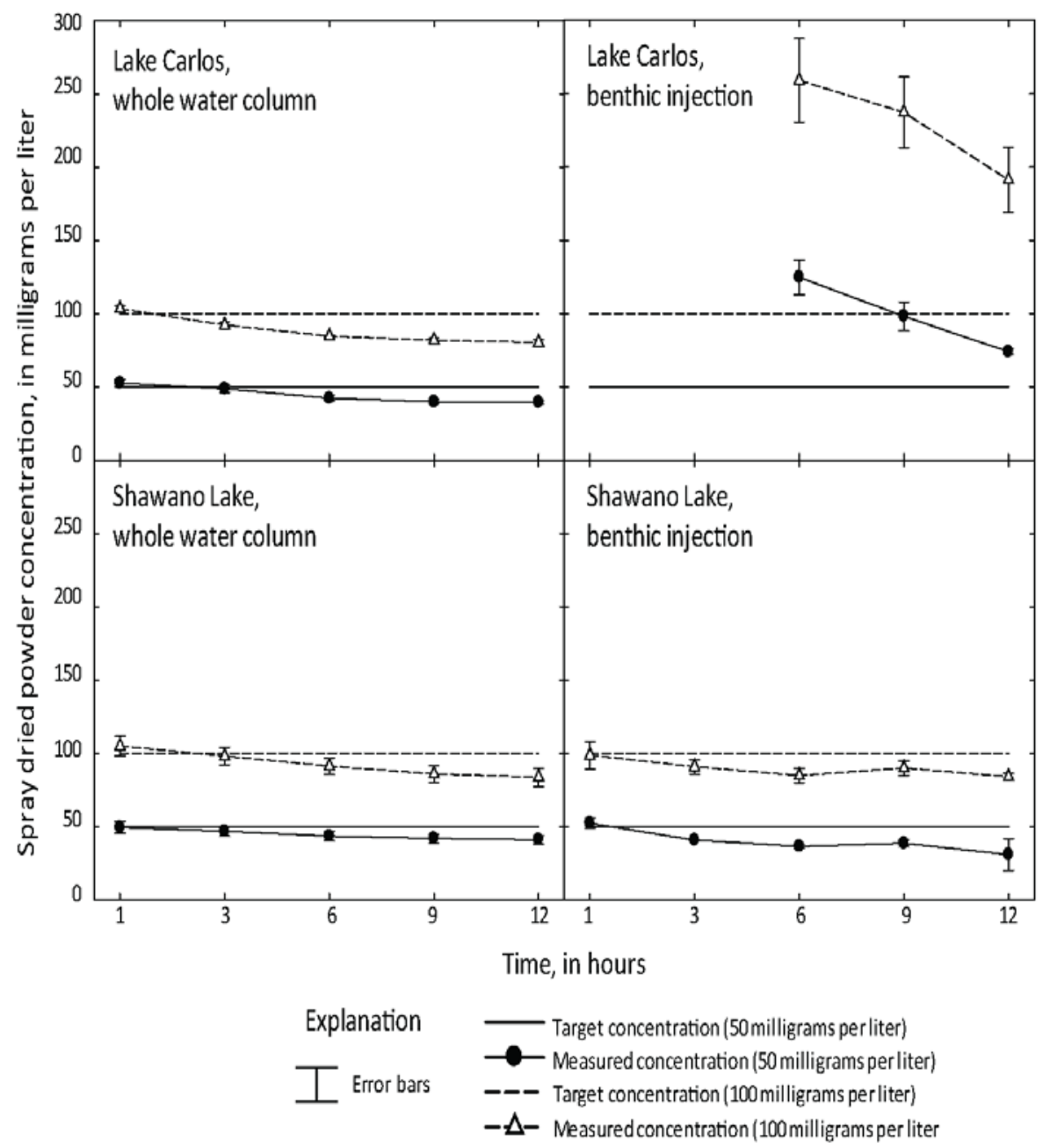

Figure 5. Mean (standard deviation) SDP active ingredient concentration of water samples collected during the exposure period. WWC graphs are from the surface samples; BI graphs are from bottom samples (Lake Carlos) and suspended samples (Shawano Lake)

\section{Zebra Mussel Survival}

Zebra mussel survival for all trials is summarized in table 4, statistical analyses are in appendix 9 (items 1-3), and survival data are in appendix 8 (items 1-5). For all trials (that is, WWC at 6, 9, and $12 \mathrm{~h}$; BI at $12 \mathrm{~h}$ ), mean survival of control groups exceeded 95 percent and zebra mussel survival in the SDP-treated groups differed $(p<0.01)$ from survival in the control groups. 
Table 4. Mean (standard deviation) percent zebra mussel survival for each lake, application type, and exposure duration.

[Means within columns and rows (for each lake) with the same letter are not significantly different $(p>0.05)$; means were compared by concentration (a-c), exposure duration (m,n) and between whole water column 12 h exposure duration and benthic injection 12 h exposure duration $(y, z)$; mg/L, milligrams per liter; h, hours; WWC, whole water column application; BI, benthic injection application]

\begin{tabular}{|c|c|c|c|c|c|}
\hline \multirow[t]{2}{*}{ Test location } & \multirow[t]{2}{*}{$\begin{array}{c}\text { Treatment } \\
\text { group (mg/L) }\end{array}$} & $6 \mathrm{~h}$ & $9 \mathrm{~h}$ & $12 \mathrm{~h}$ & \multirow{2}{*}{$\begin{array}{c}12 \mathrm{~h} \\
\mathrm{BI}\end{array}$} \\
\hline & & \multicolumn{3}{|c|}{ WWC } & \\
\hline \multirow[t]{3}{*}{ Lake Carlos } & 0 & $97.8^{\mathrm{am}}(2.2)$ & $96.9^{\mathrm{am}}(1.8)$ & $97.3^{\text {amz }}(1.0)$ & $97.3^{\mathrm{az}}(1.4)$ \\
\hline & 50 & $1.3^{\mathrm{bm}}(1.0)$ & $1.1^{\mathrm{bm}}(1.5)$ & $0.6^{\text {bmy }}(0.6)$ & $18.1^{\mathrm{bz}}(7.3)$ \\
\hline & 100 & $2.1^{\mathrm{bm}}(1.2)$ & $0.5^{\mathrm{bn}}(0.7)$ & $0.6^{\text {bny }}(0.7)$ & $18.0^{\mathrm{bz}}(10.4)$ \\
\hline \multirow[t]{3}{*}{ Shawano Lake } & 0 & $95.6^{\mathrm{am}}(2.4)$ & $95.5^{\mathrm{am}}(1.4)$ & $96.2^{\mathrm{amz}}(1.3)$ & $95.5^{\mathrm{az}}(2.3)$ \\
\hline & 50 & $12.6^{\mathrm{bm}}(10.7)$ & $10.3^{\mathrm{bm}}(9.4)$ & $2.7^{\mathrm{bnz}}(2.8)$ & $2.9^{\mathrm{bz}}(1.6)$ \\
\hline & 100 & $7.2^{\mathrm{bm}}(6.6)$ & $4.7^{\mathrm{bmn}}(5.5)$ & $2.0^{\mathrm{bnz}}(1.9)$ & $0.9^{\mathrm{cz}}(1.0)$ \\
\hline
\end{tabular}

\section{Lake Carlos Whole Water Column Trial}

Mean survival of zebra mussels in the Lake Carlos WWC SDP-treated groups ranged from 0.5 to 2.1 percent. When compared at the same exposure duration, no difference in zebra mussel survival was detected between the 50- and 100-mg/L treatment groups. When comparing the effects of exposure duration by treatment group, the only difference in zebra mussel survival detected was in the 100-mg/L treatment group, when the 6-h exposure duration group was compared to the 9-h and 12-h exposure duration groups $(p \leq 0.01)$.

\section{Shawano Lake Whole Water Column Trial}

Mean survival of zebra mussels in the Shawano Lake WWC SDP-treated groups ranged from 2.0 to 12.6 percent. When compared at the same exposure duration, no difference $(p>0.11)$ in zebra mussel survival was detected between the 50 - and $100-\mathrm{mg} / \mathrm{L}$ treatment groups. When comparing the effects of exposure duration by treatment group, no difference was detected in control group survival and in the 50-mg/L treatment group, no difference was detected when comparing the 6-h exposure duration to the 9-h exposure duration $(p=0.31)$. Differences were detected in the $50-\mathrm{mg} / \mathrm{L}$ treatment group when the 6 and 9-h exposure duration groups were compared to the 12-h exposure duration group $(p \leq 0.01)$. Differences were also detected in the 100-mg/L treatment group when the 6-h exposure duration group was compared to the 12-h exposure duration group $(p=0.01)$. No difference was detected in the 100-mg/L treatment group when comparing the 6-h exposure duration group to the 9-h exposure duration group ( $p=0.29$ ) or when comparing the 9-h exposure duration group to the 12-h exposure duration group $(p=0.10)$. 


\section{Benthic Injection Trials}

Mean survival of zebra mussels in the Lake Carlos BI trial SDP-treated groups did not differ ( $p=0.93$ ) and was 18.1 and 18.0 percent in the 50- and 100-mg/L treatment groups, respectively. Mean survival of zebra mussels in the Shawano Lake BI trial SDP-treated groups differed $(p<0.01)$ and was 2.9 and 0.9 percent in the 50 - and $100-\mathrm{mg} / \mathrm{L}$ treatment groups, respectively. Survival of zebra mussels in BI SDP-treated groups for both trials differed $(p<0.01)$ from zebra mussel survival in the control groups.

The survival of zebra mussels in the BI trials was compared to the survival of zebra mussels in the respective WWC trial 12-h exposure duration group. Survival of zebra mussels assigned to the Lake Carlos 12-h WWC trial SDP-treated groups differed $(p<0.02)$ from the survival of zebra mussels assigned to the Lake Carlos 12-h BI trial SDP-treated groups; however, after modification of the benthic injection application technique, no difference $(p=0.22)$ was detected between the survival of zebra mussel assigned to the Shawano Lake WWC 12-h exposure group and the Shawano Lake BI trial.

\section{Conclusions}

In this study, the application of the spray dried powder (SDP) formulation of Pseudomonas fluorescens (strain CL145A) at 50 and $100 \mathrm{mg} / \mathrm{L}$ (based on active ingredient) for 6-12 hours to test tanks containing lake water using either a whole water column (WWC) or benthic injection (BI) application technique significantly reduced the survival of zebra mussels. Mean survival of zebra mussels in the WWC SDP-treated groups did not exceed 12.6 percent for either trial at any exposure duration. No difference in zebra mussel survival was detected between the WWC 50- and 100-mg/L treatment groups in the Lake Carlos trial or in the Shawano Lake trial when compared at the same exposure duration. Mean survival of zebra mussels in the BI SDP-treated groups did not exceed 18.1 percent in the Lake Carlos trial and 2.9 percent in the Shawano Lake trial. After modification of the BI application method for the Shawano Lake trial, survival of zebra mussels in the BI trial did not differ ( $p=0.22$ ) compared to survival of zebra mussel in the Shawano lake WWC 12-h exposure group. In this study, the amount of SDP applied during the BI trials was 50 percent of that applied during the WWC trials. This study demonstrates that SDP has potential for use in managing dreissenid mussels in limited, open-water environments and that a benthic injection application technique to reduce the amount of SDP required to induce zebra mussel mortality may be successful in quiescent waters.

\section{References Cited}

American Public Health Association, American Water Works Association, and Water Environment Federation, 2012, Standard methods for examination of water and wastewater (22d ed.): American Public Health Association, 1360 p.

ASTM International, 2013, ASTM Standard E2455-06(2013)—Standard guide for conducting laboratory toxicity tests with freshwater mussels: West Conshohocken, Penn., ASTM International, $52 \mathrm{p}$.

Baker, S.M., and Hornbach, D.J., 1997, Acute physiological effects of zebra mussel (Dreissena polymorpha) infestation on two unionid mussels, Actinonaias ligamentina and Amblema plicata: Canadian Journal of Fisheries and Aquatic Sciences, v. 54, p. 512-519.

Birnbaum, C., 2011, NOBANIS-Invasive alien species fact sheet-Dreissena polymorpha: Online Database of the European Network on Invasive Alien Species, accessed February 13, 2014, at http://www.nobanis.org/files/factsheets/Dreissena_polymorpha.pdf. 
Burlakova, L.E., Karatayev, A.Y., and Padilla, D.K., 2000, The impact of Dreissena polymorpha (PALLAS) invasion on unionid bivalves: International Review of Hydrobiology, v. 85, no. 5-6, p. 529-541.

Emerson, K., Russo, R.C., Lund, R.E., and Thurston, R.V., 1975, Aqueous ammonia equilibrium calculations-Effect of $\mathrm{pH}$ and temperature: Journal of the Fisheries Research Board of Canada, v. 32, p. 2379-2383.

Engel, L., Valley, R., Beck, D., and Anderson, J., 2010, 2008 Sentinel Lake assessment of Lake Carlos (21-0057) in Douglas County, Minnesota: Report for Minnesota Pollution Control Agency and Minnesota Department of Natural Resources, 75 p. [Also available at http://www.pca.state.mn.us/index.php/view-document.html?gid=14962.]

Master, L., 1990, The imperiled status of North American aquatic animals: The Nature Conservancy, v. 3, no. 3, p. 5-8.

Neves, R.J., Bogan, A.E., Williams, J.D., Ahlstedt, S.A., and Hartfield, P.W., 1997, Status of aquatic mollusks in the southeastern United States-A downward spiral of diversity in Benz, G.W., and Collins, D.E., eds., Aquatic fauna in peril-The southeastern perspective: Decatur, Georgia, Southeastern Aquatic Research Institute, Lenz Design and Communications, p. 43-85.

Ricciardi, A., and Rasmussen, J.B., 1999, Extinction rates of North American freshwater fauna: Conservation Biology, v. 13, no. 5, p. 1220-1222.

Turyk, N., Foster, K., Hoverson, D., and McGinley, P., 2008, Watershed assessment of Shawano Lake, Shawano County, Wisconsin final report: University of Wisconsin Stevens Point, Center for Watershed Science and Education, 94 p. [Also available at http://www.uwsp.edu/cnrap/watershed/Documents/shawano_final_08.pdf.]

U.S. Environmental Protection Agency, 2013, Ambient water quality criteria for ammonia-Freshwater 2013: Washington, D.C., Office of Water, EPA 822-R-13-001, 242 p.

Williams, J.D., Warren, M.L., Jr., Cummings, K.S., Harris, J.L., and Neves, R.J., 1993, Conservation status of freshwater mussels of the United States and Canada: Fisheries, v. 18, no. 9, p. 6-22. 


\section{Appendix 1. Study Protocol, Amendments, and Datasheets}

\begin{tabular}{clrc}
\hline $\begin{array}{c}\text { Item } \\
\text { Number }\end{array}$ & \multicolumn{1}{c}{ Item Description } & $\begin{array}{c}\text { Number } \\
\text { of } \\
\text { Pages }\end{array}$ & $\begin{array}{c}\text { Report } \\
\text { Page } \\
\text { Number }\end{array}$ \\
\hline 1 & $\begin{array}{l}\text { Protocol: “Efficacy of Pseudomonas fluorescens (Pf-CL145A) SDP for controlling } \\
\text { settled zebra mussels on artificial substrates” }\end{array}$ & 28 & 19 \\
2 & Amendment 1: Revision of Study Protocol, Study \# AEH-12-PSEUDO-04 & 7 & 47 \\
3 & “Zebra Mussel Survival” Datasheet & 1 & 54 \\
4 & “Zebra Mussel Length” Datasheet & 1 & 55 \\
5 & “Test Chemical Stock Preparation Data Form” Datasheet & 1 & 56 \\
6 & “Conductivity and Hardness - Exposure Initiation” Datasheet & 1 & 57 \\
7 & “Alkalinity - Exposure Initiation” Datasheet & 1 & 58 \\
8 & "Water Quality - Temperature ( ${ }^{\circ}$ C) Measurements” Datasheet & 1 & 59 \\
9 & “Water Quality - pH Measurements” Datasheet & 1 & 60 \\
10 & "Water Quality - Dissolved Oxygen (mg/L) Measurements” Datasheet & 1 & 61 \\
11 & “Ammonia Sample Collection - Exposure Termination” Datasheet & 1 & 62 \\
\hline
\end{tabular}




\section{Protocol Title:}

Efficacy of Pseudomonas fluorescens (Pf-CL145A) SDP for controlling settled zebra mussels on artificial substrates

\section{Study Number: AEH-12-PSEUDO-04}

Test Facilities and Study Sponsor

Upper Midwest Environmental Sciences Center (UMESC)

Mobile Research Laboratory

US Geological Survey

2630 Fanta Reed Rd.

La Crosse, Wisconsin 54603

Proposed Experimental Start Date: August 2012

Proposed Experimental Termination Date: January 2013

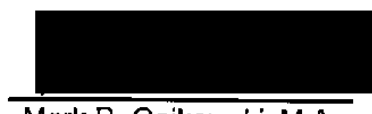

Mark P. Gaikowski, M.A.

Supervisory Biologist

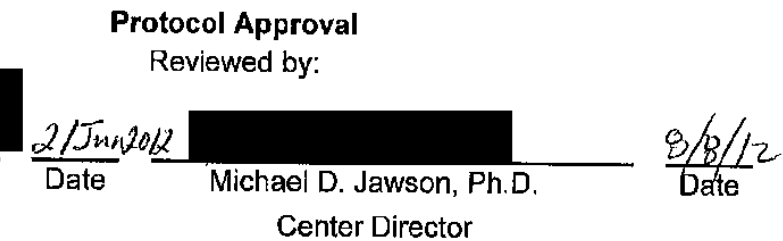

rotocol Approval

2/Jnn2012

Center Director

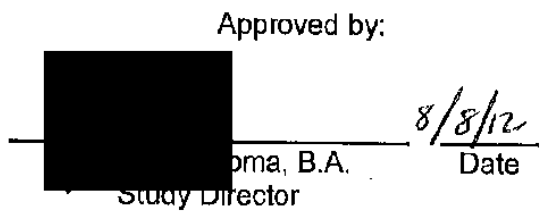

File Folder: 3

Page $\mathbf{1}$ of $\mathbf{2 8}$

Item Number: 


\section{TABLE OF CONTENTS}

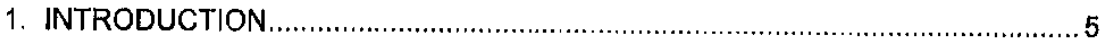

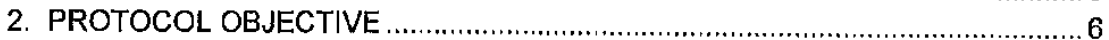

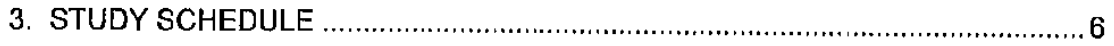

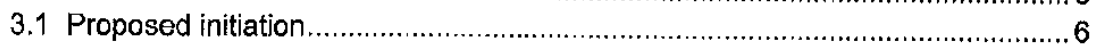

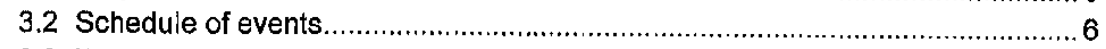

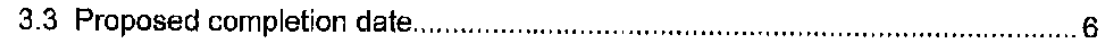

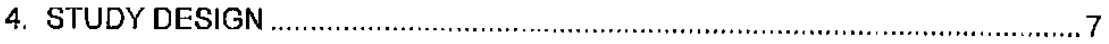

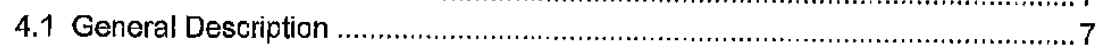

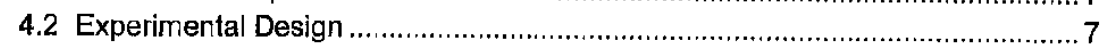

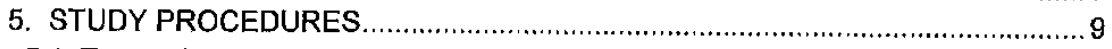

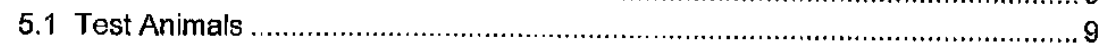

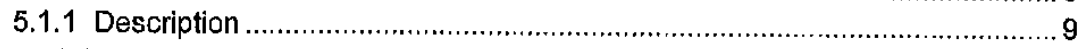

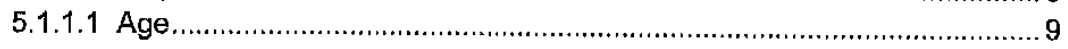

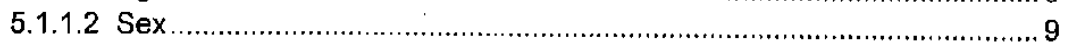

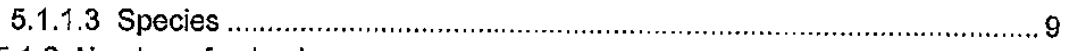

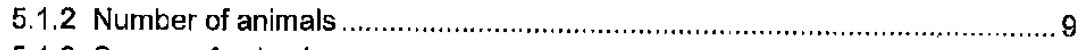

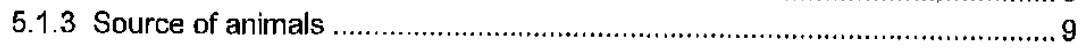

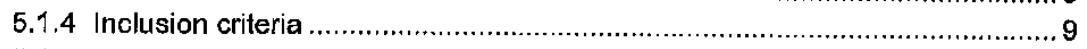

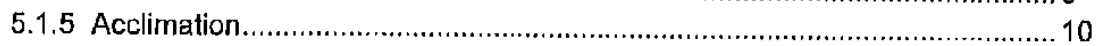

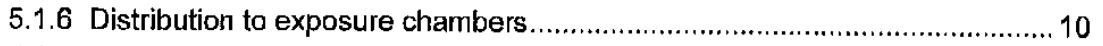

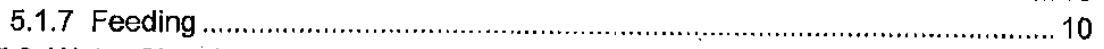

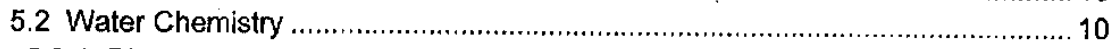

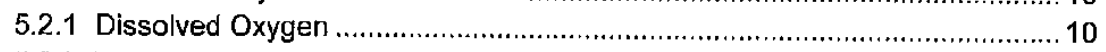

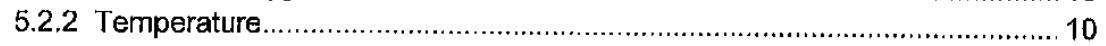

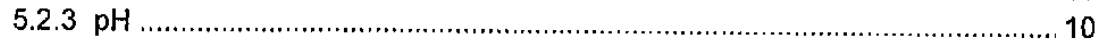

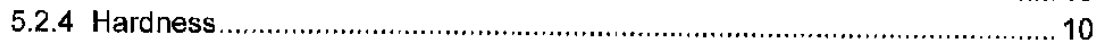

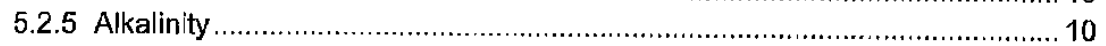

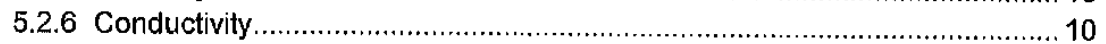

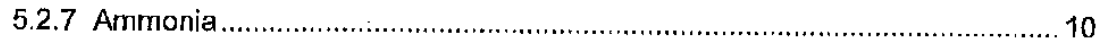

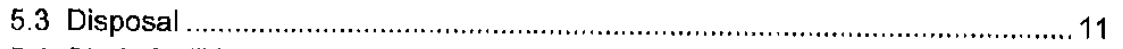

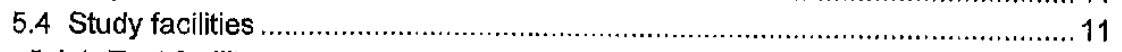

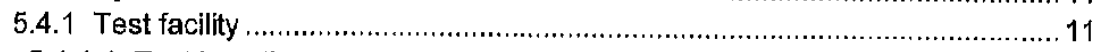

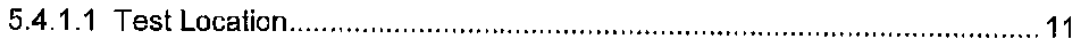

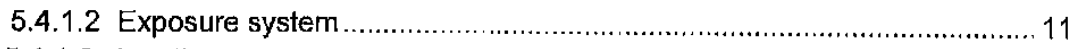

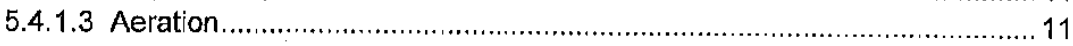

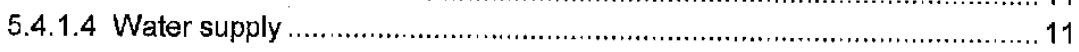

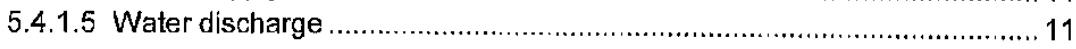

Page 2 of 28 


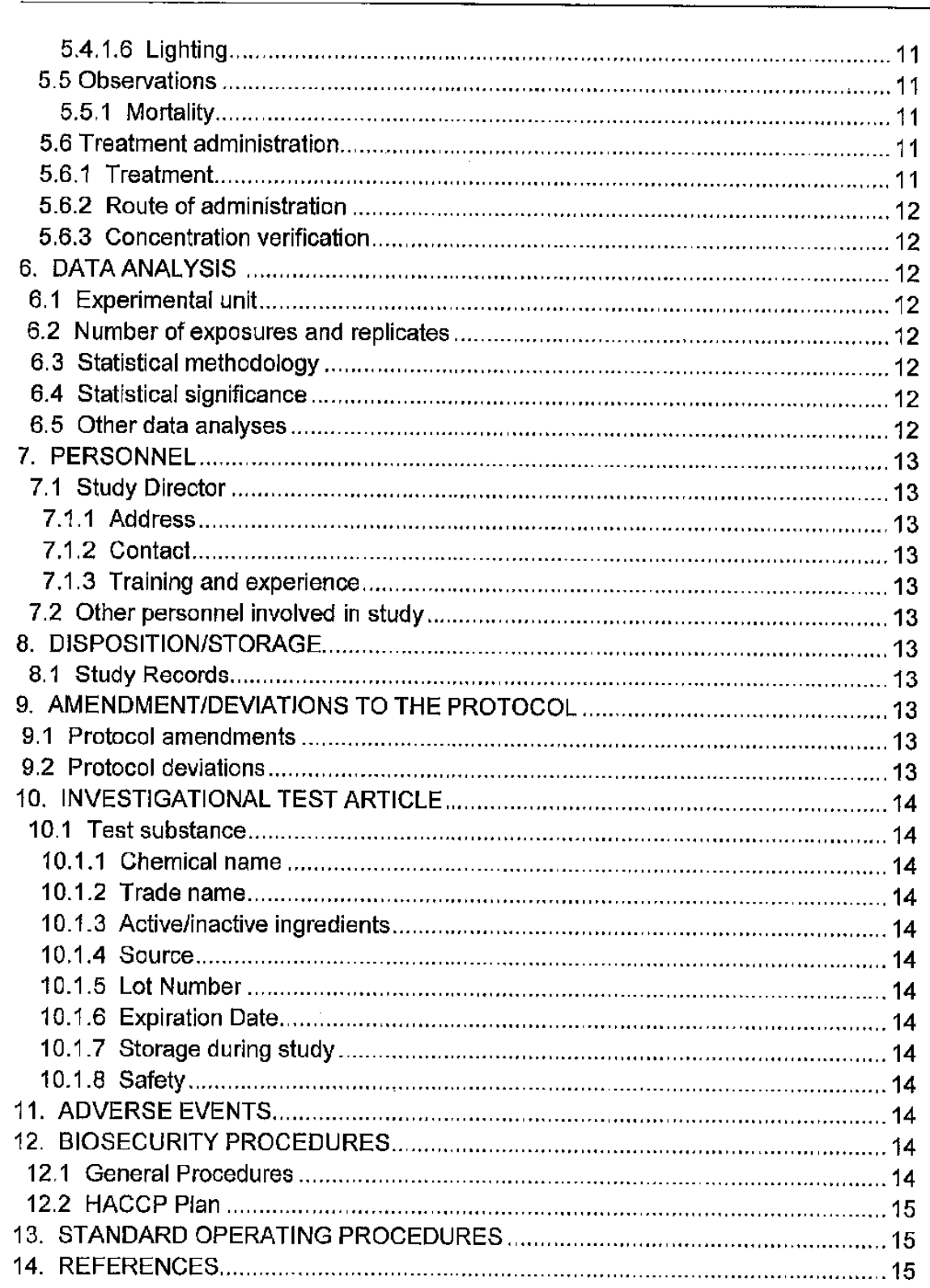

Page $\mathbf{3}$ of $\mathbf{2 8}$ 
STUDY NO. AEH-12-PSEUDO-04

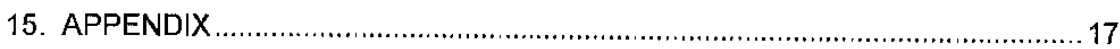

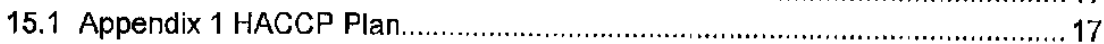

Page 4 of $\mathbf{2 8}$

Page 22 of 519 


\section{INTRODUCTION:}

Native freshwater mussel populations of North America were historically considered the most diverse in the world with about 297 recognized taxa consisting of 281 species and 16 subspecies (Williams et al., 1993). Mussels are largely sedentary in nature, relying on movement of host fish during glochidial attachment as means of transport. They are thus particularly vulnerable to a variety of anthropogenic influences including habitat degradation and alteration, pollution and overharvest. A Nature Conservancy survey (Master 1990) found 55\% of North America's mussels as extinct or imperiled compared to $7 \%$ of terrestrial species, even though terrestrial species traditionally receive far greater attention. Projections in 1999 (Ricciadi and Rasmussen, 1999) suggested that at least 127 imperiled mussel species will be lost in the next 100 years - a conservative extinction rate of $6.4 \%$ per decade given it did not take into account extirpations caused by invasive dreissenid mussels (zebra Dreissena polymorpha and quaqqa $D$. bugensis mussels).

Concerns for native mussels in the Southeast are potentially even greater given that only $25 \%$ of the 269 species historically present are reported as stable compared to the $13 \%$ presumed extinct and the 28,14 and $18 \%$ listed, respectively, as endangered, threatened or of special concern. (Neves et al,1997)

Many unionid mussels in North America were imperiled prior to epizoic colonization by zebra and quaqqa mussels though the introduction of dreissenid mussels have dramatically heightened concerns for the continued survival of native mussels. Zebra mussels were reported to be responsible for the extirpation of unionids from waters in Europe as early as 1937 (Sebestyen, 1937). Severe declines in unionid abundance in Europe (Karatayev and Burlakova, 1995; Burlakova, 1998) and North America (Haag et al, 1993; Nalepa, 1994; Ricciardi et al., 1996) have since been well documented in the literature.

The 1973 Endangered Species Act (ESA) brought forth the need to recognize, protect and recover rare mussels in the United States. The United States Fish and Wildlife Service (USFWS) develops recovery plans for threatened and endangered species which utilize a range of tools to promote recovery of the species including restoring and acquiring critical habitat, removing introduced or invasive species and captive propagation and release into historic ranges.

As of 2004, mussel propagation work was being conducted in several different facilities in 7 states as well as in Ontario, Canada (Neves, 2004). The Genoa National Fish Hatchery (GNFH) in Wisconsin has been involved in mussel recovery since 2000 , 
releasing tens of thousands of propagated subadult Higgins eye pearlymussel (Lampsilis higginsii) for recovery efforts. The GNFH produces subadult mussels using cage culture techniques. This technique involves placing glochidia laden host fish into submerged cages within natural water bodies such as the Mississippi and St. Croix Rivers. The fish are released from the cages after mussel excystment and the mussels are allowed to grow on the cage bottom for an additional 6-18 months before being harvested. Areas that were previously successful in rearing mussels using this technique have been abandoned due to the colonization and proliferation of zebra mussels.

Biologists at the New York State Museum (NYSM) Field Research Laboratory have been researching dreissenid mussel control techniques since 1991. They discovered that components of a strain of common bacterium isolated from soils (Pseudomonas fluorescens [Pf-CL145A]) are capable of causing mortality in zebra mussels. Marrone Bio Innovations ( $\mathrm{MBl}$; Davis, $\mathrm{CA}$ ) is currently developing a spray dried formulation of this bacterium called MBI-401SDP. A formulation of PF-CL145A was recently registered with the USEPA for use within closed systems such as power generating plant cooling systems. The NYSM has partnered with the USFWS (Genoa NFH) and United States Geological Survey's (USGS) Upper Midwest Environmental Sciences Center (UMESC) to determine the suitability of this product for open water zebra mussel control applications including treatment of native mussel propagation cages or native mussel beds.

Naturally occurring surface waters may be unique in their chemical and biological properties which may affect the efficacy of applied control agents such as Pf-CL145A. The research to be completed according to this protocol will assess the efficacy of various concentrations and treatment durations of Pseudomonas fluorescens (PfCL145A) for controlling settled zebra mussels (D. polymorpha) in open waters.

\section{PROTOCOL OBJECTIVE:}

To assess the efficacy of various exposure concentrations and treatment durations of Pseudomonas fluorescens (Pf-CL145A) spray dried powder (SDP) formulation for controlling settled zebra mussels (D. polymorpha) in open waters.

\section{STUDY SCHEDULE:}

3.1 Proposed initiation: August 2012

3.2 Schedule of events: A proposed schedule of events is provided in Table 1.

3.3 Proposed completion date: January 2013

Page 6 of 28 
Table 1. Proposed Schedule of Events

\begin{tabular}{cc}
\hline Date & Activity \\
\hline August 2012-Sept 2012 & substrate exposures \\
August 2012-October 2012 & substrate assessment \\
January 2013 & final report submission \\
\hline
\end{tabular}

\section{STUDY DESIGN:}

\subsection{General Description:}

Zebra mussel-encrusted, perforated aluminum substrates $(15.2 \mathrm{~cm} \times 15.2 \mathrm{~cm} \times$ $2.5 \mathrm{~cm}$ ) previously placed (10-11/2011and/or 5/2012) in two Minnesota and one Wisconsin water body will be exposed to varying concentrations and treatment durations of Pf-CL145A SDP formulation. The exposures will be conducted at Lake Carlos and Lake Pepin in Minnesota and Lake Shawano in Wisconsin with the assistance of MN or WI Department of Natural Resources. Replicated exposures will be conducted adjacent to each water body in an enclosed research trailer. Test water will be drawn from the adjacent water body. PfCL145A SDP formulation will be added to the water in the treatment tanks. The water containing $P F$-CL145A will be disposed of in compliance with the regulations of the respective DNR. Untreated water used pre- and post-exposure will be returned to the water body. The exposed substrates will be individually tagged and returned to the water body for approximately 3-4 weeks to assess post-exposure latent mortality.

\subsection{Experimental Design:}

In October and November of 2011 and again in May 2012 (depending on site) zebra mussel attachment substrates $(0.063$ " thick, type 3003 , perforated aluminum sheeting [3/16" hole, $51 \%$ open area] $15.2 \mathrm{~cm} \times 15.2 \mathrm{~cm}$ trays with 2.5 $\mathrm{cm}$ sides) were nested together in groups of 10 trays per stack (separated $\sim 2 \mathrm{~cm}$ with wooden blocking and zipped tied in place). Just prior to nesting, the trays were seeded by placing approximately 100-200 previously collected zebra mussels on the trays. The zebra mussels were collected ( $\leq 6-\mathrm{h}$ prior to use) by severing their byssal threads from rocks and other surfaces (e.g., sticks, native mussels, etc) with a scalpel; zebra mussels were maintained in a cooler until placed in an attachment substrate. At each location, approximately 60 nested trays were placed in a wire mesh cage $(\sim 0.9 \mathrm{~m}$ long $\times 0.6 \mathrm{~m}$ wide $\times 0.45 \mathrm{~m}$ high) and placed in $\sim 2 \mathrm{~m}$ of water for zebra mussel attachment and overwintering.

Substrate trays will be removed from the cages and placed in a semi-rigid plastic mesh bag $(\sim 20.3 \times 25.4 \times 5.1 \mathrm{~cm} ; 0.31 \times 0.31 \mathrm{~cm}$ openings $)$ and sealed with zip

Page 7 of $\mathbf{2 8}$ 
ties. Each substrate bag will uniquely tagged and randomly assigned to one of 9 test tanks within the mobile research trailer. At least 3 substrate bags and trays will be placed in each test tank containing up to $325 \mathrm{~L}$ of filtered $(200 \mu \mathrm{m})$ surface water. Flowing, filtered surface water will be supplied to the test tanks at a rate sufficient to achieve $\geq 1$ tank exchange per hour. The substrate bags and trays will be acclimated to conditions within the test tanks for $\geq 12-\mathrm{h}$ prior to administering a single Pf-CL145A SDP exposure. Treatment concentration (e.g. 0 [control], 50 or $100 \mathrm{mg} / \mathrm{L}$ ) will be randomly assigned to each test tank $(n=9)$ and each test tank will serve as an exposure replicate (3 replicates per treatment concentration). For each exposure day, a single treatment duration (je: 6,9 or 12-h) will be assigned for all treatment concentrations. At exposure termination the tanks will be drained of treated water, rinsed and refilled with flowing filtered surface water. Within $\mathbf{2 4} \mathrm{h}$ of exposure termination, the substrate bags and trays will be removed from the test tanks and returned to the wire mesh cages. The cages will then be placed in $\sim 2 \mathrm{~m}$ of water in the lake or river for a 3-4 week observation period. After 3-4 weeks (dependent on temperature) the cages will be removed from the water body and mussels enumerated for survival. After enumeration all test animals will be euthanized.

Figure 1. Flow chart of major study activities

Substrate trays are removed from the water body, placed in a sealed rigid plastic mesh bag and a un que indentification tag is placed on each bag Substrate bags containing trays are randomly assigned to 1 of 9 exposure tanks, a
minimum of 3 bags/trays are placed in each tank and a constant supply of surface water is supplied to each tank at $\geq 1$ tank exchange/h 
Figure 1. Continued.

Bags/trays are acclimated to test conditions for $\geq 12-h$; water inflow is ceased and static raridom exposure treatments are administered by the addition of appropriate amounts of Pf-CL145A SDP formulation; supplemental aeration is supplied.

Treatments will be a static exposure of 0 (control), 50 or $100 \mathrm{mg} / \mathrm{L}$ Pf-CL145A SDP formulation active ingredient (A.I.) with either a 6,9 or 12 -h exposure duration. Exposure

concentrations will be randomly assigned. For each exposure day, a single exposure duration $(6,9$ or 12-h) will be ass Igned with 3 replicates for each exposure concentration of 0 (control) 50 , or $100 \mathrm{mg} / \mathrm{L}$.

At exposure termination, water flow is resumed, tanks are flushed and rinsed. $\leq 24-\mathrm{h}$ post exposure termination the bags/trays are removed from the exposure tanks and the trays are placed in holding cages in $\sim 2 \mathrm{~m}$ of water

\section{5}

The cage positions are marked with GPS and a buoy, if required. After 3-4 weeks, the cages are removed from the lake and each tray is assessed for mortality, after assessment all animals are euthanized.

7

All equipment is thoroughly decontaminated prior to removal from the test site

\section{STUDY PROCEDURES}

\subsection{Test Animals}

5.1.1 Description:

\subsubsection{Age $-<18$ months}

5.1.1.2 Sex - Test animals will be used without regard to sex.

5.1.1.3 Species - (zebra mussel, Dreissena polymorpha)

5.1.2 Number of animals: Approximately 3,000-5,000 mussels, consistent with the objective of the study and contemporary scientific standards.

5.1,3 Source of animals: Animals will be collected from test location surface waters.

5.1.4 Inclusion criterion: Only trays with sufficient numbers $(n>30)$ of attached zebra mussels in apparent good health will be used.

Page $\mathbf{9}$ of $\mathbf{2 8}$ 
5.1.5 Acclimation: Mussels will be acclimated to conditions for $\geq 12-\mathrm{h}$ prior to exposure initiation.

5.1.6 Distribution to exposure tanks: One bag/tray will be distributed per exposure tank according to a predetermined randomization scheme in separate rounds. A minimum of 3 trays will be placed in each tank using a minimum of 3 distribution rounds.

5.1.7 Feeding: No supplemental feed will be offered throughout the acclimation, exposure and post-exposure holding periods.

\subsection{Water Chemistry}

5.2.1 Dissolved oxygen: Dissolved oxygen will be measured and recorded in each exposure tank during the acclimation, exposure and post-exposure holding periods. Dissolved oxygen will be measured and recorded at least once during the pre- and post-exposure periods and at least twice during the exposure period with the last measurement observed $\leq 30$ minutes prior to exposure termination (UMESC SOP AEH 394 or equivalent).

5.2.2 Temperature: Temperature will be measured and recorded in each exposure tank during the acclimation, exposure and post-exposure holding periods. Temperature will be measured and recorded at least once during the pre- and post-exposure periods and at least twice during the exposure period with the last measurement observed $\leq 30$ minutes prior to exposure termination.

5.2.3 $\mathrm{pH}: \mathrm{pH}$ will be measured and recorded in each exposure tank during the acclimation, exposure and post-exposure holding periods. $\mathrm{pH}$ will be measured and recorded at least once during the pre- and post-exposure periods and at least twice during the exposure with the last measurement observed $\leq 30$ minutes prior to exposure termination (UMESC SOP AEH 335 or equivalent).

5.2.4 Hardness: Hardness will be measured and recorded prior to exposure initiation (UMESC SOP AEH 712).

5.2.5 Alkalinity: Alkalinity will be measured and recorded prior to exposure initiation (UMESC SOP AEH 706).

5.2.6 Conductivity: Conductivity will be measured and recorded prior to exposure initiation (UMESC SOP AEH 188 or equivalent).

5.2.7 Ammonia: Samples for total ammonia-nitrogen will be collected at the termination of the exposure period for each exposure replicate. Ammonia samples will be filtered through a 0.45 micron syringe filter, acidified ( $\sim \mathrm{pH} 2.5)$ with sulfuric acid and then stored at $\sim 4^{\circ} \mathrm{C}$ until analyzed by the UMESC Long Term Resources Monitoring (LTRM) Water Quality Laboratory using the automated phenate method.

Page 10 of 28 
5.3 Disposal: All live mussels at the end of the post-exposure observation period will be euthanized and disposed by incineration or according to other state or local requirements.

5.4Study facilities:

5.4.1Teșt Facility

U.S. Geological Survey, Upper Midwest Environmental Sciences Center Mobile Research Laboratory

2630 Fanta Reed Rd

La Crosse, Wisconsin 54603

5.4.1.1 Test location: Lake Carlos, MN; Lake Pepin, MN; and Lake Shawano, WI

5.4.1.2 Exposure system: The test system consists of nine $76.2 \mathrm{~cm}$ diameter $x$ $91.4 \mathrm{~cm}$ deep fiberglass circular exposure tanks in two rows of 4 or 5 tanks. The tanks are supplied surface water through a pump, filter and headbox system. Each exposure tank will receive a continuous supply of water from a headbox during the pre- and post-exposure periods. Each tank will be uniquely identified to allow for identification treatment type and replicate number. Coding procedures will be documented in the study records.

5.4.1.3 Aeration: Supplemental aeration will be supplied during the acclimation, Pf-CL145A SDP exposures and the post exposure observation periods.

5.4.1.4 Water supply: Filtered surface water will be supplied continuously ( $\sim 5 \mathrm{~L} / \mathrm{min})$ to achieve a $\sim$ tank-volume exchange/h during the pre- and post-exposure periods. Water supply will be interrupted during the PfCL145A SDP exposures.

5.4.1.5 Water discharge: Untreated water will be returned to the surface water supply source. Pf-CL145A SDP treated water will be mechanically and/or carbon filtered and/or collected for disposal as required by DNR regulation.

5.4:1.6 Lighting: Overhead lighting ( $16 \mathrm{~h}$ light: $8 \mathrm{~h}$ dark) will be provided. 5.5 Observations:

5.5.1 Mortality: Zebra mussels that are gapping and do respond to tactile stimuli by shell closure will be coded as a mortality. Zebra mussels that have closed shells or respond to tactile stimuli by shell closure will be coded as alive.

5.6 Treatment administration:

5.6.1 Treatment: Each treatment will consist of three Pf-CL145A SDP concentrations (ie: 0 [control], 50 or $100 \mathrm{mg} / \mathrm{L} \mathrm{A.I.)} \mathrm{with} \mathrm{a} \mathrm{single} \mathrm{exposure}$

Page 11 of 28 
duration $(6,9$ or $12-\mathrm{h})$. All treatment concentrations will have three replicate exposure tanks. A minimum of 3 zebra mussel-encrusted substrate trays will be bagged and placed in each test replicate.

5.6.2 Route of administration: Exposures will be initiated by addition of an appropriate amount of a Pf-CL145A SDP stock solution. The tank will be gently mixed to achieve a uniform distribution of test material.

5.6.3 Concentration verification: Concentration will be determined spectrophotometrically. A standard curve will be prepared using a known mass of Pf-CL145A SDP. The absorbance of exposure solutions will be compared to the standard curve to determine the exposure concentration. Absorbance will be determined using a Barnstead/Thermolyne Corporation Model: Turner SP-830 Plus Beckman spectrophotometer (UMESC SOP AEH 302).

\section{DATA ANALYSIS}

6.1 Experimental unit: The experiment unit will be the exposure tank.

6.2 Number of exposures and replicates: There will be a total of 3 treatment levels

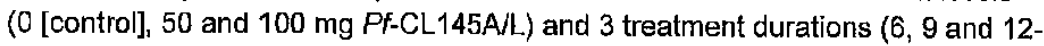
h) for each treatment level. There will be a total of 3 independent tanks for each treatment concentration and duration which will serve as the replicates. Each treatment concentration will be run concurrently for a single treatment duration. The trays from all treatment durations will be assessed for mortality at the same post exposure evaluation period.

6.3 Statistical methodology:

Survival data will be analyzed using a generalized linear mixed model (SAS PROC GLIMMIX). In every analysis, the exposure tank will be treated as the experimental unit. The change in proportion of survivors will be analyzed using a generalized linear mixed model where the distribution is binomial and the link used is the logit function.

If a significant effect of treatment is identified then pairwise comparison tests will be completed to compare each treatment group to the control group using unadjusted least squares means.

6.4 Statistical significance: Statistical significance will be declared at $p<0.05$.

6.5 Other data analyses: Statistical methods for other study data collected will include calculation of means, standard deviations and coefficients of variation. The statistical procedures used will be described in detail in the final study report.

Page 12 of 28 


\section{PERSONNEL}

\subsection{Study Director: James A. Luoma, B.A.}

7.1.1 Address: Upper Midwest Environmental Sciences Center, US Geological

Survey, 2630 Fanta Reed Rd., La Crosse, Wisconsin 54603

7.1.2 Contact: Tel: (608) 781-6391, Fax: (608) 783-6066; jluoma@usgs.gov

7.1.3 Training and experience: $\mathrm{CV}$ on file at UMESC.

7.2 Other personnel involved in study: Technical staff involved in the study will be identified in the study raw data to include study function. UMESC technical staff training and experience will be documented in CVs included in the study raw data.

\section{DISPOSITION/STORAGE}

8.1 Study Records: All data generated in the study will be recorded in bound laboratory notebooks, electronic files or kept in file folders. All data sheets, file folders, laboratory notebooks and computer disks will be encoded with the study number when the data are generated. Raw data, laboratory notebooks and electronic files (including a CD-ROM containing the annotated SAS program used for the statistical analysis, the data files, SAS log and SAS output files) generated by UMESC and contract laboratory reports will be filed in the UMESC archives (SOP No. GEN 007) of the Upper Midwest Environmental Sciences Center, La Crosse Wisconsin, before the final report is signed by the Study Director. The final report will then be signed and archived.

\section{AMENDMENT/DEVIATIONS TO THE PROTOCOL}

9.1 Protocol amendments: A signed copy of the Study Protocol will be retained on-site. Proposed amendments to the protocol shall be brought to the attention of UMESC Management. When the Study Director and Management agree verbally, the study can proceed with the change. As soon as possible, the Study Director will then prepare a written protocol amendment that is signed by the Study Director and Branch Chief. The amendment then becomes an official part of the protocol.

9.2 Protocol deviations: All deviations from this approved protocol will be documented and reviewed by the Study Director. The Study Director will make a judgment on the impact of the deviations. The Study Director will

Page 13 of 28 
notify Management, as soon as possible, of any deviations to the protocol, including their impact on the study.

\section{INVESTIGATIONAL TEST ARTICLE}

10.1 Test Substance(s): Pseudomonas fluorescens (PF-CL145A) SDP formulation 10.1.1 Chemical name: Pseudomonas fluorescens (Pf-CL145A)

10.1.2 Trade name: Zequanox

10.1.3 Active ingredients: Pseudomonas fluorescens (Pf-CL145A) is the sole active ingredient, $50 \%$ active by weight.

10.1.4 Source: Marrone Bio Innovations (MBI); Davis, CA

10.1.5 Lot number: Multiple lots are expected to be used during the exposures. Lot number(s) will be included in the test chemical log books, lab notebook, and study files.

10.1.6 Expiration date: As determined by the manufacturer. An aliquot of each lot tested will be returned to the NYSM or MBI at the conclusion of exposures for post-exposure zebra mussel bioassay tests (the standard testing protocol to assess Pseudomonas fluorescens [Pf-CL145A] formulation activity). Results of these confirmation bioassays will be used to validate the retention of activity of the Pseudomonas fluorescens (Pf-CL145A) SDP and will be included in the study files when available.

10.1.7 Storage during study: test chemical will be stored refrigerated. Test material will be transported in a cooler with ice packs to maintain proper storage temperature $\left(4-10^{\circ} \mathrm{C}\right)$

10.1.8 A NIOSH approved respirator will be used when preparing stock solutions to avoid inhalation. Protective eyewear, gloves and lab coats will be worn at all times when working with the test substance.

11. ADVERSE EVENTS: Any adverse event will be recorded in the study logbook and the Study Director will be notified.

\section{BIOSECURITY PROCEDURES}

12.1 General Procedures: All personnel involved in the study will review the UMESC biosecurity (UMESC SOP APP 075) and project HACCP plans. Testing will be conducted in a mobile laboratory with controlled access. All treated effluent water will be mechanically and/or carbon filtered and/or collected for contract disposal according to federal, state or local requirements.

Page 14 of 28 
12.2 HACCP Plan: See Appendix 1 for the HACCP plan for this project.

\section{STANDARD OPERATING PROCEDURES}

A complete list of the standard operating procedures used in the study will be included in the study guide. The follow SOP's were cited in this protocol:

UMESC SOP APP 075 - Procedures to Minimize the Risk of Transfer of Pathogens and Invasive Species

UMESC SOP AEH 188 - Accumet Portable Waterproof Conductivity meter Model \# AP75

UMESC SOP AEH 302 - Instrument Operating Procedure:

Barnstead/Thermolyne Corporation Model: Turner SP-830 Plus Beckman spectrophotometer Serial \# 1365070560781

UMESC SOP AEH 335 - Beckman Portable $\mathrm{pH} / \mathrm{mV}$ Meter, Model 250

UMESC SOP AEH 394 - YSI Handheld Dissolved Oxygen Meter, Model

55/12FT, Serials 94C17261 \& 97F0837AG

UMESC SOP AEH 706 - Determination of Total Alkalinity by the Titrimetric ( $\mathrm{pH}$

4.5) Method

UMESC SOP AEH 712 - Determination of Total Hardness

\section{REFERENCES.}

Burlakova, L.E., 1998. Ecology of Dreissena polymorpha (PALLAS) and its role in the structre and function of aquatic ecosystems. Candidate dissertation, Zoology Institute of the Academy of Science Republice Belarus, 168 p. (in Russian) in Burlakova L.E., A.Y. Karatayev and D. K. Padilla. 2000. The impact of Dreissena polymorpha (PALLAS) invasion on Unionid bivalves. Internat. Rev. hydrobiol, 85 (5-6):529-541.

Haag, W.R., D.L. Berg, D.W. Garton, and J.L. Farris, 1993. Reduced survival and fitness in native bivalves in response to fouling by the introduced zebra mussel (Dreissena polymorpha) in western Lake Erie. Canadian Journal of Fisheries and Aquatic Sciences 50(1):13-19.

Karatayev, A.Y. and L.E. Burlakova, 1995. Present and future patterns in Dreissena population development in the Narochanskaya lakes system. Vestisi Akad. Navuk Belarusi. Ser. Biyol. Navuk 3: 95098 (in Belarussian) in Burlakova L.E., A.Y. Karatayev and D. K. Padilla. 2000. The impact of Dreissena polymorpha (PALLAS) invasion on Unionid bivalves. Internat. Rev. hydrobiol. 85 (5-6):529-541.

Master, L., 1990. The imperiled status of North American aquatic animals. Biodiversity Network News 3(3):5-8.

Page 15 of 28 
Napela, T.F., 1994. Decline of native unionid bivalves in Lake St. Clair after infestation by the zebra mussel, Dreissena polymorpha. Can. J. Fish. Aquat. Sci. 51:2227-2233.

Neves R. J., 2004. Propagation of endangered freshwater mussels in North America. Journal of Conchology, special publication 3:69-80.

Neves, R.J., A.E. Bogan, J.D. Williams, S.A. Ahlstedt, and P.W. Hartfield, 1997, Status of aquatic mollusks in the southeastern United States: a downward spiral of diversity. Pages 43-85 in G.W. Benz and D.E. Collins, editors. Aquatic fauna in peril: the southeastern perspective. Southeastern Aquatic Research Institute, Lenz Design and communications, Decatur, Georgia.

Ricciardi, A. F.G. Whoriskey, and J.B. Rasamussen, 1996. Impact of the Dreissena invasion on native unionid bivalves in the upper St. Lawerance River. Can. J. Fish. Aquat. Sci. 53:1434-1444.

Ricciardi A., and J.B. Rasmussen, 1999. Extinction rates of North American freshwater fauna. Conservation Biology. Vol. 13 (5):1220-1222.

Sebestyn, O., 1937. Colonization of two new fauna-elements of Pontus-origin (Dreissena polymorpha Pall. And Corophuim cunvispinum G.O. Sars forma devium Wundsch) in Lake Balaton, Verh. Int. Ver. Theor. Angew. Limnol. 8:169-182 in Burlakova L.E., A.Y. Karatayev and D. K. Padilla. 2000. The impact of Dreissena polymorpha (PALLAS) invasion on Unionid bivalves. Internat. Rev. hydrobiol. 85 (56):529-541.

Williams, J.D., M.L. Warren Jr., K.S. Cummings, J.L. Harris, and R.J. Neves, 1993. Conservation status of freshwater mussels of the United States and Canada. Fisheries 18(9):6-22. 


\section{APPENDIX.}

15.1 Appendix 1. HACCP PLAN for the study Efficacy of Pseudomonas fluorescens (Pf-CL145A) SDP for controlling settled zebra mussels on artificial substrates

Step 1 - Activity Description

\begin{tabular}{|c|c|}
\hline $\begin{array}{l}\text { Facllity; US Geological Survey-Upper MIdwest Envirunmental Sciences } \\
\text { Center mobile research laboratory }\end{array}$ & Site: Various $-M N$ and WI \\
\hline S|te Coordlnator: Jim Ltoma & \multirow{4}{*}{$\begin{array}{l}\text { Activity: Efficacy of Pseudomonas fluorescens (PF-CL145A) SDF } \\
\text { for controlling settled zebra mussels on artificlal substrates }\end{array}$} \\
\hline Site Manager: Mark Galkowskl & \\
\hline $\begin{array}{l}\text { Address: } 2630 \text { Fanta Reed Road } \\
\text { L.a Crosse WI, } 54601\end{array}$ & \\
\hline Phone: 608-781-6322 & \\
\hline \multicolumn{2}{|c|}{ Projert Description } \\
\hline $\begin{array}{r}\text { The objectlve of this study is todetermine the efficacy of Pseudor } \\
\text { mussels on art }\end{array}$ & $\begin{array}{l}\text { onas fluorescens (Pf-CL145A) SDP for controlling seltled zebra } \\
\text { filal substrates }\end{array}$ \\
\hline
\end{tabular}

Step 2 - Potentlal Hazards: Specles which may potentlally be moved/Introduced

Vertebrates:

Multiple specles of freshwater fish, eggs and gametes found In the Mississlppl Rher and Great Lakes Basin.

Invertebrates:

Zebra mussel (Dreissena polymorpha)

Faucet snail (B/thynia tentacuiato)

Mult|ple endem|c specles found in the Mississippl River and Great Lakes Basin

Plants:

Euraslan water milfoll Myrlophyllum splcatum

Multiple endemlc species found In the Mlss|ssippi River and Great Lakes Basin

Other biologicals (dlsease, pathogen, paraslte):

Largemouth Bass Virus

Spring Viremia of Carp Virus

Bluegill Virus

Infect|cus Pancreatic Necrosls V|rus

VIral Hemorshagic Septicemla

rurunculosis Aeromonas salmonicida

Enterlc Redmouth D|sease Yorsinia rucker

Bacterial Kidney Disease Rentbacterium sulmontnor um

Other Assorted parasites/pathogens found in the Mississippl Rlver and Great Lakes Bas/r

Othor: 
STUDY NO. AEH-12-PSEUDO-04

Stap 3-Flow Dlagram

Flow dlagram outllning sequentlal tasks to complete activity/project

\begin{tabular}{|c|c|}
\hline \multirow{2}{*}{\multicolumn{2}{|c|}{ UMESC mobile research laboratory is brought to test slte and setup, water flow Initlated }} \\
\hline & \\
\hline Task 2 & Zebra mussel substrate trays are obtained from surface waters and moved into mobile laboratory \\
\hline \multicolumn{2}{|r|}{ - } \\
\hline \multirow{2}{*}{\multicolumn{2}{|c|}{ Substrate trays are exposed to test article }} \\
\hline & \\
\hline \multicolumn{2}{|l|}{$\downarrow$} \\
\hline Task 5 & Substrate trays are placed in holding cages and returned to surface waters for post-exposure holding \\
\hline \multicolumn{2}{|r|}{ 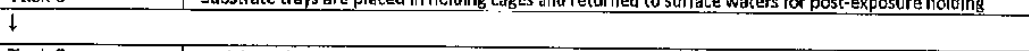 } \\
\hline Tasik 6 & Moblle traller, test tanks, hoses, pump and other equjpment is decontaminated and returned to LMESC \\
\hline \multicolumn{2}{|r|}{ - } \\
\hline Task 7 & Holdling cages and substrate trays are removed from surface waters and assessed for zebra mussel survival \\
\hline \multicolumn{2}{|r|}{ 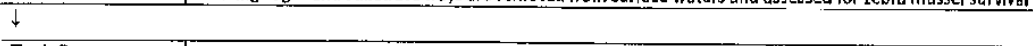 } \\
\hline Task 8 & Zebra mussels are removed from substrate trays and euthanlzed \\
\hline \multicolumn{2}{|l|}{ 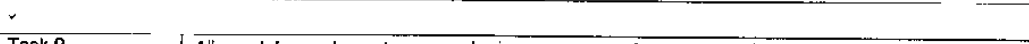 } \\
\hline Task 9 & All remalning equilpment, cages and sujstrate trays are decontaminated and returred to UMESC \\
\hline
\end{tabular}

\begin{tabular}{|c|c|c|c|c|c|}
\hline $\begin{array}{c}1 \\
\text { Tasks } \\
\text { [from HACCP Stop } 3 \\
\text { - Flow Dlagram) }\end{array}$ & $\begin{array}{c}2 \\
\text { Potentlal hazards } \\
\text { Identifled in HACCP } \\
\text { Step } 2\end{array}$ & $\begin{array}{c}\text { 3 } \\
\text { Are any potential } \\
\text { hazards probable? } \\
\text { (yes } / \text { no) }\end{array}$ & \begin{tabular}{|c|} 
\\
$\begin{array}{c}\text { Justify evaluatlan for } \\
\text { column } 3\end{array}$
\end{tabular} & $\begin{array}{c}5 \\
\text { What control } \\
\text { measures can be } \\
\text { applled to prevent } \\
\text { undesirable results? }\end{array}$ & $\begin{array}{c}\text { Is thls task a critical } \\
\text { control polnt? } \\
\text { (yes } / \mathrm{no} \text { ) }\end{array}$ \\
\hline $\begin{array}{l}\text { Task } 1 \\
\text { UMESC moblle } \\
\text { research laboratory } \\
\text { is brought to test site } \\
\text { and setup, water } \\
\text { flow Initlated }\end{array}$ & Vertebrates & yes & $\begin{array}{l}\text { Surface water } \\
\text { contains mu'tiple } \\
\text { vertebrate species }\end{array}$ & $\begin{array}{l}\text { Asstire the thorough } \\
\text { decontaminatlon all } \\
\text { equipment includilng } \\
\text { all Irternal and } \\
\text { external potentlally } \\
\text { wetted strfaces (le: } \\
\text { floors, tanks, hoses, } \\
\text { pumps, screens, nets, } \\
\text { etc) with steam, } \\
\text { chemoster|lants, or } \\
\text { other approved } \\
\text { methods prlor to } \\
\text { removal from previous } \\
\text { locatlon. Inspect alf } \\
\text { equlpment prior to set } \\
\text { up and repeat } \\
\text { decontamination if } \\
\text { warranted. }\end{array}$ & Yes \\
\hline
\end{tabular}

Page 18 of 28 
STUDY NO. AEH-12-PSEUDO-04

\begin{tabular}{|c|c|c|c|c|}
\hline \multicolumn{2}{|c|}{ Invertebrates } & $\begin{array}{l}\text { Surface water } \\
\text { contains multip'e } \\
\text { invertebrate specles } \\
\text { Include AIS }\end{array}$ & $\begin{array}{l}\text { Assure the thorough } \\
\text { decontaminatlon all } \\
\text { equlpment Induding } \\
\text { all interna, and } \\
\text { external potentlally } \\
\text { wetted surfaces fie; } \\
\text { floors, tanks, hoses, } \\
\text { pumps, screens, nets, } \\
\text { etc) with steam, } \\
\text { chemosterllants, or } \\
\text { other approved } \\
\text { methods prlor to } \\
\text { removal from previous } \\
\text { locatlon, inspect all } \\
\text { equlpment prlor to set } \\
\text { up and repeat } \\
\text { detontamination if } \\
\text { warranted. }\end{array}$ & Yes \\
\hline \begin{tabular}{|l|} 
Plants \\
\end{tabular} & yes & $\begin{array}{l}\text { Surlace water } \\
\text { contains mustiple } \\
\text { plant spectes } \\
\text { |nclud|ng AlS }\end{array}$ & $\begin{array}{l}\text { Assure the thorough } \\
\text { decontamination all } \\
\text { equipment Including } \\
\text { all Internal and } \\
\text { External potentially } \\
\text { wetted surfaces (le: } \\
\text { floors, tanks, hoses, } \\
\text { pumfs, screens, nets, } \\
\text { etc) with steam, } \\
\text { chemosterllants, or } \\
\text { other approved } \\
\text { methods prior to } \\
\text { removal from previous } \\
\text { locatlon, Inspect all } \\
\text { equipment prior to set } \\
\text { up and repeat } \\
\text { decontaminatlon lf } \\
\text { warranted. }\end{array}$ & yes \\
\hline Others & yes & $\begin{array}{l}\text { Surface water has } \\
\text { potential to transfer } \\
\text { fish diseases }\end{array}$ & $\begin{array}{l}\text { Assure the thorough } \\
\text { decortamination all } \\
\text { equlpment Including } \\
\text { all Internal and } \\
\text { external potentially } \\
\text { wetted surfaces (ie: } \\
\text { floors, tanks, hoses, } \\
\text { pumps, screens, nets, } \\
\text { etc) with steam, } \\
\text { themosterilants, or } \\
\text { other approved } \\
\text { methods prior to } \\
\text { removal from prevlous } \\
\text { location, Inspect all } \\
\text { equlpment prlor to set } \\
\text { up and repeat } \\
\text { decontamination If } \\
\text { warranted. }\end{array}$ & yes \\
\hline
\end{tabular}

Page 19 of 28

Page 37 of 519 
STUDY NO. AEF-12-PSEUDO-04

\begin{tabular}{|c|c|c|c|c|c|}
\hline \multirow[t]{3}{*}{$\begin{array}{l}\text { Task 2 } \\
\text { Zebra mussel } \\
\text { substrate trays are } \\
\text { obtalned from } \\
\text { surface waters and } \\
\text { moved into mobile } \\
\text { laboratory }\end{array}$} & Vertebrates & yes & $\begin{array}{l}\text { Surface water } \\
\text { contalns multiple } \\
\text { vertebrate specles }\end{array}$ & $\begin{array}{l}\text { Physlcal removal of } \\
\text { all visible } \\
\text { vertebrates prlor to } \\
\text { transfer Into moblle } \\
\text { research laboratory. } \\
\text { Assure the thorough } \\
\text { decontamlnatlon all } \\
\text { equipment including } \\
\text { all Internal and } \\
\text { external patentially } \\
\text { wetted sirfaces with } \\
\text { steam, } \\
\text { chemosterilants, or } \\
\text { other approved } \\
\text { methods prior to } \\
\text { removal from } \\
\text { location. }\end{array}$ & \begin{tabular}{|l|} 
Yes \\
\end{tabular} \\
\hline & Invertebrates & yos & $\begin{array}{l}\text { Surface water } \\
\text { contalns multiple } \\
\text { Inverttebrate specles } \\
\text { Include AIS }\end{array}$ & $\begin{array}{l}\text { Physical remioval of } \\
\text { all vistble } \\
\text { invertebrates not } \\
\text { required for testing } \\
\text { prlar to transfer Into } \\
\text { mobille research } \\
\text { laboratory. } \\
\text { Assure the thorough } \\
\text { decontamination all } \\
\text { equiprrent Including } \\
\text { all Internal and } \\
\text { external potentlally } \\
\text { wetled surfaces with } \\
\text { steam, } \\
\text { chemosterllants, ar } \\
\text { other approved } \\
\text { methods prlor to } \\
\text { removal from } \\
\text { locatlon. }\end{array}$ & Yes \\
\hline & Plarits & yes & $\begin{array}{l}\text { Surface water } \\
\text { contains multiple } \\
\text { plant specles } \\
\text { Including AIS }\end{array}$ & $\begin{array}{l}\text { Physlcal removal of } \\
\text { all visible plant } \\
\text { materlal prior to } \\
\text { transfer Into mnbile } \\
\text { research laboratory. } \\
\text { Assure the thorough } \\
\text { decontamination all } \\
\text { equlgment Including } \\
\text { all internal and } \\
\text { external potentially } \\
\text { wetted surfaces with } \\
\text { steam, } \\
\text { chemnosteri ants, or } \\
\text { other approved } \\
\text { methocs prior to } \\
\text { removal from } \\
\text { location. }\end{array}$ & Yes \\
\hline
\end{tabular}

Page 20 of 28 
STUDY NO. AEH-12-PSEUDO-04

\begin{tabular}{|c|c|c|c|c|c|c|}
\hline & Olhers & yes & 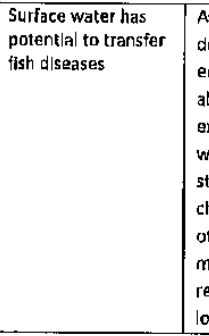 & $\begin{array}{l}\text { Assure the thorough } \\
\text { decontamination all } \\
\text { equlpment Including } \\
\text { all Internal and } \\
\text { external potentially } \\
\text { wetted surfares with } \\
\text { steam, } \\
\text { chemosterllants, or } \\
\text { other approved } \\
\text { methods prior to } \\
\text { removal from } \\
\text { location. }\end{array}$ & Yes & \\
\hline \multirow[t]{3}{*}{$\begin{array}{l}\text { Task } 3 \\
\text { Substrate trays are } \\
\text { acclimate to test } \\
\text { coroitlons with } \\
\text { flowing filtered surface } \\
\text { water }\end{array}$} & Vertebrates & ves & $\begin{array}{l}\text { Surface water contains } \\
\text { mult|ple vertebrate } \\
\text { species }\end{array}$ & \multicolumn{2}{|c|}{$\begin{array}{l}\text { Phvslcal removal of all } \\
\text { vislble vertebrates } \\
\text { prlor to transfer Into } \\
\text { moblle researcl } \\
\text { laboratory. } \\
\text { Assure the thorough } \\
\text { decontam|nation all } \\
\text { equlpment including all } \\
\text { Internal and external } \\
\text { potentlally watted } \\
\text { surfaces with steam, } \\
\text { chemosterllants, or } \\
\text { ot her approved } \\
\text { methods prlor to } \\
\text { removal from locatlon. }\end{array}$} & no \\
\hline & Invertebrates & yes & $\begin{array}{l}\text { Surface water contains } \\
\text { multiple invertebrate } \\
\text { species Ir tiude AIS }\end{array}$ & \multicolumn{2}{|c|}{$\begin{array}{l}\text { Physica removal of all } \\
\text { vislble Invertebrates } \\
\text { not required for testling } \\
\text { prlor to transfer Into } \\
\text { mobile research } \\
\text { laboratory. } \\
\text { Assure the thorough } \\
\text { decontam|nation all } \\
\text { equipment Induding all } \\
\text { internal and external } \\
\text { potentially wetted } \\
\text { surfaces with steam, } \\
\text { chemosterilants, or } \\
\text { other approved } \\
\text { methods prlor to } \\
\text { removal from location. }\end{array}$} & no \\
\hline & Plants & ves & $\begin{array}{l}\text { Surface water contalns } \\
\text { multiple plant species } \\
\text { induding AIS }\end{array}$ & \multicolumn{2}{|c|}{$\begin{array}{l}\text { Physical removal of all } \\
\text { visible plant materla! } \\
\text { prlor to transfer into } \\
\text { mobile research } \\
\text { laboratory. } \\
\text { Assure the thorough } \\
\text { decontaminatlon all } \\
\text { equipment Inclusinn all } \\
\text { internal and external } \\
\text { potentlally wetted } \\
\text { surfaces wlth steam, } \\
\text { chemosterilants, or } \\
\text { otler approved } \\
\text { methods prior to } \\
\text { removal from location. }\end{array}$} & no \\
\hline
\end{tabular}

Page 21 of 28 
STUDY NO. AEH-12-PSEUDO-04

\begin{tabular}{|l|l|l|l|l|l|}
\hline & Yes & $\begin{array}{l}\text { Surface water has } \\
\text { potentlal to transfer } \\
\text { fish diseases }\end{array}$ & $\begin{array}{l}\text { Assure the thorough } \\
\text { decontamination all } \\
\text { equipment including all } \\
\text { internal and external } \\
\text { potentlally wetted } \\
\text { surfaces wlth steam. } \\
\text { chemosterilants, or } \\
\text { other approved } \\
\text { methods prlor to } \\
\text { removal from locatlon. }\end{array}$ \\
\hline
\end{tabular}

\begin{tabular}{|c|c|c|c|c|c|c|}
\hline \multirow[t]{3}{*}{$\begin{array}{l}\text { Task } 4 \\
\text { Substrate trays are } \\
\text { exposed to test article }\end{array}$} & Vertebrates & yes & & $\begin{array}{l}\text { Surface water contains } \\
\text { mult|ple vertebrate } \\
\text { species }\end{array}$ & $\begin{array}{l}\text { Physical removal of all } \\
\text { visible vertebrates } \\
\text { prior to transler into } \\
\text { moblle research } \\
\text { laboratory. } \\
\text { Assure the thorough } \\
\text { decontaminatlon all } \\
\text { equipment including all } \\
\text { Internal and external } \\
\text { potentially wetted } \\
\text { surfaces with steam, } \\
\text { chemosterlants, or } \\
\text { other approved } \\
\text { methods prlor to } \\
\text { removal from location. }\end{array}$ & No \\
\hline & Invertebrates & yes & - & $\begin{array}{l}\text { Surface water contains } \\
\text { mult|ple Invertebrate } \\
\text { species include AIS }\end{array}$ & $\begin{array}{l}\text { Physlcal removal of all } \\
\text { vistb e invertebrates } \\
\text { not required for test|ng } \\
\text { prlor to transfer Irto } \\
\text { moblle research } \\
\text { laboratory. } \\
\text { Assure the thorough } \\
\text { dctontaminat|on all } \\
\text { eçuipment Including all } \\
\text { interna' and externa! } \\
\text { potentlally wetted } \\
\text { surfaces wlth steam, } \\
\text { chemosterilants, or } \\
\text { other approved } \\
\text { metlluds prior to } \\
\text { removal from location. }\end{array}$ & No \\
\hline & Plants & ves & . & $\begin{array}{l}\text { Surface water contains } \\
\text { multiple plant species } \\
\text { including AIS }\end{array}$ & $\begin{array}{l}\text { Fhysical removal of all } \\
\text { vislble plant material } \\
\text { prior lo transfer Into } \\
\text { mobille research } \\
\text { laboratory. } \\
\text { Assure the thorough } \\
\text { decontaminatlon all } \\
\text { equipment Including all } \\
\text { Internal and external } \\
\text { potentially wetted } \\
\text { surfaces with steam, } \\
\text { chemosterilants, or } \\
\text { other approved } \\
\text { methods prlor to } \\
\text { removal from location. }\end{array}$ & No \\
\hline
\end{tabular}

Page 22 of 28 
STUDY NO. AEH-12-PSEUDO-04

\begin{tabular}{|c|c|c|c|c|c|}
\hline & Others & yes & $\begin{array}{l}\text { Surface water has } \\
\text { potential to transfer } \\
\text { flsh diseases }\end{array}$ & $\begin{array}{l}\text { Assure the thorough } \\
\text { decortamination all } \\
\text { equlpment Including al } \\
\text { Internai and external } \\
\text { potentlally wetted } \\
\text { strfaces with steam, } \\
\text { chemosterllants, or } \\
\text { other approved } \\
\text { methods proor to } \\
\text { removal from location. }\end{array}$ & No \\
\hline \multirow[t]{3}{*}{$\begin{array}{l}\text { Task } 5 \\
\text { Substrate trays are } \\
\text { placed in holding } \\
\text { cages and returned to } \\
\text { surface waters for } \\
\text { post-exposure holding }\end{array}$} & Vertebrates & yes & $\begin{array}{l}\text { Surface water contalns } \\
\text { mult|ple vertebrate } \\
\text { specles }\end{array}$ & $\begin{array}{l}\text { Physlcal removal of all } \\
\text { vlstb'e vertebrates } \\
\text { prlor to transfer Into } \\
\text { moblle research } \\
\text { laboratory. } \\
\text { Assure the thorough } \\
\text { decontaminatlon all } \\
\text { equipment including all } \\
\text { interna and external } \\
\text { potentially wetted } \\
\text { surfaces wlth steam, } \\
\text { chemosterilants, or } \\
\text { other approved } \\
\text { methods prtor to } \\
\text { removal from location. }\end{array}$ & No \\
\hline & invertebrates & yes & $\begin{array}{l}\text { Surface water contains } \\
\text { multip e Invertebrate } \\
\text { species Include AlS }\end{array}$ & $\begin{array}{l}\text { Phys'cal removal of all } \\
\text { visible invertebrates } \\
\text { rot requilred for testing } \\
\text { prlor to transfer into } \\
\text { mobile research } \\
\text { laboratory. } \\
\text { Assure the thorough } \\
\text { decontaminatlon all } \\
\text { equipment Including all } \\
\text { internal and externa! } \\
\text { potenttally wetted } \\
\text { surfaces with steam, } \\
\text { chemosterilants, or } \\
\text { other approved } \\
\text { methcds prlor to } \\
\text { removal from location. }\end{array}$ & No \\
\hline & $\begin{array}{c}\text { Plants } \\
\text {. }\end{array}$ & yes & $\begin{array}{l}\text { Surface water contains } \\
\text { multiple plant specles } \\
\text { Including AIS }\end{array}$ & $\begin{array}{l}\text { Physlcal removal of all } \\
\text { visible plant material } \\
\text { prior to transfer Into } \\
\text { moblle research } \\
\text { laboratory. } \\
\text { Assure the thorough } \\
\text { decontam Inatlon all } \\
\text { equipment Including all } \\
\text { Internal and external } \\
\text { potentially wetted } \\
\text { surfaces with steam, } \\
\text { cherrosterilants, or } \\
\text { other approved } \\
\text { method's prlor to } \\
\text { removal from locatlon. }\end{array}$ & No \\
\hline
\end{tabular}

Page 23 of 28 
STUDY NO. AEH-12-PSEUDO-04

\begin{tabular}{|l|l|l|l|l|l|}
\hline & yes & $\begin{array}{l}\text { Surface water has } \\
\text { potential to transfer } \\
\text { flsh diseases }\end{array}$ & $\begin{array}{l}\text { Assure the therough } \\
\text { decontaminatlon all } \\
\text { equipment Including all } \\
\text { Internal and external } \\
\text { potentially wetted } \\
\text { surfaces with steam, } \\
\text { chemosterllants, or } \\
\text { other approved } \\
\text { methods prior to } \\
\text { removal from location. }\end{array}$ & nom \\
\hline
\end{tabular}

\begin{tabular}{|c|c|c|c|c|c|}
\hline \multirow[t]{4}{*}{$\begin{array}{l}\text { Task } 6 \\
\text { Mabile traller ، test } \\
\text { tanks, hoses, pump } \\
\text { and other equlpment } \\
\text { is decontamlnated and } \\
\text { returned to UMESC }\end{array}$} & Vertebrates & ves & $\begin{array}{l}\text { Surface water contalns } \\
\text { mult|ple vertebrate } \\
\text { species }\end{array}$ & $\begin{array}{l}\text { Physical remoyal of all } \\
\text { visible vertebrates. } \\
\text { Assure the thorough } \\
\text { decontamination all } \\
\text { equipment including al } \\
\text { Internal and external } \\
\text { potentially wetted } \\
\text { surfaces with steam, } \\
\text { chemosterllants, or } \\
\text { other approved } \\
\text { methods prior to } \\
\text { removal from location. }\end{array}$ & Yes \\
\hline & Invertebrates & yes & $\begin{array}{l}\text { Surface water cantalns } \\
\text { multiple invertebrate } \\
\text { specles include AIS }\end{array}$ & $\begin{array}{l}\text { Physical removal of all } \\
\text { vislble Invertebratos. } \\
\text { Assure the thorough } \\
\text { decontaminatlon all } \\
\text { equifment Including all } \\
\text { Internal and external } \\
\text { potenilally wetted } \\
\text { surfaces with steam, } \\
\text { chemosterllants, or } \\
\text { other approved } \\
\text { methods prlor to } \\
\text { removal from locatlon. }\end{array}$ & Yes \\
\hline & Plants & yes & $\begin{array}{l}\text { Surface water contains } \\
\text { multiple plant species } \\
\text { Including AIS }\end{array}$ & $\begin{array}{l}\text { Physical removal of all } \\
\text { vis'ble plant. } \\
\text { Assure the thorough, } \\
\text { decontaminatlon all } \\
\text { equipment Inciuding all } \\
\text { Internal and external } \\
\text { potentially wetted } \\
\text { surfaces with steam, } \\
\text { clemosterllants, or } \\
\text { other approved } \\
\text { methods prlor to } \\
\text { removal from location. }\end{array}$ & Yes \\
\hline & Others & ves & $\begin{array}{l}\text { Surface water has } \\
\text { potentia to transfer } \\
\text { flsh diseases }\end{array}$ & $\begin{array}{l}\text { Assure the thorough } \\
\text { decontamination all } \\
\text { equlpment including all } \\
\text { Internal and external } \\
\text { potenllally wetted } \\
\text { surfaces with steam, } \\
\text { chemosterllants, or } \\
\text { other approved } \\
\text { methods prior to } \\
\text { removal from location. }\end{array}$ & Yes \\
\hline
\end{tabular}

Page 24 of 28 
STUDY NO. AEH-12-PSEUDO-C4

\begin{tabular}{|c|c|c|c|c|c|}
\hline \multirow[t]{4}{*}{$\begin{array}{l}\text { Task } 7 \\
\text { Holding cages and } \\
\text { substrate trays are } \\
\text { removed from surface } \\
\text { waters and assessec } \\
\text { for zebra mussel } \\
\text { survival }\end{array}$} & \begin{tabular}{|l} 
Vertebrates \\
\end{tabular} & yes & $\begin{array}{l}\text { Surface water contalns } \\
\text { mult|ple vertebrate } \\
\text { species }\end{array}$ & $\begin{array}{l}\text { Physlcal removal of all } \\
\text { vistible vertebrates } \\
\text { prlor to transfer Into } \\
\text { moblle research } \\
\text { laboratory. } \\
\text { Assure the thorough } \\
\text { decontam!natlon al': } \\
\text { equlpment Including all } \\
\text { internal and external } \\
\text { potentlally wetted } \\
\text { surfaces wlth steam, } \\
\text { chemosterllants, or } \\
\text { otiner approved } \\
\text { mett.ods prlor to } \\
\text { removal from location. }\end{array}$ & Yes \\
\hline & Invertebrates & yes & $\begin{array}{l}\text { Surface water contalns } \\
\text { multiple invertebrate } \\
\text { specles include AIS }\end{array}$ & $\begin{array}{l}\text { Physica removal of all } \\
\text { vislble Invertebrates } \\
\text { not required for testing } \\
\text { prlor to transfer Into } \\
\text { moblle research } \\
\text { laboratory. } \\
\text { Assure the thorougl } \\
\text { decontam|natlon all } \\
\text { equlpment including all } \\
\text { internal and external } \\
\text { potentially wetted } \\
\text { surfaces with steam, } \\
\text { chemosterilants, or } \\
\text { other approved } \\
\text { methods prlor to } \\
\text { remcval from location. }\end{array}$ & Yes \\
\hline & Plants & yes & $\begin{array}{l}\text { Surface water contatns } \\
\text { multiple plant specles } \\
\text { Including Als }\end{array}$ & $\begin{array}{l}\text { Physical removal of all } \\
\text { vislble plant material } \\
\text { prior to transfer into } \\
\text { moblie research } \\
\text { laboratory. } \\
\text { Assure the thorough } \\
\text { decontaminatlon ail } \\
\text { equipment Including all } \\
\text { Internal and external } \\
\text { potentlally wetted } \\
\text { surfaces with steam, } \\
\text { ciem osterilants, or } \\
\text { other approved } \\
\text { methods prlor to } \\
\text { removal from location. }\end{array}$ & Yes \\
\hline & others & yes & $\begin{array}{l}\text { Surface water has } \\
\text { potentlal to transfer } \\
\text { fish diseases }\end{array}$ & $\begin{array}{l}\text { Assure the thorough } \\
\text { decontaminatlon all } \\
\text { equlpment including all } \\
\text { internal and external } \\
\text { potentlally wetted } \\
\text { surfaces with steam, } \\
\text { cherrosterllants, or } \\
\text { other approved } \\
\text { methods prlor to } \\
\text { removal from location, }\end{array}$ & yes \\
\hline
\end{tabular}

Page $\mathbf{2 5}$ of $\mathbf{2 8}$

Page 43 of 519 
STUDY NO. AEH-12-PSEUDO-04

\begin{tabular}{|c|c|c|c|c|c|}
\hline \multirow[t]{4}{*}{$\begin{array}{l}\text { Task } 8 \\
\text { Zebra mussels are } \\
\text { removed from } \\
\text { substrate trâys and } \\
\text { euthanized }\end{array}$} & Vertebrates & Yes & $\begin{array}{l}\text { Surface vater contains } \\
\text { multiple vertebrate } \\
\text { specles }\end{array}$ & $\begin{array}{l}\text { Physlcal removal of all } \\
\text { vislbie vertebrates } \\
\text { prlor to transfer Ir to } \\
\text { moblle research } \\
\text { laboratory. } \\
\text { Assure the thorougin } \\
\text { decontamination all } \\
\text { ecuipment including al! } \\
\text { interna and external } \\
\text { potentlally wetted } \\
\text { surfaces with steam, } \\
\text { chemosterilants, or } \\
\text { other approved } \\
\text { methods prfor to } \\
\text { removal from location, }\end{array}$ & Yes \\
\hline & Invertebrates & yes & $\begin{array}{l}\text { Surface water contalns } \\
\text { multiple Invertebrate } \\
\text { specles Include A|S }\end{array}$ & $\begin{array}{l}\text { Phys' cal removal of all } \\
\text { vislble Invertebrates } \\
\text { nct requlred for testling } \\
\text { prlor to transfer Into } \\
\text { moblle research } \\
\text { laboratory. } \\
\text { Assure the therough } \\
\text { decontaminatlon ali } \\
\text { equipment Including all } \\
\text { Internal and external } \\
\text { potentlally wetted } \\
\text { surfaces with steam, } \\
\text { chemosterilants, or } \\
\text { other approved } \\
\text { methods prlor to } \\
\text { removal from lacation. }\end{array}$ & Yes \\
\hline & Plants & ves & $\begin{array}{l}\text { Surface water contalns } \\
\text { multiple plant species } \\
\text { Including Ais }\end{array}$ & $\begin{array}{l}\text { Physlcal removal of all } \\
\text { vlsible plant material } \\
\text { prior to transfer into } \\
\text { moblle research } \\
\text { laboratory. } \\
\text { Assure the thorough } \\
\text { decontaminatlon all } \\
\text { equlpment Induding all } \\
\text { Internal and external } \\
\text { potentlally wetted } \\
\text { surfaces with steam, } \\
\text { chemosterilants, or } \\
\text { other approved } \\
\text { methods prior to } \\
\text { removal from location. }\end{array}$ & Yes \\
\hline & Others & yes & $\begin{array}{l}\text { Surface water has } \\
\text { potentlal to transfer } \\
\text { flsh dlseases }\end{array}$ & $\begin{array}{l}\text { Assure the thorough } \\
\text { decontaminatton all } \\
\text { equlpment Including all } \\
\text { internal and external } \\
\text { potentially wetted } \\
\text { surfaces with steam, } \\
\text { chemosterllants, or } \\
\text { other approved } \\
\text { methods prlor to } \\
\text { removal from locatlon. }\end{array}$ & yes \\
\hline
\end{tabular}

Page 26 of 28

Page 44 of 519 
STUDY NO. AEH-12-PSEUDO-04

\begin{tabular}{|c|c|c|c|c|c|}
\hline \multirow[t]{4}{*}{$\begin{array}{l}\text { Task } 9 \\
\text { All remalning } \\
\text { equipment, cages and } \\
\text { substrate trays are } \\
\text { decontaminated and } \\
\text { returned to UMESC }\end{array}$} & Vertebrates & yes & $\begin{array}{l}\text { Surface water contalns } \\
\text { multiple vertebrate } \\
\text { species }\end{array}$ & $\begin{array}{l}\text { Phys cal removal of all } \\
\text { visible vertebrates } \\
\text { prlor to transfer Into } \\
\text { moblle research } \\
\text { laboratory. } \\
\text { Assure the thorough } \\
\text { decontaminatlon all } \\
\text { equipment including all } \\
\text { Internal and cxternal } \\
\text { potentlally wetted } \\
\text { surfaces with steam, } \\
\text { chemosterilants, or } \\
\text { other approved } \\
\text { methods prior to } \\
\text { removal from location. }\end{array}$ & Yes \\
\hline & Invertebrates & ves & $\begin{array}{l}\text { Surface water contalns } \\
\text { multiple Invertejrate } \\
\text { species incluce AIS }\end{array}$ & $\begin{array}{l}\text { Physlcai removal of all } \\
\text { visible Invertebrates } \\
\text { not required for testing } \\
\text { prlor to transfer Into } \\
\text { mobile research } \\
\text { laboratory. } \\
\text { Assure the thorough } \\
\text { detontammation all } \\
\text { equlpment including all } \\
\text { Internal and external } \\
\text { potentlally wetted } \\
\text { surfaces with steam, } \\
\text { chemosterllants, or } \\
\text { other approved } \\
\text { methods prior to } \\
\text { removal from locatlon. } \\
\end{array}$ & Yes \\
\hline & Plants & ycs & $\begin{array}{l}\text { Surfece water contains } \\
\text { multiple plant specles } \\
\text { Including AIS }\end{array}$ & $\begin{array}{l}\text { Physical removal of all } \\
\text { visible plant materlal } \\
\text { prlor to transfer into } \\
\text { mabile research } \\
\text { :aboratory. } \\
\text { Assure the thorough } \\
\text { decontamlnation all } \\
\text { equlpment including all } \\
\text { Internal and external } \\
\text { potentially wetted } \\
\text { surfaces wlth steam, } \\
\text { chemosterlants, or } \\
\text { other approved } \\
\text { rethods prlor to } \\
\text { removal from location. } \\
\end{array}$ & Yes \\
\hline & Others & yes & \begin{tabular}{|l|} 
Surface water has \\
potentia! to transfer \\
flsh diseases
\end{tabular} & $\begin{array}{l}\text { Assure the thorough } \\
\text { decontarnInatlon all } \\
\text { equipment Including all } \\
\text { Internal and external } \\
\text { poter tlally wetted } \\
\text { surfaces with steam, } \\
\text { chemosterllants, or } \\
\text { other approved } \\
\text { methods prior to } \\
\text { removal from location. }\end{array}$ & ves \\
\hline
\end{tabular}

Page $\mathbf{2 7}$ of $\mathbf{2 8}$ 
STUDY NO. AEH-12-PSEUDO-04

\begin{tabular}{|c|c|c|c|c|c|c|c|c|}
\hline \multicolumn{9}{|c|}{$\begin{array}{l}\text { HACCP Plan Form } \\
\text { (all CCP's ar "yes's" from column } 6 \text { of HACCP Step } 4 \text { - Hazard Anaiys|s Worksheet) }\end{array}$} \\
\hline \multirow[b]{2}{*}{$\begin{array}{c}\text { Critica! } \\
\text { Contral } \\
\text { Point } \\
\text { (CCP) }\end{array}$} & \multirow[b]{2}{*}{$\begin{array}{l}\text { Slgnifileant } \\
\text { Hazard(s) }\end{array}$} & \multirow[b]{2}{*}{$\begin{array}{c}\text { Limits for each } \\
\text { Control Measure }\end{array}$} & \multicolumn{4}{|c|}{ Monltoring } & \multirow[b]{2}{*}{$\begin{array}{l}\text { Evaluation \& } \\
\text { Corrective } \\
\text { Actlon(s) } \\
\text { (If needed) }\end{array}$} & \multirow[b]{2}{*}{$\begin{array}{l}\text { Supporting } \\
\text { Documentation } \\
\text { (If any) }\end{array}$} \\
\hline & & & What & How & Frequency & Who & & \\
\hline $\begin{array}{l}\text { Tasks } 1,2 \\
3,6,7,8 \& 9\end{array}$ & \begin{tabular}{|c|} 
Transfer of \\
endemlc and \\
Als Ircluting \\
vertabrates, \\
invertebrates, \\
plants and \\
pathogens
\end{tabular} & \begin{tabular}{|c|} 
Transfer of \\
vertebrates, \\
Invertebrates, \\
plants and \\
pathogens must \\
not occur. All \\
equalpment must \\
be thoroughly \\
Inspected and \\
disinfected prior \\
to slie removal \\
and Inspected \\
and/or re- \\
decontaminated \\
upon setup at new \\
locatlon.
\end{tabular} & $\begin{array}{l}\text { Equlpment } \\
\text { disinfectlon }\end{array}$ & \begin{tabular}{|c|} 
Mechantcal \\
cleaning/remo \\
val, pressure \\
washing, \\
sleam \\
cleaning, \\
chemosterllant \\
or other \\
approved \\
methods. \\
\end{tabular} & $\begin{array}{c}\text { Pror to } \\
\text { equipment } \\
\text { arrival, upon } \\
\text { deployment, } \\
\text { upon } \\
\text { movement } \\
\text { from/to surface } \\
\text { water and prior } \\
\text { to departure }\end{array}$ & $\begin{array}{l}\text { Techniclans } \\
\text { lead fleld } \\
\text { supervisor }\end{array}$ & $\begin{array}{c}\text { Supervisor and' staff } \\
\text { are responslble for } \\
\text { mettiodlacal } \\
\text { decontaminatlon using } \\
\text { establlshed } \\
\text { procedures. } \\
\text { Correctlve actions } \\
\text { required to complete } \\
\text { decontamination must } \\
\text { be performed pror to } \\
\text { any equipment } \\
\text { movement from test } \\
\text { locatlon. } \\
\text { Decontamlnatlon and } \\
\text { Inspection must be } \\
\text { completed before } \\
\text { equlprnent removal } \\
\text { from test locatlon }\end{array}$ & \begin{tabular}{|c|} 
Records In log \\
books all \\
procedures used \\
for \\
decontamination
\end{tabular} \\
\hline \multicolumn{3}{|c|}{$\begin{array}{l}\text { Facillty: } \\
\text { Upper Midwest Environmental Sciences Center } \\
\text { Noblle Research Laboratory }\end{array}$} & & & \multicolumn{4}{|c|}{$\begin{array}{l}\text { Activity: Efficacy of Pseudomonas fluorescens (Pf-CL145A) SDP for } \\
\text { controlling settled zebra mussels on artificial substrates }\end{array}$} \\
\hline \multicolumn{5}{|c|}{$\begin{array}{l}\text { Address: } \\
2630 \text { Fanta Reed Road, ta Crosse, W1 } 54601\end{array}$} & & & & \\
\hline \multicolumn{3}{|c|}{$\begin{array}{l}\text { Signature: } \\
\text { HACCP Plan was followed. }\end{array}$} & & & \multicolumn{3}{|l|}{ Date: } & \\
\hline
\end{tabular}

File Folder: 3

Item Number: |

Page $\mathbf{2 8}$ of $\mathbf{2 8}$ 


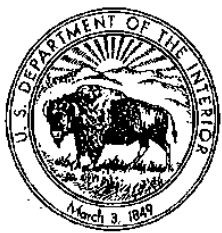

United States Department of the Interior

U.S. GEOLOGICAL SURVEY

Blological Resources Division

Upper Midwest Environmental Sciences Center

2630 Fanta Reed Road

La Crosse, Wisconsin 54603

Date: August 13, 2012

To: The Record Study Number AEH-12-PSEUDO-04

Subject: Amendment 1-Amendment to the study AEH-12-PSEUDO-04 "Efficacy of Pseudomonas fluorescens (Pf-CL145A) SDP for controlling settled zebra mussels on artificial substrates"

Revision of Study Protocol, Study \# AEH-12-PSEUDO-04 is proposed as detailed on pages 2-7 of this amendment. Revised text is indicated in bold.

This amendment details the 1) combining all exposure times into a single exposure bath with removal of substrates replicates at 6,9 and $12-h ; 2$ ) the elimination of supplemental aeration during the exposure period 3 ) the inclusion of a separate bottom layer treatment exposures if sufficient substrate trays remain after the initial whole tank exposures and 4) the delivery of test material, collection of water samples and water chemistry data.

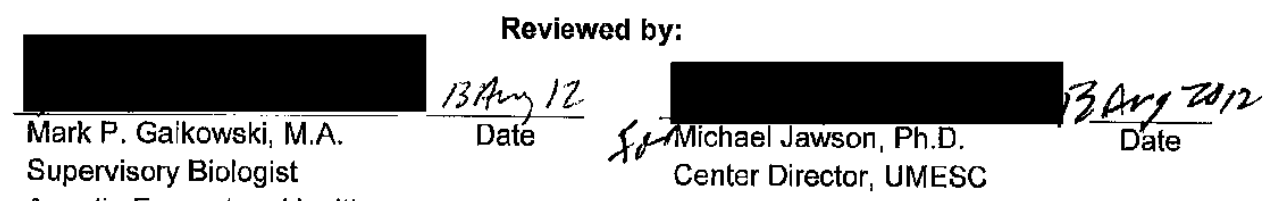

Aquatic Ecosystem Health, UMESC ${ }^{1}$

Approved by:

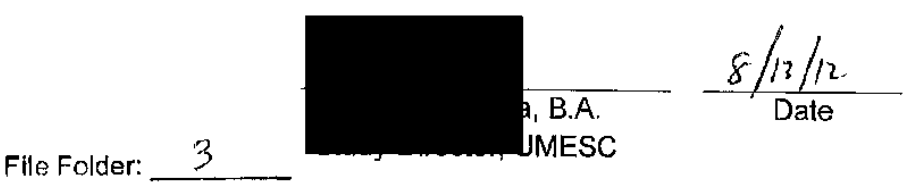
Item Number: 2

${ }^{1}$ UMESC: U.S. Geological Survey, Upper Midwest Environmental Sciences Center

Studył AEH-12-PSEUDO-04 Amendment \#1

Page 1 of 7 
Current text:

4.2 Experimental Design:

In October and November of 2011 and again in May 2012 (depending on site) zebra mussel attachment substrates $\left(0.063^{\prime \prime}\right.$ thick, type 3003 , perforated aluminum sheeting [ $3 / 16$ " hole, $51 \%$ open area] $15.2 \mathrm{~cm} \times 15.2 \mathrm{~cm}$ trays with 2.5 $\mathrm{cm}$ sides) were nested together in groups of 10 trays per stack (separated $\sim 2 \mathrm{~cm}$ with wooden blocking and zipped tied in place). Just prior to nesting, the trays were seeded by placing approximately $100-200$ previously collected zebra mussels on the trays. The zebra mussels were collected ( $\leq 6-h$ prior to use) by severing their byssal threads from rocks and other surfaces (e.g., sticks, native mussels, etc) with a scalpel; zebra mussels were maintained in a cooler until placed in an attachment substrate. At each location, approximately 60 nested trays were placed in a wire mesh cage $(\sim 0.9 \mathrm{~m}$ long $\times 0.6 \mathrm{~m}$ wide $\times 0.45 \mathrm{~m}$ high) and placed in $\sim 2 \mathrm{~m}$ of water for zebra mussel attachment and overwintering. .

Substrate trays will be removed from the cages and placed in a semi-rigid plastic mesh bag ( $20.3 \times 25.4 \times 5.1 \mathrm{~cm} ; 0.31 \times 0.31 \mathrm{~cm}$ openings) and sealed with zip ties. Each substrate bag will uniquely tagged and randomly assigned to one of $\theta$ test tanks within the mobile research trailer. At least 3 substrate bags and trays will be placed in each test tank containing up to $325 \mathrm{~L}$ of filtered $(200 \mu \mathrm{m})$ surface water. Flowing, filtered surface water will be supplied to the test tanks at a rate sufficient to achieve $\geq 1$ tank exchange per hour. The substrate bags and trays will be acclimated to conditions within the test tanks for $\geq 12$-h prior to administering a single $P f-C L 145 A$ SDP exposure. Treatment concentration (e.g. 0 [control], 50 or $100 \mathrm{mg} / \mathrm{L})$ will be randomly assigned to each test tank $(n=9)$ and each test tank will serve as an exposure replicate ( 3 replicates per treatment concentration). For each exposure day, a single treatment duration (ie: 6,9 or 12-h) will be assigned for all treatment concentrations. At exposure termination the tanks will be drained of treated water, rinsed and refilled with flowing filtered surface water. Within $24 \mathrm{~h}$ of exposure termination, the substrate bags and trays will be removed from the test tanks and retumed to the wire mesh cages. The cages will then be placed in $\sim 2 \mathrm{~m}$ of water in the lake or river for a 3-4 week observation period. After 3-4 weeks (dependent on temperature) the cages will be removed from the water body and mussels enumerated for survival. After enumeration all test animals will be euthanized.

\subsection{Water Chemistry}

5.2.1 Dissolved oxygen: Dissolved oxygen will be measured and recorded in each exposure tank during the acclimation, exposure and post-exposure holding periods. Dissolved oxygen will be measured and recorded at least once during the pre- and post-exposure periods and at least twice during the exposure period with the last measurement observed $\leq 30$ minutes prior to exposure termination (UMESC SOP AEH 394 or equivalent).

5.2.2 Temperature: Temperature will be measured and recorded in each exposure tank during the acclimation, exposure and post-exposure holding periods. Temperature will be measured and recorded at least 
once during the pre- and post-exposure periods and at least twice during the exposure period with the last measurement observed $\leq 30$ minutes prior to exposure termination.

5.2.3 $\mathrm{pH}$ : $\mathrm{pH}$ will be measured and recorded in each exposure tank during the acclimation, exposure and post-exposure holding periods. $\mathrm{pH}$ will be measured and recorded at least once during the pre- and post-exposure periods and at least twice during the exposure with the last measurement observed $\leq 30$ minutes prior to exposure termination (UMESC SOP AEH 335 or equivalent).

5.2.4 Hardness: Hardness will be measured and recorded prior to exposure initiation (UMESC SOP AEH 712).

5.2.5 Alkalinity: Alkalinity will be measured and recorded prior to exposure initiation (UMESC SOP AEH 706).

5.2.6 Conductivity: Conductivity will be measured and recorded prior to exposure initiation (UMESC SOP AEH 188 or equivalent).

5.2.7 Ammonia: Samples for total ammonia-nitrogen will be collected at the termination of the exposure period for each exposure replicate. Ammonia samples will be filtered through a 0.45 micron syringe filter, acidified ( pH 2.5) with sulfuric acid and then stored at $\sim 4^{\circ} \mathrm{C}$ until analyzed by the UMESC Long Term Resources Monitoring (LTRM) Water Quality Laboratory using the automated phenate method.

5.3 Disposal: All live mussels at the end of the post-exposure observation period will be euthanized and disposed by incineration or according to other state or local requirements.

5.4 Study facilities:

5.4.1 Test Facility

U.S. Geological Survey, Upper Midwest Environmental Sciences Center

Mobile Research Laboratory

2630 Fanta Reed Rd

La Crosse, Wisconsin 54603

5.4.1.1 Test location: Lake Carlos, MN; Lake Pepin, MN; and Lake Shawano, WI

5.4.1.2 Exposure system: The test system consists of nine $76.2 \mathrm{~cm}$ diameter $x$ $91.4 \mathrm{~cm}$ deep fiberglass circular exposure tanks in two rows of 4 or 5 tanks. The tanks are supplied surface water through a pump, filter and headbox system. Each exposure tank will receive a continuous supply of water from a headbox during the pre- and post-exposure periods. Each tank will be uniquely identified to allow for identification treatment type and replicate number. Coding procedures will be documented in the study records.

5.4.1.3 Aeration: Supplemental aeration will be supplied during the acclimation, Pf-CL145A SDP exposures and the post exposure observation periods. 
5.4.1.4 Water supply: Filtered surface water will be supplied continuously ( $\sim 5 \mathrm{~L} / \mathrm{min}$ ) to achieve a tank-volume exchange/h during the pre- and post-exposure periods. Water supply will be interrupted during the $P f$ CL145A SDP exposures.

5.4.1.5 Water discharge: Untreated water will be returned to the surface water supply source. PF-CL145A SDP treated water will be mechanically andior carbon filtered and/or collected for disposal as required by DNR regulation.

5.4.1.6 Lighting: Overhead lighting ( $16 \mathrm{~h}$ light: $8 \mathrm{~h}$ dark) will be provided. 5.5 Observations:

5.5.1 Mortality: Zebra mussels that are gapping and do respond to tactile stimuli by shell closure will be coded as a mortality. Zebra mussels that have closed shells or respond to tactile stimuli by shell closure will be coded as alive.

5.6 Treatment administration:

5.6.1 Treatment: Each treatment will consist of three PF-CL145A SDP concentrations (ie: 0 [control], 50 or $100 \mathrm{mg} / \mathrm{L} \mathrm{A.I.)}$ with a single exposure duration $(6,9$ or $12-h)$. All treatment concentrations will have three replicate exposure tanks. A minimum of 3 zebra mussel-encrusted substrate trays wil be bagged and placed in each test replicate.

5.6.2 Route of administration: Exposures will be initiated by addition of an appropriate amount of a Pf-CL145A SDP stock solution. The tank will be gently mixed to achieve a uniform distribution of test material.

5.6.3 Concentration verification: Concentration will be determined spectrophotometrically. A standard curve will be prepared using a known mass of Pf-CL145A SDP. The absorbance of exposure solutions will be compared to the standard curve to determine the exposure concentration. Absorbance will be determined using a Barnstead/Thermolyne Corporation Model: Turner SP-830 Plus Beckman spectrophotometer (UMESC SOP AEH 302).

Revised text (in bold):

4.2 Experimental Design:

In October and November of 2011 and again in May and August 2012

(depending on site and observed mussel condition) zebra mussel attachment substrates $(0.063$ " thick, type 3003 , perforated aluminum sheeting [3/16" hole, $51 \%$ open area] $15.2 \mathrm{~cm} \times 15.2 \mathrm{~cm}$ trays with $2.5 \mathrm{~cm}$ sides) were nested together in groups of 10 trays per stack (separated $\sim 2 \mathrm{~cm}$ with wooden blocking or staggered to allow open corners and zip tied in place). Just prior to nesting, the trays were seeded by placing approximately $100-200$ previously collected zebra mussels on the trays. The zebra mussels were collected ( $\leq 6-\mathrm{h}$ prior to use) by severing their byssal threads from rocks and other surfaces (e.g., sticks, native mussels, etc) with a scalpel; zebra mussels were maintained in a cooler until placed in an attachment substrate. At each location, approximately 60 nested trays were placed in a wire mesh cage $(\sim 0.9 \mathrm{~m}$ long $\times 0.6 \mathrm{~m}$ wide $\mathrm{x}$ 
$0.45 \mathrm{~m}$ high) and placed in $\sim 2 \mathrm{~m}$ of water for zebra musse] attachment. Substrate trays will be removed from the cages and placed in a semi-rigid plastic mesh bag $(\sim 20.3 \times 25.4 \times 5.1 \mathrm{~cm} ; \sim 0.31 \times 0.31 \mathrm{~cm}$ openings $)$ and sealed with zip ties. Each substrate bag will uniquely tagged and randomly assigned to one of 9 test tanks within the mobile research trailer. At least 3 substrate bags and trays will be placed in each test tank containing $350 \mathrm{~L}$ of filtered $(200 \mu \mathrm{m})$ surface water. Flowing, filtered surface water will be supplied to the test tanks at a rate sufficient to achieve $\geq 1$ tank exchange per hour. The substrate bags and trays will be acclimated to conditions within the test tanks for $\geq 12 \cdot \mathrm{h}$ prior to administering a single whole tank treatment of Pf-CL145A SDP. Treatment concentration (e.g. 0 [control], 50 or $100 \mathrm{mg} / \mathrm{L}$ ) will be randomly assigned to each test tank $(n=9)$ and each test tank will serve as an exposure replicate (3 replicates per treatment concentration). For each exposure, 9 trays will be randomly assigned to each of 3 treatment concentration replicates. At each exposure termination time point $(6,9$ and $12-h)$ three randomly selected trays will be immediately removed from each treatment replicate, rinsed with tempered surface water and returned to the wire mesh cages located in $\sim 1 \mathrm{~m}$ of water in the lake or river. Within $12 \mathrm{~h}$ of exposure termination the cages will then be relocated and placed in $\sim 2 \mathrm{~m}$ of water in the lake or river for a 3-4 week observation period.

If sufficient untreated substrate trays remain, an additional exposure will be completed in an identical fashion except that 1) the number of trays per replicate and exposure duration time point will be adjusted according to the number of avallable substrate and 2) the exposures will be conducted as a bottom layer injection designed to treat the bottom $50 \%$ of the water column within the tank. The injection design, number of trays and exposure durations tested will be recorded in the study files.

After 3-4 weeks (dependent on temperature) the cages will be removed from the water body and mussels enumerated for survival. After enumeration all test animals will be euthanized.

\subsection{Water Chemistry}

5.2.1 Dissolved oxygen: Dissolved oxygen will be measured and recorded in each exposure tank during the acclimation and exposure periods. Dissolved oxygen will be measured and recorded at least once during the acclimation period and at least twice during the exposure period with the last measurement observed $\leq \mathbf{3 0}$ minutes prior to exposure termination (UMESC SOP AEH 394 or equivalent).

5.2.2 Temperature: Temperature will be measured and recorded in each exposure tank during the acclimation and exposure period. Temperature will be measured and recorded at least once during the acclimation period and at least twice during the exposure period with the last measurement observed $\leq \mathbf{3 0}$ minutes prior to exposure termination.

5.2.3 $\mathrm{pH}: \mathrm{pH}$ will be measured and recorded in each exposure tank during the acclimation and exposure period. $\mathrm{pH}$ will be measured and 
recorded at least once during the acclimation period and at least twice during the exposure period with the last measurement observed $\leq \mathbf{3 0}$ minutes prior to exposure termination (UMESC SOP AEH 335 or equivalent).

5.2.4 Hardness: Hardness will be measured and recorded prior to exposure initiation in each headbox (UMESC SOP AEH 712).

5.2.5 Alkalinity: Alkalinity will be measured and recorded prior to exposure initiation in each headbox (UMESC SOP AEH 706).

5.2.6 Conductivity: Conductivity will be measured and recorded prior to exposure initiation in each headbox (UMESC SOP AEH 188 or equivalent).

5.2.7 Ammonia: Samples for total ammonia-nitrogen will be collected at the termination of the exposure period for each exposure replicate. Ammonia samples will be filtered through a 0.45 micron syringe filter, acidified ( $\sim \mathrm{pH} 2.5)$ with sulfuric acid and then stored at $\sim 4^{\circ} \mathrm{C}$ until analyzed by the UMESC Long Term Resources Monitoring (LTRM) Water Quality Laboratory using the automated phenate method.

5.3 Disposal: All live mussels at the end of the post-exposure observation period will be euthanized and disposed by incineration or according to other state or local requirements.

5.4 Study facilities:

5.4.1 Test Facility

U.S. Geological Survey, Upper Midwest Environmental Sciences Center

Mobile Research Laboratory

2630 Fanta Reed Rd

La Crosse, Wisconsin 54603

5.4.1.1 Test location: Lake Carlos, MN; Lake Pepin, MN; and Lake Shawano, WI

5.4.1.2 Exposure system: The test system consists of nine $76.2 \mathrm{~cm}$ diameter $x$ $91.4 \mathrm{~cm}$ deep fiberglass circular exposure tanks in two rows of 4 or 5 tanks. The tanks are supplied surface water through a pump, filter and headbox system. Each exposure tank will receive a continuous supply of water from a headbox during the acclimation period. Each tank will be uniquely identified to allow for identification treatment type and replicate number. Coding procedures will be documented in the study records.

5.4.1.3 Aeration: Supplemental aeration may be supplied during the acclimation period and will not be supplied during the Pf-CL145A SDP exposure period. Use of supplemental aeration will be documented in the study record. 
5.4.1.4 Water supply: Filtered surface water will be supplied continuously ( $\sim 5 \mathrm{~L} / \mathrm{min}$ ) to achieve a $\sim$ tank-volume exchange/h during the acclimation period. Water supply will be interrupted during the PfCL145A SDP exposures.

5.4.1.5 Water discharge: Untreated water will be returned to the surface water supply source. PF-CL145A SDP treated water will be mechanically and/or carbon filtered and/or collected for disposal as required by DNR regulation.

5.4.1.6 Lighting: Overhead lighting ( $16 \mathrm{~h}$ light:8 $\mathrm{h}$ dark) will be provided.

5.5 Observations:

5.5.1 Mortality: Zebra mussels that are gapping and do respond to tactile stimuli by shell closure will be coded as a mortality. Zebra mussels that have closed shells or respond to tactile stimuli by shell closure will be coded as alive.

5.6 Treatment administration:

5.6.1 Treatment: Each treatment will consist of three Pf-CL145A SDP concentrations (ie: 0 [control], 50 or $100 \mathrm{mg} / \mathrm{L}$ A.I.) with multiple exposure durations (6,9 and 12-h). All treatment concentrations will have three replicate exposure tanks. A minimum of 3 zebra musselencrusted substrate trays will be bagged and placed in each test replicate. Randomly selected trays will be removed from each test repllcate at the end of each exposure duration, rinsed with tempered surface water and returned to the wire mesh holding cages.

5.6.2 Route of administration: Exposures will be initiated by addition of an appropriate amount of a Pf-CL145A SDP stock solution. In whole tank treatments, the tank will be gently mixed to achieve a uniform distribution of test material. In the bottom injection treatments the appropriate amount of a Pf-CL.145A SDP stock solution will be delivered with a peristaltic pump at $\sim 1 / 4$ of the water column height $(\sim 19 \mathrm{~cm})$ using four suspended delivery tubes.

5.6.3 Concentration verification: Concentration will be determined spectrophotometrically. A standard curve will be prepared using a known mass of Pf-CL145A SDP. The absorbance of exposure solutions will be compared to the standard curve to determine the exposure concentration. Absorbance will be determined using a Barnstead/Thermolyne Corporation Model: Turner SP-830 Plus Beckman spectrophotometer (UMESC SOP AEH 302) or equivalent. Samples collected during the whole tank treatments exposures will be collected from the surface of the exposure replicates. Samples collected during the bottom layer injection exposures will be collected with a peristaltic pump from the bottom at $\sim 1 / 4$ of the water column height $(\sim 19 \mathrm{~cm})$ using four suspended collection tubes. The collected water from each delivery tube will be pooled for each replicate tank and analyzed for Pf-CL145A SDP concentration. Concentrations will be verified in each replicate within 30 minutes of initial dosing and at 3, 6, 9 and 12-h post-dosing.

File Foider: 


\section{Zebra Mussel Survival}

Test Organism: Zebra Mussels Applications Type:
Test Chemical: Pf-CL145A SDP Lot \#: $\frac{401 P 12163 \mathrm{C} \text { and 401P12164C Mix }}{\text { Holding cage GPS Coordinates: }}$
Test Location:

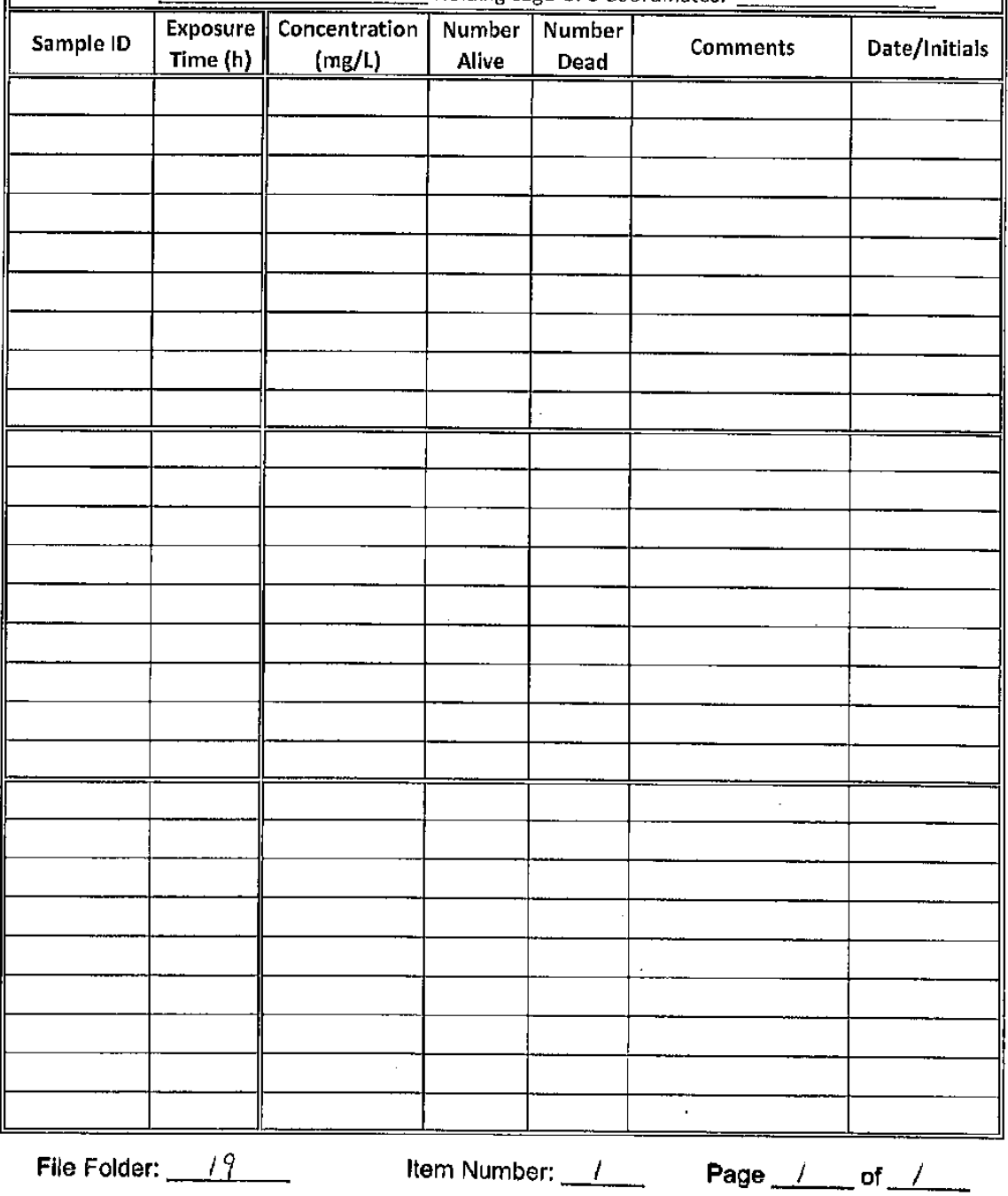


Study Number: AEH-12-PSEUDO-04 File folder: Lab book/pgs:
Reviewed by: Verifled by:

Date: Date:

\section{Zebra Mussel Lengths}

Test Organism: Zebra Mussels Applications Type: Exposure Date:

Test Chemical: Pf-CL145A SDP Lot \#: 40.1P12163C and 401P12164C Mix Test Location:

\begin{tabular}{||l|c|c|c|c|c|c|c|}
\hline \hline Sample ID & $\begin{array}{c}\text { Mussel } \\
\text { Number }\end{array}$ & $\begin{array}{c}\text { Exposure } \\
\text { Time (h) }\end{array}$ & $\begin{array}{c}\text { Conc. } \\
(\mathrm{mg} / \mathrm{L})\end{array}$ & $\begin{array}{c}\text { Shell } \\
\text { Length } \\
(\mathrm{mm})\end{array}$ & Comments & Date & Initials \\
\hline \hline
\end{tabular}

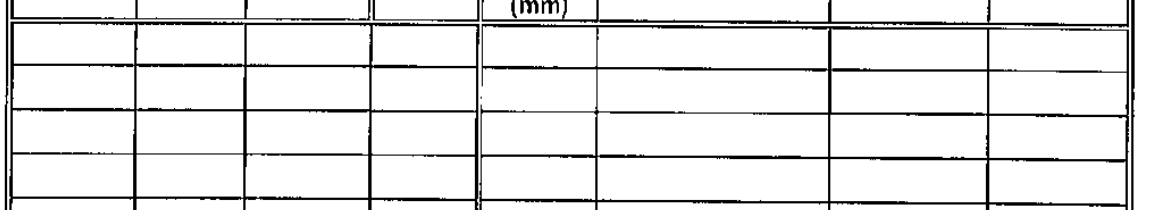

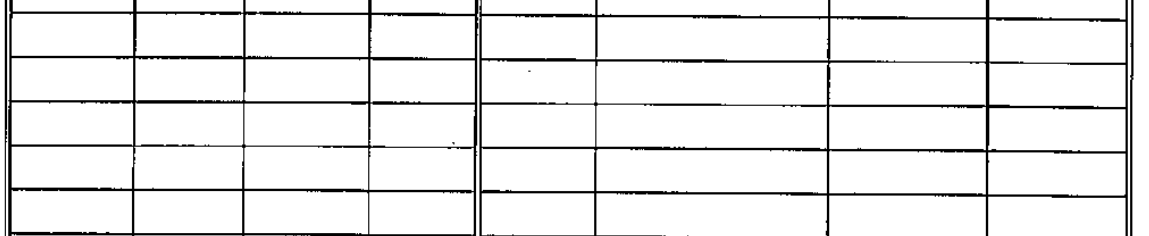

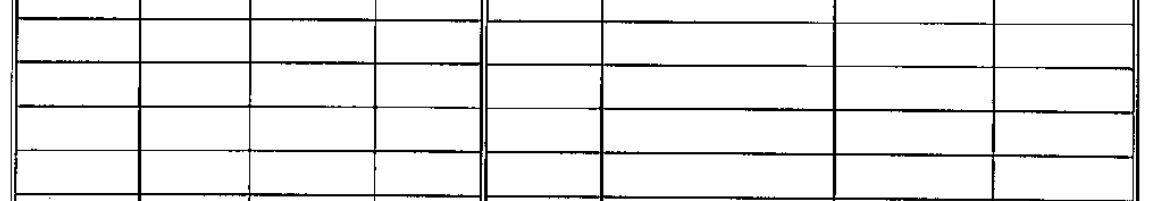

\begin{tabular}{|l|l|l|l|l|l|l|l||}
\hline & & & & & & & \\
\hline & & & & & & & \\
\hline & & & & & & & \\
\hline
\end{tabular}

\begin{tabular}{||l|l|l||l|l|l|l||}
\hline & & & & & & \\
\hline \\
\hline
\end{tabular}

File Folder: 19 Item Number: 219 Page 
Study Number: AEI1-12-PSEUDO-04

Fie Folder:

Lab book/pgs:

Reviewed by:

Verified by:

Date:

Date:

\section{Test Chemical Stock Preparation Data Form}

Test Chemical: Pseudomonas fluroescens strain 145A

Test Chemical Lot \#: 401P12163C and 401P12164C Mix Date Rec'd 7-Aug-12 Exp. Date 21-Jun-12 Test Organism : zebra musseis Test Location:

Instruments Used:

Weights of Chemical Samples:

\begin{tabular}{|c|c|c|c|c|}
\hline Sample ID & Sample Weight & Comments & Date & Initials \\
\hline & & & & \\
\hline & & & & \\
\hline & & & & \\
\hline & & & & \\
\hline & & & & \\
\hline & & & & \\
\hline & & & & \\
\hline & & & & \\
\hline & & & & \\
\hline & & & & \\
\hline
\end{tabular}

NOTE: Chemical samples to be stored refrigerated until used for stock preparation.

Stock Solution Preparation:

\begin{tabular}{|c|c|c|c|c|c|c|}
\hline Sample ID & $\begin{array}{c}\text { Dilution } \\
\text { Volume (m) }\end{array}$ & $\begin{array}{c}\text { Dillution } \\
\text { Time }\end{array}$ & Use & $\begin{array}{c}\text { Exposure } \\
\text { Time }\end{array}$ & Date & Initials \\
\hline & & & & & & \\
\hline & & & & & & \\
\hline & & & & & & \\
\hline & & & & & & \\
\hline & & & & & & \\
\hline & & & & & & \\
\hline & & & & & & \\
\hline & & & & & & \\
\hline & & & & & & \\
\hline & & & & & & \\
\hline Folder: & & Item & 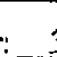 & Page & 1 & 1 \\
\hline
\end{tabular}




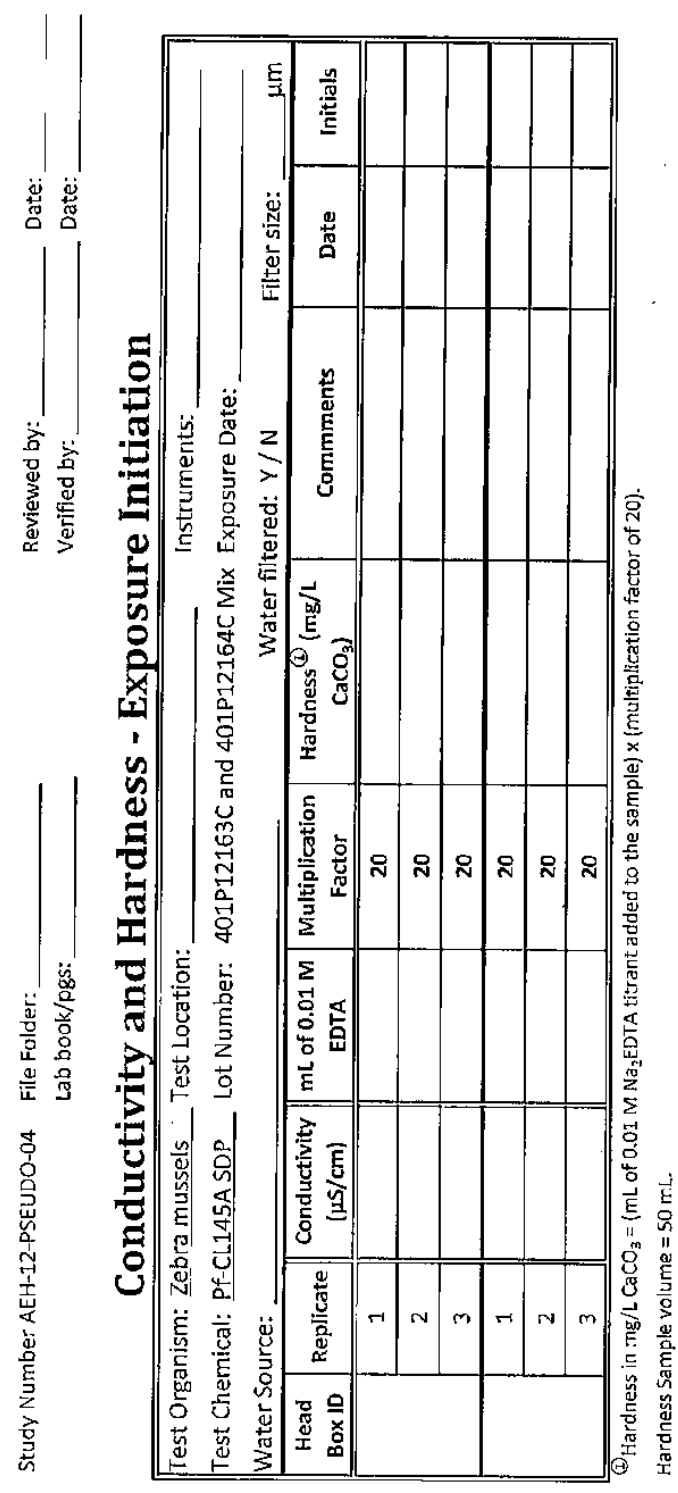

File Folder: 19 Item Number: 4 Page 11 of 1 


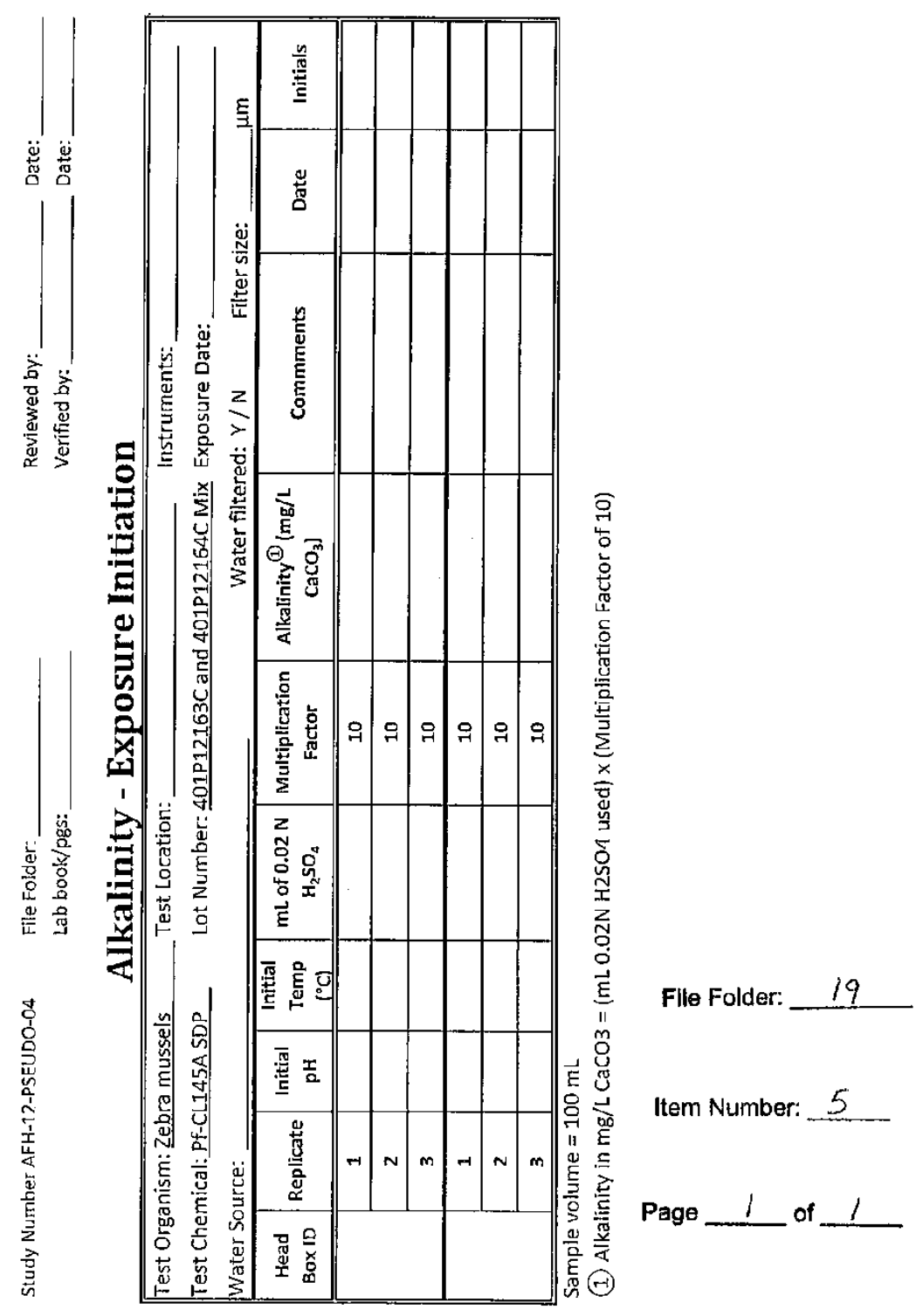




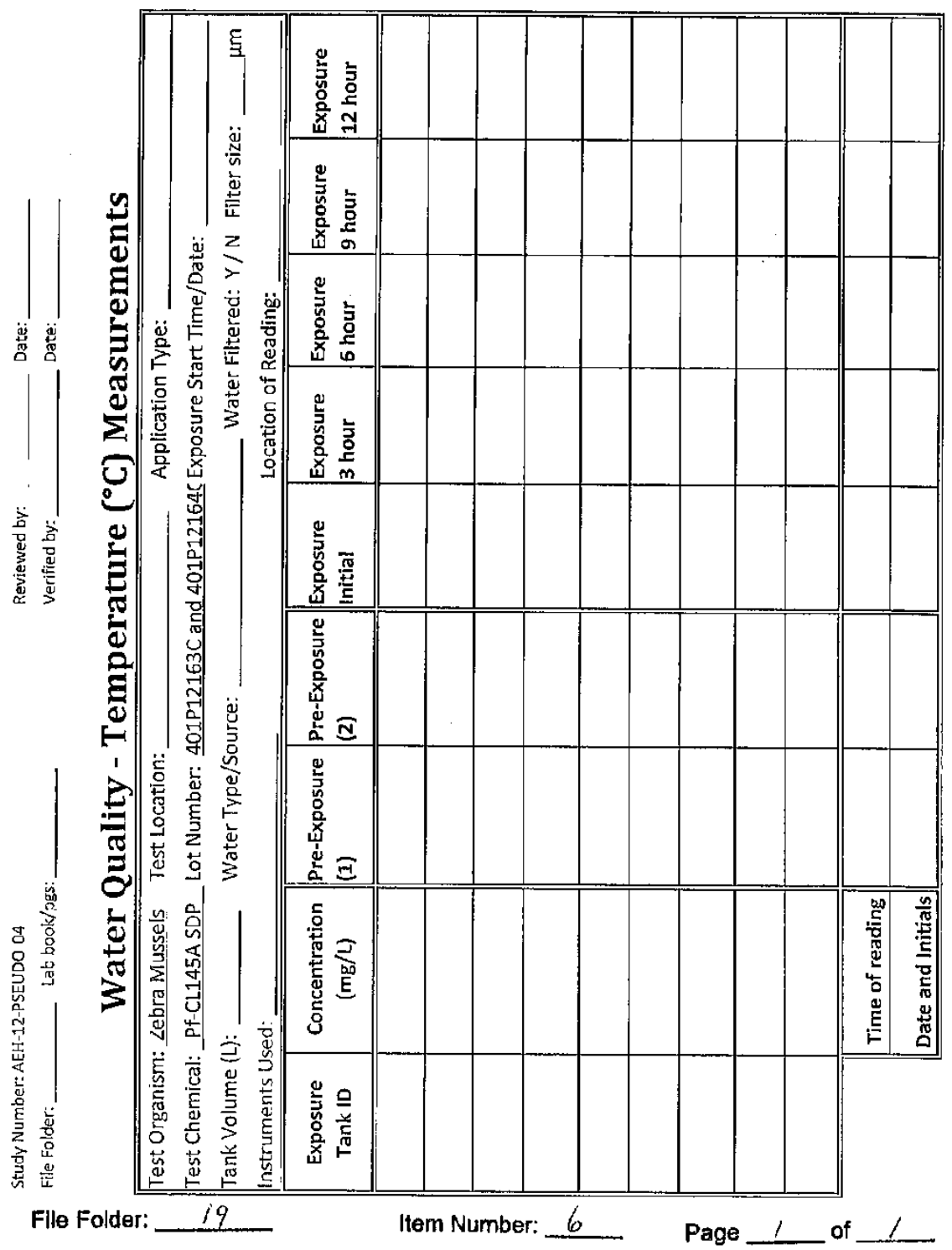

Page 59 of 519 


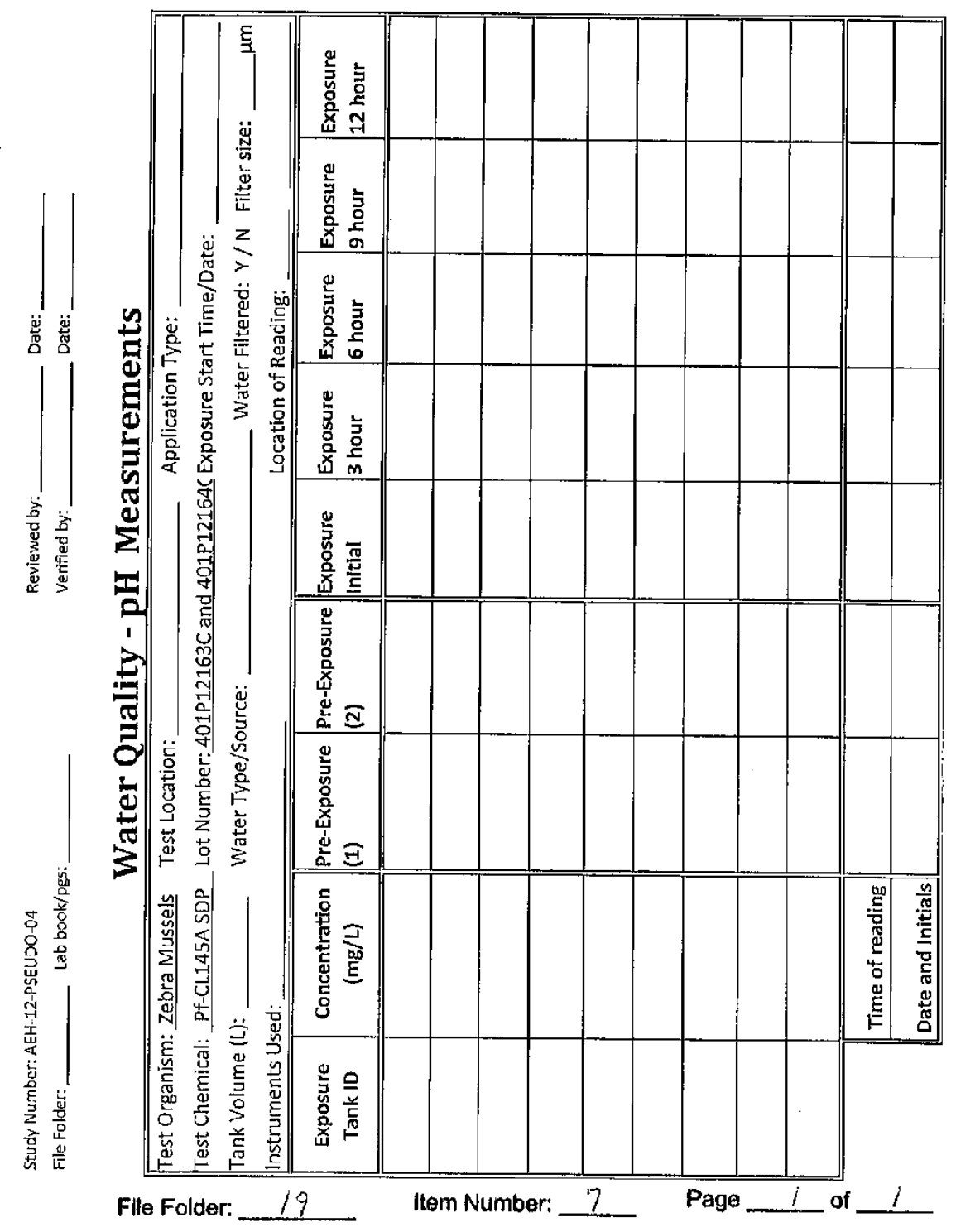




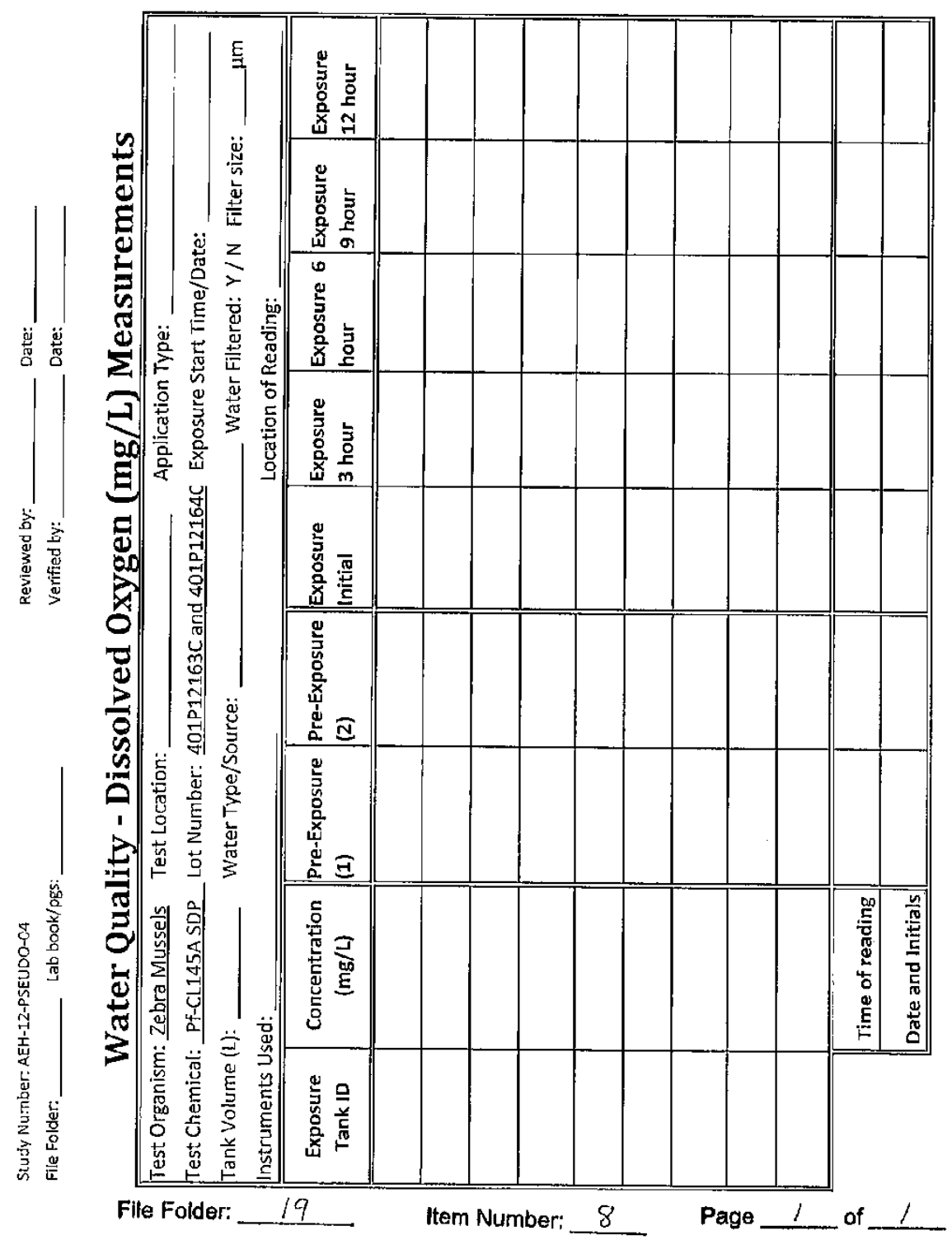

Page 61 of 519 


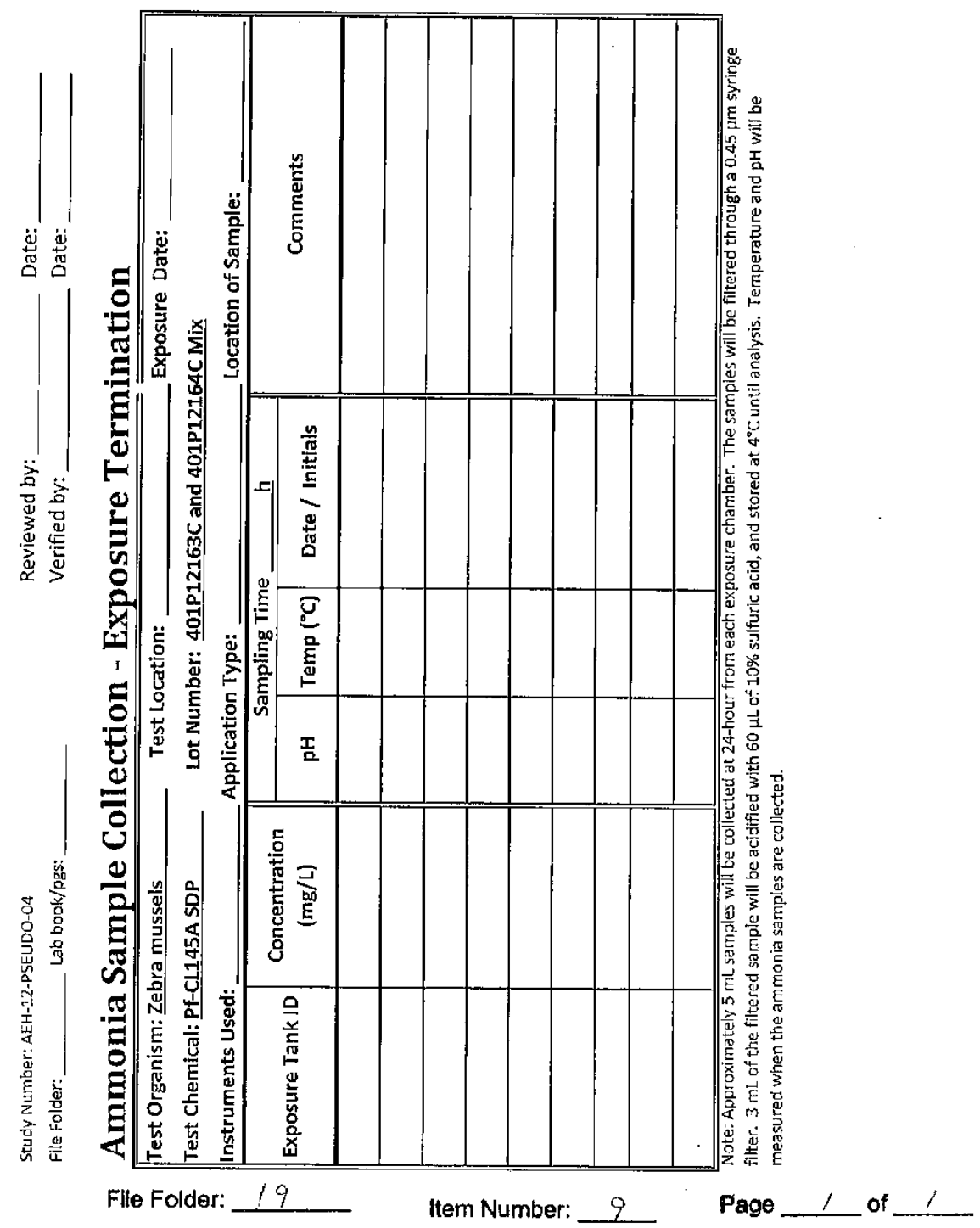

Page 62 of 519 


\section{Appendix 2. Deviations from the Study Protocol}

\begin{tabular}{clcc}
\hline $\begin{array}{c}\text { Item } \\
\text { Number }\end{array}$ & \multicolumn{1}{c}{ Item Description } & $\begin{array}{c}\text { Number } \\
\text { of } \\
\text { Pages }\end{array}$ & $\begin{array}{c}\text { Report } \\
\text { Page } \\
\text { Number }\end{array}$ \\
\hline 1 & $\begin{array}{c}\text { Deviation \#1 - Randomization of tank treatment assignment error for Shawano Lake } \\
\text { whole water body trial }\end{array}$ & 1 & 64 \\
2 & $\begin{array}{l}\text { Deviation \#2 - Randomization of substrate removal from tanks error } \\
\text { Deviation \#3 - Total ammonia-nitrogen water samples not collected at the 6 and 9 } \\
\text { hour termination during the whole water treatment at Lake Shawano } \\
\text { Deviation \#4 - Control exposure tanks not analyzed for concentration verification at } \\
\text { Lake Carlos }\end{array}$ & 1 & 65 \\
5 & $\begin{array}{c}\text { Deviation \#5 - No curriculum vitae or signature on the verification page for an } \\
\text { incidental data collector }\end{array}$ & 1 & 66 \\
6 & Deviation \#6 - Removal of Lake Pepin test location & 1 & 68 \\
\hline
\end{tabular}




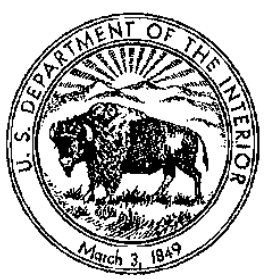

United States Department of the Interior

\author{
U.S. GEOLOGICAL SURVEY \\ Biological Resources Division \\ Upper Midwest Environmental Sciences Center \\ 2630 Fanta Reed Road \\ La Crosse, Wisconsin 54603
}

MEMORANDUM

Date: November 19, 2013

To: The Record Study Number AEH-12-PSEUDO-04

Subject: Deviation 1 to study AEH-12-PSEUDO-04 "Efficacy of Pseudomonas fluorescens (Pf-CL145A) SDP for controlling settled zebra mussels on artificial substrates"

Deviation \#1 - Randomization of Tank Treatment Assignment Error for Shawano Lake whole water body trial

For unknown reasons the randomization prepared for the experimental tank treatment assignment during the Shawano Lake whole water body trial was not followed. The treatments were applied repetitively in chronological order (e.g. tanks 1,2 , and 3 received 0,50 and $100 \mathrm{mg} / \mathrm{L}$ respectively, tanks 4,5 , and 6 received 0,50 and $100 \mathrm{mg} / \mathrm{L}$ respectively, and tanks 7,8 , and 9 received 0,50 and $100 \mathrm{mg} / \mathrm{L}$ respectively) The tank treatment assignment applied to each tank was verified with stock preparation data (File Folder 07), the water chemistry data (File Folder 11b), and the spectrophotometry data (File Folder 11c).

No adverse impacts are anticipated as a result of this deviation as the test animals (ie: bagged mussel trays) were randomly assigned to each treatment tank. Additionally, each treatment level was conducted in triplicate. Any impacts to the study as a result of this deviation will be addressed in the final report.

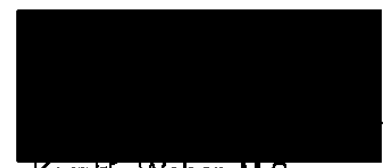

$$
-\frac{1919013}{\text { Date }}
$$

Kerry L. Weber, M.S.

Principal Investigator, UMESC

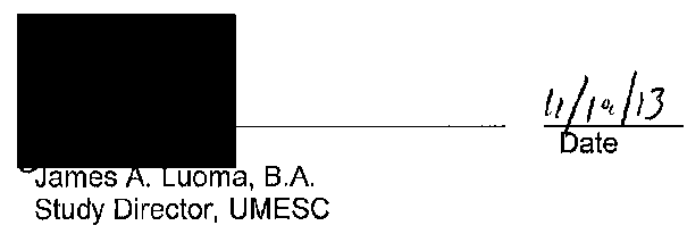

CC: UMESC QAU

Item Number: 3

File Folder: 3 - 3 Page 1 of 1 


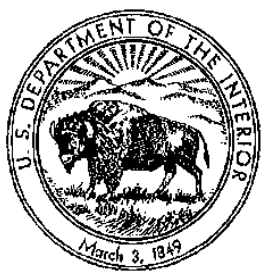

United States Department of the Interior

U.S. GEOLOGICAL SURVEY

Biological Resources Division

Upper Midwest Environmental Sciences Center

2630 Fanta Reed Road

La Crosse, Wisconsin 54603

\section{MEMORANDUM}

Date: November 19, 2013

To: The Record Study Number AEH-12-PSEUDO-04

Subject: Deviation 2 to study AEH-12-PSEUDO-04 "Efficacy of Pseudomonas fluorescens (Pf-CL145A) SDP for controlling settled zebra mussels on artificial substrates"

Deviation \#2 - Randomization of Substrate Removal from Tanks Error

Due to a programming and proc print error, the randomizations generated for the removal of substrates from exposure tanks 5, 7 and 9 for each trial (i.e., whole water body and bottom injection application for each testing location) were the same as the randomizations prepared for exposure tank 2 (i.e., the data for tank 2 was printed in error for tanks 5,7 and 9 ) from each individual trial. Therefore at 6,9 and $12 \mathrm{~h}$ post-dosing initiation, treated substrates were removed from the same location in exposure tanks $2,5,7$ and 9 .

No adverse impacts are anticipated as a result of this deviation as the test animals (ie: bagged mussel trays) were randomly assigned to each treatment tank. Additionally, each treatment level was conducted in triplicate and was randomly assigned to the exposure tanks. Any impacts to the study as a result of this deviation will be addressed in the final report.
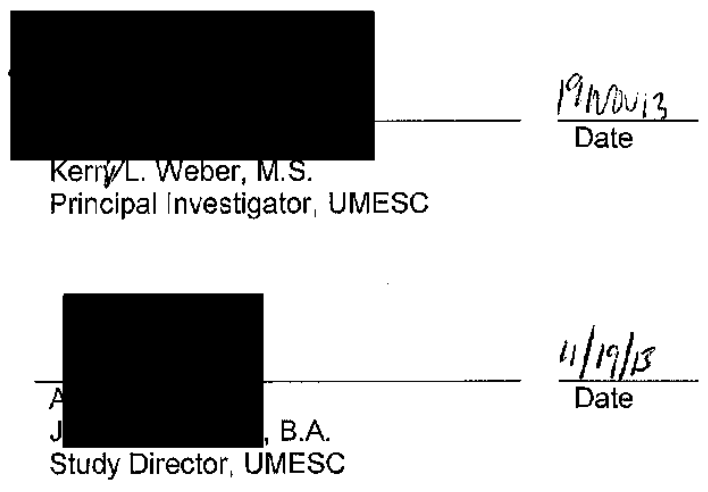

CC: UMESC QAU

Item Number: 4

File Folder: 


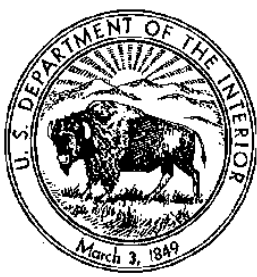

United States Department of the Interior

\author{
U.S. GEOLOGICAL SURVEY \\ Biological Resources Division \\ Upper Midwest Environmental Sciences Center \\ 2630 Fanta Reed Road \\ La Crosse, Wisconsin 54603
}

MEMORANDUM

Date: November 20, 2013

To: The Record Study Number AEH-12-PSEUDO-04

Subject: Deviation 3 to study AEH-12-PSEUDO-04 "Efficacy of Pseudomonas fluorescens (Pf-CL145A) SDP for controlling settled zebra mussels on artificial substrates"

Deviation \#3 - Total ammonia-nitrogen water samples not collected at the 6 and 9 hour termination during the whole water treatment at Lake Shawano

Section 5.2.7 of study number AEH-12-PSEUDO-04 amended protocol entitled "Efficacy of Pseudomonas fluorescens ( $P F$-CL145A) SDP for controlling settled zebra mussels on artificial substrates" states that "samples for total ammonia-nitrogen will be collected at the termination of the exposure period for each exposure replicate."

Previous studies (AEH-11-PSEUDO-01, AEH-11-PSEUDO-02 and AEH-12-PSEUDO-03) demonstrated that ammonia levels did not appreciably accumulate during 24 hour static treatments of the commercially produced Pseudomonas fluorescens ( $P f$-CL145A). Additionally, ammonia accumulatlon increases with degradation and, therefore, the greatest concentration would be at the 12 hour termination. Therefore, total ammonia-nitrogen water samples were collected only at the 12 hour exposure termination and not at 6 and 9 hour exposure termination times for the whole water treatment at Lake Shawano.

No adverse impacts are anticipated as a result of this deviation as ammonia levels from the static treatments would be highest at 12 hours. The highest observed un-ionized ammonia concentration at the 12 hour termination of the Lake Shawano whole tank treatment was $0.045 \mathrm{mg} / \mathrm{L}$ (Tank 2; $50 \mathrm{mg} / \mathrm{L}$ treatment group), a level that should not cause acute ammonia toxicity. Any impacts to the study as a result of this deviation will be addressed in the final report.

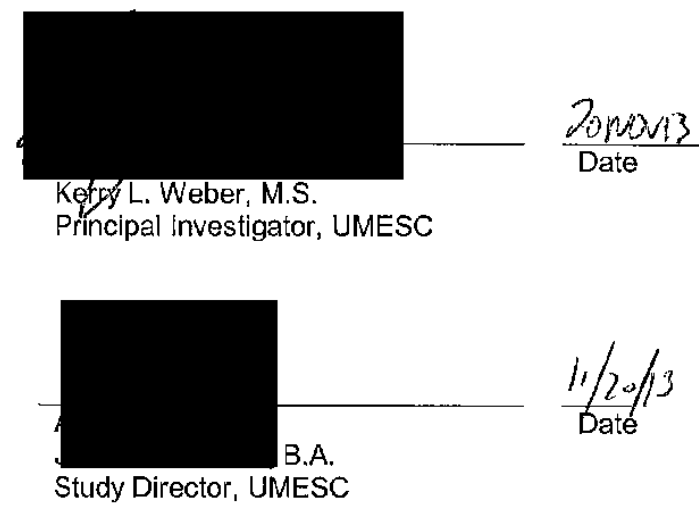

cC: UMESC QAU

File Folder: 3

Item Number:

Page 1 of 1 


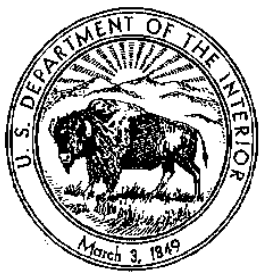

United States Department of the Interior

\section{U.S. GEOLOGICAL SURVEY}

Biological Resources Division

Upper Midwest Environmental Sciences Center

2630 Fanta Reed Road

La Crosse, Wisconsin 54603

\section{MEMORANDUM}

Date: November 20, 2013

To: The Record Study Number AEH-12-PSEUDO-04

Subject: Deviation 4 to study AEH-12-PSEUDO-04 "Efficacy of Pseudomonas fluorescens (Pf-CL145A) SDP for controlling settled zebra mussels on artificial substrates"

Deviation \#4 - Control exposure tanks not analyzed for concentration verification at Lake Carlos

Section 5.6.3 of study number AEH-12-PSEUDO-04 amended protocol entitled "Efficacy of Pseudomonas fluorescens ( $P f$-CL145A) SDP for controlling settled zebra mussels on artificial substrates" states that "concentrations will be verified in each replicate within 30 minutes of initial dosing and at 3,6, 9 and 12-h postdosing."

Water samples from the control exposure tanks were not analyzed for $P f-C L 145 A$ concentration during the Lake Carlos whole water treatment (Tanks 2, 3, and 5) or the Lake Carlos bottom injection treatment (Tanks 3,6 and 7). Samples were analyzed from all treated exposure replicates at 1, 3, 6, 9 and 12 hours for both whole water and bottom injections treatments.

No adverse impacts are anticipated as a result of this deviation as a linear, zero intercept standard curve was created from dilutions prepared from a $2,000 \mathrm{mg} / \mathrm{L}$ Pf-CL $145 \mathrm{~A}$ stock solution in $200 \mu \mathrm{m}$ filtered Lake Carlos water and the spectrophotometer was blanked using $200 \mu \mathrm{m}$ filtered Lake Carlos water. Additionally, all treatment replicates were isolated, static treatments which precluded any cross contamination. Any impacts to the study as a result of this deviation will be addressed in the final report.

File Folder: 3

CC: UMESC QAU

Item Number: 6
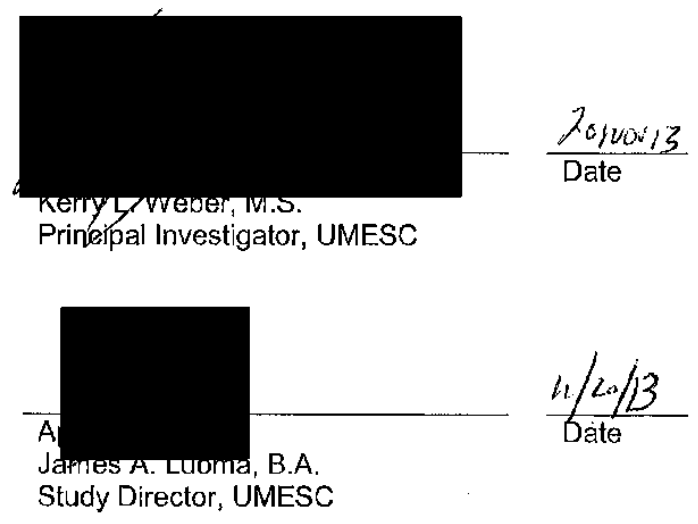

Page 1 of 1 


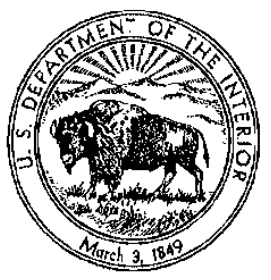

United States Department of the Interior

U.S. GEOLOGICAL. SURVEY

Biological Resources Division

Upper Midwest Environmental Sciences Center

2630 Fanta Reed Road

La Crosse, Wisconsin 54603

\section{MEMORANDUM}

Date: February 10, 2014

To: The Record Study Number AEH-12-PSEUDO-04

Subject: Deviation 5 to study AEH-12-PSEUDO-04 "Efficacy of Pseudomonas fluorescens (Pf-CL145A) SDP for controlling settled zebra musseis on artificial substrates"

Deviation \#5 - No curriculum vitae or signature on the verification page for an incidental data collector

Data entries were recorded on "Zebra Mussel Survival" forms (File Folder 14d) by an individual with the initials "ATM". The individual was Anna T. Morales, a WI DNR scientist working in Shawano County, Wisconsin. Ms Morales only participated in the enumeration of zebra mussels during the Shawano assessment conducted on October 10, 2012. Ms. Morales worked under the direct supervision of UMESC Biologists, only enumerated three samples, and completed no other study activities. No curriculum vitae or signature on the verification page were obtained; attempts to locate Ms. Morales were unsuccessful.

No adverse impacts are anticipated as a result of this deviation and any impacts to the study as a result of this deviation will be addressed in the final report.

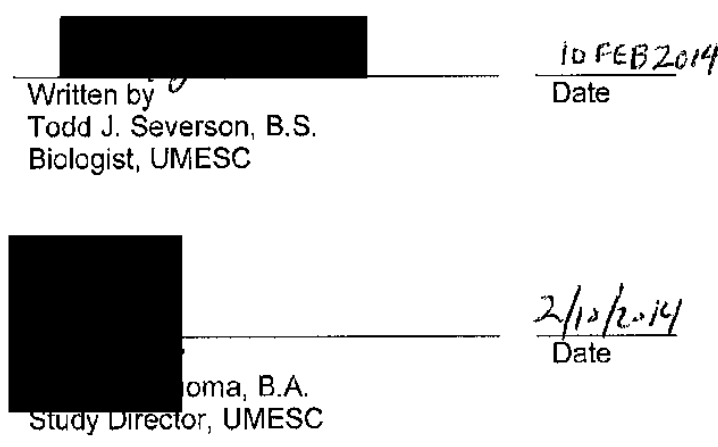

File Folder: 3

Item Number: 7

Page 1 of 1 


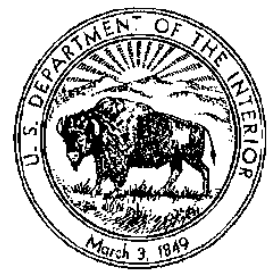

United States Department of the Interior

U.S. GEOLOGICAL SURVEY Biological Resources Division

Upper Midwest Environmental Sclences Center

2630 Fanta Reed Road

La Crosse, Wisconsin 54603

\section{MEMORANDUM}

Date: May 30,2014

To: The Record Study Number AEH-12-PSEUDO-04

Subject: Deviation 6 to study AEH-12-PSEUDO-04 "Efficacy of Pseudomonas fluorescens (Pf-CL145A) SDP for controlling settled zebra mussels on artificial substrates"

\section{Deviation \#6 - Removal of Lake Pepin test location}

A wide spread die-off of Dreissena polymorpha (zebra mussels) in the Upper Mississippi River system resulted in the need to cancel test exposures at Lake Pepin in Minnesota. Zebra mussel test animals were unavailable, with extremely few animals being found within approximately 5 river miles of the proposed test site. Exposures were successfully completed at two locations (Lake Carlos, Minnesota and Shawano Lake, Wisconsin), which resulted in sufficient data collection.

There were no adverse impacts as a result of this deviation as sufficient data was collected during the two exposures conducted to provide robust and scientifically defensible conclusions.

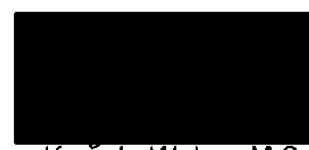

\section{$\frac{30 m \text { A- } 2 \text { dury }}{\text { Date }}$}

Kerry L. Weber, M.S

Principal Investigator, UMESC
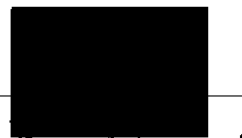

James A. Luoma, B.A.

Study Director, UMESC

Flle Folder:

Item Number: 8

Page 1 of 1 


\section{Appendix 3. Randomization Assignments}

\begin{tabular}{|c|c|c|c|}
\hline $\begin{array}{l}\text { Item } \\
\text { Number }\end{array}$ & Item Description & $\begin{array}{c}\text { Number } \\
\text { of } \\
\text { Pages }\end{array}$ & $\begin{array}{l}\text { Report } \\
\text { Page } \\
\text { Number }\end{array}$ \\
\hline 1 & $\begin{array}{l}\text { SAS generated random assignment of treatment to experimental tank (Lake Carlos; } \\
\text { whole tank treatment) }\end{array}$ & 4 & 71 \\
\hline 2 & $\begin{array}{l}\text { SAS generated random assignment of trays to test tank/position (Lake Carlos; whole } \\
\text { tank treatment) }\end{array}$ & 8 & 75 \\
\hline 3 & $\begin{array}{l}\text { SAS generated random assignment of substrate removal from tanks (Lake Carlos; } \\
\text { whole tank treatment }\end{array}$ & 30 & 83 \\
\hline 4 & $\begin{array}{l}\text { SAS generated random assignment of treatment to experimental tank (Lake Carlos; } \\
\text { bottom injection treatment) }\end{array}$ & 4 & 113 \\
\hline 5 & $\begin{array}{l}\text { SAS generated random assignment of trays to test tank/position (Lake Carlos; bottom } \\
\text { injection treatment) }\end{array}$ & 8 & 117 \\
\hline 6 & $\begin{array}{l}\text { SAS generated random assignment of substrate removal from tanks (Lake Carlos; } \\
\text { bottom injection treatment }\end{array}$ & 30 & 125 \\
\hline 7 & $\begin{array}{l}\text { SAS generated random assignment of treatment to experimental tank (Lake Shawano; } \\
\text { whole tank treatment) }\end{array}$ & 4 & 155 \\
\hline 8 & $\begin{array}{l}\text { SAS generated random assignment of trays to test tank/position (Lake Shawano; whole } \\
\text { tank treatment) }\end{array}$ & 8 & 159 \\
\hline 9 & $\begin{array}{l}\text { SAS generated random assignment of substrate removal from tanks (Lake Shawano; } \\
\text { whole tank treatment }\end{array}$ & 30 & 167 \\
\hline 10 & $\begin{array}{l}\text { SAS generated random assignment of treatment to experimental tank (Lake Shawano; } \\
\text { bottom injection treatment) }\end{array}$ & 4 & 197 \\
\hline 11 & $\begin{array}{l}\text { SAS generated random assignment of trays to test tank/position (Lake Shawano; } \\
\text { bottom injection treatment) }\end{array}$ & 8 & 201 \\
\hline 12 & $\begin{array}{l}\text { SAS generated random assignment of substrate removal from tanks (Lake Shawano; } \\
\text { bottom injection treatment }\end{array}$ & 30 & 209 \\
\hline
\end{tabular}



Efficacy of Pseudomonas fluorescens (Pf-CL145A)for controlling zebra mussesi on artifical substrat 1
AEH-12-PSEUDO-04
Random assignment of treatment to experimental tanks $8 / 1 / 12$
Treatment Location/type: Lake Carlos - whole water body $\mathrm{J}^{2}$

$\begin{array}{lrrrrl}\text { 0bs } & \text { block } & \text { tank } & x & \text { tankn } & \text { trt } \\ 1 & 1 & 3 & 0.04216 & \text { Tank } 3 & \text { control } \\ 2 & 1 & 4 & 0.04661 & \text { Tank } 4 & 50 \\ 3 & 1 & 7 & 0.24380 & \text { Tank } 7 & 100 \\ 4 & 1 & 5 & 0.34596 & \text { Tank } 5 & \text { control } \\ 5 & 1 & 1 & 0.45411 & \text { Tank } 1 & 50 \\ 6 & 1 & 6 & 0.55661 & \text { Tank } 6 & 100 \\ 7 & 1 & 2 & 0.68773 & \text { Tank } 2 & \text { control } \\ 8 & 1 & 8 & 0.83316 & \text { Tank } 8 & 50 \\ 9 & 1 & 9 & 0.89124 & \text { Tank } 9 & 100\end{array}$

File Folder: $9 a$ 


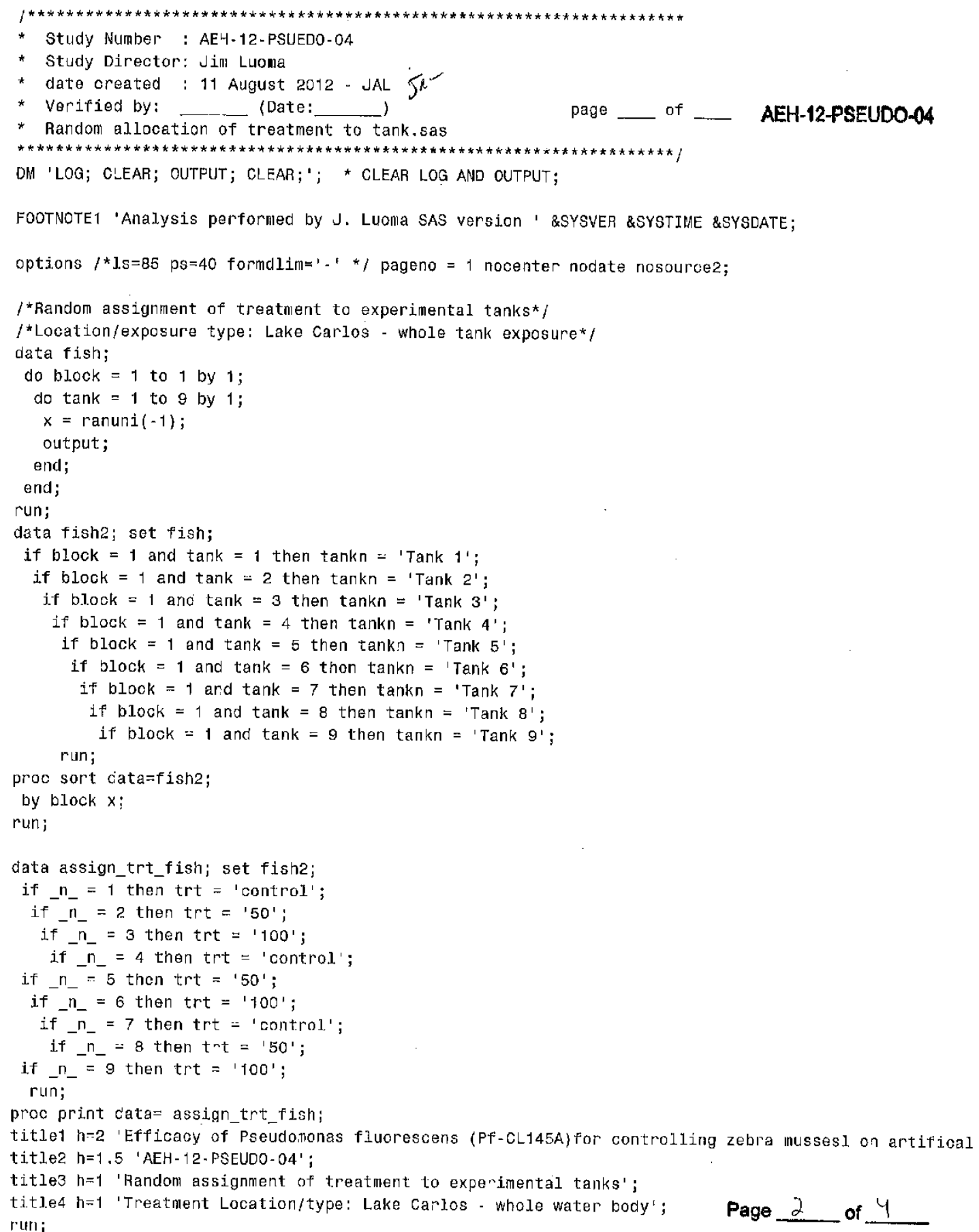




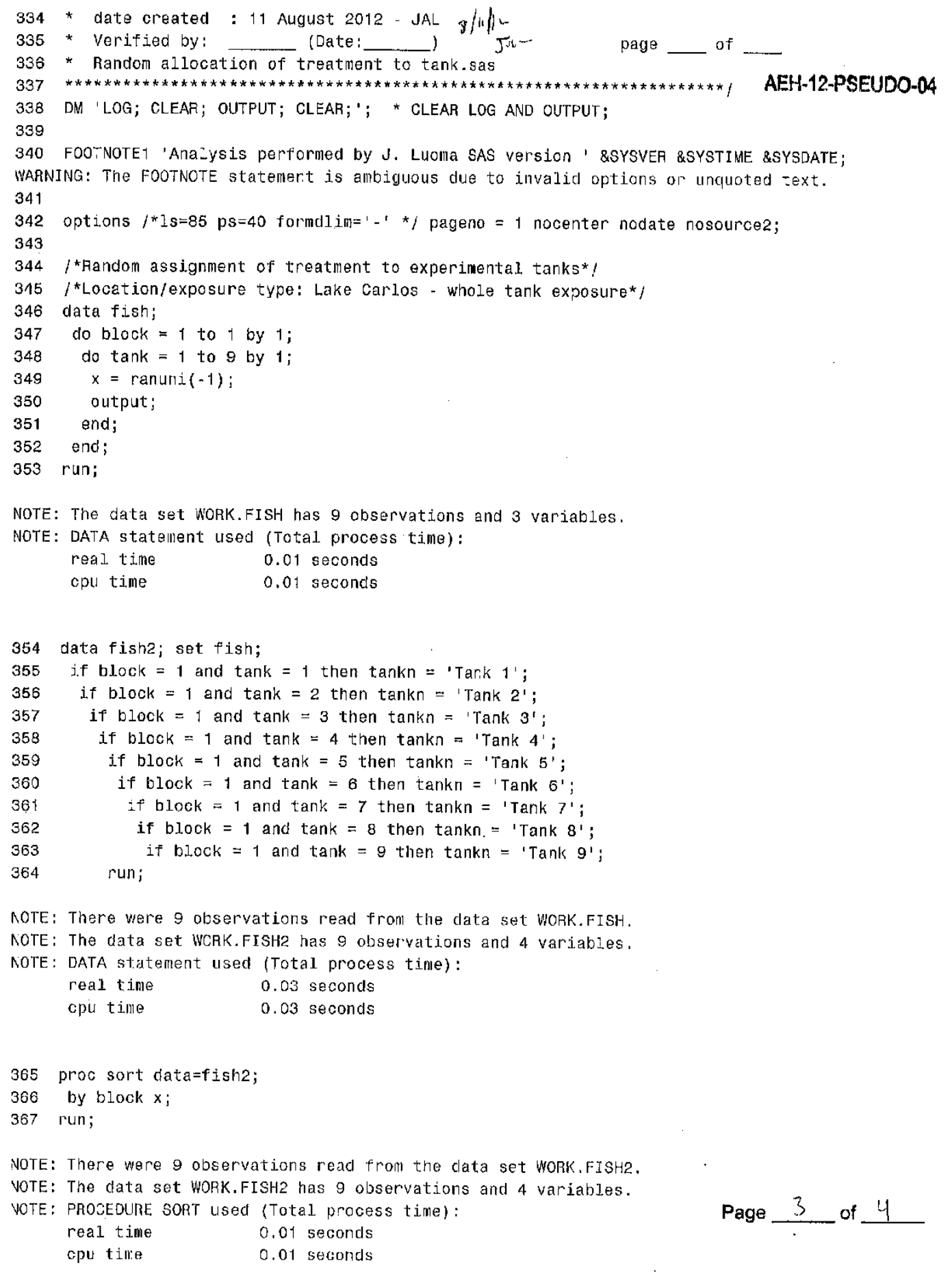




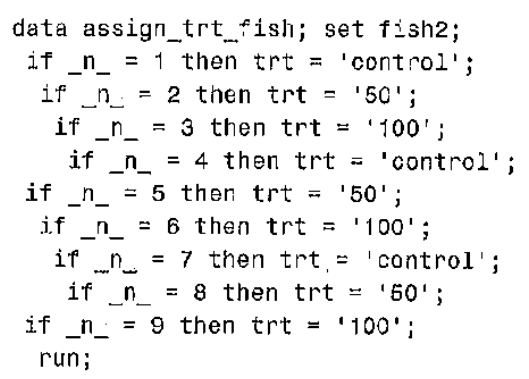

NOTE: There were 9 observatiors read froll the data set WORK. FISH2.

NOTE: The data set WORK.ASSIGN_TRT_FISH has 9 observations and 5 variables.

NOTE: DATA statement used (Total, procoss time):

real time $\quad 0.03$ seconds

cpu time $\quad 0.03$ seconds

380 proc print data= assign_trt_fish;

381 title1 $h=2$ 'Efficacy of Pseudomonas fluorescens ( $P f-C L-145 A$ ) for controlling zebra musses 1 on 381 I artifical substrates';

382 title2 h=1.5 'AEH-12-PSEUDO-04';

383 title3 $h=1$ 'Random assignment of treatment to experimental tanks';

384 title4 $\mathrm{h}=1$ 'Treatment Location/type: Lake Carlos - whole water body';

385 run;

NOTE: There were 9 observations read from the data set WORK.ASSIGN_TRT_FISH.

NOTE: PROCEOURE PRIN" used (Total process time):

real time $\quad 0.01$ seconds

cpu tille $\quad 0.01$ seconds

$8 / 15 / 12$

$\sqrt{n-}$

FF \#

Item $\mathrm{No} \frac{1}{4}$ 
Efficacy of.Psuedomonas fluorescens (Pf-CL145A)SDP for controlling zebra mussels on artifical $s$. AEH-12-PSUEDO-04

Random assignment of trays to test tank/position

Test Location/type = Lake Carlos/whole water tank treatment $8 / 1 / 12$

\begin{tabular}{|c|c|c|c|c|c|c|c|c|}
\hline Obs & $\because$ roun & roy & iposition & & tank & $x$ & row. & $\operatorname{tankn}$ \\
\hline 1 & 1 & 2 & 3 & & 2 & $0.0158 \uparrow$ & $B$ & $\bigvee 283$ \\
\hline 2 & 1 & 1 & 1 & & 5 & 0.01848 & A & $5 \mathrm{~A} 1$ \\
\hline 3 & 1 & 3 & 3 & & 3 & 0.01856 & $\mathrm{C}$ & $3 \mathrm{C3}$ \\
\hline 4 & 1 & 3 & 2 & & 9 & 0.02608 & C & 9.62 \\
\hline 5 & 1 & 2 & 2 & & 7 & 0.02978 & B & $7 \mathrm{~B} 2$ \\
\hline 6 & 1 & 3 & 1 & $\cdot$ & 7 & 0.03709 & $C$ & 701 \\
\hline 7 & 1 & 1 & 3 & & 2 & 0.06534 & A & $2 A 3$ \\
\hline 8 & 1 & 2 & 3 & & 8 & 0.08637 & B & $8 \mathrm{B3}$ \\
\hline 9 & 1 & 1 & 2 & & 9 & 0.09121 & A & $9 A 2$ \\
\hline 10 & 1 & 1 & 2 & & .1 & 0.13514 & A & $1 \mathrm{A2}$ \\
\hline 11 & 1 & 1 & 2 & $\cdots$ & $\div 7$ & 0.14899 & A & $7 \mathrm{A2}$ \\
\hline 12 & 1 & 1 & 2 & & 2 & 0.14907 & A & $2 A 2$ \\
\hline 13 & 1 & 3 & 1 & & 6 & 0.22154 & C & $6 \mathrm{C1}$ \\
\hline 14 & 1 & 2 & 3 & & $\cdot 6$ & 0.22497 & B & $6 \mathrm{~B} 3$ \\
\hline 15 & 1 & 2 & 1 & & 7. & 0.23740 & $B$ & $7 \mathrm{~B} 1$ \\
\hline 16 & 1 & 1 & 3 & & .5 & 0.24309 & A & $5 A B$ \\
\hline 17 & 1 & 3 & 3 & & B & 0.24872 & C & $\mathrm{BC}^{\circ}$ \\
\hline 18 & 1 & 2 & 3 & & 1 & 0.24915 & B & AB3 \\
\hline 19 & 1 & 1 & 2 & : : & 8 & 0.25031 & A & BA2 \\
\hline 20 & 1 & 1 & 2 & & 5 & 0.27193 & A & $5 A 2$ \\
\hline 21 & 1 & 1 & 3 & & 7 & 0.27954 & A & $7 A 3$ \\
\hline 22 & 1 & 1 & 3 & & 1 & 0.31226 & A & $1 A 3$ \\
\hline 23 & 1 & 3 & 3 & & 9 & 0.31388 & $c$ & 903 \\
\hline 24 & 1 & 1 & 3 & & 4 & 0.32192 & A & $4 A 3$ \\
\hline 25 & 1 & 1 & 1 & & 7 & 0.32805 & A & 7A1. \\
\hline 26 & 1 & 3 & 1 & & 2 & 0.33235 & $\mathrm{C}$ & $2 \mathrm{C} 1$ \\
\hline 27 & 1 & 3 & 2 & & 4 & 0.34771 & C & $4 \mathrm{C2}$ \\
\hline 28 & 1 & 2 & 1 & $\because$ & 1 & 0.35529 & B & 1B1 \\
\hline 29 & 1 & 3 & 1 & s & 9 & 0.35723 & $\mathrm{C}$ & 901 \\
\hline 30 & 1 & 1 & 1 & & 4 & 0.36690 & A & 4AI: \\
\hline 31 & 1 & 1 & 1 & $\because$ & 3 & 0.37178 & $A$ & $3 \mathrm{~A} 1$ \\
\hline 32 & 1 & 1 & 3 & & 9 & 0.38632 & A & 9A3 \\
\hline 33 & 1 & 1 & 1 & & 2 & 0.39205 & A & $2 A 1$ \\
\hline 34 & 1 & 3 & 1 & & 9 & 0.40328 & $\mathrm{c}$ & 101 \\
\hline 35 & 1 & 1 & 1 & & 9 & 0.41738 & A & gA1 \\
\hline 36 & 1 & 3 & 3 & & 6 & 0.41888 & $c$ & $6 \mathrm{C3}$ \\
\hline 37 & 1 & 2 & 1 & & 3 & 0.42874 & $B$ & 3B1 \\
\hline 38 & 1 & 1 & 2 & & 6 & 0.45875 & A & 6A2 \\
\hline 39 & 1 & 3 & 1 & & 3 & 0.45937 & C & $3 C 1$ \\
\hline 40 & 1 & 3 & 3 & & 4 & 0.48106 & C & $4 C 3$ \\
\hline 41 & 1 & 2 & 3 & & 3 & 0.50277 & $\mathrm{~B}$ & 3B3 \\
\hline 42 & 1 & 2 & 2 & & 8 & 0.51279 & B & $8 B 2$ \\
\hline 43 & 1 & 3 & 1 & & 4 & 0.52493 & C & $4 c 1$ \\
\hline 44 & 1 & 3 & 3 & & 5 & 0.53590 & $\mathrm{c}$ & $5 \mathrm{C3}$ \\
\hline 45 & 1 & 2 & 2 & & 3 & 0.55895 & B & $3 B 2$ \\
\hline 46 & 1 & 2 & 3 & & 7 & 0.57234 & B & $7 B 3$ \\
\hline
\end{tabular}

AEH-12-PSEUDO-04

start

$1500 \mathrm{~h}$

Finished

$1630 k$

to

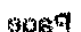

Analysis performed by J. Luoma SAS version $9.2 \quad 10: 48 \quad 11$ AUG12

Fith Folder: $9 a$ tom Number: 2

Pepe 1 of 8 
Efficacy of Psuedomonas fluorescens (Pf-CL145A)SDP for controlling zebra mussels on artifical \& 2 AEH-12- PSUEDO-04

Random assignment of trays to test tank/position

Test Location/type = Lake Carlos/whole water tank treatment $\quad 8 / \mathrm{h} / \mathrm{h} 5 \mathrm{~h}$

Obs round row position tank $x$-row_ tankn AEH-12-PSEUDO-04

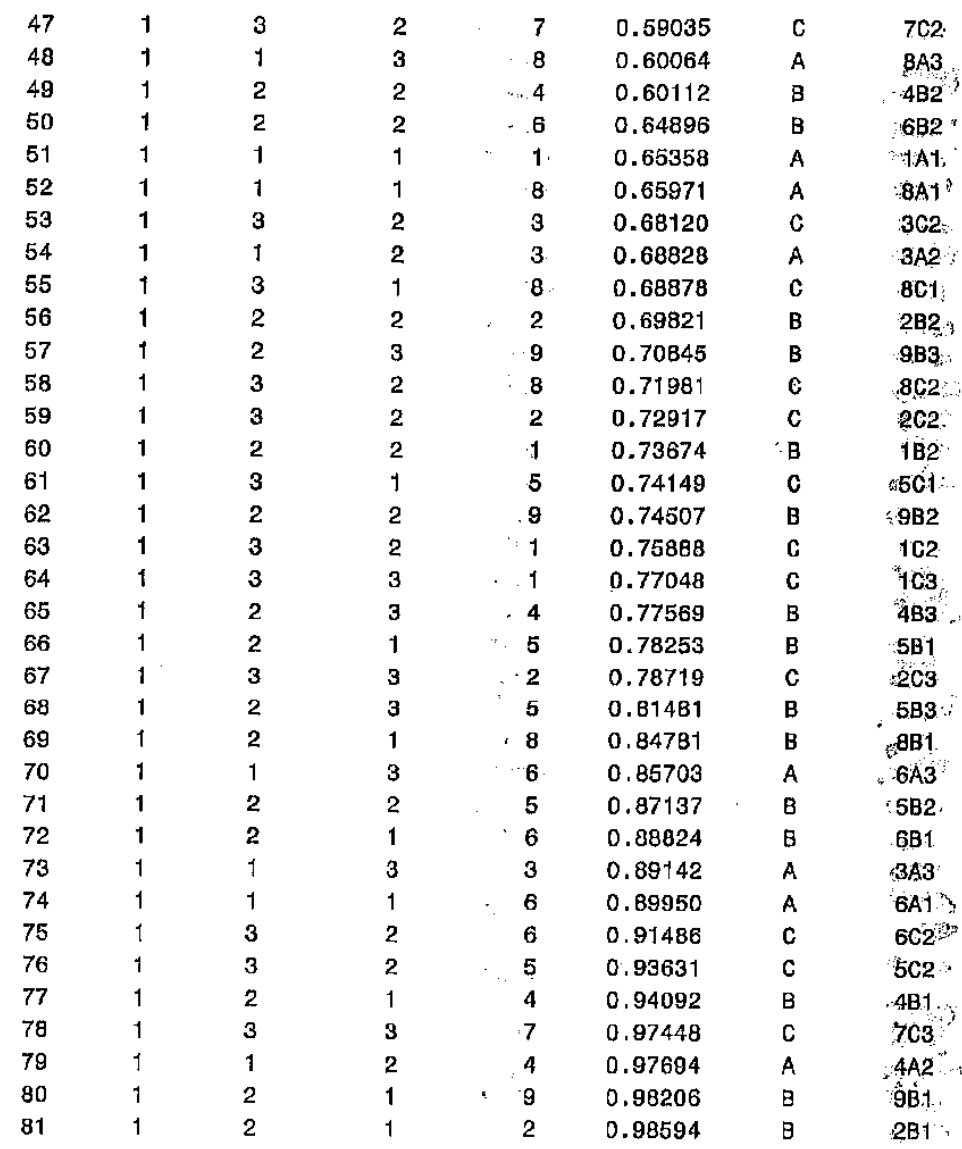




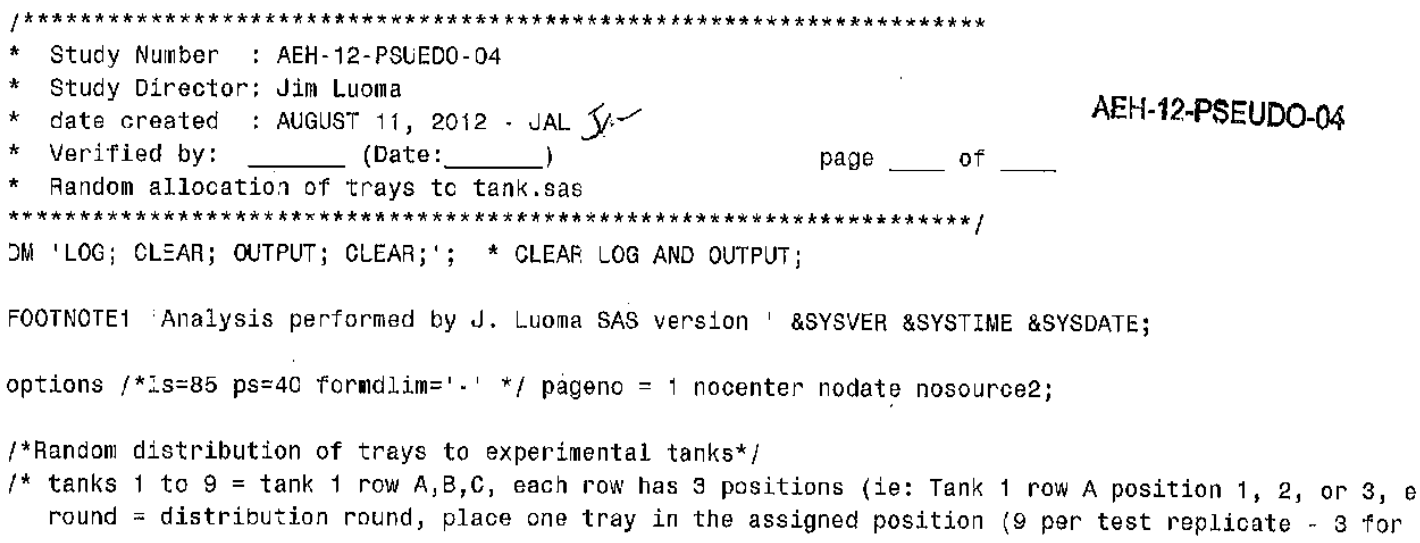



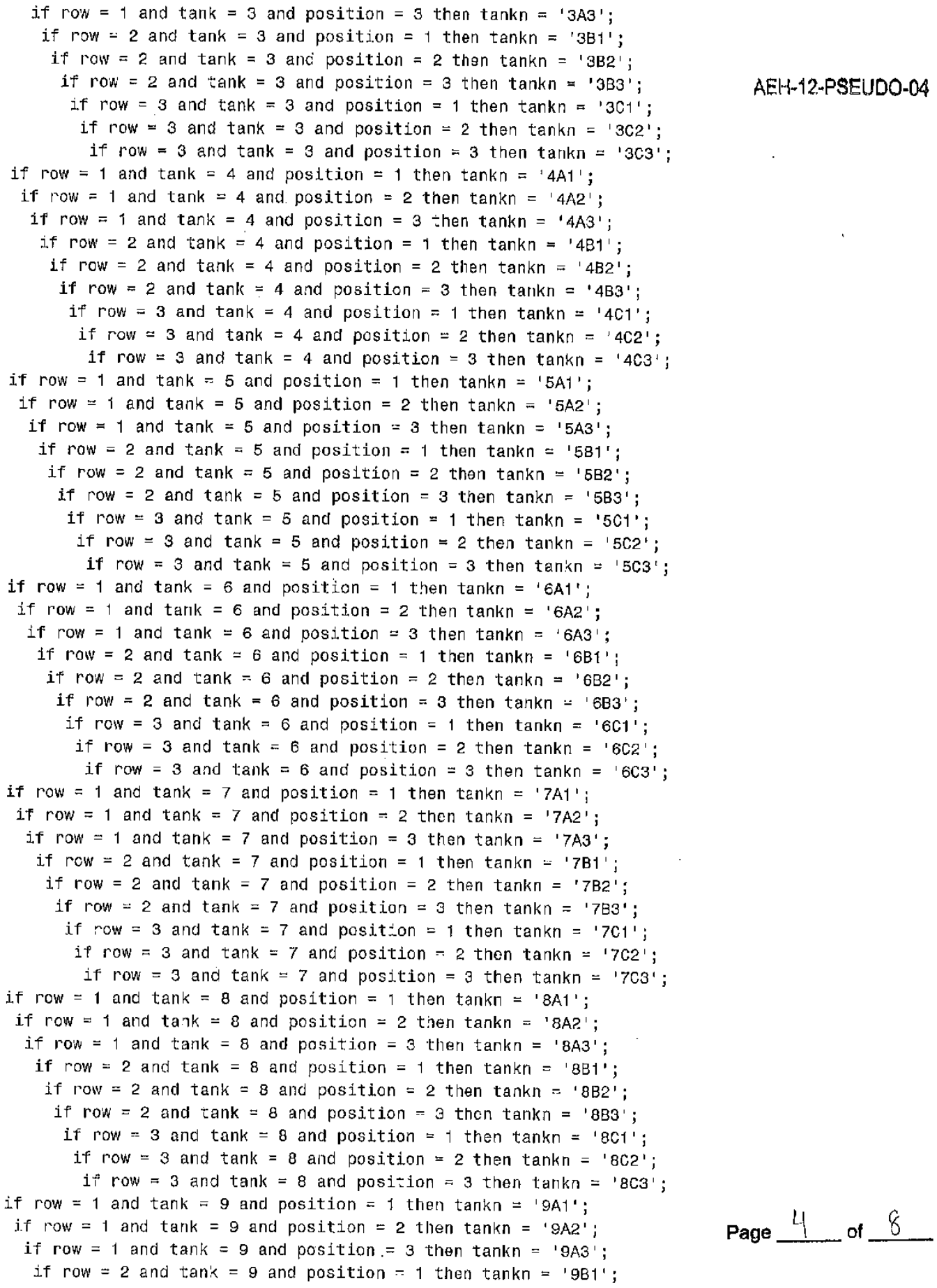


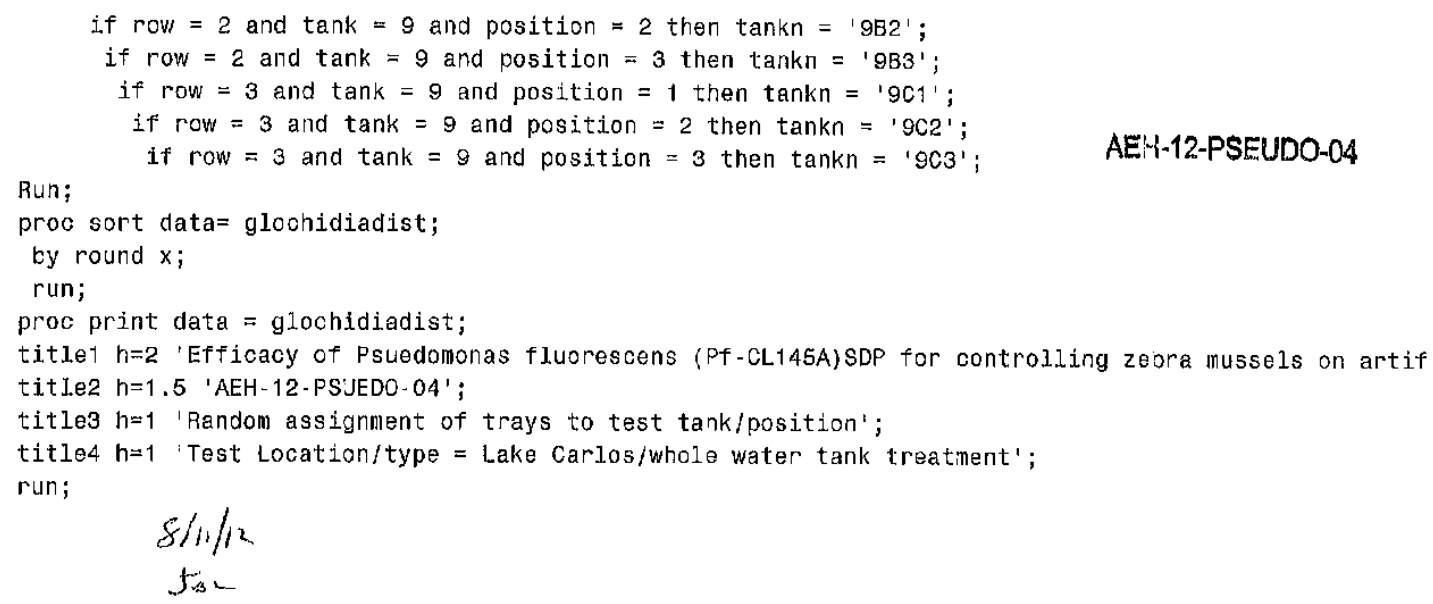

Page 5 of 8 


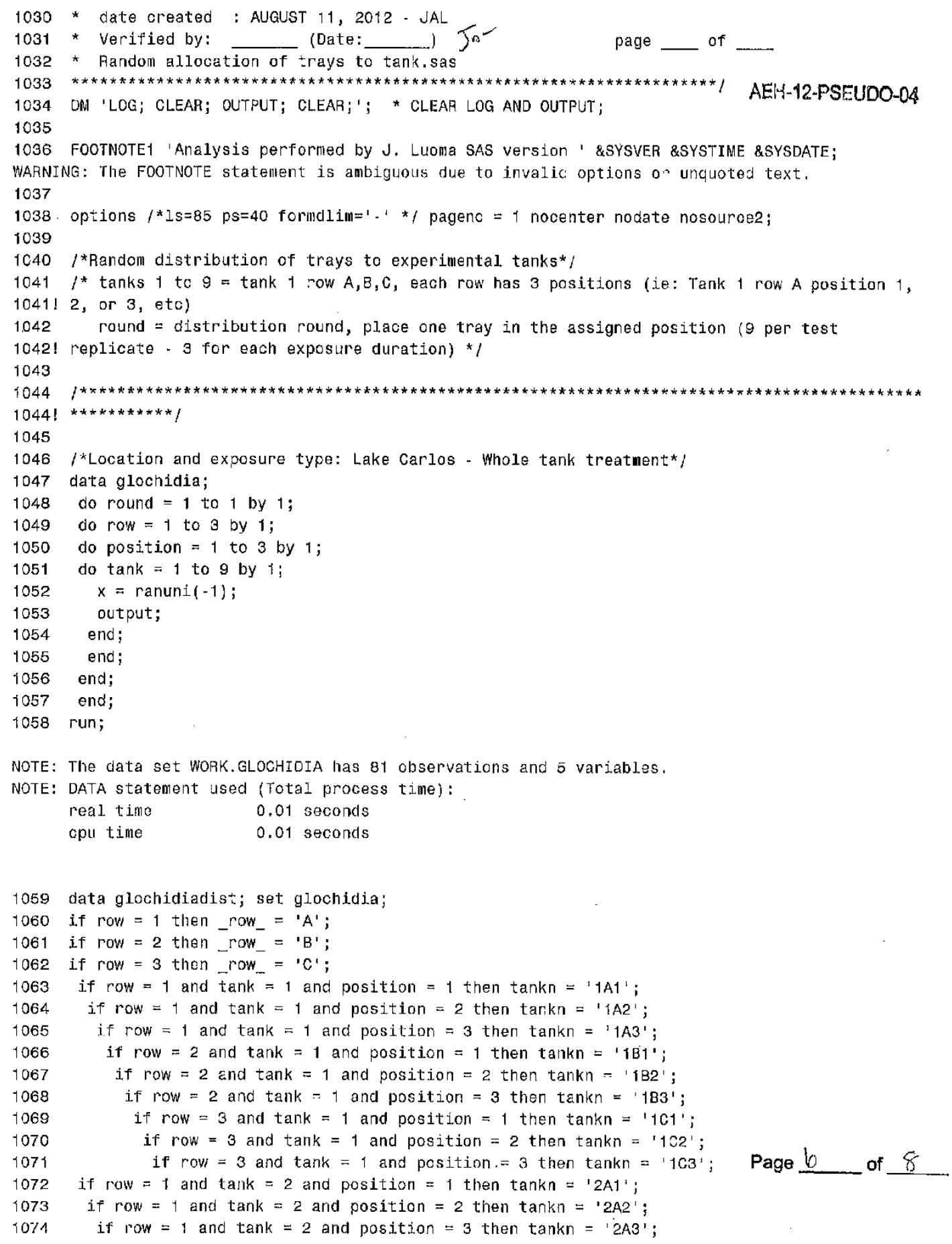




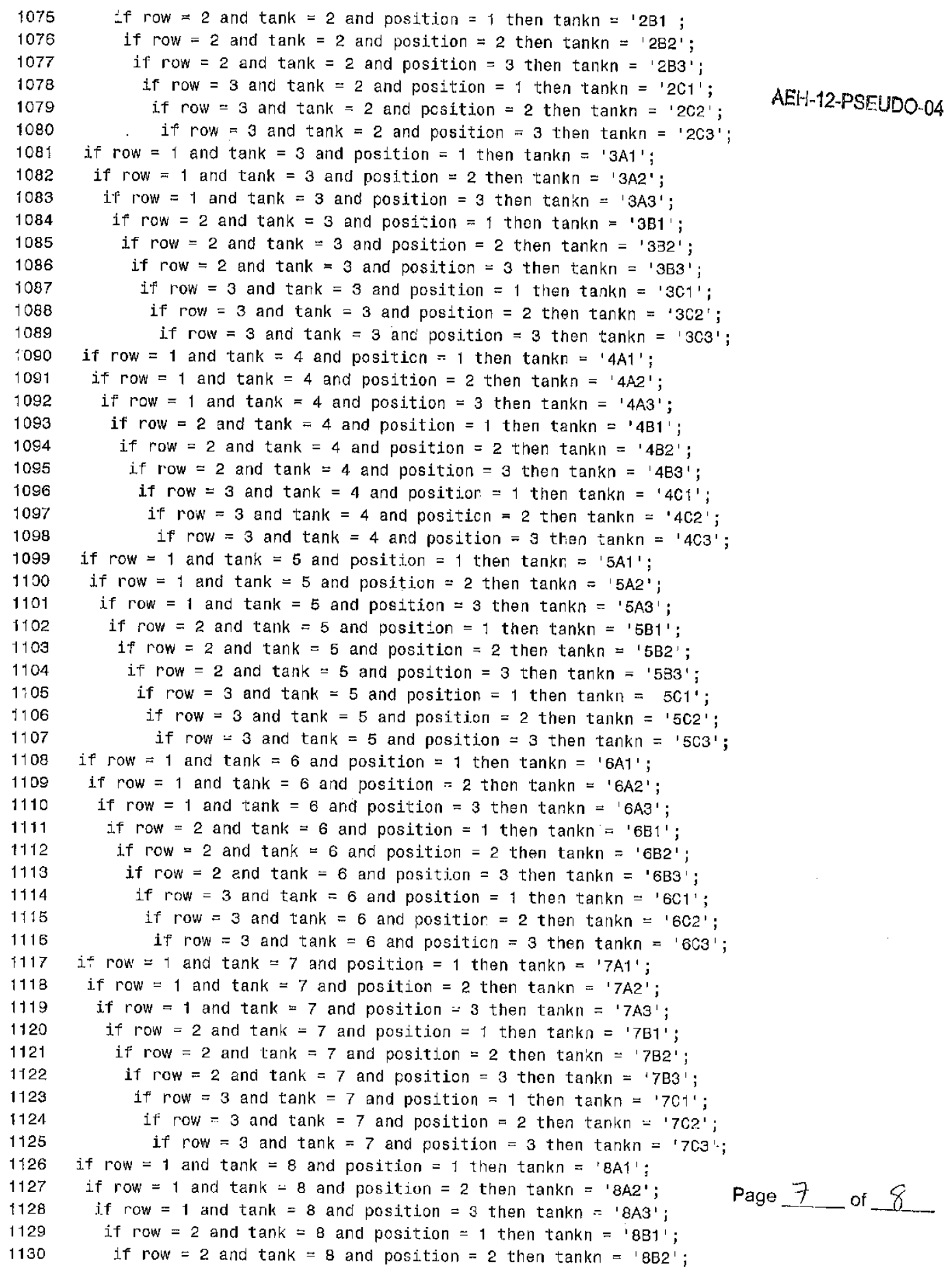




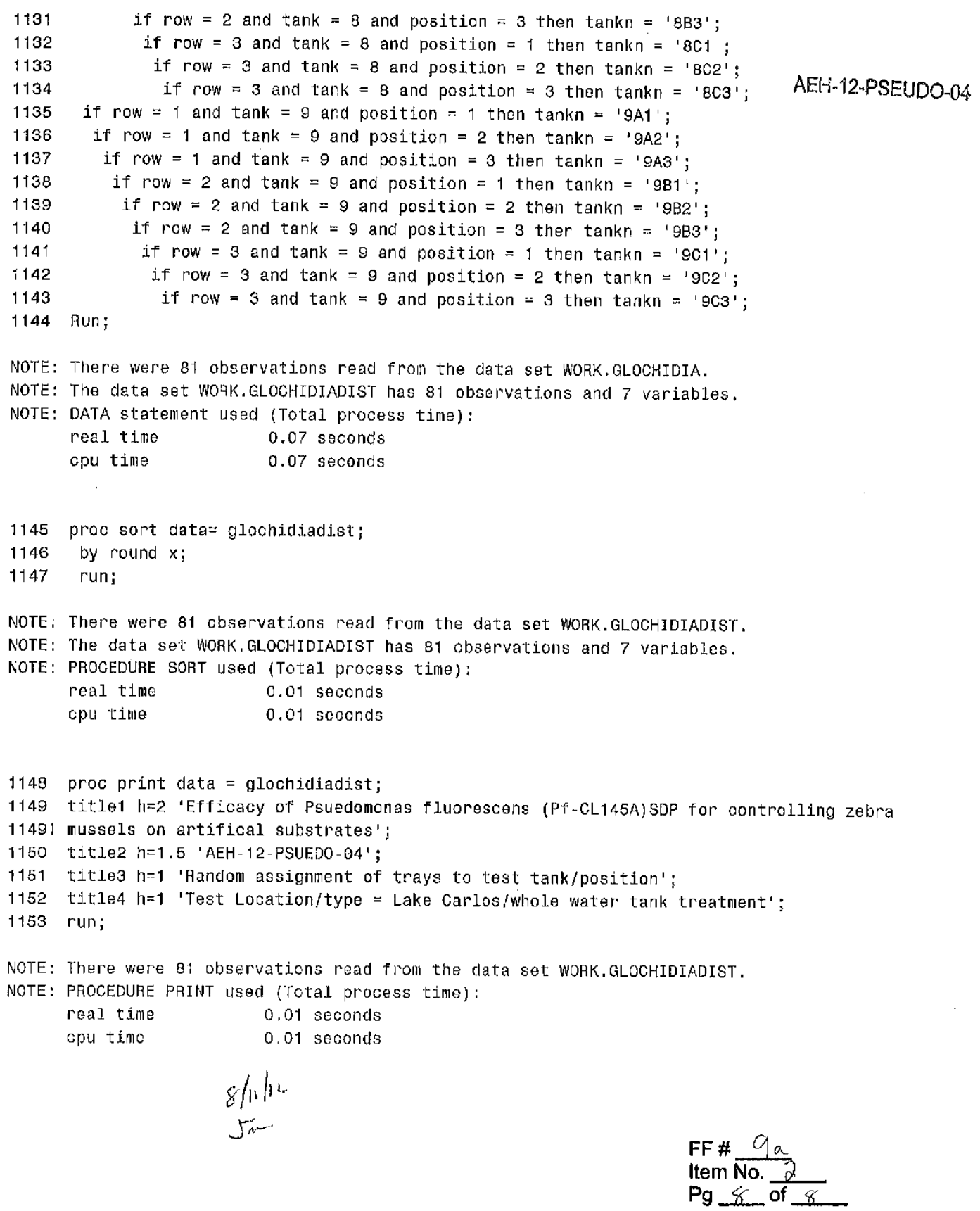


Efficacy of Pseudomonas fluorescens (Pf-CL145A)for controlling zebra mussesi on artifical substrates AEH - 12-PSEUDO-04

Random assignment of substrate removal from tanks *** TANK $1 * * * \quad 8 / 13 / 14$
Lake carlos - Whole water gody Treatment

$\begin{array}{cccccc}\text { Obs } & \text { row } & \text { position } & x & \text { tankn } & \text { trt } \\ 1 & 2 & 2 & 0.02216 & 1 \mathrm{~B} 2 & 6 \mathrm{~h} \\ 2 & 1 & 3 & 0.16367 & 1 \mathrm{A3} & 6 \mathrm{~h} \\ 3 & 1 & 2 & 0.21372 & 1 \mathrm{~A} 2 & 6 \mathrm{~h} \\ 4 & 3 & 1 & 0.27213 & 1 \mathrm{C} 1 & 9 \mathrm{~h} \\ 5 & 1 & 1 & 0.29050 & 1 \mathrm{~A} 1 & 9 \mathrm{~h} \\ 6 & 2 & 1 & 0.60999 & 1 \mathrm{~B} 1 & 9 \mathrm{~h} \\ 7 & 3 & 3 & 0.61991 & 1 \mathrm{C3} & 12 \\ 8 & 3 & 2 & 0.95789 & 1 \mathrm{C} 2 & 12 \\ 9 & 2 & 3 & 0.95946 & 1 \mathrm{~B} 3 & 12\end{array}$


Analysis performed by J. Luoma SAS version 9.2 10:20 13AUG12

Efficacy of Pseudomonas fluorescens (Pf-CL145A)for controlling zebra mussesl on artifical substrates AEH - 12-PSEUDO-04

Random assignment of substrate removal from tanks *** TANK $2 * * *$

Lake Carlos . Whole water Body Treatment

AEH-12-PSEUDO-04

$\begin{array}{cccccc}\text { Obs } & \text { row } & \text { position } & \mathrm{x} & \text { tankn } & \text { trt } \\ & & & & & \\ 1 & 2 & 2 & 0.05116 & 2 \mathrm{~B} 2 & 6 \mathrm{~h} \\ 2 & 1 & 1 & 0.15754 & 2 \mathrm{~A} 1 & 6 \mathrm{~h} \\ 3 & 3 & 1 & 0.19038 & 2 \mathrm{C} 1 & 6 \mathrm{~h} \\ 4 & 1 & 3 & 0.29438 & 2 \mathrm{A3} & 9 \mathrm{~h} \\ 5 & 3 & 3 & 0.36230 & 2 \mathrm{G} 3 & 9 \mathrm{~h} \\ 6 & 2 & 3 & 0.63280 & 2 \mathrm{~B} 3 & 9 \mathrm{~h} \\ 7 & 3 & 2 & 0.73826 & 2 \mathrm{C} 2 & 12 \\ 8 & 1 & 2 & 0.86034 & 2 \mathrm{~A} 2 & 12 \\ 9 & 2 & 1 & 0.87946 & 2 \mathrm{~B} 1 & 12\end{array}$

Tequrwh moll

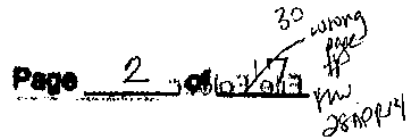


Analysis performed by $J$. Luoma SAS version 9.2 10:20 13AUG12

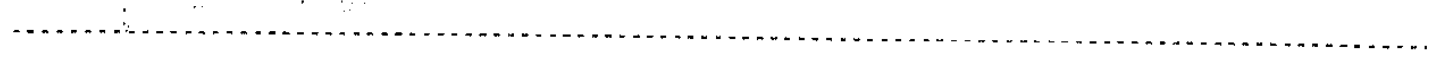

Erficacy of Pseudomonas fluorescens (PF-CL145A)for controlling zebra mussesl on artifical substrates AEH-12-PSEUDO-04

Random assignment of substrate renoval from tanks $* * *$ TANK $3 * * *$

Lake Carlos - Whole water Body Treatment

$\begin{array}{cccccc}\text { Obs } & \text { row } & \text { position } & x & \text { tankn } & \text { trt } \\ 1 & 3 & 3 & 0.09037 & 3 \mathrm{C} 3 & 6 \mathrm{~h} \\ 2 & 1 & 1 & 0.20055 & 3 \mathrm{~A} 1 & 6 \mathrm{~h} \\ 3 & 2 & 1 & 0.37245 & 3 \mathrm{~B} 1 & 6 \mathrm{~h} \\ 4 & 3 & 2 & 0.38436 & 3 \mathrm{C} 2 & 9 \mathrm{~h} \\ 5 & 1 & 3 & 0.41454 & 3 \mathrm{A3} & 9 \mathrm{~h} \\ 6 & 2 & 3 & 0.54349 & 3 \mathrm{~B} 3 & 9 \mathrm{~h} \\ 7 & 3 & 1 & 0.54506 & 3 \mathrm{C} 1 & 12 \\ 8 & 2 & 2 & 0.65481 & 3 \mathrm{~B} 2 & 12 \\ 9 & 1 & 2 & 0.86888 & 3 \mathrm{~A} 2 & 12\end{array}$

AEH-12-PSEUDO-04

to

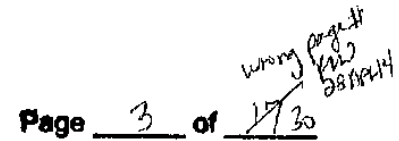

Page 85 of 519 
Analysis performed by $\mathrm{J}$. Luoma SAS version 9.2 10:20 13 AUG12

AEH-12-PSEUDO-04

Efficacy of Pseudomonas fluorescens (PF-CL145A)for controlling zebra mussesl on artifical substrates AEH-12-PSEUDO-04

Random assignment of substrate removal from tanks *** TANK $4 * * *$

Lake Carlos - Whole water Body Treatment

$\begin{array}{cccccc}\text { Obs } & \text { row } & \text { position } & x & \text { tankn } & \text { trt } \\ 1 & 3 & 1 & 0.10801 & 4 \mathrm{C} 1 & 6 \mathrm{~h} \\ 2 & 3 & 2 & 0.11324 & 4 \mathrm{C} 2 & 6 \mathrm{~h} \\ 3 & 2 & 2 & 0.23268 & 4 \mathrm{~B} 2 & 6 \mathrm{~h} \\ 4 & 1 & 3 & 0.29166 & 4 \mathrm{A3} & 9 \mathrm{~h} \\ 5 & 1 & 1 & 0.61787 & 4 \mathrm{~A} 1 & 9 \mathrm{~h} \\ 6 & 2 & 3 & 0.68056 & 4 \mathrm{B3} & 9 \mathrm{~h} \\ 7 & 3 & 3 & 0.87818 & 4 \mathrm{C3} & 12 \\ 8 & 2 & 1 & 0.87898 & 4 \mathrm{~B} 1 & 12 \\ 9 & 1 & 2 & 0.99026 & 4 \mathrm{~A} 2 & 12\end{array}$

fos

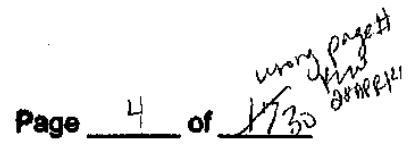

Page 86 of 519 
Analysis performed by J. Luoma SAS version 9.2 10:20 13AUGt2

AEH-12-PSEUDO-04

Efficacy of Pseudomonas fluorescens (Pf-CL145A)for controlling zebra mussesI on artifical substrates AEH-12-PSEUDO-04

Random assignment of substrate removal from tanks *** TANK $5 * * *$

Lake Carlos - Whole water Body Treatment

$\begin{array}{cccccc}\text { obs } & \text { row } & \text { position } & x & 0^{\text {tankn }} & \text { trt } \\ & & & & & \\ 1 & 2 & 2 & 0.05116 & 2 \mathrm{~B} 2 & 6 \mathrm{~h} \\ 2 & 1 & 1 & 0.15754 & 2 \mathrm{~A} 1 & 6 \mathrm{~h} \\ 3 & 3 & 1 & 0.19038 & 2 \mathrm{C} 1 & 6 \mathrm{~h} \\ 4 & 1 & 3 & 0.29436 & 2 \mathrm{~A} 3 & 9 \mathrm{~h} \\ 5 & 3 & 3 & 0.36230 & 2 \mathrm{C3} & \mathrm{gh} \\ 6 & 2 & 3 & 0.63280 & 2 \mathrm{~B} 3 & \mathrm{gh} \\ 7 & 3 & 2 & 0.73826 & 2 \mathrm{CA} & 12 \\ 8 & 1 & 2 & 0.86034 & 2 \mathrm{~A} 2 & 12 \\ 9 & 2 & 1 & 0.87946 & 2 \mathrm{~B} 1 & 12\end{array}$

(1) Tank numbers should be 5 not 2 . Yow 15ankid See Deviation $\mathrm{H2}$ for furtar clarification. Kino

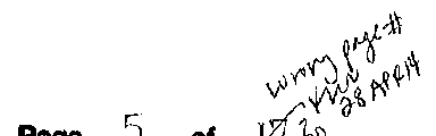

Page 5 of $1 / 30$ 
Analysis performed by J. Luoma SAS version 9.2 10:20 13AUG12

AEH-12-PSEUDO-04

Efficacy of Pseudomonas fluorescens (Pf-CL145A)for controlling zebra mussesl on artifical substrates AEH-12-PSEUD0-04

Random assignment of substrate removal from tanks $* * *$ TANK $6 * * *$

Lake Carlos - Whole water Body Treatment

$\begin{array}{cccccc}\text { Obs } & \text { row } & \text { position } & \mathrm{x} & \text { tankn } & \text { trt } \\ 1 & 1 & 3 & 0.13967 & 6 \mathrm{A3} & 6 \mathrm{~h} \\ 2 & 3 & 3 & 0.18498 & 6 \mathrm{C3} & 6 \mathrm{~h} \\ 3 & 1 & 2 & 0.22522 & 6 \mathrm{~A} 2 & 6 \mathrm{~h} \\ 4 & 3 & 1 & 0.29669 & 6 \mathrm{C} 1 & 9 \mathrm{~h} \\ 5 & 2 & 3 & 0.43557 & 6 \mathrm{~B} 3 & 9 \mathrm{~h} \\ 6 & 2 & 2 & 0.50443 & 6 \mathrm{~B} 2 & 9 \mathrm{~h} \\ 7 & 3 & 2 & 0.69851 & 6 \mathrm{C} 2 & 12 \\ 8 & 1 & 1 & 0.76815 & 6 \mathrm{~A} 1 & 12 \\ 9 & 2 & 1 & 0.83108 & 6 \mathrm{~B} 1 & 12\end{array}$

to

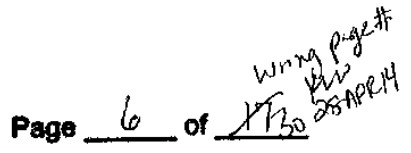

Page 88 of 519 
Analysis performed by J. Luoma SAS version 9.2 10:20 13AUG12

AEH-12-PSEUDO-04

Efficacy of Pseudomonas fluorescens (Pf-CL145A)for controlling zebra mussesl on artifical substrates AEH-12, PSEUDO-04

Randon assignment of substrate removal from tanks *** TANK $7 * * *$

Lake Carlos - Whole water Body Treatment

$\begin{array}{cccccc}\text { obs } & \text { row } & \text { position } & \times & \text { C }^{\text {tankn }} & \text { trt } \\ 1 & 2 & 2 & 0.05116 & 2 \mathrm{~B} 2 & 6 \mathrm{~h} \\ 2 & 1 & 1 & 0.15754 & 2 \mathrm{~A} 1 & 6 \mathrm{~h} \\ 3 & 3 & 1 & 0.19038 & 2 \mathrm{C1} & 6 \mathrm{~h} \\ 4 & 1 & 3 & 0.29436 & 2 \mathrm{A3} & 9 \mathrm{~h} \\ 5 & 3 & 3 & 0.36230 & 2 \mathrm{C3} & 9 \mathrm{~h} \\ 6 & 2 & 3 & 0.63280 & 2 \mathrm{~B} 3 & 9 \mathrm{~h} \\ 7 & 3 & 2 & 0.73826 & 2 \mathrm{C} 2 & 12 \\ 8 & 1 & 2 & 0.86034 & 2 \mathrm{~A} 2 & 12 \\ 9 & 2 & 1 & 0.87946 & 2 \mathrm{~B} 1 & 12\end{array}$

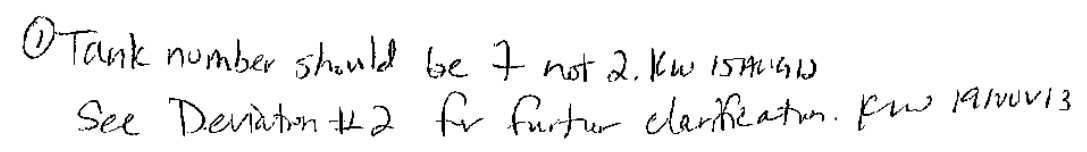

is

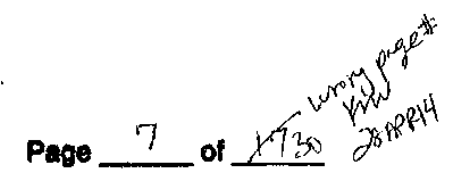


Analysis performed by $J$. Luoma SAS version 9.2 10:20 13 AUG12

Efficacy of Pseudomonas fluorescens (PF-CL145A) for controlling zebra mussesl on artifical substrates AEH-12-PSEUDO-04

Random assignment of substrate renoval from tanks *** TANK $8 * * *$

Lake Carlos - Whole water Body Treatment

$\begin{array}{cccccc}\text { Obs } & \text { row } & \text { position } & x & \operatorname{tankn} & \text { trt } \\ 1 & 2 & 3 & 0.21368 & 8 \mathrm{BB} & 6 \mathrm{~h} \\ 2 & 2 & 2 & 0.23698 & 8 \mathrm{~B} 2 & 6 \mathrm{~h} \\ 3 & 2 & 1 & 0.25634 & \mathrm{BB} 1 & 6 \mathrm{~h} \\ 4 & 1 & 3 & 0.47516 & \mathrm{BA} 3 & 9 \mathrm{~h} \\ 5 & 3 & 1 & 0.61003 & \mathrm{BC} 1 & 9 \mathrm{~h} \\ 6 & 3 & 3 & 0.61436 & 8 \mathrm{C} 3 & 9 \mathrm{~h} \\ 7 & 1 & 2 & 0.66954 & 8 \mathrm{~A} 2 & 12 \\ 8 & 3 & 2 & 0.93075 & 8 \mathrm{C} 2 & 12 \\ 9 & 1 & 1 & 0.93965 & 8 \mathrm{~A} 1 & 12\end{array}$

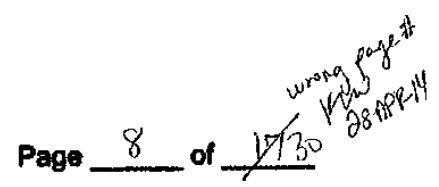


AEL-12-PSEUDO-O4

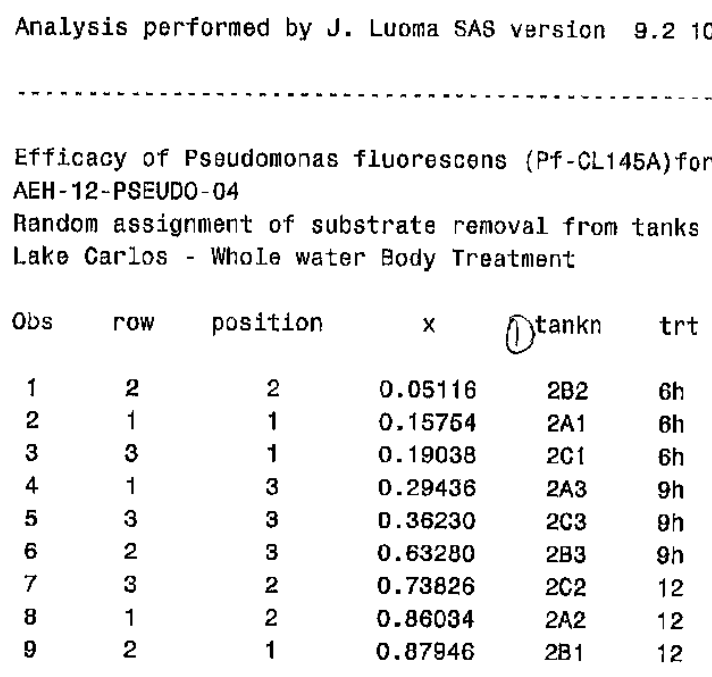

(1) Tank number shiveld be 9 not 2. kew 15 Aacig 18

See Deviaton tw 2 for further darification. pow anovis

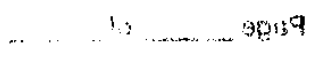

Page 9 of $L / 3_{3}$ 


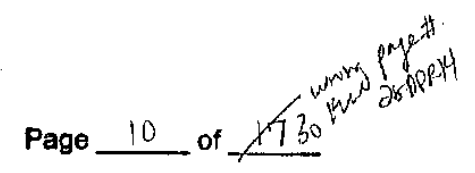




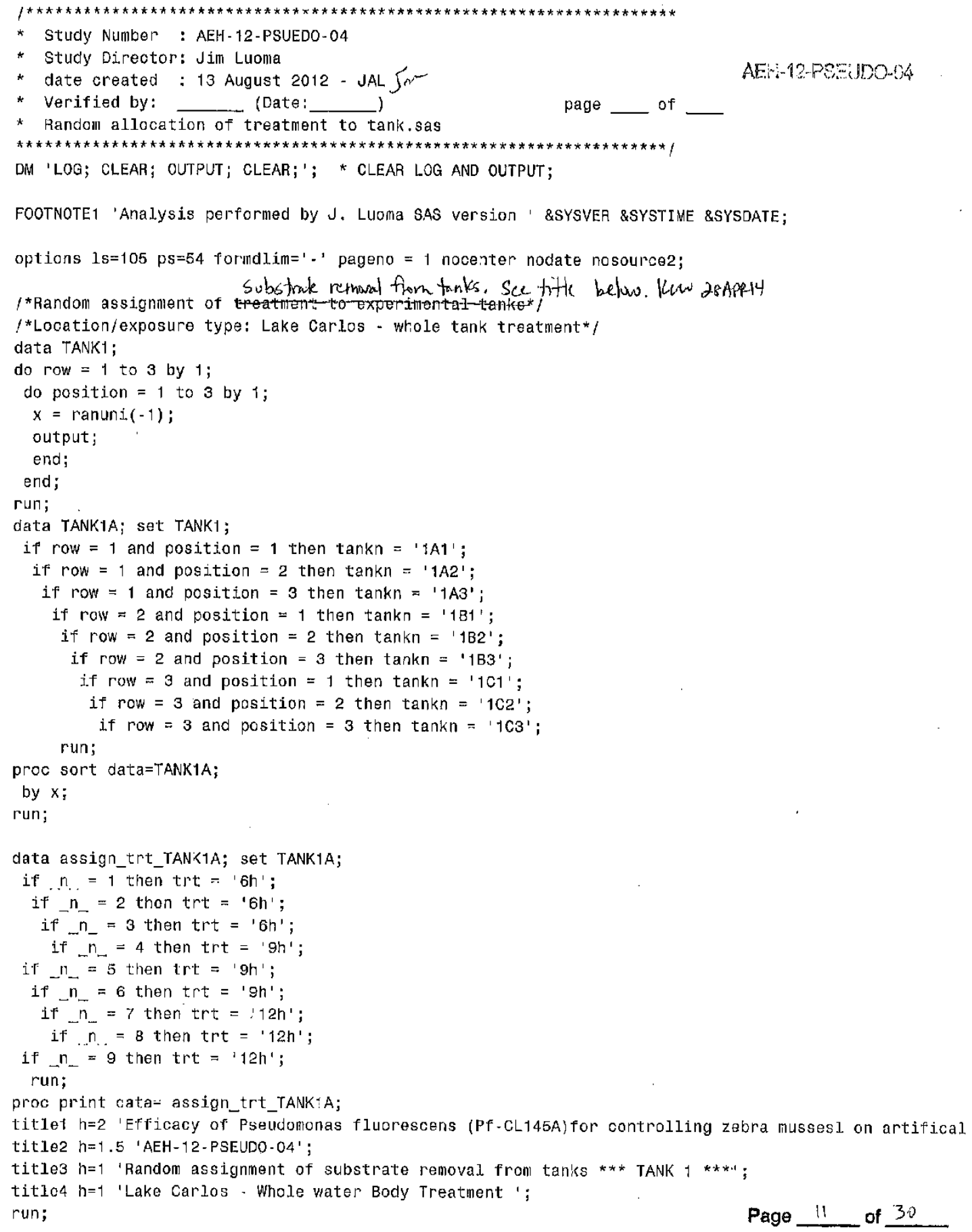




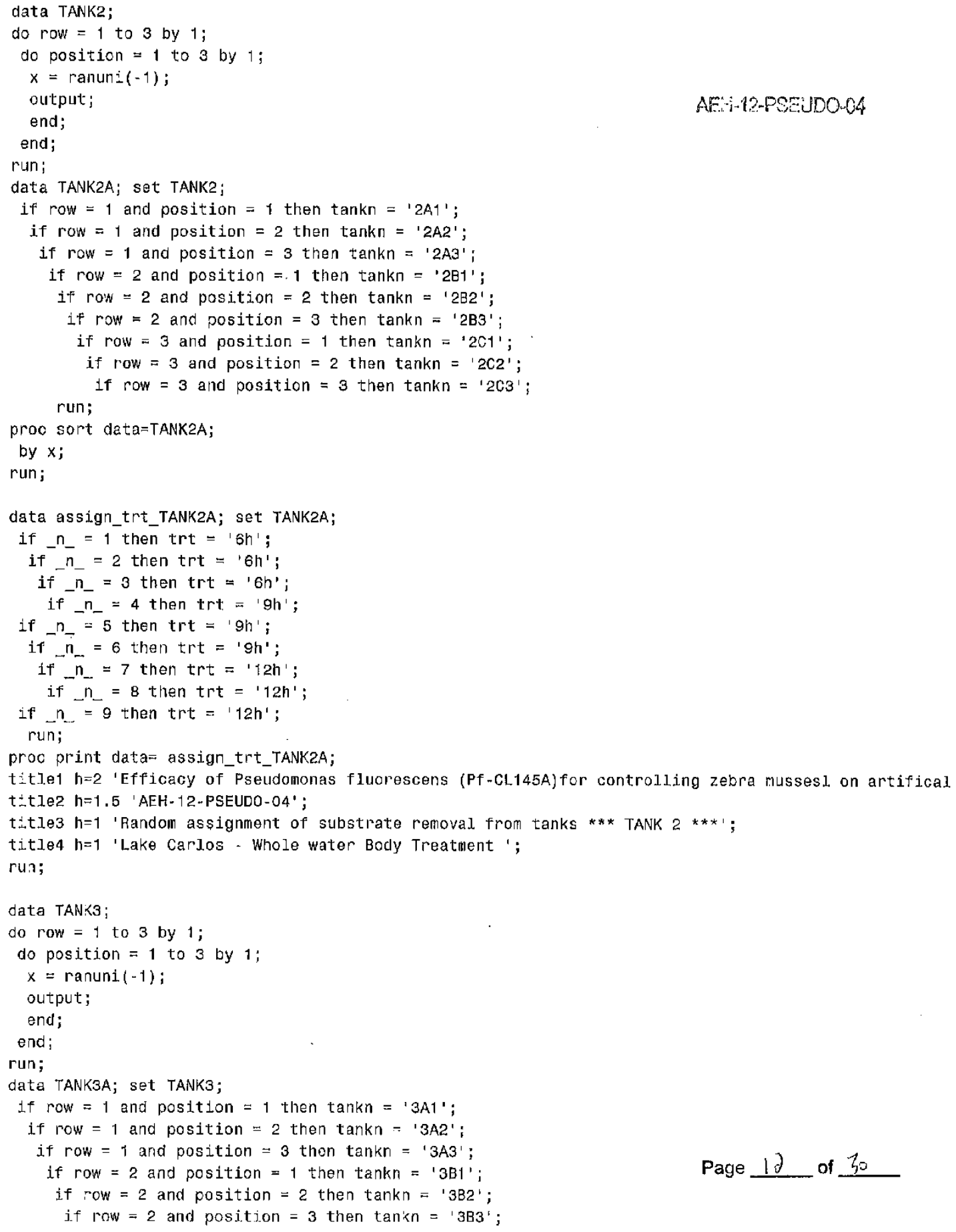




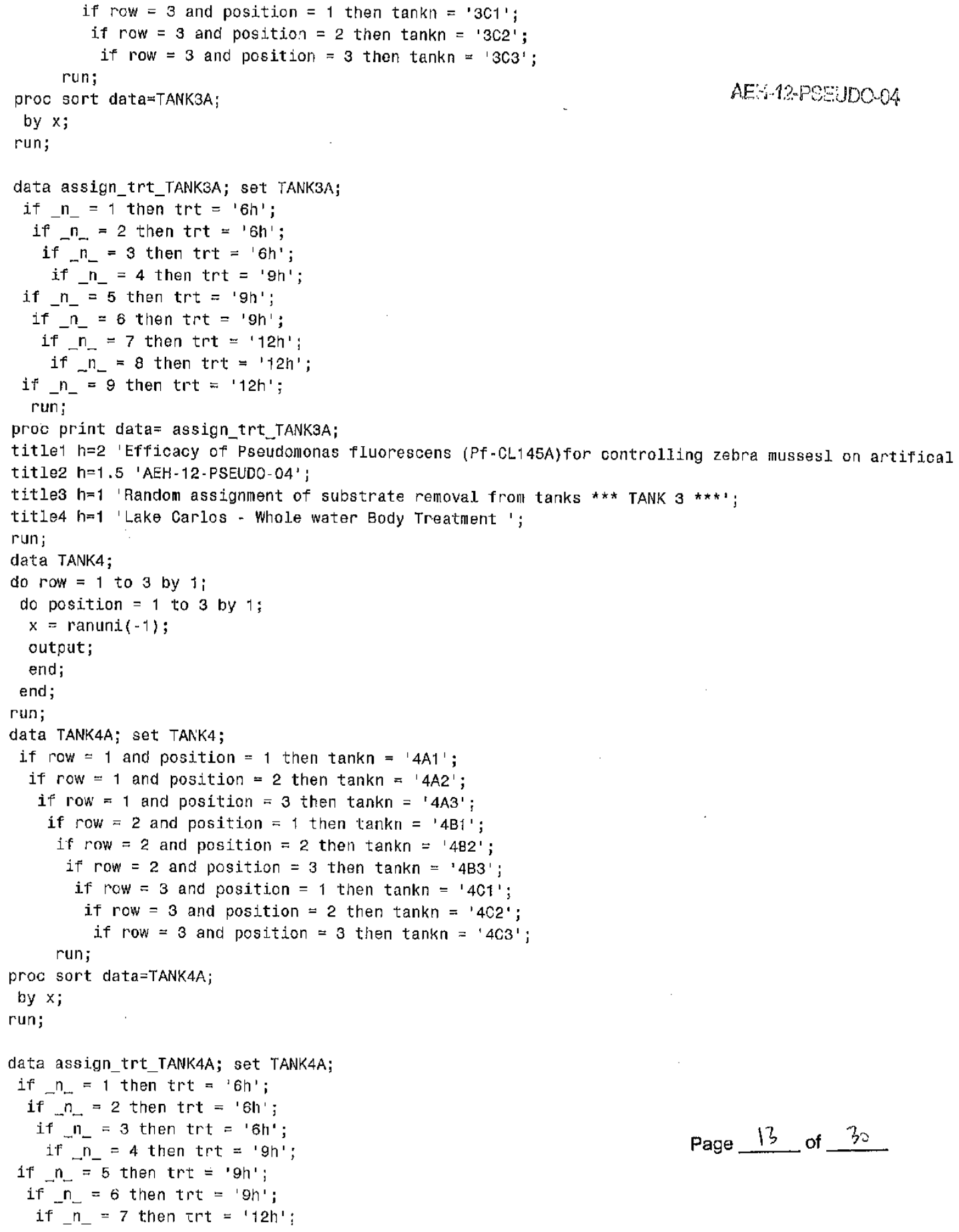




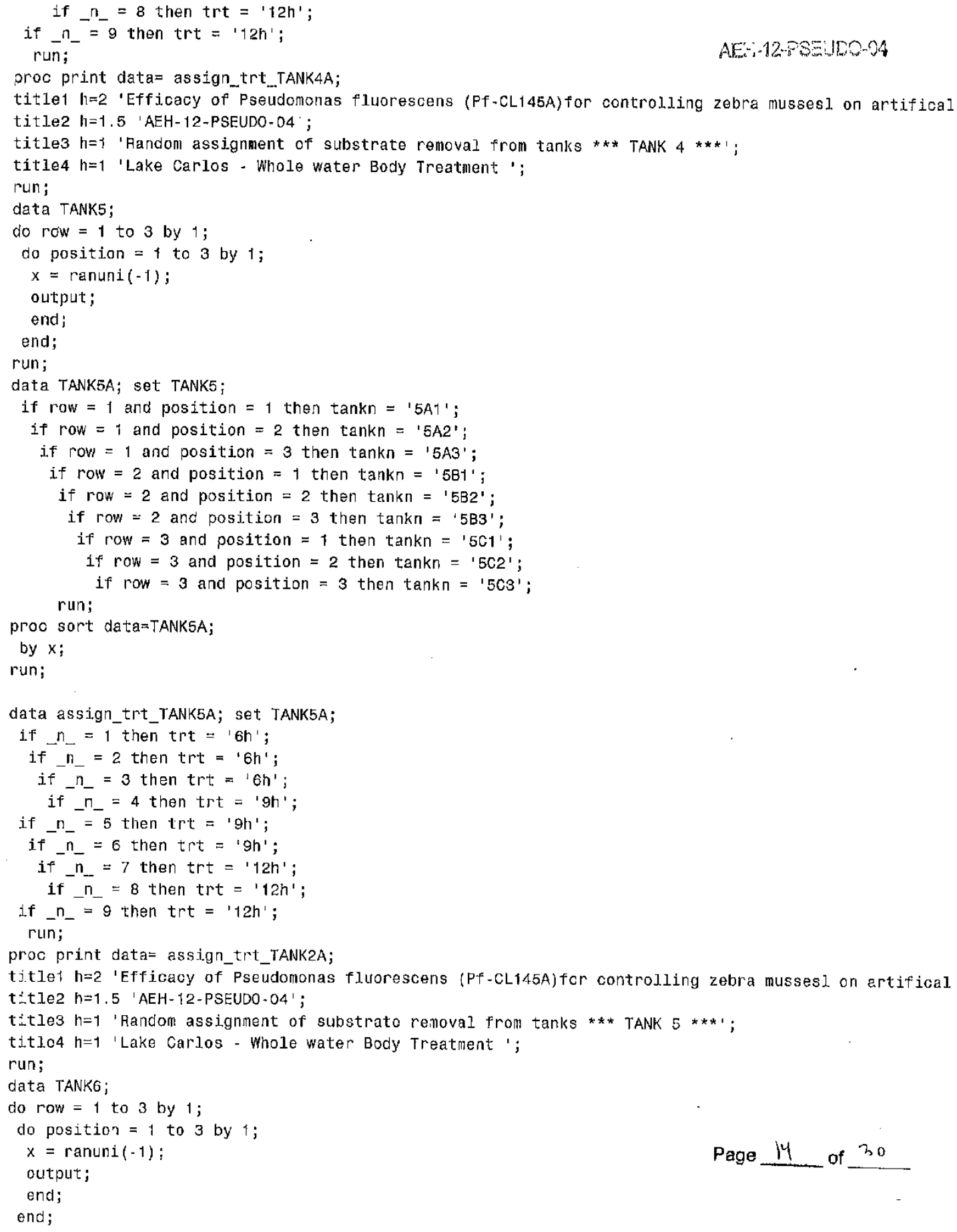

\section{Page 14 of 30}

\section{Page 96 of 519}




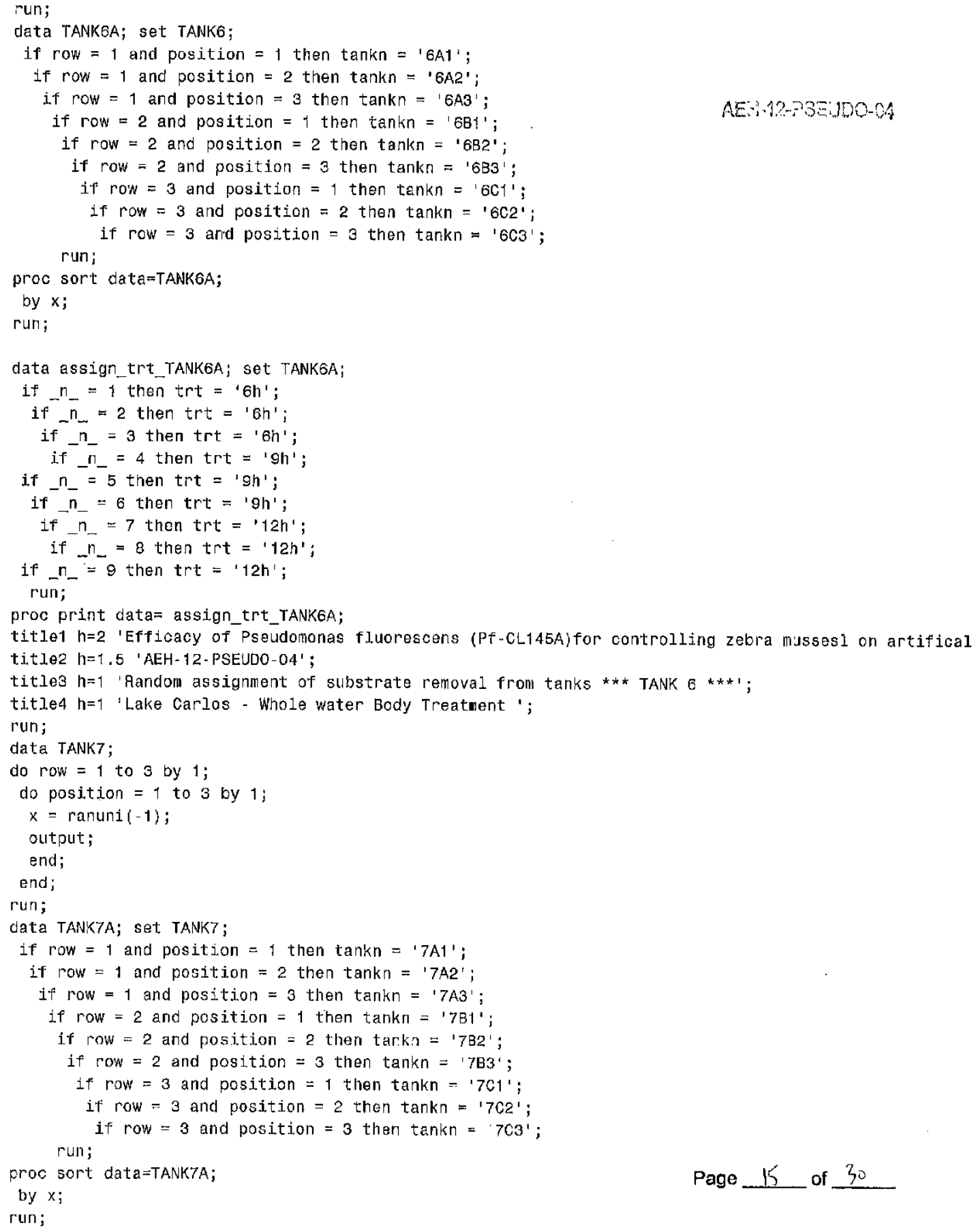




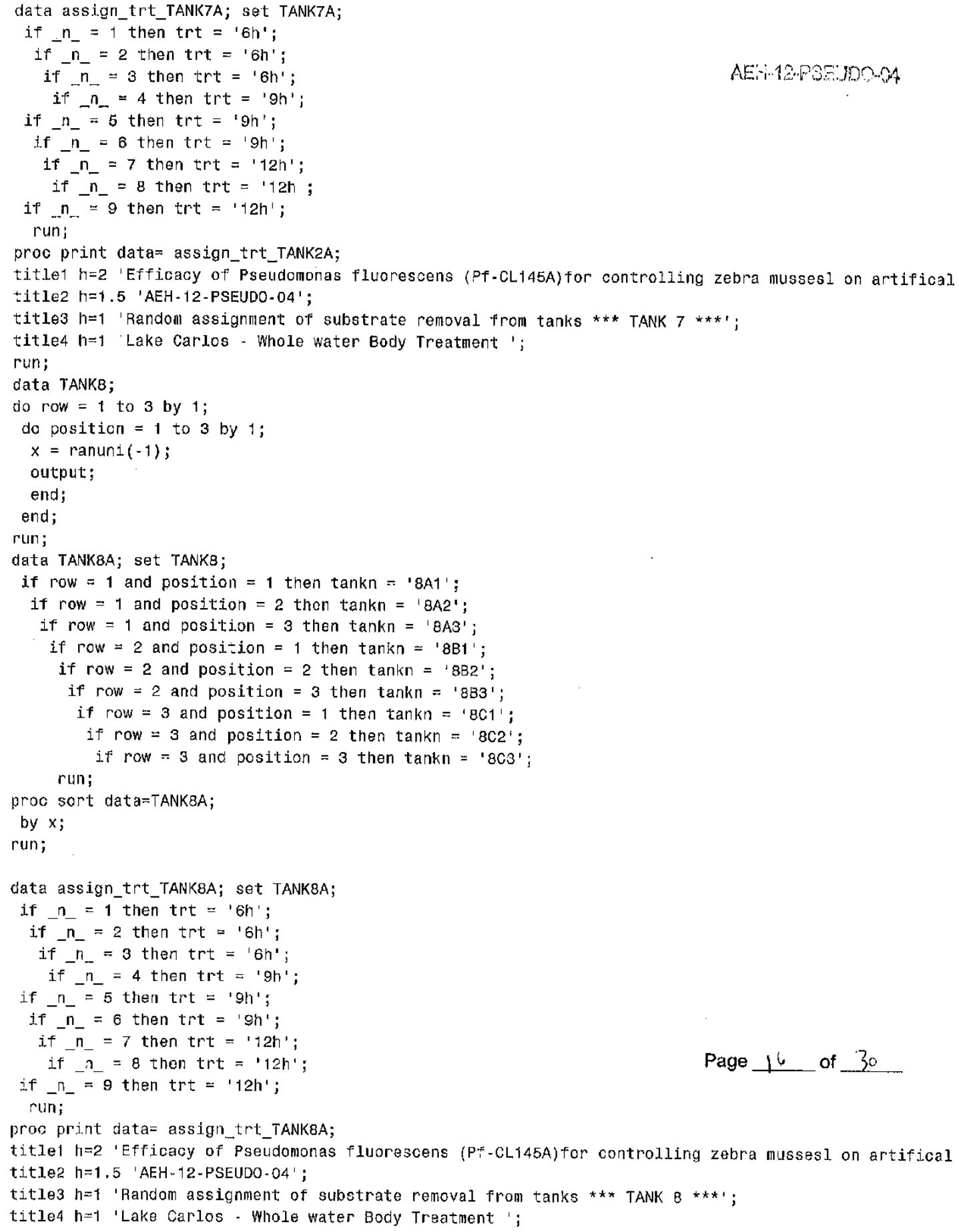




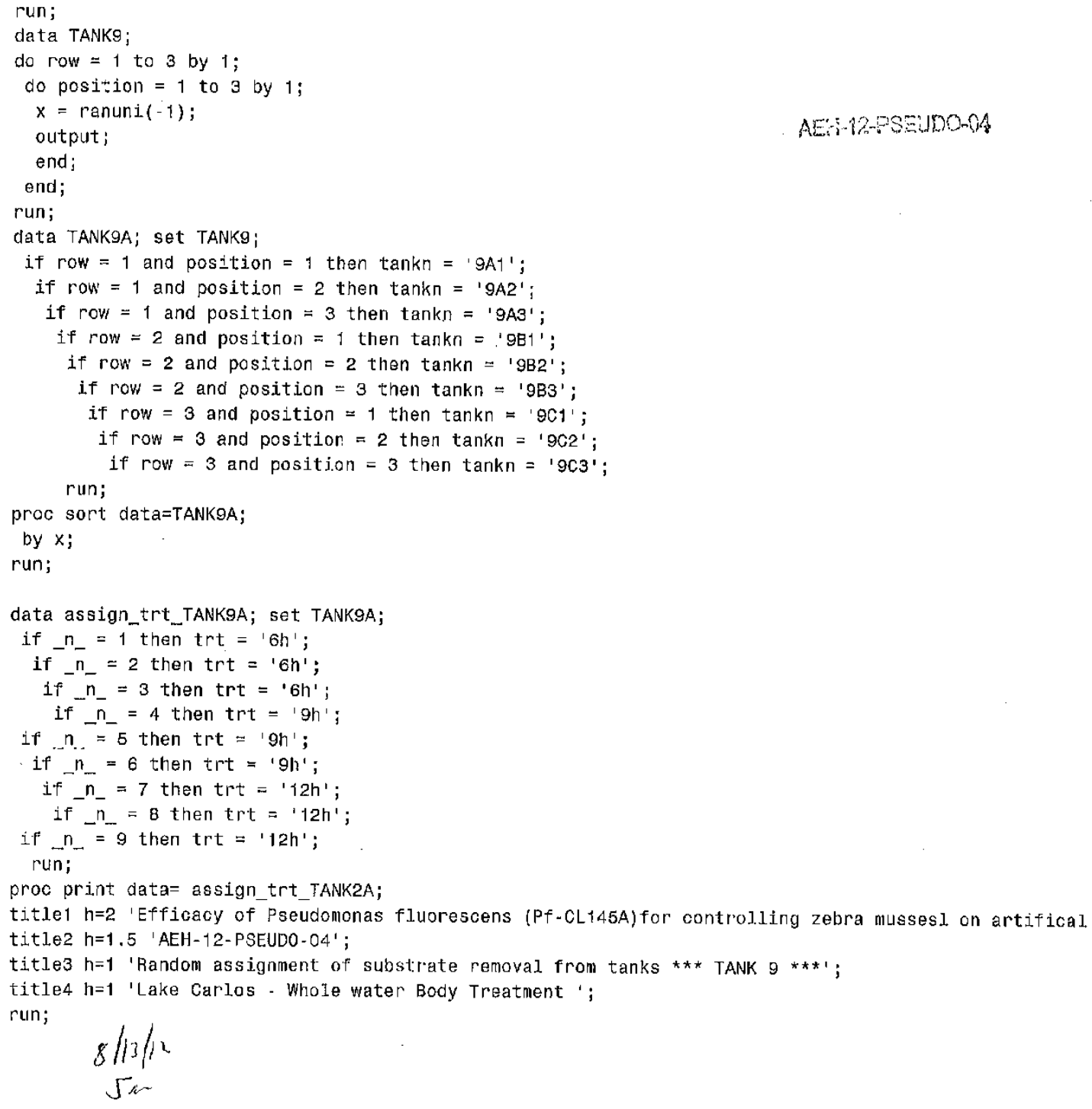




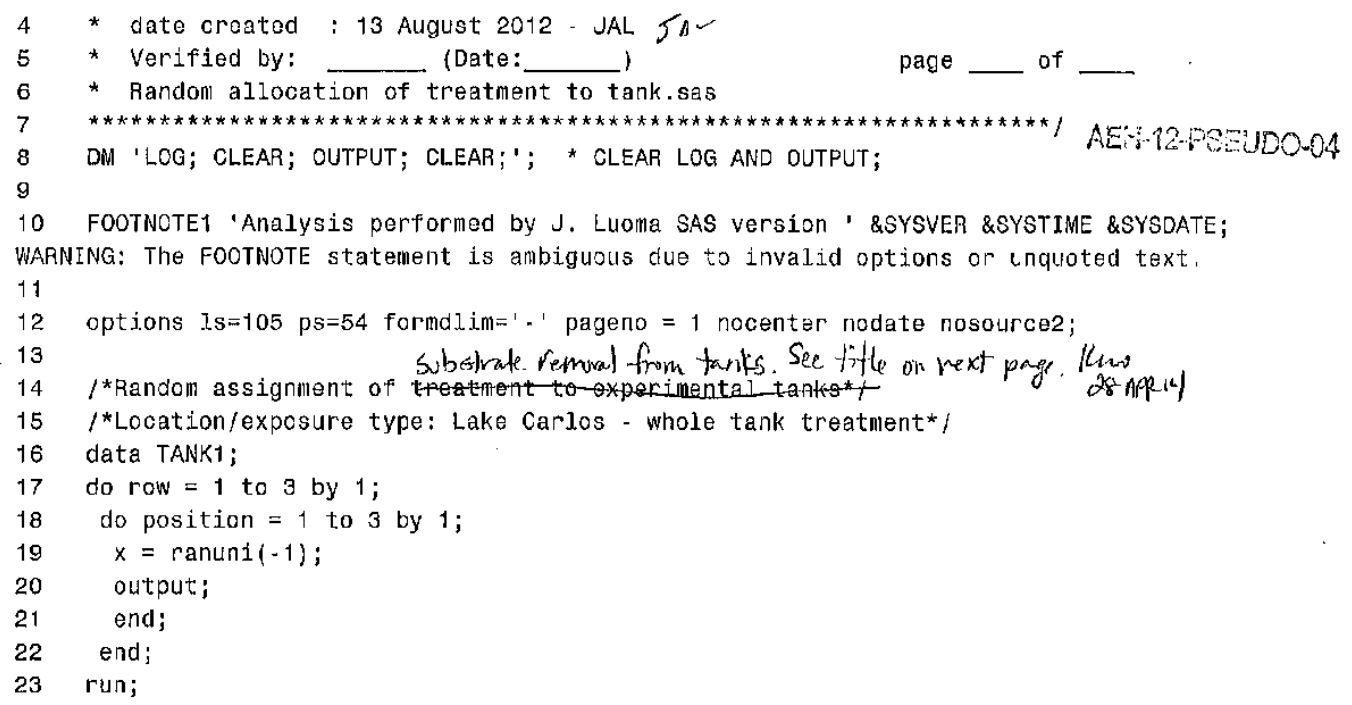

NOTE: The data set WORK.TANK1 has 9 observations and 3 variables,

NOTE: DATA statement used (Total process time):
real time
0.01 seconds
cpu time
0.01 seconds

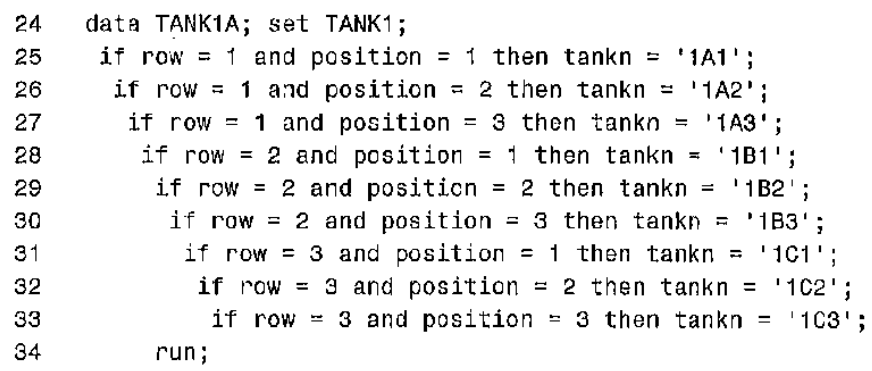

NOTE: There were $g$ observations read from the data set WORK. TANK1. NOTE: The data set WORK.TANK1A has 9 observations and 4 variables.

NOTE: DATA statenient used (Total process time):
real time
0.01 seconds

cpu time

0.01 seconds
35 proc sort data=TANKiA;
36 by $x$;
37 run;

NOTE: There were 9 observations road from the data set WORK.TANK1A.

NOTE: The data set WORK.TANK1A has 9 observations and 4 variables.

NOTE: PROCEJURE SORT used (Total process time):
real time
0.01 seconds
cpu time
0.01 seconds

Page 18 of 30 


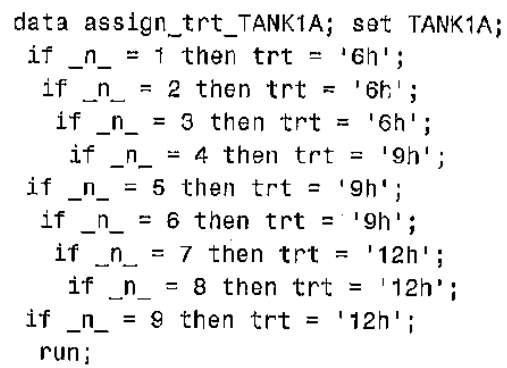

NOTE: There were 9 observations read from the data set WORK. TANK1A.

NOTE: The data set WORK.ASSIGN_TRT_TANKIA has $\theta$ observations and 5 variables.

NCTE: DATA statemont used (Total process time):

real time $\quad 0.01$ seconds

cpu time $\quad 0.01$ seconds

50 proc print data= assign_trt_TANK1A;

51 title1 $h=2$ 'Efficacy of Pseudomonas fluorescens (Pf-GL145A) for controlling zebra mussesl on 51 I artifical substrates';

52 title2 $h=1.5$ 'AEH-12-PSEUDO-04'

53 title3 $h=1$ 'Random assignment of substrate removal from tanks $* * *$ TANK $1 * * * 1$;

54 title4 h=1 'Lake Carlos - Whole water Body Treatment ';

55 run;

NOTE: There wore 9 observations read from the data set WORK.ASSIGN_TRT_TANK1A.

NOTE: PROCEDURE PRINT Lised (Total process time):

real time $\quad 0.09$ seconds

cpu time $\quad 0.03$ seconds

56 data TANK2;

do row $=1$ to 3 by 1

do position $=1$ to 3 by 1 ;

$x=\operatorname{ranuni.}(\cdot 1)$;

output;

end;

end;

64 run;

NOTE: The data set WORK. TANK? has 9 ubservations and 3 variables,

NOTE: DATA statement used (Total process time):
real time
0.00 seconds

cpu time

0.01 seconds

65 data TANK2A; set TANK2;

Page +19 of 30

if row $=1$ and position $=1$ then tankn $={ }^{2} A^{\prime}{ }^{\prime}$;

if row $=1$ and position $=2$ then tankn $=$ ' $2 \mathrm{~A} 22^{\prime} \mathrm{i}$

if row $=1$ and position $=3$ then tankn $=' 2 \mathrm{~A} 3^{\prime}$; 


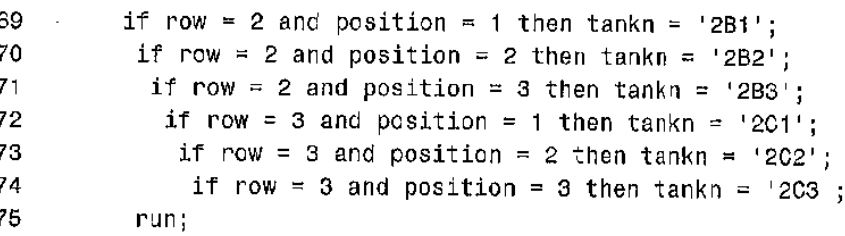

VOTE: There were 9 observations read from the data set WOAK. TANK2, NOTE: The data set WORK.TANK2A has 9 observations and 4 variables. NOTE: DATA statement used (Total process time):
real time
0.01 seconds

cpu time

0.01 seconds

proc sort data $=$ TANK2A;

by $x$;

run;

NOTE: There were 9 observations read from the data set WORK.TANK2A.

NOTE: The data set WORK.TANK2A has 9 observations and 4 variables.

NOTE: PROCEDURE SORT used (Total process time):

real time $\quad 0.00$ seconds

cpu time $\quad 0.01$ seconds

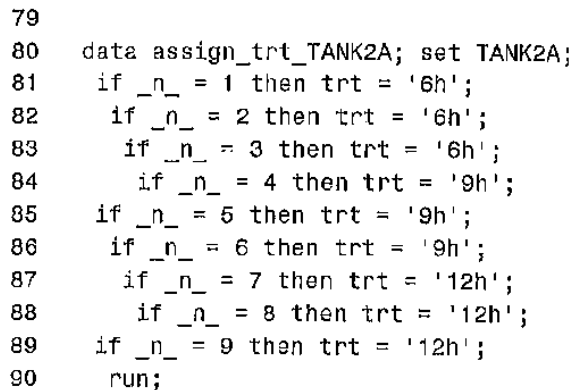

NOTE: There were 9 observations read from the data set WORK.TANK2A.

NOTE: The data set WORK.ASSIGN_TRT_TANK2A has 9 observations and 5 variables.

NOTE: DATA statement used (Total process tine):
real time
0.00 seconds

cpu $=i m e$

0.00 seconds

91 proc print data= assign_trt_TANK2A;

92 ti.tle1 $h=2$ 'Efficacy of Pseudomonas fluorescens (Pf-CL145N)for controlling zebra mussesl on

92 ! artifical substrates';

93 title2 $n=1.5$ 'AEH-12-PSEUDO-04';

S4 title3 $h=1$ 'Random assignmont of substrate removal from tanks *** TANK $2 * \star *$ ';

95 title4 h=1 'Lake Carlos - Whole water Body Treatment ';

96 run

Page 20 of 30

NOTE: There were 9 ooservations read from the data set WORK.ASSIGN_TRT_TANK2A.

NOTE: PROCEDURE PRINT used (Total process time): 
$\begin{array}{ll}\text { real time } & 0.00 \text { seconds } \\ \text { cpu time } & 0.01 \text { seconds }\end{array}$

data TANK3;

do rovl $=1$ to 3 by 1 ;

do position $=1$ to 3 by 1 ;

$x=\operatorname{ranuni}(-1)$;

output;

end;

end;

105. run;

NOTE: The data set WORK. TANK3 has 9 observations and 3 variables.

NOTE: DATA statement used (Total process time):

real time $\quad 0.00$ seconds

cpu time $\quad 0.01$ seconds

106 data TANK3A; set TANK3;

107 if row $=1$ and position $=1$ then tankn $={ }^{\prime} 3 \mathrm{~A} 1^{\prime}$;

108 if row $=1$ and position $=2$ then $\operatorname{tankn}=$ ' $3 \mathrm{~A} 2$ '

if row $=1$ and position $=3$ then tankn $={ }^{3} \mathrm{~A} \mathrm{~S}^{\prime}$;

if row $=2$ and position $=1$ then tankn = ' $3 \mathrm{~B} 1$ ' ';

if row $=2$ and position $=2$ then tankn $=$ ' $3 B 2$ ';

if row $=2$ and position $=3$ then tankn $=$ ' $3 \mathrm{~B} 3$ ';

if row $=3$ and position $=1$ then tankn $=3 \mathrm{C} 1$ ';

if row $=3$ and position $=2$ then tankn $={ }^{\prime} 3 \mathrm{C} 2$ ';

if row $=3$ and position $=3$ then $\operatorname{tankn}=' 3 \times 3$ ';

run;

NOTE: There were 9 observations read from the data set WOAK. TANK3.

NOTE: The data set WORK. TANK3A has 9 observations and 4 variables.

NOTE: DATA statement used (Total process time):

real time. $\quad 0.00$ seconds

cpu $-i m e \quad 0.01$ seconds

117 proc sort data=TANK3A;

118 by $x$;

119 run;

NOTE: There were 9 observations read from the date set WOZK. TANK3A.

NOTE: The data set WCRK.TANK3A nas 9 observations and 4 variables.

VOTE: PROCEDURE SORT used (Total, process time):
real time
0.00 seconds
cpu time
0.01 seconds

120

121 data assign_trt_TANK3A; set TANK3A;

122 if $n_{-}=1$ then trt $=' 6 \mathrm{~h}^{\prime}$;

123 if ${ }_{-} \bar{n}_{-}=2$ then trt $=$ ' $6 \mathrm{~h}$ ';

124 if $\bar{n}_{-}=3$ then trt $=$ ' $6 \mathrm{~h}$ ';

125 if $\bar{n}_{-}=4$ then trt $=' 9 h^{\prime}$; 


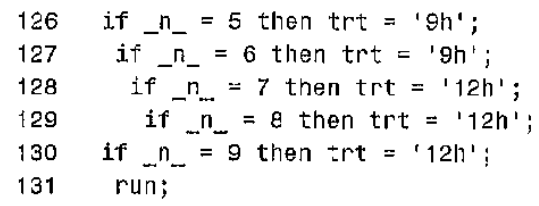

NOTE: There were 9 observations read from the data set WOR'. TANK3A.

NOTE: The data set WORK.ASSIGN_TRT_TANK3A has 9 observations and 5 variables.

NOTE: DATA statement used (Total process time):
real time
0.00 seconds
cpu time
0.01 seconds

\$32 proc print data= assign_trt_TANK3A;

133 title1 h=2 'Efficacy of Pseudomonas flucrescens (Pf-CL145A) for controlling zekra mussesl on

133 ! artifical substrates';

134 title2 $\mathrm{h}=1.5$ 'AEH-12-PSEUDO-04';

135 title $h=1$ 'Random assigninent of substrate removal from tanks *** TANK $3 * * * 1$;

136 title4 h=1 'Lake Carlos - Whole water Body Treatment: ';

137 run;

NOTE: There were 9 observations read from the data sot WORK.ASSIGN_TRT_TANK3A.

NOTE: PROCEDURE PRINT used (Total procoss time):

real time $\quad 0.00$ seconds

cpu time 0.00 seconds

138 data TANK4;

139 do row $=1$ to 3 by 1 ;

140 do position $=1$ to 3 by 1 ;

$141 \quad x=\operatorname{ranuni}(-1)$;

142 output;

143 end;

144 end;

145 run;

NOTE: The data set WORK.TANK4 has 9 observations and 3 variables.

NOTE: DATA statement used (Total process time):
real tilne
0.00 seconds
cpu time
0.00 seconds

146 data TANK4A; set TANK4;

147 if row $=1$ and position $=1$ then $\tan \kappa \mathrm{kn}={ }^{\prime} 4 \mathrm{~A} 11^{\prime}$;

148 if row $=1$ and position $=2$ then tankn $={ }^{\prime} 4 \mathrm{~A} 2^{\prime}$;

149 if row $=1$ and position $=3$ then tankn $=14 \mathrm{~A} 3{ }^{\prime}$;

150 if row $=2$ and position $=1$ then tankn $={ }^{\prime} 4 \mathrm{~B} 11^{\prime}$;

151 if row $=2$ and position $=2$ then tankn $=' A B 2^{\prime}$;

152 if row $=2$ and position $=3$ then tankn $=$ ' $4 \mathrm{~B} 33^{\prime}$;

153 if row $=3$ and position $=1$ then tankn $={ }^{\prime} 4 C 1{ }^{\prime}$;

154 if row $=3$ and position $=2$ then $\operatorname{tankn}={ }^{\prime} 4 \mathrm{CQ} 2^{\prime}$;

155 if row $=3$ and position $=3$ then $\operatorname{tankn}={ }^{\prime}$ ' $4 \mathrm{CB}{ }^{\prime}$;

156 run;

NOTE: There were 9 observations read from the data set WORK. TANK4. 
NOTE: The data set WORK. TANK4A has 9 observations and 4 variables.

NOTE: DATA statement used (Total process tille):
real time
0.00 seconds
cpu time
0.00 seconds

AE'-12.5.

157 proc sort data= -ANK4A;
158 by $x$;
159 run;

NOTE: There were 9 observations read from the data set WOAK. TANK4A.

NOTE: The data set WORK.TANK4A has 9 observations and 4 variables.

NOTE: PROCEOURE SORT used (Total process time):

real tine $\quad 0.05$ seconds

cpu time $\quad 0.00$ seconds

160

161 data assign_trt_TANK4A; set TANK4A;

162 if $n_{-}=1$ then $t r t=' 6 h^{\prime}$;

163 if $\bar{n}_{-}=2$ then trt $=16 h^{\prime}$;

164 if $n_{-}=3$ then trt $=$ ' $6 \mathrm{~h}^{\prime}$; if $\vec{n}_{-}=4$ then trt $=$ ' $9 h^{\prime}$;

if $n=5$ then trt $=$ ' $9 \mathrm{~h}$ ':

if $n_{-}=6$ then trt $=$ ' $9 \mathrm{~h}^{\prime}$;

if $n=7$ then $\operatorname{trt}=12 h^{\prime}$;

if $\bar{n}_{-}=8$ then trt $=112 h '$ ';

if $n_{-}=9$ then trt $=112 h^{\prime}$;

171 run;

NOTE: There wore 9 observations read from the data set WORK. TANK4A.

NOTE: The data set WORK.ASSIGN_TRT_TANK4A has 9 observations and 5 variables.

NOTE: DATA statement used (Total process time):
real Łine
0.00 seconds
cpu time
0.00 seconds

172 proo print data $=$ assign trt_TANK4A

173 titie1 $h=2$ 'Efficacy of Pseudomonas fluorescens (Pf-CL145A)for controlling zebra mussesl on

173 ! artifical substrates';

174 titlee $h=1.5$ 'AEH-12-PSEUDO-04';

175 ti.tle3 $h=i$ 'Randon assignment of substrate removal from tanks *** TANK $4 * * * 1$;

176 ti.t.e4 $h=1$ 'Lake Carlos - Whole water Body Treatment ';

177 run;

NOTE: There were 9 observations read from the data sel WORK.ASSIGN_TRT_TANK4A.

NOTE: PROCEDURE PRINT used (Total. process time):
real time
0.00 seconds
cpu time
0.01 seconds

178 data TANK5:

179 do row $=1$ to 3 by 1 ;

180 do position $=1$ to 3 by 1 ;

Page 23 of 30

$181 \mathrm{x}=\operatorname{ranuni}(-1)$;

182 ouzput: 
VOTE: The data set WORK. TANK5 has 9 observations and 3 variables.

NOTE: DATA statoment used (Total process time):
real time
0.01 seconds
cpu time
0.01 seconds

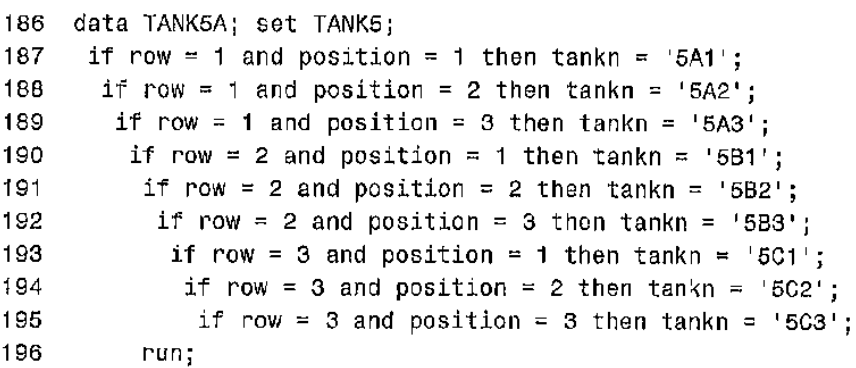

NOTE: There were 9 observations read from the data set WORK.TANK5. NOTE: Tho data set WORK.TANK5A has 9 observations and 4 variables. NOTE: DATA statement used (Total process time):

real time $\quad 0.00$ seconds

cpu tine $\quad 0.01$ seconds

197 proc sort data=TANK5A;

198 by $\mathrm{x}$;

199 run;

NOTE: There were 9 observations read from the data set WCRK.TANK5A. NOTE: The data set WORK. TANK5A has 9 observations and 4 variables.

NOTE: PROCEDURE SORT used (Total process time):

$\begin{array}{ll}\text { real time } & 0.00 \text { seconds } \\ \text { cpu time } & 0.01 \text { seconds }\end{array}$

200

201 data assign_trt_TANK5A; set TANK5A;

202 if $?=1$ then trt $=$ ' $6 \mathrm{~h}$ ';

203 if $n_{-}=2$ then trt $=$ ' $6 \mathrm{~h}$ ';

204 if $n_{-}=3$ then trt $=$ ' $6 \mathrm{~h}^{\prime}$ ';

205 if $\bar{n}_{-}=4$ then trt $=$ ' $9 \mathrm{~h}^{\prime}$ ';

206 if $n=5$ then trt $=$ ' $9 h^{\prime}$ ';

207 if $\bar{n}_{-}=6$ then $t r t=9 h^{\prime}$;

208 if $n_{-}=7$ then $\operatorname{trt}=12 \mathrm{~h}$ ';

209 if $\bar{n}_{-}=8$ then trt $=112 h^{\prime}$;

210 if $n_{-}=9$ then trt $=$ '12h';

211 run;

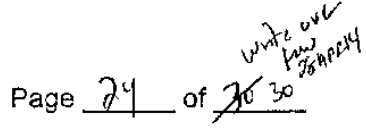

NOTE: There were 9 observations read from the data set WORK. TANK5A.

NOTE: The data set WORK.ASSIGN_TRT_TANK5A has 9 observations and 5 variables.

NOTE: DATA statement used (Total process time): 

real time
0.00 seconds
cpu time
0.01 seconds

212 proc print data= assign_trt_TANK2A;

213 titley $h=2$ 'Efficacy of Pseudomonas fuuorescens (Pf-CL145A)for controlling zebra mussesl on

213 ! artifical substrates';

214 ti.tle2 $h=1.5$ 'AEH-12-PSEUDO-04';

215 title3 $n=1$ 'Randoll assignment of substrate removal from tanks *** TANK $5 * * * 1$;

216 title $\mathrm{h}=1$ 'Lake Carlos . Whole water Body Treatment ';

217 run;

NOTE: There were 9 observations read front the data set WORK.ASSIGN_TRT_TANK2A.

NOTE: PROCEDURE PRINT used (Total process time):

roal time $\quad 0.00$ seconds

cpu time $\quad 0.00$ seconds

218 data TANK6;

219 do row $=1$ to 3 by 1 ;

220 do position $=1$ to 3 by 1 ;

$221 \quad x=\operatorname{ranuni}(-1)$;

222 output;

223 end;

224 end;

225 run;

NOTE: The data set WORK.TANK6 has 9 observations and 3 variables.

NOTE: DATA statement used (Totàl process time):
real time
0.00 seconds
cpu tilie
0.00 seconds

226 data TANK6A; set TANK6;

227 if row $=1$ and position $=1$ then tankn $=$ ' $6 \mathrm{~A} 1$ ';

228 if row $=1$ and position $=2$ then tankn $={ }^{\prime} 6 \mathrm{~A} 2^{\prime}$;

229 If row $=1$ and position $=3$ then $\operatorname{tankn}={ }^{\prime} 6 \mathrm{~A} 3{ }^{\prime}$;

if row $=2$ and position $=1$ then tankn $=$ ' $6 \mathrm{~B} 1$ ';

if row $=2$ and position $=2$ then tankn $={ }^{\prime} 6 \mathrm{~B} 2$ ';

if row $=2$ and position $=3$ then tankn $=$ ' $683^{\prime}$;

if row $=3$ and position $=1$ then tankn $=16 \mathrm{C} 11^{\prime}$

if row $=3$ and position $=2$ then tankn $={ }^{\prime} 6 \mathrm{C} 2{ }^{\prime}$;

if row $=3$ and position $=3$ then tankn $={ }^{\prime} 6 \mathrm{C} 3^{\prime}$; run;

NOTE: There were 9 observations read from the data set WOAK. TANK6.

NOTE: The data set WORK.TANK6A has 9 observations and 4 variables.

NOTE: DATA statement used (Total process time):
real time
0.00 seconds
cpu time
o. or seconds

237 proc sort data=TANKGA;

238 by $x$;

Page 25 of 30

239 run; 
NOTE: There were $\theta$ observations read from the data set WORK, TANK6A.

NOTE: The data set WORK.TANK6A has 9 observations and 4 variables.

NOTE: PROCEDURE SORT used (Total process time):

$\begin{array}{ll}\text { real time } & 0.00 \text { seconds } \\ \text { cpu time } & 0.01 \text { seconds }\end{array}$

$A E-A=0-10024$

240

241 data assign_trt_TANK6A; set TANK6A;

242 if $\mathrm{n}_{-}=1$ then trt $=$ ' $6 \mathrm{~h}$ ';

243 if $\bar{n}_{-}=2$ then trt $=$ '6h';

244 if $n_{-}=3$ then trt $=$ ' $6 \mathrm{~h}^{\prime}$;

245 if $\vec{n}_{-}=4$ then trt $=$ ' $9 \mathrm{~h}^{\prime}$;

246 if $n_{-}=5$ then trt $=' 9 \mathrm{~h}^{\prime}$;

247 if $\bar{n}_{-}=6$ then trt $=$ ' $9 \mathrm{~h}$ ';

248 if $n_{-}=7$ then trt $=112 \mathrm{~h}^{\prime}$;

249 if $\vec{n}_{-}=8$ then $t r t=12 h^{\prime}$;

250 if $n_{-}=9$ then trt $=112 \mathrm{~h}$ ';

251 run;

NOTE: There were 9 observations read from the data set WORK.TANK6A.

NOTE: The data set WORK.ASSIGN_TRT_TANK6A has 9 observations and 5 variables.

NOTE: DATA statement used (Total process time):

real time $\quad 0.00$ seconds

cpu time $\quad 0.01$ seconds

252 proc print data= assign_trt_rANK6A;

253 title1 h=2 'Efficacy of Pseudomonas fluorescens (Pf-CL.145A)for controllirg zebra mussesl on

253 ! artifical substrates';

254 title2 $h=1.5$ 'AEH-12-PSEUDO-04';

255 titles $h=1$ 'Random assignment of substrate removal from tanks *** TANK $6 * * *$;

256 title4 $\mathrm{h}=1$ 'Lako Carlos - Whole water Body Treatment ;

257 run;

NOTE: There were 9 observations read fron the data set WOAK.ASSIGN_TRT TANK6A.

NOTE: PROCEDURE PRINT used (Total process time):

$\begin{array}{ll}\text { real time } & 0.00 \text { seconds } \\ \text { cpu time } & 0.00 \text { soconds }\end{array}$

258 data TANK7;

259 do row $=1$ to 3 by 1 ;

260 do position $=1$ to 3 by 1 ;

$261 \quad x=$ ranuni $(-1)$;

262 output;

263 end

264 end;

265 run;

NOTE: The data set WORK. TANK7 has 9 observations and 3 variabies.

NOTE: DATA statenent used (Total process time):
roal tine
0.00 seconds
cpu time
0.00 seconds

Page 20 of 30 


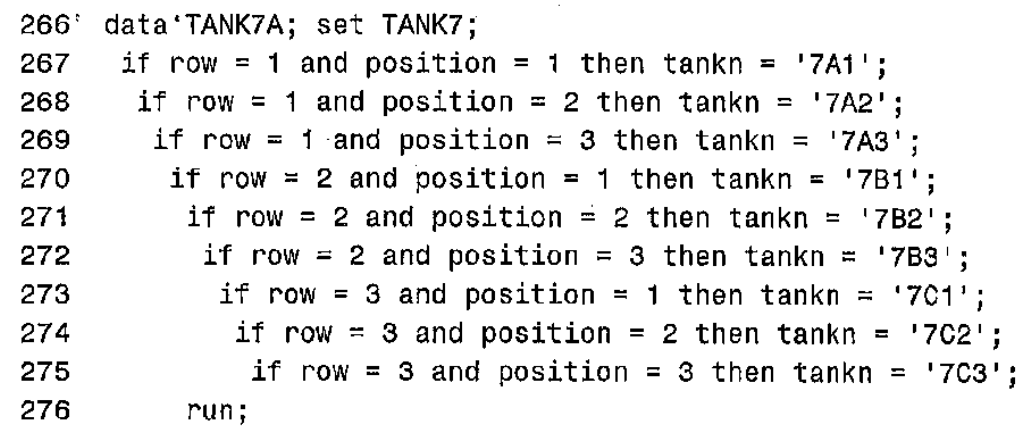

NOTE: There were 9 observations read from the data set WOAK. TANK7.

NOTE: The data set WORK. TANK7A has 9 observations and 4 variables.

NOTE: DATA statement used (Total process time):

real time $\quad 0.00$ seconds

cpu time $\quad 0.01$ seconds

277 proc sort data=TANK7A;

278 by $x$;

279 run;

NOTE: There were 9 observations read from the data set WORK.TANK7A.

NOTE: The data set WORK.TANKTA has 9 observations and 4 variables.

NOTE: PROCEDURE SORT used (Total process time):
real time
0.01 seconds
cpu time
0.00 seconds

data assign_trt_TANK7A; set TANKTA;

if $\mathrm{n}_{-}=1$ then $\operatorname{trt}=$ ' $6 \mathrm{~h}^{\prime}$;

if $\bar{n}_{-}=2$ then trt $=$ ' $6 \mathrm{~h}$ ';

if ${ }_{-} n_{-}=3$ then trt $=' 6 \mathrm{~h}^{\prime}$;

if $\bar{n}_{-}=4$ then trt $=$ ' $9 \mathrm{~h}^{\prime}$;

if $n_{-}=5$ then trt $=19 h^{\prime}$;

if $\bar{n}_{-}=6$ then trt $=\bar{n}^{\prime} 9 \mathrm{~h}^{\prime}$;

if ${ }_{-} n_{-}=7$ then trt $=112 \mathrm{~h}^{\prime}$;

if $\bar{n}_{-}=8$ then $\operatorname{trt}=12 \mathrm{~h}^{\prime}$;

if $n_{-}=9$ then $\operatorname{trt}=' 12 \mathrm{~h}^{\prime}$;

run;

NOTE: There were 9 observations read from the data set WORK. TANK7A.

NOTE: The data set WORK.ASSIGN.TRT_TANK7A has 9 observations and 5 variables.

NOTE: DATA statement used (Total process time):
real time
0.00 seconds
cpu time
0.01 seconds 
297 run;

NOTE: There were 9 observations read fron the data set WORK.ASSIGN_TRT_TANK2A.

NOTE: PROCEDURE PRINT used (Total process time):

real time $\quad 0.00$ seconds

cpu time $\quad 0.00$ seconds

$A-n+1200-15004$

298 data TANK8;

299 do row $=1$ to 3 by 1 ;

300 do position $=1$ to 3 by 1 ;

$301 \quad x=$ ranuni $(-1)$;

302 output;

303 end;

304 end;

305 run;

NOTE: The data set WORK. TANK8 has 9 observations and 3 variables.

NOTE: DATA statement used (Total process time):
real time
0.00 seconds
cpu time
0.00 seconds

306 data TANK8A; set TANKB;

307 if row $=1$ and position $=1$ then tankn $=' B A 1 '$;

308 if row $=1$ and position $=2$ then $\operatorname{tankn}={ }^{\prime} 8 \mathrm{~A} 2^{\prime}$;

309 if row $=1$ and position $=3$ then $\operatorname{tankn}={ }^{\prime} B A 3^{\prime}$;

310 if row $=2$ and position $=1$ then tankn $=$ ' $8 \mathrm{~B} 1$ ';

311 If row $=2$ and position $=2$ then tankn $=$ ' $8 \mathrm{~B} 2$ ' ;

312 if row $=2$ and position $=3$ then tankn $=$ ' $8 B 3$ ';

313 if row $=3$ and position $=1$ then tankn $=$ ' $8 \mathrm{C} 1$ '

314 if row $=3$ and position $=2$ then tankn $={ }^{\prime} 8 \mathrm{C} 2{ }^{\prime}$;

315 if row $=3$ and position $=3$ then tankn $={ }^{\prime} 8 \mathrm{C} 3^{\prime}$;

316 run:

NOTE: There were 9 observations read fron the data set WORK. TANK8.

NOTE: The data set WORK.TANKBA has 9 observations and 4 variables.

NOTE: DATA statement used (Total process time):
real time
0.00 seconds
cpu timo
0.00 seconds

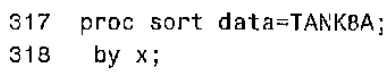

NOTE: There were 9 observations read from the data set WORK. TANK8A.

NOTE: The data set WORK.TANKBA has 9 observations and 4 variables.

NOTE: PROCEDURE SORT used (Total process time):
real time
0.00 seconds

cpJ time

0.01 seconds

321 data assign_trt TANK8A; set TANK8A;

322 if $n_{-}=1$ then $\operatorname{trt}=' 6 \mathrm{~h}^{\prime}$; 


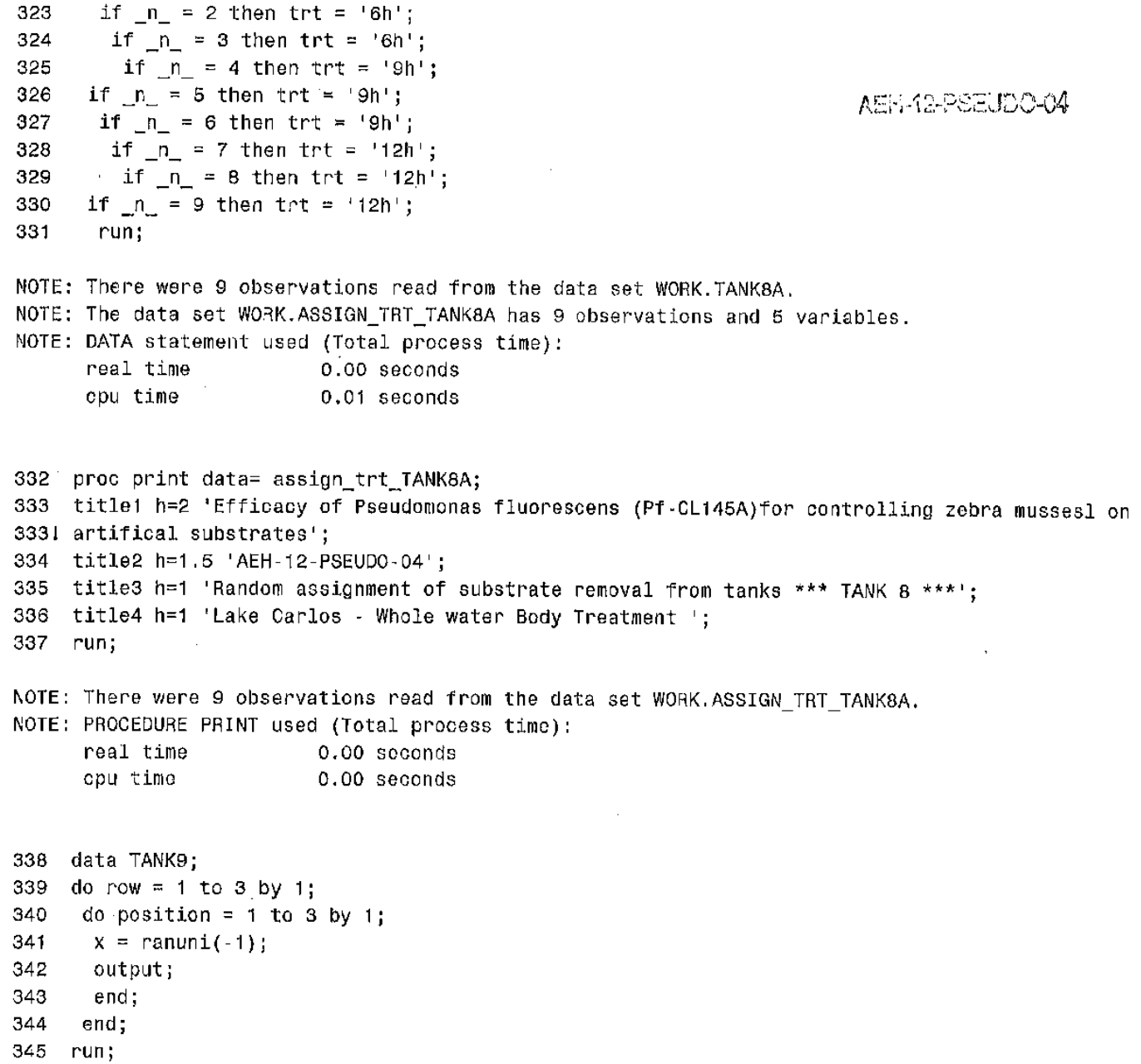




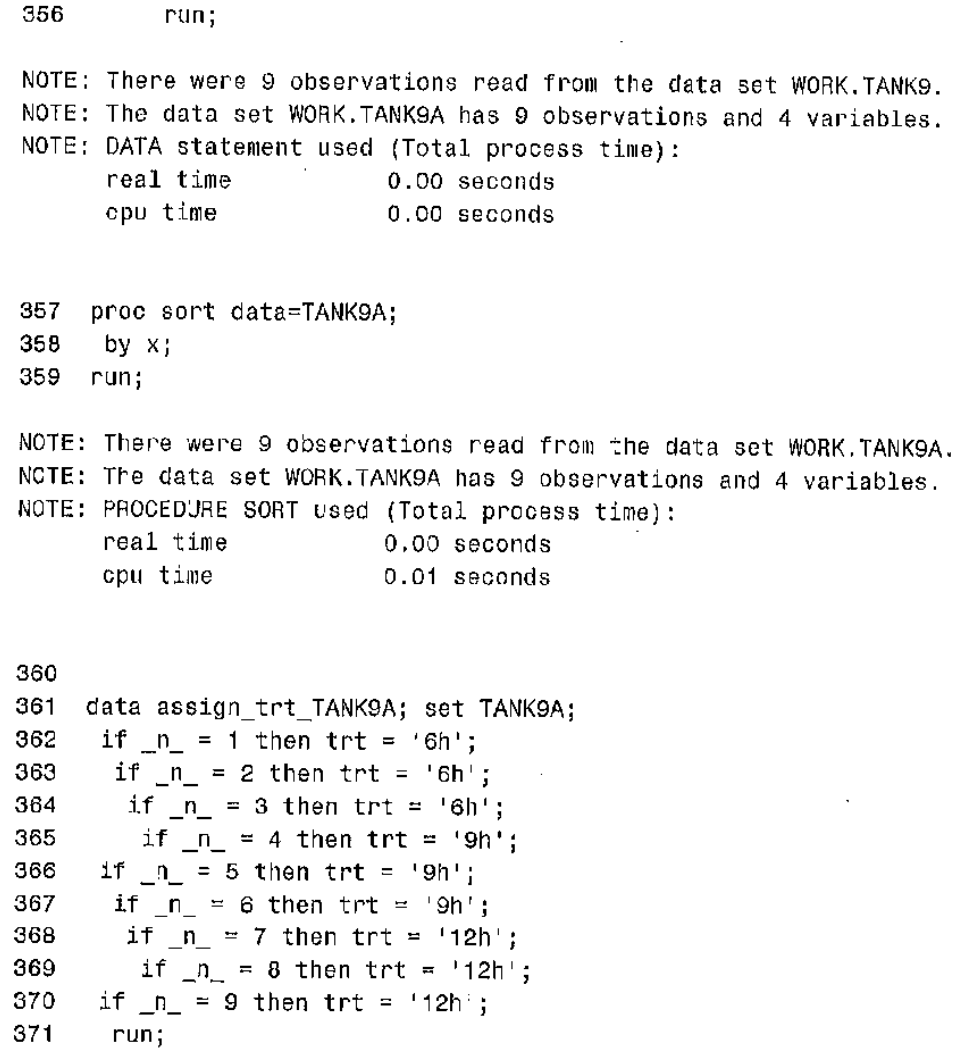

NOTE: There were 9 observations read from the data set WORK. TANK9A.

NOTE: The data set WORK.ASSIGN_TRT_TANK 9 A has 9 observations and 5 variables.

NOTE: DATA statement used (Total process time):
real time
0.00 seconds

cpu time

0.01 seconds

372 proc print data= assign trt TANK2A;

373 title1 $h=2$ 'Efficacy of Pseudomonas fluorescens (Pf-CL145A) for controlling zebra mussesl on 373 ! artifical substrates';

374 title2 $h=1.5$ 'AEH-12-PSEUDO-04',

375 title3 $h=1$ 'Random assignment of substrate renoval from tanks *** TANK $9 * * * 1$;

376 title4 $h=1$ 'Lake Carlos - Whole water Body Treatment ';

377 run;

NOTE: There were 9 observations read fron the data set WORK.ASSTGN_TRT_TANK2A.

NOTE: PROCEDURE PRINT used (Total process zime):
real time
0.00 seconds
cpu ti.ne
0.00 seconds$$
\text { 8/13/12.50. }
$$

FF $* 94$

Item No. 3

NCTE: This SAS session is using a registry in WOAk. All changes will be lost at the end of this session. 


\begin{tabular}{|c|c|c|c|c|c|c|}
\hline Obs & block & $\operatorname{tank}$ & $x$ & tankn & tret & 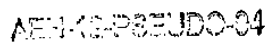 \\
\hline 1 & 1 & 6 & 0.13335 & Tank 6 & control & \\
\hline 2 & 1 & 1 & 0.20575 & Tank 1 & 50 & \\
\hline 3 & 1 & 5 & 0.28506 & Tank 5 & 100 & \\
\hline 4 & 1 & 7 & 0.34557 & Tank 7 & control & \\
\hline 5 & 1 & 4 & 0.34624 & Tank 4 & 50 & \\
\hline 6 & 1 & 2 & 0.55080 & Tank 2 & 100 & \\
\hline 7 & 1 & 3 & 0.59072 & Tank 3 & control. & \\
\hline$B$ & 1 & 8 & 0.70738 & Tank 8 & 50 & \\
\hline 9 & 1 & 9 & 0.88671 & Tank $\theta$ & 100 & \\
\hline
\end{tabular}

File Folder: $12 a$

Item Number:

Page _ـ of 4

Analysis performed by J. Luoma SAS version 9.2 08:59 11 AUG12 


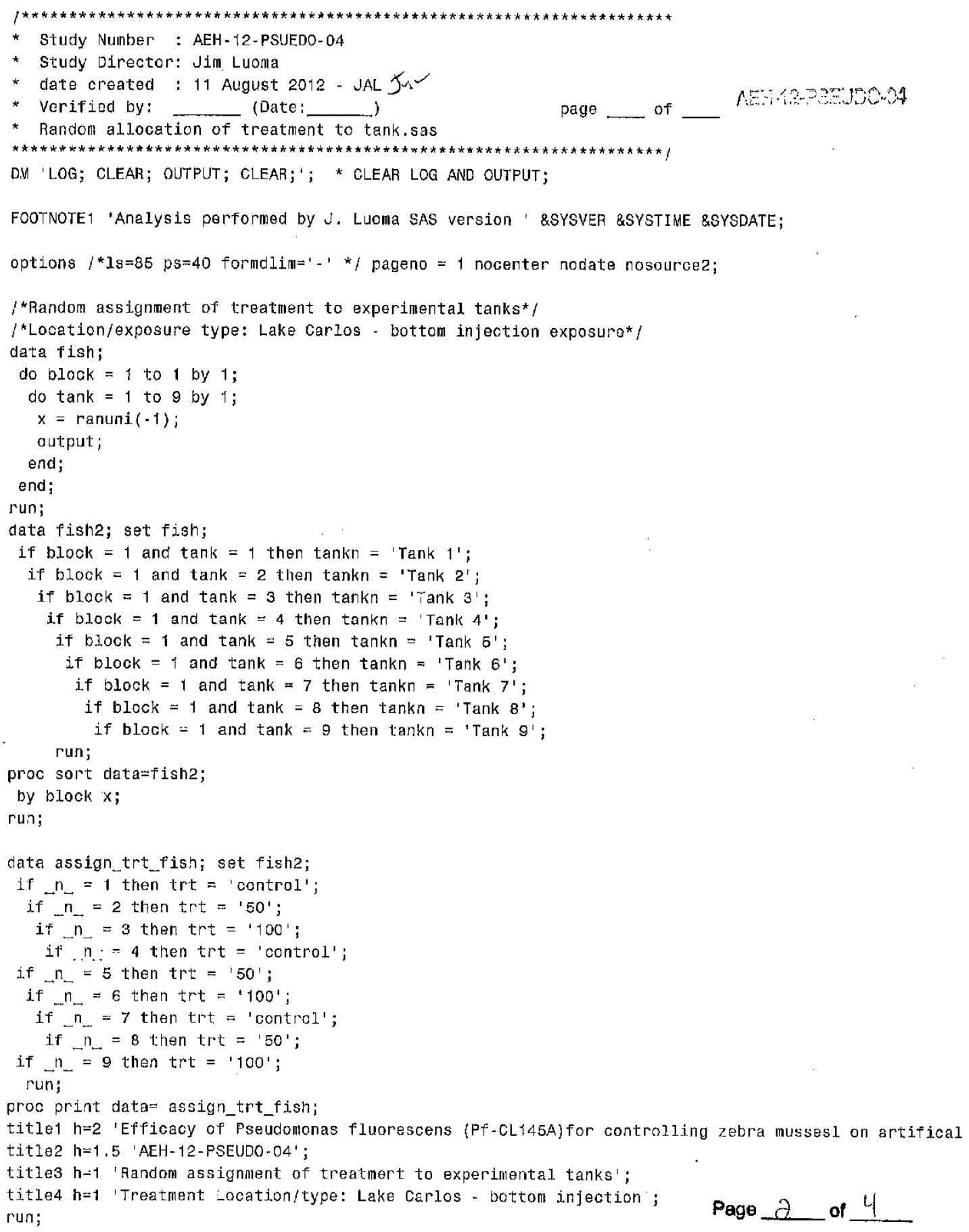

\section{Page 114 of 519}




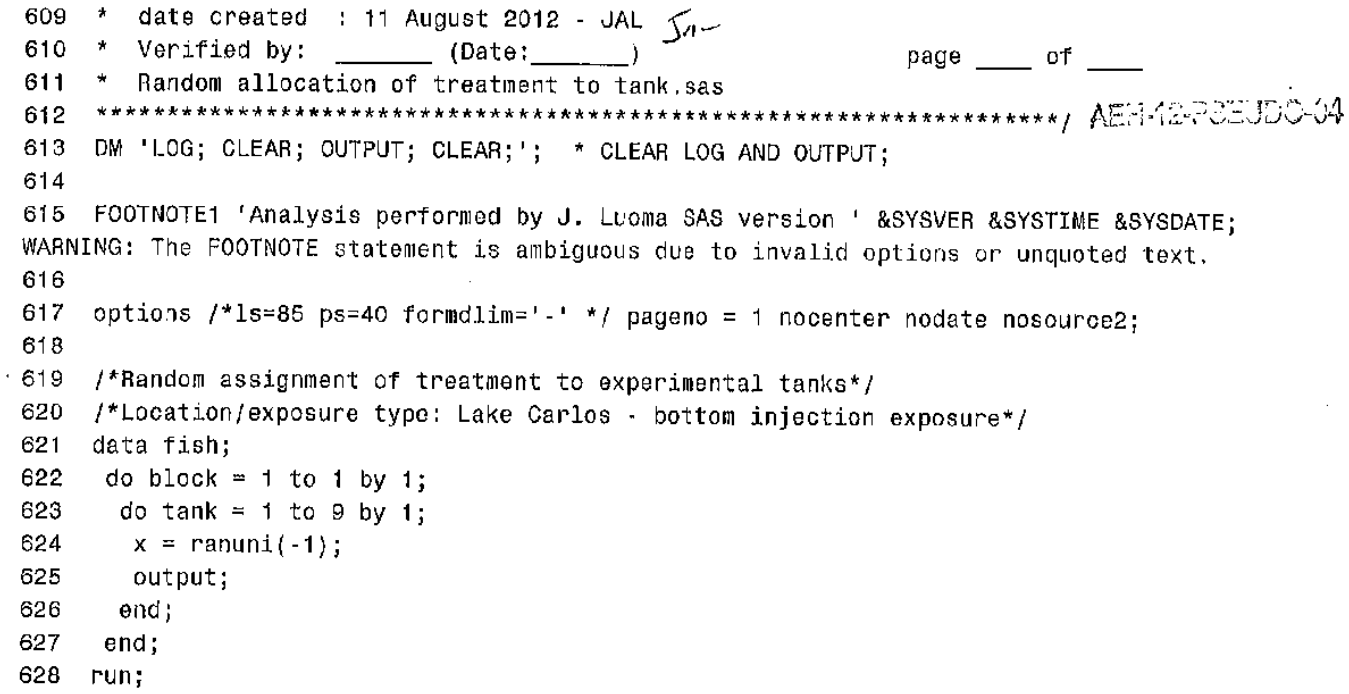




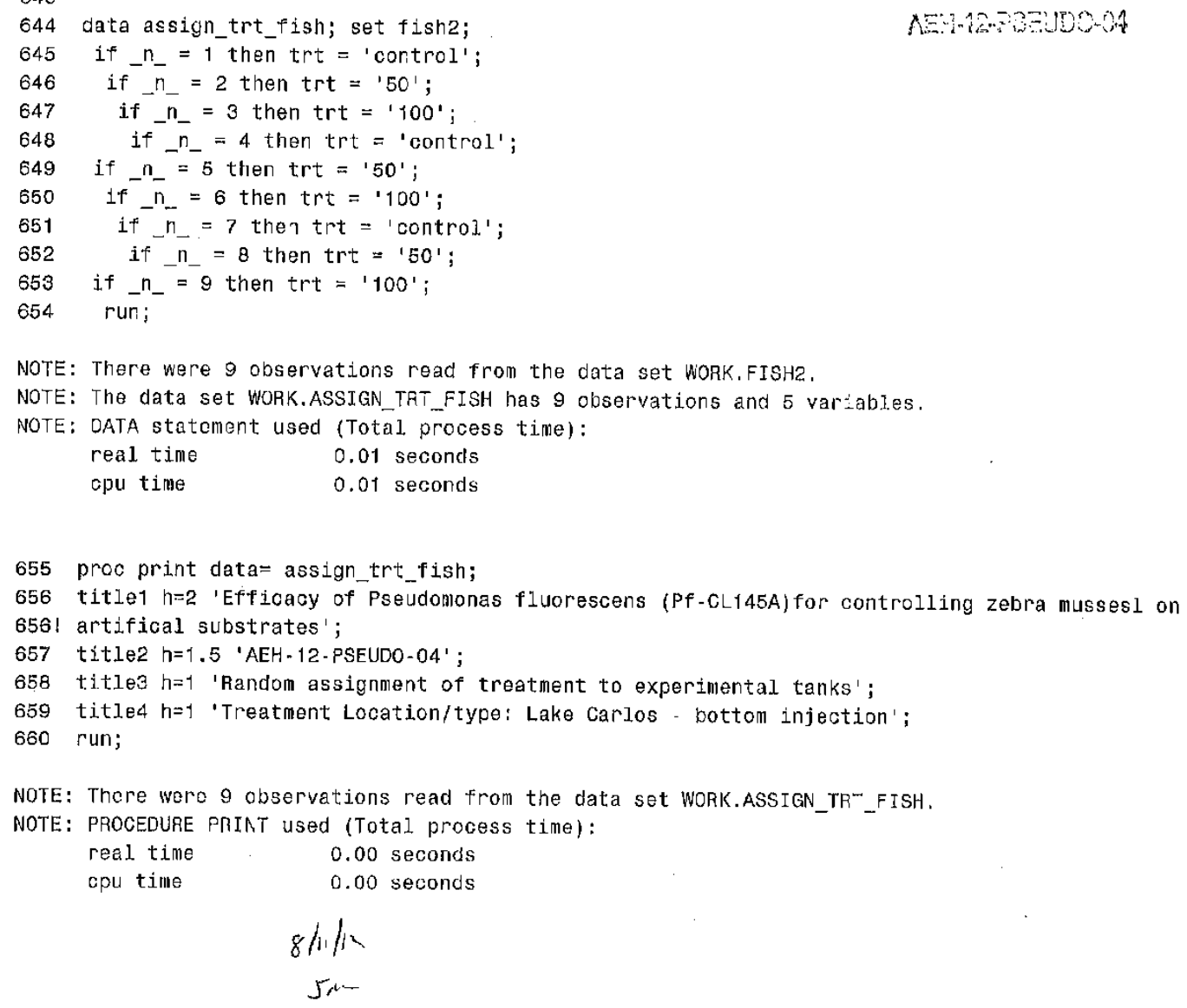

FF \# 12a 


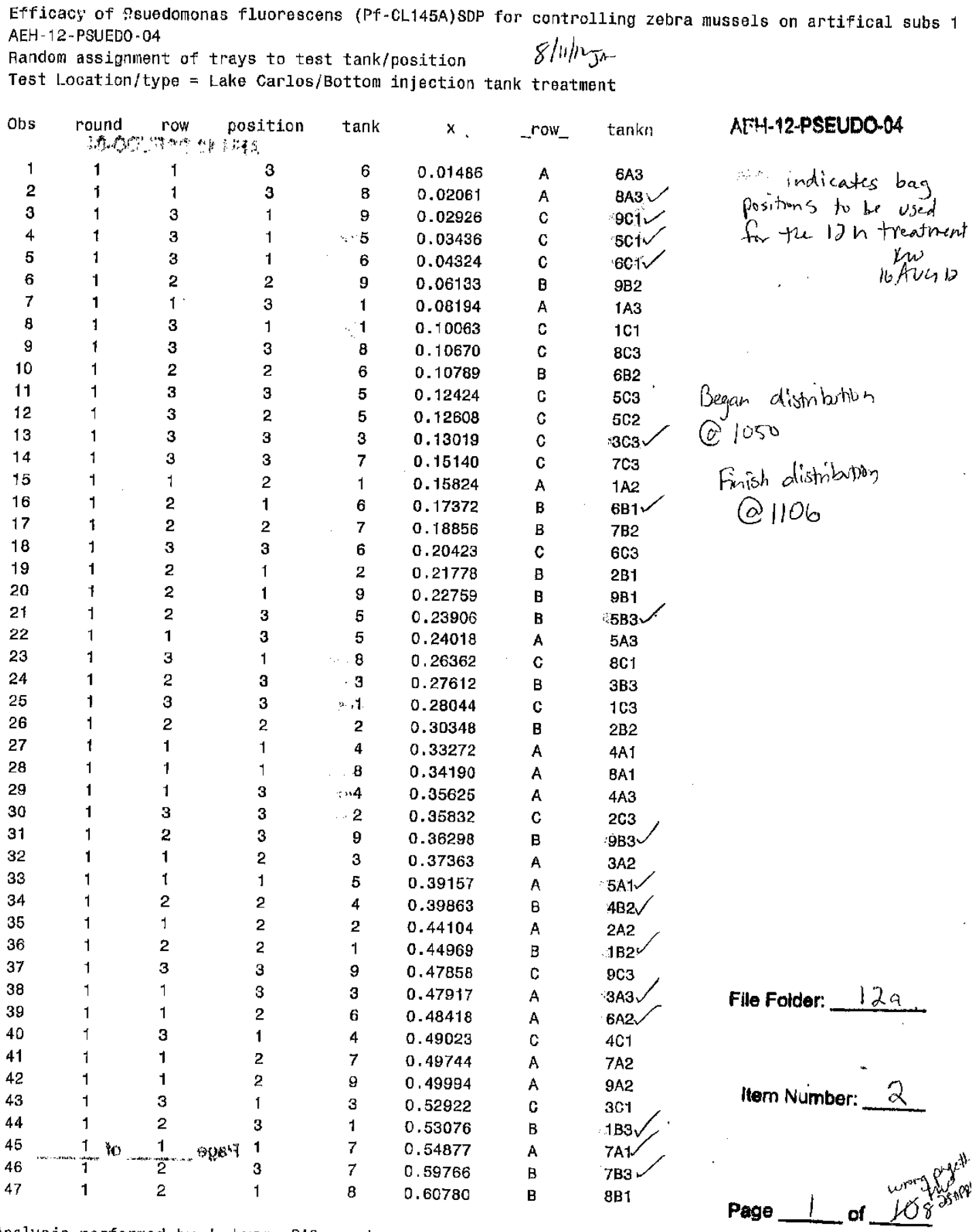




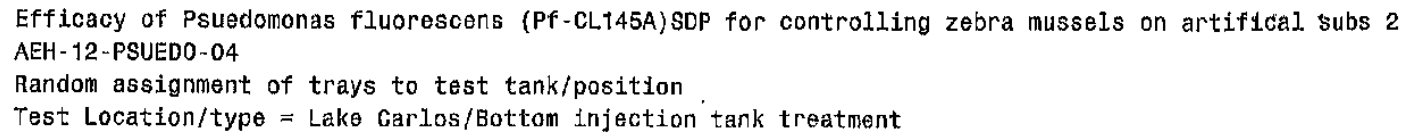

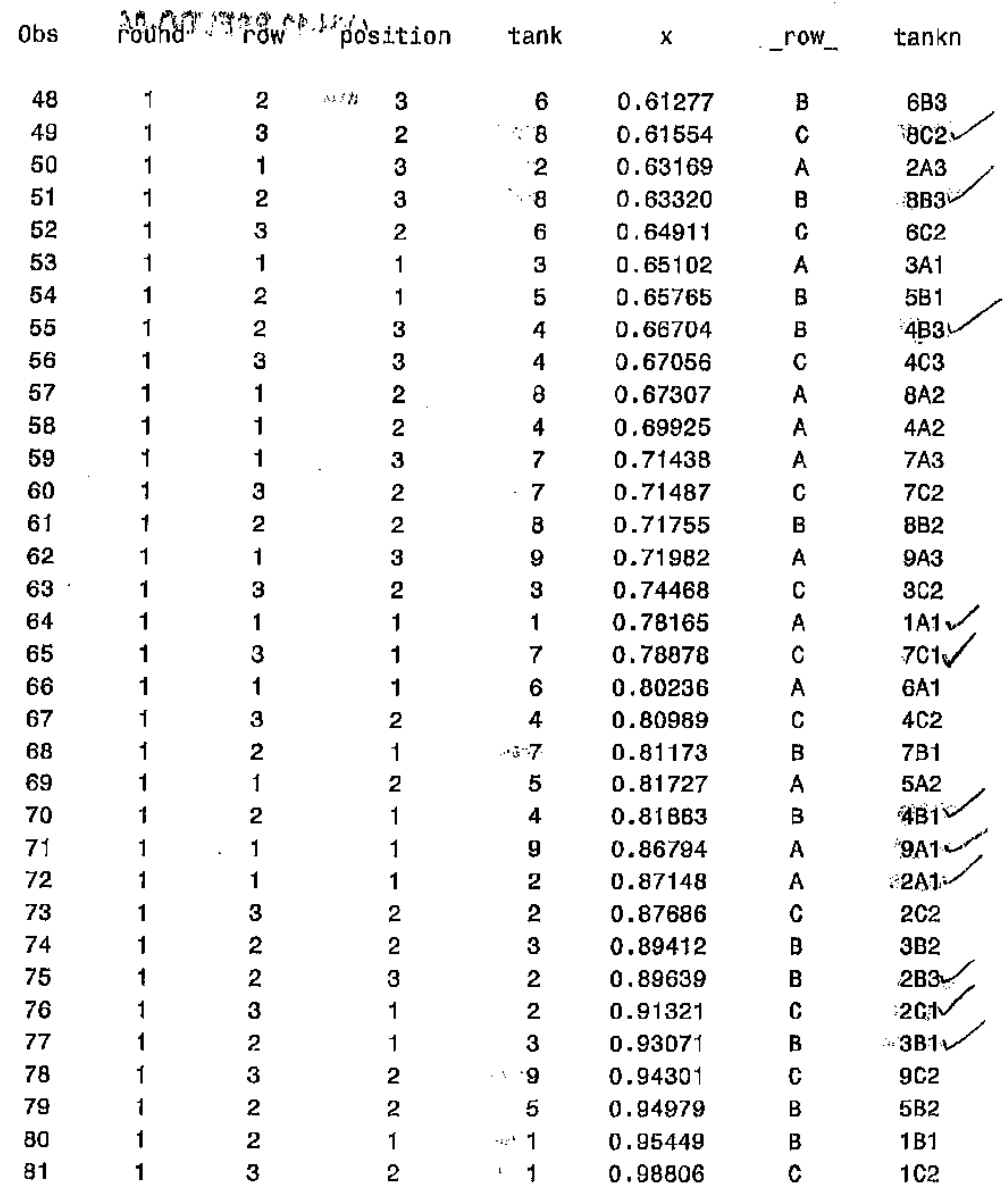

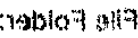

:1sdrminin inest

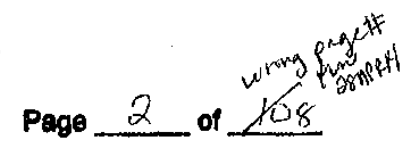

$10 . . .9 \mathrm{gk} 9$

Analysis performed by J. Luoma SAS version $9.2 \quad 10: 4811$ AUG12 Jn 


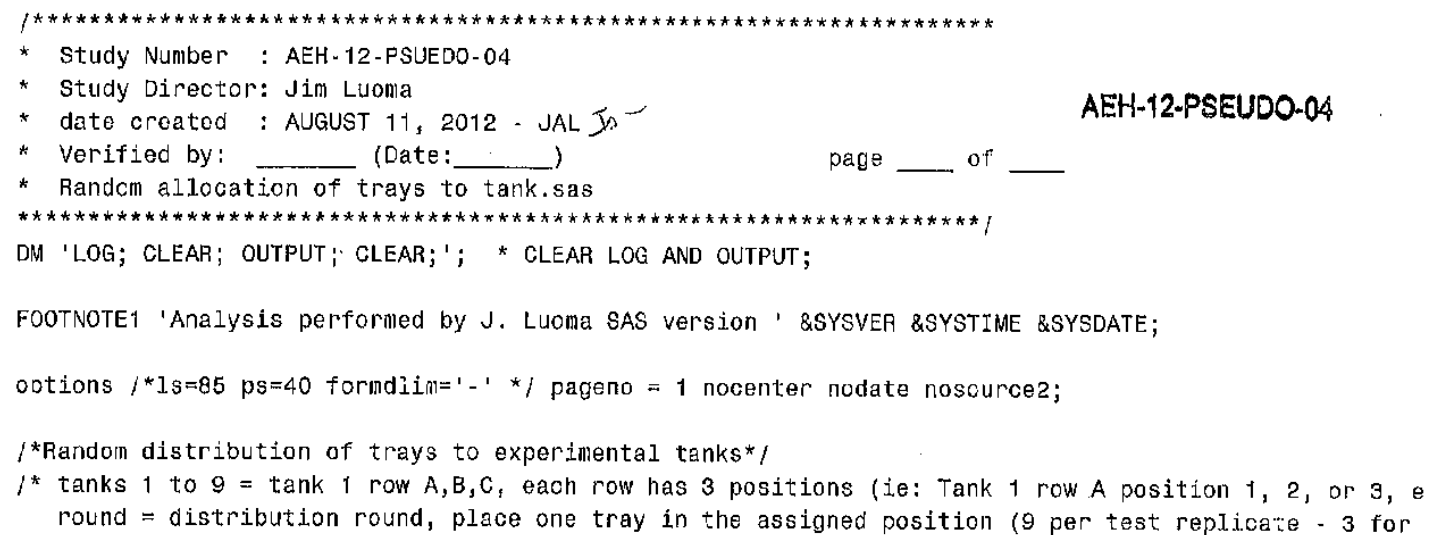




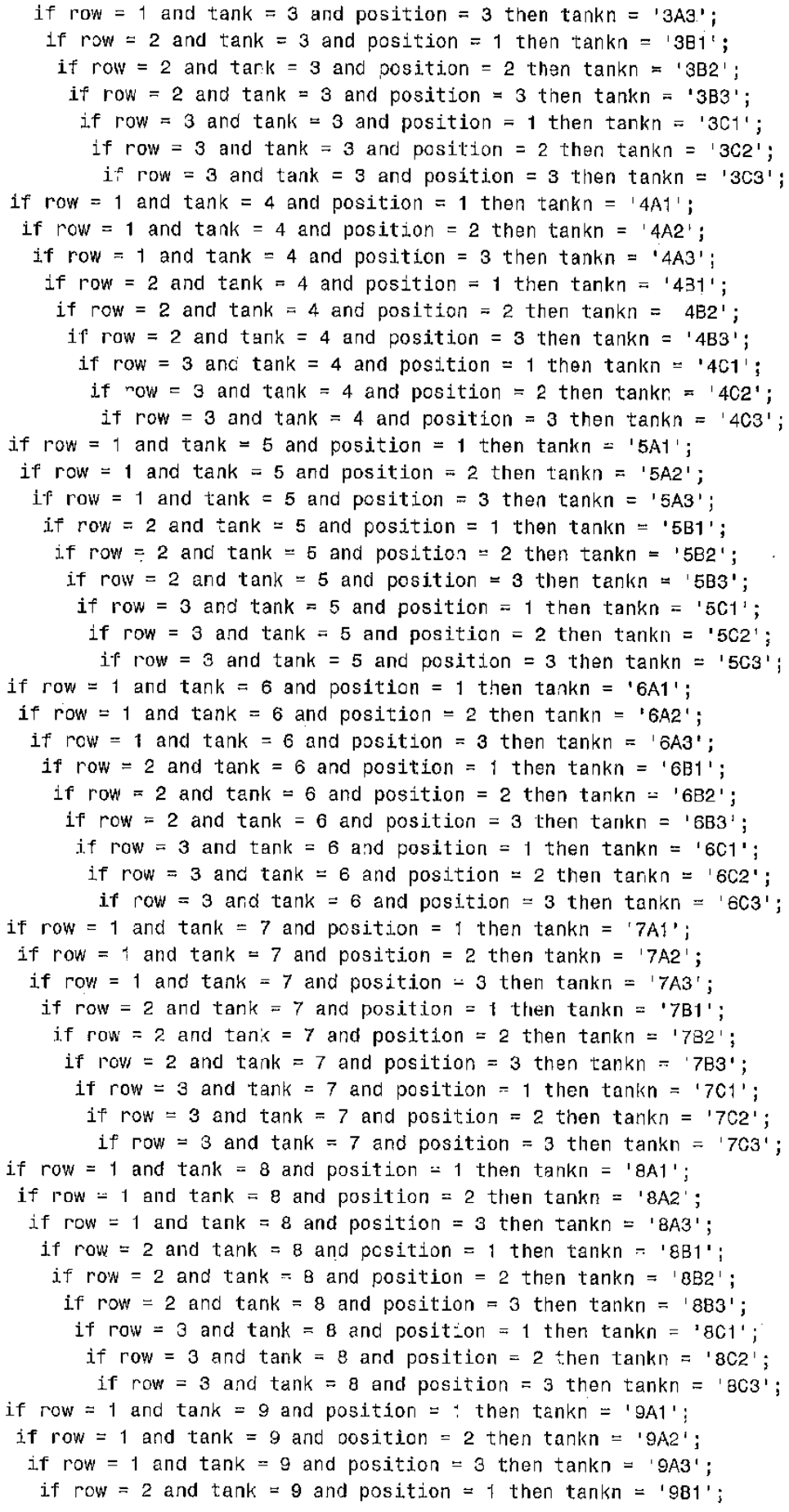




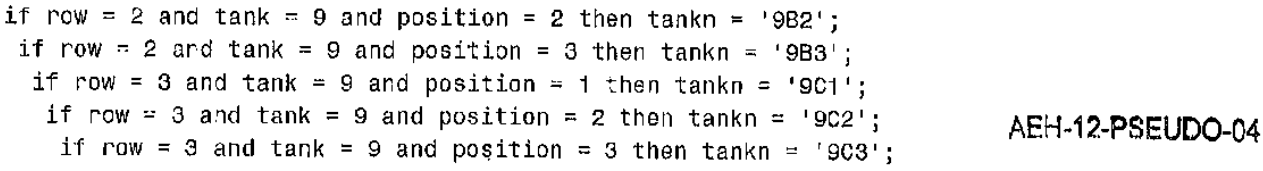

title1 h=2 'Efficacy of Psuedomonas fluorescens (Pf-CL145A) SDP for controlling zebra mussels on artif title2 $h=1.5$ 'AEH-12-PSUEDO-04';

title3 $h=1$ Randon assignment of trays to test tank/position';

ti.t.e4 $\mathrm{h}=1$ 'Test Location/type = Lake Carlos/Botton injection tank treatment';

rin:

$$
8 / 1 / 12
$$

Page $5 \quad$ of 8 


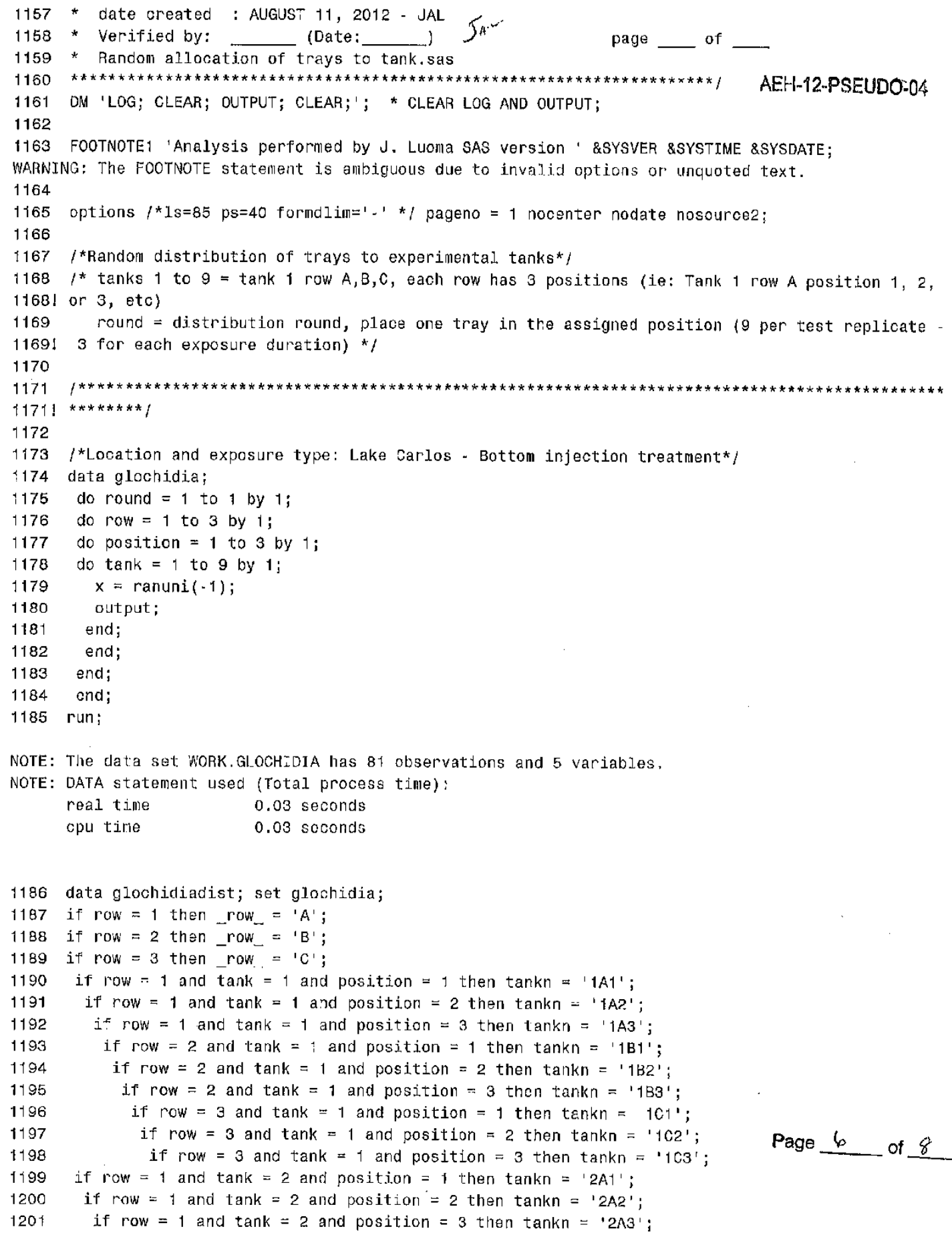


if row $=2$ and tank $=2$ and position $=1$ then tankn $={ }^{\prime} 2 \mathrm{~B} 1^{\prime}$;

if row $=2$ and tank $=2$ and position $=2$ then tankn $=2 \mathrm{~B} 2$ ';

if row $=2$ and tank $=2$ and position $=3$ then $\operatorname{tankn}={ }^{\prime} 2 \mathrm{~B} 3^{\prime}$;

if row $=3$ and tank $=2$ and position $=1$ then tankn $=201$ '

if row $=3$ and tank $=2$ and position $=2$ then tankn $=$ ' $2 \mathrm{C} 2{ }^{\prime}$;

if row $=3$ and tank $=2$ and position $=3$ then tankn $=12{ }^{\prime} 3^{\prime}$; if row $=1$ and tank $=3$ and position $=1$ then tankn $=$ ' $3 \mathrm{~A} 1$ '; if row $=1$ and tank $=3$ and position $=2$ then tankn $=3 A 2 '$; if row $=1$ and tank $=3$ and position. $=3$ then tankn $=3{ }^{\prime}{ }^{\prime}{ }^{\prime}$;

if row $=2$ and tank -3 and position $=1$ then tankn $=$ ' $3 B 1$ ';

if row $=2$ and tank $=3$ and position $=2$ then tankn $={ }^{\prime} 3 \mathrm{~B} 2{ }^{\prime}$;

if row $=2$ and $\operatorname{tank}=3$ and position $=3$ then tankn $=$ ' $3 \mathrm{~B}^{\prime}$ ';

if row $=3$ and tank $=3$ and position $=1$ then tankn $=$ ' 3 c1' ';

if roiv $=3$ and tank $=3$ and position $=2$ then tankn $={ }^{\prime} 3 \mathrm{C} 2$ ';

if row $=3$ and $\tan k=3$ and position $=3$ then tankn $=$ ' $3 \mathrm{C} 3$ '; if row $=1$ and tank $=4$ and position $=1$ then tankn $={ }^{\prime} 4 \mathrm{Al}^{\prime}$ ';

if row $=1$ and $\operatorname{tank}=4$ and position $=2$ then tankn $=$ ' $4 \mathrm{~A} 22^{\prime}$;

if row $=1$ and $\operatorname{tank}=4$ and position $=3$ then tankn $=$ ' $4 \mathrm{~A} 3^{\prime}$ ';

if row $=2$ and tank $=4$ and position $=1$ then tankn $={ }^{\prime} 4 \mathrm{~B} 1{ }^{\prime}$;

if row $\simeq 2$ and tank $=4$ and position $=2$ then tankn $=$ ' $4 \mathrm{~B} 2$ ';

if row $=2$ and tank $=4$ and position $=3$ then tankn $={ }^{\prime} 4 \mathrm{~B}^{\prime}$ ';

if row $=3$ and tank $=4$ and position $=1$ then tankn $=$ ' 4 C 1 ';

if row $=3$ and tank $=4$ and position $=2$ then tankn $={ }^{\prime} 4 \mathrm{C} 2{ }^{\prime}$;

if row $=3$ and tank $=4$ and position $=3$ then tankn $={ }^{\prime} 4 \mathrm{C}^{\prime}$ '; if row $=1$ and tank $=5$ and position $=1$ then tankn $=$ ' $5 \mathrm{~A} 1$ ';

If row $=1$ and tank $=5$ and position $=2$ then tankn $={ }^{\prime} 5 \mathrm{~A} 2$ ';

If row $=1$ and tank $=5$ and position $=3$ then tankn $={ }^{\prime} 5 \mathrm{~A} 3^{\prime}$;

if row $=2$ and tank $=5$ and position $=1$ then $\operatorname{tankn}={ }^{\prime} 5 \mathrm{~B} 1^{\prime}$ ';

if row $=2$ and tank $=5$ and position $=2$ then tankn $=$ ' 582 ';

if row $=2$ and tank $=5$ and position $=3$ then tankn $=$ ' 5 B3';

if row $=3$ and tank $=5$ and position $=1$ then tankn $={ }^{\prime} 5 \mathrm{C}_{1}{ }^{\prime}$;

if row -3 and tank $=5$ and position $=2$ then tankn $={ }^{\prime} 502$ ';

if row $=3$ and tank $=5$ and position $=3$ then tankn $={ }^{\prime} 5 \mathrm{C} 3^{\prime}$;

if row $=1$ and tank $=6$ and position $=1$ then tankn $=' 6 \mathrm{~A} 1^{\prime}$;

if row $=1$ and tank -6 and position $=2$ then tankn $={ }^{\prime} 6 A_{2}{ }^{\prime}$;

if row $=1$ and tank $=6$ and position $=3$ then tankn $={ }^{\prime} 6 \mathrm{~A}{ }^{\prime}$ ';

if row $=2$ and tank $=6$ and position $=1$ then tankn $=$ ' $6 \mathrm{~B} 1$ ' ;

if row $=2$ and $\operatorname{tank}=6$ and position $=2$ then tankn = ' $6 \mathrm{~B} 2$ ' ;

if row $=2$ and tank $=6$ and position $=3$ then tankn $=$ ' $6 B 3^{\prime}$;

if row $=3$ and $\operatorname{tank}=6$ and position $=1$ then tankn $=' 6 \mathrm{C} 1{ }^{\prime}$;

if row $=3$ and tank $=6$ and position $=2$ then tankn $={ }^{\prime} 6 \mathrm{C} 2$ ';

if row $=3$ and tank $=6$ and position $=3$ then tankn $={ }^{\prime} 6 \mathrm{C} 33^{\prime}$;

if row $=1$ and tank $=7$ and position $=1$ then tankn $=17 \mathrm{~A} 1{ }^{\prime}$;

if row $=1$ and tank $=7$ and position $=2$ then tankn $=$ ' $7 \mathrm{~A} 2$ ';

if row $=1$ and tank -7 and position $=3$ then tankn $=$ ' $7 \mathrm{~A} 3^{\prime}$;

if row $=2$ and tank $=7$ and position $=1$ then tankn $=' 7 \mathrm{~B} 1 '$ '

if row $=2$ and tank $=7$ and position $=2$ then tankn $=17 \mathrm{~B} 2 '$;

if row $=2$ and tank $=7$ and position $=3$ then tankn $=' 7 \mathrm{~B} 3^{\prime}$;

if row -3 and tank $=7$ and position $=1$ then tankn $=17 \mathrm{C1}^{\prime}$ ';

if row $=3$ and $\operatorname{tank}=7$ and cosition $=2$ then tankn $={ }^{\prime} 7 \mathrm{C} 2{ }^{\prime}$;

if row $=3$ and $\operatorname{tank}=7$ and position $=3$ then tankn $=1703$ ';

if row $=1$ and tank $=8$ and position $=1$ then tankn $=$ ' $B A 1$ ';

if row $=1$ and tank $=8$ and position $=2$ then tankn $=18 \mathrm{~A} 2^{\prime}$ ';

If row $=1$ and tank $=8$ and position $=3$ then tarkn $=$ ' $B A 3^{\prime}$;

if row $=2$ and tank $=8$ and position $=1$ then $\operatorname{tankn}={ }^{\prime} B B_{1} 1$; if row $=2$ and tank $=8$ and position $=2$ then tankn $={ }^{\prime} 8 \mathrm{~B} 2{ }^{\prime}$;
AEH-12-PSEUDO-04

Page 7 of 8 


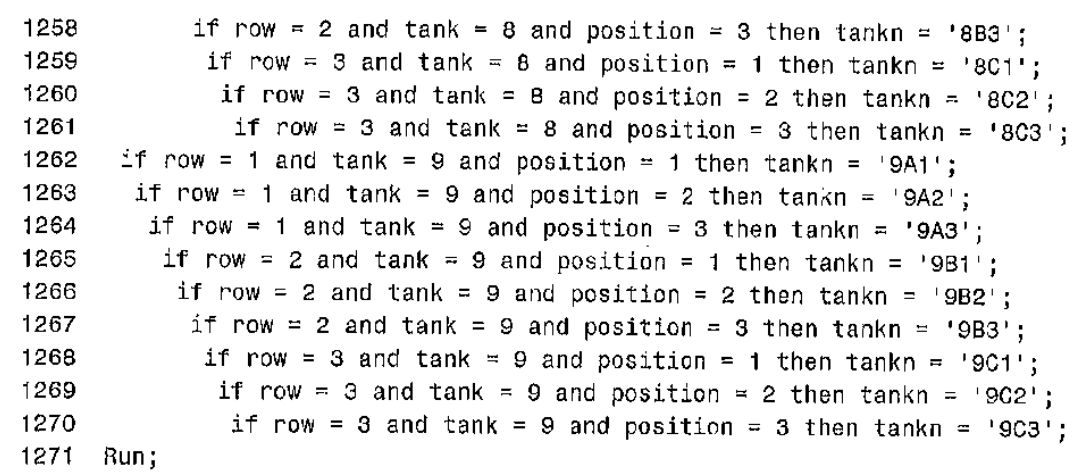

NOTE: There were 81 observations read from the data set wORK.GLOCHIDIA.

NOTE: Tre data set WORK.GLOCHIDIADIST has 81 observations and 7 variables.

NOTE: DATA statement usod (Total process tine):

real time $\quad 0.07$ seconds

cpu time $\quad 0.07$ seconds

1272 proc sort data= glochidiadist;

1273 by round $x$;

1274 run;

NOTE: There were 81 observations read from the data set WORK,GLOCHIDIAJIST,

NOTE: The data set WORK.GLOCHIDIADIST has 81 observations and 7 variables.

NOTE: PROCEDURE SORT used (Totaz process time):
real time
0.01 seconds
cpu time
0.01 seconds

1275 proc print data $=$ glochidjadist;

1276 title1 h=2 'Efficacy of Psuedomonas fluorescens (PF-CL145A)SOP for controlling zebra miussels

1276 I on artifical substrates';

1277 title2 $n=1.5$ ' $\mathrm{AEH}-12$-PSUEDO-04';

1278 titles $h=1$ 'Random assignment of trays to test tank/position'

1279 title4 $h=1$ 'Test Location/type = Lake Carlos/Bottom injection tank treatment';

1280 run:

NOTE: There were 81 observations read from the data set WORK.GLOCHIDIADIST.

NOTE: PROCEDURE PRINT used (Total process time):
real time
0.01 scconds
cpu time
0.01 seconds
$8 / 11 / 2$
5,2

FF \# $12 \alpha$

Item No. $\partial$

$\mathrm{Pg} 8$ of 8 
Efficacy of Pseudomonas fluorescens (Pf-CL145A)for controlling zebra mussesl on artifical substrates AEH-12-PSEUDO-04

Random assignment of substrate removal from tanks *** TANK $1 * * *$

Lake Carlos - bottom injectïon Treatment

$8 / 13 / 12$ AEH-12-PSEUDO-04

\begin{tabular}{|c|c|c|c|c|}
\hline obs & row & position & $x$ & $\tan k n$ \\
\hline 7 & 3 & 2 & 0.00578 & $1 \mathrm{C} 2$ \\
\hline 2 & 3 & 3 & 0.07326 & $1 \mathrm{CB}$ \\
\hline 3 & 3 & & 0.24288 & $1 \mathrm{c} 1$ \\
\hline 4 & 1 & 2 & 0.27838 & $1 \mathrm{~A} 2$ \\
\hline 5 & 1 & 3 & 0.28017 & $1 \mathrm{AB}$ \\
\hline 6 & 2 & 1 & 0.53070 & $1 \mathrm{B1}$ \\
\hline 7 & 2 & 2 & 0.61838 & 182 \\
\hline 8 & 2 & 3 & 0.68756 & 1B3 \\
\hline 9 & 1 & 1 & 0.81146 & $1 \mathrm{~A} 1$ \\
\hline
\end{tabular}

Si

Dosing for bistom injection is occurring for $12 \mathrm{~h}$ with no 6 or $9 \mathrm{~h}$ sampliteg. itw ib tilib 


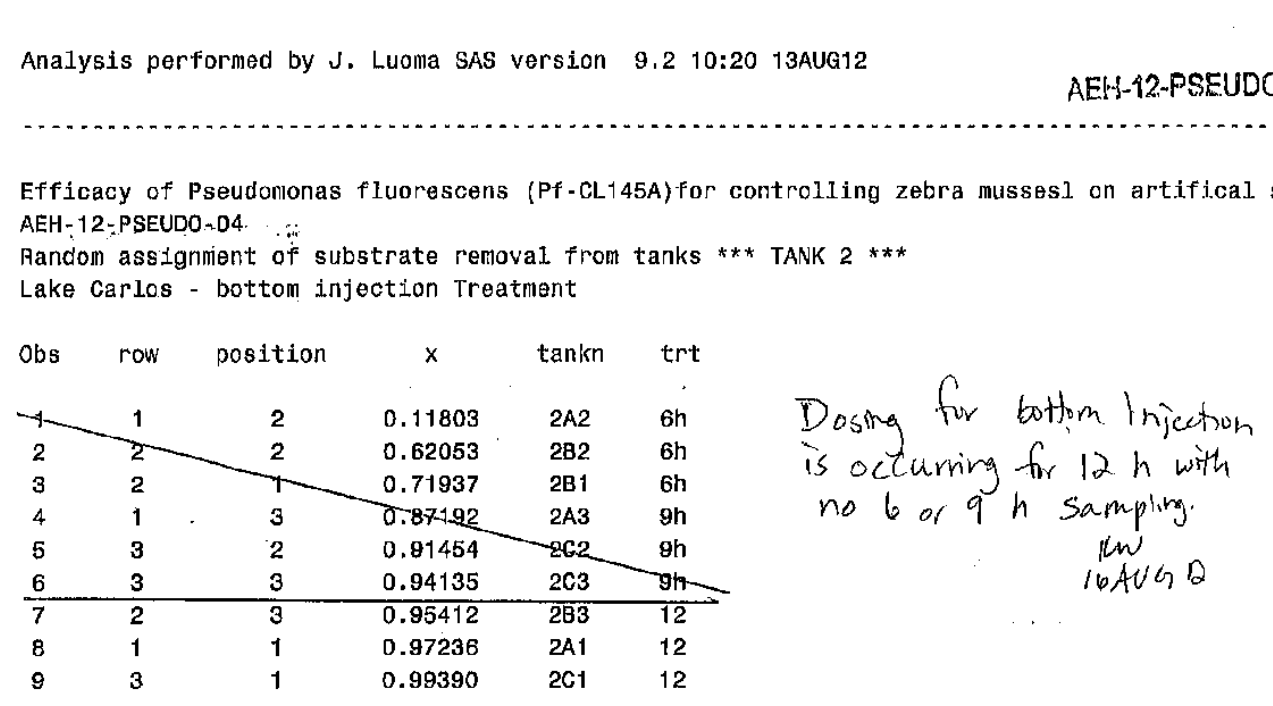

Page 2 of 30 
nalysis performed by $J$. Luoma SAS version 9.2 10:20 13AUG12

AEH-12.PSEUDO-04

Efficacy of Pseudomonas fluorescens ( $\mathrm{Pf}-\mathrm{CL} 145 \mathrm{~A}$ )for controlling zebra musses1 on artifical substrates AEH-12-PSEUDO-04

Fandom assignment of substrate removal from tanks $* * *$ TANK $3 * * *$

Lake Carlos - bottom injection Treatment

\begin{tabular}{cccccc} 
Obs & row & position & $x$ & tankn & trt \\
r & 3 & 2 & 0.19914 & $3 \mathrm{C} 2$ & $6 \mathrm{~h}$ \\
2 & 1 & 2 & 0.28840 & $3 \mathrm{~A} 2$ & $6 \mathrm{~h}$ \\
3 & 2 & 3 & 0.38093 & $3 \mathrm{~B} 3$ & $6 \mathrm{~h}$ \\
4 & 3 & 1 & 0.43250 & $3 \mathrm{C} 1$ & $9 \mathrm{~h}$ \\
5 & 1 & 1 & 0.57180 & $3 \mathrm{~A} 1$ & $9 \mathrm{~h}$ \\
6 & 2 & 2 & 0.66134 & $3 \mathrm{~B} 2$ & $\mathrm{gh}$ \\
\hline 7 & 3 & 3 & 0.71983 & $3 \mathrm{C} 3$ & 12 \\
8 & 1 & 3 & 0.82826 & $3 \mathrm{A3}$ & 12 \\
9 & 2 & 1 & 0.88773 & $3 \mathrm{~B} 1$ & 12
\end{tabular}

Dosing for bottom injection is occuring frr lah with no 6 or $9 h$ sampling.

thw Io Augh

.

Page 3 of 30 
Analysis performed by J. Luoma SAS version $9.2 \quad 10: 2013 A U G 12$

AEH-12-PSEUDO-04

Efficacy of Pseudomonas fluorescens (Pf-CL145A)for controlling zebra mussesl on artifical substrates AEH-12-PSEUDO- 04

Fandom assignment of substrate removal from tanks $* * *$ TANK $4 * * *$

Lake Carlos - bottom injection Body Treatment

\begin{tabular}{|c|c|c|c|c|c|c|}
\hline obs & row & position & $x$ & $\operatorname{tankn}$ & $\operatorname{trt}$ & \\
\hline$\therefore=$ & 1 & 2 & 0.04712 & $4 \mathrm{~A} 2$ & $6 \mathrm{~h}$ & Dosth tor bottom injection is \\
\hline 2 & & 3 & 0.06272 & $4 \mathrm{C3}$ & $6 h$ & occurrim for $12 \mathrm{~h}$ with $\mathrm{n}_{0}$ \\
\hline 3 & 3 & & 0.14155 & $4 \mathrm{C} 1$ & $6 h$ & 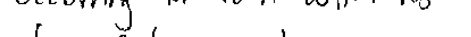 \\
\hline 4 & 3 & 2 & 0.28865 & $4 C 2$ & 9h & w or $9 \mathrm{~h}$ sampling. \\
\hline 5 & 1 & 1 & 0.45448 & $-4 A 1$ & $9 \mathrm{~h}$ & 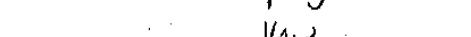 \\
\hline 6 & 1 & 3 & 0.50193 & $4 A 3$ & ah & 100,412 \\
\hline 7 & 2 & 1 & 0.73873 & $\overline{4 B 1}$ & 12 & \\
\hline 8 & 2 & 3 & 0.88940 & 4B3 & 12 & \\
\hline 9 & 2 & 2 & 0.94491 & $4 \mathrm{~B} 2$ & 12 & \\
\hline
\end{tabular}

Page 4 of 30

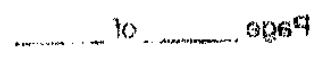


Analysis performed by $J$. Luoma SAS version 9.2 10:20 13AUG12

AEF-12-PSEUDO-04

Efficacy of Pseudomonas fluorescens (Pf-CL145A)for controlling zebra mussesl on artifical substrates AEH - 12 -PSEUDO-04

Random assignment of substrate removal from tanks $* * *$ TANK $5 * * *$

Lake carlos - bottom injection Treatment

\begin{tabular}{|c|c|c|c|c|c|}
\hline Obs & row & position & $x$ & (1) tankn & trt \\
\hline & 1 & 2 & 0.11803 & $2 A 2$ & $6 \mathrm{~h}$ \\
\hline 2 & 2 & 2 & 0.62053 & $2 B 2$ & $6 \mathrm{~h}$ \\
\hline 3 & 2 & & 0.71937 & 281 & $6 \mathrm{~h}$ \\
\hline 4 & 1 & 3 & $0: 87192$ & $2 A 3$ & $9 \mathrm{~h}$ \\
\hline 5 & 3 & 2 & 0.91454 & $-2 \mathrm{C2}$ & $9 \mathrm{~h}$ \\
\hline 6 & 3 & 3 & 0.94135 & $2 \mathrm{C3}$ & $8 \mathrm{~h}$ \\
\hline 7 & 2 & 3 & $0 . \overline{95412}$ & $2 \mathrm{B3}$ & 12 \\
\hline 8 & 1 & 1 & 0.97236 & $2 A 1$ & 12 \\
\hline 9 & 3 & 1 & 0.99390 & $2 \mathrm{C}^{-1}$ & 12 \\
\hline
\end{tabular}

$$
\begin{aligned}
& \text { Thank in should be } 5 \text { not } 2 \text {. Ku nutio } \\
& \text { See Deviation } \# 2 \text { for } \\
& \text { furtuer clarification. 10w panovis }
\end{aligned}
$$

is $\arg \cos ^{2}$

Page 5 of 30 


\section{AEH-12.PSEUDO-04}

Analysis performed by $J$, Luoma SAS version 9.2 10:20 13AUG12

Efficacy of Pseudomonas fluorescens (Pf-CL145A)for controlling zebra mussesi on artifical substrates AEH-12-PSEUDO-04

Random assignment of substrate removal from tanks *** TANK $6 * * *$

Lake carlos - bottom injection Treatment

\begin{tabular}{|c|c|c|c|c|c|c|}
\hline Obs & row & position & $x$ & tankn & trt & \\
\hline & 3 & 2 & 0.08423 & $6 \mathrm{C} 2$ & $6 \mathrm{~h}$ & for bothm injection is \\
\hline 2 & & 3 & 0.16043 & $6 \mathrm{c3}$ & $6 \mathrm{~h}$ & yentor is \\
\hline 3 & 1 & 3 & 0.20065 & $6 A 3$ & $6 \mathrm{~h}$ & $2 \mathrm{~h}$ with no bor $9 \mathrm{~h}$ \\
\hline 4 & 1 & 1 & 0.29470 & 6A1 & $9 \mathrm{~h}$ & 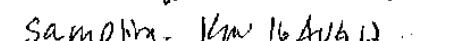 \\
\hline 5 & 2 & 2 & 0.30688 & $6 B 2$ & 9h & \\
\hline 6 & 2 & $\cdots$ & 0.35165 & $6 \mathrm{B3}$ & an & \\
\hline 7 & 1 & 2 & 0.38714 & 6A2 & 12 & \\
\hline 8 & 3 & 1 & 0.60130 & $6 C 1$ & 12 & \\
\hline 9 & 2 & 1 & 0.64342 & $6 B 1$ & 12 & \\
\hline
\end{tabular}


AEH-12-PSEUDO-04

Analysis performed by J. Luoma SAS version $9.2 \quad 10: 20 \quad 13 A U G 12$

Efficacy of Pseudomonas fluorescens (Pf-CL145A)for controlling zebra musses1 on artifical substrates AEH-12-PSEUDO- 04

Random assigrment of substrate removal from tanks $* * *$ TANK $7 * * *$

Lake Carlos - bottom injection Treatment

\begin{tabular}{lccccc} 
Obs & row & position & $x$ & Otankn & trt \\
\hline & 1 & 2 & 0.11803 & $2 \mathrm{~A} 2$ & $6 \mathrm{~h}$ \\
2 & 2 & 2 & 0.62053 & $2 \mathrm{~B} 2$ & $6 \mathrm{~h}$ \\
3 & 2 & 1 & 0.71937 & $2 \mathrm{~B} 1$ & $6 \mathrm{~h}$ \\
4 & 1 & 3 & 0.87192 & $2 \mathrm{~A} 3$ & $9 \mathrm{~h}$ \\
5 & 3 & 2 & 0.91454 & $2 \mathrm{~S} 2$ & $9 \mathrm{~h}$ \\
6 & 3 & 3 & 0.94135 & $2 \mathrm{CB}$ & $8 \mathrm{~h}$ \\
\hline 7 & 2 & 3 & 0.95412 & $2 \mathrm{~B} 3$ & 12 \\
8 & 1 & 1 & 0.97236 & 241 & 12 \\
9 & 3 & 1 & 0.99390 & $2 \mathrm{C} 1$ & 12
\end{tabular}

Dasing for botform injection is

for id with no $k$ or $9 h$

sampling.

$$
\text { fow } 14 \text { Av4 } 12
$$

$$
\begin{aligned}
& \text { (1) Tank D should be } 7 \text { nt } 2 \text {. kw } \\
& \text { I I A Aug, D } \\
& \text { See Deviation }+2 \text { fr. } \\
& \text { furtor clarification. How 19nowi3 }
\end{aligned}
$$

1.........................

Page 7 of 30 


\section{AFIT-12-PSEUDO-04}

Analysis performed by J. Luoma SAS version $9.2 \quad 10: 201$ BAUG12

Efficacy of Pseudomonas fluorescens (Pf-CL145A) for controlling zebra mussesl on artifical substrates AEH-12-PSEUDO- 04

Fandom assignment of substrate removal from tanks $* * *$ TANK $8 * * *$

Lake Carlos - bottom injection Treatment

\begin{tabular}{|c|c|c|c|c|c|c|}
\hline Obs & row & position & $x$ & $\operatorname{tankn}$ & trt & \\
\hline y & 3 & 1 & 0.06104 & $8 \mathrm{c} 1$ & $6 \mathrm{~h}$ & for bottim injection is \\
\hline 2 & 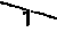 & 1 & 0.13492 & BA1 & $6 \mathrm{~h}$ & ith he hor $h$ \\
\hline 3 & 3 & & 0.25814 & 8c3 & $6 h$ & Yoh with to 6 or 1 n \\
\hline 4 & 2 & 1 & -0.54811 & $8 B 1$ & $9 \mathrm{~h}$ & KW IGAUGD \\
\hline 5 & 2 & 2 & 0.66886 & 882 & $9 h$ & \\
\hline 6 & $t$ & 2 & 0.72715 & $8 \mathrm{A2}$ & $2 \mathrm{~h}$ & \\
\hline 7 & 2 & 3 & $0 . \overline{81389}$ & $8 \overline{83}$ & 12 & \\
\hline 8 & 3 & 2 & 0.87980 & $8 \mathrm{C} 2$ & 12 & \\
\hline 9 & 1 & 3 & 0.89938 & $8 \mathrm{A3}$ & 12 & \\
\hline
\end{tabular}


AEH-12.PSEUDO-04

Analysis performed by J. Luoma SAS version $0.210: 2013 A \cup G 125{ }^{2}$ AEH-12PSEUDO-C: NL, 29APQ2014

Efficacy of Pseudomonas fluorescens (Pf-CL145A)for controlling zebra mussesl on artifical substrates AEH-12-PSEUDO-04

Random assignment of substrate removal from tanks *** TANK $9 * * *$

Lake Carlos - bottom injection Treatment

\begin{tabular}{|c|c|c|c|c|c|}
\hline Obs & row & position & $x$ & $\tan k n$ & $\operatorname{trt}$ \\
\hline & 1 & 2 & 0.11803 & $2 A 2$ & $6 \mathrm{~h}$ \\
\hline 2 & 2 & 2 & 0.62053 & $2 \mathrm{~B} 2$ & $6 \mathrm{~h}$ \\
\hline 3 & 2 & 7 & 0.71937 & 2B1 & $6 h$ \\
\hline 4 & 1 & 3 & 0.871 .92 & $2 A 3$ & $9 \mathrm{~h}$ \\
\hline 5 & 3 & 2 & 0.91454 & -362 & $9 \mathrm{~h}$ \\
\hline 6 & 3 & 3 & 0.94135 & $2 \mathrm{C3}$ & -94 \\
\hline 7 & 2 & 3 & 0.95412 & $2 \mathrm{B3}$ & 12 \\
\hline 8 & 1 & 1 & 0.97236 & $2 A 1$ & 12 \\
\hline 9 & 3 & 1 & 0.99390 & $2 \mathrm{C} 1$ & 12 \\
\hline
\end{tabular}

Dosing for bottem injection is for lash with no 6 or 9 h Simpling. Now Io Aucold

(1)Tank ID should be 9 not 2 . kn 16 Aveq See Deviation \#2 for furluer clarifization. kno ianovis is

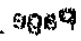

Page 9 of 30 
AEH-12.PSEUDOO-04

Analysis performed by $J$. Luoma SAS version 9.2 10:20 13AUG12

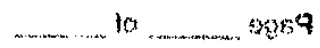

Page 10 of 30 


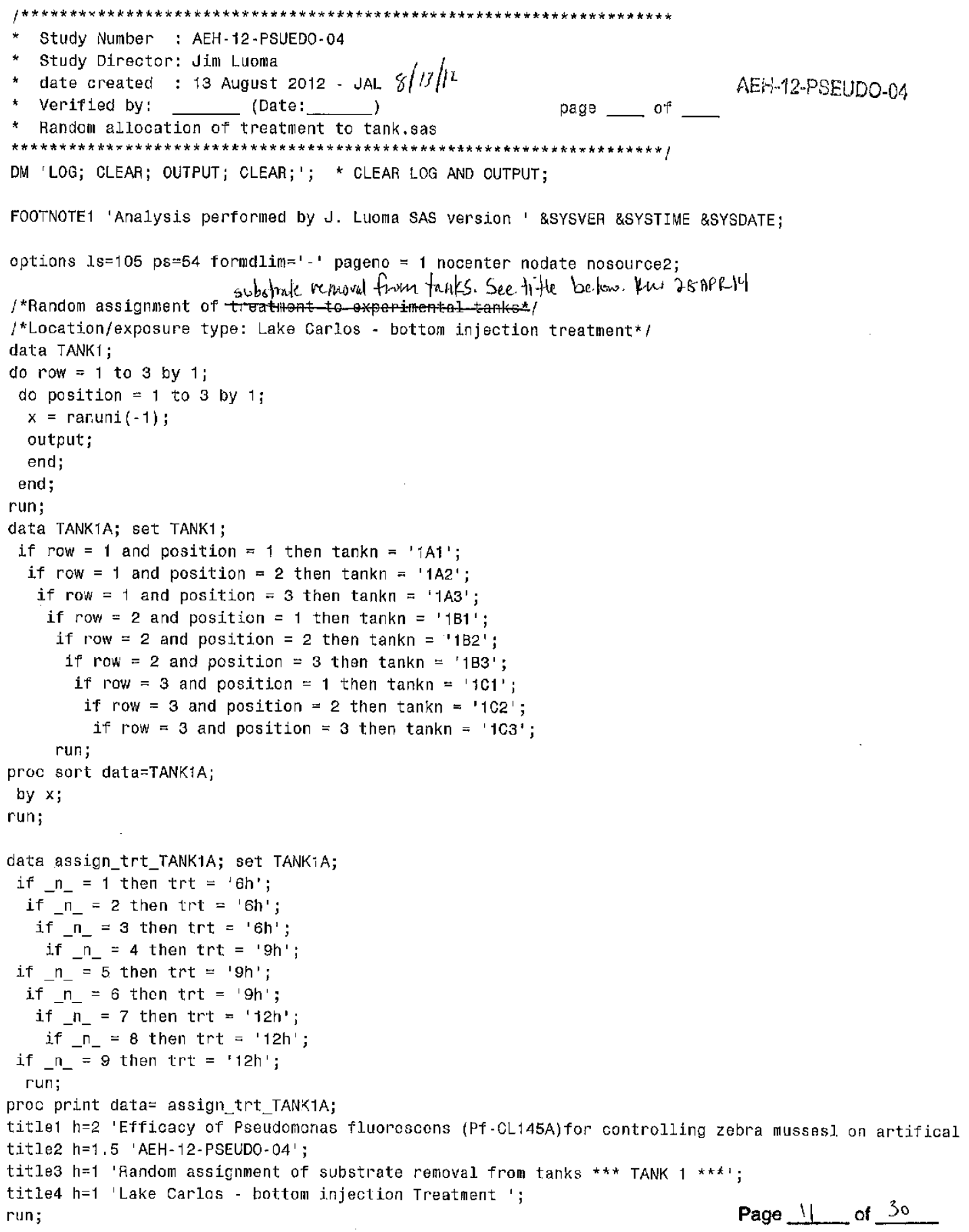


data TANK2;

do row $=1$ to 3 by 1 ;

do position $=1$ to 3 by 1 ;

$x=\operatorname{ranuni}(-1)$;

output;

AFI-12PSEJDO-04

end;

end;

run;

data TANK2A; set TANK2;

if row $=1$ and position $=1$ then tankn $=' 2 A 1 '$;

if row $=1$ and position $=2$ then tankn $=' 2 A 2^{\prime}$;

if row $=1$ and position $=3$ then $\operatorname{tankn}={ }^{\prime} 2 \mathrm{~A} \mathbf{3}^{\prime}$;

if row $=2$ and position $=1$ then tankn $={ }^{\prime} 2 \mathrm{~B}_{1} \mathrm{I}^{\prime}$;

if row $=2$ and position $=2$ then $\operatorname{tankn}=$ ' 282 ';

if row $=2$ and position $=3$ then tankn $={ }^{\prime} 283^{\prime}$;

if row $=3$ and position $=1$ then tankn $=' 2 \mathrm{C} 1$ ';

if row $=3$ and position $=2$ then tankn $=' 2 \mathrm{C} 2$ ';

run;

if row $=3$ and position $=3$ then tankn $=12 \mathrm{C} 3 '$;

proc sort data=TANK2A;

by $x$;

run;

data assign_trt_TANK2A; set TANK2A;

if $\mathrm{n}_{-}=1$ then $\operatorname{trt}=$ ' $^{\prime} 6 \mathrm{~h}^{\prime}$;

if _n_ $=2$ then trt $=$ ' $6 \mathrm{~h}^{\prime}$;

i.f $\bar{n}_{-}=3$ then trt $=16 \mathrm{~h}$ ';

if $n_{-}=4$ then trt $=$ ' 9 ' ';

if $\mathrm{n}_{-}=5$ then $\operatorname{trt}={ }^{\prime} 9 \mathrm{~h}^{\prime}$;

if $n_{-}=6$ then trt $=$ ' $9 \mathrm{~h}$ ';

if $\bar{n}_{-}=7$ then trt $=12 \mathrm{~h}^{\prime}$;

if $n_{-}=8$ then trt $=12 \mathrm{~h}$ ' :

if $n_{-}=9$ then $\operatorname{trt}=' 12 h^{\prime}$;

run;

proc print data $=$ assign_trt_TANK2A;

title1 h=2 'Efficacy of Pseudomonas fluorescens (Pf-CL145A)for controlling zebra mussesl on artifical title2 $h=1.5$ 'AEH-12-PSEUDO-04';

title3 $h=1$ 'Random assignment of substrate removal from tanks *** TANK $2 * * * '$;

title4 $h=1$ 'Lake Carlos - bottom injection Treatmert ';

run;

data TANK3;

do row $=1$ to 3 by 1 ;

do position $=1$ to 3 by 1 ;

$x=\operatorname{ranuni}(-1)$;

output;

end;

end;

run;

data TANK3A; set TANKB;

if row $=1$ and position $=1$ then tankn $=$ '3A1';

if row $=1$ and position $=2$ then tankn $=$ ' $3 A 2{ }^{\prime}$;

if row $=1$ and position $=3$ then tankn $=$ ' 3 A ' ';

if row $=2$ and position $=1$ then tankn $=$ '3B1';

if row $=2$ and position $=2$ then tankn $=$ ' $3 B 2$ ';

Page 12 of 30

if row $=2$ and position $=3$ then tankn $={ }^{\prime 3 B 3^{\prime}}$; 


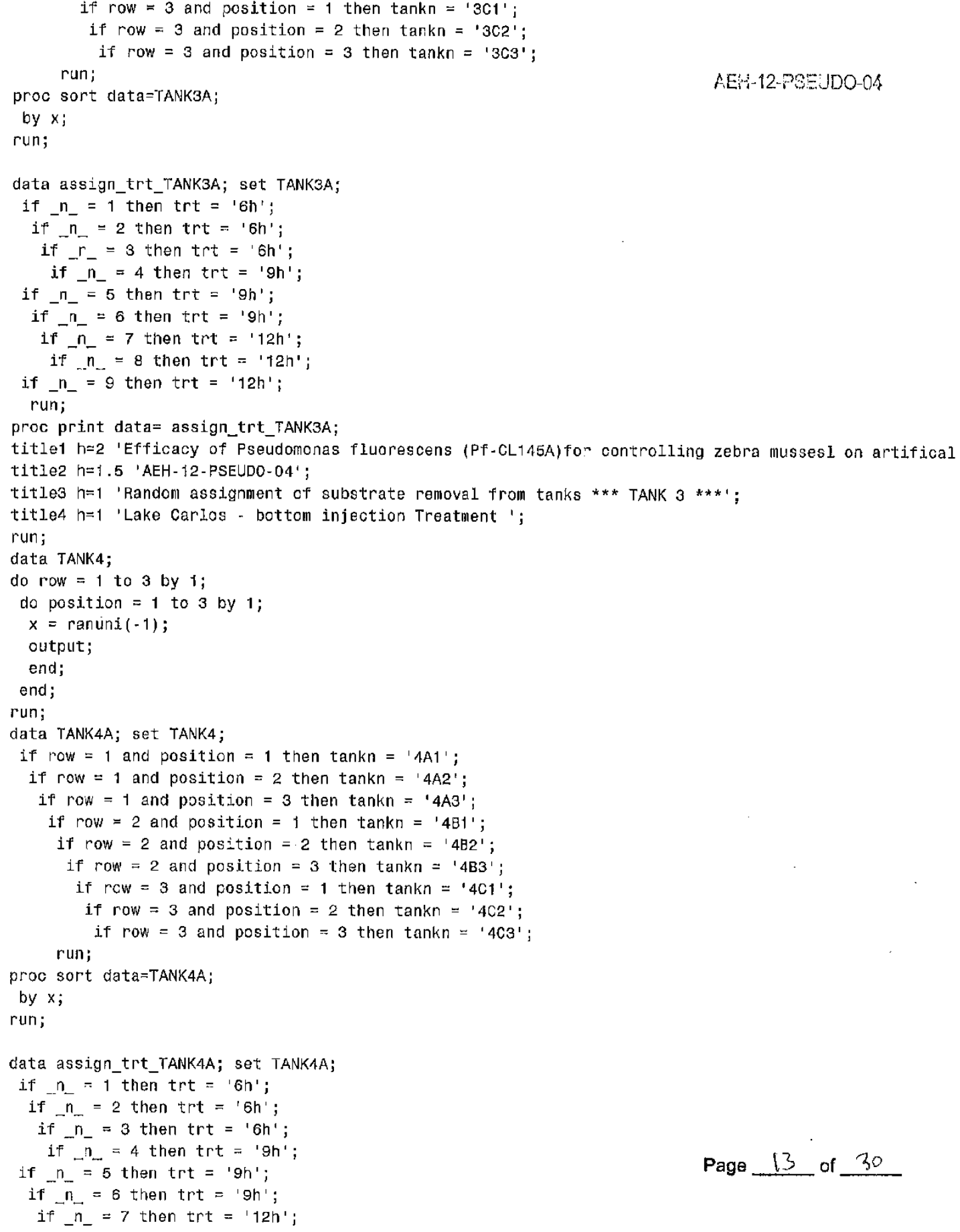




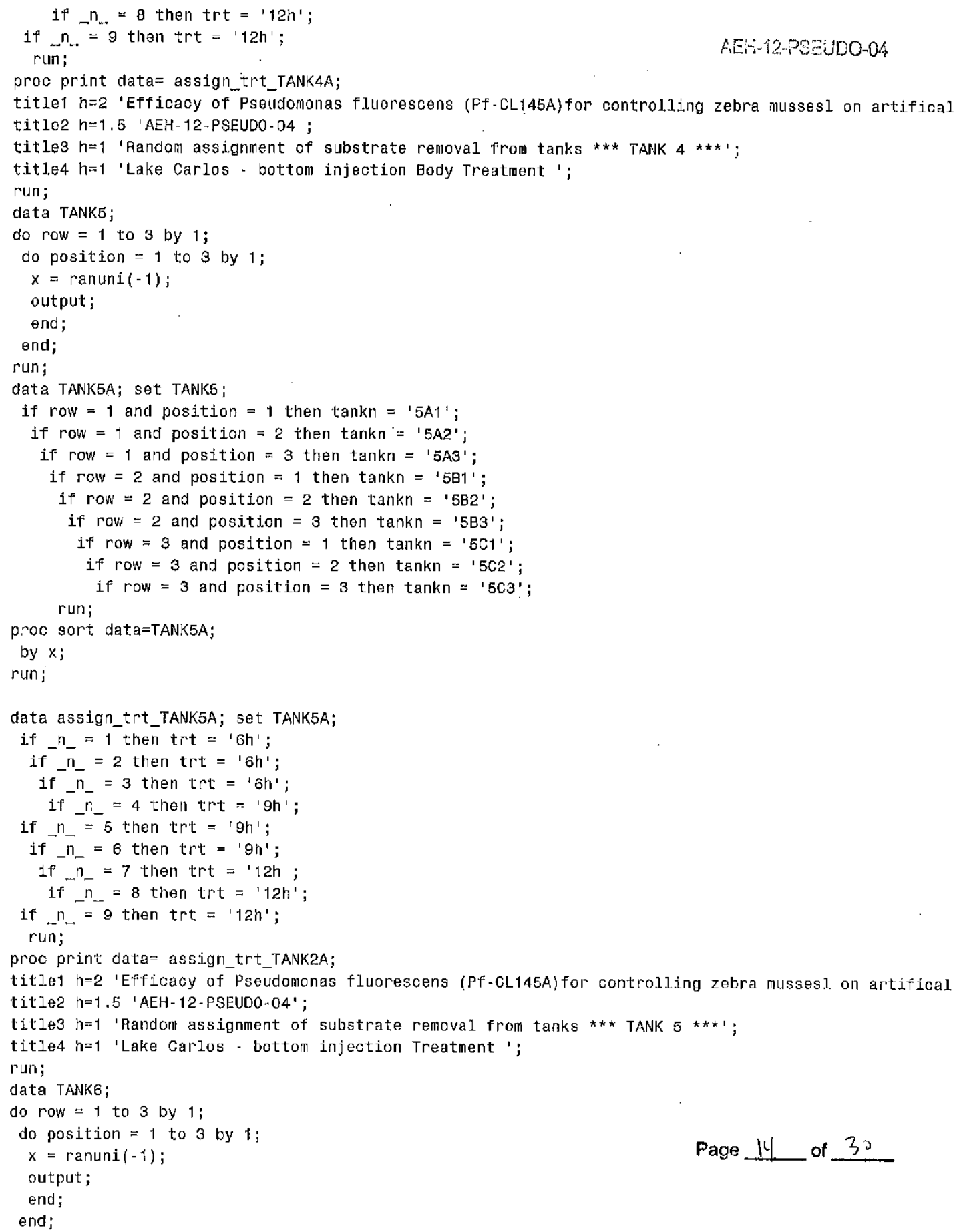

Page 14 of 30 


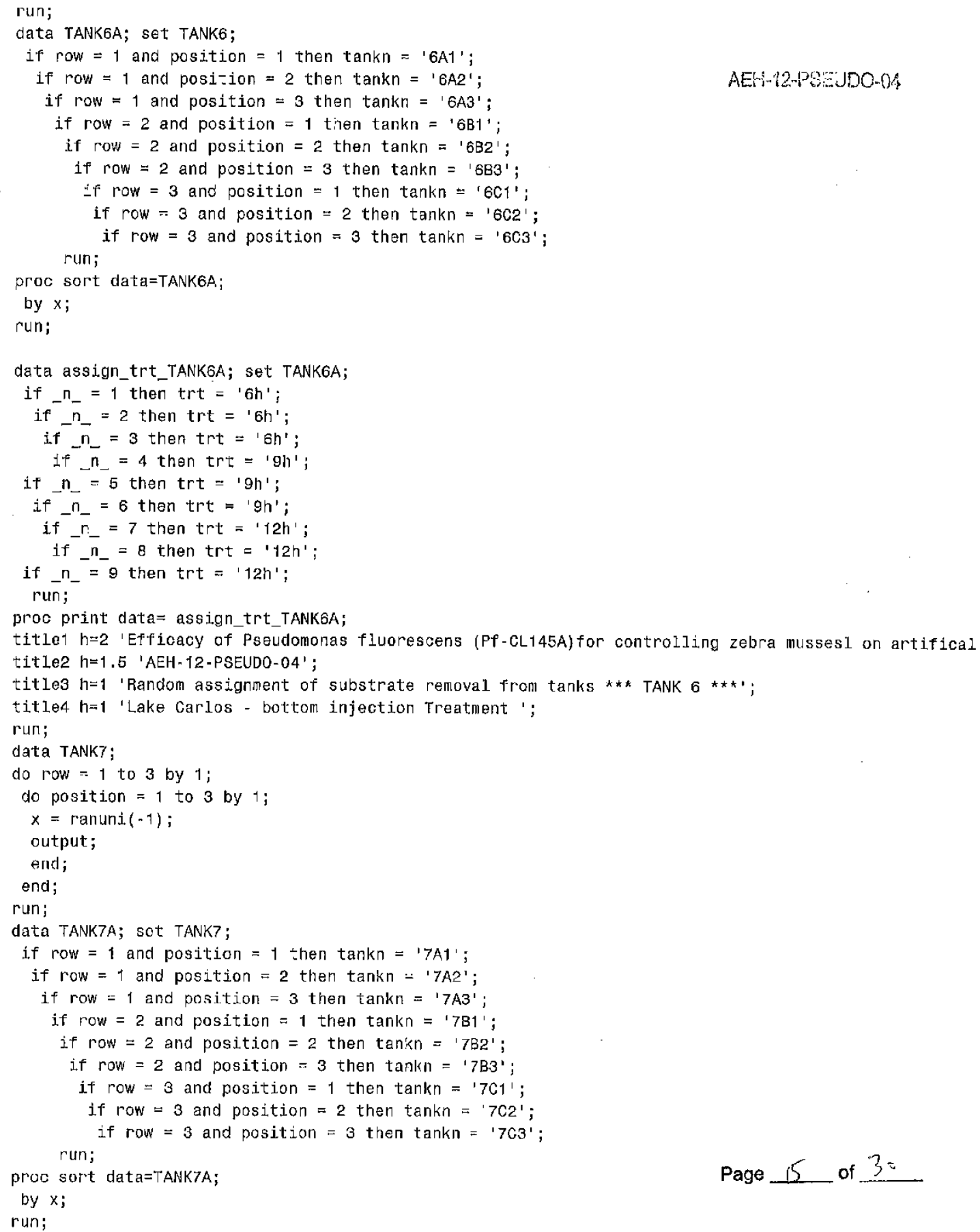




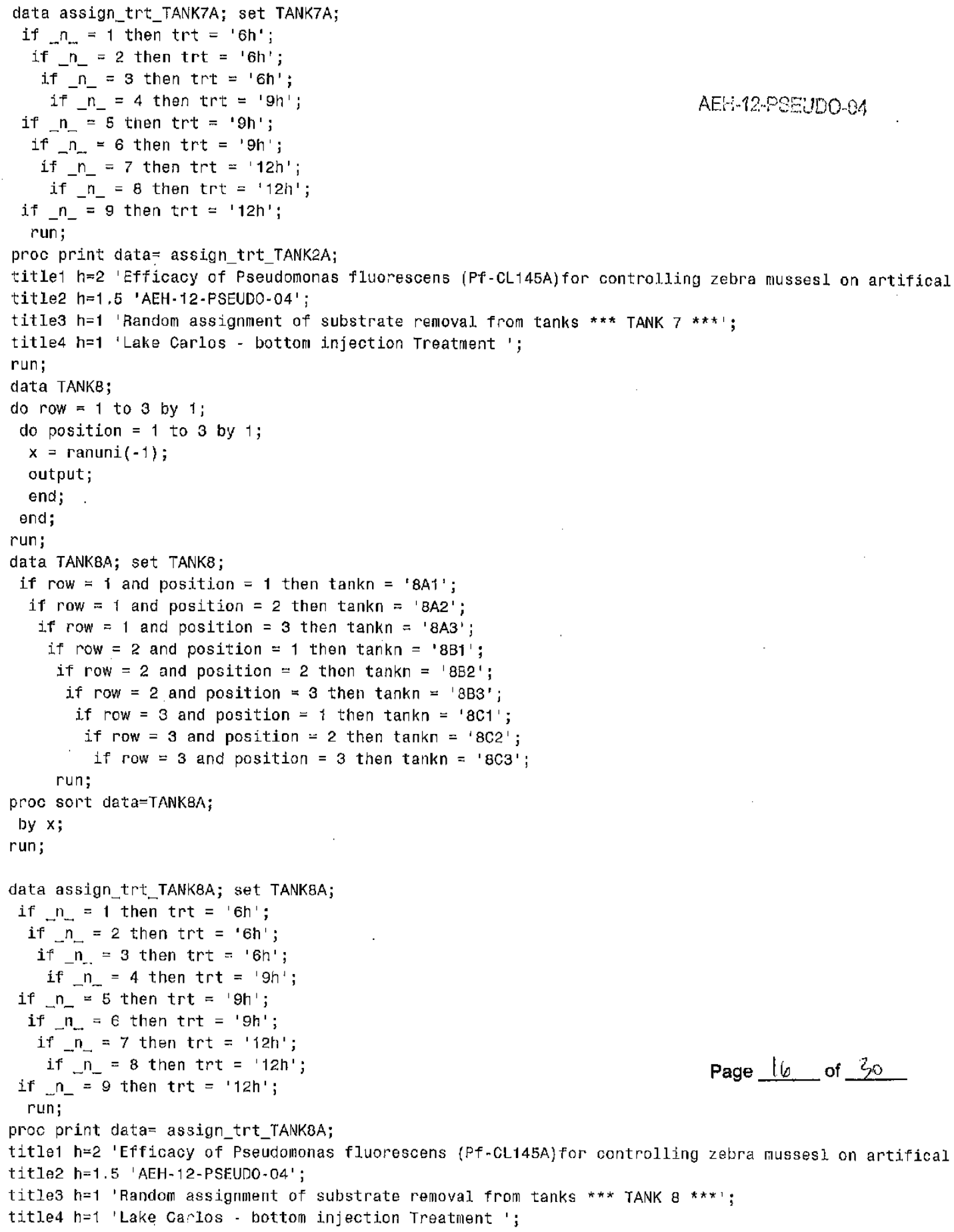




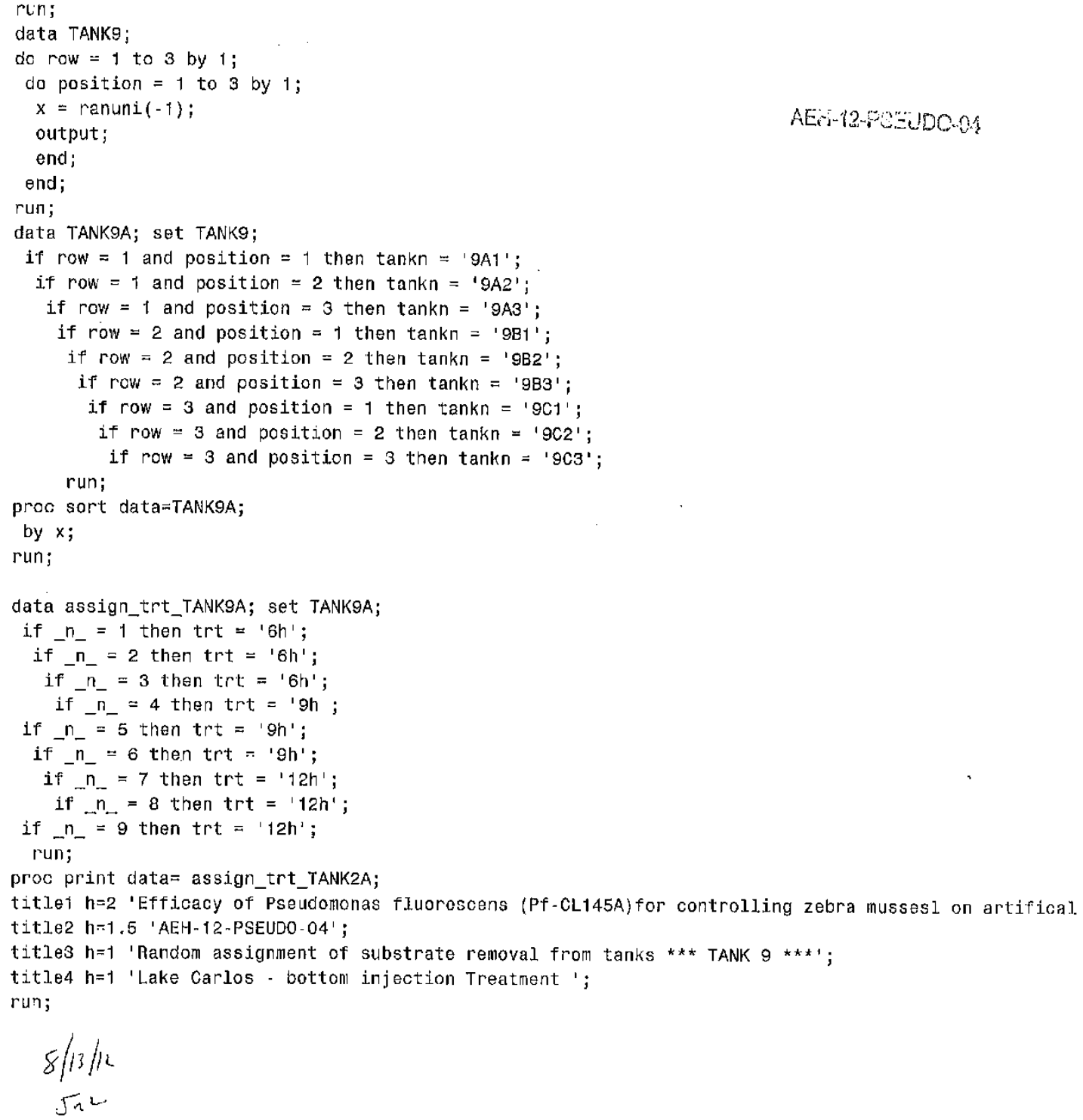




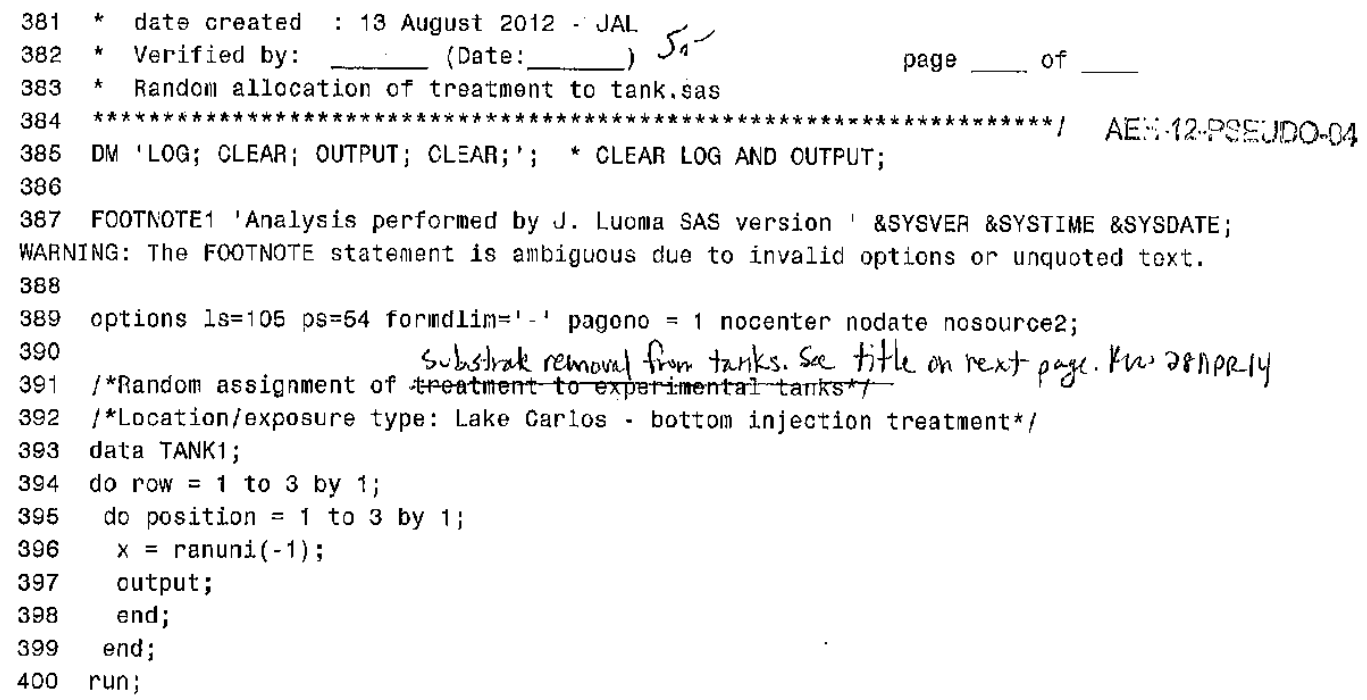

NOTE: There were 9 observations read from the data set WOAK. TANK1A. NOTE: The data set WORK. TANK1A has 9 observarions and 4 variables. NOTE: PROCEDURE SORT used (Total process time):

Page 18 of 30 


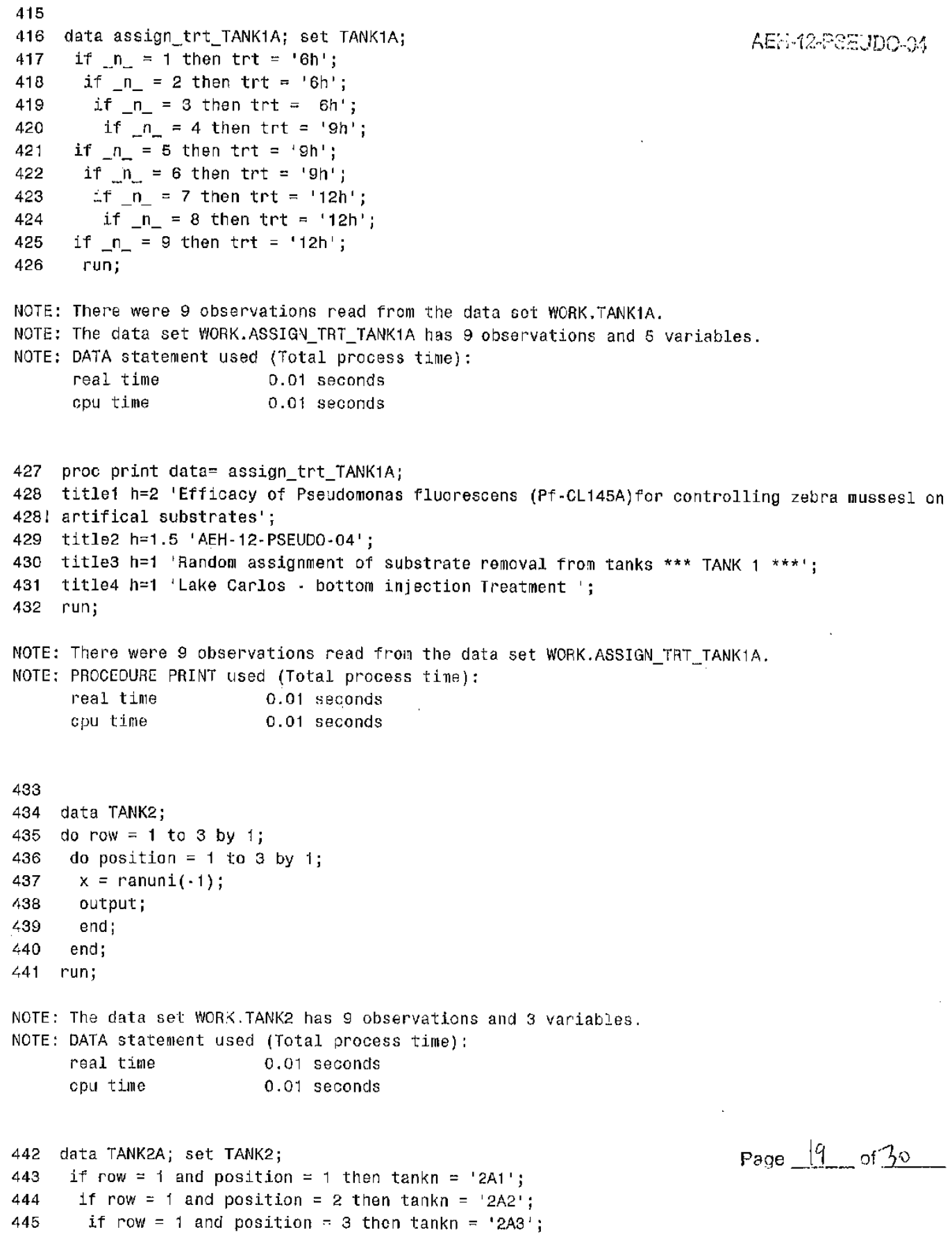




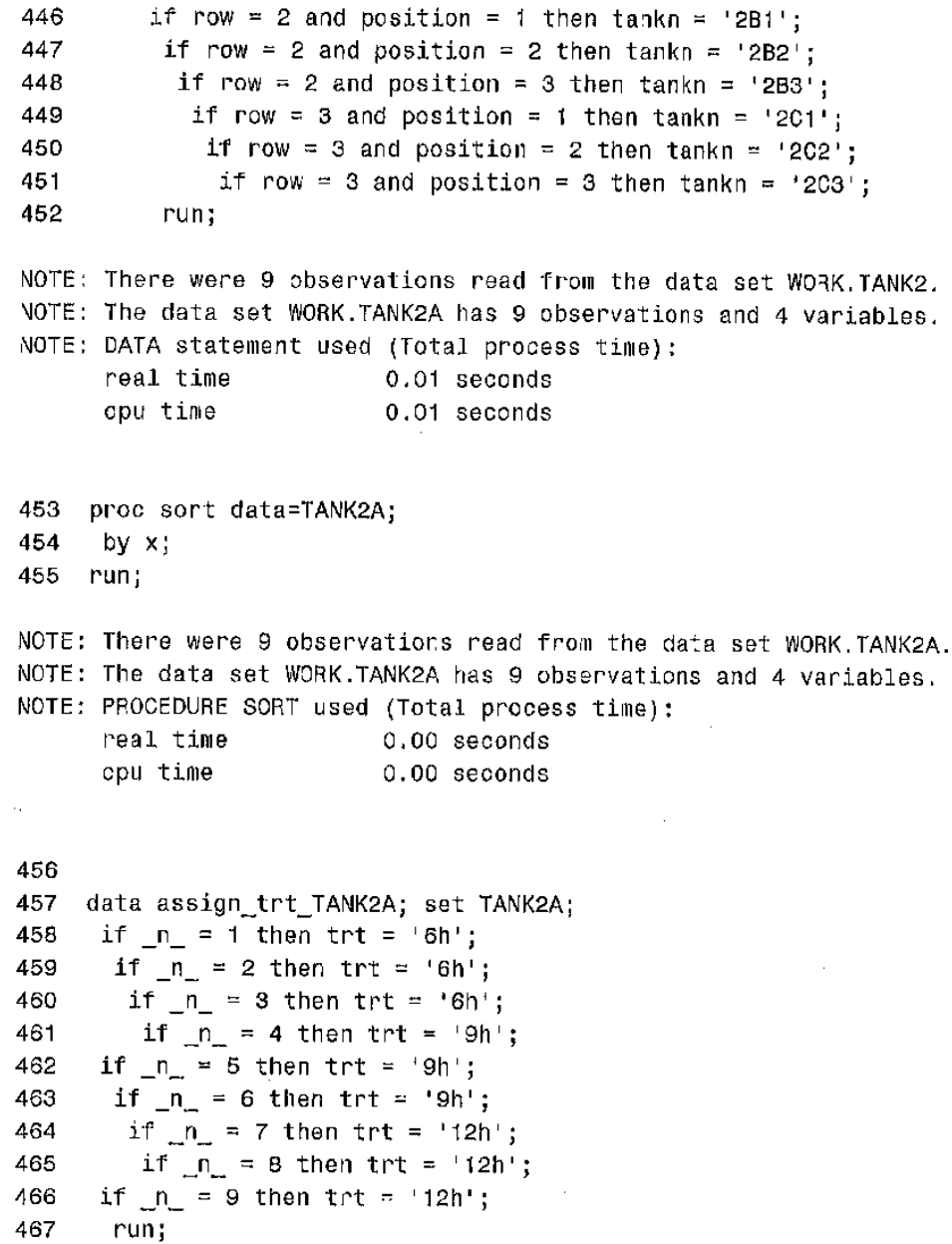

NOTE: There were 9 observations read from the data set WOAK.TANK2A.

NOTE: The data set WORK.ASSIGN_TRT_TANK2A has 9 observations and 5 variables.

NOTE: DATA statement used (Total process time):

real time $\quad 0.01$ seconds

cpt time $\quad 0.01$ seconds

468 proc print data= assign_trt_TANK2A;

469 titlet $h=2$ 'Efficacy of Pseudomonas fluorescens (Pf-CL145A) for controlling zcbra mussesl on

469 ! artifical substrates';

470 title2 $h=1.5$ 'AEH-12-PSEUDO-04';

471 title3 $h=1$ 'Random assignment of substrate removal from tanks *** TANk $2 * * * 1$;

472 title4 $h=1$ 'Lake Carlos - bottom injection Treatment ';

473 run;

NOTE: There were 9 observations read fron the data set WOFK.ASSIGN TH'. TANK2A.

Page 20 of 30 NOTE: PROCEDURE PRINT Lised (Total process time): 
$\begin{array}{ll}\text { real time } & 0.00 \text { seconds } \\ \text { cpu time } & 0.00 \text { seconds }\end{array}$

474

475 data TANK3;

476 do row $=1$ to 3 by 1 ;

477 do position $=1$ to 3 by 1 ;

$478 \quad x=$ ranuni $(-1)$;

479 output;

480 end;

481 end;

482 run;

NOTE: The data set WORK.TANK3 has 9 observations and 3 variables.

NOTE: DATA statement used (Total process time):

real time $\quad 0.01$ seconds

cpu time $\quad 0.01$ seconds

483 data TANK3A; set TANK3;

484 if row $=1$ and position $=1$ then tankn = '3A1';

485 if row $=1$ and position $=2$ then tankn $=$ ' $3 \mathrm{~A} 2$ ';

486 if row $=1$ and position $=3$ then tankn $=13 \mathrm{~A} 3^{\prime}$;

487 if row $=2$ and position $=1$ then tankn = '3B1';

488 if row $=2$ and position $=2$ then tankn $=$ ' $3 \mathrm{~B} 2$ '.

489 if row $=2$ and position $=3$ then tankn $={ }^{\prime} 3 \mathrm{~B}^{\prime}$ ';

490

491

492

493

the 1 then tankn = '3c1'. if row $=3$ and position $=2$ then tankn $=' 3 \mathrm{C2}$ ';

if row $=3$ and position $=3$ then tankn $=$ ' $3 \mathrm{C} 3$ '; run;

NOTE: There were 9 observations read from the data set WORK. TANK3 NOTE: The data set WOKK.TANK3A has 9 observations and 4 variables. NOTE: DATA statement used (Total process time) :
real tine
0.03 seconds
cpu time
0.03 scoonds

494 proc sort data=TANK3A;

495 by $x$;

496 run;

NOTE: There were 9 observations read from the data set WORK. TANK3A, NOTE: The data set WORK. TANK3A ras 9 observations and 4 variables. NOTE: PROCEOURE SORT used (TOtal process time):
real time
0.01 seconds

spu time

0.01 seconds

497

498 data assign_trt_TANKBA; set TANK3A;

499 if $n_{-}=1$ then trt $=$ ' $6 \mathrm{~h}$ ';

500 if ${ }_{-}{ }_{-}=2$ then $\operatorname{trt}=' 6 \mathrm{~h}^{\prime}$;

501 if $\bar{n}_{-}=3$ then tht $=6 h^{\prime}$;

502 if $\bar{n}_{-}=4$ then trt $=9 \mathrm{~h}^{\prime}$;

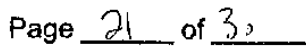




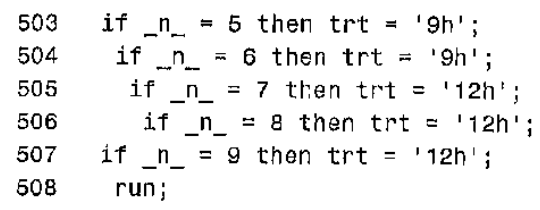

NOTE: There were 9 observations read from the data set WORK. TANK3A.

NOTE: The data set WORK.ASSIGN_TRT_TANK3A has 9 observations and 5 variables.

NOTE: DATA statement used (Total process time):
real tim
0.01 seconds
cpu time
0.01 seconds

509 proc print data= assign_trt_TANK3A;

510 title1 h=2 'Efficacy of Pseudomonas fluorescens (Pf-CL145A)for controlling zebra mussesi on

5101 artifical substrates';

511 title2 $h=1.5$ 'AEH-12-PSEUDO-04';

512 titles $h=1$ 'Random assignment of substrate removal from tanks $* * *$ TANK $3 * * * 1$;

513 title $4 \mathrm{~h}=1$ 'Lake Carlos - bottom injection Treatment ';

514 run;

NOTE: There were 9 observations read fron the data set WORK.ASSIGN_TRT_TANK3A.

NOTE: PROCEDURE PRINT used (TotaI process time):

real time $\quad 0.00$ seconds

cpu time $\quad 0.00$ seconds

515 data TANK4;

516 do row $=1$ to 3 by 1 ;

517 do position $=1$ to 3 by 1 ;

$518 \quad x=\operatorname{ranuni}(-1) i$

519 output;

520 end;

521 end;

522 run;

NOTE: The data set WORK.TANK4 has 9 observations and 3 variables.

NOTE: DATA statement used (Total process time):
real time
0.01 seconds
cpu time
0.01 seconds

523 data TANK4A; set TANK4;

524 if row $=1$ and position $=1$ then tankn $=$ ' $4 \mathrm{~A} 1$ ';

525 if row $=1$ and position $=2$ then tankn $=$ ' $4 \mathrm{~A} 2$ ';

526 if row $=1$ and position $=3$ then tankn $={ }^{\prime} 4 \mathrm{~A} 33^{\prime}$;

527 if row $=2$ and position $=1$ then tankn $={ }^{\prime} 4 B 1 '$;

528 if row $=2$ and position $=2$ then tankn $=14 \mathrm{~B} 2$ ',

529 if row $=2$ and position $=3$ then tankn $={ }^{\prime} 4 \mathrm{~B} 3{ }^{\prime}$;

530 if row $=3$ and position $=1$ tren tankn $={ }^{\prime} 4011^{\prime}$;

531 if row $=3$ and position $=2$ then tankn $={ }^{\prime}{ }^{\prime} 4 \mathrm{C} 2^{\prime}$;

532 if row $=3$ and position $=3$ then tankn $=14 \mathrm{CC} 3^{\prime}$,

533 run;

Page 22 of 30

NCTE: There were 9 observations read from the data set WORK.TANK4. 
NOTE: The data set WORK.TANK4A has 9 observations and 4 variables.

NOTE: DATA statenent: used (Total process tine) :
real time
0.01 seconds
cpu time
0.01 seconds

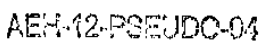

534 proc sort data=TANK4A;

535 by $x$;

536 run;

NOTE: There were 9 observations read from the data set WORK. TANK4A.

NOTE: The data set WORK. TANK4A has 9 observations and 4 variables.

NOTE: PROCEDJRE SORT used (Total process time):

real time 0.01 seconds

cpu time $\quad 0.01$ seconds

537

538 data assign_trt_TANK4A; set TANK4A;

539 if $n_{-}=1$ then $\operatorname{trt}=$ ' $6 \mathrm{~h}$;

540 if $\bar{n}_{-}=2$ then trt $=$ ' $6 h^{\prime}$;

541 if $n_{-}=3$ then trt $={ }^{\prime} 6 \mathrm{~h}^{\prime}$;

542 if $\bar{n}_{-}=4$ then trt $={ }^{\prime}$ ' $9 h^{\prime}$;

543 if $\mathrm{n}_{-}=5$ then trt $=$ ' $9 \mathrm{~h}^{\prime}$;

54.4 if $\vec{n}_{-}=6$ then $\operatorname{trt}=$ ' $9 \mathrm{~h}^{\prime}$ ';

545 if ${ }_{-}{ }_{-}=7$ then $\operatorname{trt}=' 12 \mathrm{~h}$ ';

546 if $\bar{n}_{-}=8$ then trt $=112 h^{\prime}$;

547 if $n=9$ then $\mathrm{trt}=112 \mathrm{~h}$ ';

548 run;

NOTE: There were $\theta$ observations read from the data set WORK.TANK4A.

NOTE: The data set WORK.ASSIGN_TRT_TANK4A las 9 observations and 5 variables.

NOTE: DATA statement used (Total process tirle):

real time $\quad 0.03$ seconds

cpu tine $\quad 0.03$ seconds

549 proc print data= assign_trt_TANK4A;

550 titlel $h=2$ 'Efficacy of Pseudomonas fluorescens (Pf-CL145A)for controlling zebra mussesl on

550 ! artifical substrates';

551 title2 $h=1.5$ 'AEH-12-PSEUDO-04';

552 title3 $h=1$ 'Pandom assignment of substrate removal from tanks $* * *$ TANK $4 * * * 1$;

553 title4 $\mathrm{h}=1$ 'Lake Carlos - bottom injection Body Treatment ';

554 run;

NOTE: There were 9 observations read from the data set WORK.ASSIGN_TRT_TANKAA.

NOTE: PROCECURE PRINT used (Total process tille):

real time $\quad 0.00$ seconds

cpu time $\quad 0.00$ seconds

555 data TANK5;

556 do row $=1$ to 3 by 1 ;

557 do position $=1$ to 3 by 1 ;

Page 23 of 30

$558 \quad x=\operatorname{ranuni}(-1)$;

559 output: 
NOTE: The data set WORK.TANK5 has 9 observations and 3 variables.

NOTE: DATA statenent used (Total process time):
real time
0.03 seconds
cpu time
0.03 seconds

563 data TANK5A; set TANK5;

564 if row $=1$ and position $=1$ then tankn $=$ ' $5 \mathrm{~A} 1$ '

565 if row $=1$ and position $=2$ then tankn $=$ ' $5 \mathrm{~A} 2$ ';

566 if row $=1$ and position $=3$ then tankn $={ }^{\prime} 5 \mathrm{~A} 3^{\prime}$;

567 if row $=2$ and position $=1$ then tankn $=' 5 B 1^{\prime}$;

568 if row $=2$ and position $=2$ then tankn $=' 582$ '.

569 If row $=2$ and position $=3$ then tankn $={ }^{\prime} 583^{\prime}$;

570 i.f row $=3$ and position $=1$ then $\operatorname{tankn}={ }^{\prime} 5 \mathrm{C} 1^{\prime}$;

571 j.f row $=3$ and position $=2$ then $\operatorname{tankn}={ }^{\prime} 5 \mathrm{C} 2^{\prime}$;

572 If row $=3$ and position $=3$ then tankn $={ }^{\prime} 5 \mathrm{C} 3{ }^{\prime}$ '.

573 run;

NOTE: There were 9 observations read from the data set WORK. TANK5. NOTE: The data set WORK.TANK5A has 9 observations and 4 variables.

NOTE: DATA statement used (Total process time):
reas time
0.01 seconds
cpu time
0.01 seconds

574 proc sort data=TANK5A;

575 by $x$;

576 run;

NOTE: There were 9 observations read from the data set WORK. TANK5A. NOTE: The data set WORK. TANK5A has 9 observations and 4 variables. NOTE: PROCEDURE SORT used (Total process Time):
roal time
0.00 seconds
cpu time
0.00 seconds

577

578 data assign_trt_TANK5A; set TANK5A;

579 if $\mathrm{n}_{-}=1$ then trt $=$ ' $6 \mathrm{~h}$ ';

580 if $n_{-}=2$ then trt $=' 6 h ' ;$

581 . if $n_{-}=3$ then $t r t=' 6 h '$ ';

582 if ${ }^{n}{ }_{-}=4$ then trt $=$ ' $g$ h';

583 if $\mathrm{n}_{-}=5$ then trt $=$ ' $9 \mathrm{~h}$ ';

584 if ${ }^{n}{ }_{-}=6$ then trt $=$ '9h';

585 if $\bar{n}_{-}=7$ then trt $=12 h^{\prime}$;

586 if ${ }^{n}{ }_{-}=8$ then trt $=112 \mathrm{~h}$ '

587 if $n_{-}=9$ then trt $=$ '12h'.

588 run;

Page 24 of 30

NOTE: There were 9 observations read from the data set WORK. TANK5A.

NO'E: The data set VIORK.ASSIGN_TRT_TANK5A has 9 observations and 5 variables.

NO-E: DATA statement used (Total process time): 


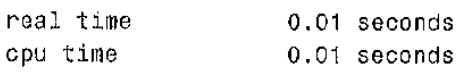

589 proc print data= assign_trt_TANK2A;

590 title1 h=2 'Efficacy of Pseudomonas fluorescens (Pf-CL145A)for controlling zebra mussesl on $590 !$ artifical substrates'

591 title2 h=1.5 'AEH-12-PSEUDO-04';

592 title3 $h=1$ 'Random assignment of substrate removal from tanks *** TANK $5 * * * 1$;

593 ti.tle4 $h=1$ 'Lake carlos - bottom injection Treatment ';

594 run;

NOTE: There were 9 okservations read from the data set WORK.ASSIGN_TRT_TANK2A.

NOTE: PROCEDURE PRINT used (Total process tine):
real time
0,01 seconds
cpu time
0.01 seconds

595 data TANK6;

596 do row $=1$ to 3 by 1 ;

597 do position $=1$ to 3 by 1 ;

$598 \quad x=\operatorname{ranuni}(-1)$;

599 output;

600 end;

601 end;

602 run;

NOTE: The data set: WORK. TANK6 ras 9 observations and 3 variables.

NOTE: DATA statement used (Totai process time):
real time
0.01 seconds
cpu time
0.01 seconds

603 data TANK6A; set TANK6;

604 if row $=1$ and position $=1$ then tankn $={ }^{\prime} 6 \mathrm{~A} \mathrm{I}^{\prime}$;

605 if row $=1$ and position $=2$ then tankn $=' 6 \mathrm{~A} 2$ '

606 If row $=1$ and position $=3$ then tankn $={ }^{\prime} 6 \mathrm{A3} \mathrm{I}^{\prime}$;

607 if row $=2$ and position $=1$ then tankn $=$ ' $6 \mathrm{~B} 1$ ';

608 if row $=2$ and position $=2$ then tankn $=$ ' $6 \mathrm{~B} 22^{\prime}$;

609 if row $=2$ and position $=3$ then tankm $={ }^{\prime} 6 \mathrm{~B} 3$ ';

610 if row $=3$ and position $=1$ then tankn $=16 \mathrm{C} 1{ }^{\prime}$;

611 If row $=3$ and position $=2$ then tankn $={ }^{\prime} 6 \mathrm{C} 2{ }^{\prime}$;

612 if row $=3$ and position $=3$ then tankn $={ }^{\prime} 6 \mathrm{C} 3{ }^{\prime}$;

613 rin;

NOTE: There were 9 observations read from the data set WORK.TANK6.

NOTE: The data set WORK. TANKGA has 9 observations and 4 variables.

NOTE: DATA statement used (Total process time):
real time
0.01 seconds
cpu time
0.01 seconds

614 proc sort data=TANK6A;

615 by $x$;

616 run;

Page 25 of 30 
NOTE: There were 9 observations read from the data set WORK. TANK6A.

NOTE: The data set WORK. TANKGA has 9 observations and 4 variables.

NOTE: PROCEDURE SORT LIsed (Tota1 process time):

$\begin{array}{ll}\text { real time } & 0.01 \text { seconds } \\ \text { cpu time } & 0.01 \text { seconds }\end{array}$

617

618 data assign_trt_TANK6A; set TANK6A;

619 if $n_{-}=1$ then trt $=16 h^{\prime}$;

620 if $n_{-}=2$ then trt $=$ ' $6 h^{\prime}$ ';

621 if $\bar{n}_{-}=3$ then trt $=$ ' $6 \mathrm{~h}$ ';

622 if _n_ $=4$ then $\operatorname{trt}=' 9 \mathrm{n}^{\prime}$;

623 if $\mathrm{n}_{-}=5$ then $\mathrm{trt}=$ ' $9 \mathrm{~h}^{\prime}$ ';

624 j.f_n_ $=6$ then $\operatorname{trt}=\mathrm{H}^{\prime} 9 \mathrm{~h}^{\prime}$;

625 if $\bar{n}_{-}=7$ then trt $=112 \mathrm{~h}$ ';

626 if $\bar{n}_{-}=8$ then trt $=112 \mathrm{~h}$ ';

627 if $n_{-}=9$ then $t r t=12 h^{\prime}$;

628 run;

NOTE: There were 9 observations read from the data set WORK. TANK6A.

NOTE: The data set WOFK.ASSIGN_TRT_TANK6A has 9 observations and 5 variables.

NOTE: DATA statement used (Total process time):

real timie $\quad 0.03$ seconds

cpu tille $\quad 0.03$ seconds

629 proc print data= assign trt TANK6A;

630 titlet h=2 'Efficacy of Pseudomonas fluorescons (Pf-CL145A) for controlling zebra musses.1. on

$630 !$ artifical substrates' :

631 title2 $\mathrm{h}=1.5$ 'AEH-12-PSEUDO-04';

632 title3 $h=1$ 'Random assignnent of substrate removal from tanks *** TANK $6 * * * 1$;

633 title4 $h=1$ 'Lake Carlos - bottom injection Treatment ';

634 run;

NOTE: There were 9 observations read from the data set WORK.ASSIGN_TRT_TANKGA.

NOTE: PROCECURE PRINT usod (Totai process time):
roal time
0.01 seconds
срu ¿ime
0.01 seconds

635 data TANK7;

636 do row $=1$ to 3 by 1 ;

637 do position $=1$ to 3 by 1 ,

$638 \quad x=\operatorname{ranuni}(-1)$;

639 output:

640 end;

641 end;

642 run;

NOTE: The data sot WORK.TANK7 has 9 observations and 3 variables.

NOTE: DATA statement used (Total process time):
real time
0.01 seconds
cpul time
0.01 seconds

Page 20 of 30 


643
644
645
646
647
648
649
650
651
652
653

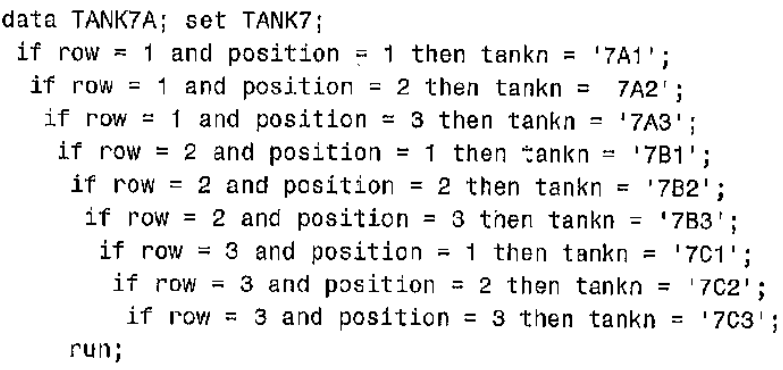

NOTE: There were 9 observations read fron the data set WORK. TANK7.

NOTE: The data set WORK.TANK7A has 9 observations and 4 variables.

NOTE: DATA statement used (Total process time):

real time $\quad 0.01$ seconds

cpli time $\quad 0.01$ seconds

654 proc sort data=TANK7A;

655 by $x$;

656 run;

NOTE: There were 9 observations read from the data set WORK. TANK7A.

NOTE: The data set WORK. TANK7A has 9 observations and 4 variables.

NOTE: PROCEDURE SORT. used (Total process time):

real time $\quad 0.01$ seconds

cpu time $\quad 0.01$ seconds

657

658 data assign_trt_TANK7A; set TANK7A;

659 if $\mathrm{n}_{-}=1$ then trt $=$ ' $6 \mathrm{~h}$ ';

660 if $n=2$ then trt $=$ ' $6 h^{\prime}$;

661 i.f $\bar{n}_{-}=3$ then $\operatorname{tr} t={ }^{\prime} 6 h^{\prime}$;

662 if $n_{-}=4$ then $\operatorname{trt}=$ ' $\mathrm{gh}$ ';

663 if $n_{-}=5$ then trt $=$ ' $9 \mathrm{~h}^{\prime}$;

664 if $n=6$ then $\operatorname{trt}={ }^{\prime} 9 \mathrm{n}$ ';

665 if $r_{-}=7$ then trt $=12 h^{\prime}$;

666 if $n_{-}=8$ then trt $=' 12 h^{\prime}$

667 if $n_{-}=9$ then $\operatorname{trt}=112 h^{\prime}$;

668 run;

NOTE: There were 9 observations read from the data set WORK. TANK7A.

NOTE: The data sot WORK.ASSIGN_TRT_TANK7A has 9 observations and 5 varjables.

NOTE: DATA statement used (Total process time):
real time
0.01 seconds

cpu time $\quad 0.01$ seconds

Page 27 of 30

669 proc print data= assign trt_TANK2A;

670 title1 $h=2$ 'Efficacy of Pseudomonas fluorescens (Pf-GL145A) for controlling zebra mussesl on

670 ! artifical substrates';

671 title2 $h=1.5$ 'AEH-12-PSELDO-04';

672 title3 $h=1$ 'Random assignment of substrate removal from tanks $* * *$ TANK $7 * * * 1$;

673 title4 $h=1$ 'Lake Carlos - bottom injection Treatment '; 
674 run;

NOTE: There were 9 observations read from the data set WOAK.ASSIGN_TRT_TANK2A.

NOTE: PROCEDURE PRINT used (Total process tinie):
real time
0.00 seconds
cpu time
0.00 seconds

675 data TANK8;

676 do row $=1$ to 3 by 1 ;

677 do position $=1$ to 3 by 1 ;

$678 \quad x=\operatorname{ranuni}(-1)$;

679 output:

680 end;

681 end;

682 run;

NOTE: The data set WCRK.TANK8 has 9 observations and 3 variables.

NOTE: DATA statement used (Total process time):
real time
0.01 seconds

cpu time

0.01 seconds

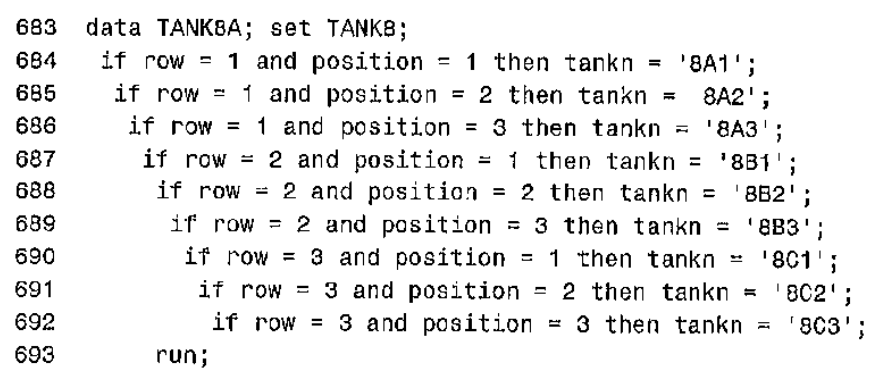

NOTE: There were 9 observations read fron the data set WORK. TANKB.

NOTE: The data set WORK. TANKBA has 9 observations and 4 variables.

NOTE: DATA statement used (Total process time):

real time $\quad 0.03$ seconds

cpu time $\quad 0.03$ seconds

694 proc sort data=TANK8A;

695 by $x$;

696 run;

NOTE: There were 9 observations read from the data set WORK.TANK8A.

NOTE: The data set WORK. TANK8A has 9 observations and 4 variables.

NOTE: PROCEDURE SORT used (Total process time):
real time
0.01 seconds
cpu tine
0.01 seconds

Page 28 of 30

697

698 data assign_trt_TANKBA; set TANKBA;

699 if $n_{-}=1$ then trt $=$ ' $6 \mathrm{~h}$ '; 


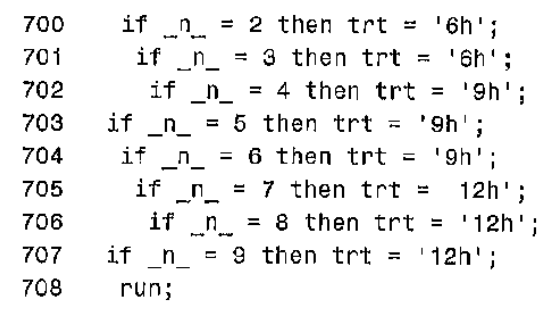

NOTE: There were 9 observations read from the data set WORK. TANKBA.

NOTE: The data set WORK.ASSIGN_TRT_TANK8A has 9 observations and 5 variablos.

NOTE: DATA statement used (Total process tine):

real tinis $\quad 0.01$ seconds

opu time $\quad 0.01$ seconds

709 proc print data= assign_trt_TANK8A;

710 title1 $h=2$ 'Efficacy of Pseudomonas fluorescens (Pf-CL145A) for controlling zebra mussesl on

710 ! artifical substrates';

711 title2 $h=1.5$ 'AEH-12-PSEUDO-04';

712 title $3 \mathrm{~h}=1$ 'Random assignment of substrate removal from tanks $* * *$ TANK 8 ***1;

713 title4 h=1 'Lake Carlos - bottom injection Treatment ';

714 run;

NOTE: There were 9 observations read from the data set WORK.ASSIGN_TAT_TANKBA.

NOTE: PROCEDURE PRINT used (Total process time):
real time
0.00 seconds
apu time
0.00 seconds

715 data TANK9;

716 do row $=9$ to 3 by 1 ;

717 do position $=1$ to 3 by 1 ;

$718 \quad x=\operatorname{ranuni}(-1)$;

719 output;

720 end;

721 end;

722 run;

NOTE: The data set WORK.TANK9 has 9 observations and 3 variables.

NOTE: DATA statement used (Total process time):

$\begin{array}{ll}\text { real time } & 0.01 \text { seconds } \\ \text { cpu time } & 0.01 \text { seconds }\end{array}$

723 data TANK9A; set TANK9;

724 if row $=1$ and position $=1$ then $\tan \kappa n=' 9 \mathrm{~A} 11^{\prime}$;

725 if row $=1$ and position $=2$ then tankn $=$ ' $9 \mathrm{~A}^{\prime}$ ';

726 i.f row $=1$ and position $=3$ then tankn $=' 9 \mathrm{AB}{ }^{\prime}$;

727 if row' $=2$ and posttion $=1$ then tankn $=9 \mathrm{~B} 1 \mathrm{I}^{\prime}$;

728 if row $=2$ and position $=2$ then tankn $={ }^{\prime} 9 \mathrm{~B} 2{ }^{\prime}$;

729 if row $=2$ and position $=3$ then tankn $=$ '9B3';

730 if row $=3$ and position $=1$ then $\operatorname{tankn}={ }^{\prime} 9 C 1^{\prime}$;

731 if row $=3$ and position $=2$ then tankn $={ }^{\prime} 9 \mathrm{G} 2$ ';

732

if row $=3$ and position $=3$ then tarkn $=$ ' $9 \mathrm{C}^{\prime} \mathrm{s}^{\prime}$;

Page 29 of 30 
NOTE: There were 9 observations read froll the data set WORK. TANK9.

NOTE: The data set WORK. TANK9A has 9 observations and 4 variables.

NOTE: DATA statement used (Total process time):

real time $\quad 0.01$ seconds

cpu time $\quad 0.01$ seconds

734 proc sort data=TANK9A;

735 by $x$;

736 run;

NOTE: There were 9 observations read from the data set WORK. TANK9A.

NOTE: The data set WORK.TANKSA has 9 observations and 4 variables.

NOTE: PROCECURE SORT used (Total process time) :
real time
0.0 : seconds
opu $\because$ ime
0.01 seconds

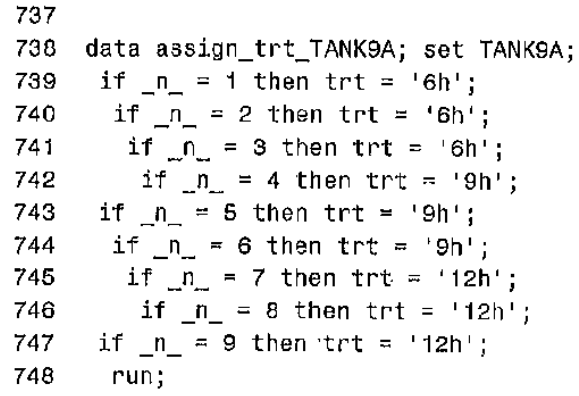

NOTE: This SAS session is using a registry in WORK. All changes will be lost at the end of this sess: 


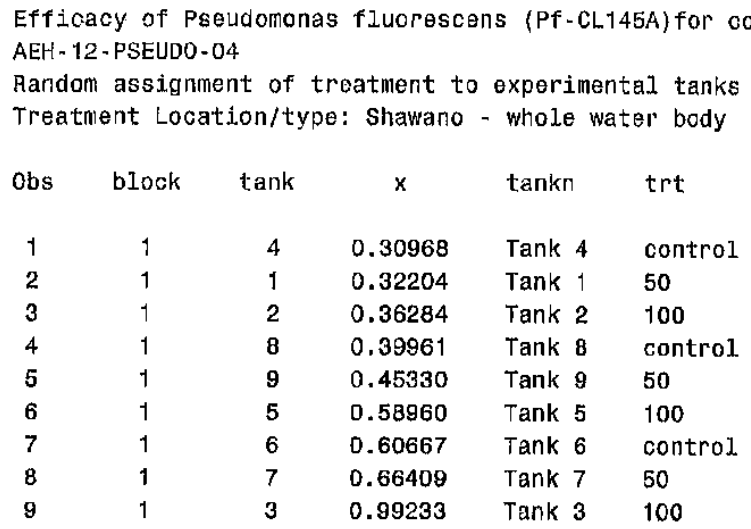

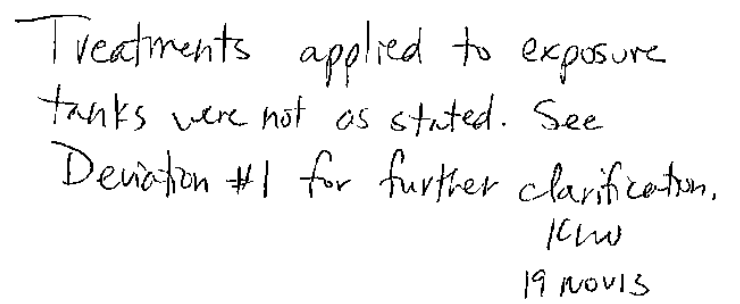

File Folder:

Nem Number:

Page 1

Analysts performed by J. Luona sas version $9.208: 5911$ AUG12 Jiv 


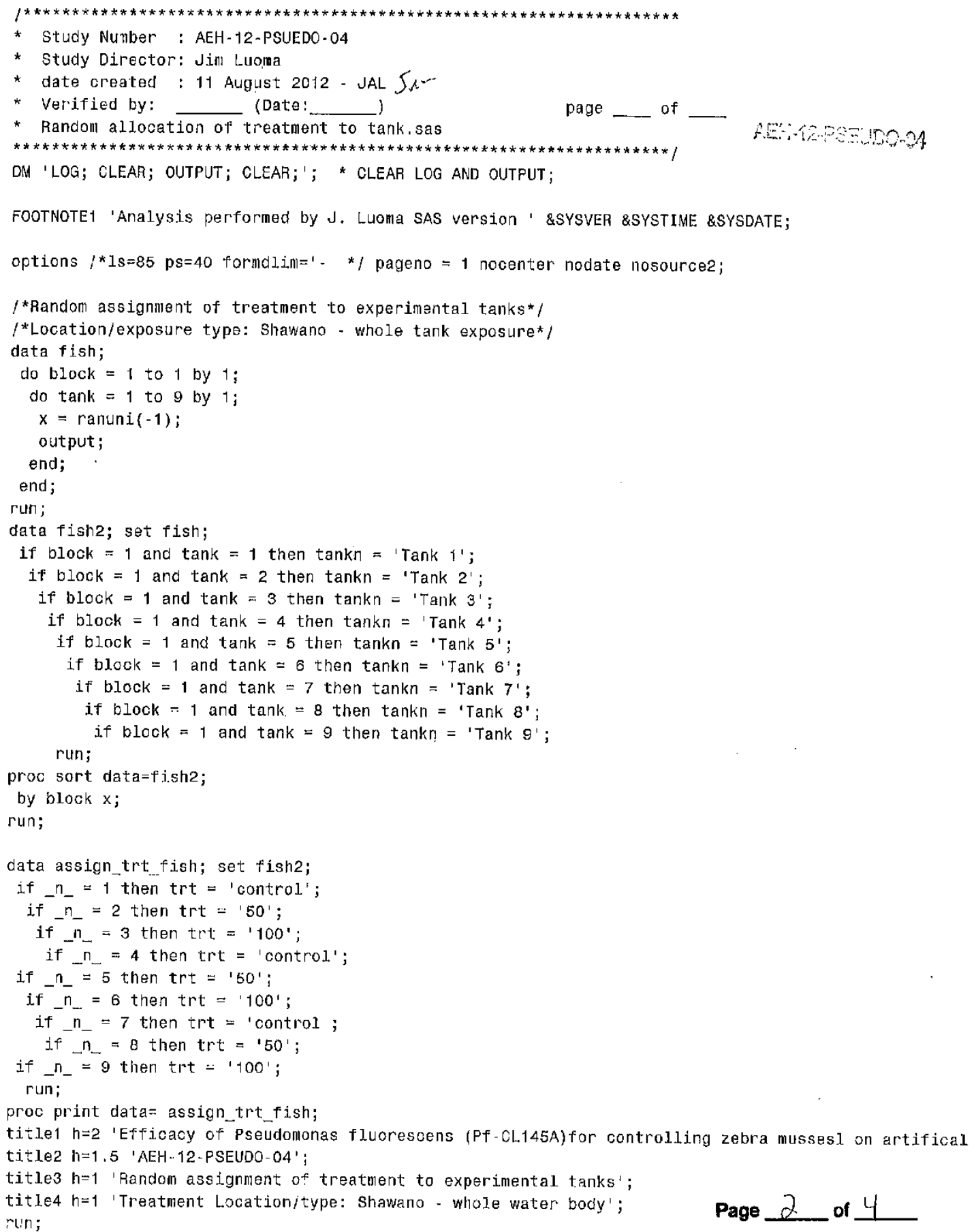

\section{Page 156 of 519}




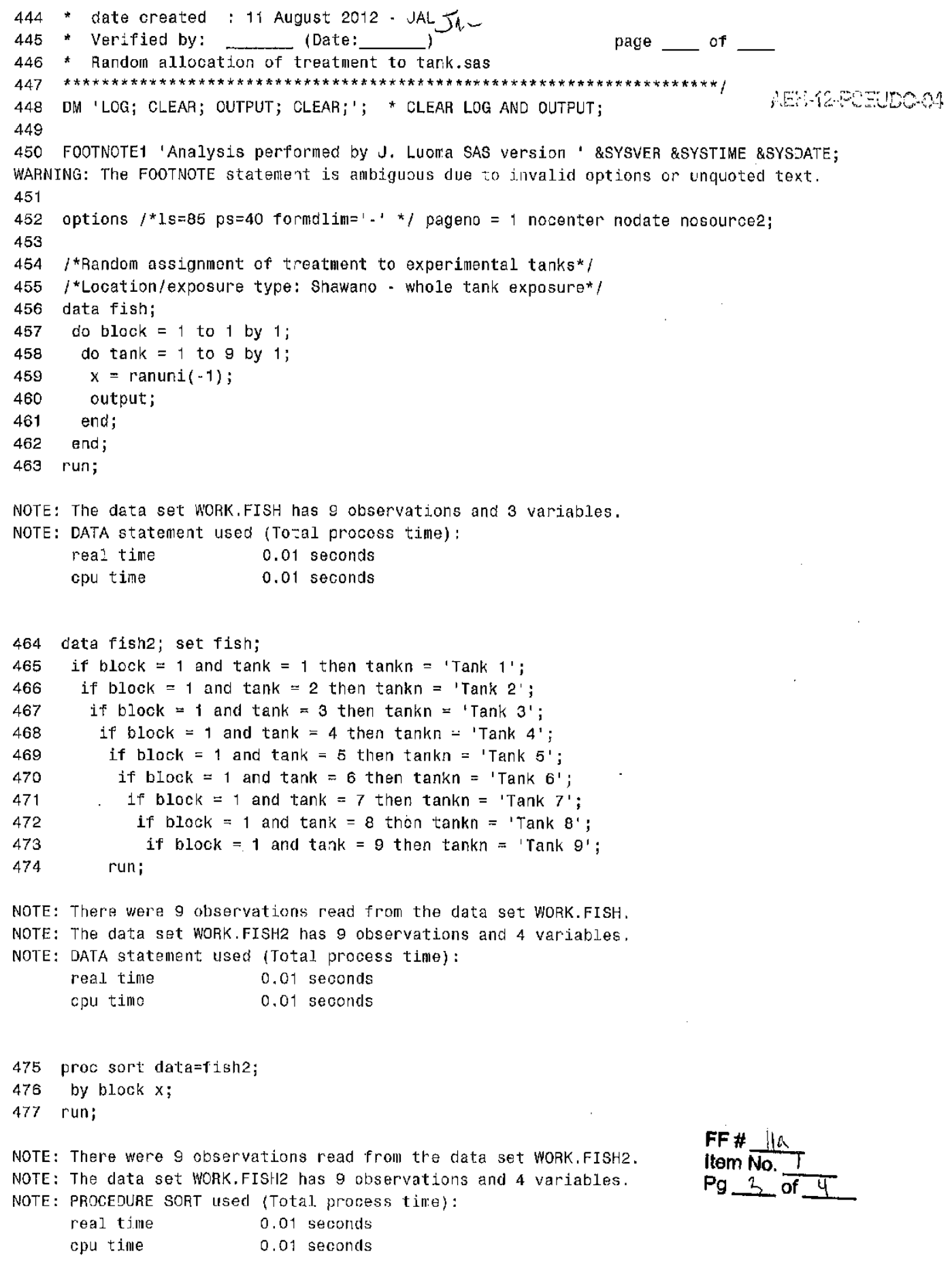

NOTE: There were 9 observations read from the data set WORK.FISH.

NOTE: The data set WORK.FISH2 has 9 observations and 4 variables.

NOTE: DATA statement used (Total process time):
real time
0.01 seconds

cpu timo

0.01 seconds

475 proc sort data=fish2;

476 by block $x$;

477 run;

NOTE: There were 9 observations read from the data set WORK.FISH2.

NOTE: The data set WORK.FISH2 has 9 observations and 4 variables.

NOTE: PROCEDURE SORT used (Total. process tine):

FF \#

Item No. T
real time
0.01 seconds
cpu tine
0.01 seconds

$\mathrm{Pg} 3$ of 4 


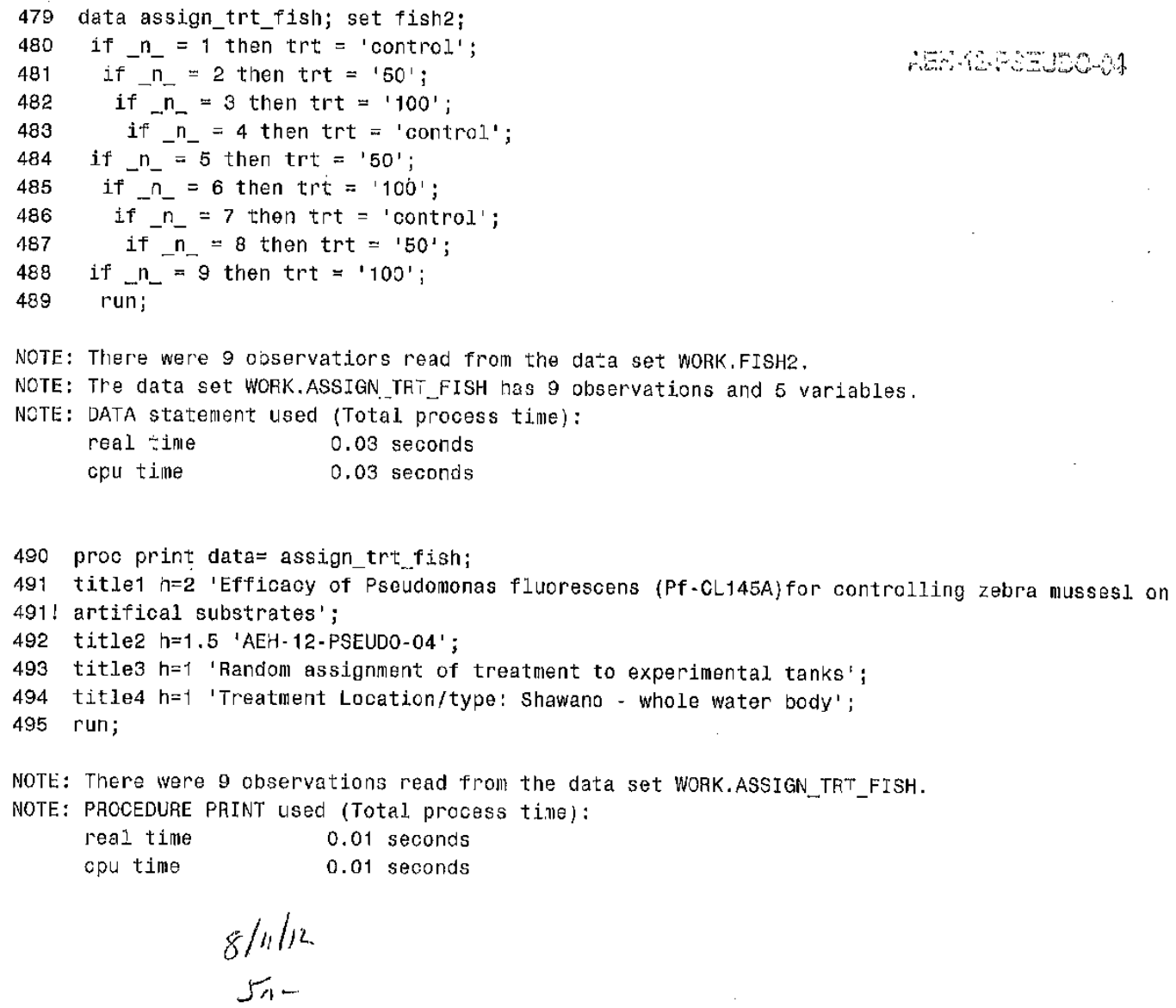

FF\# Ha

Item No.

$\mathrm{Pg} 4$ of 4 


\begin{tabular}{|c|c|c|c|c|c|c|c|c|}
\hline \multicolumn{8}{|c|}{$\begin{array}{l}\text { Random assignment of trays to test tank/position } \\
\text { Test Location/type = Shawano whole water column tank treatment }\end{array}$} & \multirow{2}{*}{$\begin{array}{l}\text { s/h/n } \\
5 \text { REH }\end{array}$} \\
\hline obs & round & row & position & tank & $x$ & _row_ & $\operatorname{tankn}$ & \\
\hline 1 & 1 & 1 & 1 & 2 & 0.00522 & A & $2 A 1$ & \multirow{36}{*}{$\begin{array}{c}\text { Distribution } \\
\text { began at } 1345 \\
\text { termikated at } 1500 \\
\text { 15W } \\
\text { SSEPT2012 }\end{array}$} \\
\hline 2 & 1 & 2 & 3 & 3 & 0.00709 & $\mathrm{~B}$ & 383 & \\
\hline 3 & 1 & 3 & 1 & 1 & 0.00785 & c & $1 \mathrm{C} 1$ & \\
\hline 4 & 1 & 3 & 2 & .3 & $0.0222 \theta$ & $c$ & $3 \mathrm{C} 2$ & \\
\hline 5 & 1 & 3 & 3 & 1 & 0.02239 & c & 103 & \\
\hline 6 & 1 & 1 & 3 & 7 & 0.02452 & A & $7 A 3$ & \\
\hline 7 & 1 & 2 & 3 & 4 & 0.02735 & B & $4 \mathrm{BB}$ & \\
\hline 8 & 1 & 1 & 1 & 4 & 0.02946 & A & $4 A 1$. & \\
\hline 9 & 1 & 2 & 3 & 5 & 0.05448 & B & $5 \mathrm{~B} 3$ & \\
\hline 10 & 1 & 1 & 2 & 8 & 0.06361 & A & $B A 2$ & \\
\hline 11 & 1 & 2 & 2 & 1 & 0.06658 & B & 182 & \\
\hline 12 & 1 & 2 & 1 & 8 & 0.06729 & B & 8B1. & \\
\hline 13 & 1 & 3 & 3 & 9 & 0.06782 & C & $9 c 3$ & \\
\hline 14 & 1 & 3 & 3 & 6 & 0.06930 & c & 663 & \\
\hline 15 & 1 & 1 & 2 & 7 & 0.07870 & A & $7 A 2$ & \\
\hline 16 & 1 & 2 & 2 & 3 & 0.08066 & B & $3 \mathrm{~B} 2$ & \\
\hline 17 & 1 & 1 & 3 & 3 & 0.08124 & A & $3 A B$ & \\
\hline 18 & 1 & 1 & 3 & 2 & 0.09097 & A & $2 \mathrm{A3}$ & \\
\hline 19 & 1 & 3 & 2 & - 8 & 0.09876 & c & $8 \mathrm{C} 2 \ldots$ & \\
\hline 20 & 1 & 2 & 2 & 7 & 0.11058 & B & $7 \mathrm{~B} 2$ & \\
\hline 21 & 1 & 3 & 3 & 5 & 0.11777 & c & $5 \mathrm{C3}$ & \\
\hline 22 & .1 & 3 & 1 & 9 & 0.12717 & c & 901 & \\
\hline 23 & 1 & 2 & 1 & 43 & 0.13584 & B & $3 B 1$. & \\
\hline 24 & 1 & 3 & 2 & 7 & 0.14704 & c & $7 \mathrm{C2}$ & \\
\hline 25 & 1 & 3 & 1 & 7 & 0.15021 & c & 701 & \\
\hline 26 & 1 & 2 & 3 & 2 & 0.15352 & B & $2 \mathrm{B3}$ & \\
\hline 27 & 1 & 1 & 3 & $=4$ & 0.16437 & A & $4 A 3$ & \\
\hline 28 & 1 & 1 & 2 & 3 & 0.19665 & A & $3 A 2$ & \\
\hline 29 & 1 & 2 & 3 & 9 & 0.21558 & B & $9 \mathrm{B3}$ & \\
\hline 30 & 1 & 3 & 2 & . 4 & 0.22984 & c & $4 \mathrm{C} 2$ & \\
\hline 31 & 1 & 2 & 1 & 7 & 0.26785 & B & $7 \mathrm{~B} 1$ & \\
\hline 32 & 1 & 2 & 2 & 5 & 0.27061 & B & $5 \mathrm{~B} 2$ & \\
\hline 33 & 1 & 3 & 1 & $\therefore 2$ & 0.27844 & $c$ & $2 \mathrm{Cl}^{\circ}$ & \\
\hline 34 & 1 & 3 & 3 & 4. & 0.29757 & c & $4 \mathrm{CB}$ & \\
\hline 35 & 1 & 2 & 2 & 4 & 0.32852 & B & $4 \mathrm{~B} 2$ & \\
\hline 36 & 1 & 1 & 2 & 5 & 0.33047 & A & $5 A 2$ & \\
\hline 37 & 1 & 3 & 3 & 2 & 0.36000 & c & $2 \mathrm{C3}$ & \multirow{2}{*}{ File Fotder: $\|_{a}$} \\
\hline 38 & 1 & 1 & 2 & 2 & 0.36047 & A & $2 A 2$ & \\
\hline 39 & 1 & 1 & 3 & 1 & 0.38105 & A & $1 \mathrm{AB}$ & \multirow{3}{*}{$* \quad-$} \\
\hline 40 & 1 & 2 & 2 & 6 & 0.39888 & B & $6 \mathrm{~B} 2$ & \\
\hline 41 & 1 & 1 & 2 & 1 & 0.40441 & A & $1 \mathrm{~A} 2$ & \\
\hline 42 & 1 & 2 & 1 & 6 & 0.42695 & B & $6 \mathrm{~B} 1$ & \multirow{2}{*}{ Item Number: 2} \\
\hline 43 & 1 & 3 & 1 & 8 & 0.43967 & c & $8 \mathrm{Cl}$ & \\
\hline 44 & 1 & 1 & 2 & 6 & 0.44954 & A & $6 \mathrm{~A} 2$ & \\
\hline 45 & 1 & 3 & 2 & 6 & 0.47464 & $c$ & $6 \mathrm{C} 2$ & \\
\hline 46 & 19 & 1 & 969 & 8 & 0.48023 & A & $8 \mathrm{~A} 1$ & \\
\hline 47 & 1 & 1 & 1 & 7 & 0.48398 & A & $7 A 1$ & Page 1 of 8 \\
\hline
\end{tabular}



Efficacy of Psuedomonas fIuorescens (Pf-CL145A)SDP for controliing zebra mussels on artifical subs 2
AEH-12-PSUED0-04

Random assignment of trays to test tank/position

Test Location/type = Shawano whole water column tank treatment

\begin{tabular}{|c|c|c|c|c|c|c|c|c|c|c|}
\hline $\mathrm{Ob}_{5}$ & round & row & position & & tank & $x$ & _row_ & tankn & & 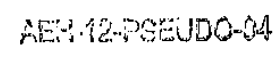 \\
\hline 48 & 1 & 2 & 3 & & 8 & 0.48900 & B & 8BO & & \\
\hline 49 & 1 & 2 & 2 & & 2 & $0.505+1$ & $B$ & $2 \mathrm{B2}$ & & \\
\hline 50 & 1 & 2 & 2 & & 8 & 0.52181 & $\mathrm{~B}$ & $8 \mathrm{~B} 2$ & & \\
\hline 51 & 1 & 1 & 3 & & 9 & 0.52488 & A & $9 A 3$ & & \\
\hline 52 & 1 & 2 & 3 & . & $\cdots 6$ & 0.53810 & $B$ & $6 \mathrm{~B} 3$ & & \\
\hline 53 & 1 & 2 & 2 & & 9 & 0.54061 & $\mathrm{~B}$ & $\mathrm{BB} 2$ & & \\
\hline 54 & 1 & 2 & 1 & & 4 & 0.56338 & B & $4 \mathrm{~B} 1$ & & \\
\hline 55 & 1 & 3 & 3 & & 7 & 0.60611 & $\mathrm{C}$ & 703 & & \\
\hline 56 & 1 & 3 & 1 & & 3 & 0.60927 & C & 301 & & \\
\hline 57 & 1 & 1 & 2 & & 4 & 0.62026 & A & $4 \mathrm{A2}$ & & \\
\hline 58 & 1 & 1 & 3 & & 5 & 0.63012 & A & $5 \mathbf{A B}$ & & \\
\hline 59 & 1 & 1 & 1 & & 6 & 0.65625 & A & $6 \mathrm{A1}$ & & \\
\hline 60 & 1 & 3 & 2 & $\therefore$ & 9 & 0.66940 & c & 902 & & \\
\hline 61 & 1 & 3 & 1 & $\because$ & 4 & 0.68710 & $\mathrm{c}$ & $4 C 1$ & & \\
\hline 62 & 1 & 3 & 1 & $:$ & 5 & 0.69054 & $\mathrm{c}$ & $6 \mathrm{Cl}$ & & \\
\hline 63 & 1 & 1 & 3 & . & $\cdot 6$ & 0.71055 & A & $6 A 3$ & $\sigma^{w}$ & $\because$ \\
\hline 64 & 1 & 3 & 2 & $\therefore$ & 1 & 0.73661 & C & $1 \mathrm{C2}$ & & \\
\hline 65 & 1 & 2 & 3 & $\therefore$ & 1 & 0.75311 & $B$ & IB3: & & \\
\hline 66 & 1 & 3 & 1 & 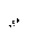 & 6 & 0.76155 & $c$ & $6 \mathrm{C} 1$ & & \\
\hline 67 & 1 & 1 & 3 & & 8 & 0.76411 & A & $B A 3$ & & \\
\hline 68 & 1 & 2 & 1 & & 5 & 0.76527 & $\mathrm{E}$ & $5 \mathrm{~B} 1$ & & \\
\hline 69 & 1 & 2 & 1 & & 2 & 0.76674 & B & $2 \mathrm{~B} 1$ & $\cdots$ & \\
\hline 70 & 1 & 3 & 3 & & 8 & 0.77423 & $\mathrm{C}$ & $8 \mathrm{C3}$ & & $\therefore$ \\
\hline 71 & 1 & 3 & 3 & & 3 & 0.79781 & $c$ & $\mathrm{BC3}$ & & \\
\hline 72 & 1 & 2 & 3 & $\cdot$ & 7 & 0.81154 & B & $\mathrm{t}_{\mathrm{B}}$ & & . \\
\hline 73 & 1 & 2 & 1 & $\therefore$ & 9 & 0.82249 & B & 981 & $" s$ & \\
\hline 74 & 1 & 1 & 2 & & 9 & 0.83782 & A & $9 A 2$ & $i$ & \\
\hline 75 & 1 & 1 & 1 & " & 3 & 0.85976 & A & $3 \mathbf{A 1}_{3}$ & & \\
\hline 76 & 1 & 3 & 2 & & 5 & 0.86941 & C & $5 \mathrm{C} 2$ & & \\
\hline 77 & 1 & 1 & 1 & & 5 & 0.89111 & A & $5 A 1$ & & \\
\hline 78 & 1 & 2 & 1 & & 1 & 0.89793 & B & 1B1 & & \\
\hline 79 & 1 & 1 & 1 & $s$ & 9 & 0.90048 & A & $9 A 1$ & & \\
\hline 80 & 1 & 1 & 1 & 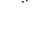 & 1 & 0.91950 & A & 1A1 & & $\therefore$ \\
\hline 81 & 1 & 3 & 2 & $s$ & 2 & 0.98447 & C & $2 C 2$ & & \\
\hline
\end{tabular}

ireblom gll

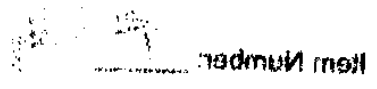

to.

$\operatorname{ogm} 9$

Page 2 of 8

inalysis performed by $J$. Luoma sAs version $9.2 \quad 10: 48$ 11AUa12 


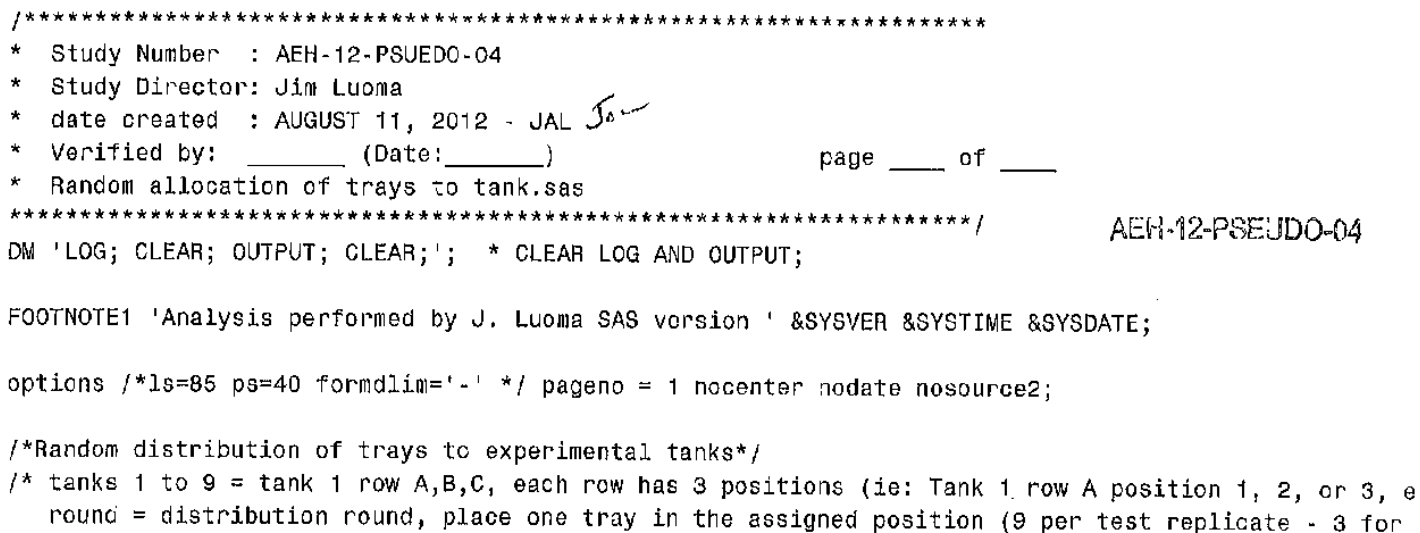




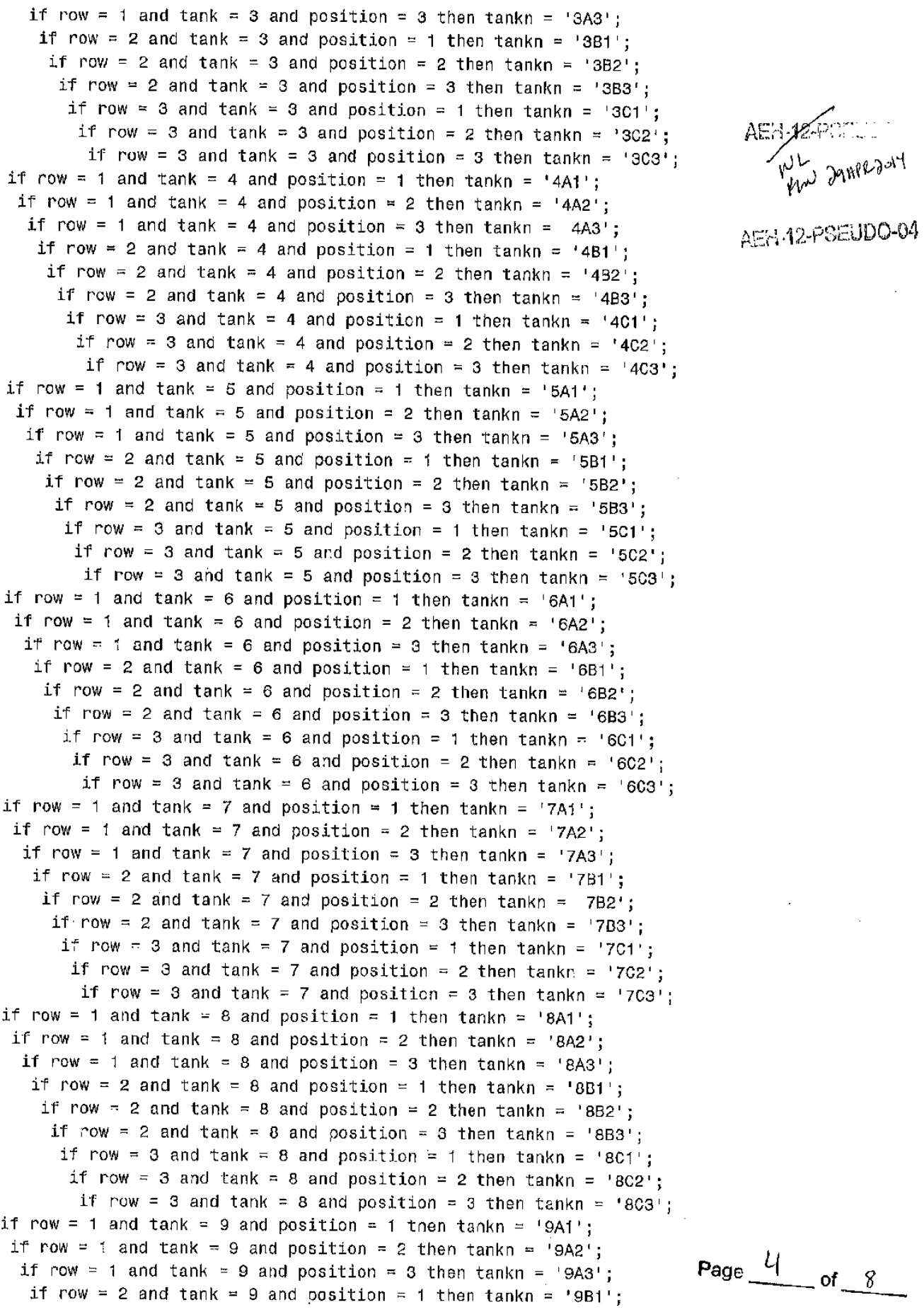




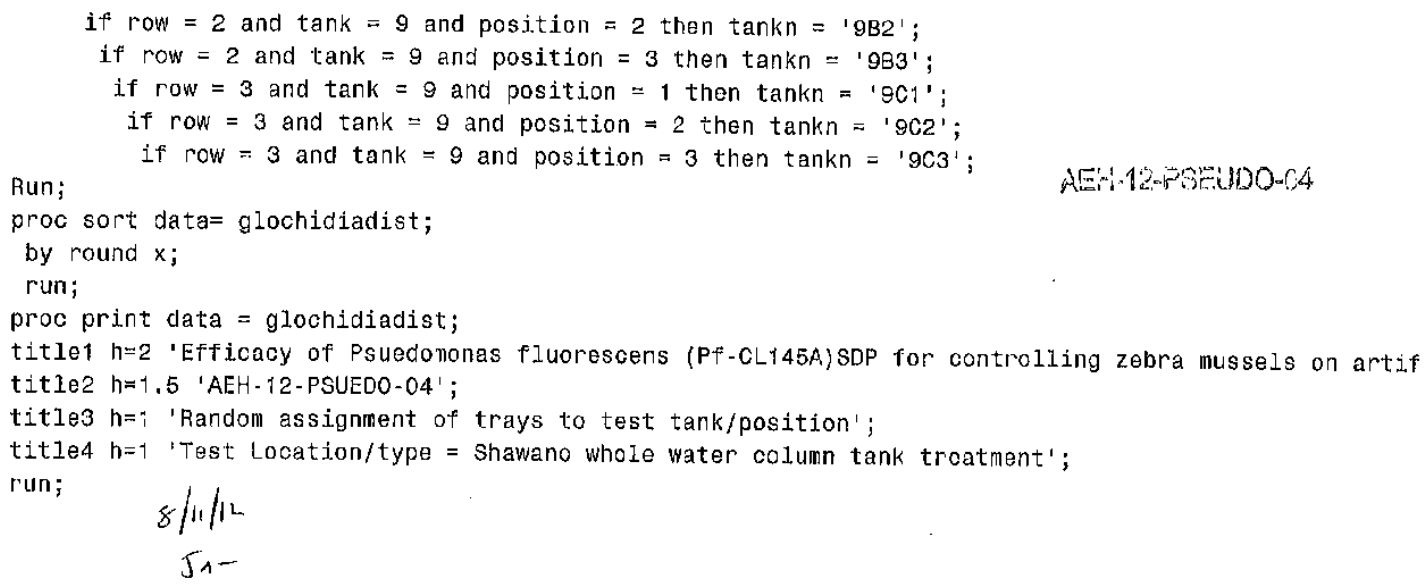




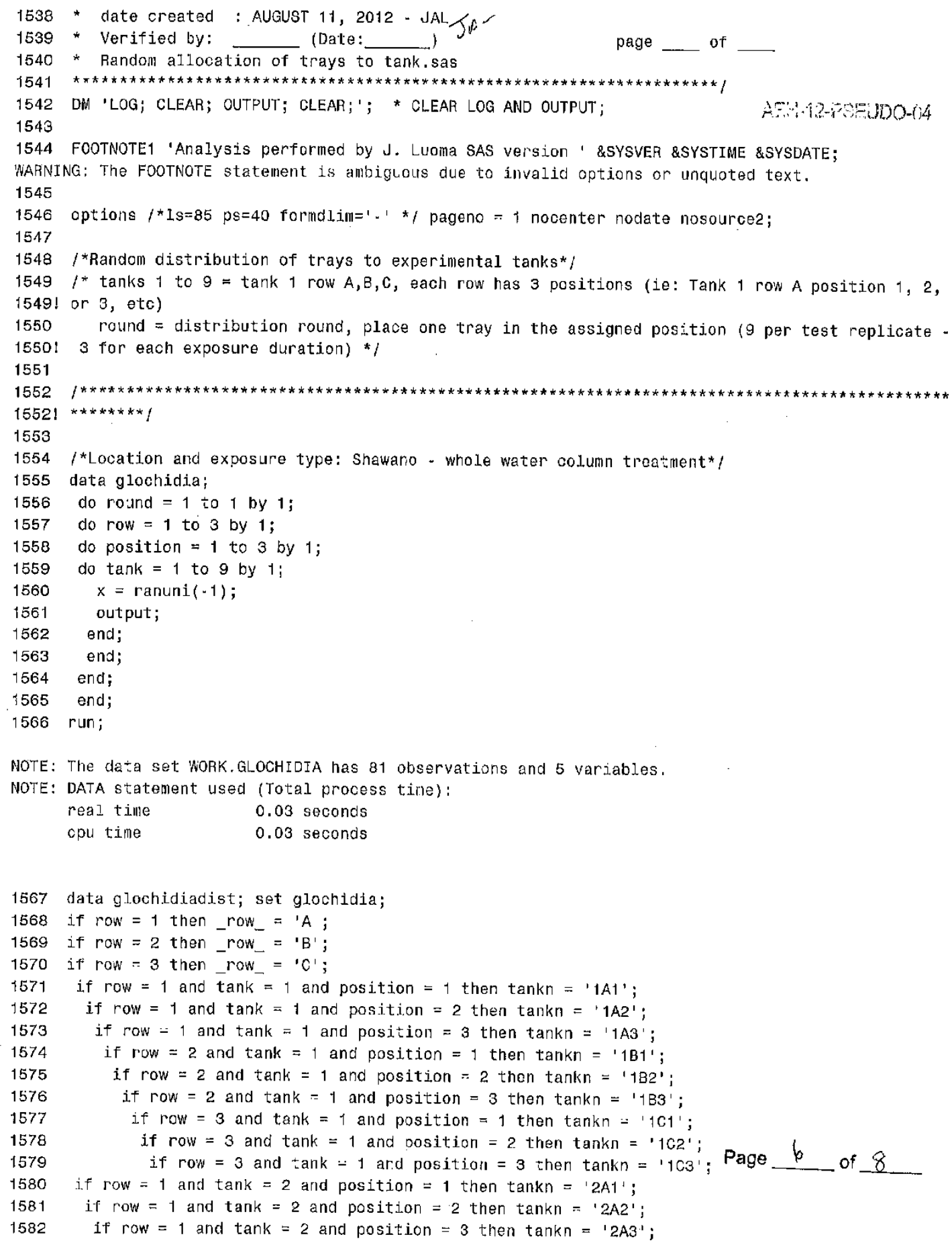




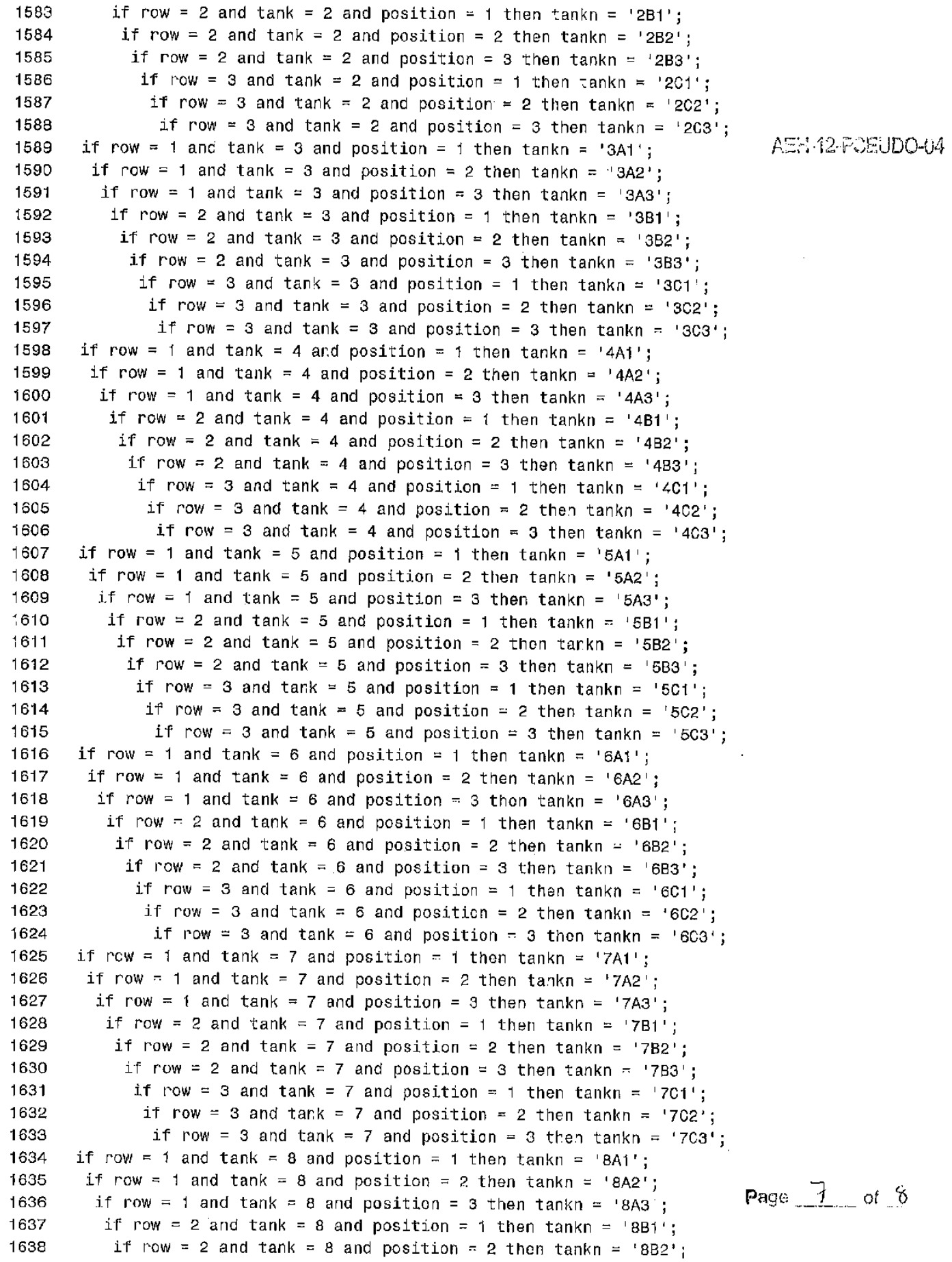




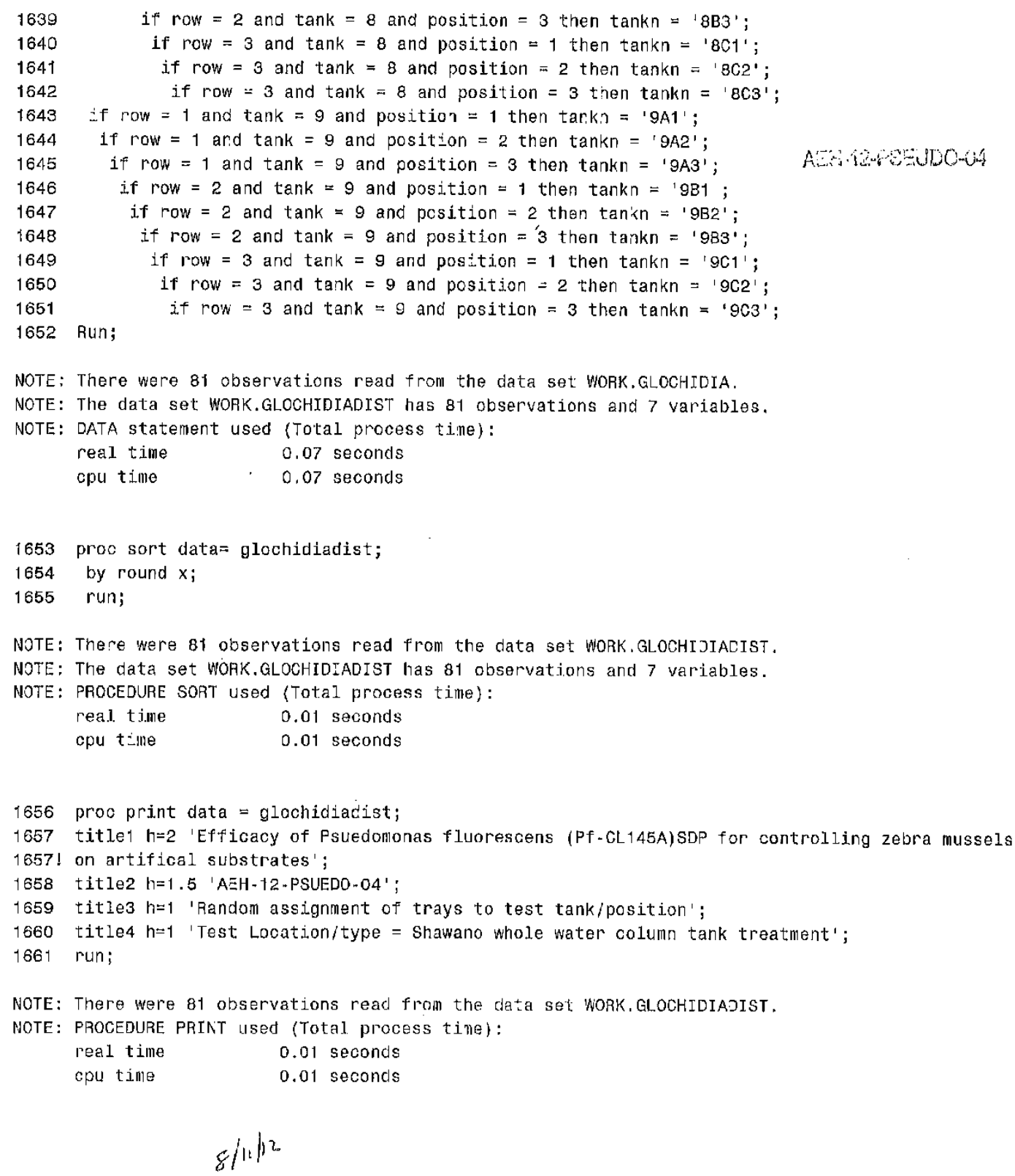

FF \# lla

Item No.

$\mathrm{Pg}$ /6 of 8 
Efficacy of Pseudomonas fluorescens (Pf-CL145A)for controlling zebra mussesl on artifical substrates AEH - 12-PSEUDO-04

Random assignment of substrate removal from tanks *** TANK $1 * * *$
Shawano - whole water body Treatment

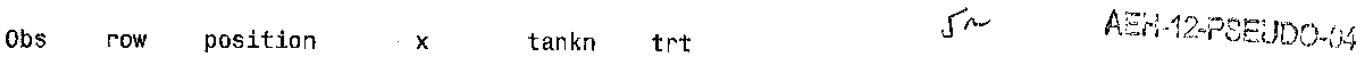

$\begin{array}{llllll}1 & 1 & 1 & 0.03194 & 1 \mathrm{~A} 1 & 6 \mathrm{~h} \\ 2 & 2 & 1 & 0.03514 & 1 \mathrm{~B} 1 & 6 \mathrm{~h} \\ 3 & 2 & 2 & 0.28180 & 1 \mathrm{~B} 2 & 6 \mathrm{~h} \\ 4 & 3 & 1 & 0.49047 & 1 \mathrm{C} 1 & 9 \mathrm{~h} \\ 5 & 1 & 3 & 0.70588 & 1 \mathrm{A3} & 9 \mathrm{~h} \\ 6 & 2 & 3 & 0.72184 & 1 \mathrm{~B} 3 & 9 \mathrm{~h} \\ 7 & 3 & 3 & 0.75338 & 1 \mathrm{C3} & 12 \\ 8 & 3 & 2 & 0.80186 & 1 \mathrm{C} 2 & 12 \\ 9 & 1 & 2 & 0.95124 & 1 \mathrm{~A} 2 & 12\end{array}$

10 ............

File Folter: Ha Hem Number: 3 Page 1 of 30 
Analysis performed by $\checkmark$. Luoma SAS version 9.2 10:20 13 AUG12

Efficacy of Pseudomonas fluorescens (Pf-CL145A) for controlling zebra mussesl on artifical substrates AEH-12-PSEUDO-04

Random assignment of substrate removal from tanks *** TANK $2 * * *$

Shawano - whole water body Treatment

$\begin{array}{cccccc}\text { Obs } & \text { row } & \text { position } & x & \text { tankn } & \text { trt } \\ 1 & 1 & 1 & 0.19283 & 2 \mathrm{~A} 1 & 6 \mathrm{~h} \\ 2 & 2 & 1 & 0.22382 & 2 \mathrm{~B} 1 & 6 \mathrm{~h} \\ 3 & 3 & 1 & 0.24148 & 2 \mathrm{C} 1 & 6 \mathrm{~h} \\ 4 & 2 & 2 & 0.28394 & 2 \mathrm{~B} 2 & 9 \mathrm{~h} \\ 5 & 1 & 3 & 0.29763 & 2 \mathrm{~A} 3 & 9 \mathrm{~h} \\ 6 & 3 & 3 & 0.62146 & 2 \mathrm{C} 3 & 9 \mathrm{~h} \\ 7 & 3 & 2 & 0.71062 & 2 \mathrm{C} 2 & 12 \\ 8 & 1 & 2 & 0.79088 & 2 \mathrm{~A} 2 & 12 \\ 9 & 2 & 3 & 0.99840 & 2 \mathrm{~B} 3 & 12\end{array}$

AEHTLPSEUDOMA

Page 2 of 30

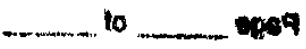


Analysis performed by $J$. Luoma SAS version 0.2 10:20 13AUG12

Efficacy of Pseudomonas fluorescens (Pf-CL145A)for control.1ing zebra mussesl on artifical substrates AEH-12-PSEUDO- 04

Randon assignnent of substrate removal from tanks *** TANK $3 * * *$

Shawano - whole water body Treatment

$\begin{array}{cccccc}\text { Obs } & \text { row } & \text { position } & \mathrm{x} & \text { tankn } & \text { trt } \\ 1 & 2 & 3 & 0.25496 & 3 \mathrm{~B} 3 & 6 \mathrm{~h} \\ 2 & 2 & 2 & 0.28609 & 3 \mathrm{~B} 2 & 6 \mathrm{~h} \\ 3 & 1 & 1 & 0.35372 & 3 \mathrm{~A} 1 & 6 \mathrm{~h} \\ 4 & 2 & 1 & 0.41249 & 3 \mathrm{~B} 1 & 9 \mathrm{~h} \\ 5 & 3 & 3 & 0.48955 & 3 \mathrm{C} 3 & 9 \mathrm{~h} \\ 6 & 3 & 2 & 0.61938 & 3 \mathrm{C} 2 & 9 \mathrm{~h} \\ 7 & 1 & 2 & 0.63052 & 3 \mathrm{~A} 2 & 12 \\ 8 & 1 & 3 & 0.88938 & 3 \mathrm{A3} & 12 \\ 9 & 3 & 1 & 0.99249 & 3 \mathrm{C} 1 & 12\end{array}$

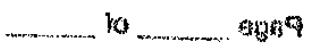

Page 3 of 30 
AnaIysis performed by J. Luoma SAS version 9.2 10:20 13AUG12

Efficacy of Pseudomonas fluorescens (Pf-CL145A) for controlling zebra mussesl on artifical substrates AEH-12-PSEUDO-04

Random assignment of substrate removal from tanks *** TANK $4 * * *$

Shawano - whole water body Treatment

$\begin{array}{cccccc}\text { Obs } & \text { row } & \text { position } & \mathrm{x} & \text { tankn } & \text { trt } \\ 1 & 1 & 1 & 0.23640 & 4 \mathrm{A1} & 6 \mathrm{~h} \\ 2 & 2 & 1 & 0.26349 & 4 \mathrm{~B} 1 & 6 \mathrm{~h} \\ 3 & 1 & 2 & 0.38924 & 4 \mathrm{A2} & 6 \mathrm{~h} \\ 4 & 1 & 3 & 0.56305 & 4 \mathrm{A3} & 9 \mathrm{~h} \\ 5 & 2 & 2 & 0.56965 & 4 \mathrm{~B} 2 & 9 \mathrm{~h} \\ 6 & 3 & 1 & 0.70154 & 4 \mathrm{Cl} & 8 \mathrm{~h} \\ 7 & 3 & 2 & 0.70889 & 4 \mathrm{C} 2 & 12 \\ 8 & 2 & 3 & 0.76343 & 4 \mathrm{~B} 3 & 12 \\ 9 & 3 & 3 & 0.83244 & 4 \mathrm{C3} & 12\end{array}$

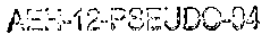

to

aper

Page 4 of 30 
Analysis performed by J. Luoma SAS version 9.2 10:20 13AUG12

Efficacy of Pseudomonas fIuorescens (Pf-CL145A)for controling zebra mussesl on artifical substrates AEH-12-PSEUDO-04

Random assignment of substrate removal from tanks $* * *$ TANK $5 * * *$

Shawano - whole water body Treatment

A $=\cdots$ -

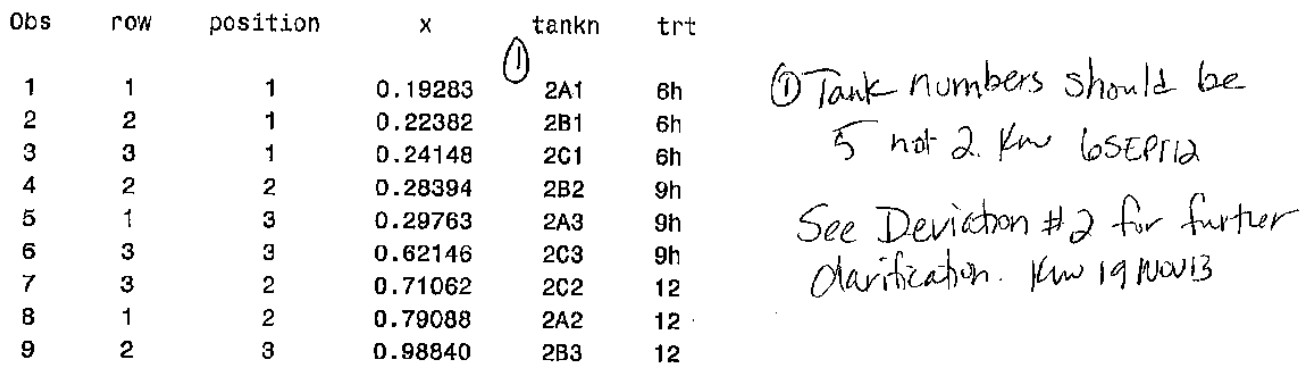

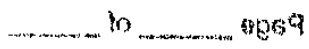

Page 5 of 30 
Efficacy of Pseudomonas fluorescens (Pf-Cl-145A)for controlling zebra musses] on artifical substrates AEH-12-PSEUDO-04

Random assignment of substrate removal. from tanks *** TANK $6 * * *$

Shawano - whole water body Treatment

$\begin{array}{cccccc}\text { Obs } & \text { row } & \text { position } & x & \text { tankn } & \text { trt } \\ 1 & 1 & 2 & 0.14871 & 6 \mathrm{~A} 2 & 6 \mathrm{~h} \\ 2 & 1 & 3 & 0.45067 & 6 \mathrm{A3} & 6 \mathrm{~h} \\ 3 & 1 & 1 & 0.47774 & 6 \mathrm{~A} 1 & 6 \mathrm{~h} \\ 4 & 2 & 1 & 0.54650 & 6 \mathrm{~B} 1 & 9 \mathrm{~h} \\ 5 & 3 & 2 & 0.57203 & 6 \mathrm{C} 2 & 9 \mathrm{~h} \\ 6 & 2 & 2 & 0.57288 & 6 \mathrm{~B} 2 & 9 \mathrm{~h} \\ 7 & 3 & 3 & 0.63457 & 6 \mathrm{C} 3 & 12 \\ 8 & 2 & 3 & 0.66328 & 6 \mathrm{~B} 3 & 12 \\ 9 & 3 & 1 & 0.82805 & 6 \mathrm{C} 1 & 12\end{array}$


Analysis performed by $J$. Luoma SAS version $9.2 \quad 10: 2013 A U G 12$

Efficacy of Pseudomonas fluorescens (Pf-CL145A) for controlling zebra mussesi on artifical substrates AEH - 12-PSEUDO-04

Fiandom assignment of substrate removal from tanks $* * *$ TANK $7 * * *$

Shawano - whole water body Treatment

\begin{tabular}{|c|c|c|c|c|c|c|}
\hline obs & row & position & $x$ & $\operatorname{tankn}$ & trt & \\
\hline 1 & 1 & 1 & 0.19283 & (1) $2 A 1$ & $6 \mathrm{~h}$ & \\
\hline 2 & 2 & 1 & 0.22382 & $2 \mathrm{~B} 1$ & $6 \mathrm{~h}$ & (1) lank numbers should be \\
\hline 3 & 3 & 1 & 0.24148 & $2 \mathrm{C}_{1}$ & $6 h$ & $1 \mathrm{CEP} / \mathrm{C}$ \\
\hline 4 & 2 & 2 & 0.28394 & 2B2 & $9 \mathrm{~h}$ & Row GSETI \\
\hline 5 & 1 & 3 & 0.29763 & $2 A 3$ & $9 \mathrm{~h}$ & \\
\hline 6 & 3 & 3 & 0.62146 & $2 \mathrm{C3}$ & 9h & \\
\hline 7 & $\mathbf{3}$ & 2 & 0.71062 & $2 \mathrm{C} 2$ & 12 & $\mathrm{orm}^{3}$ \\
\hline 8 & 1 & 2 & 0.79088 & $2 A 2$ & 12 & janovis \\
\hline 9 & 2 & 3 & 0.98840 & $2 \mathrm{~B} 3$ & 12 & \\
\hline
\end{tabular}

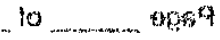

Page 7 of 30 
Analysis performed by $J$. Luona SAS version $9.2 \quad 10: 2013$ AUG12

AES-12-PSEUDO-04

Efficacy of Pseudomonas fluorescens (Pf-CL145A)for controling zebra mussesl on artifical substrates AEH-12-PSEUDO-04

Random assignment of substrate removal fromi tanks $* * *$ TANK $8 * * *$

Shawano - whole water body Treatment

$\begin{array}{cccccc}\text { Obs } & \text { row } & \text { position } & x & \text { tankn } & \text { trt } \\ 1 & 2 & 3 & 0.12552 & 8 \mathrm{BB} & 6 \mathrm{~h} \\ 2 & 1 & 3 & 0.14940 & 8 \mathrm{A3} & 6 \mathrm{~h} \\ 3 & 3 & 1 & 0.28780 & 8 \mathrm{C} 1 & 6 \mathrm{~h} \\ 4 & 1 & 1 & 0.31796 & 8 \mathrm{~A} 1 & 9 \mathrm{~h} \\ 5 & 3 & 2 & 0.36760 & 8 \mathrm{C} 2 & 9 \mathrm{~h} \\ 6 & 2 & 1 & 0.45120 & 8 \mathrm{~B} 1 & 9 \mathrm{~h} \\ 7 & 1 & 2 & 0.48872 & 8 \mathrm{~A} 2 & 12 \\ 8 & 3 & 3 & 0.73228 & 8 \mathrm{CB} & 12 \\ 9 & 2 & 2 & 0.93485 & 8 \mathrm{~B} 2 & 12\end{array}$

$10 . . .909$

Page 8 of 30 
Analysis performed by J. Luoma SAS version 9.2 10:20 13AUG12 Jy-

Efficacy of Pseudomonas fluorescens (Pf-GL145A) for controlling zebra nussesl on artifical substrates AEH-12-PSEUDO- 04

Random assignment of substrate removal from tanks *** TANK $9 * * *$

Shawano - whole water body Treatment

\begin{tabular}{|c|c|c|c|c|c|c|}
\hline obs & row & position & $x$ & tankn & trt & \\
\hline 1 & 1 & 1 & 0.19283 & (1) $2 A_{1}$ & $6 \mathrm{~h}$ & 11 \\
\hline 2 & 2 & 1 & 0.22382 & $U_{2 \mathrm{~B} 1}$ & $6 h$ & (1) lank numbers should be \\
\hline 3 & 3 & 1 & 0.24148 & $2 \mathrm{C}_{1}$ & $6 \mathrm{~h}$ & 9 t 2 (w 6 SePT 1$)$ \\
\hline 4 & 2 & 2 & 0.28394 & 282 & $9 n$ & iot $\alpha$. Wh $6>0+1 / 2$ \\
\hline 5 & 1 & 3 & 0.29763 & $2 A 3$ & $9 \mathrm{~h}$ & \\
\hline 6 & 3 & 3 & 0.62146 & $2 \mathrm{C3}$ & $9 \mathrm{~h}$ & See \\
\hline 7 & 3 & 2 & 0.71062 & $2 \mathrm{C2}$ & 12 & 1 \\
\hline 8 & 1 & 2 & 0.79088 & $2 \mathrm{~A} 2$ & 12 & er clartic \\
\hline 9 & 2 & 3 & 0.98840 & $2 \mathrm{~B} 3$ & 12 & \\
\hline
\end{tabular}




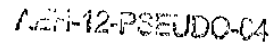

Analysis performed by $J$. Luoma SAS version 9.2 10:20 13 AUG12

Page 10 of 30 


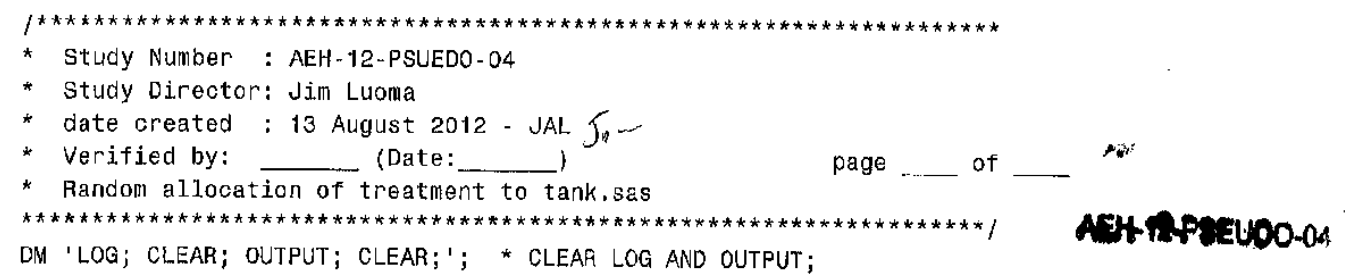

FOOTNOTE1 'Analysis performed by J. Luoma SAS version' \&SYSVER \&SYSTIME \&SYSDATE;

options $1 \mathrm{~s}=105 \mathrm{ps}=54$ formdlim=' - ' pageno = 1 nocenter nodate nosource2;

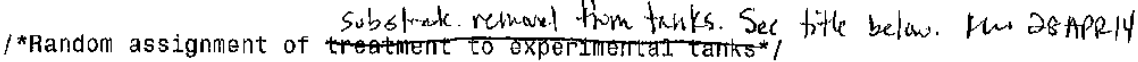

I*Location/exposure type: Shawano - whole tank treatment*I

data TANK1;

do row $=1$ to 3 by 1 ;

do position $=1$ to 3 by 1 ;

$x=\operatorname{ranur} i(-1)$;

output;

end;

end;

run;

data TANK1A; set TANK1;

if row $=1$ and position $=1$ then tankn $=$ ' $1 \mathrm{~A} 1$ ';

if row $=1$ and position $=2$ then tankn $={ }^{\prime} 1{ }^{\prime} 2^{\prime}$;

if row $=1$ and position $=3$ then tankn $=11 \mathrm{~A} 3^{\prime}$;

if row $=2$ and position $=1$ then tankn $=11 \mathrm{~B} 1$ ';

if row $=2$ and position $=2$ then tankn $={ }^{\prime} 1 \mathrm{~B} 2{ }^{\prime}$;

if row $=2$ and position $=3$ then tankn $=' 1 \mathrm{~B} 3{ }^{\prime}$;

if row $=3$ and position $=1$ then tankn $=11 \mathrm{ct}$ ';

if row $=3$ and position $=2$ then tankn $=1 \mathrm{C2}$ '; run;

if row $=3$ and position $=3$ then $\operatorname{tankn}=1103$ ';

proc sort data=TANK1A;

by $\mathrm{x}$;

ru?;

data assign_trt_TANKTA; set TANK1A;

if ${ }^{n}$ - $=1$ then $\operatorname{trt}=$ ' $6 \mathrm{~h}$ ';

if $n_{.}=2$ then $\operatorname{trt}={ }^{\prime} 6 h^{\prime}$;

if $n_{-}=3$ then trt $=$ ' $6 \mathrm{~h}^{\prime}$;

if $n_{-}=4$ then trt $=$ ' $9 h^{\prime}$;

if $n^{n}=5$ then trt $=$ ' $9 \mathrm{~h}$ ';

if $n_{-}=6$ then $\operatorname{trt}={ }^{\prime} 9 h^{\prime}$;

if $n=7$ then trt $=112 \mathrm{~h}$ '

if $\vec{n}_{-}=8$ then $\operatorname{trt}=112 \mathrm{n}^{\prime}$;

if $n_{-}=9$ then trt $=12 \mathrm{~h}$ ';

Page 11 of 30

run;

proc print data $=$ assign_trt_TANK1A;

ti:let $h=2$ 'Efficacy of Pseudononas fluorescens (Pf-CL.145A)for controlling zebra mussesl on artifical title2 $h=1.5$ 'AEH-12-PSEUDO-04';

title $3 h=1$ ' Zandom assignment of substrate removal from tanks $* * *$ TANK $1 * * *$ ';

title4 $h=1$ 'Shawano - whole water body Treatment ';

run; 


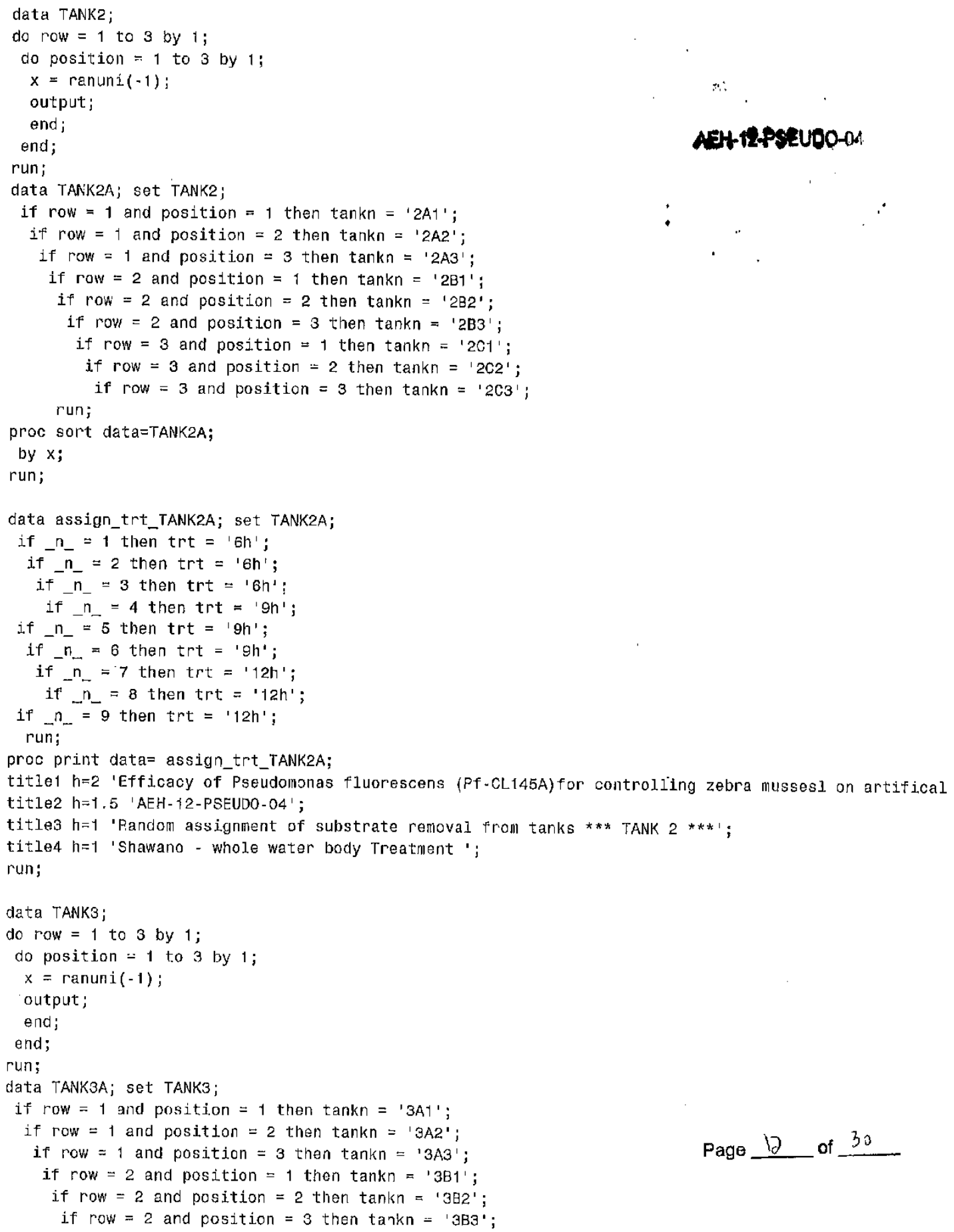




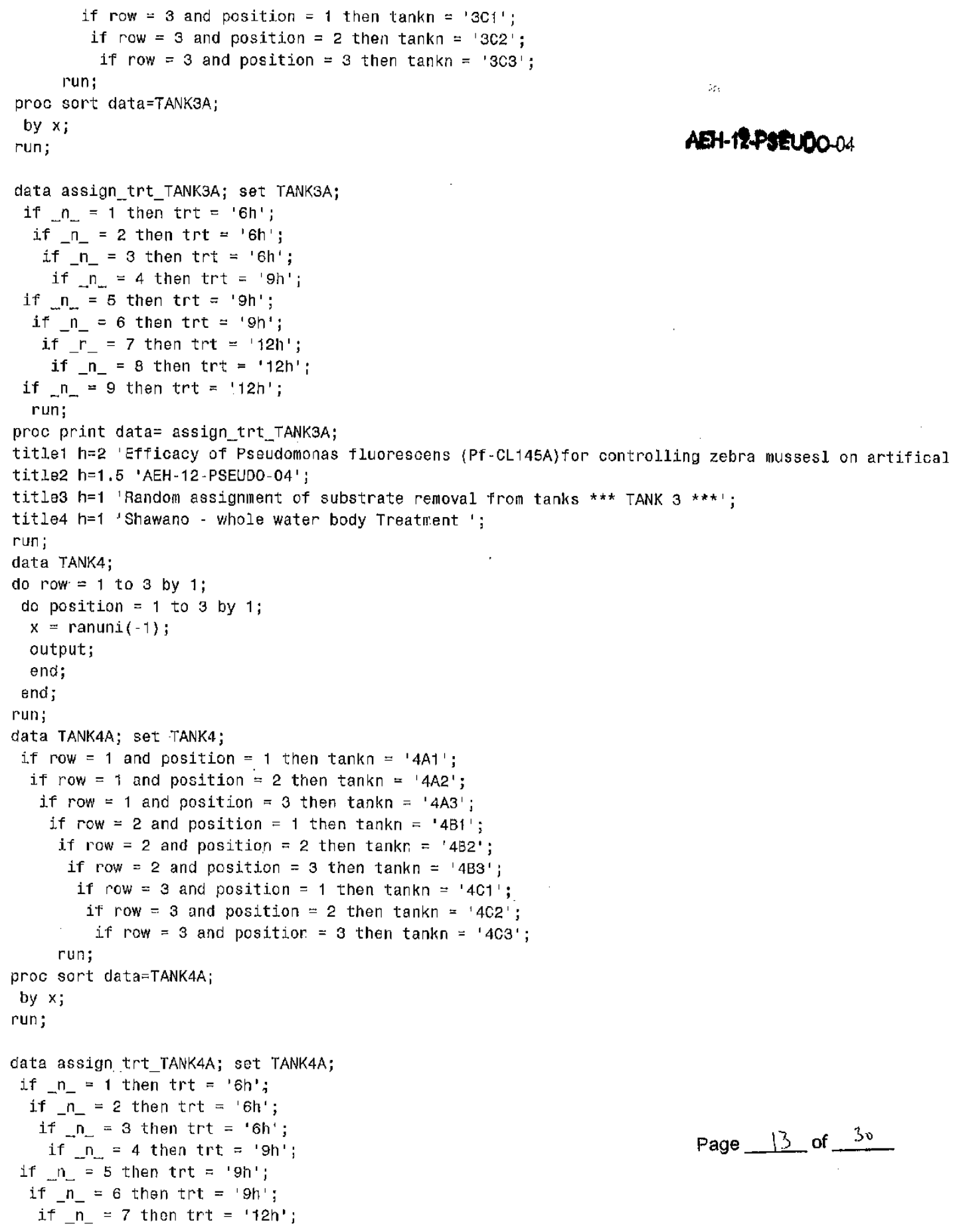




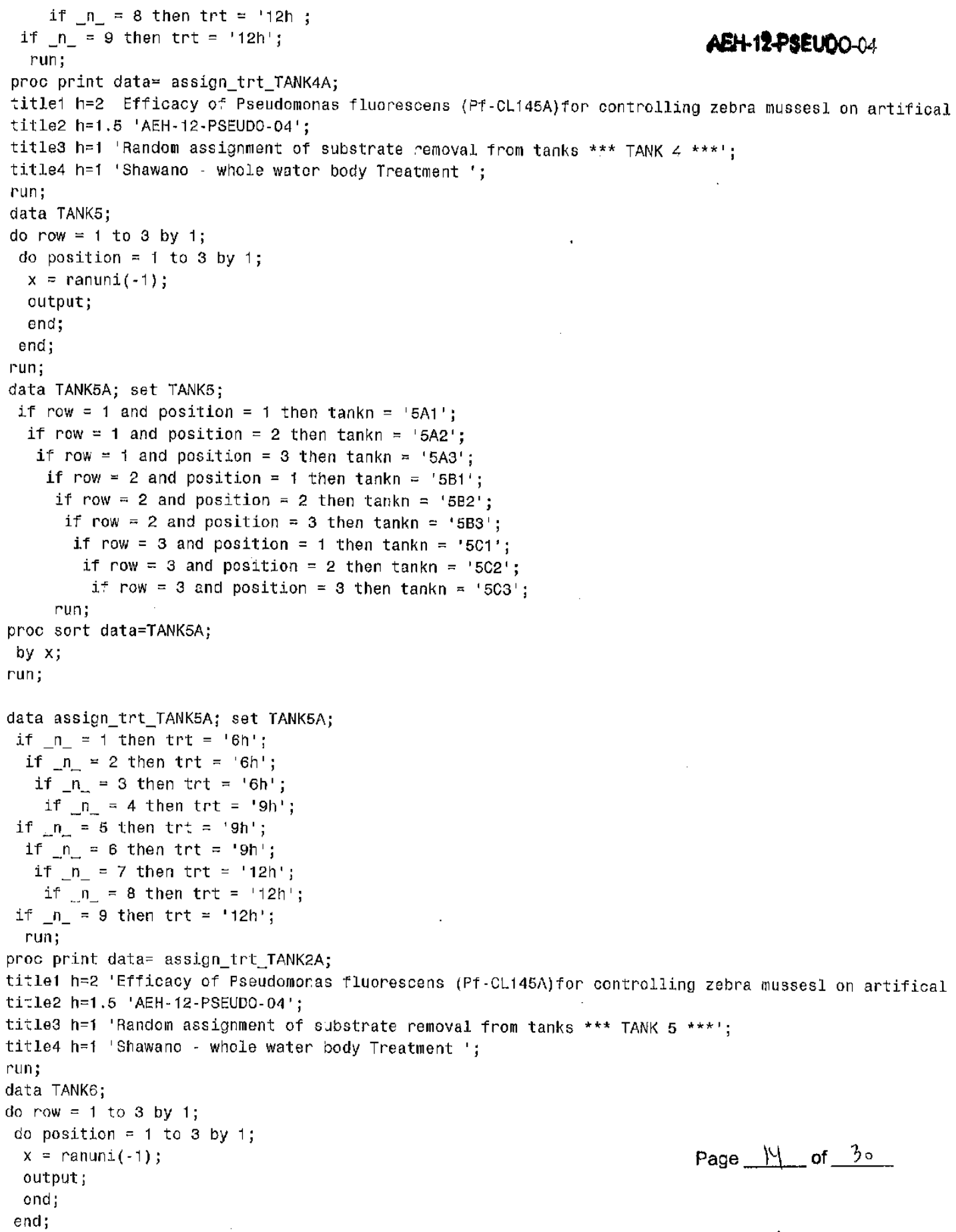




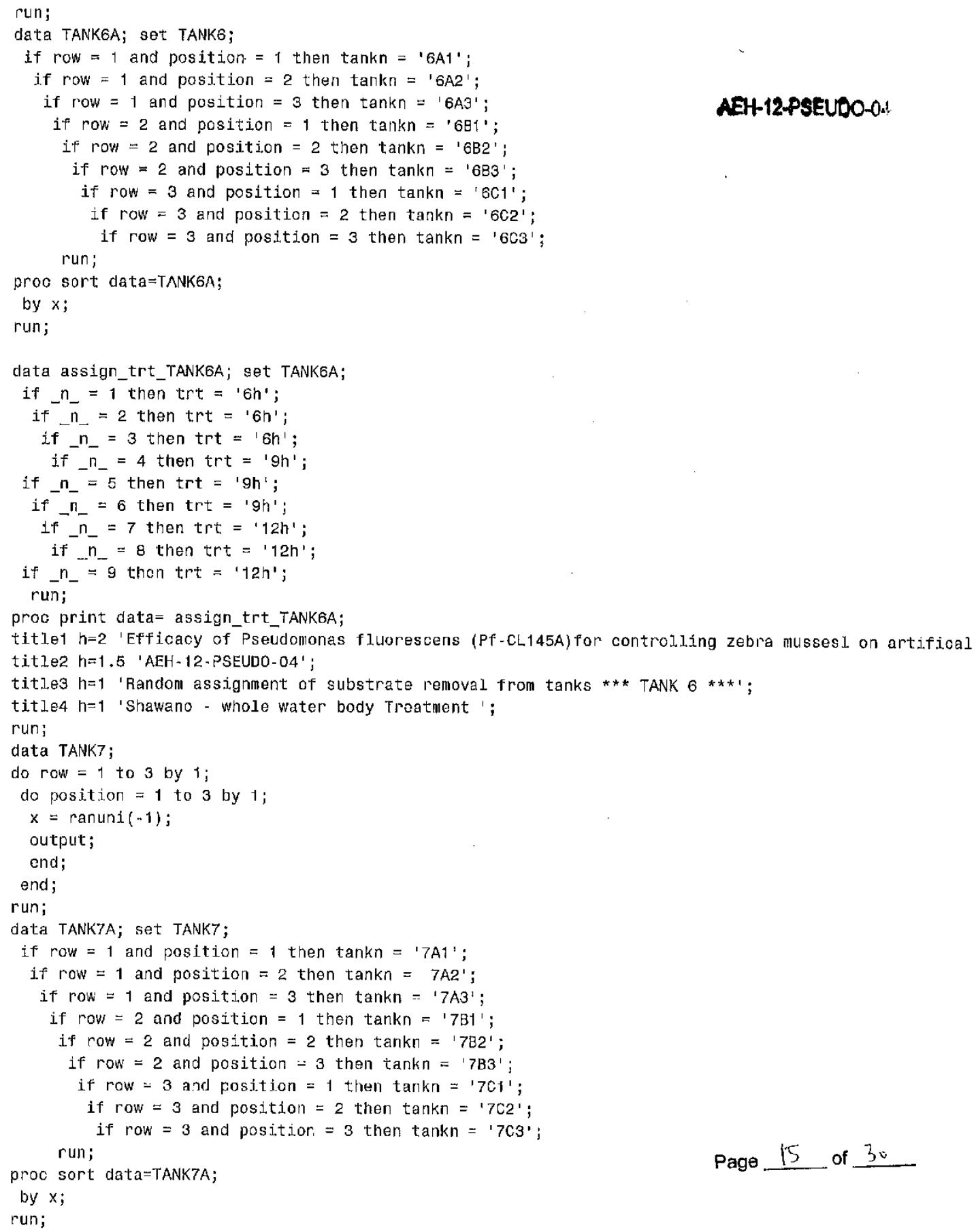




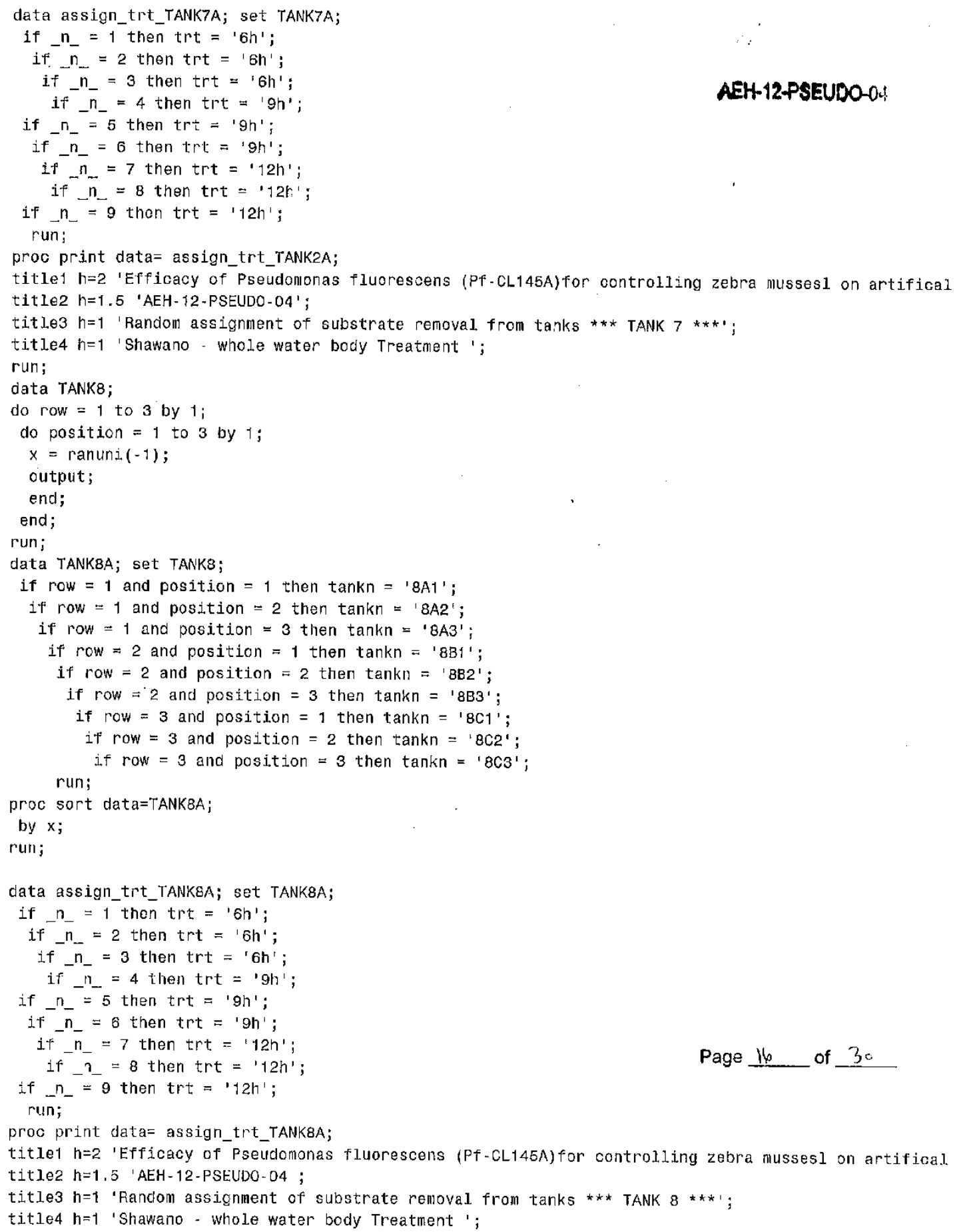

\section{AEH-12PSEUDOOA}

Page 16 of 30 


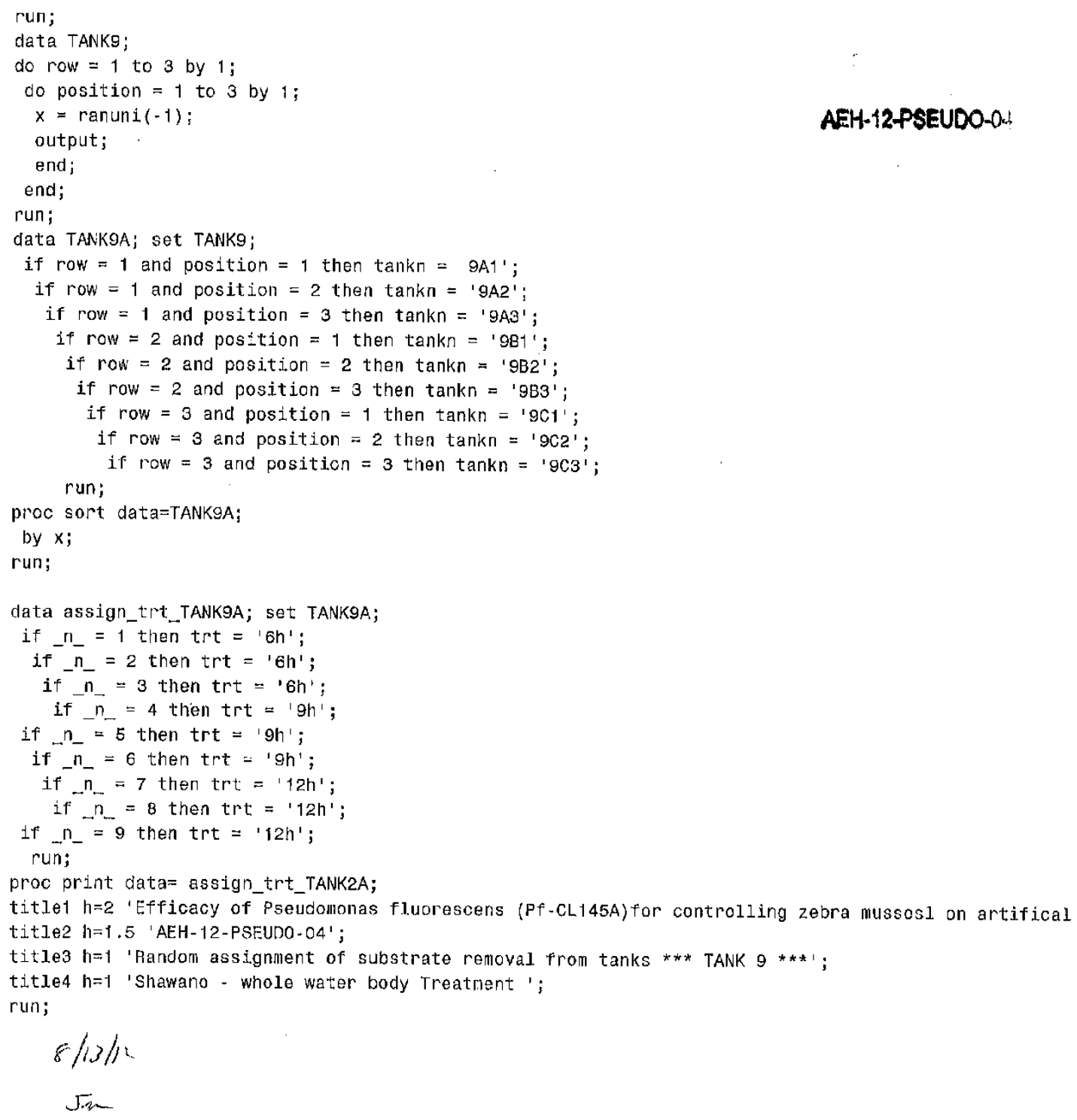




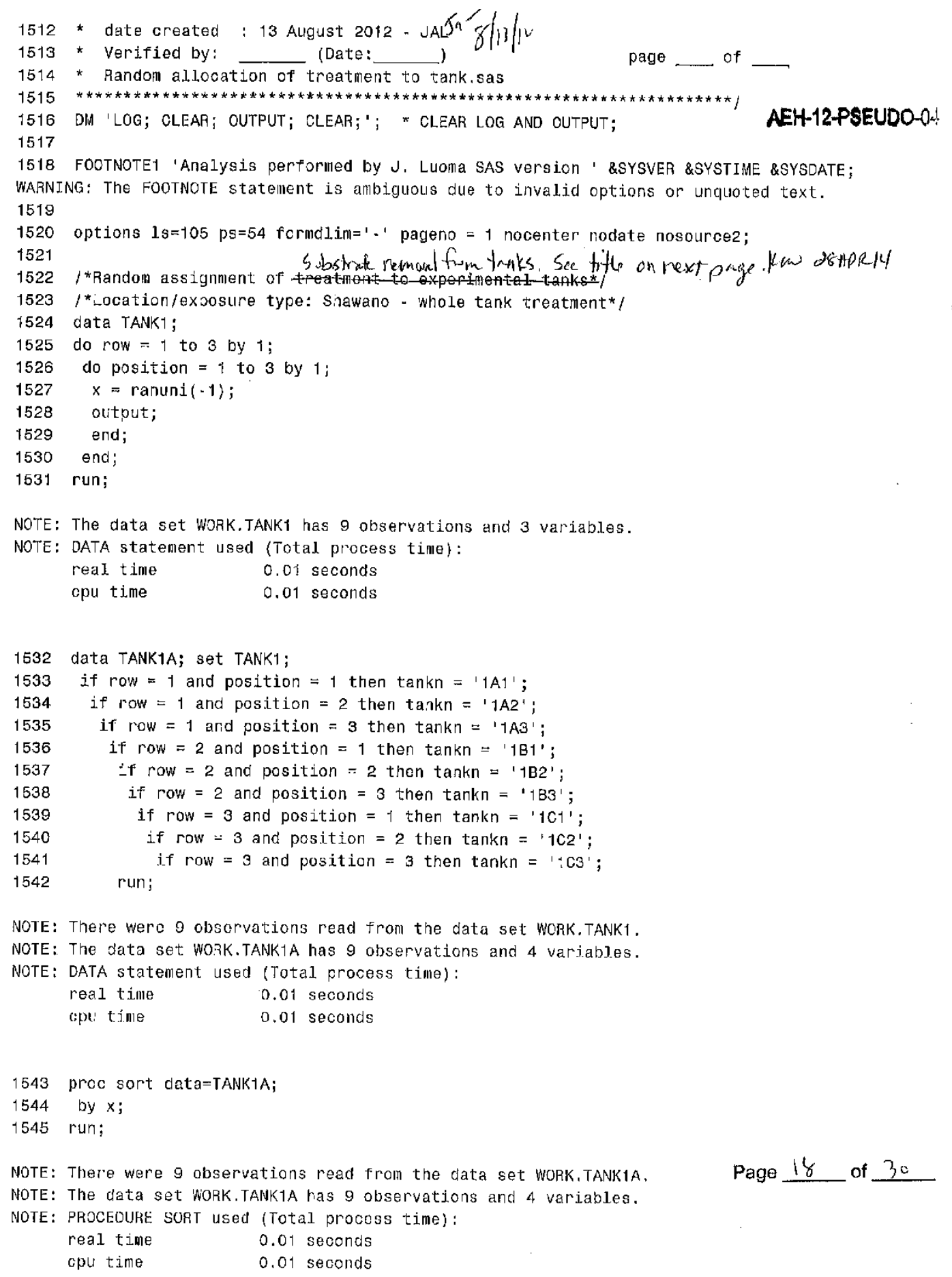




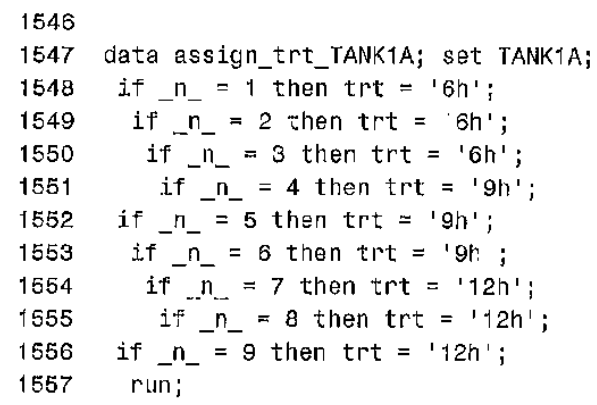

NOTE: There were 9 observations read from the data set WORK. TANK1A.

NOTE: The data set WORK.ASSIGN_TRT_TANK1A has 9 observations and 5 variables.

NOTE: DATA statement used (Total process time):

real time $\quad 0.01$ seconds

cpu time $\quad 0.01$ seconds

1558 proc print data= assign_trt_TANK1A

1559 titlel $h=2$ 'Efficacy of Fseudomonas fluorescens (Pf-CL145A)for controlling zebra mussesl on

1559 ! artifical substrates';

1560 title2 $h=1.5$ 'AEH-12-PSEUDO-04';

1561 titles $h=1$ 'Random assignment of substrate removal from tanks *** TANK $1 * * * 1$;

1562 ti.tle4 $h=1$ 'Shawano. Whole water body Treatment ';

1563 run;

NOTE: There were 9 observations read from the data set WORK.ASSIGN_TRT_TANK1A.

NOTE: PROCEOURE PRINT used (Total process time):
real timie
0.00 seconds
cpu time
0.00 seconds

1564

1565 data TANK2;

1566 do row $=1$ to 3 by $1 ;$

1567 do position $=1$ to 3 by 1 ;

$1568 \quad x=$ ranuni $(-1)$;

1569 output;

1570 end;

1571 end;

1572 run:

NOTE: The data set WOR.T.TANK2 has 9 observations and 3 variables.

NOTE: DATA statement used (Total process time):

real time $\quad 0.00$ seconds

cpu time $\quad 0.00$ soconds

1573 data TANK2A; set TANK2;

Page 19 of 30

1574 if row $=1$ and position $=1$ then tankn $={ }^{2} 2 \mathrm{~A} 1{ }^{\prime}$

1575 if row $=1$ and position $=2$ then $\operatorname{tankn}={ }^{\prime} 2 \mathrm{~A}^{\prime}$ ';

1576 if row $=1$ and position $=3$ then tankn $={ }^{\prime} 2 \mathrm{~A}^{\prime}{ }^{\prime}$; 


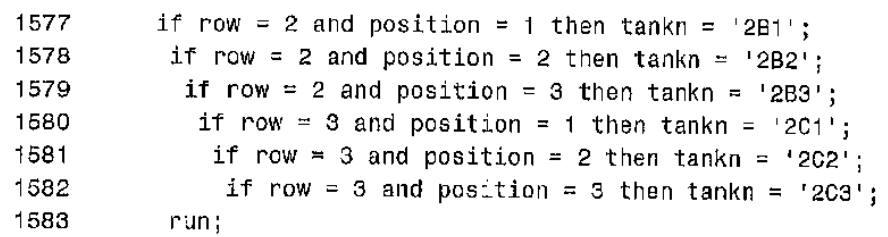


$\begin{array}{ll}\text { real time } & 0.01 \text { seconds } \\ \text { cpu ime } & 0.01 \text { seconds }\end{array}$

1606 data TANK3;

1607 do row $=1$ to 3 by 1 ;

1608 do position $=1$ to 3 by 1 ;

$1609 \quad x=\operatorname{ranuni}(-1)$;

1610 output:

1611 ond

1612 end;

1613 run;

NOTE: The data set WORK.TANK3 has 9 observations and 3 variables.

NCTE: DATA statement used (Total process time):
roal timo
0.01 seconds
cpu time
0.01 seconds

1614 data TANK3A; set TANK3

1615 if row $=1$ and position $=1$ then tankn $=$ ' $3 A^{\prime} 1^{\prime}$;

1616 if row $=1$ and position $=2$ then tankn $=3 \mathrm{~A} 2$ ';

1617 if row $=1$ and position $=3$ then tankn $={ }^{\prime} 3 \mathrm{A3} \mathrm{I}^{\prime}$;

1618 if row $=2$ and position $=1$ then tankn $=$ ' 381 '

1619 if row $=2$ and position $=2$ then tankn $={ }^{\prime} 3 \mathrm{~B} 22^{\prime}$;

1620 if row $=2$ and position $=3$ then tankn $=$ ' $3 B 3^{\prime}$;

1621

1622

1623

1624

if row $=3$ and position $=1$ then $\operatorname{tankn}=1301$ '

if row $=3$ and position $=2$ then $\operatorname{tankn}={ }^{\prime} 3 \mathrm{C} 22^{\prime}$;

if row $=3$ and position $=3$ then tankn $=$ ' $3 \mathrm{C} 3$ '; run

NOTE: There were 9 observations read from the clata set WORK. TANK3, NOTE: The data set WORK.TANK3A has 9 observations and 4 variables. NOTE: DATA statement used (Total process time):
real time
0.01 seconds

cpu time

$0.0^{\circ}$ seconds

1625 proc sort data=TANK3A;

1626 by $x$;

1627 run;

NOTE: Thare vere 9 observations read from the data set WORK. TANK3A.

NOTE: The data set WORK.TANK3A has 9 observations and 4 variables.

NOTE: PROCEDURE SORT used (Tutal process time):

real time $\quad 0.01$ seconds

cpu ine $\quad 0.01$ seconds

1629 data assign trt TANK3A; set TANK3A;

1630 if $n_{-}=1$ then $\operatorname{trt}={ }^{n} 6 h^{\prime}$;

1631 if $n=2$ then trt $={ }^{\prime} 6 \mathrm{~h}^{\prime}$;

1632 if $n_{-}=3$ then trt $=$ ' $6 h^{\prime}$;

1633 if $n=4$ then trt $=19 h^{\prime}$;

Page 21 of 30 


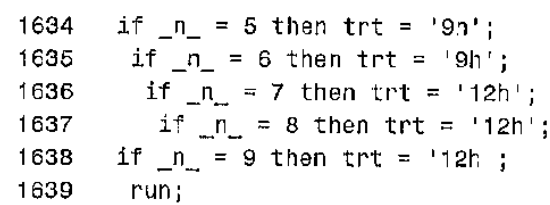

NOTE: There were 9 observations read from the data set WORK. TANK3A.

NOTE: The cata set VORK.ASSIGV_TRT_TANK3A has 9 observations and 5 variables.

NOTE: DATA statement used (Total process $t$ ime):
real time
0.03 seconds

cpu time

0.03 seconds

1640 proc print data= assign_trt_TANKBA;

1641 title1 h=2 'Efficacy of Pseudomonas fluorescens (Pf-CL145A)for controlling zebra mussesl on

16411 artifical substrates';

1642 title2 $h=1.5$ 'AEH-12-PSEUDO-04';

1643 titles $h=1$ 'Random assignment of substrate removal fron tanks $* \star$ * TANK $3 * \star \star 1$;

1644 title4 $h=1$ 'Shawano - whole water body Treatment ';

1645 run;

NOTE: There were 9 observations read from the data set WORK.ASSIGN_TRT_TANK3A.

NOTE: PROCEDURE PRINT used (Total process time):
real time
0.00 seconds
cpu time
0.00 seconds

1646 data TANK4;

1647 do row $=1$ to 3 by 1 ;

1648 do position $=1$ to 3 by 1 ;

$1649 \quad x=\operatorname{ranuni}(-1)$;

1650 output;

1651 end;

1652 end;

1653 run;

NOTE: The data sot WORK.TANK4 has 9 observations and 3 variables.

NOTE: DATA statement used (Total process time):
real time
0.01 seconds
cpu time
0.01 seconds

1654 data TANK4A; set TANK4;

1655 if row $=1$ and positior $=1$ then $\operatorname{tankn}=$ '4A1';

1656 if row $=1$ and position $=2$ then tankn $=$ ' $4 \mathrm{~A} 2^{\prime}$;

1657 if row $=1$ and position $=3$ then tankn $=$ ' $4 \mathrm{~A} 3^{\prime}$;

1658 if row $=2$ and position $=1$ then tankn $=481^{\prime}$;

1659 If row $=2$ and position $=2$ then tankn $={ }^{\prime} 4 \mathrm{~B} 2{ }^{\prime}$;

1660 if row $=2$ and position $=3$ then tankn $={ }^{\prime} 483^{\prime}$;

1661 if row $=3$ and position $=1$ then tankn $={ }^{\prime} 4 \mathrm{C} 1^{\prime}$;

1662 if row $=3$ and position $=2$ then tankn $=$ ' $4 \mathrm{C2}$ ';

1663 if row $=3$ and position $=3$ then tankn $={ }^{\prime} 4 \mathrm{C} 3{ }^{\prime}$;

1664 run;

NOTE: There were 9 observations read from the data set WORK. TANK4. 
NOTE: The data set WORK.TANK4A has 9 observations and 4 variables.

NOTE: DATA statentent used (Total process time):
real time
0.01 seconds

cpu time

0.01 seconds

AEH-12-PSEUDO-OH

1665 proc sort data=TANK4A;
1666 by $x$;
1667 run;

NOTE: There werc 9 observations read from the data set WORK. TANK4A.

NOTE: The data set WORK.TANK4A has 9 observations and 4 variables.

NOTE: PROCEDURE SORT used (Total process time):

real time $\quad 0.01$ seconds

cpu time 0.01 seconds

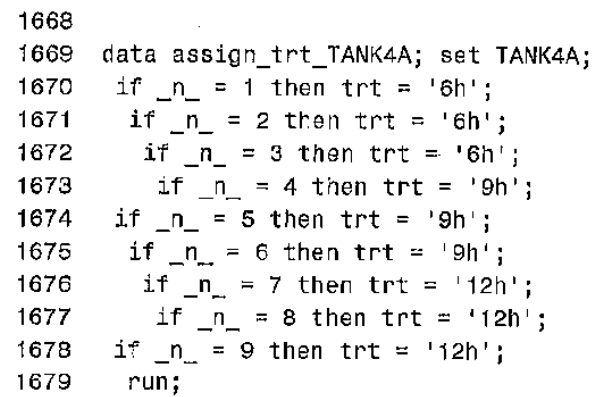

NOTE: There were 9 observations read from the data set WORK. TANK4A.

NOTE: The data set WORK.ASSIGN_TRT_TANK4A has 9 observations and 5 variables.

NOTE: DATA statement used (Total process time):

real tinte $\quad 0.01$ seconds

cpu time $\quad 0.01$ seconds

1680 proc print data= assign_trt_TANK4A;

1681 title1 h=2 'Efficacy of Pseudomonas fluorescens (Pt’-CL145A)for controlling zebra mussesl on 1681 ! artifical substrates';

1682 title2 $n=1.5$ 'AEH-12.PSEUDO-04';

1683 title3 $h=1$ 'Random assignment of substrate removal from tanks *** TANK $4 * * * 1$;

1684 title4 $h=1$ 'Shawano - whole water body Treatment ';

1685 run;

NOTE: There were 9 observations read fron the clata set WORK.ASSIGN TRT TANKAA,

NOTF: PROCEDURE PRINT used (Total process time):
real time
0.01 seconds
cpu time
0.01 seconds

1686 data TANK5;

1687 do row $=1$ to 3 by 1 ;

Page 23 of 30

1688 do position $=1$ to 3 by 1 ;

$1689 x=\operatorname{ranuni}(-1)$;

1690 cutput; 


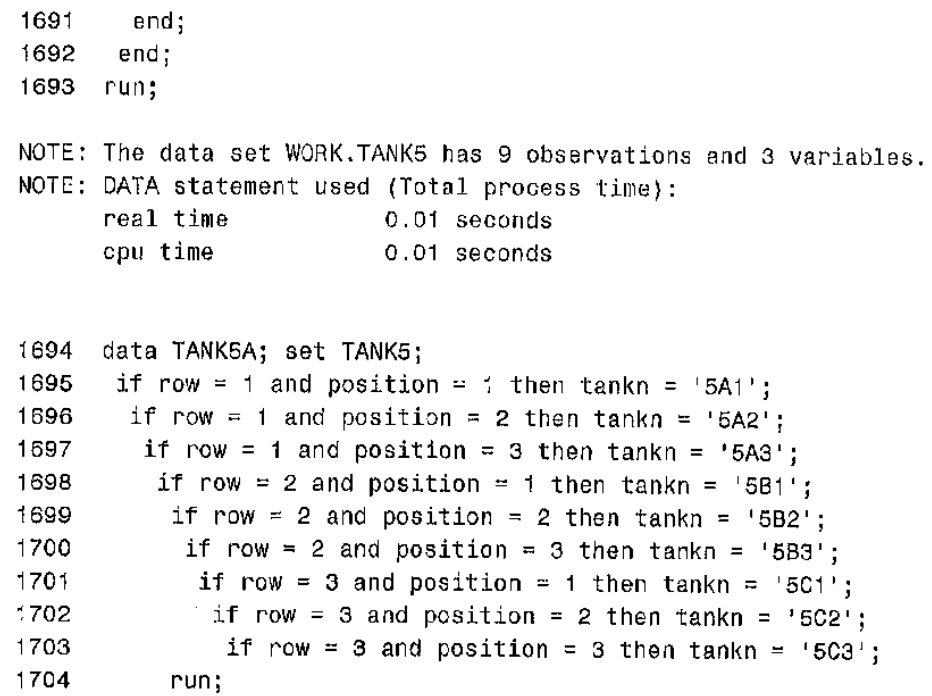

NOTE: There were 9 observations read fron the data set WORK. TANK5. NOTE: The data set WORK. TANK5A has 9 observations and 4 variables. NOTE: DATA statement used (Total process time):

rea: time 0.03 seconds

cpu time $\quad 0.03$ seconds

1705 proc sort data=TANKSA;

1706 by $x$ :

1707 run;

NOTE: There were 9 observations read from the data set WOAK. TANK5A. NOTE: The data set WORK. TANK5A has 9 observations and 4 variables. NOTE: PROCEDURE SORT used (Tatal process time):
real time
0.01 seconds
cpu time
0.01 seconds

1708

1709 data assign_trt TANKEA; set TANK5A;

1710 if ${ }^{n}{ }_{-}=1$ then trt $=$ ' $63^{\prime}$ '

1711 if $\vec{n}_{-}=2$ then trt $=$ ' $6 \mathrm{~h}$ ';

1712 if $n_{-}=3$ then trt $=' 6 h '$;

1713 if $n_{-}=4$ then trt $=$ ' $9 h^{\prime}$ ';

1714 if $\Omega_{-}=5$ then trt $=$ ' $^{n}$ ' ' $^{\prime}$;

1715 if $n_{-}=6$ then trt $=$ ' 9 h ;

1716 if $n_{-}=7$ then trt $=12 \mathrm{~h}$ ';

$1717 \quad f_{-} n_{-}=8$ then trt $=112 h^{\prime}$;

1718 if $n_{-}=9$ then $\operatorname{trt}=112 h^{\prime}$;

1719 run;

Page 24 of 30

NOTE: There were 9 observations read from the data set WORK. TANK5A.

NOTE: The data set WORK.ASSIGN_TRT. TANK5A has 9 observatiors and 5 variables.

NOTE: DATA statement used (Total process tine): 
$\begin{array}{ll}\text { real time } & 0.01 \text { seconds } \\ \text { cpu time } & 0.01 \text { seconds }\end{array}$

AEH-12-PSEUDO-0.1

1720 proc print data $=$ assign_trt_TANK2A;

1721 title1 $h=2$ 'Efficacy of Pseudomonas fluorescens (Pf-CL145A)for controlling zebra musses1 on

$1721 !$ artifical substrates';

1722 title2 $h=1.5$ 'AEH-12-PSEUDO-04';

1723 title3 $h=1$ 'Random assignment of substrate removal from tanks *** TANK $5 * * * 1$;

1724 title4 $h=1$ 'Shawano - whole water body Treatment ',

1725 run;

NOTE: There were 9 observations read from the data set WORK.ASSIGN_TRT TANK2A.

NOTE: PROCEDURE PRINT used (Tota1 process time):
real time
0.00 seconds

cpu time

0.00 seconds

1726 data TANK6;

1727 do row $=1$ to 3 by 1 ;

1728 do position $=1$ to 3 by $i$

$1729 \quad x=\operatorname{ranuni}(-1)$;

1730 output;

1731 end

1732 end;

1733 run:

NOTE; The data set WORK. TANK6 has 9 observations and 3 variatles.

NOTE: DATA statement used (Total process time):

real time $\quad 0.01$ seconds

cpu time $\quad 0.01$ seconds

1734 data TANKGA; set TANK6;

1735 if row $=1$ and position $=1$ then tankn $=$ ' $6 \mathrm{~A} 1$ ';

1736 if row $=1$ and position $=2$ then tankn $=$ ' $6 \mathrm{~A} 2$ ';

1737 if row $=1$ and position $=3$ then tankn $=' 6 \mathrm{~A} 3{ }^{\prime}$

1738 if row $=2$ and position $=1$ then tankn $=' 6 \mathrm{~B} 1{ }^{\prime}$;

1739 if row $=2$ and position $=2$ then tankn $=$ ' $6 \mathrm{~B} 2$ ';

1740 if row $=2$ and position $=3$ then $\operatorname{tankn}={ }^{\prime} 6 \mathrm{~B} 3^{\prime}$;

1741 if row $=3$ and position $=1$ then tankn $=' 6 \mathrm{C} 1$ ';

1742

1743

1744

if row $=3$ and position $=2$ then $\operatorname{tankn}=' 6 \mathrm{C} 2$ '

if row $=3$ and position $=3$ then tankn $=' 6 \mathrm{C} 3 '$; run;

NOTE: There were 9 observations read from the data set WORK. TANK6.

NOTE: The data set WORK.TANKCA has 9 observations and 4 variables.

NOTE: DATA statement used (Total process time):
real time
0.03 seconds
cpu time
0.03 seconds

1745 proc sort data=TAUK6A;

Page 25 of 30

1746 by $x$;

1747 run; 
NOTE: There were 9 observations read from the data set WORK. TANK6A.

NOTE: The data set WORK.TANK6A has 9 observations and 4 variables.

NOTE: PROCEDURE SORT used (Total process time):
real time
0.01 seconds
срu time
0.01 seconds

AEH-12.PSEUDO-0.1

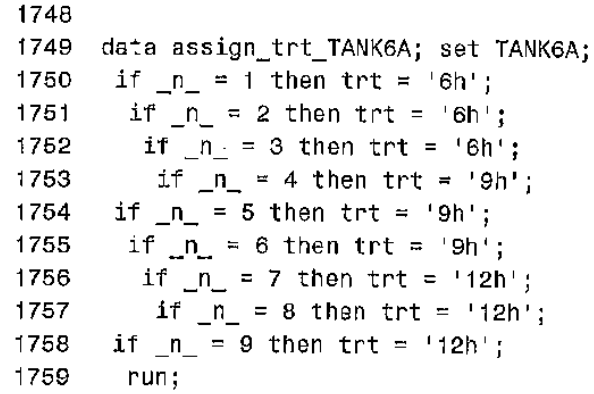

NOTE: There were 9 observations read from the data set WORK. TANK6A.

NOTE: The data set WORK.ASSIGN_TRT_TANK6A has 9 observations and 5 variables.

NOTE: DATA statement used (Total prooess time):

real time. $\quad 0.03$ seconds

cpu time $\quad 0.03$ seconds

1760 proc print data= assign_trt_TANK6A;

1761 title1 $h=2$ 'Efficacy of' Pseudomonas fiuorescens (Pf-C'-145A)for controlling zebra mussesl on 1761 ! artifical substrates';

1762 title2 $h=1.5$ 'AEH-12-PSEUDO-04';

1763 titles $h=1$ 'Random assignment of substrate removal from tanks $\star \star \star$ TANK $6 \star \star \star 1$;

1764 title4 $h=1$ 'Shawano - whole water body Treatment ';

1765 run;

NOTE: There were 9 observations read from the data sot WORK.ASSIGN_TRT_TANK6A.

NOTE: PROCELURE PHINT used (Total process time):
real time
0.00 seconds
cpu time
0.00 seconds

1766 data TANK7;

1767 do row $=1$ to 3 by 1 ;

1768 do position $=1$ to 3 by 1 ;

$1769 \quad x=\operatorname{ranuni}(-1)$;

1770 output;

1771 end;

1772 end

1773 run;

NOTE: The clata set WORK, TANK7 has 9 observations and 3 variaoles.

NOTE: DATA statement used (Total process time):
real time
0.01 seconds
cpu time
0.01 seconds

Page 26 of 30 
1774 data TANK7A; set TANK7;

1775 if row $=1$ and position $=1$ then tankn $={ }^{\prime} 7 \mathrm{~A} 1^{\prime}$;

1776 if row $=1$ and position $=2$ then tankn $=17 \mathrm{~A} 2^{\prime}$;

1777 if row $=1$ and position $=3$ then tankn $=$ ' $7 \mathrm{AB}^{\prime}$ ';

1778 If row $=2$ and position $=1$ then tankn $=' 7 \mathrm{~B} 1$ ' ;

1779 if row $=2$ and position $=2$ then tankn $={ }^{\prime} 7 \mathrm{B2}^{\prime}$;

1780 if row $=2$ and position $=3$ then $\operatorname{tankn}={ }^{\prime} 7 \mathrm{~B} 3{ }^{\prime}$;

1781

If rov $=3$ and position $=1$ then taakn $=1701 '$;

if row $=3$ and position $=2$ then tankn $=' 7 \mathrm{C2}{ }^{\prime}$;

1783

1784 un;

\section{AEH-12-PSEUDO-0.}

NOTE: There were 9 observations read from the data set WORK. TANK7.

NOTE: The data set WOAK.TANKTA has 9 observations and 4 variables.

NOTE: DATA statement used (Total process time):

real time $\quad 0.01$ seconds

cpu time $\quad 0.01$ seconds

1785 proc sort data=TANK7A;

1786 by $x$;

1787 run;

NOTE: There were 9 observations read from the data set WORK.TANK7A.

NOTE: The data set WORK. TANK7A has 9 observations and 4 variables.

NOTE: PROCEDURE SORT used (Tota? process time):

$\begin{array}{ll}\text { real time } & 0.01 \text { seconds } \\ \text { cpu time } & 0.01 \text { seconds }\end{array}$

1788

1789 data assign_trt_TANK7A; set TANKYA;

1790 if $n_{-}=1$ then trt $={ }^{\prime} 6 h^{\prime}$;

1791 if $n_{-}=2$ then trt $=$ ' $6 \mathrm{~h} '$;

1792 if $n_{-}=3$ then trt $=$ ' $6 \mathrm{~h}$ ';

1793 if $\bar{n}_{-}=4$ then trt $=$ ' $9 \mathrm{~h}^{\prime}$;

1794 if $r_{-}=5$ then trt $={ }^{\prime} 9 \mathrm{~h}^{\prime}$;

1795 if $\bar{n}_{-}=6$ then trt $=$ ' $9 \mathrm{~h}$ ';

1796 if $\mathrm{n}_{-}=7$ then trt $=112 \mathrm{~h}^{\prime}$;

1797 if $\bar{n}_{-}=8$ then trt $=12 \mathrm{~h}$;

1798 if $n_{-}=9$ then $\operatorname{trt}=112 \mathrm{~h}^{\prime}$;

1799 run;

NOTE: There vere 9 observations read from the data set WORK. TANK7A.

NOTE: The data set WORK.ASSIGN_TRT_TANK7A has 9 observations and 5 variables.

NOTE: DATA statement used (Total process time):

real tine 0.03 seconds

срu tine $\quad 0.03$ seconds

1800 proc print data= assign_trt_TANK2A;

1801 title1 $h=2$ 'Efficacy of Pseudomonas fluorescens (Pf..CL145A)for controlling zebra nussesl on

18011 artifical substrates';

1802 title2 $\mathrm{h}=1.5$ 'AEH 12 -PSEUDO-04';

1803 titles $h=1$ 'Random assignment of substrate removal from tanks *** TANK $7 * * * 1$;

1804 title4 $h=1$ 'Shawano - whole water body Treatment '; 
1805 run;

NOTE: There were 9 ooservations read from the data set WOR

NOTE: PROCEDURE PRINT used (Total process time):

real tine $\quad 0.01$ seconds

cpu time $\quad 0.01$ seconds

AEH-12-PSEUDO-0.1

1806 data TANK8;

1807 do row $=1$ to 3 by 1 ;

1808 do position $=1$ to 3 by 1 ;

$1809 \quad x=$ ranuni $(-1)$;

1810 output;

1811 end

1812 end;

1813 run;

NOTE: The data set WORK.TANKB has 9 observations and 3 variables.

NOTE: DATA statement used (Total process time):

real timo $\quad 0.01$ seconds

cpu time $\quad 0.01$ seconds

1814 data TANK8A; set TANKB;

1815 if row $=1$ and position $=1$ then tankn $=' 8 \mathrm{~A} 1$ '

1816 if row $=1$ and position $=2$ then tankn $=$ ' $8 \mathrm{~A} 2$ ';

1817 If row $=1$ and position $=3$ then $\operatorname{tankn}={ }^{\prime} 8 \mathrm{~A}^{\prime}{ }^{\prime}$;

1818 if row $=2$ ard position $=1$ then tankn $={ }^{\prime} 8 \mathrm{~B} 11^{\prime}$;

1819 if row $=2$ and position $=2$ then tankn $=$ ' $882^{\prime}$ ';

1820 if row $=2$ and position $=3$ then tankn $=' 883 '$;

1821 if row $=3$ and position $=1$ then tankn $=$ ' $B \mathrm{C} 11^{\prime}$;

1822 if row $=3$ and position $=2$ then tankn $=$ ' $8 \mathrm{C} 2$ ';

1823 if row $=3$ and position $=3$ then $\operatorname{tankn}={ }^{\prime} 8 \mathrm{C} 3{ }^{\prime}$

1824 run;

NOTE: There were 9 observations read from the data set WORK. TANK8.

NOTE: The data set WORK. TANKBA has 9 observations and 4 variables.

NOTE: DATA statement used (Total process time):
real time
0.01 seconds
cpu time
0.01 scoonds

1825 proc sort data=TANK8A;

1826 by $x$;

1827 run;

NOTE: There were 9 observations read from the data set WORK. TANKBA.

NOTE: The data set WORK.TANKBA has 9 observations and 4 variables.

NOTE: PROCEDURE SORT used (Total process time):
real time
0.01 seconds

cpu time

0.01 seconds

1828

1829 data assign_trt_TANK8A; set TANK8A;

1830 if $n_{-}=1$ then trt $=$ ' $6 h^{\prime}$ '; 


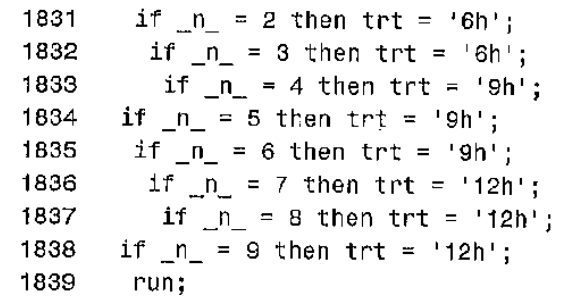

NOTE: There were 9 observations read from the data set WORK. TANKBA.

NOTE: The clata set WORK.ASSIGN_TRT_TANK8A has 9 observations and 5 variables.

NOTE: DATA statement used (Total process $=$ ime):
roal tine
0.01 seconds
cpu time
0.01 seconds

1840 proc print data= assign_trt_TANKBA;

1841 title1 h=2 'Efficacy of Pseudomonas fluorescens (Pf-CL145A)for controlling zebra mussesl on 1841 ! artifical substrates';

1842 title2 $h=1,5$ 'AEH-12-PSEUDO-04';

1843 title3 $h=1$ 'Pandom assignment of substrate renoval from tanks *** TANK $8 * * *$ ';

1844 titie4 $h=1$ 'Shawano - whole water body Treatment ';

1845 run;

NOTE: There were 9 observations read from the data set WORK.ASSIGN_TRT_TANKBA.

NOTE: PROCEDURE PRINT used (Total process time):

real time $\quad 0.01$ seconds

cpu time $\quad 0.01$ seconds

1846 data TANK9;

1847 do row $=1$ to 3 by 1 ;

1848 do position $=1$ to 3 by 1 ;

$1849 \quad x=\operatorname{ranuni}(-1)$;

1850 output;

1851 end

1852 end;

1853 run;

NOTE: The data set WORK. TANKS has 9 observations and 3 variables.

NOTE: DATA statement used (Total process time):
real tinte
0.03 seconds
cpu time
0.03 seconds

1854 data TANK9A; set TANK9;

1855 if row $=1$ and position $=1$ then tankn $=$ ' $9 A 1$ ';

1856 if row $=1$ and position $=2$ then tankn $=$ ' $9 \mathrm{~A} 2^{\prime}$ ';

1857 if row $=1$ and position $=3$ then tankn $=9 \mathrm{AB}^{\prime}$;

1858 If row $=2$ and position $=1$ then $\operatorname{tankn}=$ ' $9 \mathrm{~B} 1$ ';

1859 if row $=2$ and position $=2$ then tankn $=$ ' 982 ';

1860 if row $=2$ and position $=3$ then tankn $=$ '9B3'

1861 if row $=3$ and position $=1$ then tankn $=$ ' $9 \mathrm{C} 1$ ' ;

1862 If row $=3$ and position $=2$ then tankn $=19 \mathrm{C} 2 '$;

1863 if row $=3$ and position $=3$ then tankn $=19 \mathrm{c} 3{ }^{\prime}$;

Page 29 of 30 


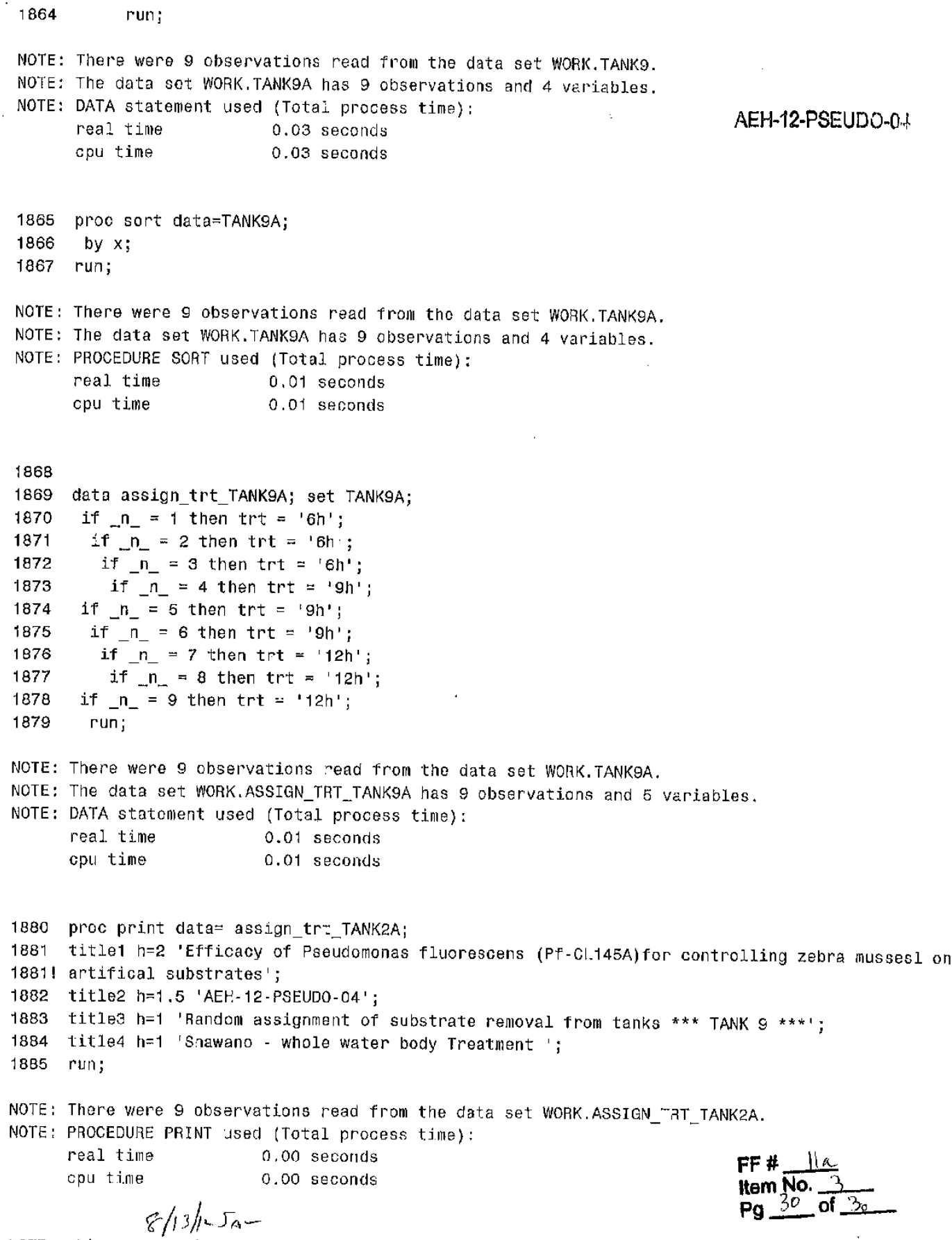

FF \# lla $\mathrm{Pg} 30$ of 30

NOTE: This SAS session is usirg a registry in WORK. All changes will be lost at the end of this sess 


\begin{tabular}{|c|c|c|c|c|c|c|c|}
\hline \multicolumn{6}{|c|}{$\begin{array}{l}\text { Handom assignment of treatment to experimental tanks } \\
\text { Treatment Location/type: Shawano - bottom injection }\end{array}$} & \multirow{2}{*}{$\begin{array}{c}8 / \mathrm{h} / \mathrm{h}^{2} \\
\mathrm{~F}^{2}-\end{array}$} & \multirow[b]{2}{*}{ AEH-12-PSEUD0-0 } \\
\hline Obs & block & tank & $x$ & $\operatorname{tankn}$ & trt & & \\
\hline 1 & 1 & 9 & 0.13021 & Tank 9 & control & & \\
\hline 2 & 1 & 2 & 0.15287 & Tank 2 & 50 & & \\
\hline 3 & 1 & 4 & 0.23351 & Tank 4 & 100 & & \\
\hline 4 & 1 & 1 & 0.26174 & Tank 1 & control & & \\
\hline 5 & 1 & 7 & 0.29656 & Tank 7 & 50 & & \\
\hline 6 & 1 & 5 & 0.52465 & Tank 5 & 100 & & \\
\hline 7 & 1 & 8 & 0.67430 & Tank 8 & control & & \\
\hline 8 & 1 & 3 & 0.91450 & Tank 3 & 50 & & \\
\hline 9 & 1 & 6 & 0.99600 & Tank 6 & 100 & & \\
\hline
\end{tabular}

Item Number: 1

Page 1 - of 4

Analysis performed by $J$. Luoma SAS version 9.2 08:59 11AUG12 


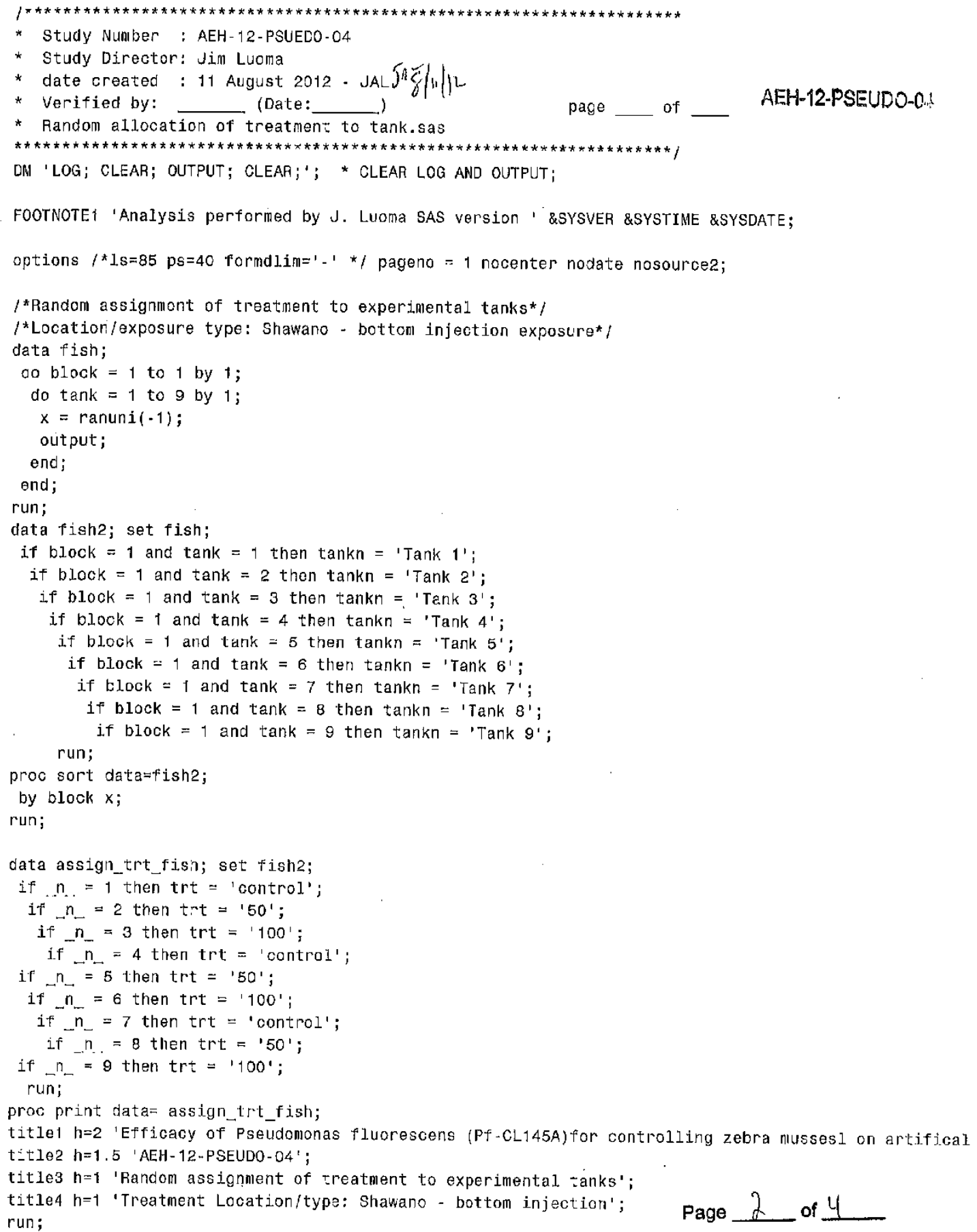




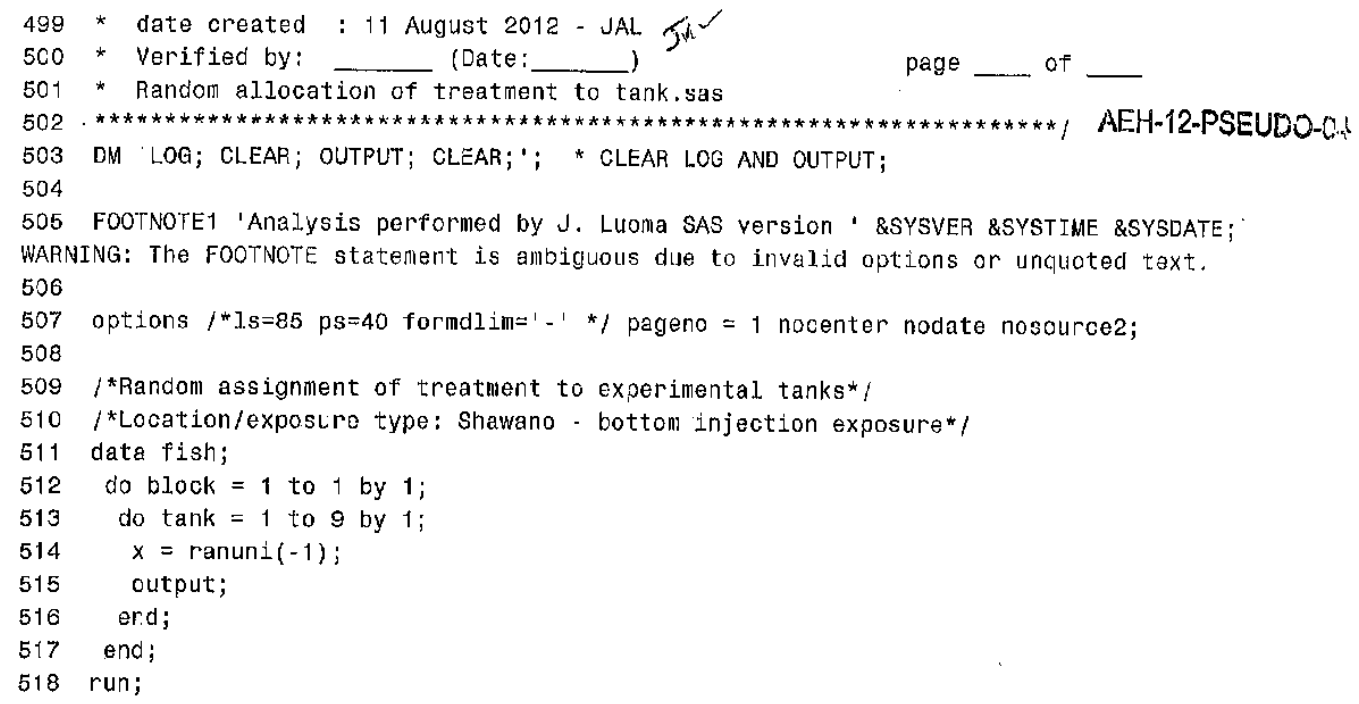

VOTE: There were 9 observations read from the data set WORK. FISH. NOTE: The data set WORK.FISH2 has 9 observations and 4 variables. NOTE: DATA statement used (Total process time):
real zime
0.01 seconds

cpu tine

0.01 seconds

530 proc sort data=fish2;

53 y by block $x$;

532 run;

NCTE: There were 9 observations read froll the data set WORK.FISH2. NCTE: The data set WORK.FISH2 has 9 observations and 4 variables.

NOTE: PROCEDURE SORT used (Total process time):
real time
0.01 seconds
cpu time
0.01 seconds

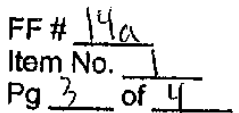




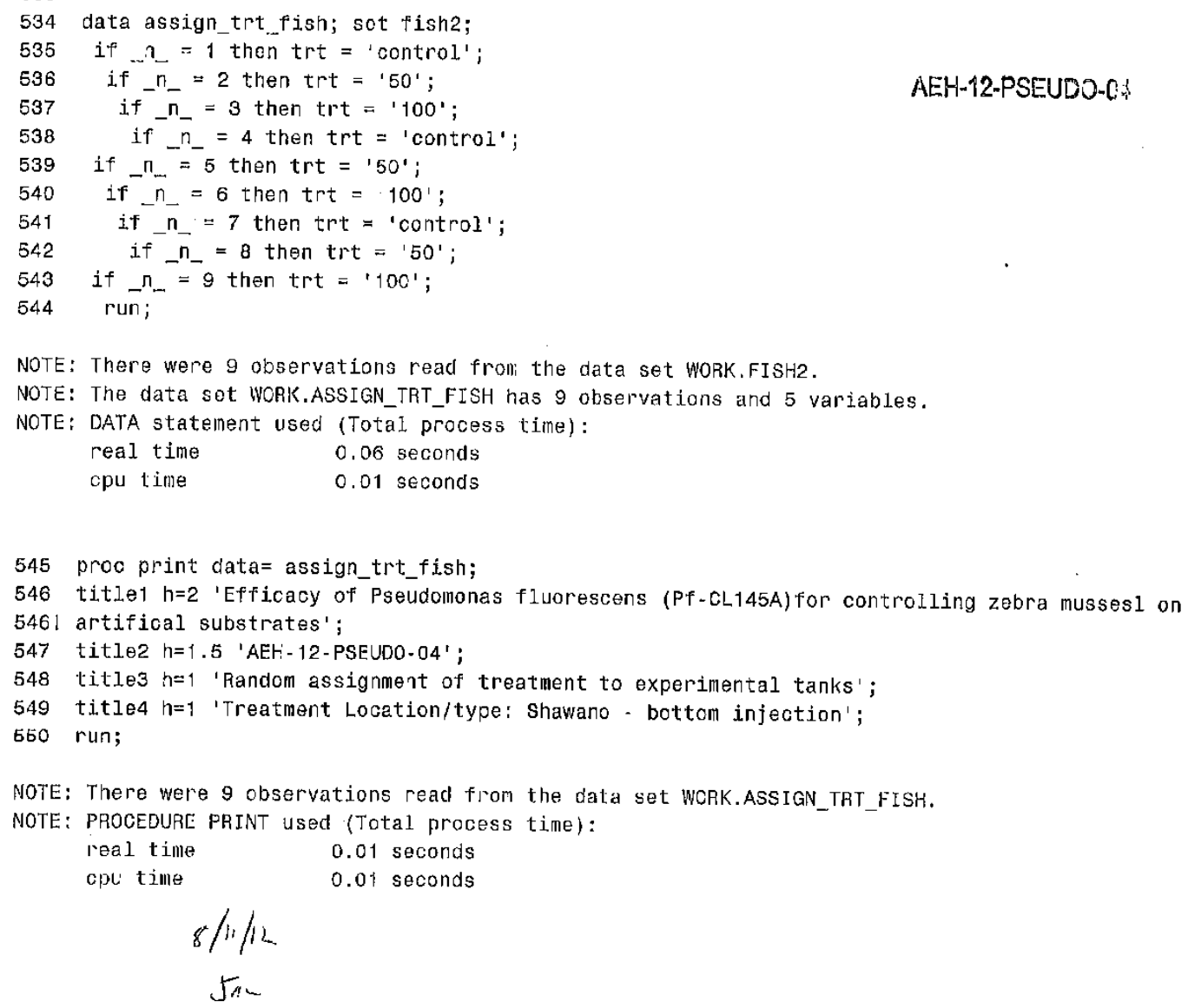


Effigacy of Psuedomonas fluorescens (Pf-CL145A)SDP for controlling zebra mussels on artifical subs 1 .
AEH-12-PSUED0-04

Random assignment of trays to test tank/position Test Location/type = Shawano bottom injection tank treatment $8 / h / 12$

\begin{tabular}{|c|c|c|c|c|c|c|c|c|}
\hline \multirow[b]{2}{*}{ Obs } & \multirow[b]{2}{*}{ round } & \multirow[b]{2}{*}{ row } & \multirow[b]{2}{*}{ positition } & \multirow[b]{2}{*}{$\operatorname{tank}$} & \multicolumn{4}{|c|}{$3 / 2$} \\
\hline & & & & & $x$ & _row_ & tankn & AEH-12-PSEUDO-OA \\
\hline 1 & 1 & 1 & 2 & 8 & 0.00810 & A & $8 \mathrm{~A} 2$ & \\
\hline 2 & 1 & 3 & 3 & 2 & 0.03556 & c & $2 \mathrm{C3}$ & \\
\hline 3 & 1. & $1 \ldots$ & 2 & 6 & 0.03743 & A & $6 \mathrm{~A} 2$ & irc \\
\hline 4 & $1: \because$ & 2 & $\therefore 1$ & 5 & 0.03887 & B & $5 B 1$ & \\
\hline 5 & 1 & 1 & 1 & $\therefore 2$ & 0.06626 & A & $2 A 1$ & ins tolse \\
\hline 6. & 1 & 2 & 1 & $=2$ & 0.06878 & B & $2 \mathrm{~B} 1 \mathrm{C}$ & used for 12.4 \\
\hline 7 & 1 & 1 & 1 & 1 & 0.08357 & A & $1 \mathrm{~A} 1$ & treatinent. \\
\hline 8 & 1 & 1 & 2 & $\therefore \quad-1$ & 0.09108 & $A$ & $1 \mathrm{~A} 2$ & kw \\
\hline 9 & 1 & 2 & 2 & 9 & 0.12185 & B & $9 \mathrm{~B} 2$ & FSEPT/2 \\
\hline 10 & 1 & 2 & 3 & 1 & 0.12192 & B & $1 \mathrm{B3}$ & \\
\hline 11 & 1 & 3 & 1 & $\therefore 4$ & 0.14635 & $G$ & $4 \mathrm{Ct}$ & \\
\hline 12 & 1 & 1 & 2 & 2 & 0.17909 & $A$ & $2 A 2$, & \\
\hline 13 & 1 & 1 & 3 & 6 & 0.19369 & A & $6 A 3$ & \\
\hline 14 & 1 & 1 & Э & $\ldots 4$ & 0.19825 & $A$ & $4 A 3$ & bution \\
\hline 15 & 1 & 2 & 2 & 6 & 0.20476 & B & 682 & beacan at \\
\hline 16 & 1 & 2 & 3 & $B$ & 0.21165 & $B$ & $8 B 3^{2}$ & \\
\hline 17 & 1 & 3 & 1. & .6 & 0.22199 & $\mathrm{C}$ & $6 C 1$ & \\
\hline 18 & 1 & 3 & 3 & " 5 & 0.22663 & C & $5 \mathrm{CB}$ & abdithon \\
\hline 19 & 1 & 3 & 3 & 1 & 0.23721 & C & $\begin{array}{l}103 v \\
483 y\end{array}$ & complete at \\
\hline 20 & 1 & 2 & 3 & 4 & $\begin{array}{l}0.23995 \\
0.25274\end{array}$ & $\begin{array}{l}B \\
B\end{array}$ & $\begin{array}{l}4 \mathrm{~B} 3 \mathrm{v} \\
1 \mathrm{~B} 2\end{array}$ & complete as \\
\hline $\begin{array}{l}21 \\
22\end{array}$ & $\begin{array}{l}1 \\
1\end{array}$ & $\begin{array}{l}2 \\
1\end{array}$ & $\begin{array}{l}2 \\
3\end{array}$ & $\begin{array}{l}1 \\
7\end{array}$ & $\begin{array}{l}0.25274 \\
0.25387\end{array}$ & A & $7 A 3$ & 1033. Kan \\
\hline $\begin{array}{l}22 \\
23\end{array}$ & $\begin{array}{l}1 \\
1\end{array}$ & $\begin{array}{l}1 \\
1\end{array}$ & $\begin{array}{l}3 \\
3\end{array}$ & $\begin{array}{l}7 \\
5\end{array}$ & $\begin{array}{l}0.25387 \\
0.27644\end{array}$ & A & $5 A 3$ & \\
\hline 24 & 1 & 3 & 2 & 2 & 0.27738 & C & $2 \mathrm{C} 2$ & \\
\hline 25 & 1 & 3 & 2 & $\quad 4$ & 0.28281 & $c$ & $4 \mathrm{C} 2$ & \\
\hline 26 & 1 & 1 & 3 & $\mathbf{8}$ & 0.28295 & A & $8 A 3 \mathrm{~V}$ & \\
\hline 27 & 1 & 3 & 2 & , $\mathbf{5}$ & 0.29153 & c & $5 \mathrm{C} 2$ & \\
\hline 28 & 1 & 3 & 1 & 2 & 0.30627 & C & $2 c 1$ & \\
\hline 29 & 1 & 1 & 2 & 5 & 0.32998 & A & $5 \mathrm{~A} 2 \mathrm{~V}^{\prime}$ & \\
\hline 30 & 1 & 2 & 1 & 1 & 0.33341 & B & $1 B 1$ & \\
\hline 31 & 1 & 1 & 3 & 1 & 0.34612 & A & $\begin{array}{l}1 \mathrm{ABO} \\
7 \mathrm{~B} B \mathrm{C}\end{array}$ & \\
\hline 32 & 1 & 2 & 3 & $\therefore 7$ & 0.36184 & B & $\begin{array}{l}7 \mathrm{~B} 3 \mathrm{C} \\
9 \mathrm{~B} 3 \mathrm{~V}\end{array}$ & \\
\hline 33 & 1 & $\begin{array}{l}2 \\
3\end{array}$ & $\begin{array}{l}3 \\
3\end{array}$ & $\begin{array}{r}9 \\
7\end{array}$ & $\begin{array}{l}0.43563 \\
0.44991\end{array}$ & $\begin{array}{l}B \\
C\end{array}$ & $\begin{array}{l}9 \mathrm{B3} \mathrm{i} \\
7 \mathrm{C3}\end{array}$ & \\
\hline 34 & 1 & $\begin{array}{l}3 \\
1\end{array}$ & $\begin{array}{l}3 \\
1\end{array}$ & $\begin{array}{l}7 \\
5\end{array}$ & $\begin{array}{l}0.44991 \\
0.47095\end{array}$ & $A$ & $5 \mathrm{~A} 1$ & \\
\hline $\begin{array}{l}35 \\
36\end{array}$ & $\begin{array}{l}1 \\
1\end{array}$ & $\begin{array}{l}1 \\
1\end{array}$ & 3 & $\begin{array}{l}5 \\
2\end{array}$ & $\begin{array}{l}0.47095 \\
0.47688\end{array}$ & A & $2 A 3$ & 140 \\
\hline $\begin{array}{l}36 \\
37\end{array}$ & $\begin{array}{l}1 \\
1\end{array}$ & $\begin{array}{l}1 \\
2\end{array}$ & 3 & 3 & 0.47977 & B & 3B3 & Flle Folder: \\
\hline 38 & 1 & 3 & 3 & 4 & 0.55220 & c & $4 c 3$ & \\
\hline 39 & 1 & 2 & 2 & 7 & 0.55570 & B & 782 & \\
\hline 40 & 1 & 1 & 1 & 6 & 0.56422 & A & $6 \mathrm{~A} 1$ & \\
\hline 41 & 1 & 3 & 3 & 3 & 0.56430 & C & $3 \mathrm{C3}$ & \\
\hline 42 & 1 & 2 & 1 & 9 & 0.56500 & B & $9 \mathrm{~B}+\mathrm{N}$ & llom Number: 2 \\
\hline 43 & 1 & 3 & 2 & 8 & 0.57148 & c & $8 \mathrm{C} 2$ & \\
\hline 44 & 1 & 1 & arset? & 4 & 0.60142 & A & $4 A Z^{\prime}$ & \\
\hline 45 & $1^{+m a n a m}$ & 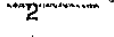 & 2 & 8 & 0.60482 & 日 & 8B2 & ' \\
\hline 46 & 1 & 1 & 3 & 3 & 0.60611 & A & $3 A 3$ & Pama \\
\hline 47 & 1 & 2 & 3 & 5 & 0.62329 & B & $5 \mathrm{~B} 3 \checkmark$ & Page \\
\hline
\end{tabular}

Analysis per-rormed by $J$. Luoma SAS version 0.2 10:48 11AUG12 

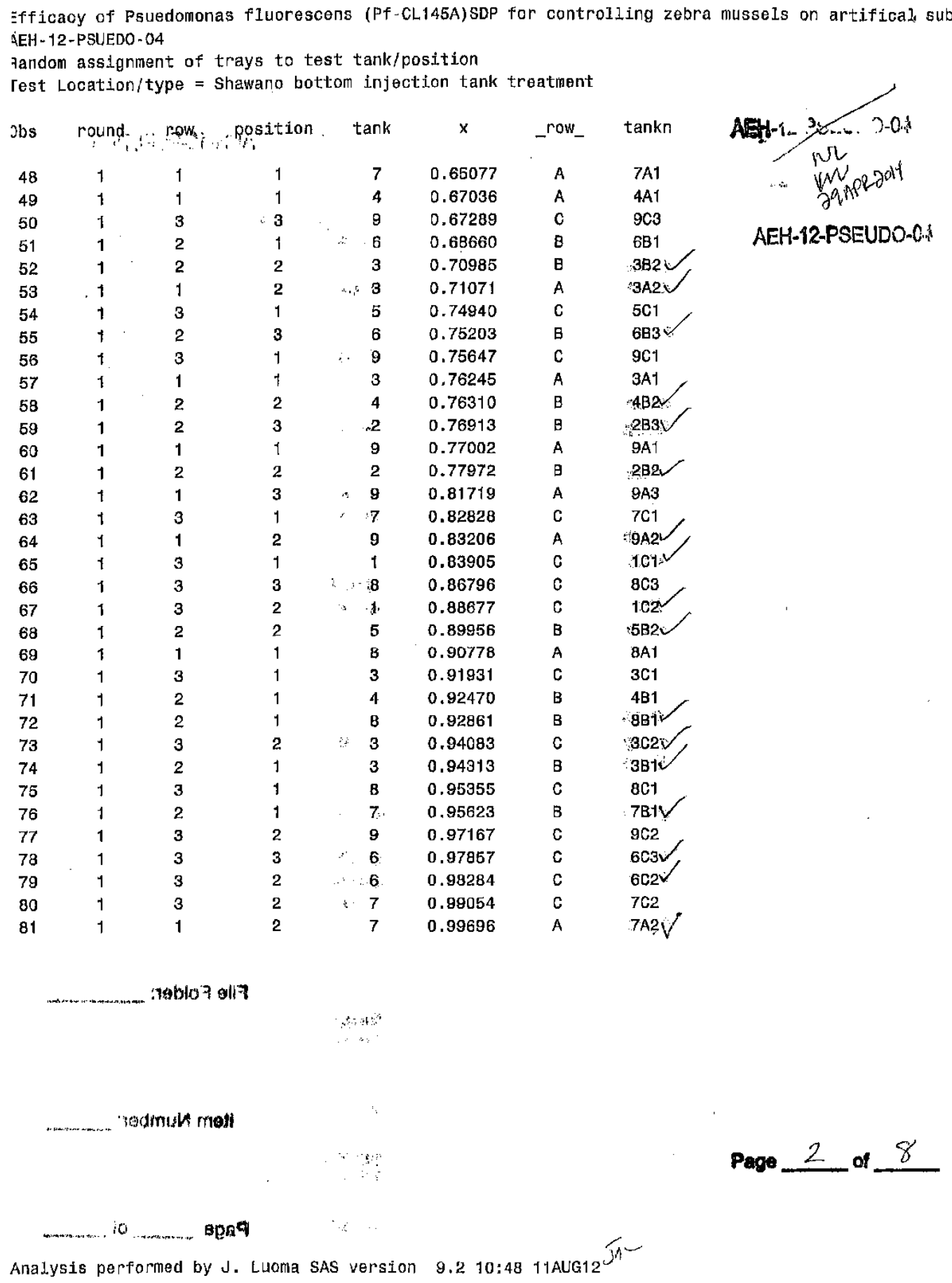


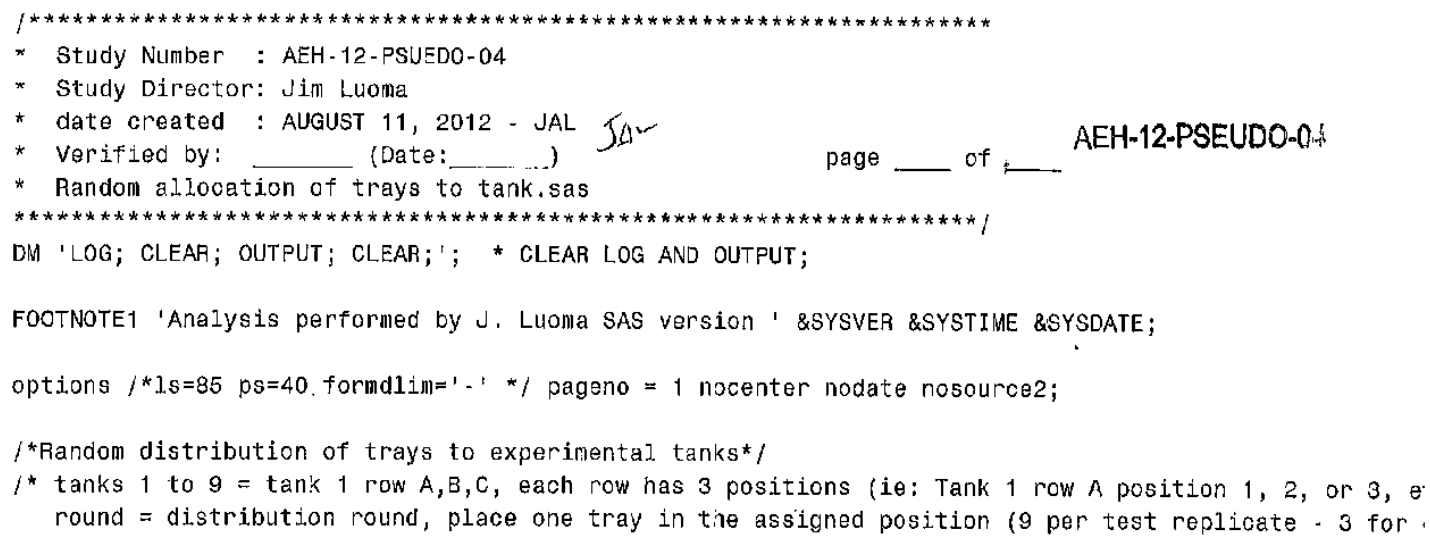




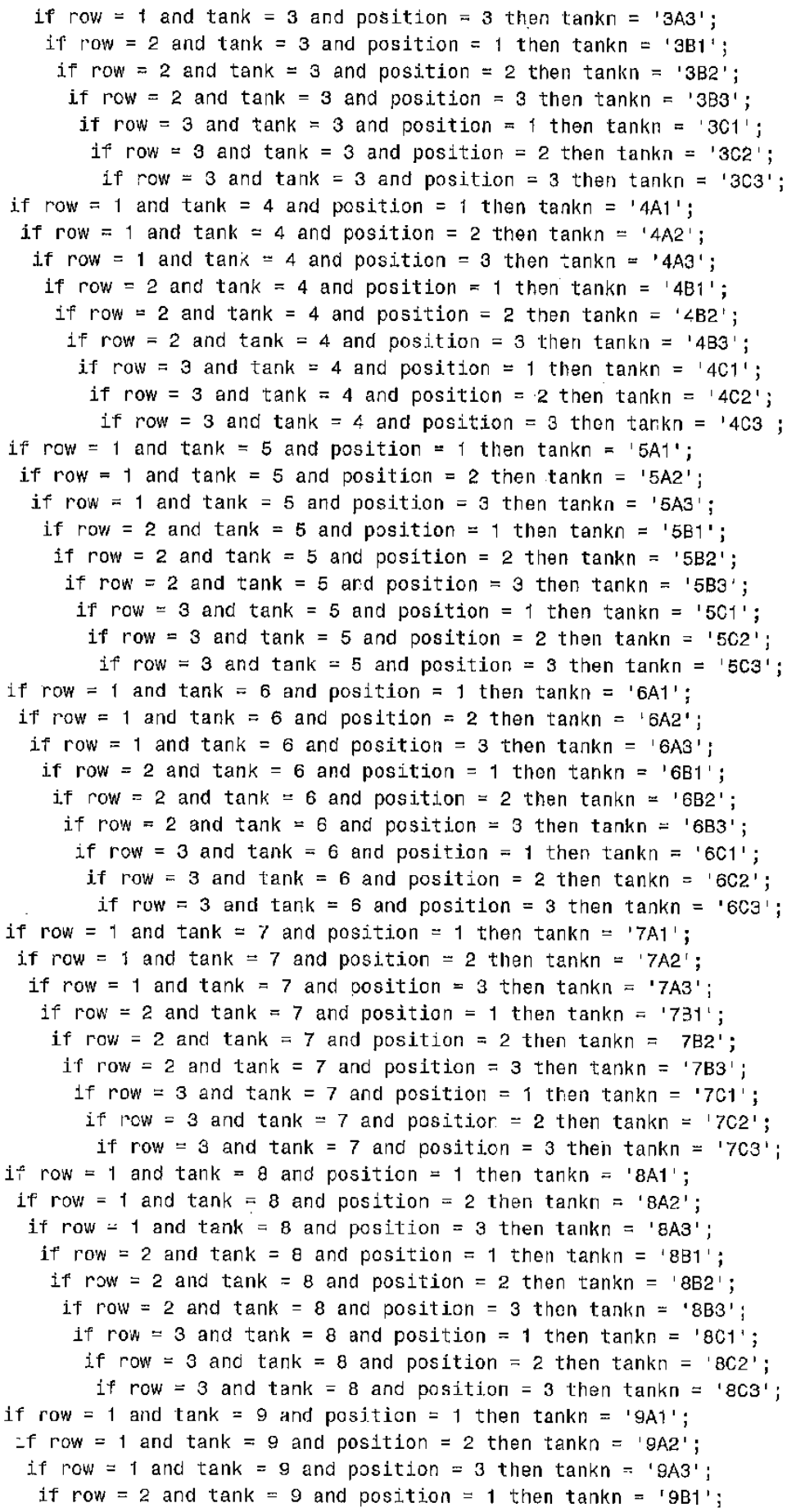




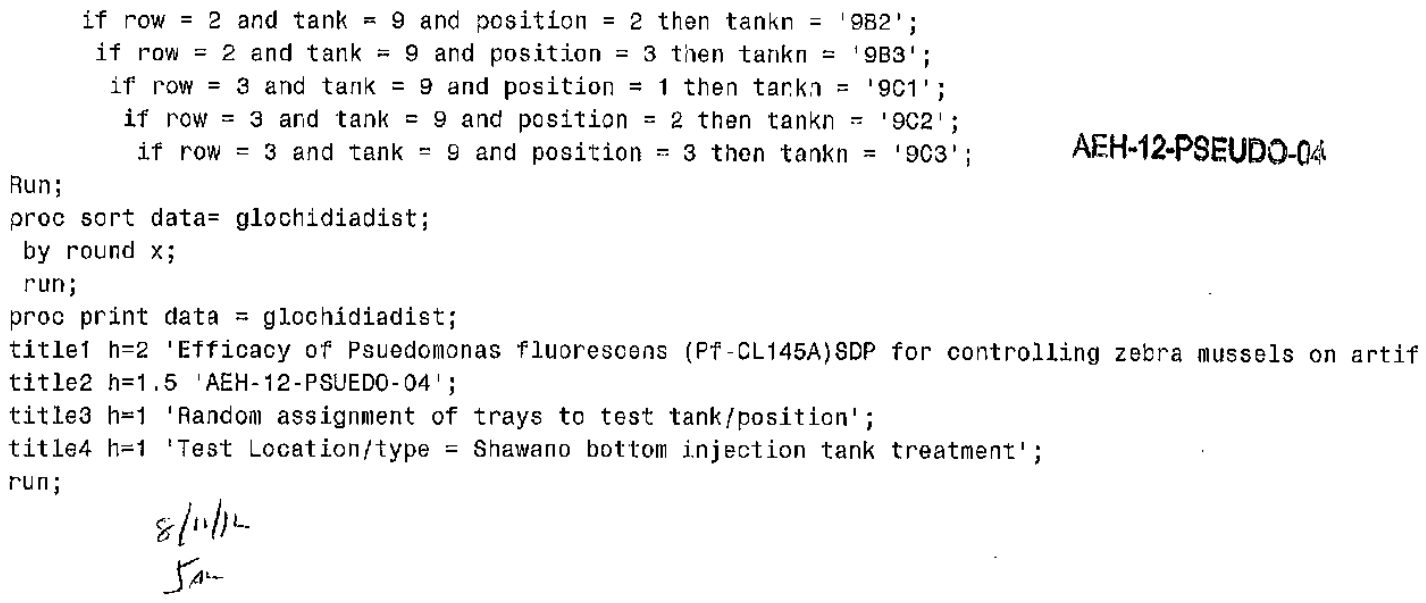




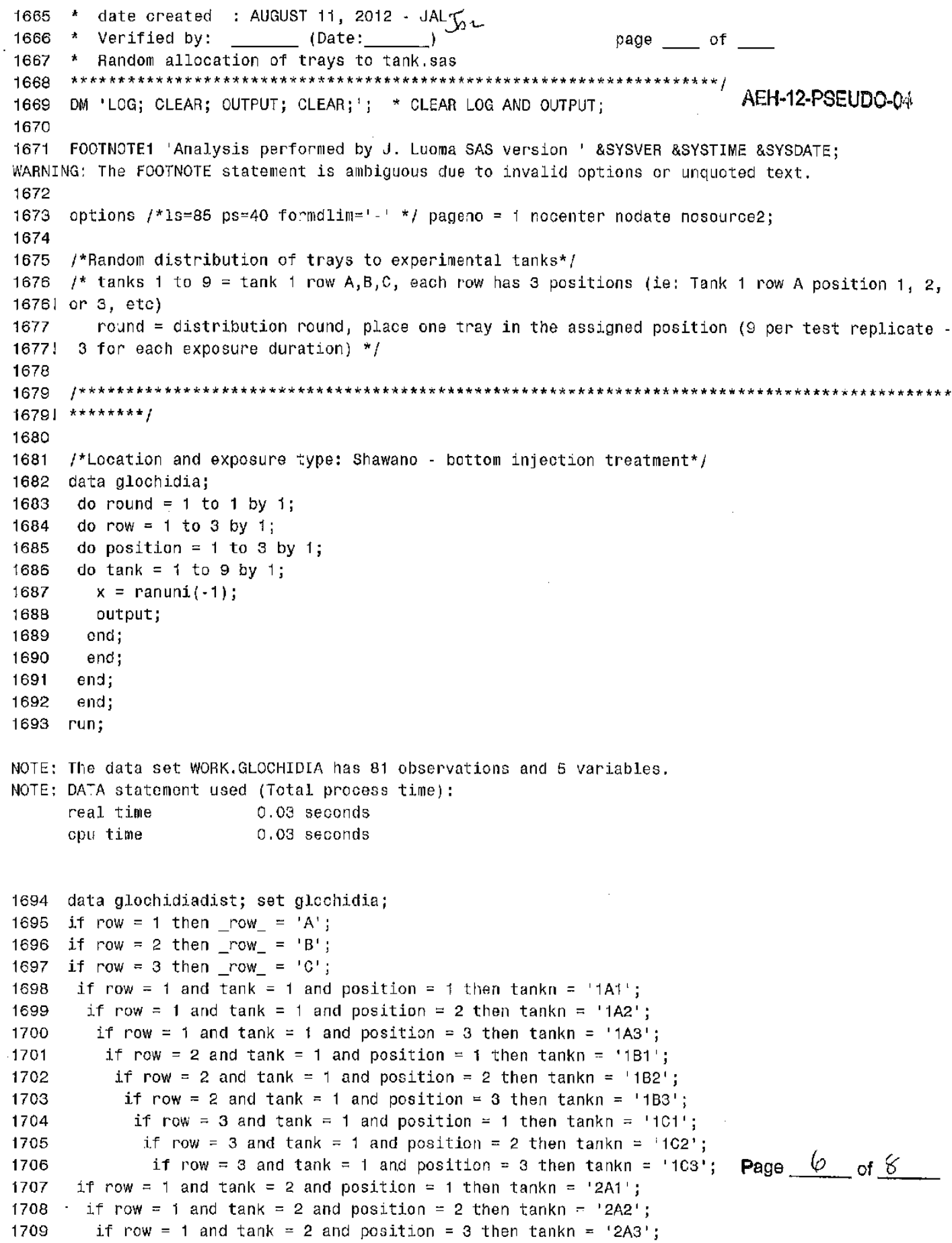




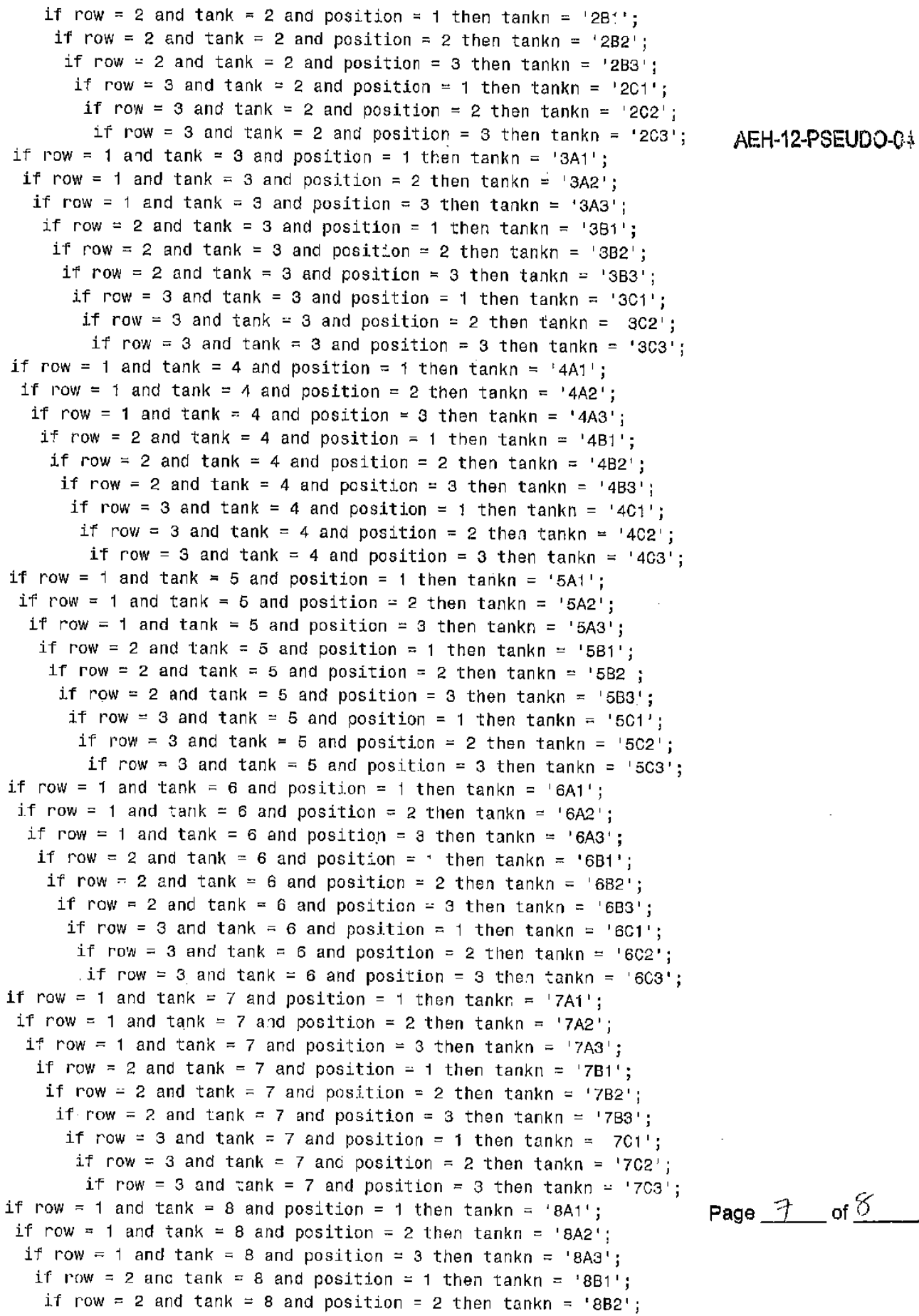




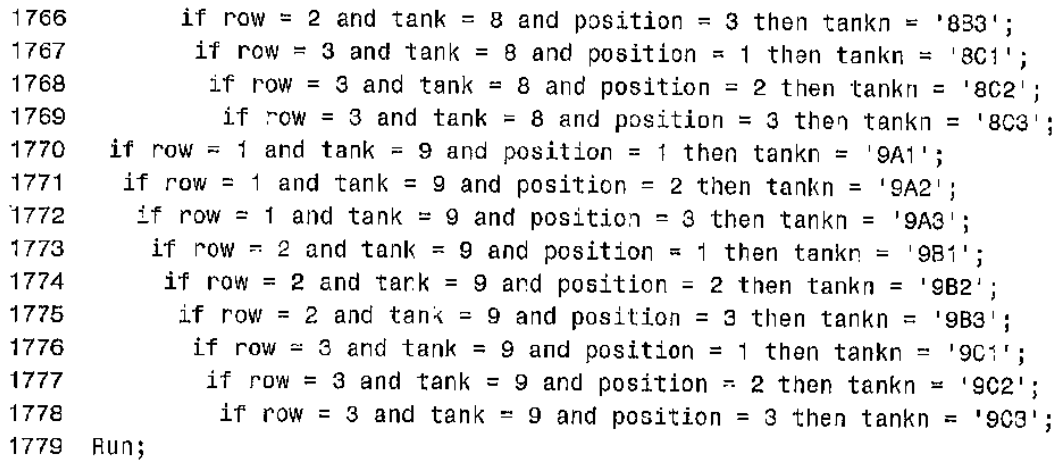

NOTE: There were 81 observations read from the data set WORK.GLOCHIDIA.

NOTE: The data set WORK.GLOCHIDIADIST has 81 observations and 7 variables.

NOTE: DATA statement used (Total process time):
real time
0.07 seconds

opu time

0.07 seconds

1780 proc sort data $=$ g.tochidiadist;

1781 by round $x$;

1782 run;

NOTE: Thero were 81 observations read from the data set WORK.GI.OCHILIADIST.

NOTE: The data set WORK.GLOCHIDIADIST has 81 observations and 7 variables.

NOTE: PROCEDURE SORT used (Total process time):

real tine $\quad 0.01$ seconds

cpu time $\quad 0.01$ seconds

1783 proc print data = glochioiadist;

1784 titlel $h=2$ 'Efficacy of Psuedomonas fliorescens (Pf-CL145A)SDP for controlling zebra mussols

1784 ! on artifical substrates';

1785 title2 $h=1.5$ 'AEH-12-PSUEDO-04',

1786 title3 $h=1$ 'Random assignment of trays to test tank/position';

1787 title4 $\mathrm{h}=1$ 'Test Location/type = Shawano bottom injection tank treatment'.

1788 run;

NOTE: There were 81 observations read fron the data set WORK.GLOCHIDIADIST.

NOTE: PROCEDURE PRINT used (Total process time):

real time $\quad 0.01$ seconds

cpu time $\quad 0.01$ seconds

$$
8 / 1 / 12
$$

$\sqrt{4} \mathrm{c}$

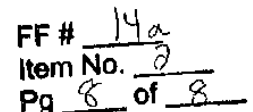


Efficacy of Pseudomonas fluorescens (Pf.CLI45A) for controlling zebra mussesl on artifical substrates AEH-12-PSEUDO-04

Random assignment of substrate removal from tanks $* * *$ TANK $1 * * *$

Shawano - bottom injection Treatment

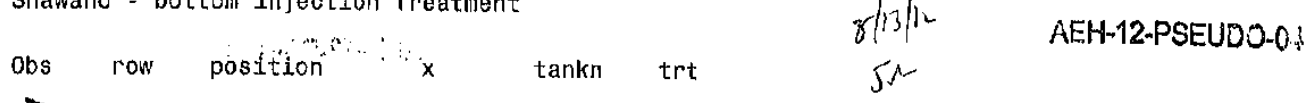

\begin{tabular}{|c|c|c|c|c|c|c|}
\hline 1 & 7 & 1 & 0.00558 & $1 \mathrm{~A} 1$ & $6 h$ & \\
\hline 2 & 2 & 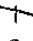 & 0.07369 & $1 \mathrm{B1}$ & $6 h$ & bottom injection \\
\hline 3 & 2 & 3 & 0.10023 & $1 \mathrm{B3}$ & $6 \mathrm{~h}$ & \\
\hline 4 & 2 & 2 & 0.27940 & $-4+B 2$ & $9 \mathrm{~h}$ & Extra \\
\hline 5 & 1 & 2 & 0.37473 & $1 \mathrm{A2}$ & $9 h=$ & bag from $9 \mathrm{~h}$ will be \\
\hline 6 & 1 & 3 & 0.48168 & 1A3 & $9 \mathrm{~h}$ & ujed byt removed at \\
\hline 7 & 3 & 2 & 0.62620 & $1 \mathrm{C} 2$ & 12 & 1) \\
\hline 8 & 3 & 3 & 0.84572 & $1 \mathrm{C3}$ & 12 & 12 h. Hw 7 SePt \\
\hline 9 & 3 & 1 & 0.87154 & $1 \mathrm{C} 1$ & 12 & \\
\hline
\end{tabular}

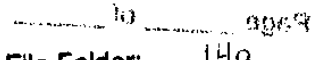

File Folder: $\quad 14 a$

Item Number: 3

Page 1 of 30 
Analysis performed by $J$. Luoma SAS version $9.210: 2013 A U G 12$ jn -

Efficacy of Pseudomonas fluorescens (Pf-CL145A)for controlling zebra mussesl on artifical substrates AEH - 12-PSEUDO - 04

Random assignment of substrate removal from tanks *** TANK 2 *** Shawano - bottom injection Treatment

\section{AEH-12-PSEUDO-0}

\begin{tabular}{|c|c|c|c|c|c|c|}
\hline Obs & row & position & $x$ & $\operatorname{tankn}$ & trt & \\
\hline 1 & & 3 & 0.26233 & $2 A 3$ & $6 \mathrm{~h}$ & \\
\hline 2 & 3 & & 0.28933 & $2 \mathrm{C} 1$ & $6 \mathrm{~h}$ & \\
\hline 3 & 3 & 3 & 0.41823 & $2 \mathrm{C3}$ & $6 \mathrm{~h}$ & * See note on pagel \\
\hline 4 & 1 & 1 & 0.56759 & $2 A 1$ & $9 \mathrm{~h}$ & $f-H a r d i$ \\
\hline 5 & 3 & 2 & 0.60252 & $2 \mathrm{C2}$ & $2 \mathrm{~h}$ & - randomizativin \\
\hline 6 & 1 & 2 & 0.63382 & $2 \mathrm{~A} 2$ & $9 \mathrm{~h}$ & (w) 7 steplo \\
\hline 7 & 2 & 1 & 0.64069 & 281 & 12 & \\
\hline 8 & 2 & 3 & 0.80439 & $2 B 3$ & 12 & \\
\hline 9 & 2 & 2 & 0.92279 & $2 B 2$ & 12 & \\
\hline
\end{tabular}

Page 2 of 30

................... Thothot ahy 
Analysis performed by $J$. Luoma SAS version $9.210: 2013 A U G 12 S^{n-}$

Efflcacy of Pseudomonas fluorescens (Pf-CL145A) for controlling zebra mussesl on artifical substrates AEH- 12 - PSEUDO-04

Random assignment of substrate removal from tanks $* * *$ TANK $3 * * *$

Shawano - bottom injection Treatment.

AEH-12-PSEUDO-0i

\begin{tabular}{llllll} 
Obs & row & position & \multicolumn{1}{l}{ tankn } & trt \\
1 & 1 & 1 & 0.16703 & $3 \mathrm{~A} 1$ & $6 \mathrm{~h}$ \\
2 & 3 & 3 & 0.19671 & $3 \mathrm{CC}$ & $6 \mathrm{~h}$ \\
3 & 1 & 3 & 0.21867 & $3 \mathrm{~A} 3$ & $6 \mathrm{~h}$ \\
4 & 2 & 3 & 0.23119 & $3 \mathrm{~B} 3$ & $9 \mathrm{~h}$ \\
5 & 3 & 1 & 0.72793 & $3 \mathrm{C} 1$ & $6 \mathrm{~h}$ \\
\hline 6 & 1 & 2 & 0.80420 & $3 \mathrm{~A} 2$ & $9 \mathrm{~h}$ \\
7 & 2 & 1 & 0.80905 & $3 \mathrm{~B} 1$ & 12 \\
8 & 3 & 2 & 0.88712 & $3 \mathrm{C} 2$ & 12 \\
9 & 2 & 2 & 0.96360 & $3 \mathrm{~B} 2$ & 12
\end{tabular}

* See note on page 1

of randomization tho

7 SEP 12

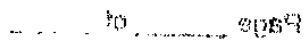

Page 3 of 30 
Analysis performed by $J$. Luoma SAS version 9.2 10:20 13AUG12 $/ \Omega^{-}$

Efficacy of Pseudomonas fluorescens (Pf-CL145A) for controling zebra mussesl on artiflcal substrates AEH-12-PSEUDO- 04

Random assignment of substrate removal from tanks *** TANK 4 ***

Shawano - bottom injection Treatment

\begin{tabular}{llllll} 
Obs & row & position & $x$ & tankn & trt \\
\hline 1 & 1 & 3 & 0.06803 & $4 A 3$ & $6 h$ \\
2 & 1 & 1 & 0.08714 & $4 A 1$ & $6 h$ \\
3 & 2 & 1 & 0.26140 & $4 B 1$ & $6 h$ \\
4 & 3 & 2 & 0.28491 & $4 C 2$ & $9 h$ \\
5 & 3 & 1 & 0.45781 & $4 C 1$ & $9 h$ \\
\hline 6 & 2 & 3 & 0.46232 & $4 B 3$ & $9 h$ \\
7 & 1 & 2 & 0.47420 & $4 A 2$ & 12 \\
8 & 2 & 2 & 0.64459 & $4 B 2$ & 12 \\
9 & 3 & 3 & 0.74556 & $4 C 3$ & 12.
\end{tabular}

AEH-12.PSEUDOOO

* See note or page lof randomization
\[ \begin{array}{c}\text { KW } \\ 75012\end{array} \] 
Analysis performed by J. Luoma SAS version $9.2 \quad 10: 20$ 13AUG12 Ja

\section{AEH-12-PSEUDO-QS}

Efficacy of Pseudomanas fluorescens (Pf-CL145A) for controlling zebra mussesl on artifical substrates AEH-12-PSEUDO-04

Random assignment of substrate removal from tanks $* * *$ TANK $5 * * *$

Shawano - bottom injection Treatment

\begin{tabular}{|c|c|c|c|c|c|}
\hline obs & row & position & $x$ & tankn & $\operatorname{tr} t$ \\
\hline 1 & 1 & 3 & 0.26233 & $012 A 3$ & $6 \mathrm{~h}$ \\
\hline 2 & 3 & 1 & 0.28933 & $2 \mathrm{C} 1$ & $6 \mathrm{~h}$ \\
\hline 3 & 3 & $3:$ & 0.41823 & $2 \mathrm{CB}$ & $6 \mathrm{~h}$ \\
\hline 4 & 1 & 1 & 0.56769 & $2 A 1$ & $9 \mathrm{~h}$ \\
\hline 5 & 3 & 2 & 0.60252 & $2 \mathrm{C} 2$ & $-9 b_{2}$ \\
\hline 6 & 1 & 2 & 0.63382 & $2 A 2$ & $9 n$ \\
\hline 7 & 2 & 1 & 0.64069 & 281 & 12 \\
\hline 8 & 2 & 3 & 0.80439 & $2 \mathrm{~B} 3$ & 12 \\
\hline $\mathbf{9}$ & 2 & 2 & 0.92279 & $2 \mathrm{~B} 2$ & 12 \\
\hline
\end{tabular}

$$
\begin{aligned}
& \text { * See note on page } \\
& 1 \text { of randomization } \\
& \text { kw } \\
& 7 \text { sepda } \\
& \text { Q1) Tank nombers should } \\
& \text { be } 5 \text { not 2. kw 7sepix } \\
& \text { See Deriation } \$ 2 \text { for } \\
& \text { furtur clanifiation } \\
& \text { kno } \\
& \text { 1900N13 }
\end{aligned}
$$




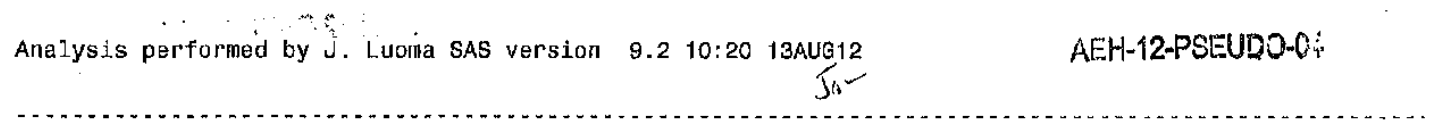

Efficacy of Pseudomonas fluorescens (P.f-CL145A)for controlling zebra mussesl on artifical substrates AEH-12-PSEUDO-04

Random assignment of substrate removal from tanks *** TANK $6 * * *$

Shawano - botton injection Treatment

\begin{tabular}{lllcll} 
Obs & row & position & $x$ & tankn & trt \\
1 & 2 & 1 & 0.18641 & $6 \mathrm{~B} 1$ & $6 \mathrm{~h}$ \\
2 & 3 & 1 & 0.22996 & $6 \mathrm{C} 1$ & $6 \mathrm{~h}$ \\
3 & 1 & 3 & 0.40217 & $6 \mathrm{~A} 3$ & $6 \mathrm{~h}$ \\
4 & 1 & 2 & 0.48348 & $6 \mathrm{~A} 2$ & $9 \mathrm{~h}$ \\
5 & 1 & 1 & 0.48881 & $6 \mathrm{~A} 1$ & $8 \mathrm{~h}$ \\
\hline 6 & 3 & 2 & 0.70464 & $6 \mathrm{C} 2$ & $9 \mathrm{~h}$ \\
7 & 2 & 3 & 0.76432 & $6 \mathrm{B3}$ & 12 \\
8 & 3 & 3 & 0.93288 & $6 \mathrm{C3}$ & 12 \\
9 & 2 & 2 & 0.96790 & $6 \mathrm{~B} 2$ & 12
\end{tabular}

* See note on page 1 of

randonitation kw 7 SEP12 
Analysis performed by.J. Luoma sas version 9.2 10:20 13aUg12 Jn'

Efficacy of Pseudomonas fluorescens (Pf-CL145A)for controlling zebra mussesl on artifical substrates AEH - 12 - PSEUDO-04

Aandom assignment of substrate removal from tanks $* * *$ TANK $7 * * *$

Shawano - botton injection Treatment

\begin{tabular}{|c|c|c|c|c|c|}
\hline Obs & row & position & $x$ & tankn & trt \\
\hline 1 & & 3 & 0.26233 & $2 A 3$ & $6 \mathrm{~h}$ \\
\hline 2 & 3 & 7 & 0.28933 & $2 \mathrm{C} 1$ & $6 \mathrm{~h}$ \\
\hline 3 & 3 & 3 & $0.4 \times 823$ & $2 \mathrm{C3}$ & $6 \mathrm{~h}$ \\
\hline 4 & 1 & 1 & 0.56759 & $2 A 1$ & $9 \mathrm{~h}$ \\
\hline 5 & 3 & 2 & 0.60252 & $2 \mathrm{C} 2$ & $-9 h$ \\
\hline 6 & 1 & 2 & 0.63382 & $2 \mathrm{~A} 2$ & gh \\
\hline 7 & 2 & 1 & 0.64069 & $2 B 1$ & 12 \\
\hline 8 & 2 & 3 & 0.80439 & 283 & 12 \\
\hline 9 & 2 & 2 & 0.92279 & 2B2 & 12 \\
\hline
\end{tabular}

* See note on page 1

of roudsmization.

kiw 7sep/2

Wank nombers should be

7 nil 2 Kuw 7 Sepla

See Deviation 2 年

furter clarification.

cmi

innovis

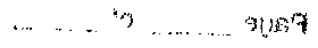

Page 7 of 30 
Analysis performed by j.'Luoma sas version $9.210: 2013 A U G 1 J^{2}$

AEH-12-PSEUDO-C4

Efficacy of Pseudomonas fluorescens (Pf-CL145A) for controliling zebra mussesl on artifical substrates AEH - 12 - PSEUDO - 04

Randoin assignment of substrate removal from tanks *** TANK 8 **

Shawano - bottom injection Treatment

\begin{tabular}{|c|c|c|c|c|c|}
\hline Obs & row & position & $x$ & $\tan k n$ & trit \\
\hline 1 & 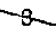 & 2 & 0.05681 & $8 \mathrm{C} 2$ & $6 \mathrm{~h}$ \\
\hline 2 & 1 & 2 & 0.07331 & $8 A 2$ & $6 \mathrm{~h}$ \\
\hline 3 & 3 & 1 & Or 33534 & $8 C 1$ & $6 h$ \\
\hline 4 & 3 & 3 & 0.41579 & $-8 \mathrm{cc} 3$ & 9h \\
\hline 5 & 1 & 1 & 0.48937 & $8 \mathrm{A1}$ & $-2 h$ \\
\hline 6 & 1 & 3 & 0.54741 & $8 \mathrm{AB}$ & $9 h$ \\
\hline 7 & 2 & 3 & 0.62872 & $8 B 3$ & 12 \\
\hline 8 & 2 & 2 & 0.64996 & $8 B 2$ & 12 \\
\hline 9 & 2 & 1 & 0.73310 & BB1 & 12 \\
\hline
\end{tabular}

* See note on page 1

of randomization. Hes $75 P_{12}$

ㄷ….... m .

Page 8 of 30 
Analysis performed by J. Luoma sAs version $9.210: 2013 A U G 12 \mathrm{~J}^{2}$.

AEH-12-PSEUDO-C:

Efficacy of Pseudomionas fluorescens (Pf-CL145A) for controlling zebra mussesl on artifical substrates AEH-12-PSEUDO-04

Random assignment of substrate removal from tanks *** TANK $9 * * *$

Shawano - bottom injection Treatment

\begin{tabular}{rrrrrr} 
Obs & row & position & $x$ & tankn & trt \\
1 & & & & & \\
2 & 3 & 3 & 0.26233 & $(1) 2 \mathrm{A3}$ & $6 \mathrm{~h}$ \\
3 & 3 & 3 & 0.28933 & $6 \mathrm{C} 1$ & $6 \mathrm{~h}$ \\
4 & 1 & 1 & 0.56759 & $2 \mathrm{~A} 1$ & $9 \mathrm{~h}$ \\
5 & 3 & 2 & 0.60252 & $2 \mathrm{C} 2$ & 9h \\
\hline 6 & 1 & 2 & 0.63382 & $2 \mathrm{~A} 2$ & $9 \mathrm{~h}$ \\
7 & 2 & 1 & 0.64069 & $2 \mathrm{~B} 1$ & 12 \\
8 & 2 & 3 & 0.80439 & $2 \mathrm{~B} 3$ & 12 \\
9 & 2 & 2 & 0.92279 & $2 \mathrm{~B} 2$ & 12
\end{tabular}

$$
\begin{aligned}
& \text { * See note on page } 1 \text { of } \\
& \text { randornitation then 7sepid } \\
& \text { (0) Tank numbers should be } \\
& 9 \text { not } 2 . \mathrm{km} 7 \text { Sep } 12 \\
& \text { See Deviation } 42 \text { for } \\
& \text { furtur clarification, } \\
& \text { ins } \\
& \text { provis }
\end{aligned}
$$

$\ldots \ldots+\ldots \ldots$

Page 9 - 


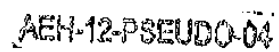

Analysis performed by J. Luoma SAS version 9.2 10:20 13AUG12 $\mathrm{JN}^{-}$

Page 10 of 30 \%s 


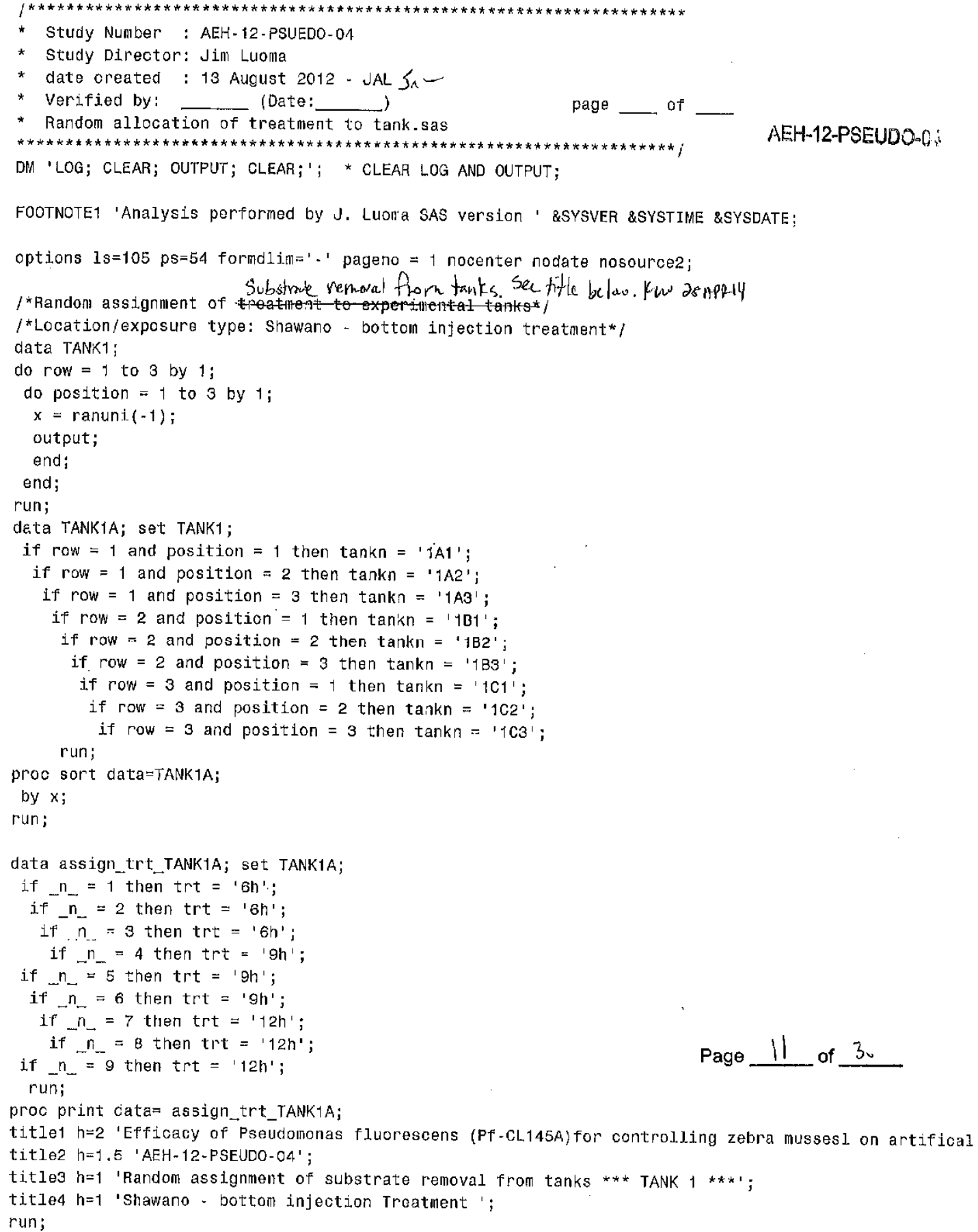




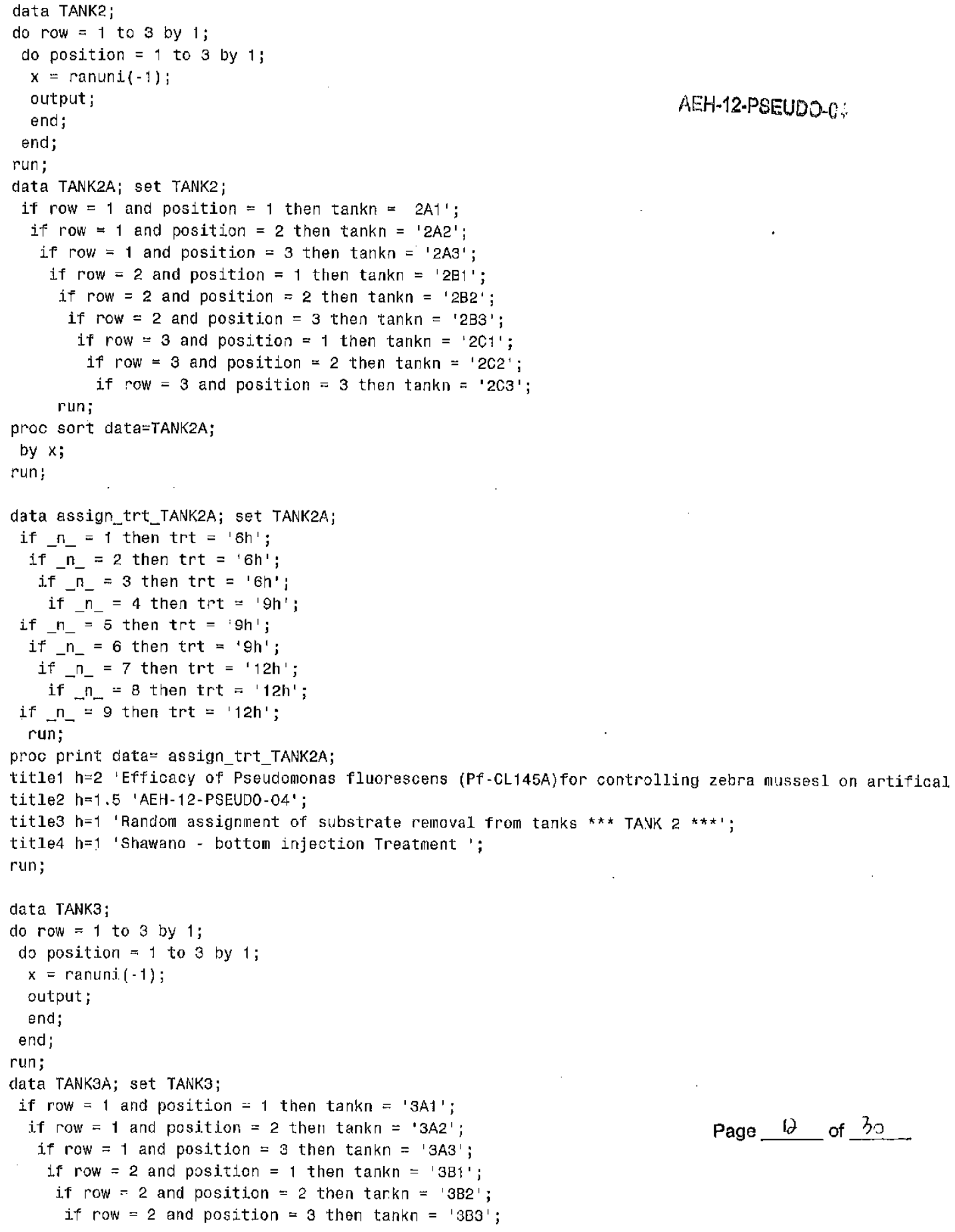




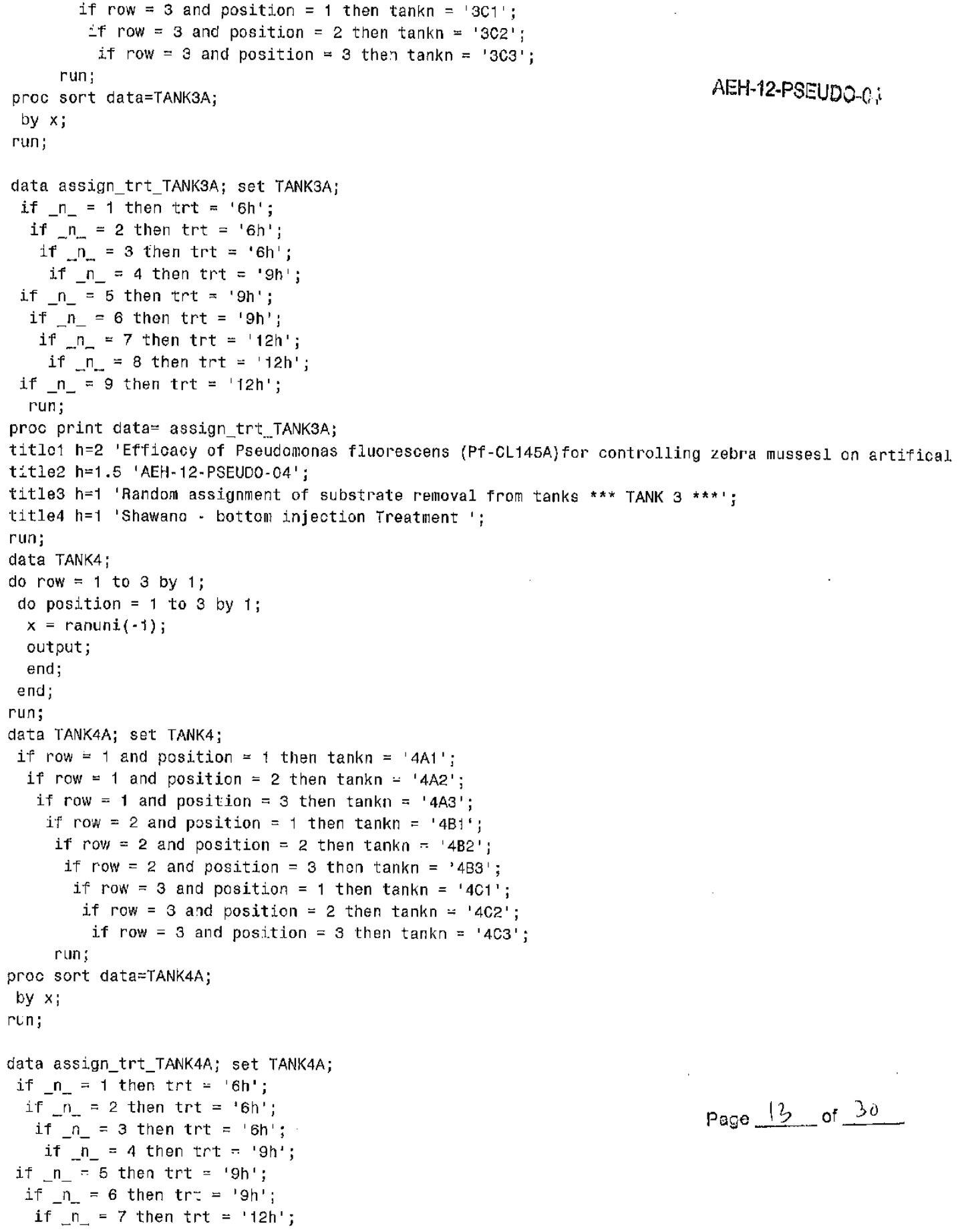




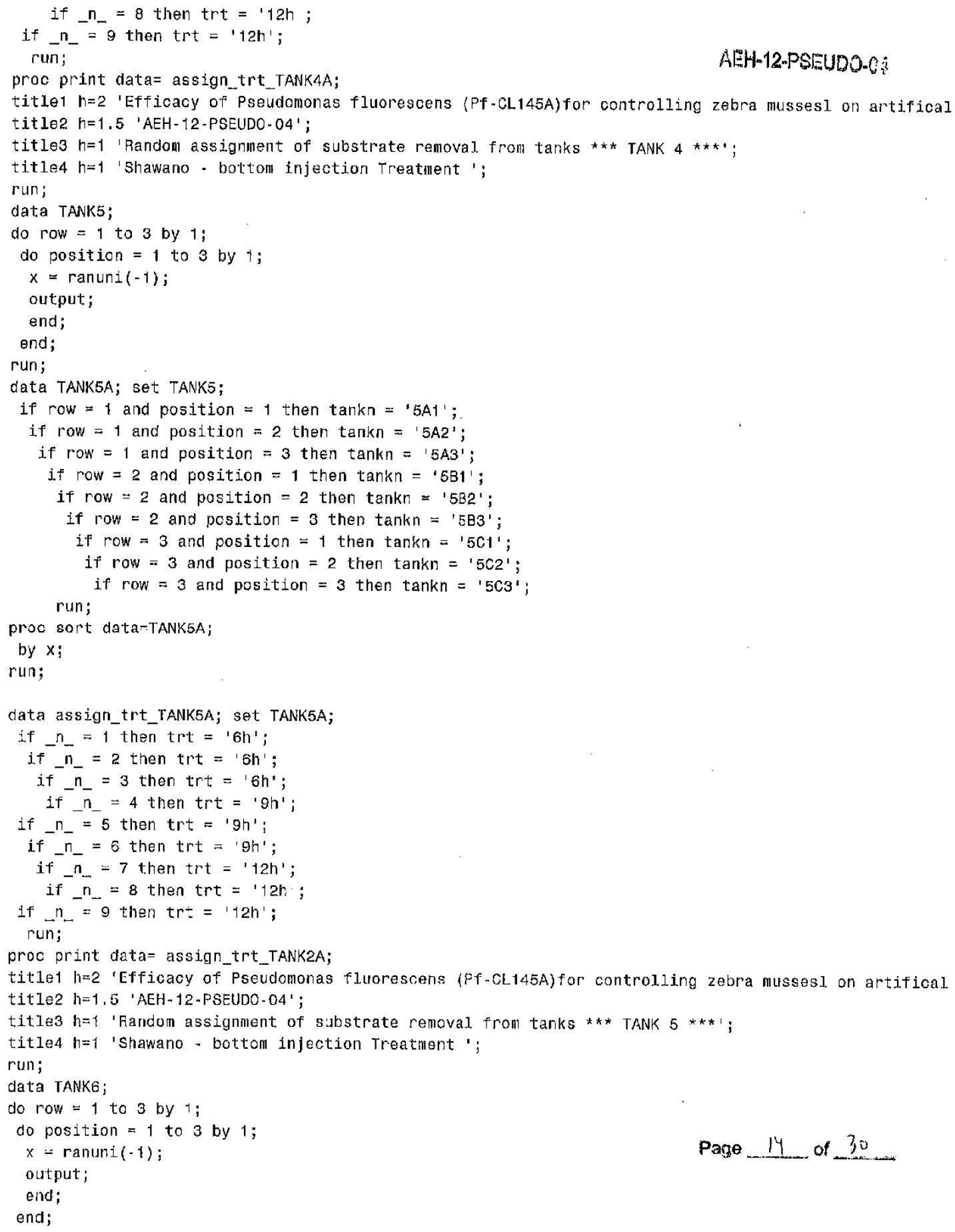




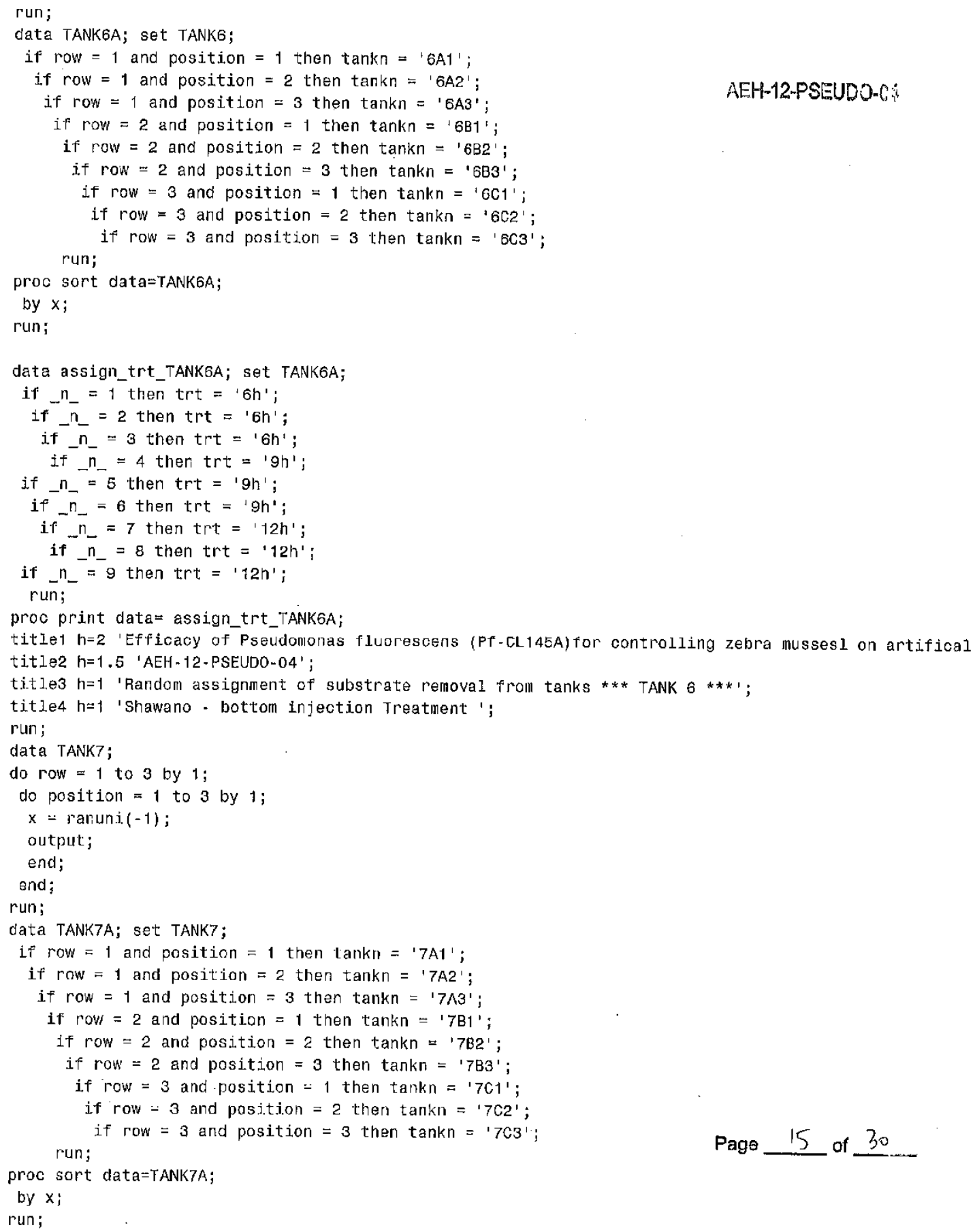




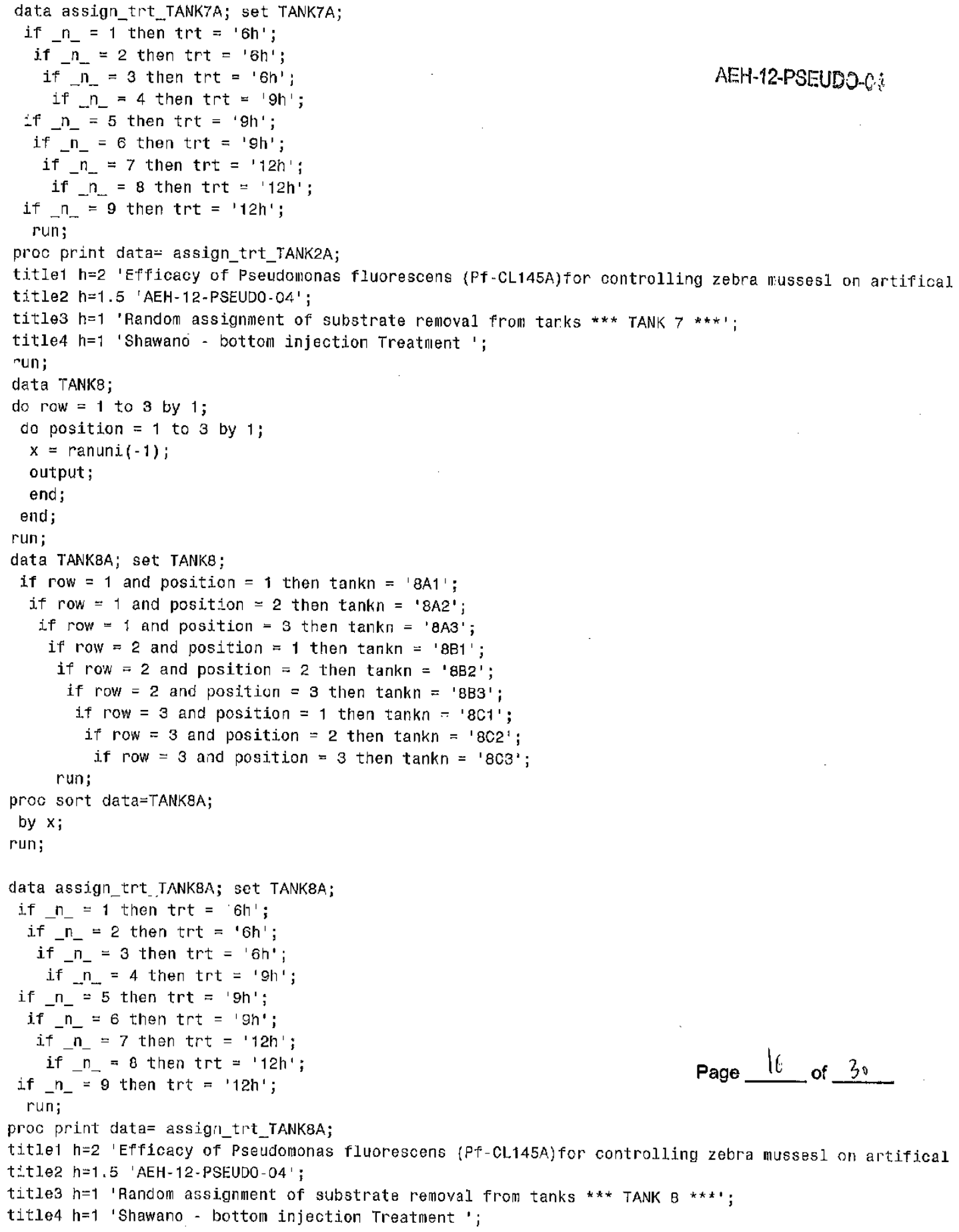




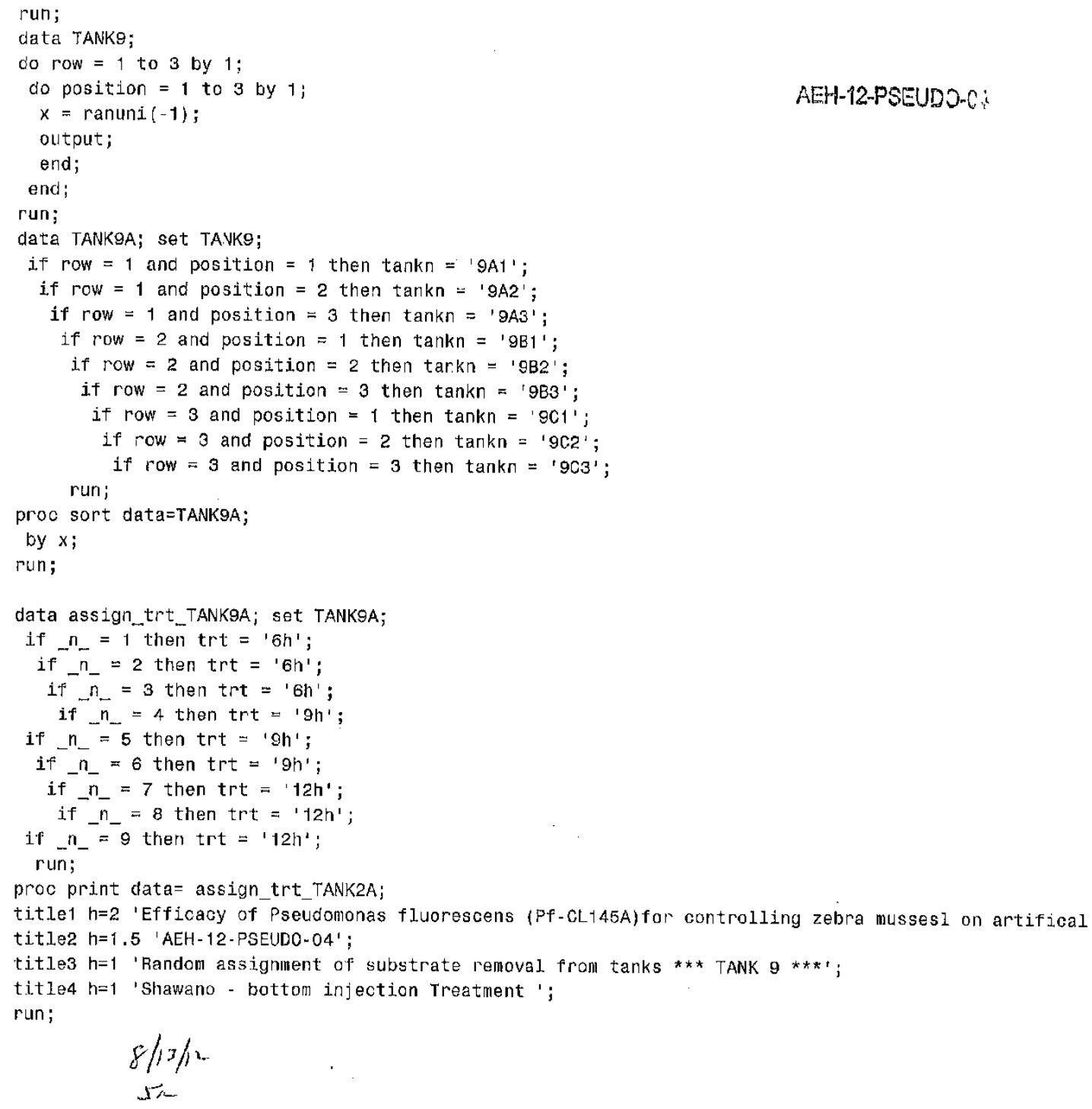




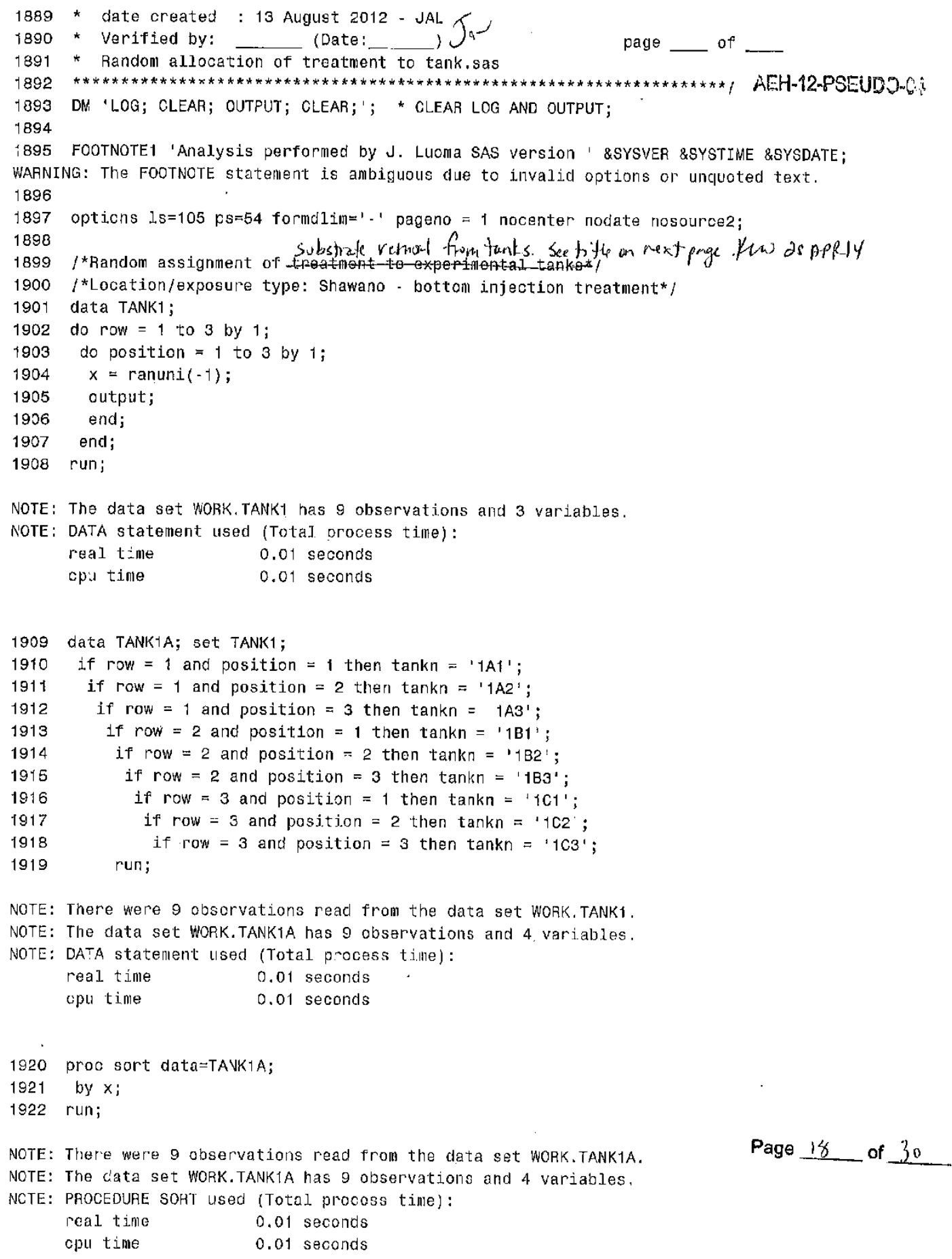




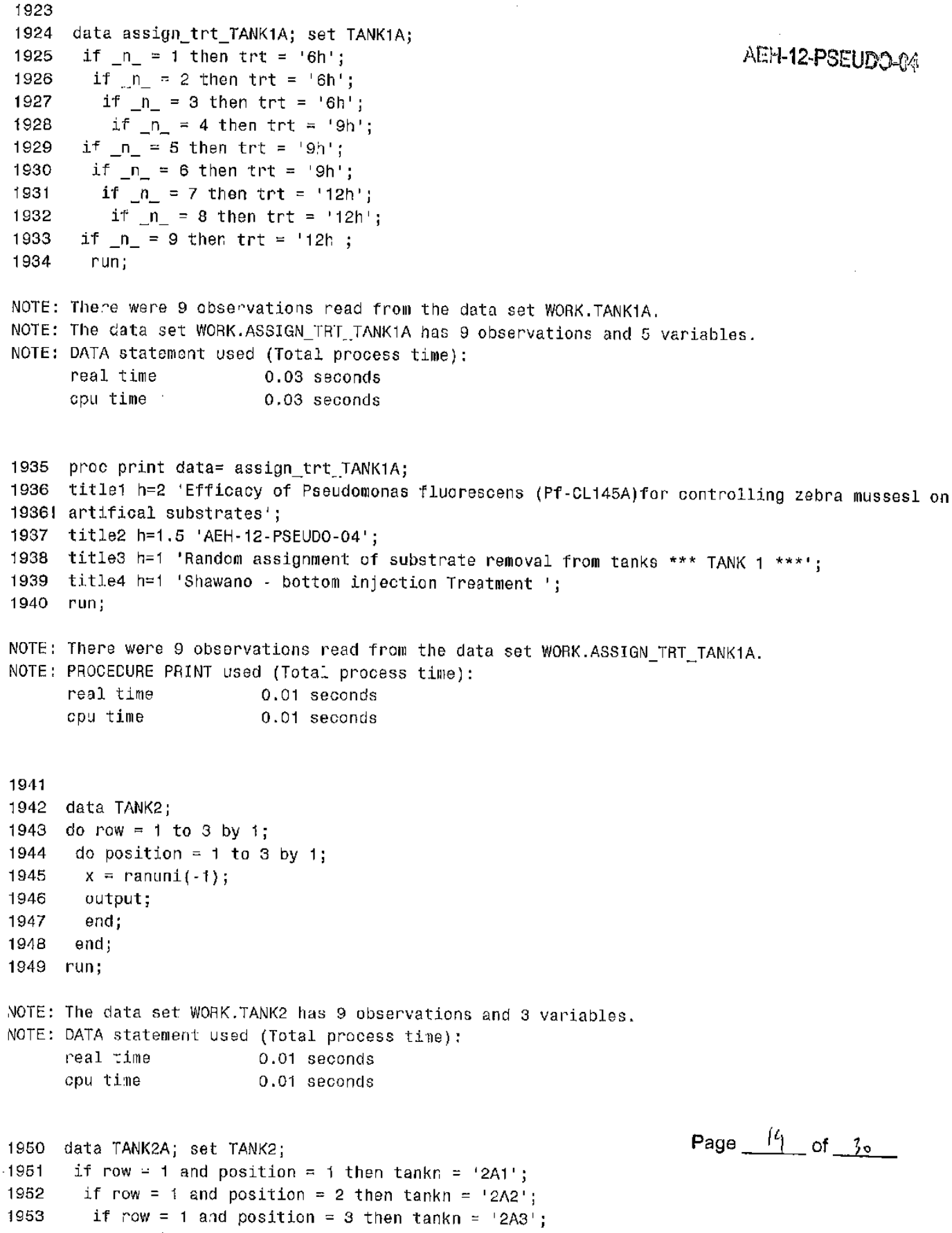




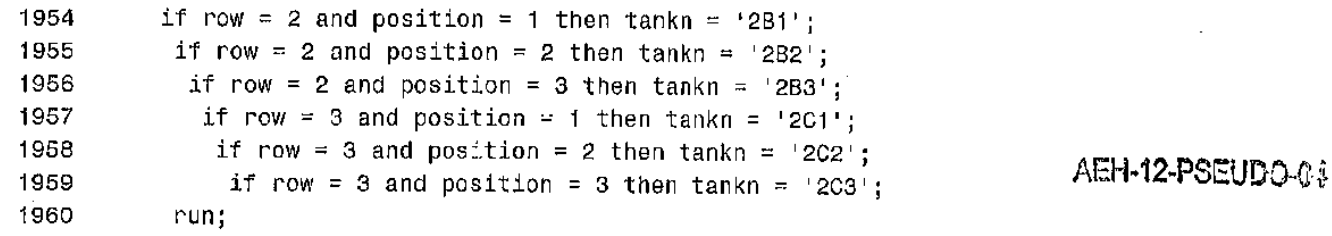

NOTE: There were 9 observations read from the data set WORK. TANK2.

NOTE: The data set WCRK. TANK2A has 9 observations and 4 variables.

NOTE: DATA statement used (Total process time):

real time $\quad 0.01$ seconds

cpu time $\quad 0.01$ seconds

1961 proc sort data=TANK2A:

1962 by $x$;

1963 run;

NOTE: There wero 9 observations read from the data set WORK. TANK2A.

NOTE: The ciata set WORK.TANKRA has 9 observations and 4 variaoles.

NOTE: PROCEDURE SORT used (Total process time):
real time
0.00 seconds
cpu time
0.00 seconds

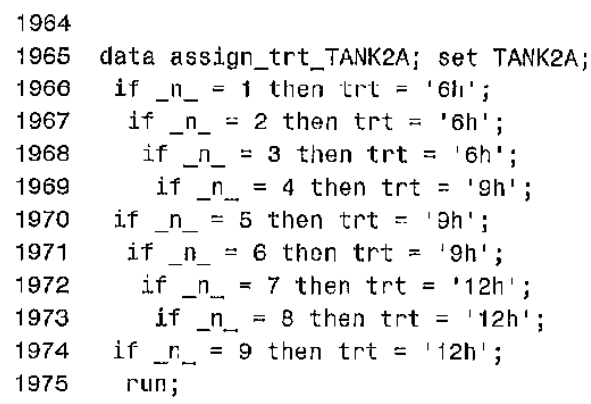

NOTE: There were 9 observations read from the data set WORK. TANK2A.

NOTE: The data set WORK.ASSIGN_TRT TANK2A has 9 observations and 5 variables.

NOTE: DATA statement used (Total process tine):
real time
0.01 seconds
cpus time
0.01 seconds

1976 proc print data= assign_trt_TANK2A;

1977 title1 h=2 'Efficacy of Pseudomonas fluorescens (Pf..CL.145A)for controlling zebra mussesl on

1977 artifical substrates';

1978 tit1e2 $h=1.5$ 'AEH-12-PSEUDO-04';

1979 title3 $h=1$ 'Ranclom assigninent of substrate removal from tanks *** TANK $2 * * *$ ';

1980 title4 $h=1$ 'Shavano - bottom injection Treatmont ';

1981 run;

NOTE: There were 9 observatiors read from the data set WORK.ASSIGN_TRT_TANK2A.

Page 20 of 30 VOTE: PROCEDURE PRINT used (Total process time): 
$\begin{array}{ll}\text { real time } & 0.00 \text { seconds } \\ \text { cpu time } & 0.00 \text { seconds }\end{array}$

1982

1983

1984

1985

1986

1987

1988

1989

1990

NOTE: The data set WORK. TANK3 has 9 observations and 3 variables.

NOTE: DATA statement used (Total process time):

real time $\quad 0.01$ seconds

cpu time $\quad 0.01$ seconds

data TANKBA; set TANK3;

if row $=1$ and position $=1$ then tankn $=$ ' $3 \mathrm{~A} 11^{\prime}$;

if row $=1$ and position $=2$ then tankn $={ }^{\prime} 3 \mathrm{~A} 2^{\prime}$;

if row $=1$ and position $=3$ then ta.kn $=' 3 \mathrm{~A} 3$ ';

if row $=2$ and position $=1$ then $\operatorname{tankn}={ }^{\prime} 3 \mathrm{~B} 1$ ';

if row $=2$ and position $=2$ then $\operatorname{tankn}=$ ' $3 \mathrm{~B} 2{ }^{\prime}$;

if row $=2$ and position $=3$ then tankn $={ }^{\prime} 3 \mathrm{~B} 3{ }^{\prime}$;

if row $=3$ and position $=1$ then tankn $=$ ' $3 \mathrm{C} 1$ ';

if row $=3$ and position $=2$ then tankn $=' 3 \mathrm{C} 2 '$ ';

if row $=3$ and position $=3$ then tankn $=' 303$ ';

run;

NOTE: There were 9 observations read from the data set WORK. TANK3.

NOTE: The data set WORK. TANK3A has 9 observations and 4 variables.

NOTE: DATA statement used (Total process time):
real time
0.03 seconds
cpu time
0.03 seconds

2002 proc sort data=TANK3A;

2003 by $x$;

2004 run;

NOTE: There were 9 observations read from the data set WOaK. TANK3A.

NOTE: The data sot WORK. TANK3A has 9 observations and 4 variables.

NOTE: PROCEDURE SORT used (Total process time):
real time
0.01 seconds
cpu time
0.01 seconds

2005

2006 data assign_trt_TANK3A; set TANK3A;

2007 if $n_{-}=1$ then trt $=$ ' $6 h^{\prime}$;

2008 if _n_ $=2$ then trt $=$ ' $6 h^{\prime}$;

2009 if $\bar{n}_{-}=3$ then trt $=' 6 \mathrm{~h}$ ';

2010 if $\vec{n}_{-}=4$ then trt $=$ ' $9 \mathrm{~h}^{\prime}$; 


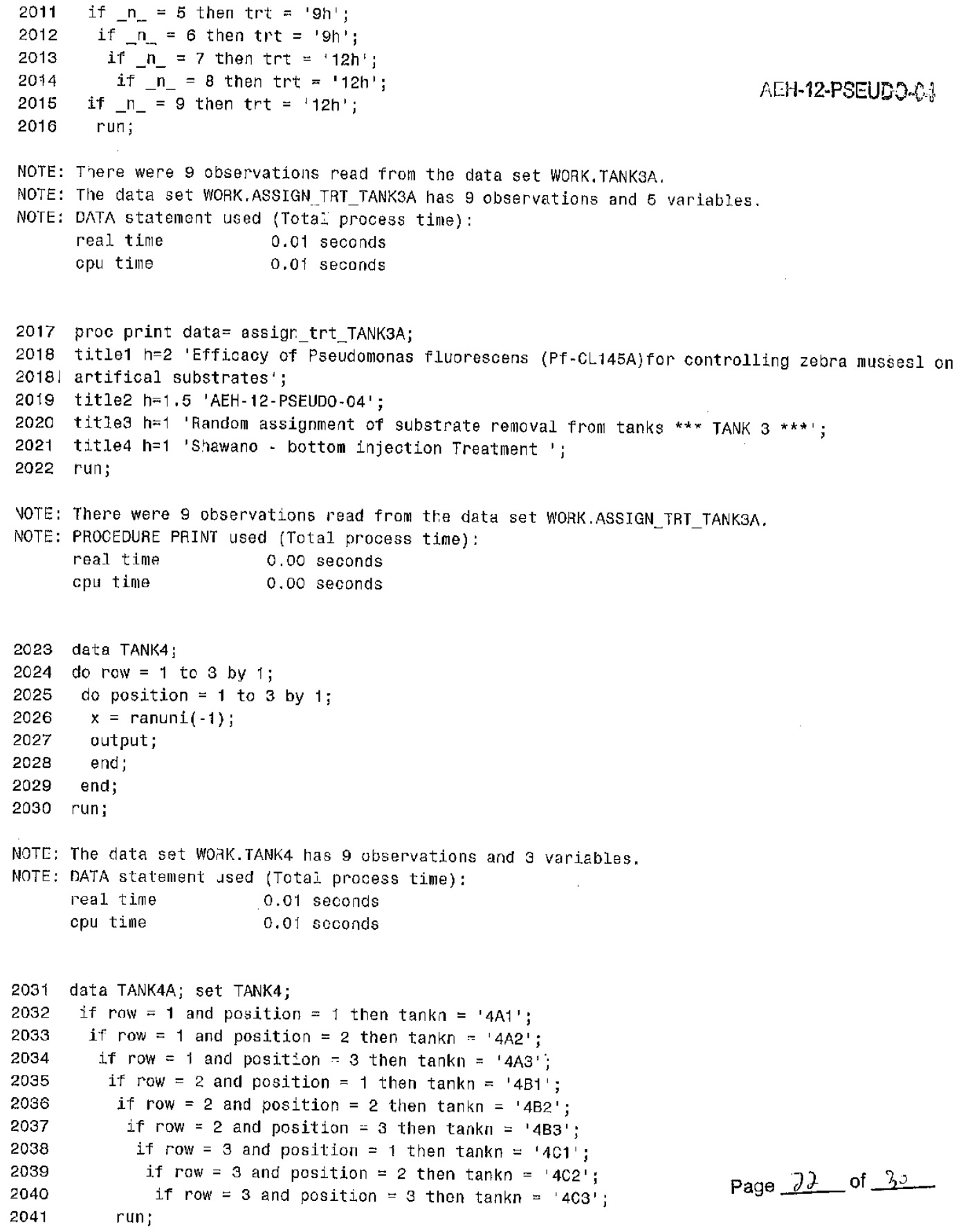

NOTE: Thoro wore 9 observations read from the data set wORK. TANK4. 
NOTE: The clata set WORK.TANK4A has 9 observations and 4 variables.

NOTE: DATA statement used (Total process time):
real time
0.01 seconds
cpd time
0.01 seconds

AEH-12-PSEUDS-O

2042 proc sort data=TANK4A;

2043 by $x$;

2044 run

NOTE: There were 0 observations read from the data set WORK. TANK4A.

NOTE: The data set WORK.TANK4A has 9 observations and 4 variables.

NOTE: PROCEDURE SORT used (Total process tille):

real time $\quad 0.01$ seconds

cpu time $\quad 0.01$ seconds

2045

2046 data assign_trt__ANK4A; set TANK4A;

2047 if $n_{-}=1$ then trt $=' 6 h^{\prime}$;

2048 if $n_{-}=2$ then trt $={ }^{\prime} 6 \mathrm{~h}^{\prime}$;

2049 if $n_{-}=3$ then trt $={ }^{\prime} 6 h^{\prime}$;

2050 if $\bar{n}_{-}=4$ then trt $={ }^{\prime} 9 \mathrm{~h}^{\prime}$;

2051 if $n_{-}=5$ then trt $=$ ' $Q \mathrm{~h}^{\prime}$;

2052 if $\bar{n}_{-}=6$ then trt $=$ ' $9 \mathrm{~h}^{\prime}$;

2053 if $\mathrm{n}_{-}=7$ then trt $=112 \mathrm{~h}$ ';

2054 if $\bar{n}_{-}=8$ then trt $=112 \mathrm{~h} '$;

2055 if $n_{-}=9$ then trt $=12 h^{\prime}$.

2056 run;

NOTE: There were 9 observations read from the data set WORK. TANK4A.

NOTE: The data set WORK.ASSIGN_TRT_TANK4A has 9 observaticns and 5 variables.

NOTE: DATA statement used (Total process time):
real time
0.01 seconds
cpu $\leftarrow \mathbf{i m e}$
0.01 seconds

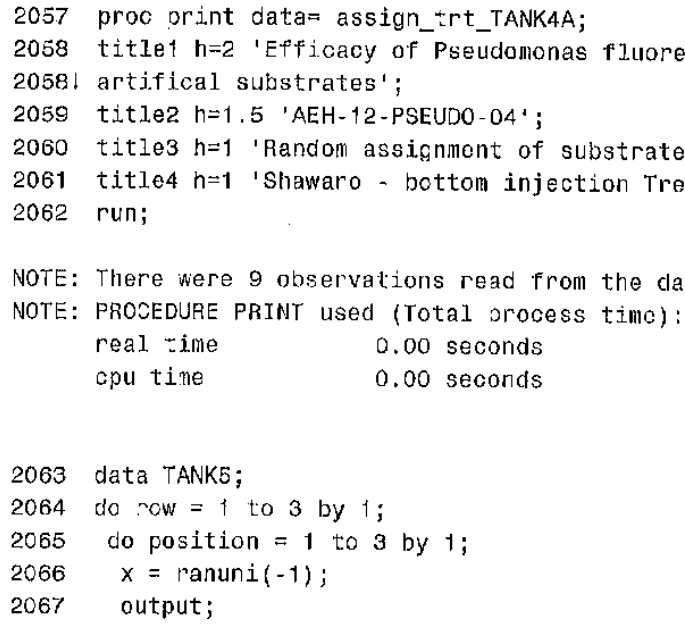




$\begin{array}{ll}2068 & \text { end; } \\ 2069 & \text { end; } \\ 2070 & \text { run; }\end{array}$

NOTE: The data set NORK. TANKS has 9 observations and 3 variabjes.

NOTE: DATA statement used (Total process time):
real tine
0.01 seconds
cpu time
0.01 seconds

2071 data TANK5A; set TANK5;

2072 if row $=1$ and position $=1$ then tankn = ' $5 \mathrm{~A} 1$ ';

2073 if row $=1$ and position $=2$ then tankn $=15 \mathrm{~A} 2$ ';

2074 if row $=1$ and position $=3$ then tankn $=' 5 \mathrm{~A} 3$ ' ;

2075 if row $=2$ and position $=1$ then tankn $=$ ' $5 B 1$ ';

2076 if row $=2$ and position $=2$ then tankn $=$ ' $5 \mathrm{B2}$ ';

2077 if row $=2$ and position $=3$ then tankn $=$ ' $5 \mathrm{~B} 3{ }^{\prime}$;

2078 If row $=3$ and position $=1$ then tankn $=' 5 \mathrm{C} 1$ '

2079 If row $=3$ and position $=2$ then tankn $=' 5 \mathrm{C} 2$ ';

2080

2081

if row $=3$ and position $=3$ then tankn $=' 503$ '; run;

NOTE: There were 9 observations read from the data set WORK. TANK5. NOTE: The data set WORK.TANK5A has 9 observations and 4 variables.

NOTE: DATA statement ised (Total process time):

real time $\quad 0.01$ seconds

cpu time $\quad 0.01$ seconds

2082 proc sort data=TANK5A;

2083 by $x$;

2084 run;

NOTE: There were 9 observations read from the data set WORK. TANK5A.

NOTE: The data set WORK. TANK5A has 9 observations and 4 variables.

NOTE: PROCEDURE SORT used (Total process time):

real time $\quad 0.00$ seconcls

cpu time $\quad 0.00$ seconds

2085

2086 data assign_trt. TANK5A; sot TANK5A;

2087 if $r_{-}=1$ then trt $={ }^{\prime} 6 h^{\prime}$;

2088 if $\bar{n}_{-}=2$ then trt $={ }^{\prime} 6 h^{\prime}$;

2089 if $n_{-}=3$ then trt $=$ ' $6 \mathrm{~h}$ ';

2090 if $\bar{n}_{-}=4$ then tr.t $=9 \mathrm{~h}^{\prime}$;

2091 if $n_{-}=5$ then trt $=19 \mathrm{n}^{\prime}$;

2092 if $n_{-}=6$ then trt $=$ ' $9 \mathrm{~h}^{\prime}$;

2093 if $n_{-}=7$ then trt $=112 h^{\prime}$;

2094 if ${ }^{n}+=8$ then trt $=112 h^{\prime}$;

2095 if $n_{-}=9$ then trt $=112 \mathrm{~h}$ ';

2096 run;

Page 24 of 30

NOTE: There were 9 observations read from tre data set WORK. TANK5A.

NOTE: The data set WORK.ASSIGN_TRT_TANK5A has 9 observations and 5 varjables.

NOTE: DATA statement used (Total process time): 
$\begin{array}{ll}\text { real time } & 0.01 \text { seconds } \\ \text { cpu time } & 0.01 \text { seconds }\end{array}$

AEH-12-PSEUEDC:

2097 proc print data= assign trt TANK2A;

2098 tithle1 h=2 'Efficacy of Pseudomonas fluorescens (Pf.CL145A)for controlliog zebra mussesl on 2098 ! artifical substrates';

2099 title2 $h=1.5$ 'AEH-12-PSEUDO-04';

2100 title3 $h=1$ 'Random assignment of substrate removal srom tanks *** TANK $5 * * * 1$;

2101 title4 $h=1$ 'Shawano - botton injection Treatment ' ;

2102 run;

NOTE: There were 9 observations read from the data set WORK.ASSIGN_TRT_TANK2A.

NOTE: PROGEDURE PRINT used (Total process time):

real time $\quad 0.01$ seconds

cpu time $\quad 0.01$ seconds

2103 data TANK6;

2104 do row $=1$ to 3 by 1 ;

2105 do position $=1$ to 3 by 1 ;

$2106 \quad x=$ ranuni $(-1)$;

2107 output;

2108 end;

2109 end;

2110 run;

NOTE: The data set WORK. TANK6 has 9 observations and 3 variables.

NOTE: DATA statement usod (Total process time):
real time
0.01 seconds
cpu tine
0.01 seconds

2111 data TANK6A; set TANK6;

2112 if row $=1$ and position $=1$ then tankn $=' 6 \mathrm{~A} 1$ ';

2113 if row $=1$ and position $=2$ then tankn $=$ ' $6 \mathrm{~A} 2$ ';

21.14 if row $=1$ and position $=3$ then tankn $={ }^{\prime} 6 \mathrm{~A} 3^{\prime}$;

2115 if row $=2$ and position $=1$ then tankn $=' 6 B 1 '$;

2116 if row $=2$ and position $=2$ then tankn $={ }^{\prime} 6 \mathrm{~B} 2$ ';

2117 if row $=2$ and position $=3$ then tankn $={ }^{\prime} 6 \mathrm{~B} 3^{\prime}$;

2118 If row $=3$ and position $=1$ then tankn $=' 6 C 1$ ';

2119 if row $=3$ and position $=2$ then tankn $=$ ' $6 \mathrm{C} 2$ ';

2120

2121

if row $=3$ and position $=3$ then tankn $={ }^{\prime} 6 \mathrm{CC} 3^{\prime}$; run;

NOTE: There were 9 observations read from the data set WORK. TANK6.

NOTE: The clata set WORK. TANK6A has 9 observations and 4 variables.

NOTE: DATA statement used (Total process time):
real time
0.01 seconds
cpu time
0.01 seconds

2122 proc sort data=TANK6A;

Page 25 of 30

2123 by $x$;

2124 run; 
NOTE: There were 9 observations read from the data set WORK. TANKGA.

NOTE: The data set WORK.TANKGA has 9 observations and 4 variables.

NOTE: PROCEDURE SORT used (Total process tinc):

$\begin{array}{ll}\text { real time } & 0.00 \text { seconds } \\ \text { opu time } & 0.00 \text { seconds }\end{array}$

AEH-12-PSEUDNA:

2125

2126 data assign_trt_TANK6A; set TANK6A;

2127 if _n_ = 1 then trt $=$ ' $6 \mathrm{~h} '$;

2128 if $\bar{n}_{-}=2$ then trt $=$ ' $6 \mathrm{~h}$;

2129 if $\bar{n}_{-}=3$ then trt $=$ ' $6 \mathrm{~h}$ ';

$2130 \quad$ if $\bar{n}_{-}=4$ then trt $=$ ' $9 \mathrm{~h}^{\prime}$;

2131 if $n_{-}=5$ then trt $=$ 'gh':

2132 if $\bar{n}_{-}=6$ then trt $={ }^{\prime} 9 \mathrm{~h}^{\prime}$;

2133 if $n_{-}=7$ then $t r t=12 h^{\prime}$;

2134 if $\bar{n}_{-}=8$ then trt $=112 \mathrm{~h}$ ';

2135 if $n_{-}=9$ then trt $=12 \mathrm{~h}^{\prime}$;

2136 run;

NOTE: There were $\mathbf{g}$ observations read from the data set WOAK. TANKEA.

NOTE: The data set WORK.ASSIGN_TRT_TANK6A has 9 observations and 5 variables.

NOTE: DATA statement used (Total process time):

real time $\quad 0.01$ seconds

cpu time $\quad 0.01$ seconds

2137 proc print data= assign_trt_TANK6A;

2138 title1 h=2 'Efficacy of Pseudomonas fluorescens (PF-GL145A)for controlling zebra mussesl on

21381 artifical substrates';

2139 title2 $h=1.5$ 'AEH-12-PSEUDO-04';

2140 ti.tles $h=1$ 'Random assignment of substrate removal from tanks *** TANK $6 * * * 1$;

2141 ti.tle4 $\mathrm{h}=1$ 'Shawano - bottom injection Treatment ';

2142 run;

NOTE: There were 9 observations read from the data set WOAK.ASSIGN_TRT_TANK6A.

NOTE: PAOCECURE PRINT used (Total process time):

real time $\quad 0.00$ seconds

cpu time $\quad 0.00$ seconds

2143 data TANK7;

2144 do row $=1$ to 3 by 1 ;

$2: 45$ do position $=1$ to 3 by 1 ;

$2: 46 \quad x=\operatorname{ranuni}(-1)$;

2147 output;

2148 end;

2149 end;

2150 run;

NOTE: The data set WORK. TANK7 has 9 observations and 3 variables.

NOTE: DATA statement used (Total process time):
real time
0.01 seconds
cpu time
0.01 seconds

Page $\partial x$ of 30 


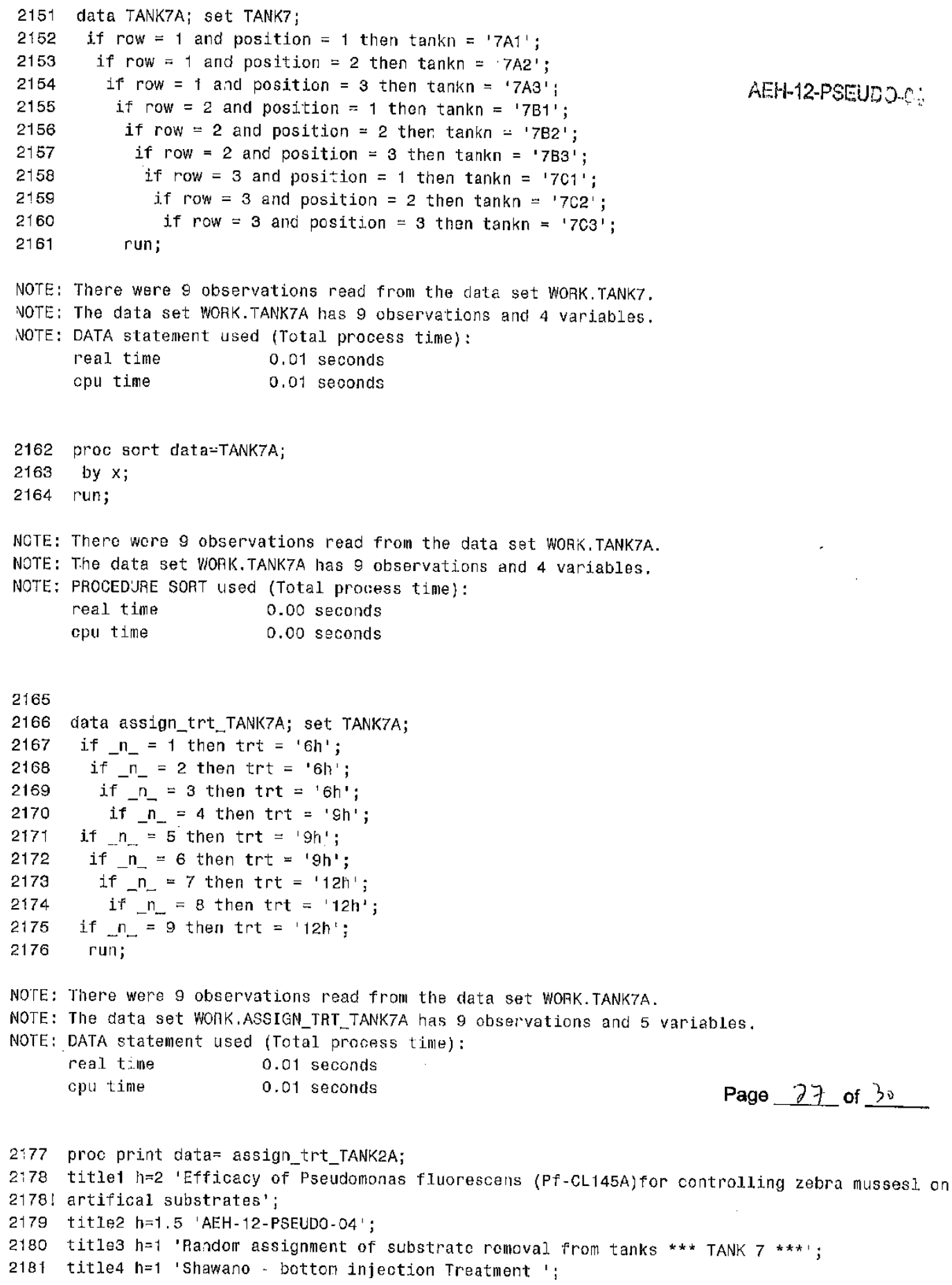


2182 run;

NOTE: There were 9 observations read from the data set WORK.ASSIGN_TRT_TANK2A.

NOTE: PROCEDURE PRINT used (Total process time):
real time
0.00 seconds
cpu time
0.00 seconds

AEH 12-PSEUSOS:

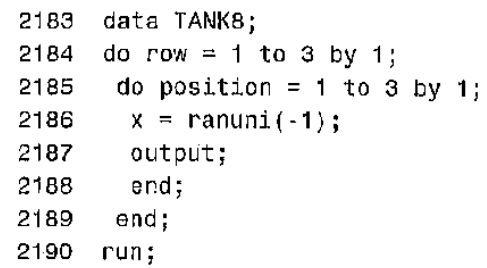

NOTE: The data sot WORK. TANK8 has 9 observations and 3 variables.

NOTE: DATA statement used (Total process time):
real time
0.01 seconds
cpu time
0.01 seconds

2191 data TANK8A; sot TANK8;

2192 if row $=1$ and position $=$; then $\operatorname{tankn}={ }^{\prime} 8 \mathrm{~A} 1$ ';

2193 if row $=1$ and position $=2$ then tankn $=$ ' 8 A2';

2194 if row $=1$ and position $=3$ then tankn $={ }^{\prime} \mathrm{BA}^{\prime}$ ';

2195 if row $=2$ and position $=1$ then tankn $=$ ' $8 \mathrm{~B} 1$ ';

2196 If row $=2$ and position $=2$ then tankn $={ }^{\prime} 8 \mathrm{~B} 2{ }^{\prime}$;

2197 if row $=2$ and position $=3$ then tankn $=$ ' $8 \mathrm{BB}$ ';

2198 if row $=3$ and position $=1$ then tankn $=$ ' $8 \mathrm{C} 1$ ' ';

2199 If row $=3$ and position $=2$ then tankn $=$ ' $8 \mathrm{C} 2$ ';

2200

2201 if row $=3$ and position $=3$ then tankn $=' 8 \mathrm{Cs}$ ';

NOTE: There were 9 observations read from the data sot WORK. TANK8, NOTE: The data set NORK. TANKBA has 9 observations and 4 variables. NOTE: DATA statement used (Total process time):

real time $\quad 0.01$ seconds

cpu time $\quad 0.01$ seconds

2202 proc sort data=TANK8A;

2203 by $x$;

2204 run;

NOTE: There were 9 observations read from the data set WOFK. TANK8A.

NOTF: The data set WORK. TANK8A has 9 observations and 4 variables.

NOTE: PROCECURE SORT used (Total process timo):

real time $\quad 0.00$ seconds

cpu time $\quad 0.00$ seconds

Page 28 of 30

2205

2206 data assign_trt_TANK8A; set TANK8A;

2207 if $n_{-}=1$ then trt $=$ ' $6 h^{\prime}$; 


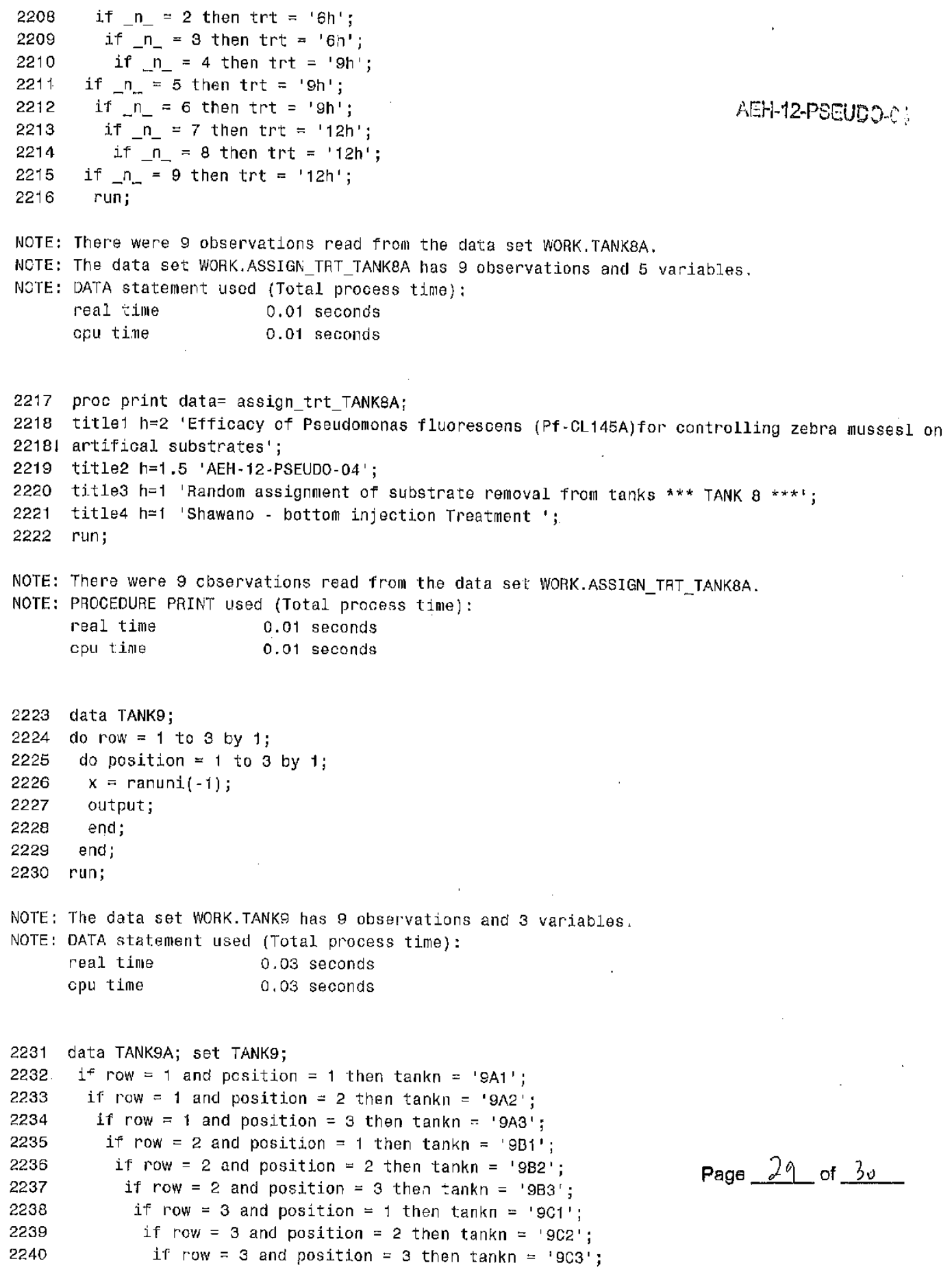

NOTE: There were 9 observations read from the data set WORK.TANKBA.

NOTE: The data set WORK.ASSIGN_TAT_TANK8A has 9 observations and 5 variables.

NOTE: DATA statement used (Total process tine): 


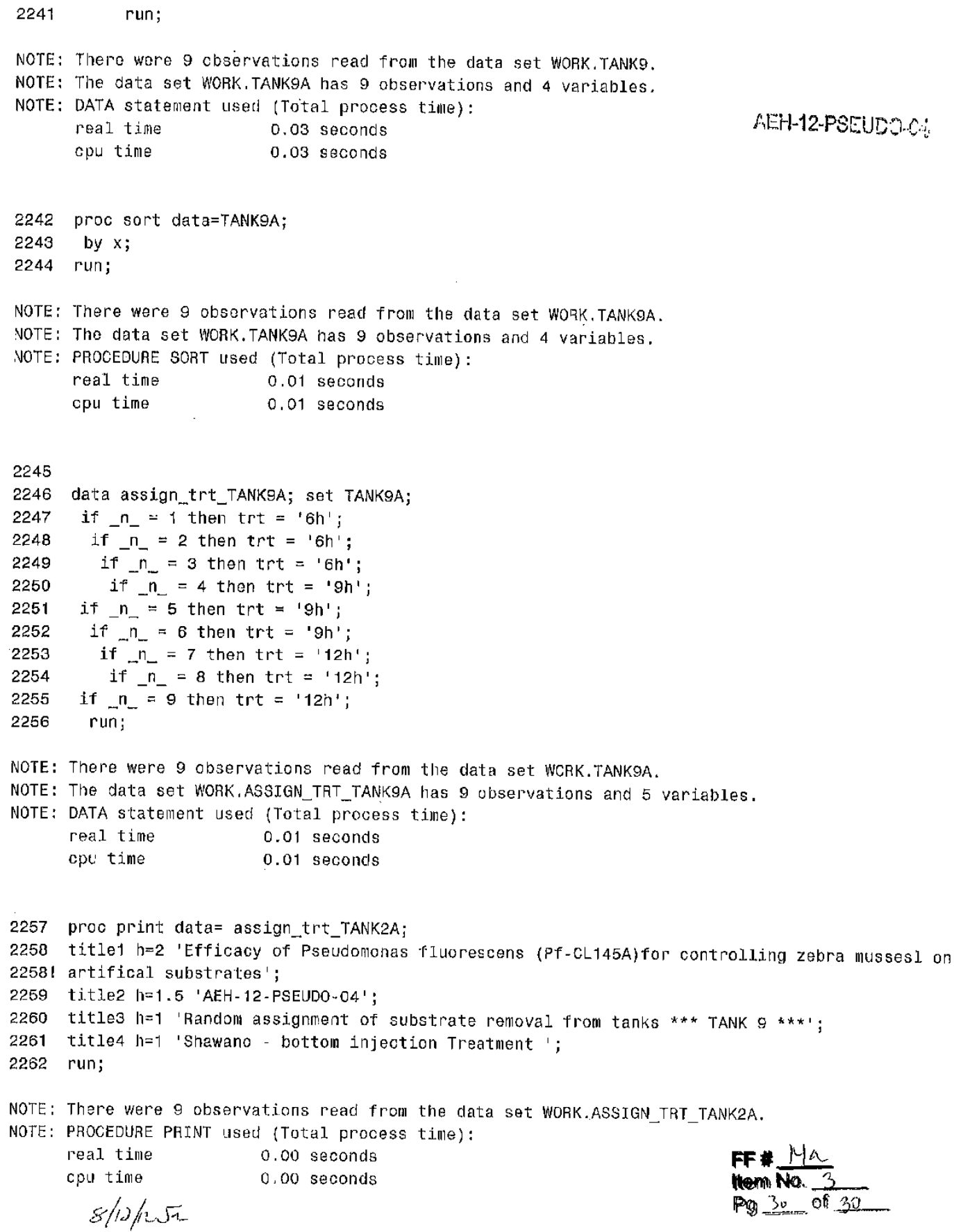

NOTE: This SAS session is using a registry in wORK. All changes will be lost a: the end of this sess 


\section{Appendix 4. Test Article Information}

\begin{tabular}{|c|c|c|c|}
\hline $\begin{array}{l}\text { Item } \\
\text { Number }\end{array}$ & Item Description & $\begin{array}{c}\text { Number } \\
\text { of } \\
\text { Pages }\end{array}$ & $\begin{array}{l}\text { Report } \\
\text { Page } \\
\text { Number }\end{array}$ \\
\hline 1 & Material Safety Data Sheet: MBI-401 SDP & 2 & 240 \\
\hline 2 & $\begin{array}{l}\text { MBI-401 SDP (lots \# 401P12163C and 401P12164C) Test Article: UPS Next Day Air } \\
\text { Label (shipped to Jim Luoma) }\end{array}$ & 1 & 242 \\
\hline 3 & MBI-401 SDP (lot \# 401P12163C) Test Article: Certificate of Analysis & 1 & 243 \\
\hline 4 & MBI-401 SDP (lot \# 401P12164C) Test Article: Certificate of Analysis & 1 & 244 \\
\hline 5 & “Test Chemical Stock Preparation Data Form” Datasheet (Lake Carlos) & 1 & 245 \\
\hline 6 & “Test Chemical Stock Preparation Data Form” Datasheet (Lake Shawano) & 1 & 246 \\
\hline 7 & $\begin{array}{l}\text { FedEx Priority Overnight Airbill (shipped to Denise Mayer) for post-treatment product } \\
\text { validation - dated January 8, } 2013\end{array}$ & 1 & 247 \\
\hline 8 & $\begin{array}{l}\text { NYSM Post -Treatment Product Validation Assay MBI-401 SDP lot \#(s) 401P12163C } \\
\text { and 401P12164C Mix }\end{array}$ & 3 & 248 \\
\hline 9 & $\begin{array}{l}\text { Copy of test article log book for MBI-401 SDP; lot \#(s) 401P12163C and 401P12164C } \\
\text { Mix; Container } 1 \text { of } 6 \text { (Used for Lake Carlos) }\end{array}$ & 5 & 251 \\
\hline 10 & $\begin{array}{l}\text { Copy of test article log book for MBI-401 SDP; lot \#(s) 401P12163C and 401P12164C } \\
\text { Mix; Container } 2 \text { of } 6 \text { (Used for Lake Carlos) }\end{array}$ & 4 & 256 \\
\hline 11 & $\begin{array}{l}\text { Copy of test article log book for MBI-401 SDP; lot \#(s) 401P12163C and 401P12164C } \\
\text { Mix; Container } 3 \text { of } 6 \text { (Used for Lake Shawano) }\end{array}$ & 4 & 260 \\
\hline 12 & $\begin{array}{l}\text { Copy of test article log book for MBI-401 SDP; lot \#(s) 401P12163C and 401P12164C } \\
\text { Mix; Container } 4 \text { of } 6 \text { (Used for Lake Shawano) }\end{array}$ & 4 & 264 \\
\hline 13 & $\begin{array}{l}\text { Copy of test article log book for MBI-401 SDP; lot \#(s) 401P12163C and 401P12164C } \\
\text { Mix; Container } 6 \text { of } 6 \text { (Used for Lake Carlos water analysis by RMB Environmental } \\
\text { Laboratories) }\end{array}$ & 4 & 268 \\
\hline
\end{tabular}




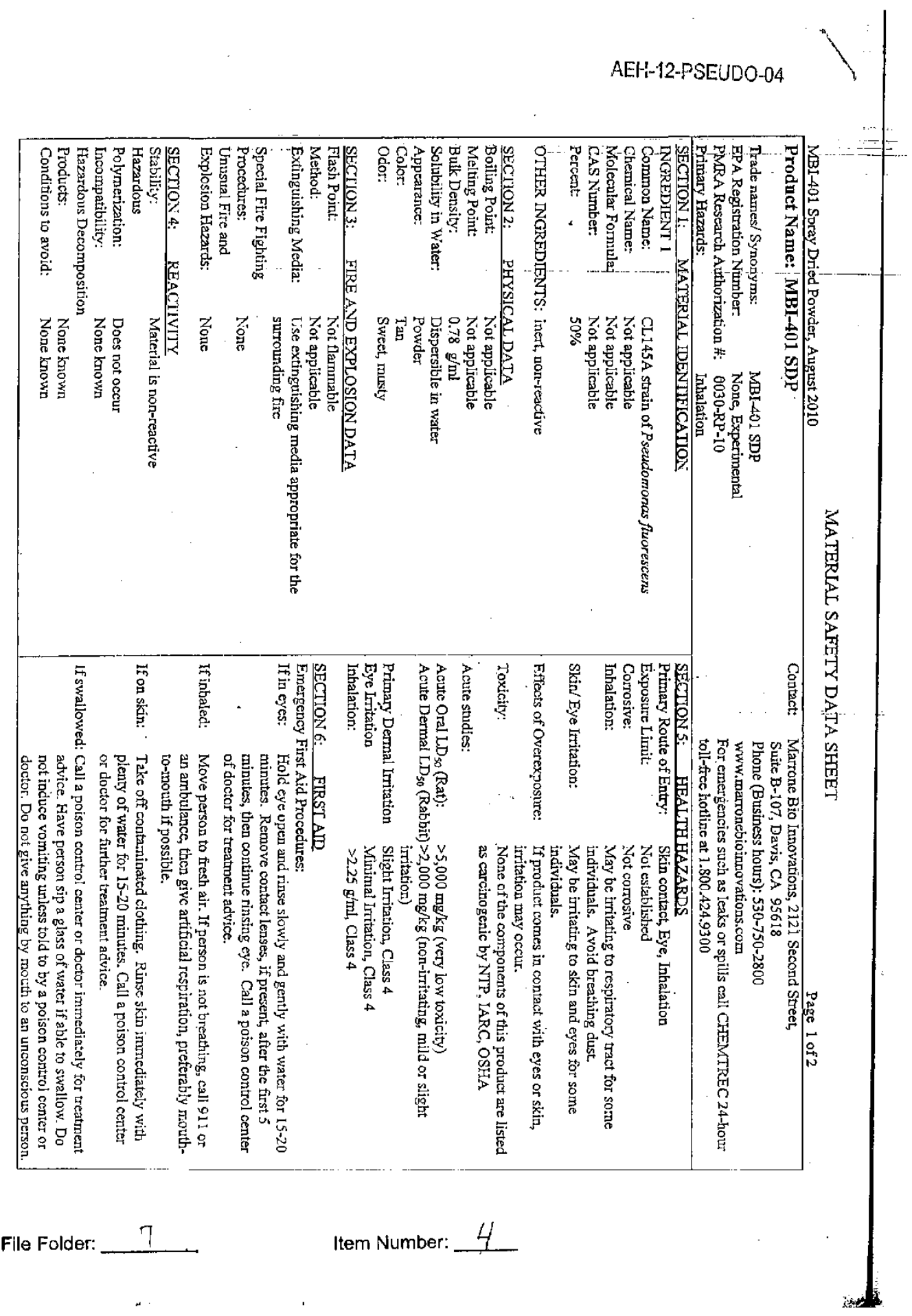

Page 240 of 519 


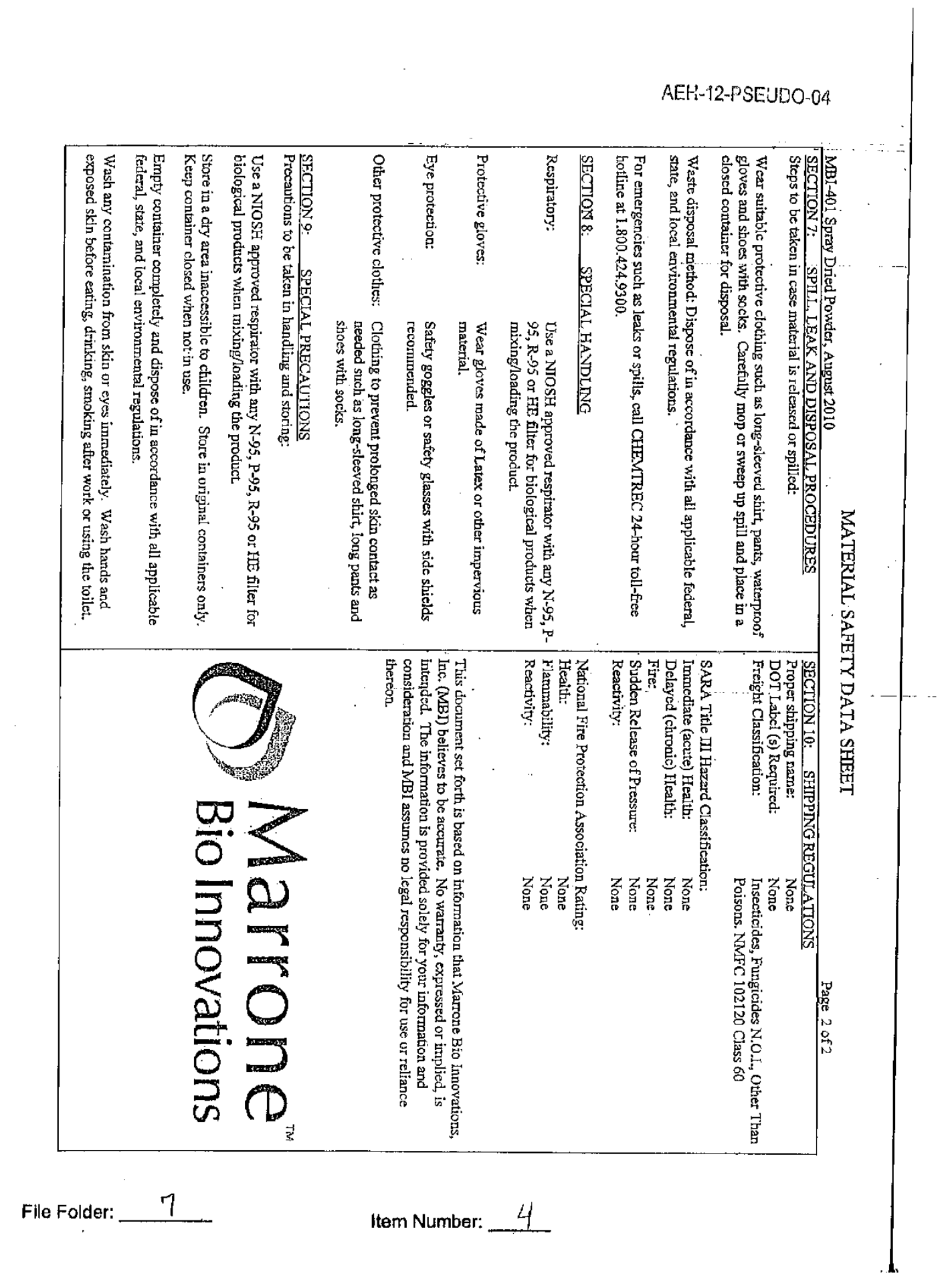




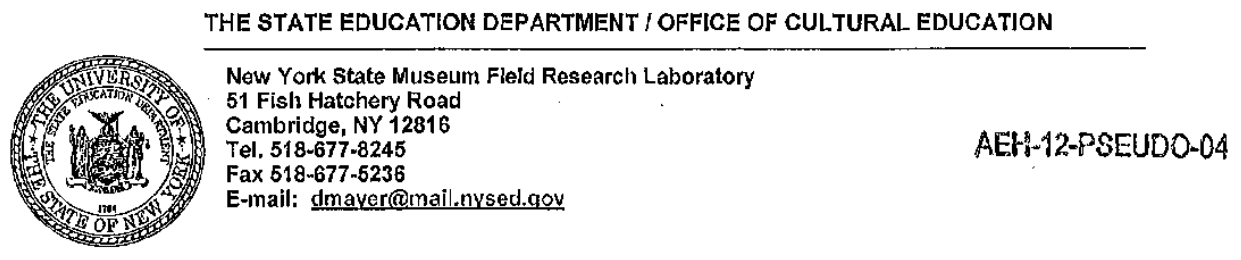

PACKING LIST

DATE OF SHIPMENT - 2012/08/06

Ship from:

Denise Mayer

New York State Museum

Field Research Laboratory

51 Fish Hatchery Road

Cambridge, NY 12816
Ship To:

Jim Luoma

USGS UMESC

2630 Fanta Reed Road

La Crosse, WI 54603

Shipping Method: UPS Next Day Air

Required by: 2012/08/07

Item Description:

MBI-401 SDP Lot \# 401P12163C and 401P12164C Mix in six containers of approximately 0.5 $\mathrm{Kg}$ each (total weight $3 \mathrm{Kg}$ ):

Packaged by D. Mayer.

Shipped on ice. Store at $4^{\circ} \mathrm{C}$, protected from light.

Manufactured: 06-21-2012

Expiration date 06-21-2013

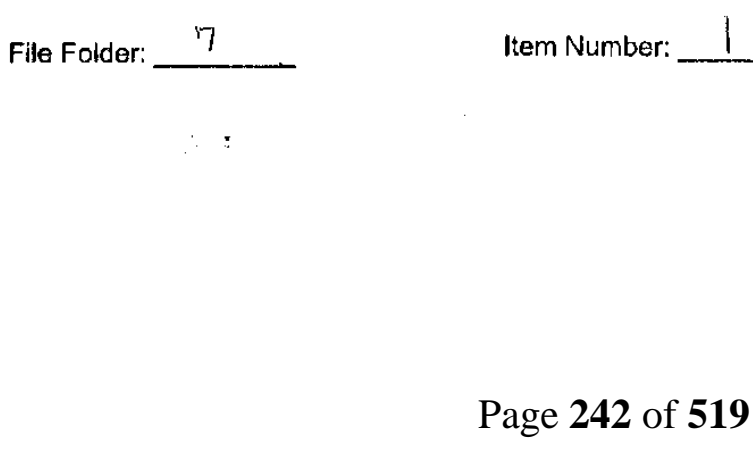




\section{(1) Marrone Bio Innovations}

AEF-12-PSEUDO-04

\section{CERTIFICATE OF ANALYSIS}

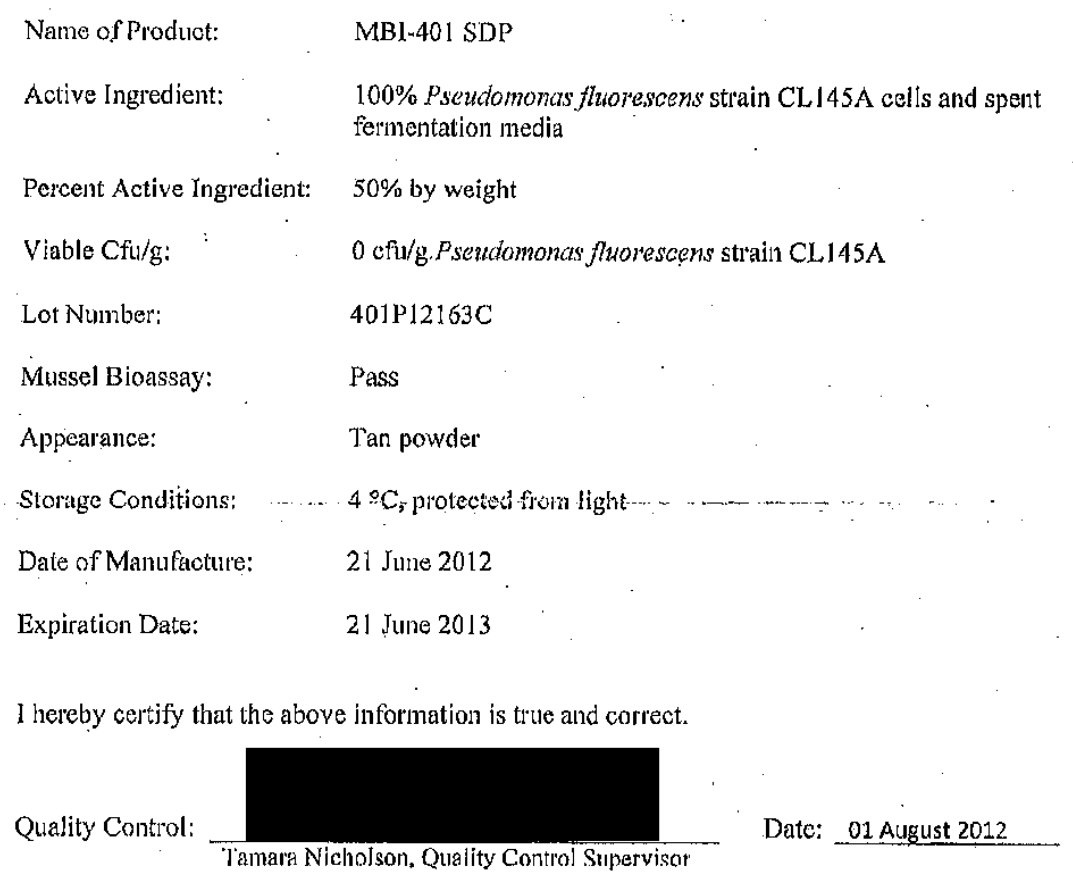

2121 Second Street, Suite B-107

File Folder: 7 $\begin{array}{r}\text { - Dnvis, CA 95618 Phone: 530-750-2800 } \\ \text { wirong irem rumber. } 1 / 27 / 14 \\ \hline 2 \text { is correct. Trs }\end{array}$ ltem Number: $x_{2} 2$ is correct. Page 1 


\title{
CERTIFICATE OF ANALYSIS
}

\author{
AEH-12-PSEUDO-04
}

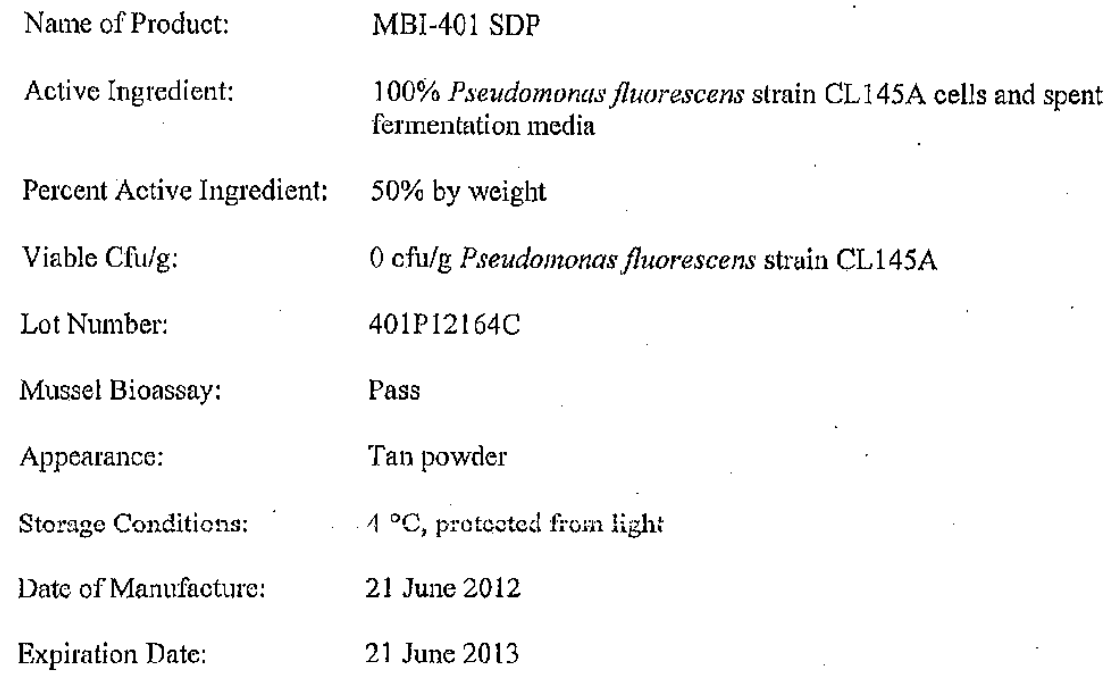

I hereby certify that the above information is true and correct.

Quality Control:

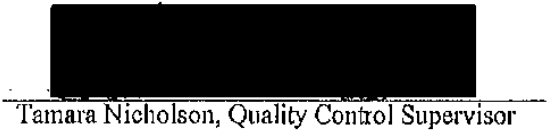

Date: 01 August 2012

2121 Second Street, Suite 13-107

File Folder:
- Davis, CA 95618

Item Number: 3
- Phone: 530-750-2800

Page 1 of 1 
Study Number: AEH-12-PSEUDO-04 Flle folder: 7 _ Lab book/pgs: E-loci pagesill

Revlewed by:_Th _ Date: 16 TaN14 Verifled by: $\sqrt{2}$ Date: $2 / 11 / 4$

\section{Test Chemical Stock Preparation Data Form}

\section{Test Chemical: $\rho_{\text {seudomonas fluroescens strain } 145 \mathrm{~A}}$}

Test Chemical Lot \#: 401P12163C and 401P12164C Mix Date Rec'd 7-Aug-12 Exp. Date 21-Jun-12 Test Organism : zebra mussels Test Location: Lake Curlus, Alexandriz, in w

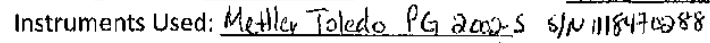

Sartorids CPA. $225 D$ S/N JUADO130

Weights of Chemical Samples:

\begin{tabular}{|c|c|c|c|c|}
\hline Sample ID & Sample Welght & Comments & Date & Inltials \\
\hline Aralytical 1 & 2,600075 & $\begin{array}{l}\text { Whole water } \\
\text { body ku } 15 \text { and (avl. }\end{array}$ & 12. AuG 1.2 & KW \\
\hline Analytical $\# 2$ & 2.00035 & $J$ & $\hat{1}$ & $\uparrow$ \\
\hline Stock & 35,00 & & & \\
\hline Slock $\# 2$ & 35,03 & & & \\
\hline Stock $=3$ & 35 & & & \\
\hline Stok +44 & 70 & & & \\
\hline Stot +15 & $70.00 \mathrm{~s}$ & & & \\
\hline $5 \sin +16$ & 70.044 & 7 & & \\
\hline Stook 47 & 75,015 & 7 & V & $\sqrt{1}$ \\
\hline Stokt 48 & 150.054 & $\angle$ & id fual & fin \\
\hline
\end{tabular}

NOTE: Chemical samples to be stored refrigerated until used for stock preparation.

Stock Solution Preparation:

\begin{tabular}{|c|c|c|c|c|c|c|}
\hline Sample ID & $\begin{array}{c}\text { Dllution } \\
\text { volume (mL) }\end{array}$ & $\begin{array}{c}\text { Dllution } \\
\text { Time } \\
\end{array}$ & Use & $\begin{array}{c}\text { Exposure } \\
\text { Time } \\
\end{array}$ & Date & Initials \\
\hline Aralutical $\geqslant 1$ & 500 & 0800 & 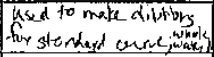 & $d y-$ & 152,1412 & iw \\
\hline Stock $\# 1$ & 8000 & 0945 & Tank I whoke body & 0945 & $\hat{\imath}$ & \\
\hline Stock \#2 & 8000 & 0 & Tank 8 whole body & 0954 & & \\
\hline Stock +3 & 8000 & $0-$ & Tank 4 whoke body & 0958 & & \\
\hline Stock t.4 & 8000 & $0 \ldots$ & Tank 9 whole body & 1007 & & \\
\hline Stock $\$ 5$ & 8000 & $0-$ & Tank 7 wholebady & 1010 & $\psi$ & \\
\hline Slock 46 & 8000 & D- & Tank 6 whislebarly & 1015 & 15 AnL 12 & kew \\
\hline fullyticel $\neq 2$ & 500 & 2330 & 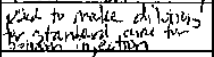 & - & $17-1 x_{4} 12$ & fun \\
\hline Stock 业8 & 2500 & 0940 & $100 \mathrm{mo} / \mathrm{L}$ tanks & (6) & BA AUG 12 & kno \\
\hline Sfock t 7 & 2500 & 1015 & $50 \mathrm{ma} / \mathrm{l}$ tarks & (2) & 1714412 & How \\
\hline
\end{tabular}

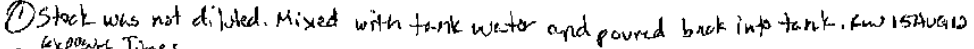
(2) $\frac{\text { Txpouste Tines }}{\text { Tank } 9-1008}$

Tank $2-1021$ Thk 4 - 0416

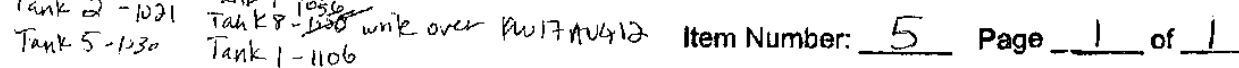


Study Number; AEH-12-PSEUDO-04

Reviewed by: TrS Date: $16 \mathrm{TAN} / \mathrm{A} \mid$

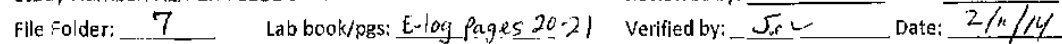

\section{Test Chemícal Stock Preparation Data Form}

Test Chemical: Pseudomonas fluroescens straln 145A Test Chemical Lot \#: 401P12163C and 401P12164C Mix Date Rec'd 7-Aug-12 Exp. Date 21-Jun-12 Test Organism : zebra mussels Test Location: Sha huano Instruments Used: Metllev Toledo PG 2002-5 5/N118470288 Sartorins CPA $225 D$ S/W 26450930

Weights of Chemical Samples:

\begin{tabular}{|c|c|c|c|c|}
\hline Sample ID & Sample Weight & Comments & Date & Initlals \\
\hline Analytial $* 1$ & 2,00072 & & $27 A x 0_{2}$ & $k w$ \\
\hline Analytizal +2 & 2.0002 & & $\pi$ & $?$ \\
\hline Stock til & 35.10 & & & \\
\hline$S+2, k+2$ & 35,06 & & & \\
\hline Stork +3 & 35.079 & & & \\
\hline Sto $t+14$ & 70.029 & & & \\
\hline Stodc. $\# 5$ & 70.049 & & & \\
\hline Stack $k+6$ & $70.02 \mathrm{c}$ & & & \\
\hline Stock $t 7$ & 6090 & & $V$ & 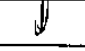 \\
\hline Stook +8 & 120.07 & & 27muan & $Y e$ \\
\hline
\end{tabular}

NOTE: Chemical samples to be stored refrigerated until used for stock preparation.

Stock Solution Preparation:

\begin{tabular}{|c|c|c|c|c|c|c|}
\hline Sample ID & $\begin{array}{c}\text { Dilution } \\
\text { volume (mL) }\end{array}$ & $\begin{array}{c}\text { Dilution } \\
\text { Time }\end{array}$ & Use & $\begin{array}{c}\text { Exposure } \\
\text { Time } \\
\end{array}$ & Date & Initials \\
\hline Analytical +1 & 500 & 0800 & $\begin{array}{l}\text { Sfyodard curve } \\
\text { for whole tanik }\end{array}$ & While & 6 SEP 12 & pw \\
\hline Stock $\# 1$ & 8000 & 0855 & Tank 2 whole & 09001 & 1 & 1 \\
\hline $5 \sin k+2$ & 8000 & 0902 & Tanic 8 whold & 0905 & & \\
\hline$S t o c k-13$ & 8000 & 0906 & Tank 5 whed & 0909 & & \\
\hline Stock $=$ 非 & 8000 & 0913 & Tank 9 unde & 0915 & & \\
\hline Stock 45 & 8000 & 0917 & Tank 3 whode & 0920 & $\sqrt{ }$ & $\sqrt{ }$ \\
\hline Stock $k \neq b$ & 8000 & 0922 & Tank 4 bude & 0925 & (c) SEPI) & kw \\
\hline Anay liticaltea & 500 & 0745 & Standord for bortton & bottom & 8Steid & $m$ \\
\hline Shoul 7 & 12000 & 0809 & $50 \mathrm{~m} y / 2 \operatorname{tanks}$ & $0841-090$ & j & $\downarrow$ \\
\hline Stok 48 & 12000 & 0900 & $100 \mathrm{mg} / \mathrm{L}$ tanks & $0907-7^{\circ} 939$ & ssep 12 & kw \\
\hline
\end{tabular}

Item Number: 7 Page 1 of 1 


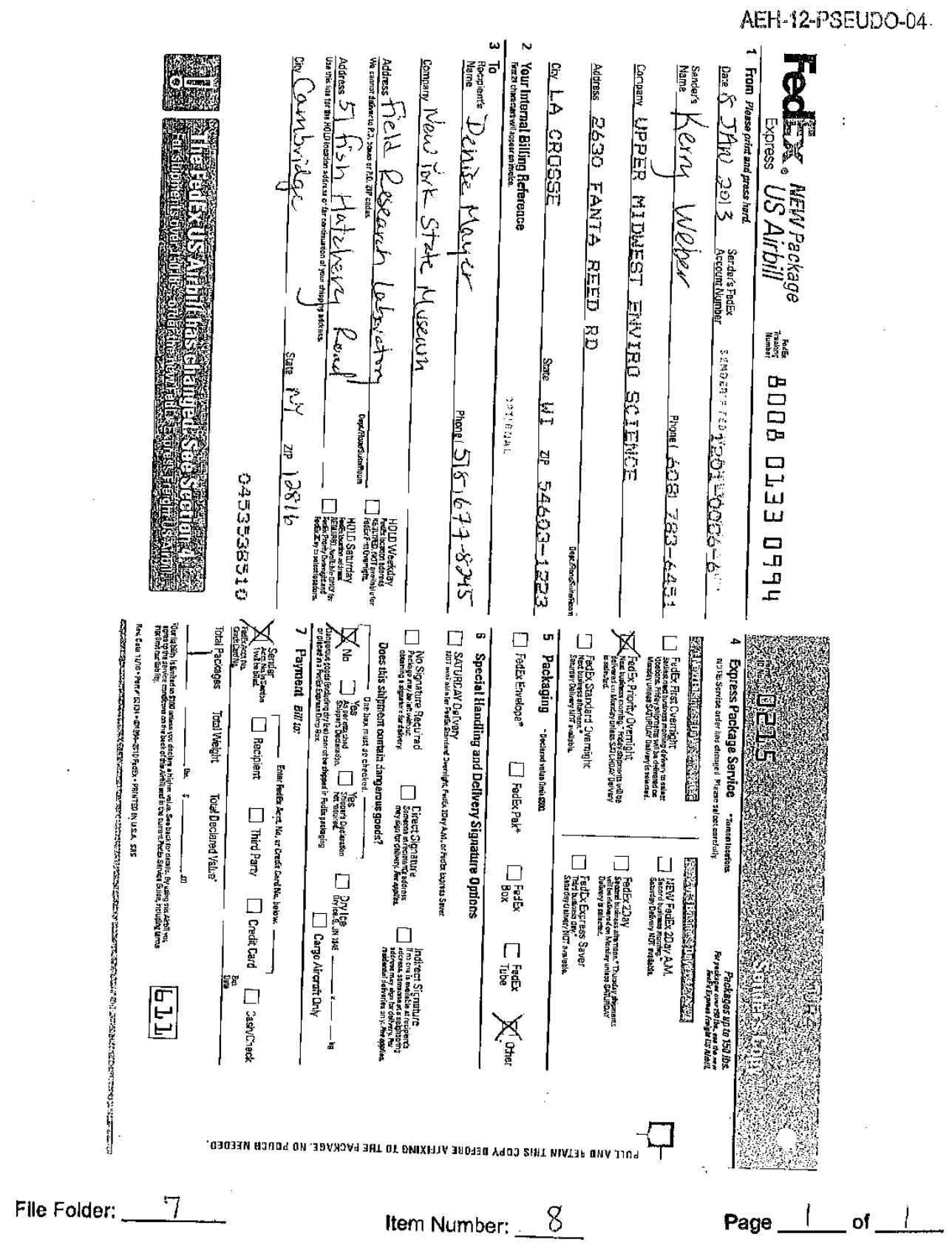




\section{NYSM Post-Treatment Product Validation Assay MBI-401 SDP 401P12163C and 401P12164C Mix}

(USGS Study \#AEH-12-PSEUDO-04 Field trials at Shawano and Carlos in 2012)

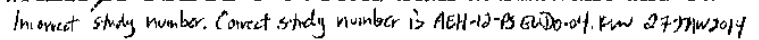

Date product received from USGS: 2013/01/09

Date of start of test: $2013 / 01 / 09$

AEH-12-PSEUDO-04

BACKGROUND: As standard protocol for the USEPA project, each time a batch of Zequanox product is used in a test a UMESC, a portion of the product is bioassayed by the NYSM to validate toxicity post-treatment.

- MBI-401 SDP 401P12163C and 401P12164C Mix

- USGS Study \#AEH-13- PSEUDO-04 Field trials at Shawano and Carlos in 2012 iniored sthaly vimber. Courcet stimd

PURPOSE: Post-test product validation of MBI-401 SDP 401P12163C and 401P12164C Mix used in AEH-13-PSEUDO-04 Field Trials at lakes Shawano and Carlos.

\section{MATERIALS AND METHODS:}

Preparation of product for testing:

Product was shipped under cold condicions and held in the laboratory refrigerator at $4^{\circ} \mathrm{C}$ until use. Within 30 min of treatment application, prepare each at treatment stock of each MBI-401 formulated product:

MBI-401 SDP 401P12163C and 401P12164C Mix (SDP - 50\% active ingredient): $3 \mathrm{~g}$ of the powder from each sample was added slowly to a beaker with dilution water with stirring on a stir plate for even suspension and then the total volume was adjusted to $30 \mathrm{ml}$ with dilution water. The suspension was transferred to a $50 \mathrm{ml}$ centrifuge and stored in refrigerator until ready to use. The suspension, when evenly dispersed was $100 \mathrm{mg}$ product $/ \mathrm{ml}$ or $50 \mathrm{mg} \mathrm{a.i./ml}$. For $200 \mathrm{ppm}$ a.i. treatments in testing jars, $2 \mathrm{ml}$ wcre added to cach testing jar $(500 \mathrm{ml})$.

Cambridge Cr (Standard for Positive Controls):

As an efficacy standard, we used Pf-CL145A killed CF that was maintained at $-80^{\circ} \mathrm{C}$ (Cambridge CF), Since its production in 2005, this material has been valuable as a reference standard. The Cambridge CF was produced in 2005 (2005-0027) in 100-L batches 10,11 and 12 and E-beaned to kill the cells. The solution, at $110 \mathrm{mg} / \mathrm{ml}$ dry weight, is stored in $1 \mathrm{~cm}$ thick sheets in the Cambridge ultrafreezer at $-80^{\circ} \mathrm{C}$. A section of the sheet was broken off and weighed to determine volume (ca. $1 \mathrm{~g}=1 \mathrm{ml}$ ).

For this bioassay, a positive control suspension was produced on 06/18/2012 from the frozen blocks described above and dispensed into multiple 50- $\mathrm{ml}$ centrifuge tubes for single-use treatment of bioassays. The dry weight of the matcrial was $68.15 \mathrm{mg} / \mathrm{ml}$; therefore $1.5 \mathrm{ml}$ was added to each testing jar to treat at $200 \mathrm{ppm}$.

Mussel collections:

Mussels were scraped from substrates (rocks) in the field and brought back to the lab in coolers. Mussels were held unchlorinated water in aquaria with aeration and filtration (Whisper filters) at $10 \pm 2^{\circ} \mathrm{C}$. Approximately one week before the test, jars of mussels were placed in aquaria containing $10 \pm 2^{\circ} \mathrm{C}$ tap water with low aeration in the laboratory, wrapped in towels to slow warming, and allowed to warm to ambient laboratory temperature $\left(20 \pm 1^{\circ} \mathrm{C}\right)$, after which filtration and high aeration was applied to the aquarium. One day prior to treatment exposure, mussels were carefully examined and placed into testing jars and allowed to attach overnight at ambient laboratory temperature (ca. $20^{\circ} \mathrm{C}$ ).

File Folder: 7

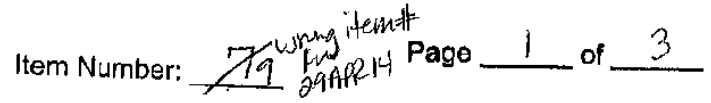


2013-0001 MBI-401 SDP 401P12163C and 64C Mix Post-test from 2012 field trials Validation -2 -

Mussel collection and handling:

AEH-12-PSEUDO-04

\begin{tabular}{|c|c|c|c|c|}
\hline Species & Collection site & Collection date & Date in lab $\left(20^{\circ} \mathrm{C}\right)$ & Picked for test \\
\hline $\begin{array}{c}\text { Zebra } \\
\text { mussels }\end{array}$ & $\begin{array}{c}\text { Hedges Lake } \\
\text { (Washington County) }\end{array}$ & $11 / 07 / 2012$ & $01 / 02 / 2013$ & $01 / 08 / 2013$ \\
\hline
\end{tabular}

\section{Experimental design:}

For validation of efficacy the following treatments will be set up:

Zebra mussels (25 mussels/jar):

3 - Untreated Control $(A, B, C)$

3-200 ppm (a.i.) Cambridge CF Positive Control (Pf-CLi45A killed cclls) (A, B, C)

$3-200 \mathrm{ppm}$ (a.i.) $401 \mathrm{P} 12163 \mathrm{C}$ and $401 \mathrm{P} 12164 \mathrm{C}$ Mixed (A, B, C)

Total of 9 testirg jars.

Testing jar bioassay protocol:

On the day prior to treatment $(01 / 08 / 2013)$ mussels were carefully examined and 25 mussels placed into each testing jar containing ca. $100 \mathrm{ml}$ aerated hard water and allowed to attach overnight. The next morning (01/09/2013), unattached mussels were removed and replaced with attached mussels from an extra glass Petri dish. Water was replaced with $500 \mathrm{mll}$ fresh aerated hard water.

After at least one hour, the treatment was applied. The optical density of each jar was mcasured in duplicate $\left(A_{660 \mathrm{~nm}}\right.$ Genesys Spectrophotometer).

After $24 \mathrm{hr}$ of treatment, mussel mortality was checked and mussels were transferred to square plastic dishes with fresh aerated hard water. Mortality was checked and recorded each day with water replacements, for an additional 7 days ( 8 days tolal). On the final day of mortality checks, 20 mussels were measured from the untreated controls using a caliper.

Results:

Mussel length: Zebra mussels $19.65 \pm 2.36 \mathrm{~mm}$.

Optical density of treatments:

\begin{tabular}{|c|c|}
\hline Treatment & Mean $( \pm S D)$ OD $\left(\mathrm{A}_{660 \mathrm{~nm}}\right)$ \\
\hline \hline Untreated Control & $0.001 \pm 0.001$ \\
\hline Cambridge CF (Positive Control) & $0.171 \pm 0.010$ \\
\hline MBI-401 SDP 401P12163C and 401P12164C Mixed & $0.226 \pm 0.004$ \\
\hline
\end{tabular}

Zebra mussel mortality: Mussels were treated in triplicate testing jars $\left(500 \mathrm{ml}\right.$ ) at $20^{\circ} \mathrm{C}$ for $24 \mathrm{hr}$ and mortality was recorded for a total of 55 days.

\begin{tabular}{|c|c|}
\hline Treatment & $\begin{array}{c}\text { Mean } \% \text { mortality } \\
( \pm \mathrm{SD})\end{array}$ \\
\hline \hline Untreated Control & $0.0 \pm 0.0 \%$ \\
\hline Cambridge CF (Positive Control) & $73.3 \pm 8.3 \%$ \\
\hline MBl-401 SDP 401P12163C and 401P12164C Mixed & $70.7 \pm 4.6 \%$ \\
\hline
\end{tabular}

Page 2 of 3 
AEH $\{2$ PSEUDO-04

2013-0001 MBI-401 SDP 401P12163C and 64C Mix Post-test from 2012 field trials Validation -3 -

MBI-401 SDP 401P12163C and 401P12164C Mixed PASSED the post-test bioassay validation (71\% mortality). Untreated control mortality was $0 \%$.

File Folder: $\quad 7$

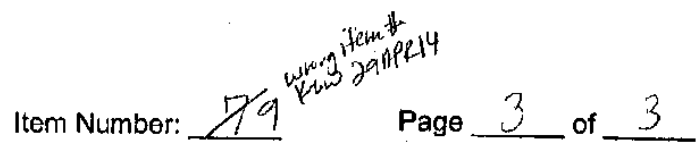

Page $\mathbf{2 5 0}$ of $\mathbf{5 1 9}$ 
CHEMICAL LOG BOOK

Marrone Bio Innovations

Davis, California

\author{
LOT NUMBERS: \\ 401P12163C \\ and \\ 401P12164C \\ (Received Mixed in Containers)
}

Container 1 of 6

Fille Folder: $17 \mathrm{a}$

ltem Number:

Page _1 of 5 
AEH-12-PSELDO-04

\section{IIGNATURE PAGE}

All personnel making an entry in this log must fill out the form below in accordance with SOP GEN 009

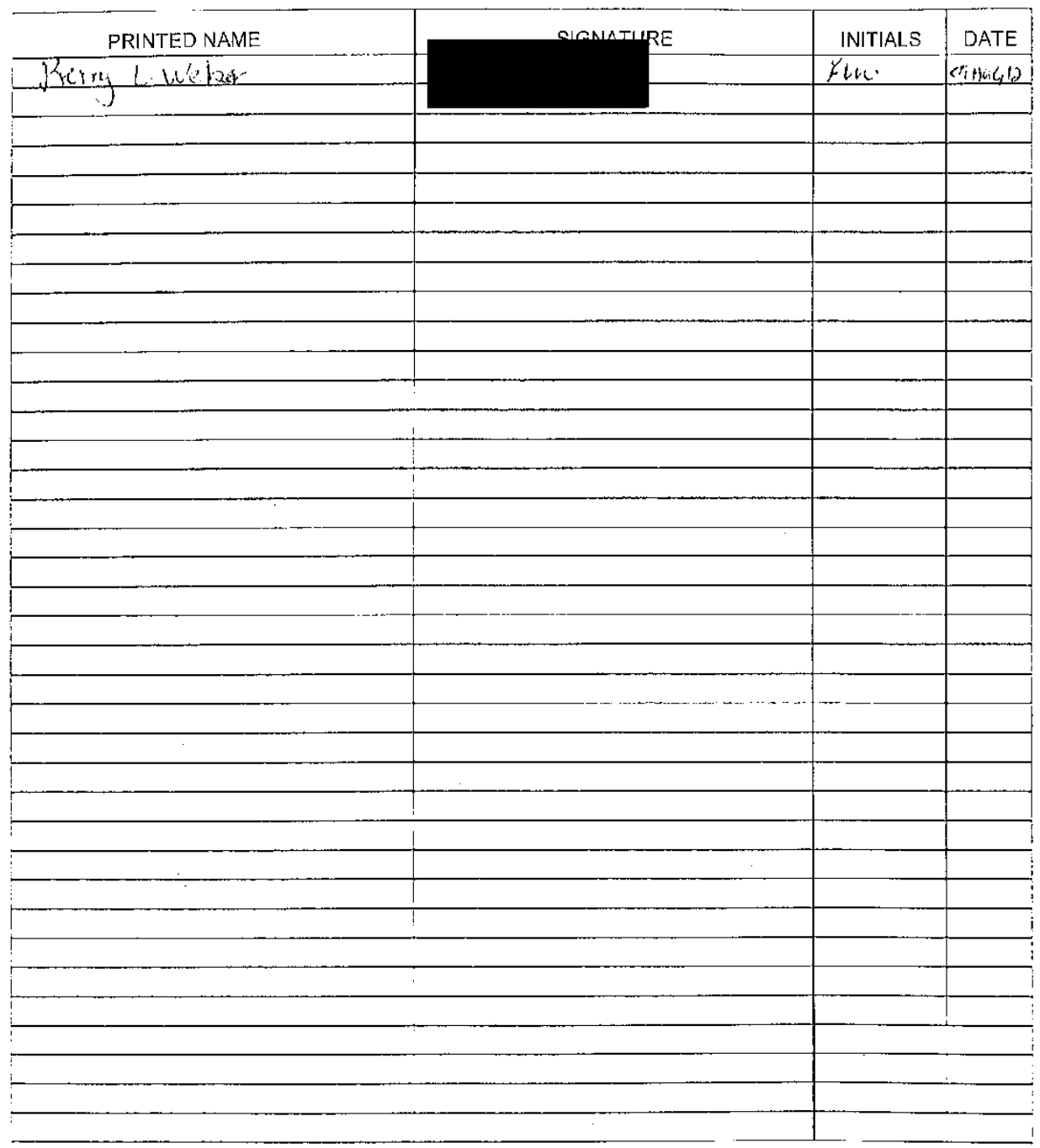

"This Use and Maintenance l.og Book has been inspected and fo. und th he incompliance with SOP GEN 009 Inspected and sealed on

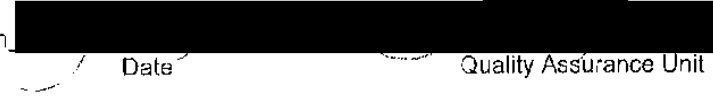

Page 2 of 5 


\section{DRIGIAAL}

SOP NO. GEN 012.3

Page 5 of 7

Jorm CLN 012.3a

Page 1 of 1

TEST CHEMICAL DATA FORM

Tcst Chemical (Chemical Name) M(bi-4oi SDP

Circle ouc: Test Article Control Article

Trade Name of Test Chemical (Synonyms) Pf.CL. ILSA; Zxputhex

Source of Test Chemical (Manufacturer) Matrirs Bo Lrilovetions

Storage Location Refrigeratir

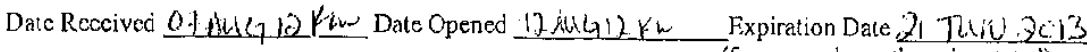
4013.21036

(5 years unless otherwise stated)

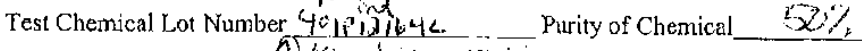

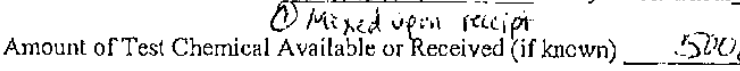

Initial Mass (with cover on) of Test Chemical and Container $541.45 \mathrm{c}$

Characterization of Test Chemical: Color _. Tan

Physicai State: liquid solid $x$

Chemical Abstract Service Number _ W J A

Solid Form: powder $x$ crystal _ pellet

Manufacturer Certificate of Analysis Yes $\underset{X}{ }$ No

Additional Comments about the Tesl Chemical:

QTest clemicel vos mixed by manfather ot shifed to we in

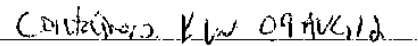

Sample Placed in Archives: Yes _ No X (Entries should also be made on Form GEN 012.b)

Archive Location _______. Material Safety Data Sheet Available: Yes $\underline{X}$ No

Signature of Study Director or designee iniliating Test Chemical Use Log and date:

Signature Date 0961.2

Page 3 of 5 
AEH-12-PSEUDO-04

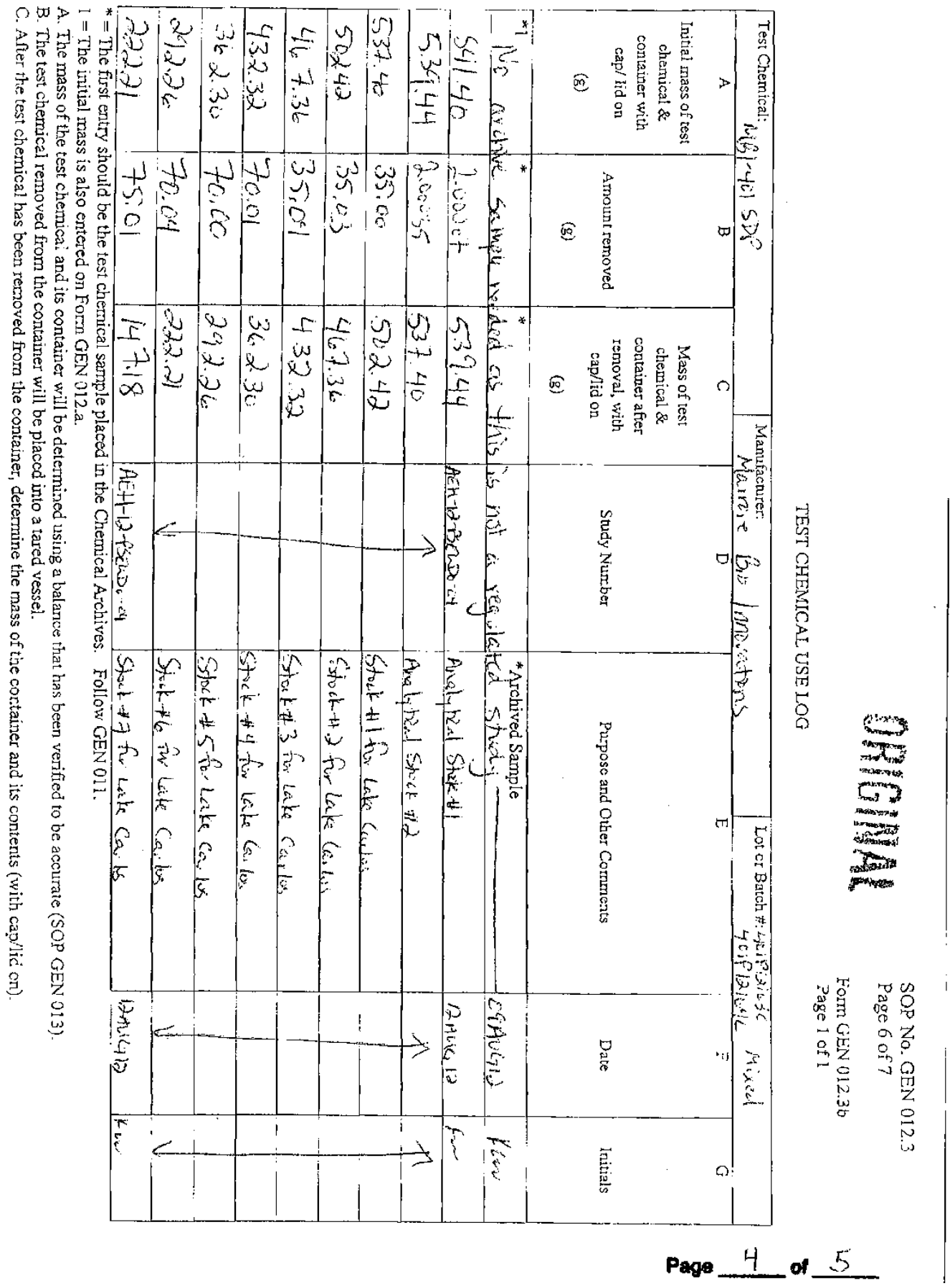


AEH-12-PSELDO-04

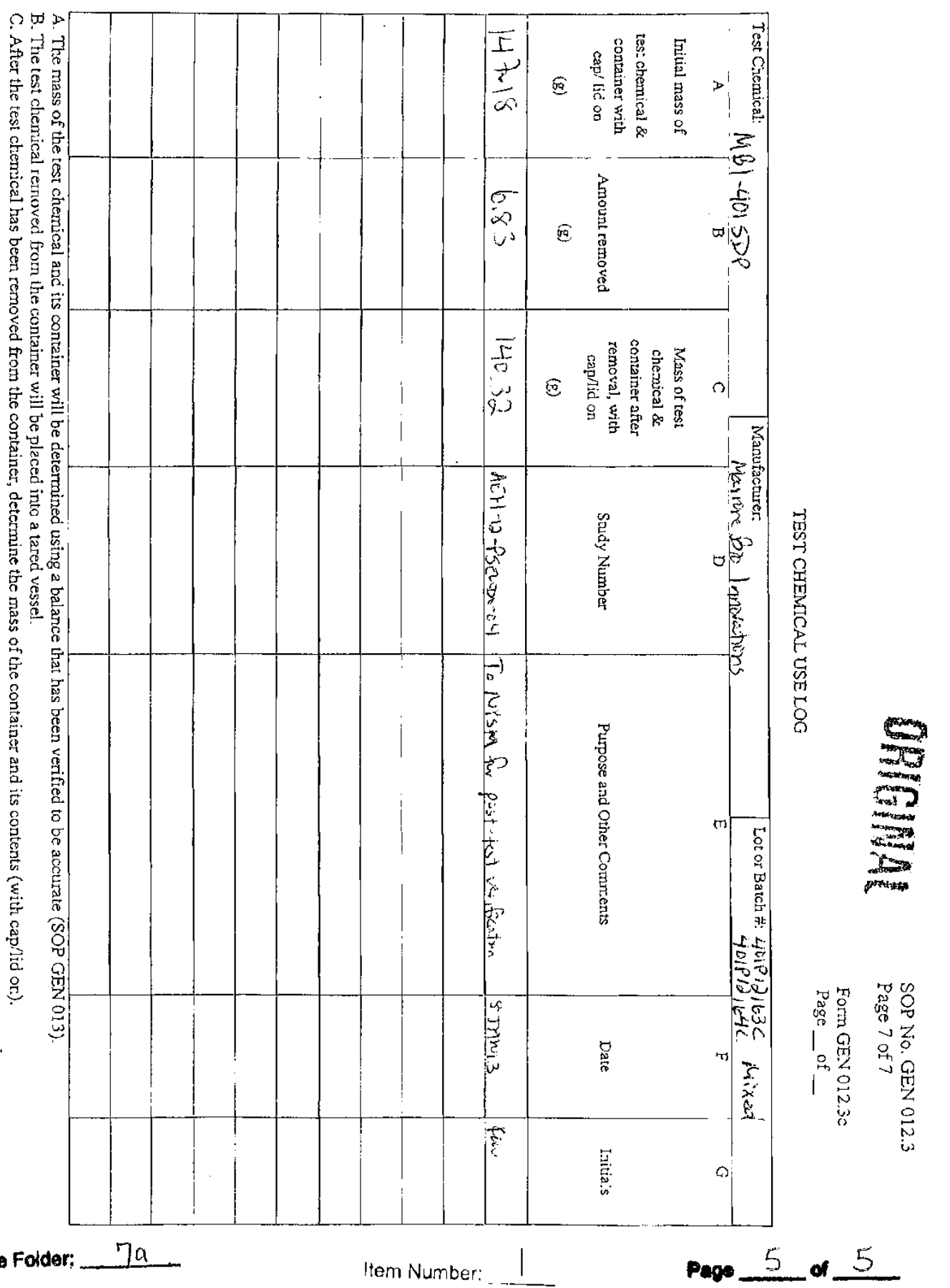




\section{CHEMICAL LOG BOOK}

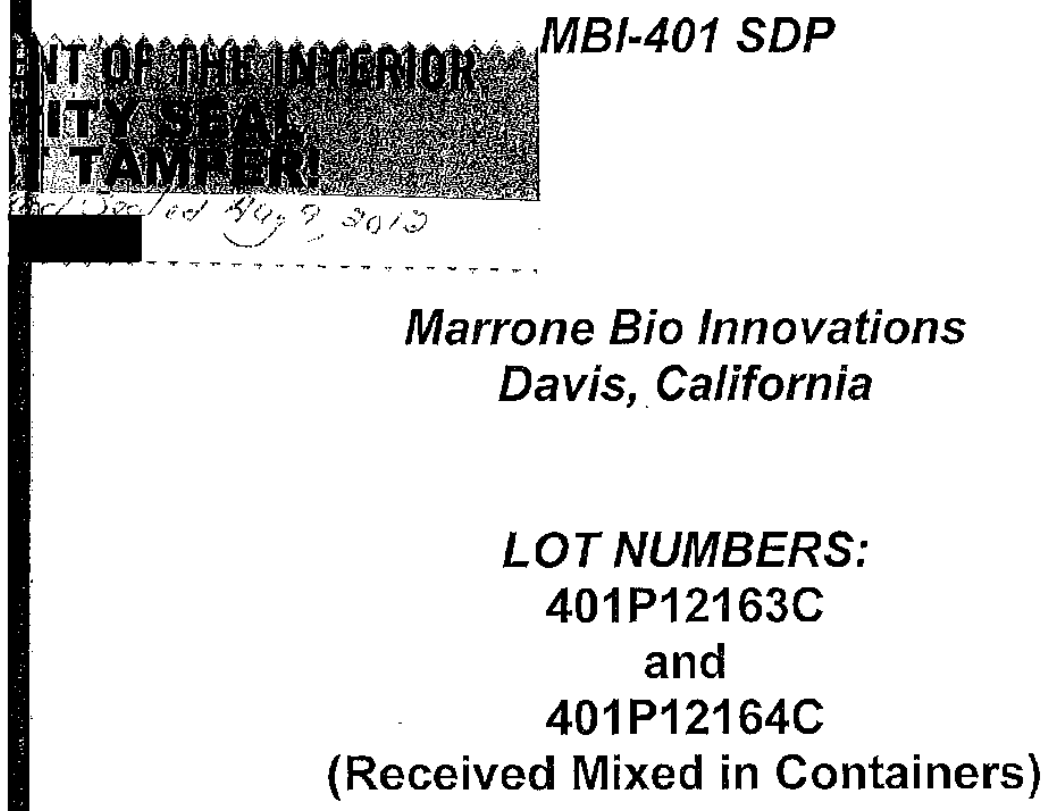

Container 2 of 6

File Folder; $\quad 7 a$

Item Number: 2

Page 1 of 4 


\section{SIGNATURE PAGE}

AEH-12-PSEUDO-04

All personnel making an entry in this log must fill out the form selow in accordance with SOP GEN 009.

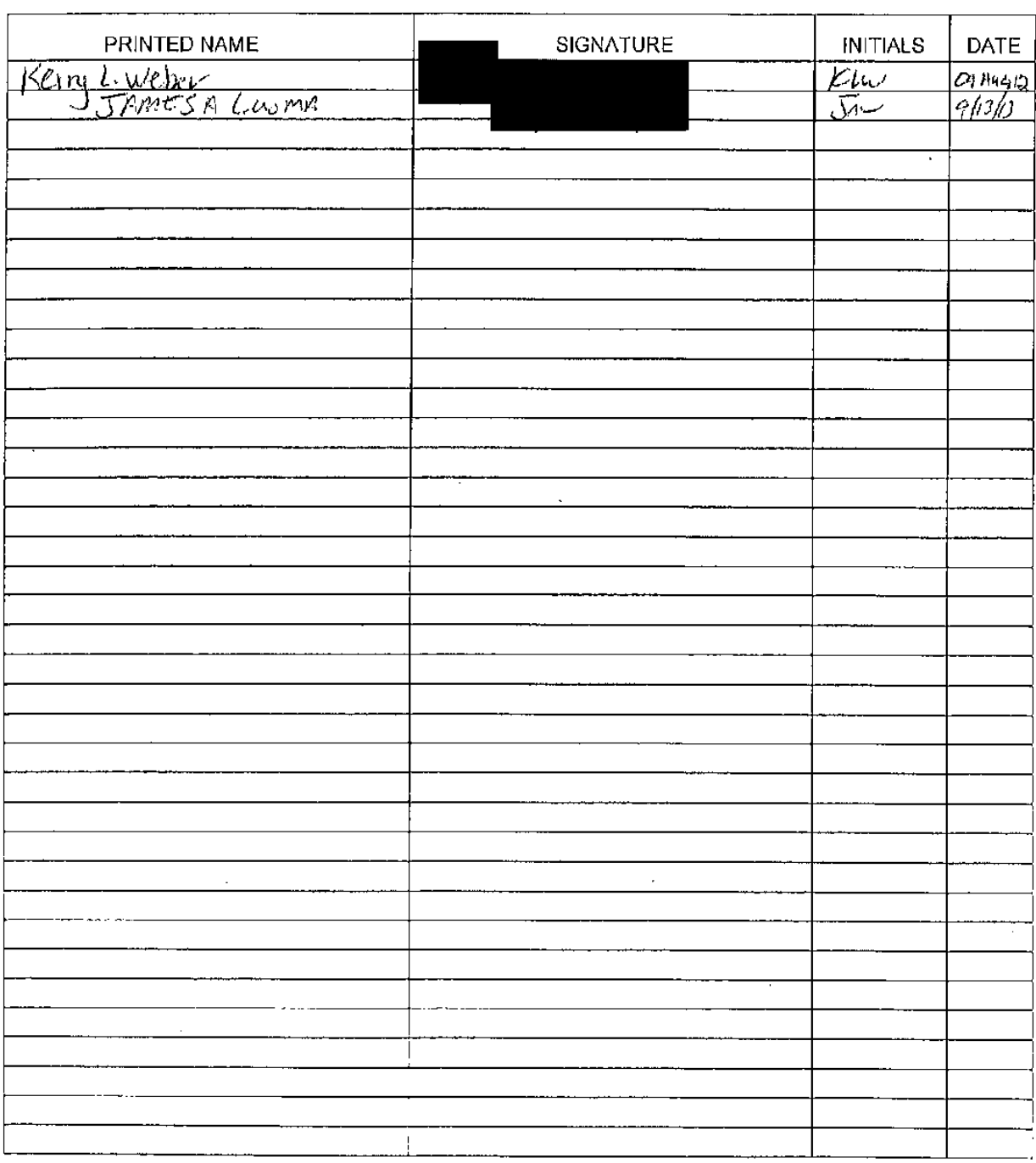

"This Use and Maintenance Log Book has boon inspected and found to be in compliance with SOP GEN 009

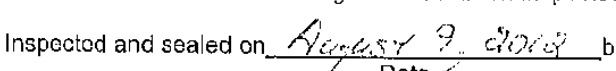

Date

Page 2 of 4 
SOP No. GEN 012.3

Page 5 of 7

TEST CIIENICAL DATA IORM

Page 1 of 1

Test Chemical (Chemical Name) MGi-40! SDP

Circle ouc: (Test Article Control Article

Trade Name of Test Chemical (Synonyms) Pf-CL 1457 ; 2 quemex

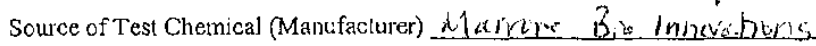

Storage Location Refigeathr

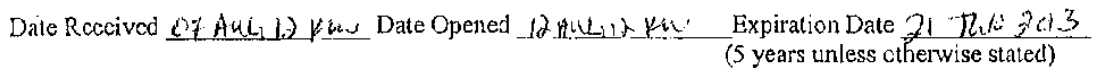

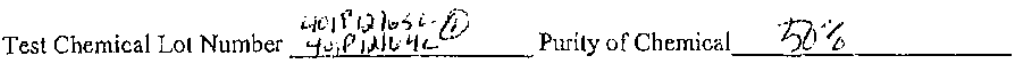

Amount of Test Chemical Available or Received (if known) $2520 \mathrm{~g}$

Initial Mass (with cover on) of Test Chemical and Container $5,56.52 \mathrm{~g}$

Characterization of Test Chemical: Color $\frac{\text { Jah }}{\text { Physical State: liquidi_esolid } X}$

Chemical Abstract Servicc Number___N/A

Solid Form: powder $X \overline{c r y s t a l}_{\text {_ pellet }}$

Manufacturer Certificale of Analysis Yes丈 No _-

Additional Comments about the Test Chemical:

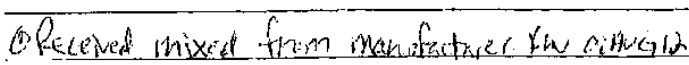

Sample Placed in Archives: Yes _.. No $\triangle$ (Entries should also be made on Porm GEN 012.b)

Archive Location Matcrial Safety Data Sheet Available: Yes X No

Signature of Study Director or designee initiating Test Chemical Use Log and date:

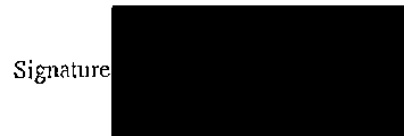

Datc $\left(C_{1}+\mathrm{KCO}_{2}\right.$

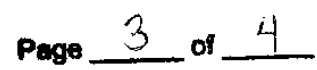




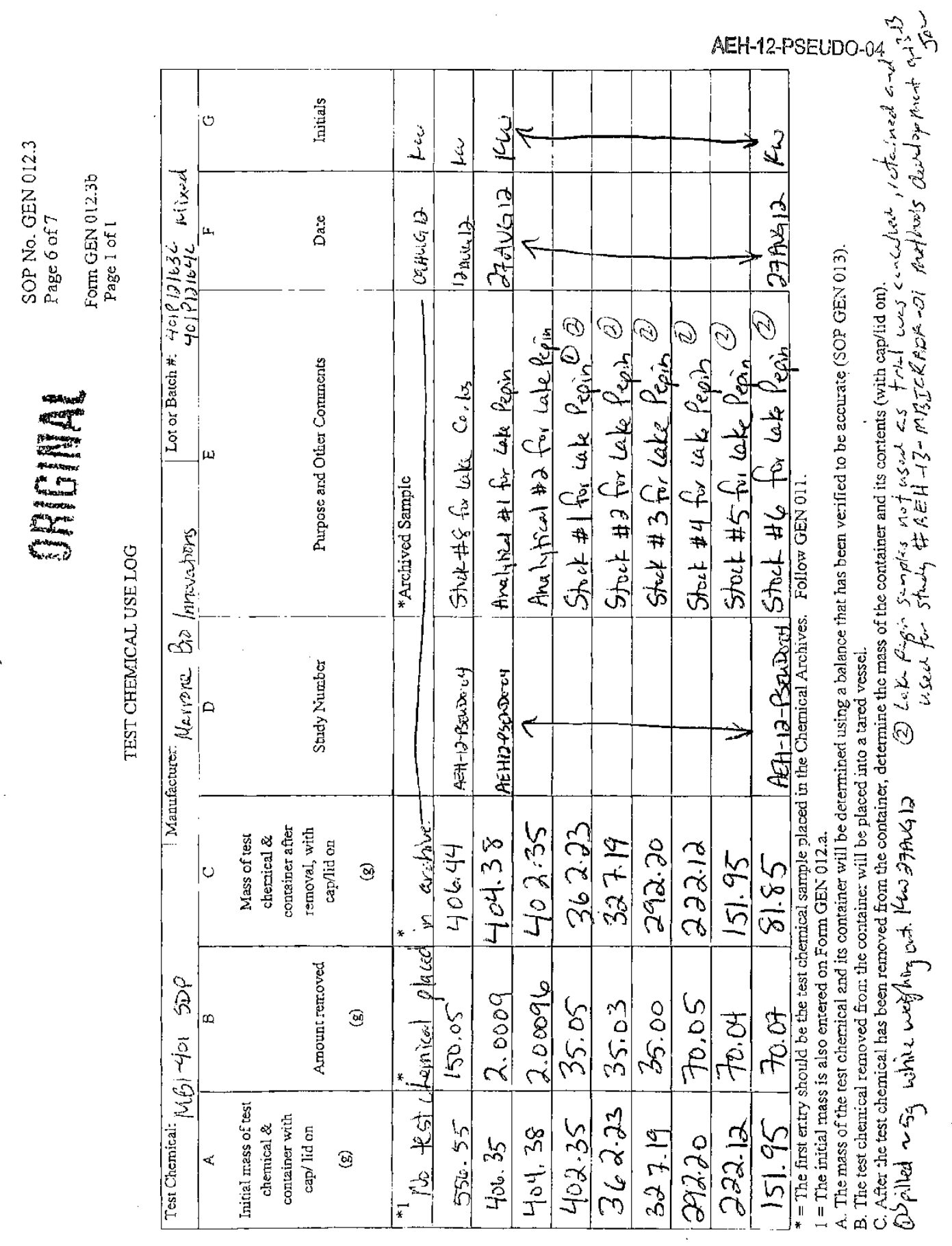

Flle Folder: $7 a$

Item Number: 2

Page 4 of 4 


\section{CHEMICAL LOG BOOK}

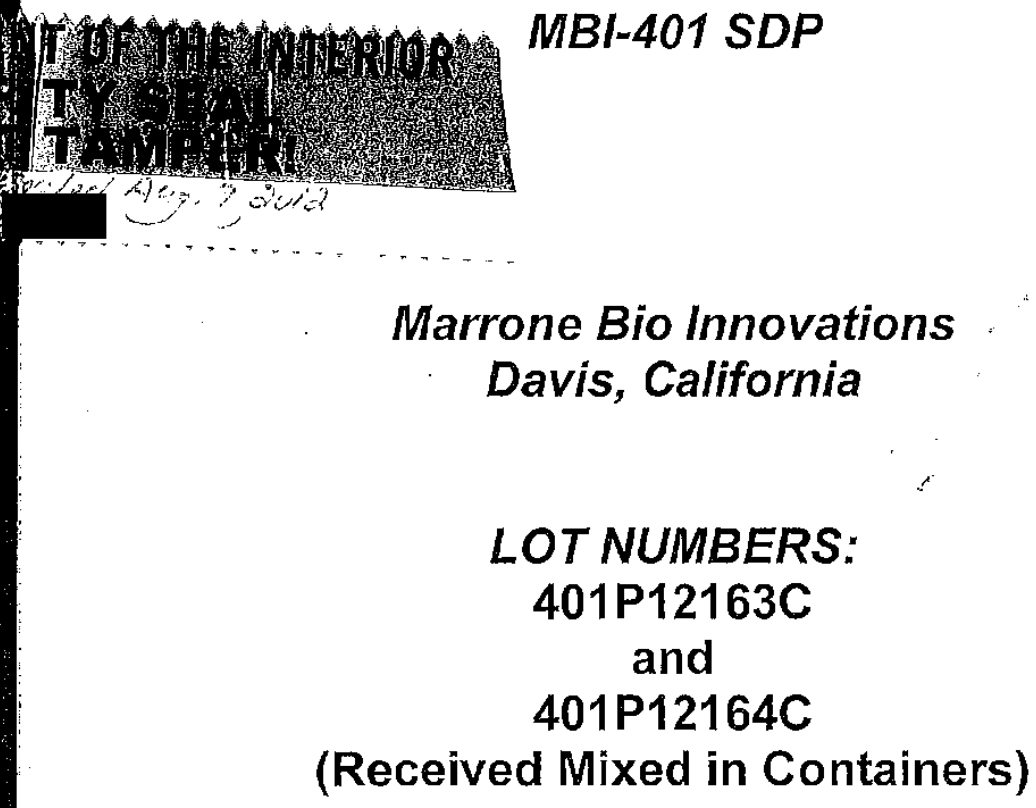

Container 3 of 6

File Folder:

Item Number: 3

Page 1 of 4 


\section{AEH-12-PSELDO-04}

SIGNATURE PAGE

All personnel making an entry in this log must fill out the form below in accordance with SOP GEN 009

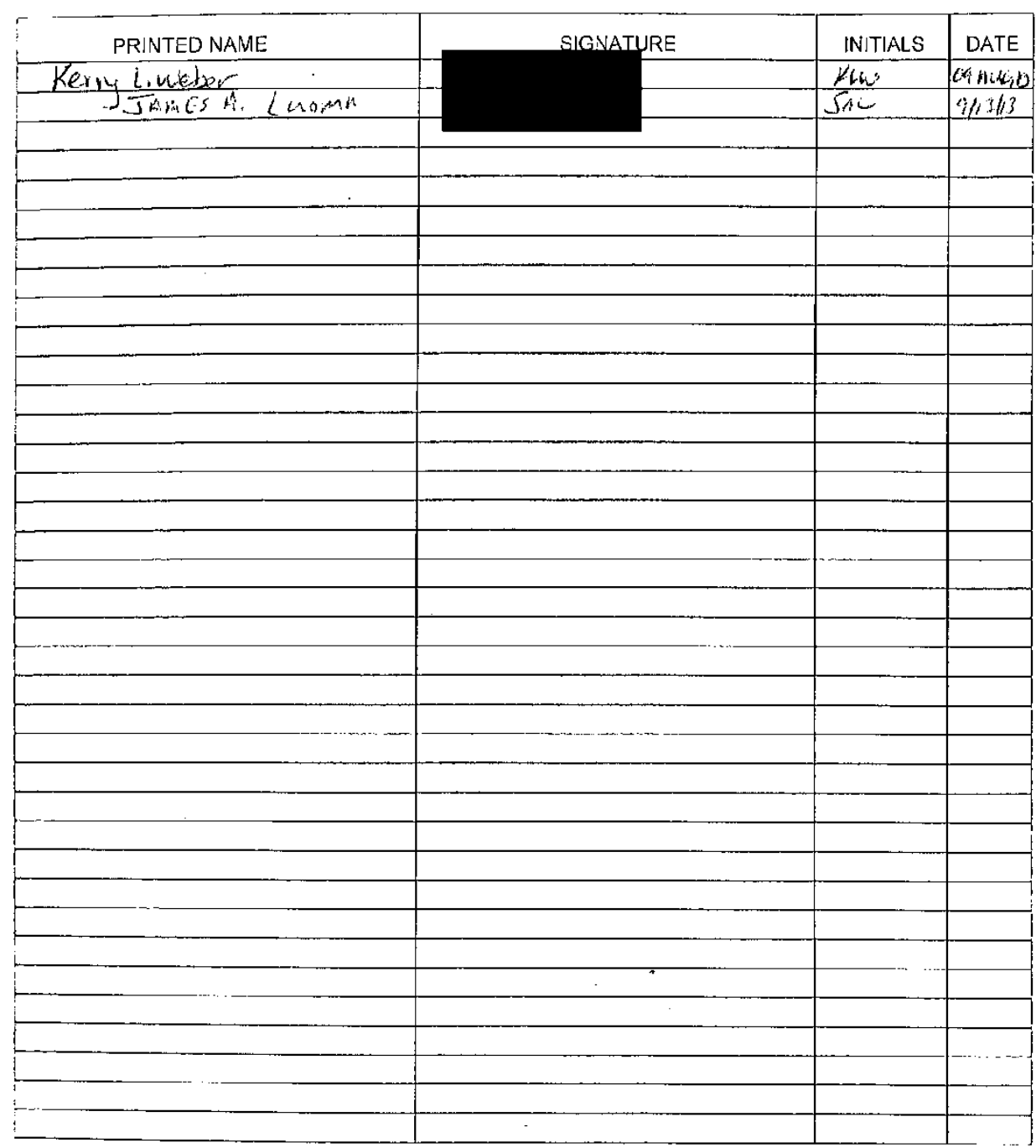

"This Use and Maintenance Log Book has beer inspected and found to be in compliance with SOP GEN 009 Inspected and sealec on $/ 1, \ldots$

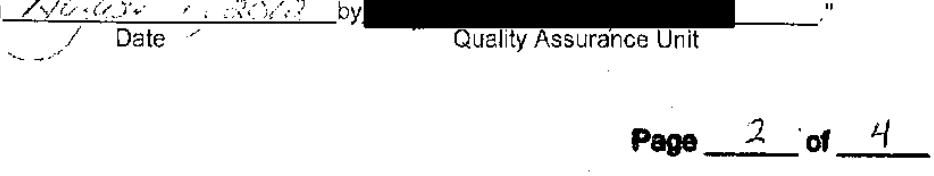




\section{DPIGIAAL}

TEST CHEMICAL DATA FORM
AEH-12-PSELDDO-04

SOP No. GEN 012.3

Page 5 of 7

Form CFN 0j2.3a

Page I of 1

Test Chemical (Chemical Name) $M(3)-4015) P$

Circle one: Test Article Control Article

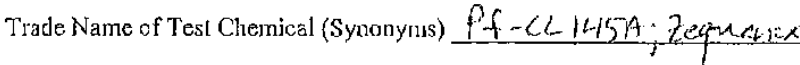

Source of Test Chemical (Manufacturer) Marrowe Bio lronevetions

Storage Location Refingerator.

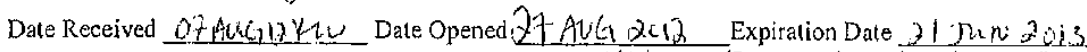

fiw (5 years unless otherwise stated)

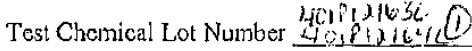
Purity of Chemical $50 \%$

Amount of Test Chemical Ayailable or Received (if known)

25205

Initial Mass (with cover on) of Test Chemical and Container $536.87 \mathrm{~g}$

Characterization of Test Chemical: Color Tan

Physical Statc; liquid solid $\underline{x}$

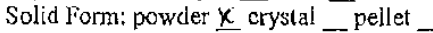

Chemical Abstract Service Number _ U U/A

Manufacturer Certificate of Analysis YesX_ No _-

Additional Comments about the Test Chenical:

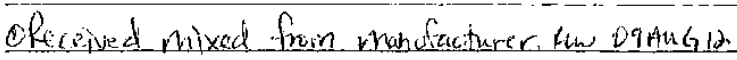

Sample Placed in Archives: Yes _ No $X_{\text {_ }}$ (Entries should also be made on Form GEN 012.b)

Archive Location Material Safety Data Saeet Available: Yes $\not$ No

Signature of Study Directur or designee initiating Test Chemical Use Log and date:

Signatur

Date ESH Hal

Page 3 of 4 


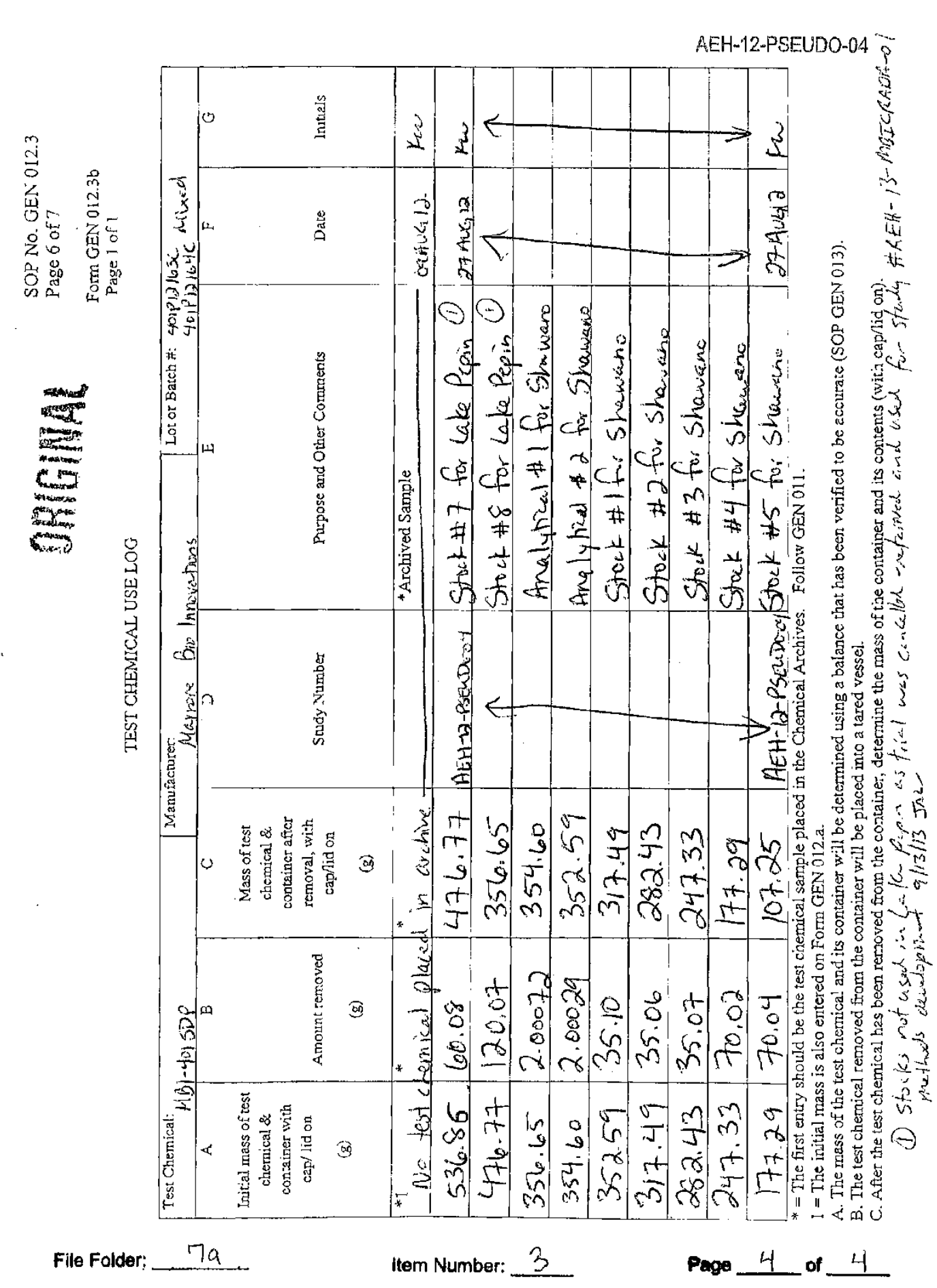

Page 263 of 519 


\title{
CHEMICAL LOG BOOK
}

\author{
MBI-401 SDP
}

Marrone Bio Innovations

Davis, California

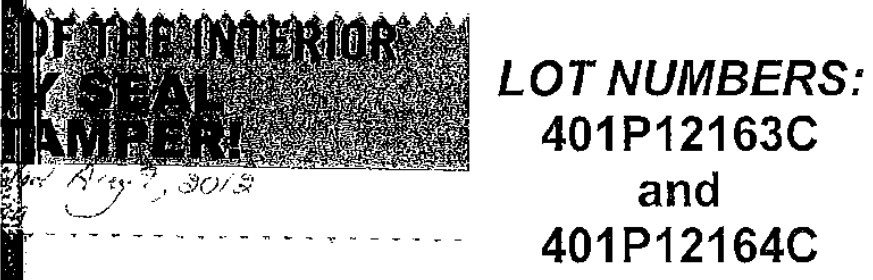

(Received Mixed in Containers)

Container 4 of 6

File Folder: $7 a$

Item Number: 4

Page 1 of 4 
SIGNATURE PAGE

AEH-12-PSEUDO-04

All personnel making an ertry in this log must fill out the form below in accordance with SOP GEN 009.

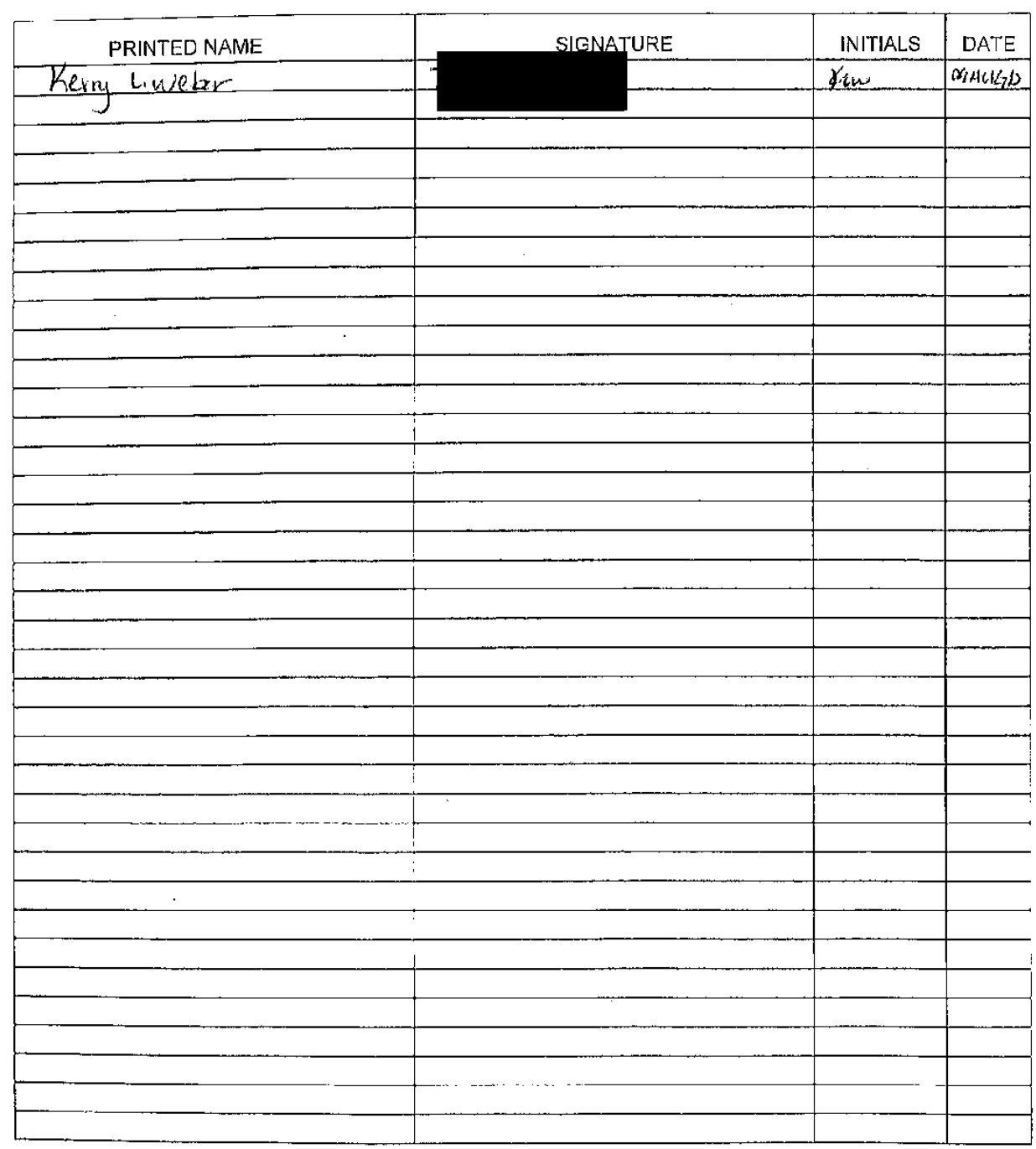

"This Use and Mairtenance Log Book has been inspected and found to be in compliance with SOP GEN 009.

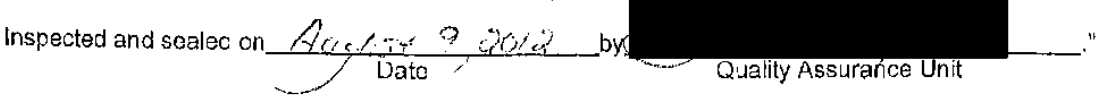

Page 2 of 4 


\section{gRGGHAL}

AEH-12-PSELDO-04

SOP No. GEN 012.3

Page 5 of 7

Form GEN 012.3a

Page $l$ of 1

TEST CHEMYCAL DATA IRORM

Test Chemical (Chemical Name) MSt-4t, Spe

Circle oue: Cost Ártic Con Control Article

Trade Name of Test Chomical (Synonyms) Pf-LL MLSPA; Z, eceprehix

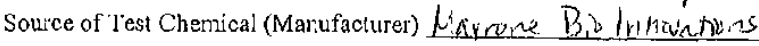

Storage Location Se fingerater

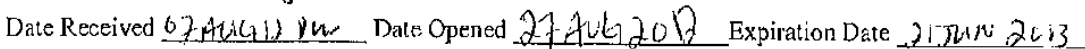
(ow (5 years unless ctherwise stated)

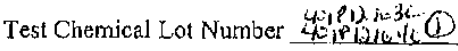
Purity of Chemical $50 \%$

Amount of Test Chemical Available or Received (if known)

$-520$

Initial Mass (witt: cover on) of Test Chemical and Container $540.24 \mathrm{~g}$

Characterization of Test Chemical: color Tan

Physical State: liquid _ solid $x$

Chemical Abstract Service Number_ N / it

Solid Form: powder $X$ crystal _ pellet

Manufacturer Certificate of Analysis Yes X. No

Additional Comments about the Test Chemical:

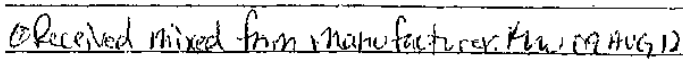

Sample Placed in Archives: Yes __ NoX_ (Entries should also be made on Form GEN 012.b)

Archive Location Material Safety Data Sheet Available: Yes $\underline{K}$ No

Signature of Study Director or designee initialing Test Chemical Use log and date:

Signature

Date $09+4612$

Page 3 of 4 


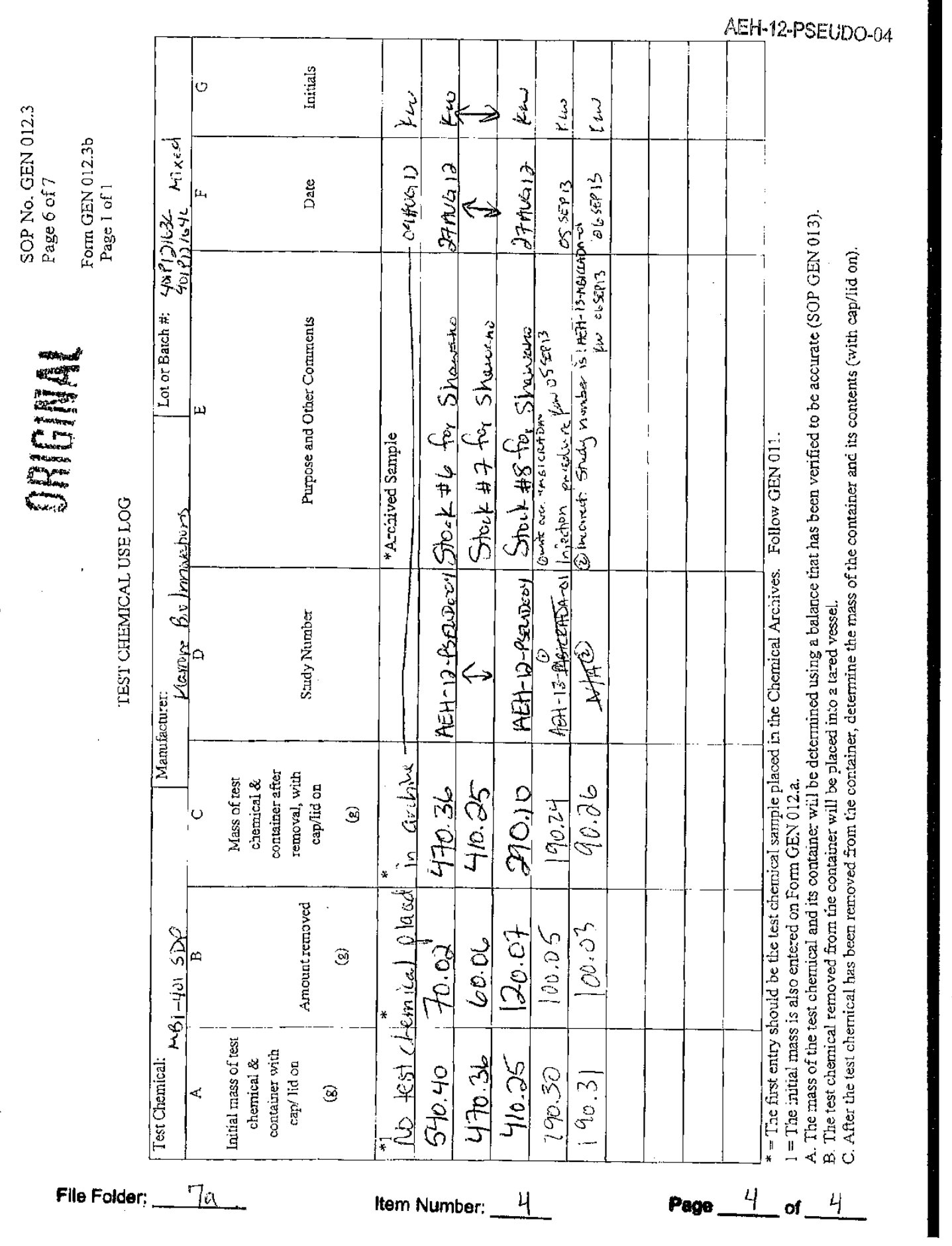




\title{
CHEMICAL LOG BOOK
}

\author{
MBI-401 SDP
}

Marrone Bio Innovations

Davis, California

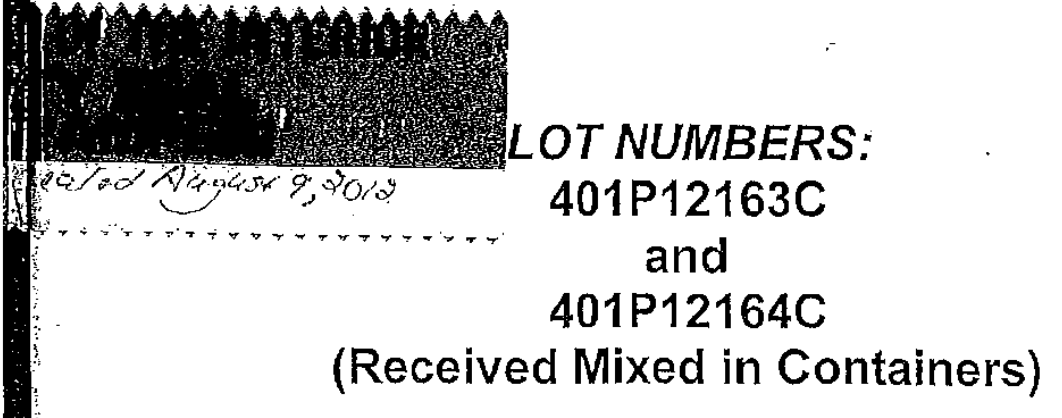

Container 6 of 6

File Folder: $7 \mathrm{7a}$

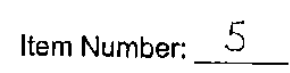

Page 1 of 4 
All personnel making an entry in this log must fill out the form below in accordance with SOP GEN 009.

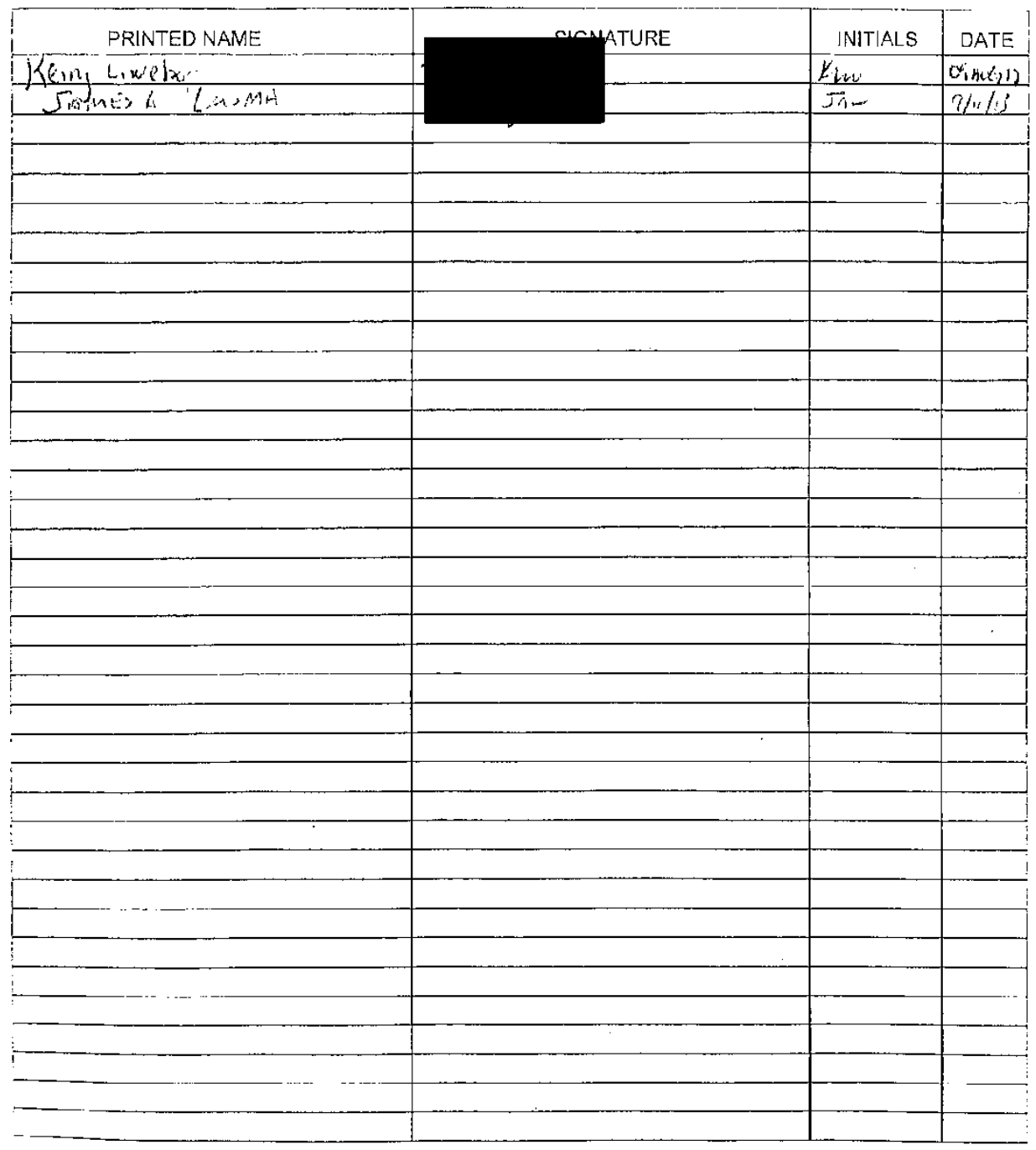

"This Use and Maintenance Log Book has peen inspected and found to be in compliance with SOP GEN 009. Inspected and sealed on $\frac{1}{\text { Date }}$ by

$$
\text { Page } 2 \text { of } 4
$$




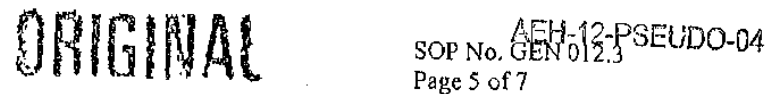 \\ Form GEN 012.3a \\ Page $l$ of 1 \\ TEST CHEMICAL DATA FORM}

Test Chemical (Chemical Name) MBI- 401 SDP

Circle one: Test Articlo Control Article

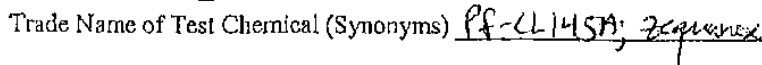

Source of Test Clemical (Manufacturer) Marnice Bo Inncuations

Storage Location_Refingeratur.

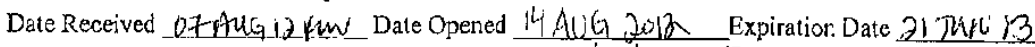

puw (5 years unless otherwise stated)

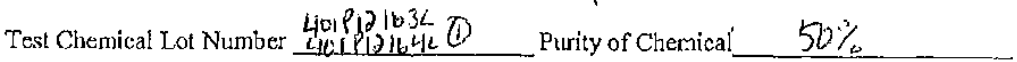

Amount of Test Chemical Available or Received (if known) _.2500

Initial Mass (with cover on) of'Test Chemical and Container $\frac{539.20 \mathrm{~g}}{\mathrm{~g}}$

Characterization of Test Chemical: Color Ten

Physical Stale: liquid__ solid $\underline{\underline{ }}$

Chemical Abstract Service Number_NUA

Solid Form: powcier $\underline{X}$ crystal _.. pellet

Manufacturer Certificate of Analysis Yes $\underline{X}$ No.

Additional Comments about the Test Chenical:

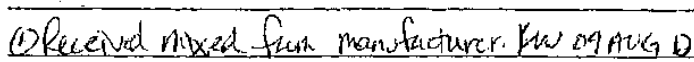

Sample Placed in Archives: Yes _. No X (Entries should also be mace on Form GEN 012.b)

Archive Location Material Safecy Data Sheet Available: Yes $X$ No

Signature of Study Director or designee initiating Test Chemical Use Log and date:

Signature Dite Dafućll

Page 3 of 4 


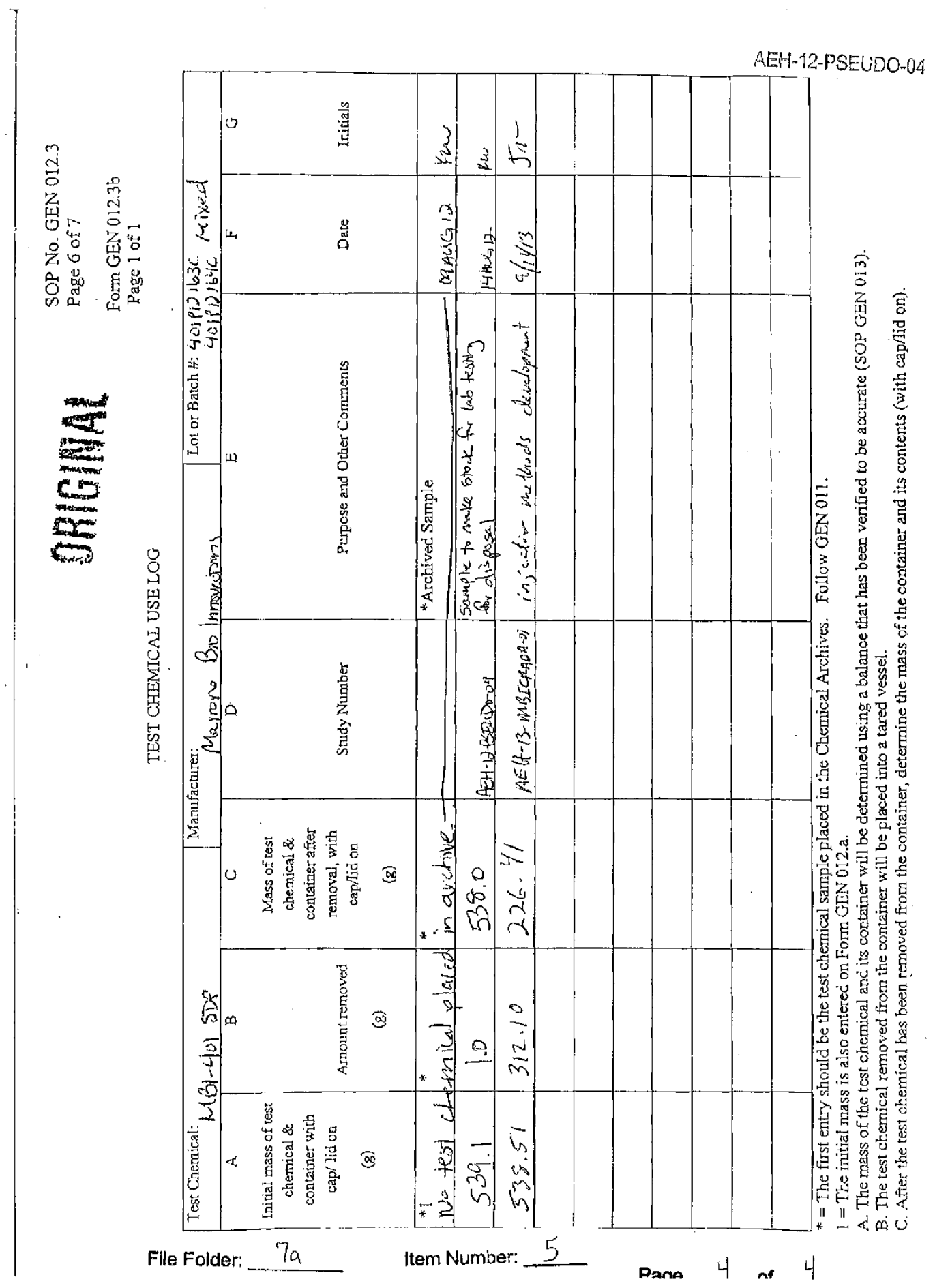

Page 271 of 519 


\section{Appendix 5. Test Animal Information}

\begin{tabular}{clcc}
\hline $\begin{array}{c}\text { Item } \\
\text { Number }\end{array}$ & \multicolumn{1}{c}{ Item Description } & $\begin{array}{c}\text { Number } \\
\text { of } \\
\text { Pages }\end{array}$ & $\begin{array}{c}\text { Report } \\
\text { Page } \\
\text { Number }\end{array}$ \\
\hline 1 & Approval for Housing and Care of Test Animals During Experiments & 1 & 273 \\
2 & Zebra Mussel Lengths - Lake Carlos (Whole Water Column) - Data Summary & 4 & 274 \\
3 & Zebra Mussel Lengths - Lake Carlos (Bottom Injection) - Data Summary & 2 & 278 \\
4 & Zebra Mussel Lengths - Lake Shawano (Whole Water Column) - Data Summary & 4 & 280 \\
5 & Zebra Mussel Lengths - Lake Shawano (Bottom Injection) - Data Summary & 2 & 284 \\
\hline
\end{tabular}


SOP NO. GEN 134.4

Page 4 of 4

ORIGINAL

Form GEN $134.4 a$

Page 1 of 1

\section{APPROVAL FOR HOUSING AND CARE OF TEST ANIMALS DURING EXPERIMENTS}

This protocol has been examined by the Animal Care and Use Committee for consistency with the Animal Welfare Act (7 U.S.C. 2131 et. seg.) and with rules governing the use of test animais at the Upper Midwest environmental Sciences Center. La Crosse.

We, the undersigned, find this protocol to be acceptable as it is represented.

Protocol Number: AE $12-12-$ PSEUDO-O4

Principal Investigator: Janves. A. heoma

Study Director:

Protocol Titie: Effecary of Psesidomonas therescens (Pf-CL (45A) SDP for contioling sotted febramissels on axhicial australes

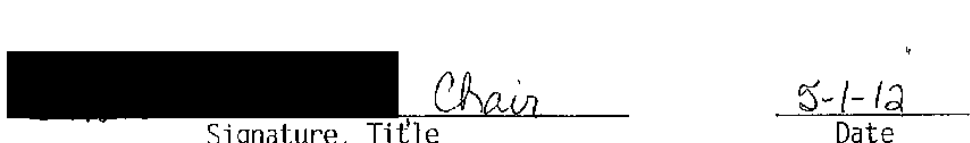

To additional Acu reviow neaded because no vestebates are pare of this sture,

Signature. Tit?e

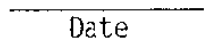

Signature. Title

Date

Signature. Tit:le

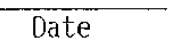

* File the original signed copy of this form in the study file for each study and the Chair. Anima: Care and Use Committee gets a copy.

File Folder: 6

Item Number: 1.

Page 1 of 1 


\begin{tabular}{|c|c|c|c|}
\hline \multirow{5}{*}{$\begin{array}{l}\text { Study Number: AEH-12-PSEUDO-04 } \\
\text { Electron'c Lab Notebook (pages 30) } \\
\text { Data Source: File Folder: } 15 \\
\text { Forms: "Zebra Mussel Lengtl-s" }\end{array}$} & Action & Date & Initials \\
\hline & Created.... & 28-Oct.13 & KLW. $\mathbb{R}^{\prime}$ \\
\hline & Revlsed...... & 12- *eb-14 & TJS TS TS \\
\hline & Revlewed.. & $12\} E B, 4$ & $m$ \\
\hline & Certified... & $2 \sqrt{1 x^{2}}$ & $J^{2}$ \\
\hline Fille Name: See filenames as stated below & & & \\
\hline
\end{tabular}

Zebra Mussel Lengths - Lake Carlos (Whole Water Column)

Test Artlcle: MBl 401 SDP [Pseudomonas fluorescens $P f$-CL. 145A (SOP)]

Artlcle Lot H: 401P12163C and 401P12164C Mlx

Exposure Date: August 15, 2012

Test Location: Lake Carlos, Alexandria, MN

Treatment Type: Whole Tark

Overa'l Lata Summary

\begin{tabular}{|c|c|c|c|c|c|c|c|c|c|}
\hline & $0 \mathrm{mg} / \mathrm{h}$ & $\begin{array}{c}6 \mathrm{~h} \\
50 \mathrm{mg} / \mathrm{l} \\
\end{array}$ & $100 \mathrm{mg} / \mathrm{L}$ & $0 \mathrm{mg} / \mathrm{L}$ & $\begin{array}{c}9 \mathrm{~h} \\
50 \mathrm{mg} / \mathrm{L} \\
\end{array}$ & $100 \mathrm{mg} / \mathrm{L}$ & $0 \mathrm{mg} / \mathrm{L}$ & $\begin{array}{r}12 \mathrm{\eta} \\
50 \mathrm{mg} / \mathrm{h} \\
\end{array}$ & $100 \mathrm{mg} / \mathrm{L}$ \\
\hline $\begin{array}{c}\text { Mean (mm) } \\
\text { (STD) }\end{array}$ & $\begin{array}{l}1 . .51 \\
(2.41)\end{array}$ & $\begin{array}{l}12,41 \\
(2,64)\end{array}$ & $\begin{array}{l}10.65 \\
(2.85)\end{array}$ & $\begin{array}{l}21.26 \\
(2.44)\end{array}$ & $\begin{array}{l}11.64 \\
(2.61)\end{array}$ & $\begin{array}{l}11.37 \\
(2.74)\end{array}$ & $\begin{array}{l}11.79 \\
(2.76)\end{array}$ & $\begin{array}{l}11.33 \\
(2.82)\end{array}$ & $\begin{array}{l}10.67 \\
(2.48)\end{array}$ \\
\hline Minkmum & 6.15 & 6.94 & 6.00 & 6.43 & 6.70 & 6.43 & 6.19 & 6.04 & 6.02 \\
\hline Maximum & 19.47 & 17.79 & 28.33 & 17.17 & 18.10 & 18.19 & 19.03 & 21.27 & 17.78 \\
\hline \begin{tabular}{|c|}
$\begin{array}{c}\text { Grand Mean } \\
\text { (STD) }\end{array}$ \\
\end{tabular} & & $\begin{array}{l}11.53 \\
(0.71) \\
\end{array}$ & & & $\begin{array}{l}11.42 \\
{[0.16 !} \\
\end{array}$ & & & $\begin{array}{l}11,26 \\
(0.46)\end{array}$ & \\
\hline
\end{tabular}

Data Explanatlon:

After survival analysls, one tray from each treatment level $(0,50$ and $100 \mathrm{mg} /$ L $)$ at each exposure termination time poirt $(6,9$ and $12 \mathrm{~h})$ was retained for nussel length analysls. All animals were measured tor length. All lengths were reported except for those from mussels with broken shelis or those $<6 \mathrm{~mm}$.

$6 \mathrm{~h}$ Mussel Length Data

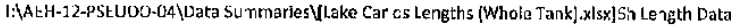

$9 \mathrm{~h}$ Mussel Length Dat

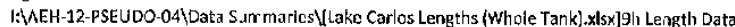

$12 \mathrm{~h}$ MLissel Length Da:e

I: \AEH-12-PSEUDO-04\}Data Summarles\[Lake Carlos Lengths (Whole Jank).xlsx)12h Length Data

Data anomalies and deviations:

NONE

Flle Folder: 15

Item Number $\quad 1$

Page. 1 ol 4

Page 274 of 519 

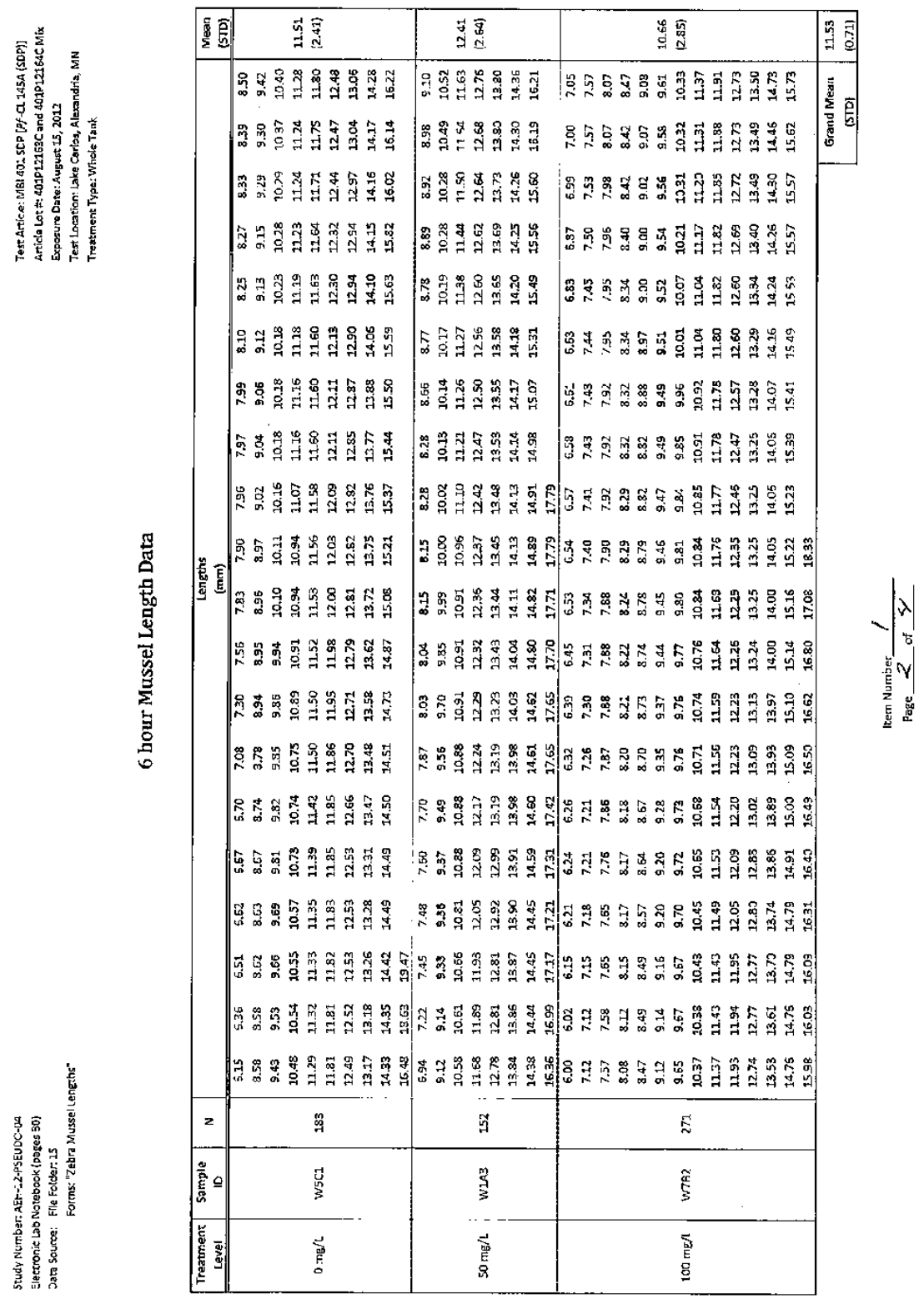

Page 275 of 519 


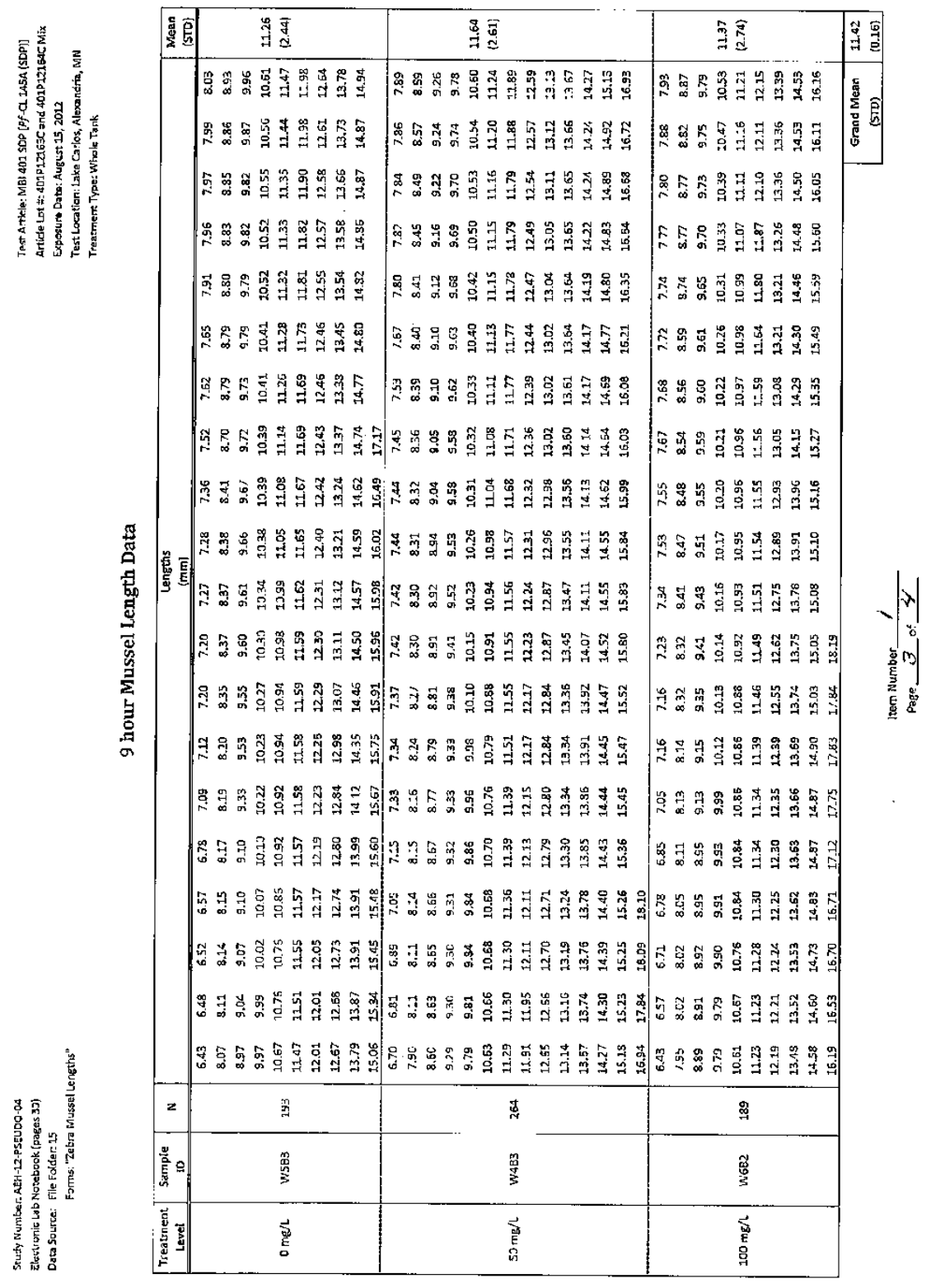




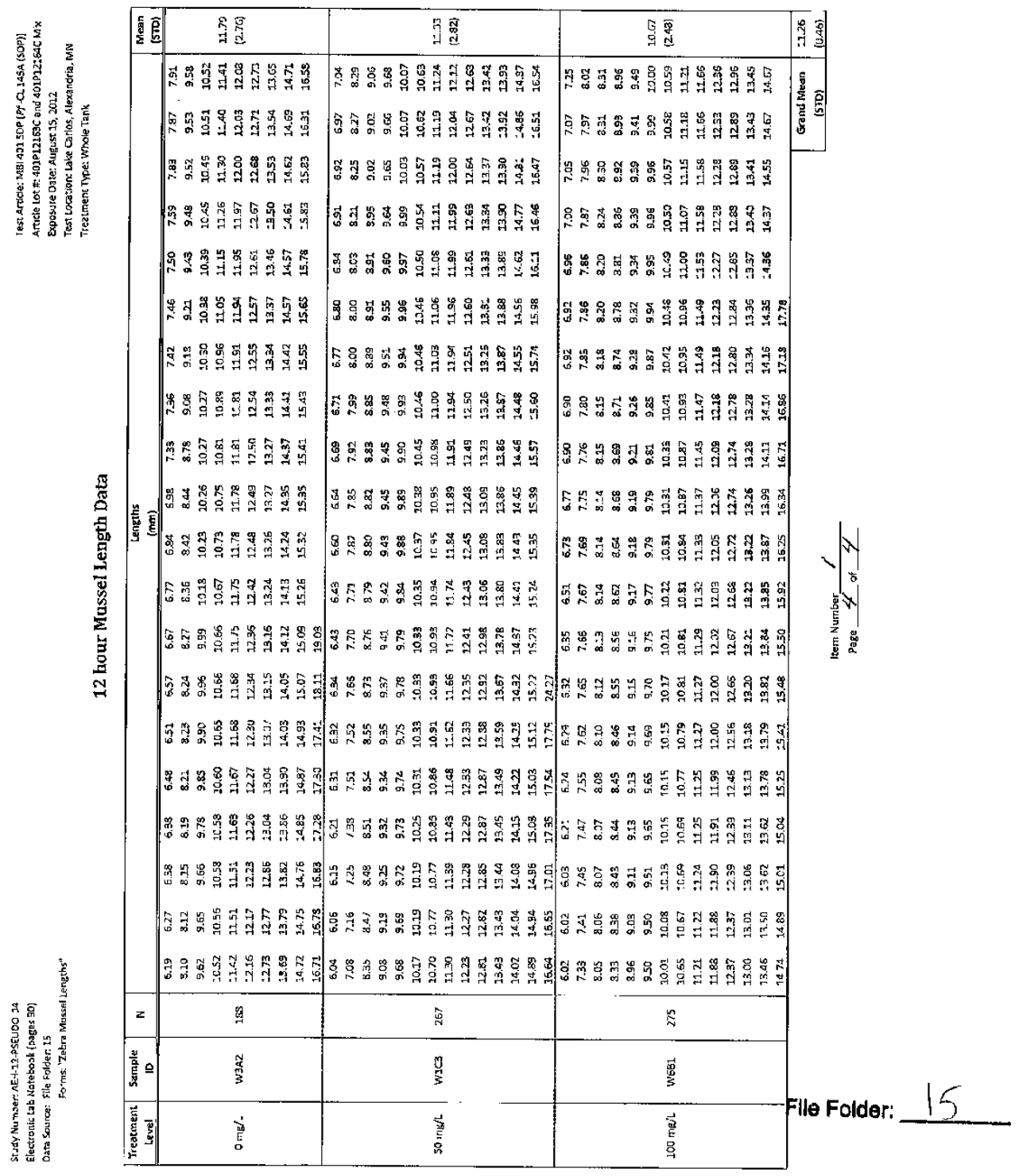

Page $\mathbf{2 7 7}$ of $\mathbf{5 1 9}$ 


\begin{tabular}{|c|c|c|c|}
\hline Study Number: AEH-12-PSELD0-04 & Actior & Date & In.tlais \\
\hline Electronlc Lab Notebook (pages 30) & Created...... & $28-$ Oct-13 & KLW TK \\
\hline Data Source: Flle Folder: is & Revised...... & 12 -Feb-14 & TJS 傮 \\
\hline \multirow[t]{2}{*}{ Forms: "Zebra Mussel Lengths' } & Revlewed... & 12 FEBIL & $\pi$ \\
\hline & Certifled... & $2 / 19 / 14$ & $5 h^{2}$ \\
\hline
\end{tabular}

Zebra Mussel Lengths - Lake Carlos (Bottom Injection)

Test Ar:lele: $\mathrm{ABC} 401$ SOP ipseudomanas fluorescens Pf - $\mathrm{CL}$ 145A [SDP)]

Art|cle Lot If: A01P1.2163C and 401P12164C Mlx

Exposure Date: August 17, 2012

Test Locatlon: Lake Carlos, Alexandria, MN

Treatment Type: Bottons Injectlon

Overall Data Summary:

\begin{tabular}{|c|c|c|c|}
\hline & $0 \mathrm{mg} / \mathrm{L}$ & $\begin{array}{c}12 \mathrm{~h} \\
50 \mathrm{mg} / \mathrm{h}\end{array}$ & $100 \mathrm{mg} / \mathrm{L}$ \\
\hline $\begin{array}{c}\text { Mean (mm) } \\
\text { (STDj) }\end{array}$ & $\begin{array}{l}12.34 \\
(2.70)\end{array}$ & $\begin{array}{l}11.22 \\
(2.66)\end{array}$ & $\begin{array}{l}11.99 \\
(2.88)\end{array}$ \\
\hline Minimum & 6.2 & 6.37 & 6.18 \\
\hline Maximum & 20.71 & 20.06 & $\therefore 8.94$ \\
\hline $\begin{array}{c}\text { Grand Mea } \\
\text { (STO) }\end{array}$ & & $\begin{array}{l}11.85 \\
10.47) \\
\end{array}$ & \\
\hline
\end{tabular}

Data Explanatlon:

After strvival analysis, one tray from each treatment level 10,50 and $100 \mathrm{mg} / \mathrm{L}$ ) from the $12 \mathrm{~h}$ exposure termination tlme polnt was retained for mussel length analysis. All aninala were measured for length. All lengths were reported except for those from mussels with broken shells or those $<6 \mathrm{~mm}$.

Data anomalles and devatlons

NUNE

Flle Folder: 15

Item Numbet 2

Page 1 of 2

Page 278 of 519 


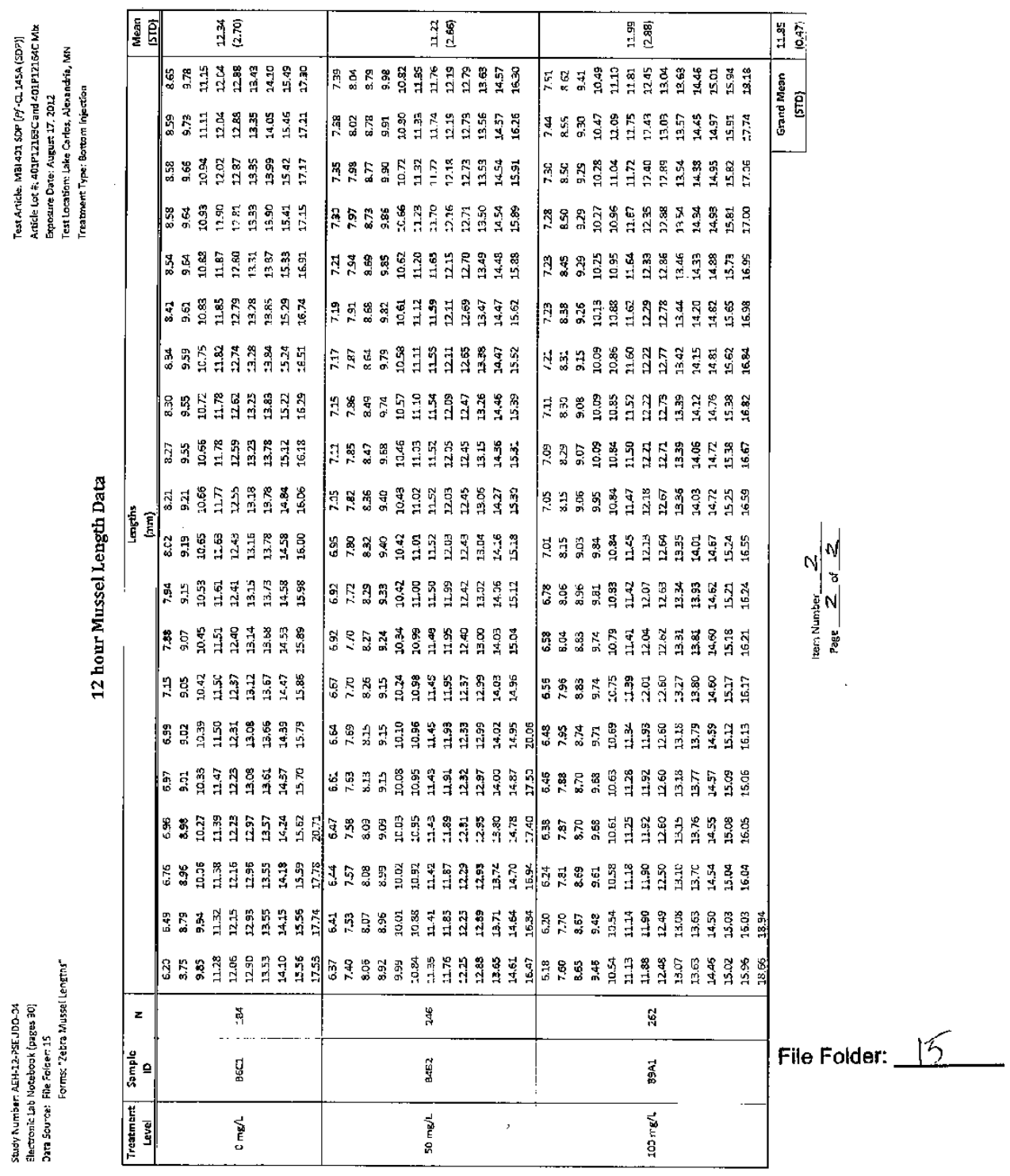




\begin{tabular}{|c|c|c|c|}
\hline \multirow{5}{*}{$\begin{array}{l}\text { Study Number: AEH-12-PSEUDO-04 } \\
\text { Electronlc Lab Notebook (pages } 30 \text { ) } \\
\text { Data Source: Flie Folder: } 16 \\
\text { Forms: "Zebra Mussel Lengths" }\end{array}$} & Action & Date & Inittals \\
\hline & Created...... & 28-0ct-13 & KLW +7, \\
\hline & Revised........... & 13-Feb-14 & TIS 723 \\
\hline & Revlewed... & $13 E E B H$ & $7 \% 5$ \\
\hline & Cer!lfled... & allisilin & JAL" \\
\hline Flle Name: See fllenanes as stated below & & & \\
\hline
\end{tabular}

Zebra Mussel Lengths - Lake Shawano (Whole Water Column)

Test Artlde: MBI 401 SDP [Pseudomonas fluorescens Pf $\cdot \mathrm{Cl}$ 145A 'SDP)]

Article Lot H: 401P12163C and 401P12164C Mix

Exposure Datc: September 6, 2012

Test Location: Lake Shawanc, Shawano, wI

Treatment Type; Whole Tank

Qverall Data Summary:

\begin{tabular}{|c|c|c|c|c|c|c|c|c|c|}
\hline & \multicolumn{3}{|c|}{$6 \mathrm{~h}$} & \multicolumn{3}{|c|}{$9 \mathrm{~h}$} & \multicolumn{3}{|c|}{$12 \mathrm{~h}$} \\
\hline & $0 \mathrm{mg} / \mathrm{h}$ & $50 \mathrm{mg} / \mathrm{l}$ & $100 \mathrm{mg} / \mathrm{h}$ & $0 \mathrm{mg} / \mathrm{h}$ & $50 \mathrm{mg} / \mathrm{L}$ & $100 \mathrm{mg} / \mathrm{l}$ & $0 \mathrm{mg} / \mathrm{L}$ & $50 \mathrm{mg} / \mathrm{h}$ & $100 \mathrm{mg} / \mathrm{L}$ \\
\hline Mean (mm) & 18.33 & 18.88 & 18.80 & 18.65 & 18.67 & 18.89 & 18.67 & 17,87 & 18.28 \\
\hline & $(3,10)$ & & $(2.77)$ & $(2.64)$ & [2.71] & (2.94) & $(2,52)$ & (2.82) & (9.22) \\
\hline MIn!mum & 7.49 & 8.13 & 11.64 & 8.33 & 11.02 & 6.23 & 13.28 & 7.57 & 6.55 \\
\hline Maximum & 26.57 & 35.04 & 26.34 & 27.72 & 26.42 & 27.77 & 25,44 & 26.62 & 29.30 \\
\hline $\begin{array}{c}\text { Grand Mean } \\
\text { (STO) }\end{array}$ & & $\begin{array}{l}18.67 \\
(0.24)\end{array}$ & & & $\begin{array}{l}18.74 \\
(0,11)\end{array}$ & & & $\begin{array}{l}18.27 \\
(0.33)\end{array}$ & \\
\hline
\end{tabular}

Data Explanation:

After survival analysis, oric tray from each treatment level 10,50 and $: 00 \mathrm{mg} / L)$ at each exposure termination time point $(6,9$ and $12, \mathrm{~h})$ was retalned for inussel length analysls. All anirnals were measured for length. All lengths were reported except for tinose from mussels with broken she is or those $<6 \mathrm{~mm}$

$6 \mathrm{~h}$ Mussel Length Data

I: (AEH-12-PSEUDO-04\Data Summerlest[Lake Shawano Lengths (Whole Tank).xlsx] Gh Length Data

9 h Mussel Length Data

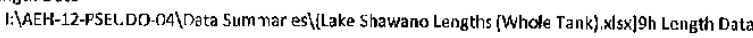

$12 \mathrm{~h}$ Mussel Length Data

I:AEH-12-PSEUDO-04\Data Surmmarles\}|Lake Shawano Lengths (Whole Tank).xisx|12h Length Data

Data anomolies and devlations:

NONE

File Folder:

Item Number $\frac{1}{1}$

Page $\mathbf{2 8 0}$ of $\mathbf{5 1 9}$ 

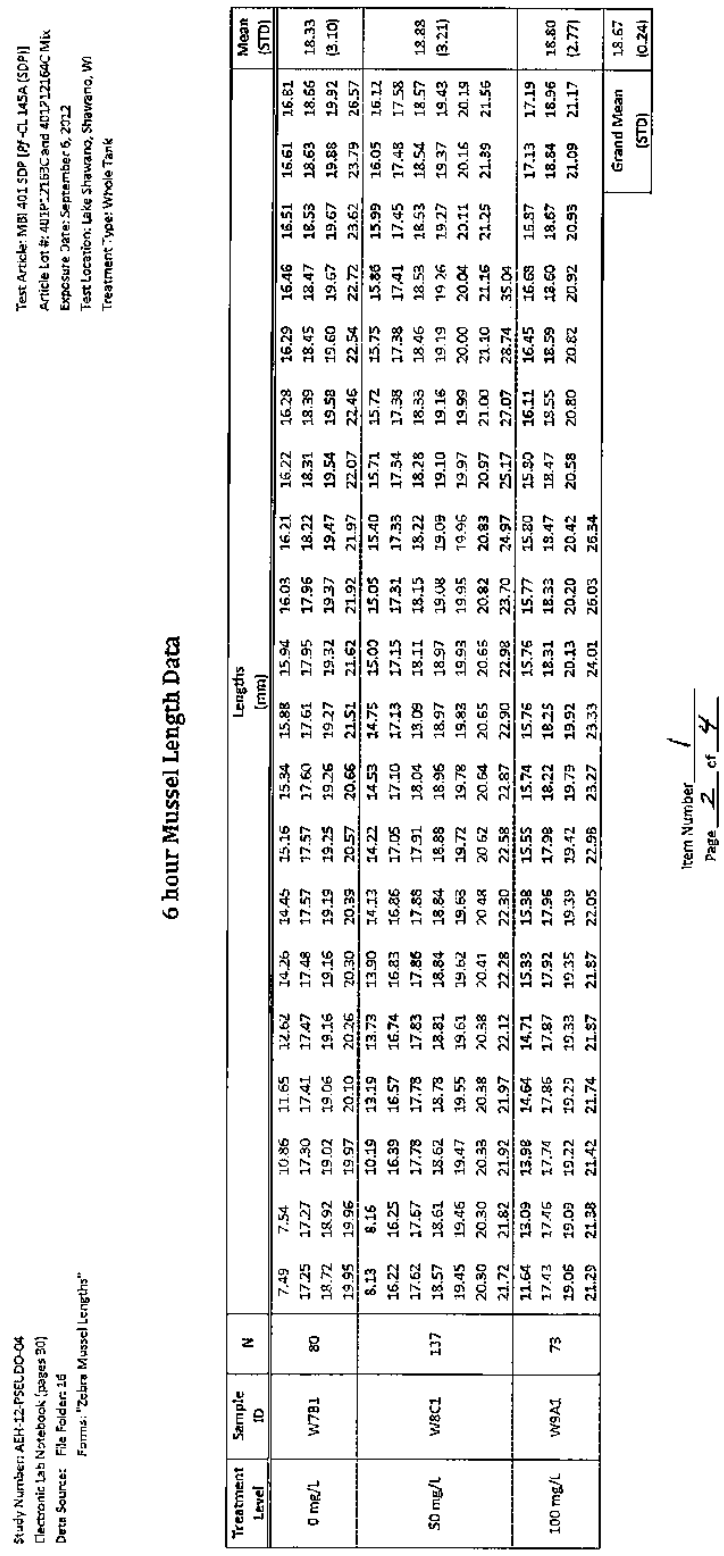

Page 281 of 519 

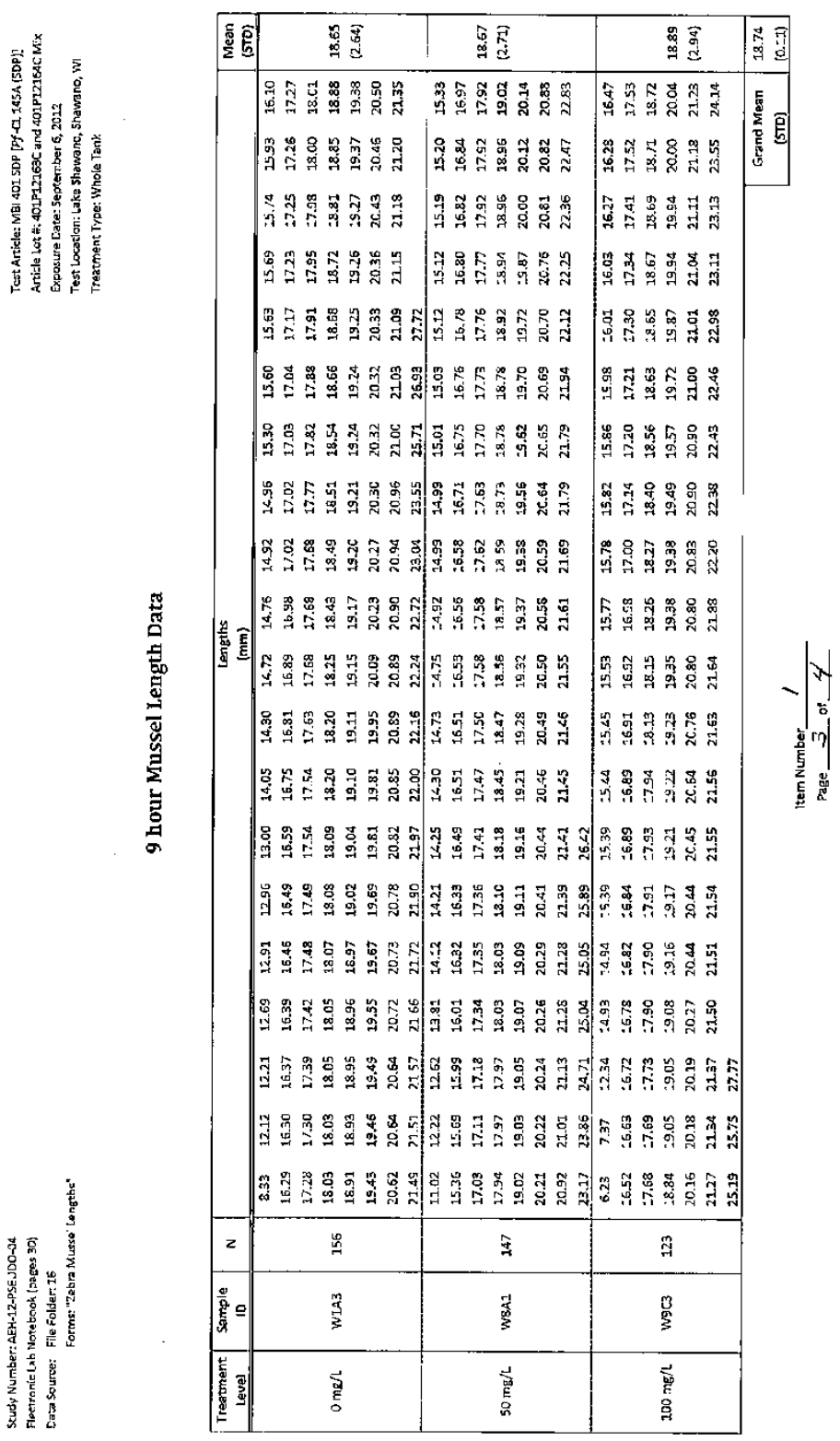

Page 282 of 519 

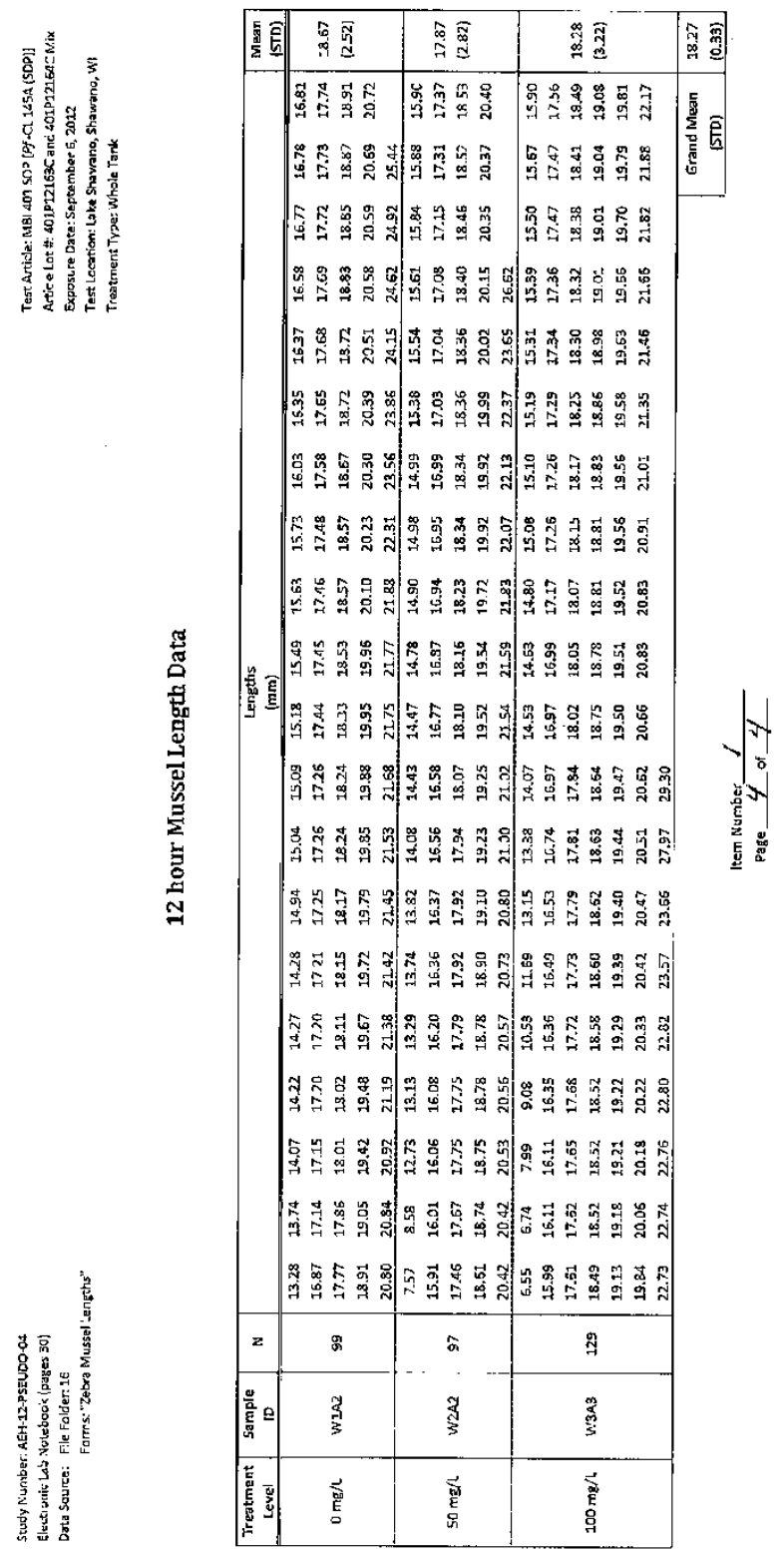

File Folder: 16 


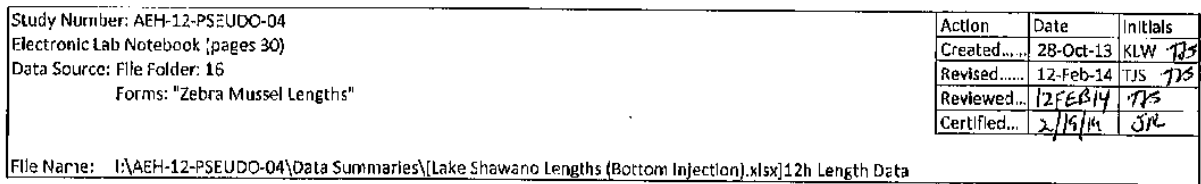

Zebra Mussel Lengths - Lake Shawano (Bottom Injection)

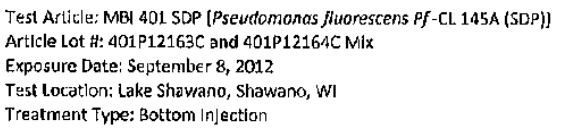

Qverall Data Summary:

\begin{tabular}{|c|c|c|c|}
\hline & \multicolumn{3}{|c|}{$12 \mathrm{~h}$} \\
\hline Mean $(\mathrm{mm})$ & 18.92 & 18.59 & 18.52 \\
\hline (STD) & (2.58) & (2.71) & $\{2.81\}$ \\
\hline Min|mum & 13.68 & 9.86 & 9.00 \\
\hline Maximum & 30.12 & 29.01 & 30.72 \\
\hline $\begin{array}{l}\text { Grand Mean } \\
\text { (STD) }\end{array}$ & & $\begin{array}{l}18.68 \\
(0.18)\end{array}$ & \\
\hline
\end{tabular}

Dasa Explanation:

After survival analysis, one tray from each treatment level $(0,5)$ and $100 \mathrm{mg} / L \mathrm{~L}$ trom the $12 \mathrm{~h}$ exposure termlratlon time point was retaincd for mussel length analysls. All animals were measured for length. All lengths were reported except for those from mussels with broken shells or those $<6 \mathrm{~mm}$.

Data anomalles and deviations:

NONE

\author{
Fille Folder: 16 \\ Item Number $\frac{2}{1}$ of 2
}

Page 284 of 519 


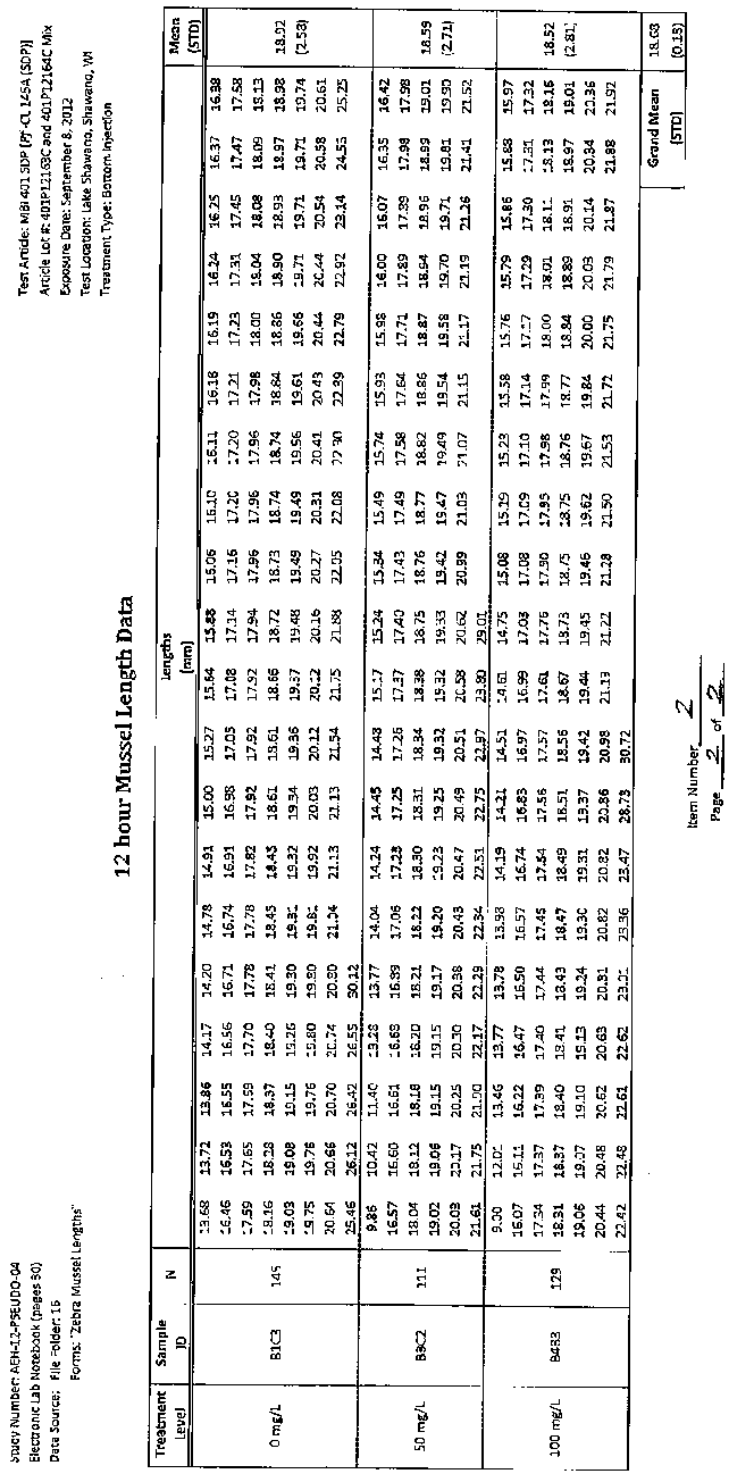

File Folder: 16 


\section{Appendix 6. Water Quality}

\begin{tabular}{clcc}
\hline $\begin{array}{c}\text { Item } \\
\text { Number }\end{array}$ & \multicolumn{1}{c}{ Item Description } & $\begin{array}{c}\text { Number } \\
\text { of } \\
\text { Pages }\end{array}$ & $\begin{array}{c}\text { Report } \\
\text { Page } \\
\text { Number }\end{array}$ \\
\hline 1 & Pre-Exposure Water Chemistry - Lake Carlos - Whole Tank - Data Summary & 2 & 287 \\
2 & Exposure Water Chemistry - Lake Carlos - Whole Tank - Data Summary & 2 & 289 \\
3 & Exposure Un-ionized Ammonia - Lake Carlos - Whole Tank - Data Summary & 3 & 291 \\
4 & Pre-Exposure Water Chemistry - Lake Shawano - Whole Tank - Data Summary & 2 & 294 \\
5 & Exposure Water Chemistry - Lake Shawano - Whole Tank - Data Summary & 2 & 296 \\
6 & Exposure Un-ionized Ammonia - Lake Shawano - Whole Tank - Data Summary & 3 & 298 \\
7 & Pre-Exposure Water Chemistry - Lake Carlos - Bottom Injection - Data Summary & 2 & 301 \\
8 & Exposure Water Chemistry - Lake Carlos - Bottom Injection - Data Summary & 2 & 303 \\
9 & Exposure Un-ionized Ammonia - Lake Carlos - Bottom Injection - Data Summary & 3 & 305 \\
10 & Pre-Exposure Water Chemistry - Lake Shawano - Bottom Injection - Data Summary & 2 & 308 \\
11 & Exposure Water Chemistry - Lake Shawano - Bottom Injection - Data Summary & 2 & 310 \\
12 & Exposure Un-ionized Ammonia - Lake Shawano - Bottom Injection - Data Summary & 3 & 312 \\
13 & Temperature Data Loggers (HOBOs) Summary - Lake Carlos & 2 & 315 \\
14 & Temperature Data Loggers (HOBOs) Summary - Lake Shawano & 2 & 317 \\
\hline
\end{tabular}




\begin{tabular}{|c|c|c|c|}
\hline \multirow{3}{*}{$\begin{array}{l}\text { Study Number: AEH-12-PSEUDO-04 } \\
\text { Electrcnlc Lab Notebock (page 10) } \\
\text { Data Source: Flle Folder: } 9 \mathrm{~b} \\
\text { Forms: } \quad \text { See form names as stated below }\end{array}$} & Action & Date & In|ttals \\
\hline & Created,...... & 19-Oct-13 & KLW $/ W$ \\
\hline & \begin{tabular}{|l|} 
Revlewed.. \\
Certified..
\end{tabular} & $\frac{10 \mathrm{DECI}}{12 / 10 / 14}$ & WN \\
\hline
\end{tabular}

\section{Pre-Exposure Water Chemistry}

Test Artcle: MBI 401 SDP [Pseudomonas fluorescens $P f \cdot C L$ 145A (SDP)]

Art|cle Lot \#: 101P12163C and 401P12164C MIX

Fxposure Date: August 15, 20.12

Test Location: Lake Car os, Alexandria, MN

Treatment Type: Whole Tank

Data Explanation:

Forms titled "Conductlvity and Hardness - Exposure Initiatlon" and "Alkalinlty - Exposure Inltlatlon"; Conductlvity, hardness, and alkalinity were measured prior to dosing. Samples were collected from both headboxes used to flll Indiv dual tanks. Samples were analyzed in triplicate.

Forms titied "Water Quality - Temperature ("C) Measurements", "Water Quality - pH Measurements" and "Water Quality - Dissolved Oxygen (mg/L) Meas.urements": Temperature, $\mathrm{pH}$ ard dissolved oxygen levels were measured prlor to dosing. Measurements were observed in each exposure tark.

Data anomalics and devlatlons:

NONE

File Folder: 96

Item Numper $\frac{1}{2}$ 

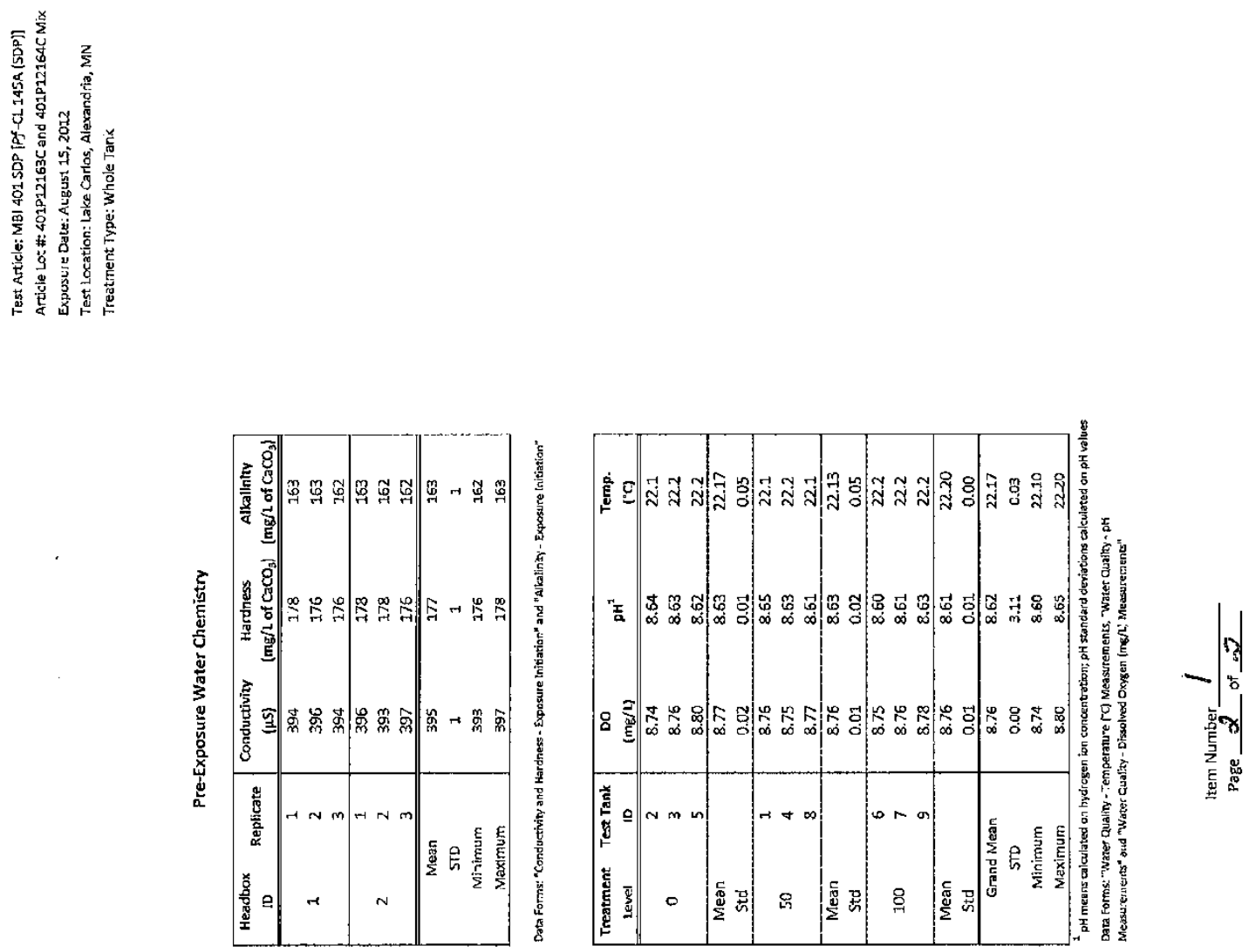

File Folder: $q b$ 
Study Number: AEH-12-PSEUDO-DA

Electronic Lab Notebook (page 11)

Data Source: File Folder: $9 b$

Forms: "Water Quality - Temperature $\left({ }^{\circ} \mathrm{C}\right)$ Measurements"

"Water Quality - pH Measurements"

"Water Quality - Dissolved Oxygen (mg/L) Measurements"

\begin{tabular}{|l|l|l|}
\hline Action & Date & Initials \\
\hline
\end{tabular}

\begin{tabular}{|c|c|c|}
\hline Created...... & 19-Oct-13 KLW WW \\
\hline ReVed & KL.
\end{tabular}

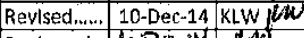

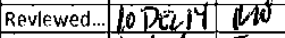

Certifled... $12 / 10 / 14.52-$

File Name: I:\AEH-12-PSEUDO-04\Data Summarles \Water chem!(Lake Carlos Water Chem (Whole Tank) 11-26-14.xlsx] Exposure Water Chem

Exposure Water Chemistry

Test A-ticle: MB| 401 SDP [Pseudomonas fluorescens Pf $-\mathrm{C}$. 145A (SDP)]

Article Lot \#: 401P12163C and 401P12164C Mix

Exposure Date: Augusl 15, 2012

Test Locatlon: Lake Carlos, Alexandria, MN

Treatment Type: Whole Tank

Da:a Explanatlon:

Waler chemlstry meas urements (dissolved oxygen, $\mathrm{oH}$ and temperature) were observed for all test tanks at $0,3,6,9$ and $12 \mathrm{~h}$ after dosing. NOTE: $0 \mathrm{~h}$ measurements were observed from 30 minutes to $1 \mathrm{~h}$ after dosing.

Data anomalles and deviations:

NONE

File Folder:

Item Number o 2

Page $\mathbf{2 8 9}$ of 519 


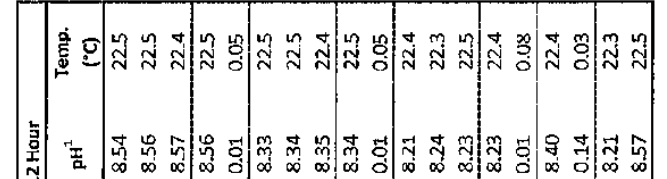

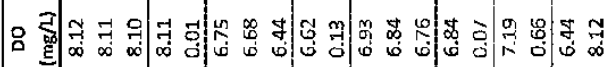

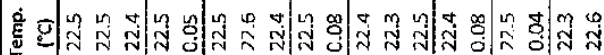

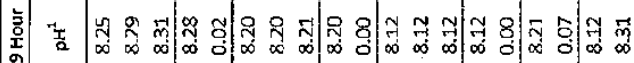

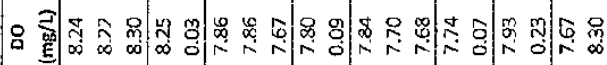

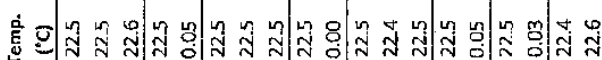

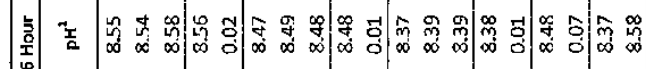

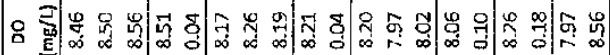

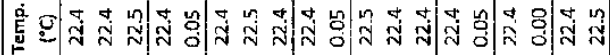

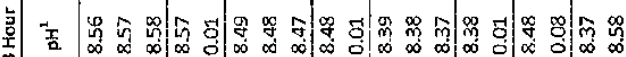
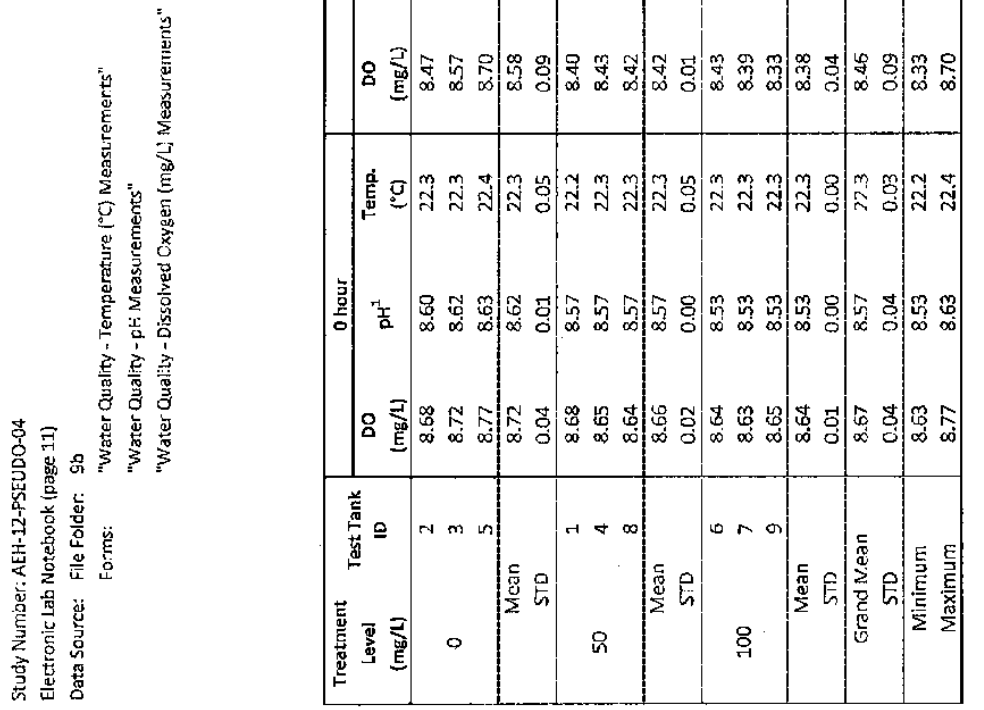

File Folder: $9 b$ 
Study Number: AE:H-12-PSEUDO-04

Electronic Lab Notebook (page 11)

TAN Data Source: LTRMP Report (File Folder 17)

$\mathrm{pH}$ and Temperature Data Source; Water Quality forms (file Folder 9b)

\begin{tabular}{|l|l|l|}
\hline Actlon & Date & |nitials \\
\hline Created & $19-O c t-13$ & LWhitho \\
\hline
\end{tabular}

\begin{tabular}{|c|c|c|}
\hline Created...... & 19-Oct-13 KLWlW \\
\hline Revised
\end{tabular}

\begin{tabular}{l|c|c|}
\hline Revised...... & 10-Dec-14 KLW & LW \\
\hline
\end{tabular}

Reviewed... 100 FCL 14.5

\begin{tabular}{l|l|l|l|l|l|}
\hline Certlfied..... &, $2 / 10 / 14$ \\
\hline
\end{tabular}

File Name: Ii\AEH-12-PSEUDO-04\Data Summaries'Water chem [Lake Carlos Water Chem (Whole Tank) 11-26-:4.xlsx]Ammonia Data

Exposure Un-ionized Ammonia

Test Article; MBI 401. SDP [Pseudomonas fluorescens Pf-CL 145A (STF)]

Article Lot \#: 401P12163C and 401P12164C Mix

Exposure Date: Augsst 15, 2012

Test Location: Lake Carlos, Alexandria, MN

Treatment Type: Whole Tank

Data Explanation

1) Water samples were collected at 6,9 and $1.2 \mathrm{~h}$ from each exposure tank. Samples were $0.45 \mathrm{\mu m}$ filtered, acidlfied with sulfuric acid, and analyzed

by the $4500-\mathrm{NH}_{3}$ G. Automated Phenate Method (5tandard Methods for the Examination of Water and Wastewater, 21st Edition, 2005) on a

Technicon Autoanalyzer II by the UMESC water quality laboratory.

2) The un-ionized ammonia fractions were calculated using the sample pH and temperature according to the formula in Emerson et al. (1975),

Data Anomalles and Deviations:

NONE

File Folder: $q b$ 
Study Number: AEH-12-PSEUDO-04

Electronlc Lab Notebook (page 11)

TAN Data Source: LTRMP Report (File Folder 17)

$\mathrm{pH}$ and Temperature Data Source: Water Quality Forms (Flle Folder 9b)

Test Artic e: MB: A01 SDP (PF CLL 1A5A (SDP)

Artlde Lo: : : 401PJ2163 and 401P12164C Ml|x

Exposure Date: August 15, 2012

Test Locatlon: Lake Carlos, Alexandrla, MN

Treatment Type: Whole Tank

Exposure Un-ionized Ammonia

\begin{tabular}{|c|c|c|c|c|c|c|c|}
\hline $\begin{array}{c}\text { Sample } \\
\text { Tirnt } \\
\end{array}$ & $\begin{array}{c}\text { Treatment } \\
\text { Lovel } \\
\text { (eng/L) }\end{array}$ & $\begin{array}{l}\text { Test Tank } \\
\text { ID }\end{array}$ & $\mathrm{pH}^{\mathrm{I}}$ & $\begin{array}{c}\text { Temperature } \\
(\mathrm{C})\end{array}$ & $\begin{array}{c}\text { TAN } \\
\text { as } \mathrm{NH}_{3}-\mathrm{N}(\mathrm{m} \mathrm{g} / \mathrm{L})\end{array}$ & $\begin{array}{l}\text { Un-lon zed } \\
\text { Fraction }\end{array}$ & $\begin{array}{c}\mathrm{NH}_{3} \\
(\mathrm{mef} / \mathrm{L})^{3}\end{array}$ \\
\hline \multirow{15}{*}{6} & \multirow{3}{*}{0} & 2 & 8.55 & 22.5 & 0.174 & 0.144 & 0.025 \\
\hline & & 3 & 8.54 & 22.5 & 0.172 & 0.141 & 0.024 \\
\hline & & 5 & 8.58 & 22,6 & 0.167 & 0.154 & 0,026 \\
\hline & \multirow{2}{*}{\multicolumn{2}{|c|}{$\begin{array}{c}\text { Mean } \\
\text { std }\end{array}$}} & 8.56 & 22.5 & 0.171 & 0.146 & 0.025 \\
\hline & & & 0.02 & 0.0 & $0.0 \times 3$ & 0.005 & 0.001 \\
\hline & \multirow{3}{*}{50} & 1 & 8,17 & 22.5 & 0.225 & 0.123 & 0.028 \\
\hline & & 4 & 8.49 & 22.5 & 0.229 & 0.128 & 0.029 \\
\hline & & 8 & 8.48 & 22.5 & 0.215 & 0.125 & 0.027 \\
\hline & \multicolumn{2}{|l|}{ Mean } & 8.48 & 22,5 & 0.223 & 0.125 & 0.028 \\
\hline & \multirow{2}{*}{\multicolumn{2}{|c|}{ std }} & 0.01 & 0.0 & 0.006 & 0.002 & 0.001 \\
\hline & & & 8.37 & 22.5 & 0.262 & $0.1 \mathrm{CO}$ & 0.026 \\
\hline & \multirow[t]{2}{*}{100} & 7 & 8.39 & 22.4 & 0.272 & $0.1 \mathrm{CA}$ & 0.028 \\
\hline & & 9 & 8.39 & 22.5 & 0.270 & $0.1 C 4$ & 0.023 \\
\hline & \multirow{2}{*}{\multicolumn{2}{|c|}{$\begin{array}{c}\text { Mean } \\
\text { std }\end{array}$}} & 8.38 & 22.5 & 0.268 & $0.1 \mathrm{C} 3$ & 0.028 \\
\hline & & & 0.01 & 0.0 & 0.004 & 0.002 & 0.001 \\
\hline \multirow{15}{*}{9} & \multirow{3}{*}{0} & 2 & 8.25 & 22.5 & 0.171 & 0.078 & 0.013 \\
\hline & & 3 & 8.29 & 22.5 & 0.169 & 0.085 & 0.014 \\
\hline & & 5 & 8.31 & 22.4 & 0.180 & 0,088 & 0,015 \\
\hline & \multirow{2}{*}{\multicolumn{2}{|c|}{$\begin{array}{l}\text { Mean } \\
\text { std }\end{array}$}} & 8.28 & 22.5 & 0.173 & 0.083 & 0.014 \\
\hline & & & 0.02 & 0.0 & 0.005 & 0.004 & 0.001 \\
\hline & \multirow{3}{*}{50} & 1 & 8.20 & 22,5 & 0.225 & 0.070 & 0.015 \\
\hline & & 4 & 8.20 & 22.6 & 0.223 & 0.070 & 0.016 \\
\hline & & 8 & 8.21 & 22.4 & 0.231 & 0.071 & 0.016 \\
\hline & \multirow{3}{*}{$\begin{array}{l}\text { Mean } \\
\text { std }\end{array}$} & & 8.20 & 22.5 & 0.226 & 0.070 & 0,016 \\
\hline & & & 0.00 & C.1 & 0.003 & 0.000 & 0.000 \\
\hline & & -6 & 8.12 & 22.4 & 0.273 & 0.658 & 0.016 \\
\hline & \multirow[t]{2}{*}{100} & 7 & 8.12 & 22.3 & 0.273 & 0.058 & 0.015 \\
\hline & & 9 & 8.12 & 22.5 & 0.273 & 0.059 & 0,015 \\
\hline & \multirow{2}{*}{\multicolumn{2}{|c|}{$\begin{array}{c}\text { Mean } \\
\text { std }\end{array}$}} & 8.12 & 22,4 & 0.273 & 0.058 & 0.015 \\
\hline & & & $0.0 \mathrm{C}$ & 0.1 & $0.00 \mathrm{C}$ & 0,000 & 0,000 \\
\hline \multirow{15}{*}{12} & \multirow{3}{*}{0} & 2 & 8.54 & 22.5 & 0.224 & 0.141 & 0.032 \\
\hline & & 3 & 8.56 & 22.5 & 0.254 & $0.14 \%$ & 0.037 \\
\hline & & 5 & 8.57 & 22,4 & 0.256 & 0.149 & 0.038 \\
\hline & \multirow{2}{*}{\multicolumn{2}{|c|}{$\begin{array}{l}\text { Mean } \\
\text { std }\end{array}$}} & 8.56 & 22.5 & 0.245 & 0.146 & 0.036 \\
\hline & & & 0.01 & 0.0 & 0.015 & 0.003 & 0.003 \\
\hline & \multirow{3}{*}{50} & 1 & 8.33 & 22,5 & 0.318 & 0.092 & 0.029 \\
\hline & & 4 & 8.34 & 22.5 & 0.331 & 0.094 & 0.031 \\
\hline & & 8 & 8.35 & 22.4 & 0.334 & 0.c95 & 0.032 \\
\hline & \multirow{2}{*}{\multicolumn{2}{|c|}{$\begin{array}{l}\text { Mean } \\
\text { std }\end{array}$}} & 8.34 & 22.5 & 0.328 & 0.094 & 0.031 \\
\hline & & & 0.01 & 0.0 & 0.007 & 0.001 & 0.001 \\
\hline & \multirow{3}{*}{100} & 6 & 8,21 & 22.4 & 0.354 & 0.071 & 0.025 \\
\hline & & 7 & 8.24 & 22.3 & 0.339 & 0.075 & 0.025 \\
\hline & & 9 & 8.23 & 22,5 & 0.333 & 0.075 & 0.025 \\
\hline & \multirow{2}{*}{\multicolumn{2}{|c|}{$\begin{array}{l}\text { Mean } \\
\text { std }\end{array}$}} & 8.23 & 22.4 & 0.342 & 0.074 & 0.025 \\
\hline & & & 0.01 & 0.1 & 0.009 & 0.002 & 0.000 \\
\hline
\end{tabular}

${ }^{1} \mathrm{pH}$ means calculated on hydrogen ion concentration; $\mathrm{pH}$ standard deviations calc, ated on $\mathrm{pH}$ values

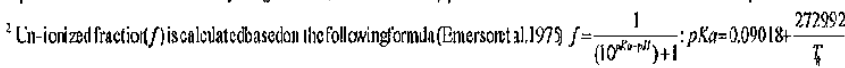

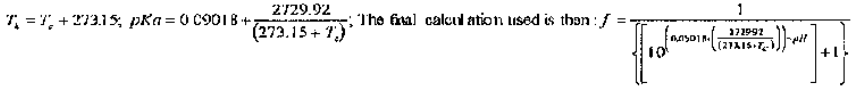

${ }^{3}$ Un-lonlzed ammonla is calculated based on the following formula: Un-ionized amnonla $=f$ * TAN (mg $/ L$ )

Item Number $\frac{3}{2} \frac{3}{\text { Page of } 2.3}$

Page 292 of 519 


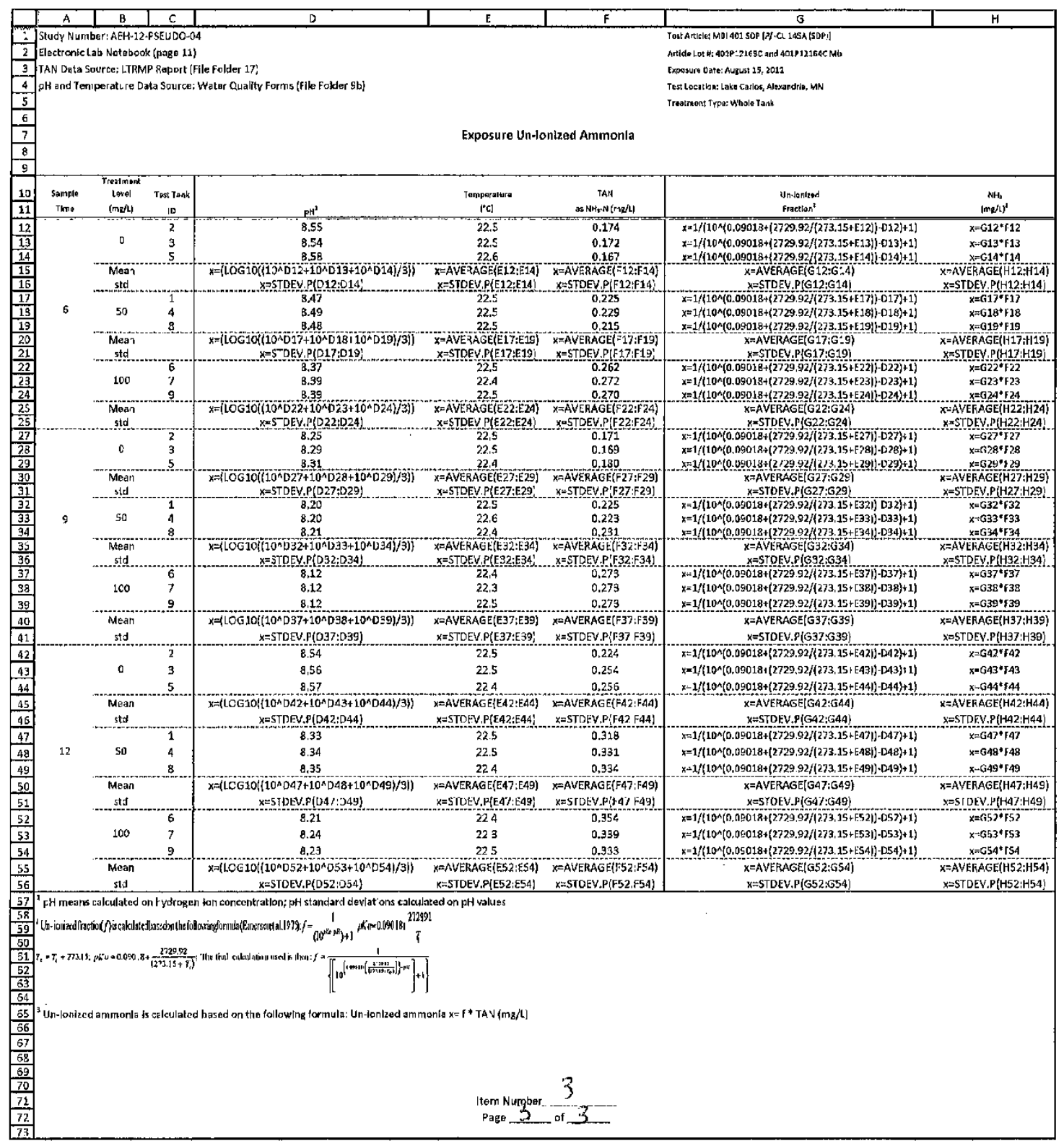

File Folder:

95 


\begin{tabular}{|c|c|c|c|}
\hline \multirow{4}{*}{\begin{tabular}{|l} 
Study Vumber: $A$ EH-12-PSEUDO-04 \\
Electronic Lab Notebook (page 21) \\
Da:a Source: Fille Folder: $11 \mathrm{~b}$ \\
Forms: See form names as stated below
\end{tabular}} & Action & Date & Initia|s \\
\hline & C'eated..... & 19-0ct-13 & KLW $/ M$ \\
\hline & Revlsed..... & 10-Dec-14 & KLWWO \\
\hline & \begin{tabular}{|l|} 
Revlewed... \\
Certifled... \\
\end{tabular} & $\frac{10 p 21^{4}}{12 / 10}$ & $\frac{4 n}{5 n v}$ \\
\hline
\end{tabular}

\section{Pre-Exposure Water Chemistry}

Test Artlcle: MBI 401 SDP [Pseudomonas fluorescens Pf $-C L$ 145A (SDP)]

Article Lot \#: $401 \mathrm{P} 12163 \mathrm{C}$ and $401 \mathrm{P} 12164 \mathrm{C}$ Mix

Exposure Date: Seotember 6, 2012

Test Location: Lake Shawano, Shawano, wi

Treatment Type: Whole Tank

Data Explanation:

Forms titled "Corductivity and Hardress - Exposure Initlatlon" and "A kalinlty - Exposure Initlation": Conductivity, hardness, and alkallnity were measured prior to dosing. Samples were collected from both headboxes used to fill indiv dual tanks, Samples were analyzed In triolicate.

Forms titled "Water Quality - Temperature ("C) Measurements", "Water Quality - pH Measurements" and "Water Quallty - Dissolved Oxygen (mg/L) Measurements": Temperature, $\mathrm{pH}$ and cissolved oxygen tevels were measured prior to dosing. Measurements were observed in each exposure sank.

Data anomalles and deviatlons:

NONE

File Folder: $11 b$

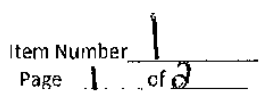



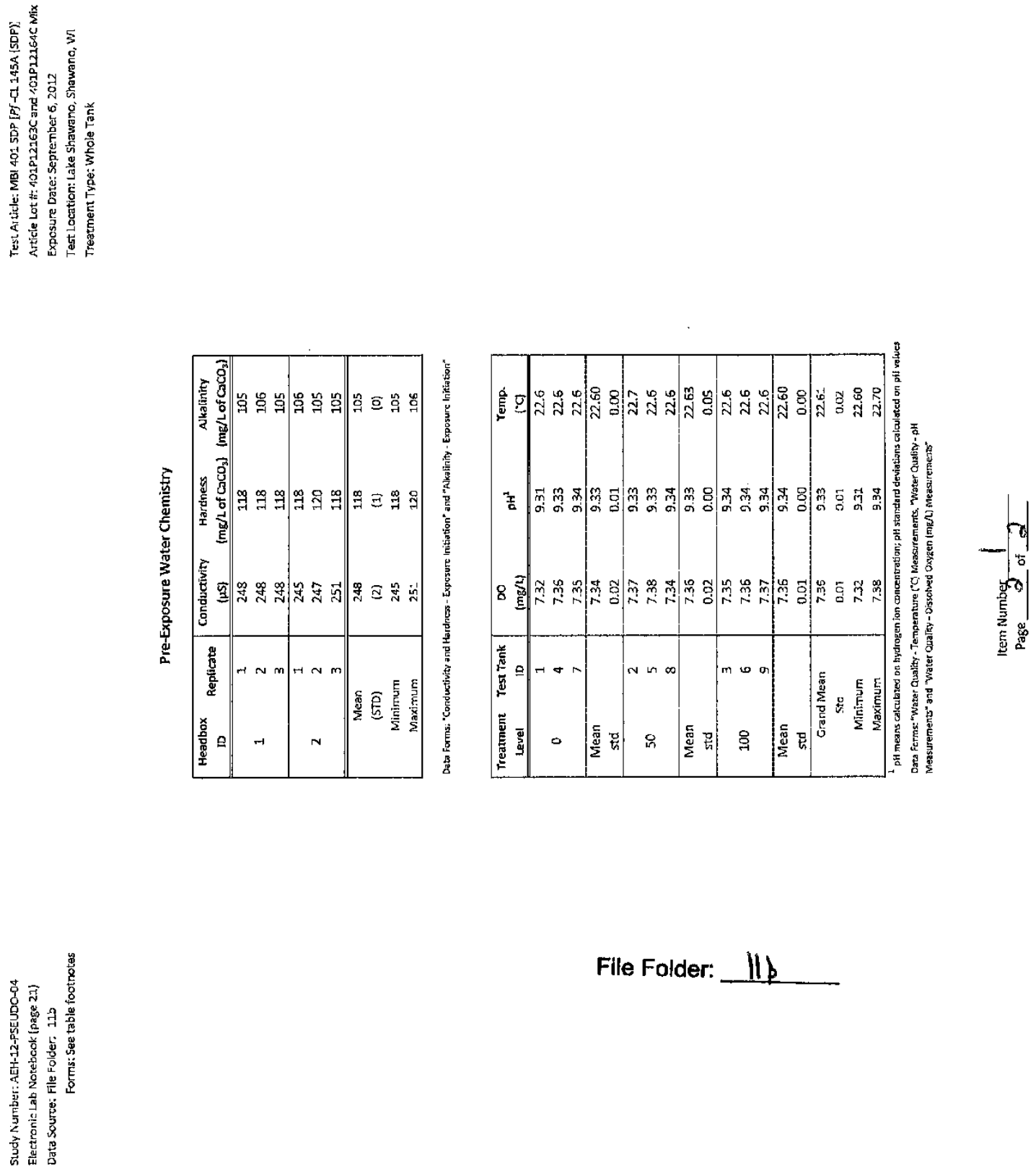

File Folder: $\|_{k}$ 


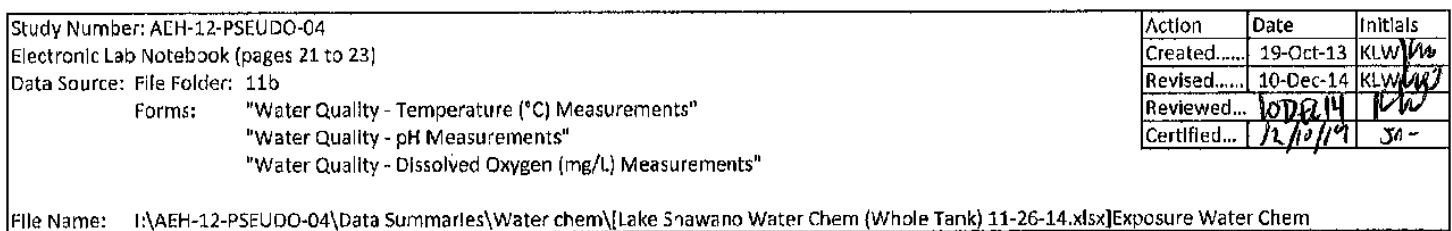

Exposure Water Chemistry

Test Article: MBI 401 SDP [Pseudomonas fluorescens Pf - CL 145A (SDP)]

Article Lot $\mathrm{H}$ : 401P12163C and 401P12164C Mix

Exposure Date: September 6, 2012

Test Location: Lake S awano, Shawano, WI

Treatment Type: Whole Tank

Data Explanation:

Water cremistry measurements (dlssolved oxygen, $\mathrm{pH}$ and temperature) were observed for all test tanks at $0,3,6,9$ and $12 \mathrm{~h}$ after dosing

NOTE: $0 \mathrm{~h}$ measurements were observed from 30 minules to $1 \mathrm{~h}$ after dosing.

Data anomalies and devlatlons:

NONE

File Folder:

Item Number 2

Page 296 of 519 

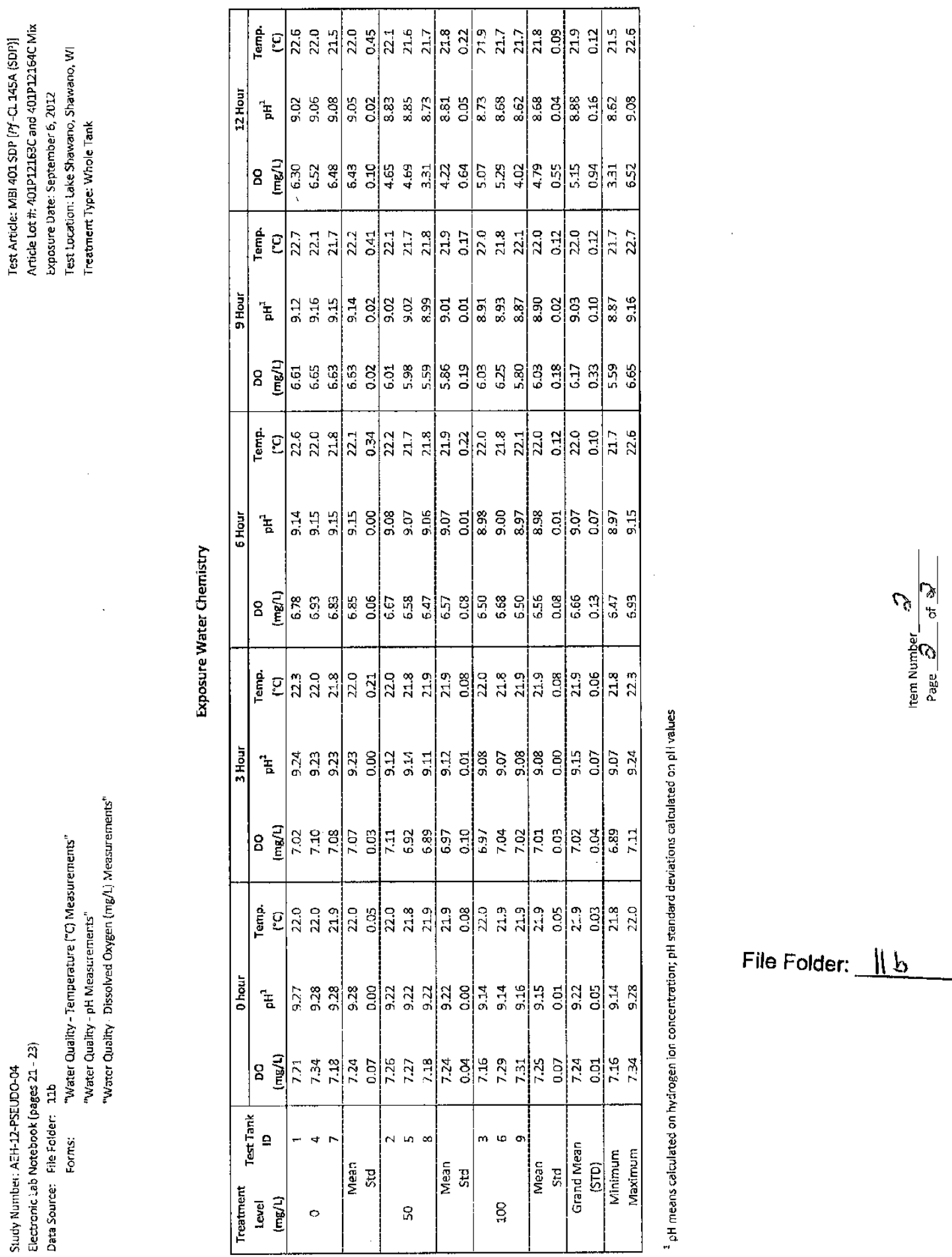

File Folder: $\| b$ 
Study Number: AEH-12-PSEUDO 04

\begin{tabular}{|c|c|c|}
\hline Action & Date & Initials \\
\hline Created...... & 19-0ct-13 & KLW $(\mathrm{MA})$ \\
\hline Revised...... & 10-Dec-14 & KLW $W^{\prime}$ \\
\hline Reviewed... & LODAM & $W$ \\
\hline Certified...... & $12 / 10 / 11$ & $5 A 2$ \\
\hline
\end{tabular}

File Name: 1: \AEH-12-PSEUDO-04\Data Summaries WWater chem \[Lake Shawano Water Chem (Whole Tank) 11-26-14.xlsx

Exposure Un-ionized Ammonia

Test Article: MB| 401 SDP [Pseudomonas fluorescens Pf -CL 145A (SDP)]

Article Lot \#: 401P12163C and 401P12164C Mix

Exposure Date: September 6, 2012

Test Location: Lake Shawano, Shawano, Wl

Treatment Type: Whole Tank

Data Explanation:

1) Water samples were collected at $12 \mathrm{~h}$ from each exposure tank. Samples were $0.45 \mu \mathrm{m}$ filtered, acidified with sulfuric acid, and analyzed by the $4500-\mathrm{NH}_{3}$ G. Automated Phenate Method (Standard Methods for the Examination of Water and Wastewater, 21st Edition, 2005) on a Technicon Autoanalyzer II by the UMESC water quality laboratory.

2) The un-ionized ammonia fractions were calculated using the sample $\mathrm{pH}$ and temperature according to the formula in Emerson et al. (1975).

Data Anomalies and Deviations:

1) Water samples were not collected at 6 and $9 \mathrm{~h}$ from the exposure tanks for un-ionized ammonia analysis. See Deviation \#3 for further clarification.

File Folder: 116

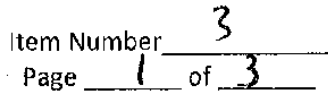


Study Number; AEH-12-PSEUDO-04

Electronic Lab Notebook (page 23)

TAN Data Source: LTRMP Report (File Folder 17)

$\mathrm{pH}$ and Temperature Data Source: Water Quality Forms (File Folder 11b)
Test Article: MB 401 SDP [Pf-CL 145A (SDP)] Art|cle Lot \#: 401P12163C and 401P12164C Mix Exposure Date: September 6, 2012

Test Location: Lake Shawano, Shawano, WI Treatment Type: Whole Tank

Exposure Un-ionized Ammonia

\begin{tabular}{|c|c|c|c|c|c|c|}
\hline $\begin{array}{c}\text { Treatment Level } \\
{[\mathrm{mg} / \mathrm{L})}\end{array}$ & $\begin{array}{c}\text { Test Tark } \\
\text { ID }\end{array}$ & $\mathrm{pH}^{1}$ & $\begin{array}{c}\text { Terperature } \\
\left.1^{\circ} \mathrm{C}\right) \\
\end{array}$ & $\begin{array}{c}\text { TAN } \\
\text { as } \mathrm{NH}_{3}-\mathrm{N}(\mathrm{mg} / \mathrm{l})\end{array}$ & $\begin{array}{l}\text { Un-ionized } \\
\text { Eraction }\end{array}$ & $\begin{array}{c}\mathrm{NH}_{3} \\
(\mathrm{mg} / \mathrm{L})^{3}\end{array}$ \\
\hline \multirow{3}{*}{0} & 1 & 9.02 & 22.6 & 0.133 & 0.334 & 0.044 \\
\hline & 4 & 9.06 & 22.0 & 0.126 & 0.344 & 0.043 \\
\hline & 7 & 9.08 & 21.5 & 0.123 & 0.347 & 0.043 \\
\hline Mean & & 9.05 & 22,0 & 0.127 & 0.342 & 0.043 \\
\hline Std & & 0.02 & 0.4 & 0.004 & 0.006 & 0.001 \\
\hline \multirow{3}{*}{50} & 2 & 8.83 & 22.1 & 0.188 & 0.238 & 0,045 \\
\hline & 5 & 8.85 & 21.6 & 0.176 & 0.239 & 0.042 \\
\hline & 8 & 8.73 & 21.7 & 0.198 & 0.194 & 0,038 \\
\hline Mean & & 8.81 & 21,8 & 0.187 & 0.224 & 0.042 \\
\hline Std & & 0.05 & 0.2 & 0,009 & 0.021 & 0.003 \\
\hline \multirow{3}{*}{100} & 3 & 8.73 & 21.9 & 0.226 & 0.196 & 0.044 \\
\hline & 6 & 8.68 & 21.7 & 0.223 & 0.177 & 0.039 \\
\hline & 9 & 8.62 & 21.7 & 0.241 & 0.157 & 0.038 \\
\hline Mea? & & 8.68 & 21.8 & 0.230 & 0.177 & 0.041 \\
\hline Std & & 0.04 & 0.1 & 0.008 & 0.016 & 0.003 \\
\hline
\end{tabular}

${ }^{1} \mathrm{pH}$ means calculated on hydrogen lon concentration; $\mathrm{pH}$ standard deviations calculated on $\mathrm{pH}$ values

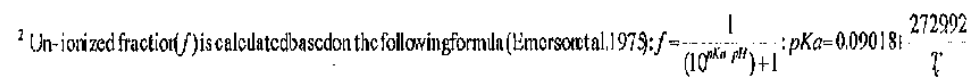

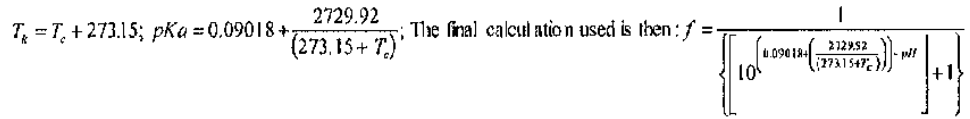

${ }^{3}$ Un-ionized ammonla is calculated based on the following formula: Un-ionized ammonia $x=f *$ TAN $\{\mathrm{mg} / \mathrm{l}$.

Item Number $\frac{3}{2}$ Page $\frac{3}{3}$ 


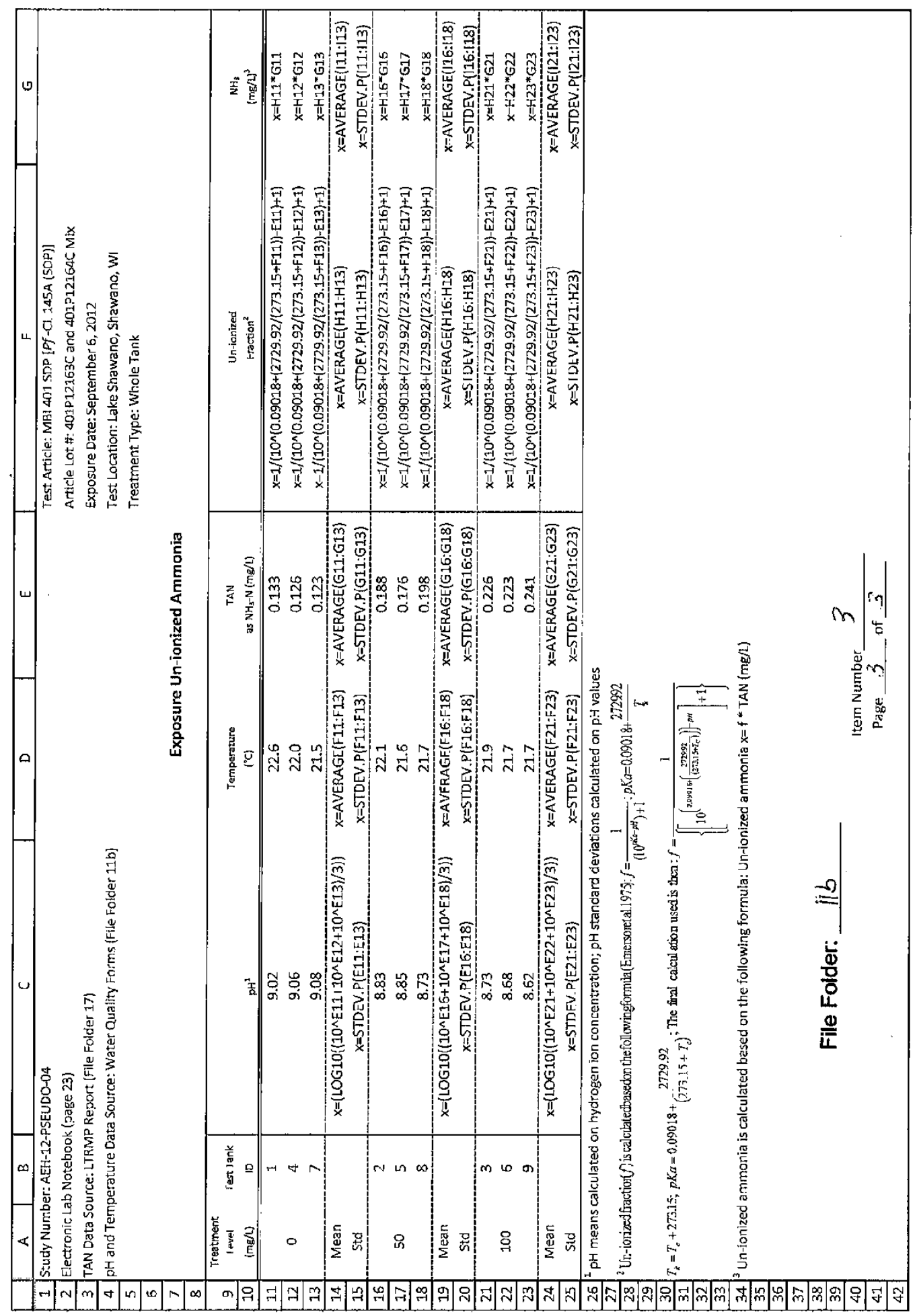

Page 300 of 519 


\begin{tabular}{|c|c|c|c|c|}
\hline \multirow{5}{*}{\multicolumn{2}{|c|}{$\begin{array}{l}\text { Study Number: AEr-12-PSEUDO-04 } \\
\text { Elcctroric Lab Notebock (page 14) } \\
\text { Data Source: Flle Folder: } 12 \mathrm{~b} \\
\text { Forn's: } \\
\end{array}$}} & Action & Date & Initlals \\
\hline & & Created...... & 19-Oct-13 & $K L W, \| w$ \\
\hline & & Revised...... & 10-Dec-14 & KLWF \\
\hline & & Revlewed... & $10 T 221$ & 112 \\
\hline & & Certlfled... & $14 / 10 / 14$ & $x^{2}$ \\
\hline
\end{tabular}

Pre-Exposure Water Chemtstry

Test Article: MB| 401 SDP [Pseudomonas fluorescens Pf -CL 145A (SDP)]

Article Lot H: 401P12163C and 401P:216AC MIX

Expcsure Date: August 17, 2012.

Test Locatlon: Lake Car os, Alexandria, MN

Treatment Type: Bot:or Injection

Data Explanation:

Forms titled "Conductlvity and Hardness - Exposure Inltiation" and "Alkalinity - Exposure Initiation": Conductivity, hardness, and alkallnity were measured prior to dosing. Samples were collected from both headboxes used to fill individual tanks. Samples were analyzed In triplicate.

Forms titled "Water Quality - Temperature ("C) Measurements", "Water Quality - pH Measurerients" and "Water Quality - Dissolved Oxygen (mg/L)

Meas srements": Temperature, $\mathrm{pH}$ and dissolved oxygen levels were measured prlor to dosing. Measurements were observed in each exposure tank.

Déta anomalies and devlations:

NONE

File Folder: $12 b$

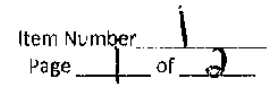

Page 301 of 519 

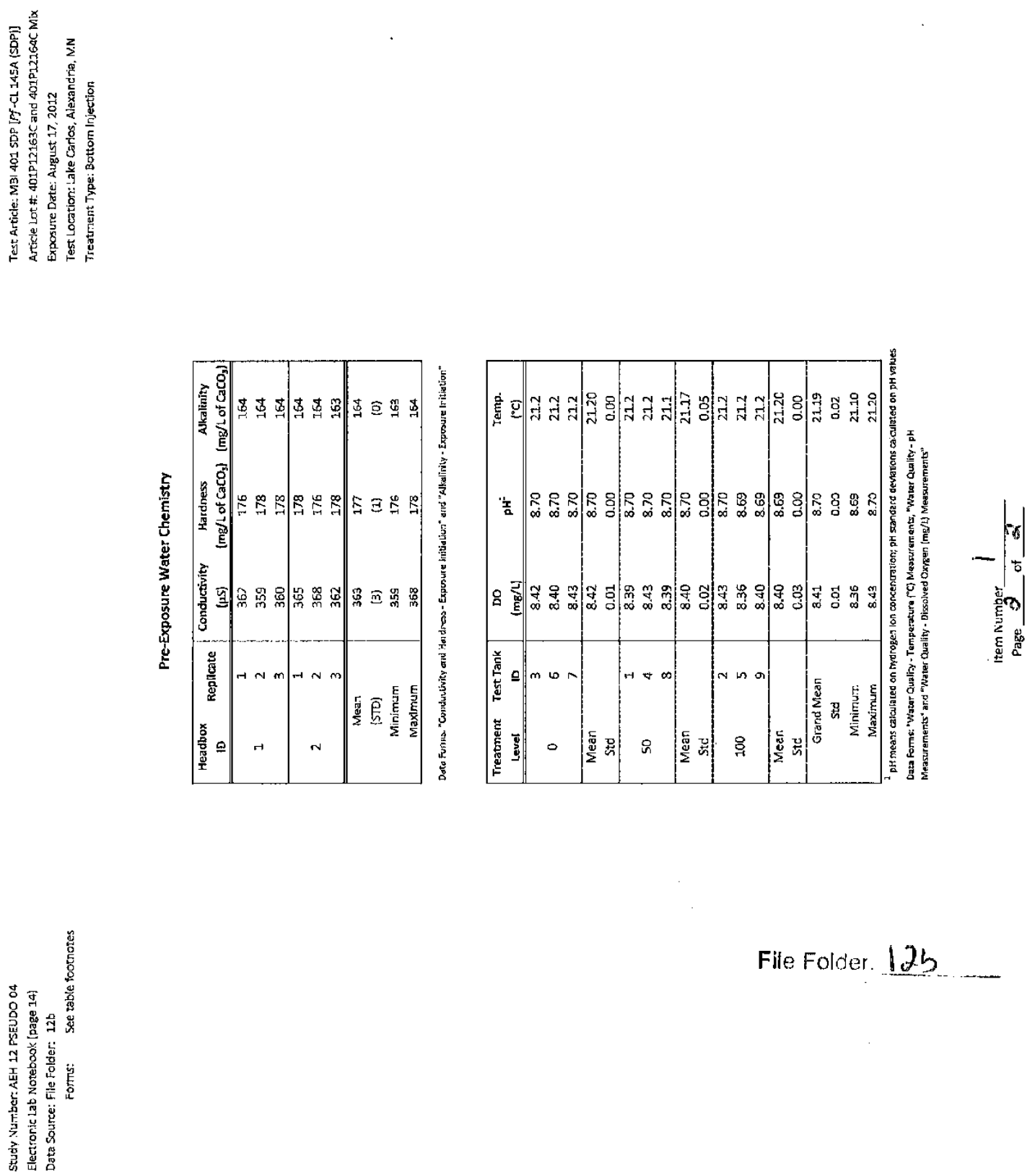

File Folder. $12 b$ 


\begin{tabular}{|c|c|c|c|c|}
\hline & Action & Date & Inltia|s \\
\hline & Electronic Lab Notebook (pages 15 to 16) & Created ....... & $19-0 \mathrm{ct}-13$ & KLW $/ 10$ \\
\hline \multirow{2}{*}{$\begin{array}{c}\text { Data Source: Flle Folder: } \\
\text { Forms: }\end{array}$} & "Water Quallty - Temperature $\left({ }^{\circ} \mathrm{C}\right)$ Measurements" & Reviewed... & 100214 & 100 \\
\hline & "Water Quality - Dissolved Oxygen (mg/L) Measurements" & & & \\
\hline
\end{tabular}

Exposure Water Chemistry

Test Arllcle: MBI 401 SDP [Pseudomonas fluorescens Pf -CL 145A (SDP)]

Article Lot \#: 401P12163C and 401P12164C MIX

Exposure Date: August 17, 2012

Test Localion: Lake Carlos, Alexandrla, MN

Treat ment Type: Bottom Injection

Dato Explanation:

Water chem/stry measurements (dissolved oxygen, $\mathrm{pH}$ and temperature) were observed for all test tanks at $0,3,6,9$ and $12 \mathrm{~h}$ after dosing. NOTE: $0 \eta$ measurements were observed from $30 \mathrm{~m} /$ nutes to $1 \mathrm{~h}$ after dosing.

Data a omalies anc devlations:

NONE

File Folder: $12 b$

Item Number $\partial$

Page_1_ of 9

Page 303 of 519 

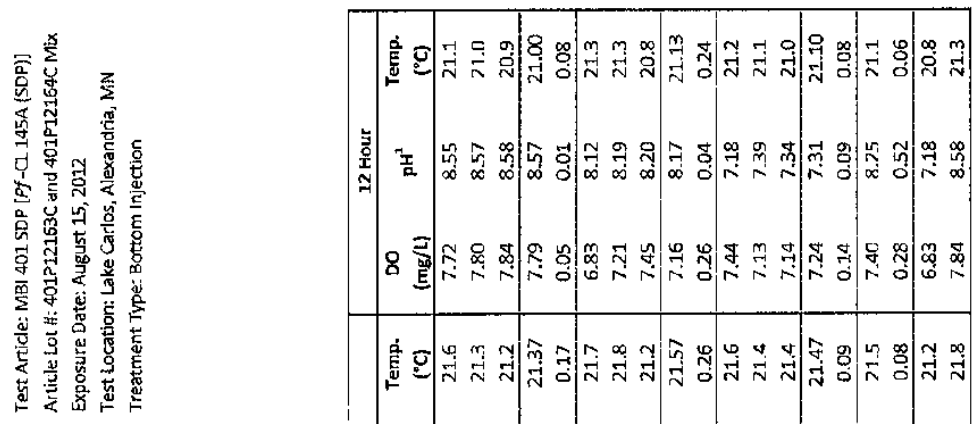

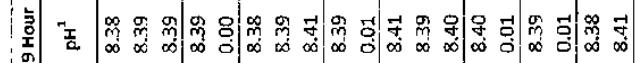

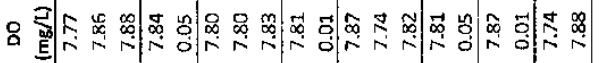

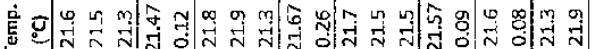

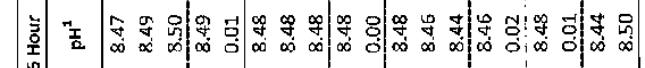

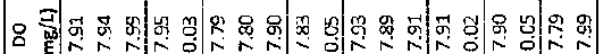

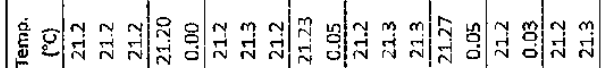

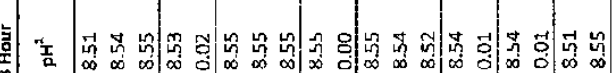
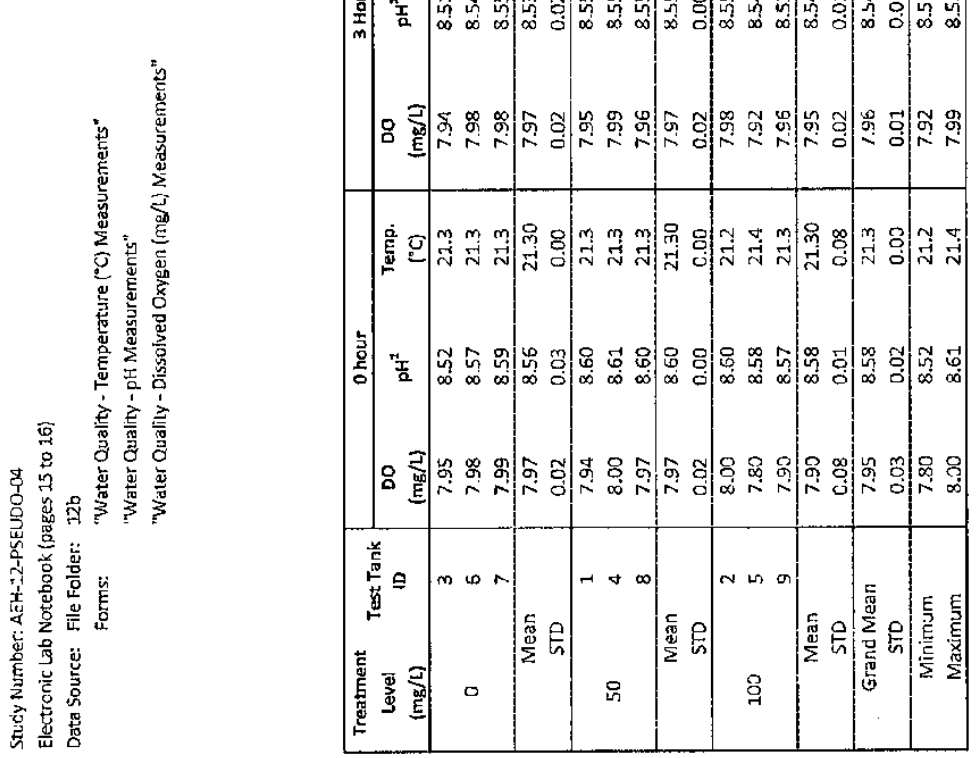

File Folder: $1 \partial b_{2}$ 


\begin{tabular}{|c|c|c|c|}
\hline \multirow{4}{*}{$\begin{array}{l}\text { StLdy Number: AEH-12-PSEUDO-04 } \\
\text { Electronic Lab Notebook (page 16) } \\
\text { TAN Data Source: LTRMP Report (File Folder } 17 \text { ) } \\
\text { pH and Temperature Data Source: Water Quality Forms (Flle Folder 12b) }\end{array}$} & Created...... & 19-Oct-13 & KLinign \\
\hline & Revlsed...... & 10-Dec-14 & $K L W \| W$ \\
\hline & Reviewed... & $1020 \times 4$ & ho \\
\hline & Certified...... & $12 / 10 / 13$ & $3=$ \\
\hline
\end{tabular}

\section{Exposure Un-ionized Ammonia}

Test Article: MBI 401 SDP [Pseudomonas fiworescens of -CL 145A (SDP)]

Article Lot \#: 401P12163C and 4C1P12164C Mix

Exposure Date: August 17, 2012

Test Locatlon: Lake Carlos, Alexandria, MN

Treatment Type: Bottom Injection

Data Explanation:

1) Water samples were collected at $12 \mathrm{~h}$ from each exposure tank. Samples were $0.45 \mu \mathrm{m}$ filtered, acidified with sulfuric acld, and analyzed by the $4500-\mathrm{NH}_{3} \mathrm{G}$. Automated Phenate Method (Standard Methods for the Examlnation of Water and Wastewater, 21st Edition, 2005) on a Technicon Autoanalyzer il by the UMESC water quallty laborato $y$.

2) The un-ionized ammonla fractions were calculated using the sample pH and temperature according to the formula in Emerson et al. (1975).

Data Anomalles and Devlations:

NONE

File Folder: $12 b$

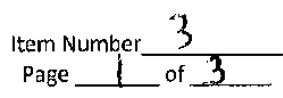


Study Number: A EH-12-PSEUDO-03

Electron/c Lab Notebook (page 16)

TAN Data Source: LTRMP Report (File Folder 17)

$\mathrm{pH}$ and Temperature Data Source: Water Quality Forms (File Folder 12B)
Test Article: MBI 401 SDP [Pf -CL $\approx 45 A$ (SDP)] Artlcle Lot \#: 401P12.163C and 401P1.7.164C Mix

Exposure Date: August 17, 2012

Test Location: Lake Carlos, Alexandria, MN

Treatment Type: Bottom Injection

Exposure Un-ionized Ammonia

\begin{tabular}{|c|c|c|c|c|c|c|}
\hline $\begin{array}{c}\text { Treatment Level } \\
\left(\mathrm{m} \mathrm{m}_{3}, L\right)\end{array}$ & $\begin{array}{c}\text { Test Tank } \\
\text { ID } \\
\end{array}$ & $\mathrm{pH}^{1}$ & $\begin{array}{c}\text { Temperature } \\
\left({ }^{\circ} \mathrm{C}\right) \\
\end{array}$ & $\begin{array}{c}\text { TAN } \\
\text { as } \mathrm{NH}_{3}-\mathrm{N}(\mathrm{mg} / \mathrm{L}) \\
\end{array}$ & $\begin{array}{l}\text { Un-lonlzed } \\
\text { Fraction }\end{array}$ & $\begin{array}{c}\mathrm{NH}_{\mathrm{s}} \\
(\mathrm{mg} / \mathrm{L})^{3}\end{array}$ \\
\hline \multirow{3}{*}{0} & 3 & 8.55 & 21.1 & 0.232 & 0.132 & 0.031 \\
\hline & 6 & 8.57 & 21.0 & 0.207 & 0.137 & 0.028 \\
\hline & 7 & 8.58 & 20.9 & 0.210 & C. 138 & 0.029 \\
\hline Mean & & 8.57 & 21.0 & 0.216 & 0.136 & 0.029 \\
\hline std & & 0.01 & 0.08 & 0.01 & 0.00 & 0.00 \\
\hline \multirow{3}{*}{50} & 1 & 8.12 & 21,3 & 0.403 & c.054 & 0.022 \\
\hline & 4 & 8.19 & 21.3 & 0.415 & 0.063 & 0.026 \\
\hline & 8 & 8.20 & 20.8 & 0.385 & 0.062 & 0.024 \\
\hline Mean & & 8.17 & 21.1 & 0.401 & 0.060 & 0.024 \\
\hline std & & 0.04 & 0.24 & 0.01 & 0.00 & 0.00 \\
\hline \multirow{3}{*}{100} & 2 & 7.18 & 21.2 & 1.486 & 0.006 & 0.010 \\
\hline & 5 & 7.39 & 21.1 & 1.123 & 0.010 & 0.012 \\
\hline & 9 & 7.34 & 21.0 & 1.504 & 0.009 & 0.014 \\
\hline Mean & & 7.31 & 21.1 & 1.371 & 0.009 & 0.012 \\
\hline std & & 0.09 & 0.08 & 0.18 & 0.00 & 0.00 \\
\hline
\end{tabular}

${ }^{1} \mathrm{pH}$ means calculated on hycrogen ton concentration; $\mathrm{pH}$ standard deviations calculated on $\mathrm{pH}$ values

${ }^{2}$ Un-ionized fraction(f) is calcuntedbasedon the lollowingformula(Emersoltal.1975): $f=\frac{1}{\left(10^{p k i-p H}\right)+1}: p K a=0.09018+\frac{272992}{T}$

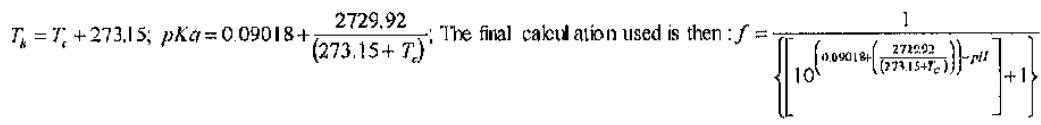

${ }^{3}$ Un-ionlzed ammonia Is calculated based on the following formula: Un-ionized ammonia $=f *$ TAN (mg/L)

Item Number 3

Page 2 of 3 


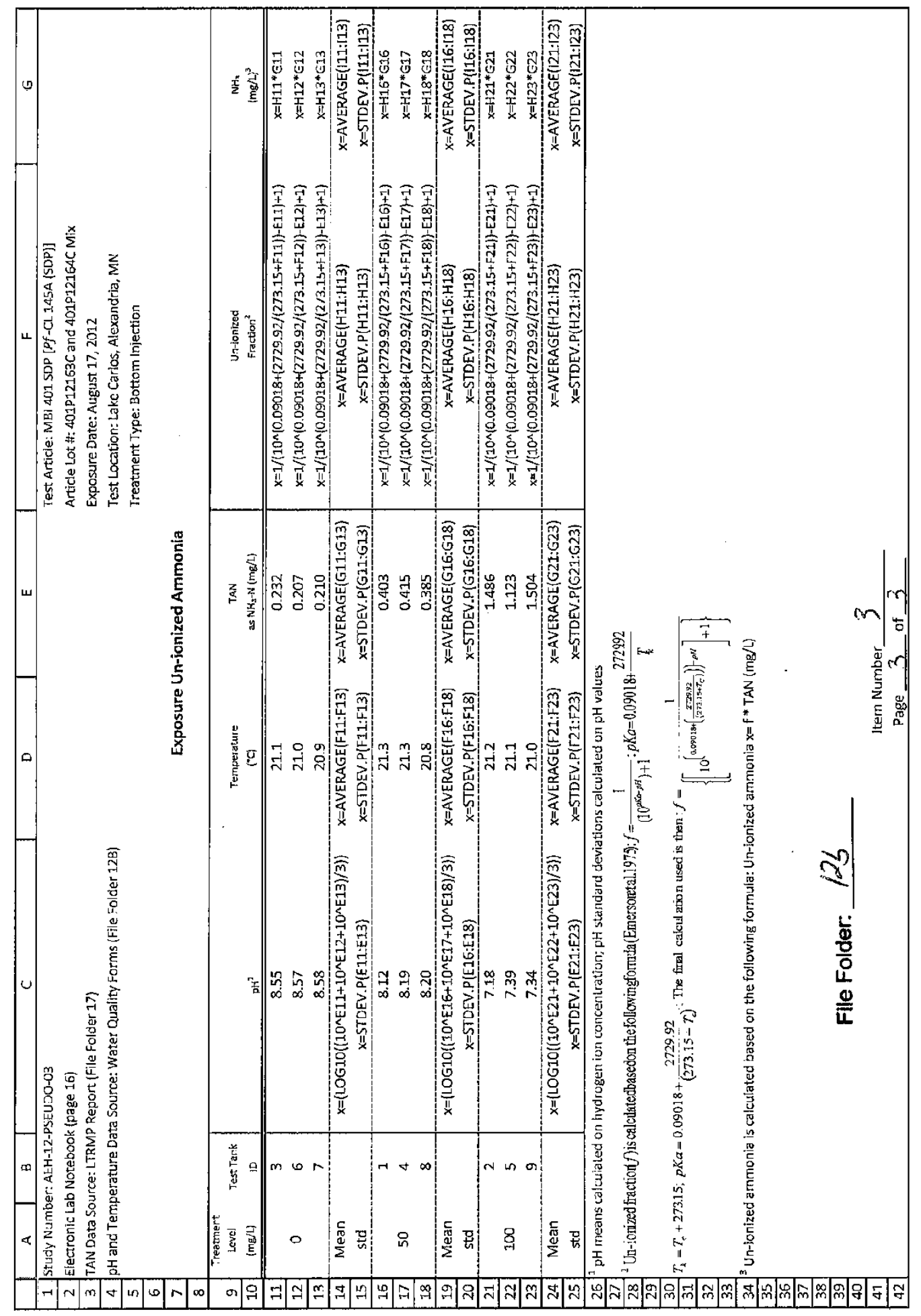

Page 307 of 519 


\begin{tabular}{|c|c|c|c|c|}
\hline \multirow{2}{*}{\multicolumn{2}{|c|}{$\begin{array}{l}\text { Study N umber: AEH-1.2-PSEUDO-04 } \\
\text { Electronlc Lab Notebook (page 25) } \\
\text { Data Source: Flle Folder: } 14 \mathrm{~b}\end{array}$}} & Action & Datc & Initials \\
\hline & & Created....... & 19moct-13 & KLWVIW \\
\hline \multicolumn{2}{|c|}{ Forms: See form names as stated kelow } & Revlewed. & 101204 & $8 w$ \\
\hline
\end{tabular}

Pre-Exposure Water Chemistry

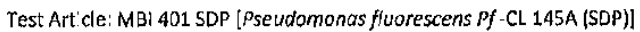

Article Lot \#: 401P12163C and 401P12164C Mix

Exposure Date: September 8, 2012

Test Location: Loke Sרawano, Shawano, wI

Treatrent Type: Bottom Injection

Data Explanation:

Forms titled "Conductlvity and Hardness - Exposure Initbatlon" and "Alkalinity - Exposure Initlatlon": Conductivity, hardness, and alkallnity were measured prlor to 'dcsing. Samples were collected from both headboxes used to fill individual tanks. Samples were analyzed in triplicate.

Forms titled "Water Quality - Temperature $\left({ }^{\circ} \mathrm{C}\right)$ Measurements", "Water Quality - pH Measurements" and "Water Quality - Dissolved Oxygen (mg/L) Masasurements": Temperature, $\mathrm{pH}$ and dissolved oxygen levels were measured prlor to dosing. Measurcments were observed in each exposure tar.k.

Deta anomalles and devlations:

NONE

Fille Folder: $14 \mathrm{~b}$

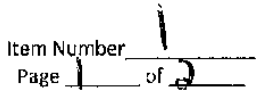



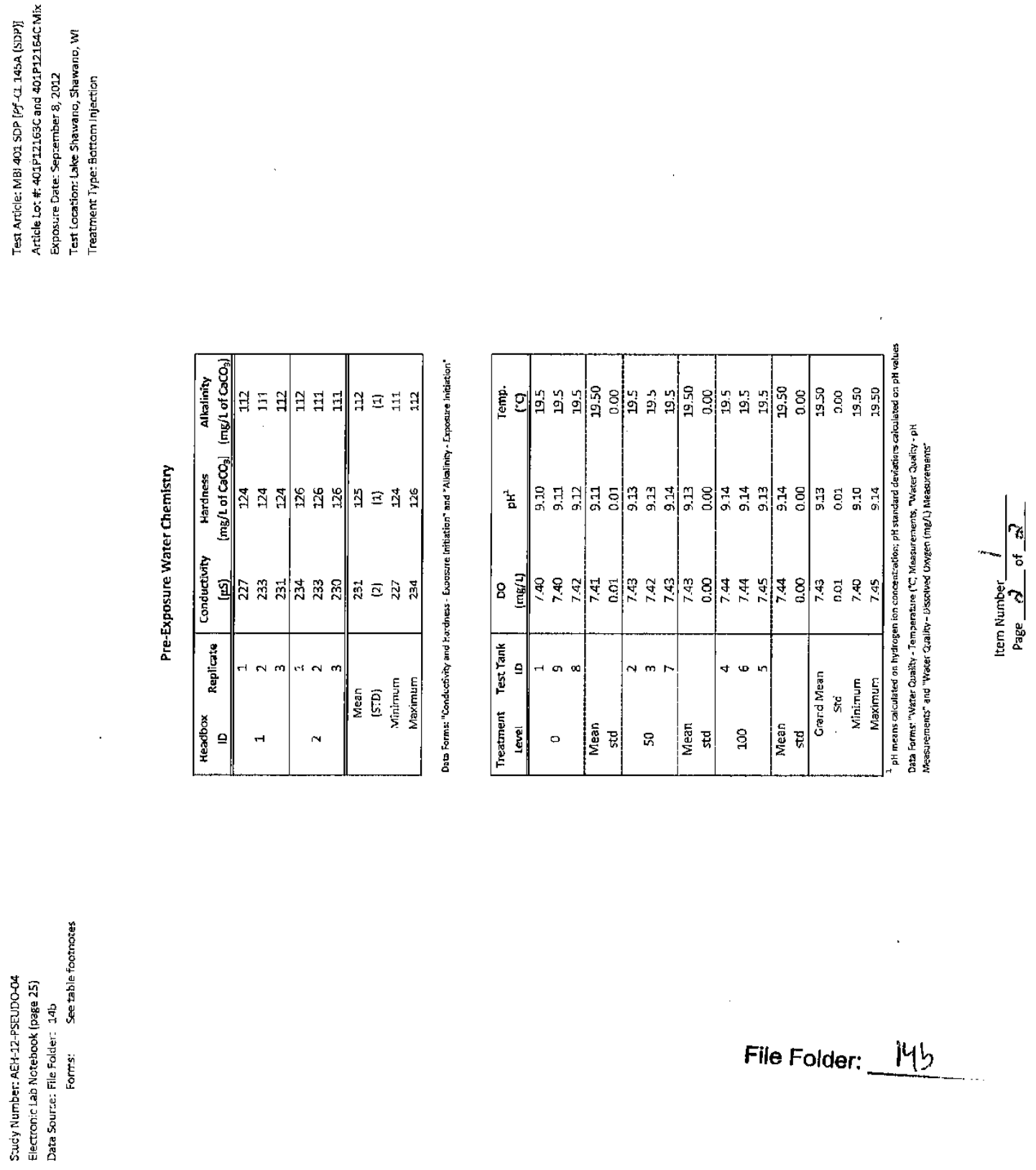

File Folder: $14 b$ 


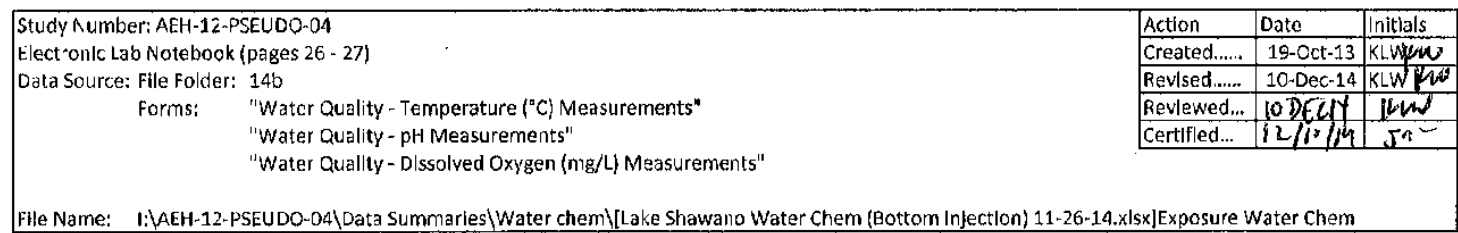

Exposure Water Chemlstry

Test Article: MBI 4C1 SDP [Pseudomenas fluorescens of -CL 145A (SDP)]

Art|cle Lot \#: 401P12163C and 401P12164C Mlx

Exposure Date: September 8, 2012

Test Locatlon: La<e Shawano, Shawano, wl

Treatment Type: Bottom injection

Da:a Explanation:

Water chemlstry measurements (dissolved oxygen, $\mathrm{oH}$ and temperature) were observed for all test tanks at $0,3,6,9$ and $12 \mathrm{~h}$ after dosing.

NOTE: $0 \mathrm{~h}$ measurements were ubserved from 30 minutes to $1 \mathrm{~h}$ after dosing.

Da:a anomalies and devlationș:

NONE

File Folder: 146

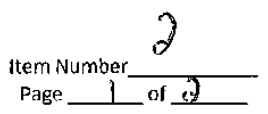

Page 310 of 519 

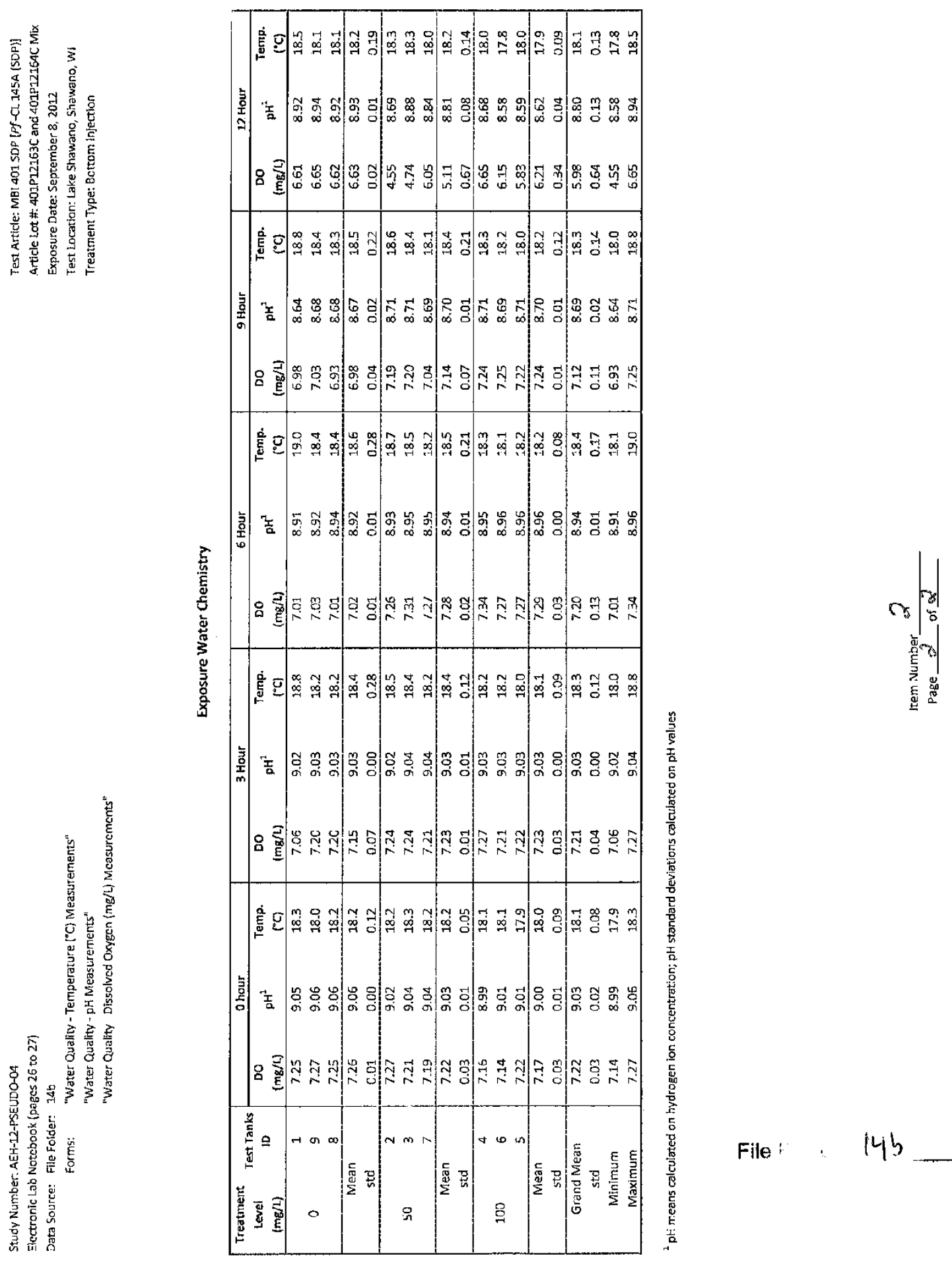

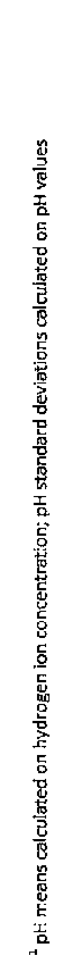

File: $\quad 14 b$ 


\begin{tabular}{|c|c|c|c|}
\hline \multirow{5}{*}{$\begin{array}{l}\text { Study Number: AEH-12-PSEUDO } 04 \\
\text { Electronic Lab Notebook (page 27) } \\
\text { TAN Data Source: LTRMP Report (File Folder 17) } \\
\text { pH and Temperature Data Source: Water Quality Forms (File Folder 14b) }\end{array}$} & Action & Date & Initials \\
\hline & Created...... & $19-$ Oct-13 & KLW WWO \\
\hline & Revlsed....... & 10-Dec-14 & KLW $/ \mathbb{W}^{2}$ \\
\hline & Reviewed... & TODE & WW \\
\hline & Certified....... & $12 / 10 / 14$ & $50-$ \\
\hline
\end{tabular}

Exposure Un-ionized Ammonia

Test Article: MBI 401 SDP [Pseudomonas fluorescens Pf $-\mathrm{CL}$ 145A (SDP)]

Article Lot \#: 401P12163C and 401P12164C Mix

Exposure Date: September 8, 2012

Test Location: Lake Shawano, Shawano, WI

Treatment Type: Bottom injection

Data Explanation:

1) Water samples were collected at $12 \mathrm{~h}$ from each exposure tank. Samples were $0.45 \mu \mathrm{m}$ filtered, acidified with sulfuric acid, and analyzed by the 4500- $\mathrm{NH}_{3} \mathrm{G}$. Automated Phenate Method (Standard Methods for the Examination of Water and Wastewater, 21st Edition, 2005) on a Technicon Autoanalyzer II by the UMESC water quality laboratory.

2.) The un-ionized ammonia fractions were calculated using the sample $\mathrm{pH}$ and temperature according to the formula in Emerson et al. (1975).

Data Anomalies and Deviations:

NONE

File Folder: $14 b$

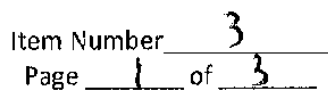


Study Number: AEH-12-PSEUDO-04

Electronic Lab Notebook (page 27)

-AN Lata Source: LTRMP Report (File Folder 17)

$\mathrm{pH}$ and Temperature Data Source: Water Quality Forms (File Folder 14b)
Test Article: MBI 401 SDP [Pf - Cl. 145A (SDP)] Article Lot $H:$ 401P12163C and 4J1P12164C Mix Exposure Date: September 8, 2012 Test Location: Lake Shawano, Shawano, WI Treatment Type: 3ottom In]ection

Exposure Un-ionized Ammonia

\begin{tabular}{|c|c|c|c|c|c|c|}
\hline $\begin{array}{c}\text { Treatment Level } \\
(\mathrm{mg} / \mathrm{h})\end{array}$ & $\begin{array}{c}\text { Test -ark } \\
\text { D }\end{array}$ & $\mathrm{pH}^{1}$ & $\begin{array}{c}\text { Temperature } \\
\left({ }^{\circ} \mathrm{C}\right)\end{array}$ & $\begin{array}{c}\text { TAN } \\
\text { as } \mathrm{VH}_{2}-\mathrm{N}(\mathrm{mg} / \mathrm{h})\end{array}$ & $\begin{array}{l}\text { Un-ionized } \\
\text { Fractlon }\end{array}$ & $\begin{array}{c}\mathrm{NH}_{3} \\
(\mathrm{ng} / \mathrm{L})^{3}\end{array}$ \\
\hline \multirow{3}{*}{0} & 1 & 8.92 & 18.5 & C.061 & 0.228 & 0.0 .14 \\
\hline & 9 & 8.94 & 18.1 & C. 072 & 0.231 & 0.017 \\
\hline & 8 & 8.92 & 18.1 & 0.056 & 0.223 & 0,012 \\
\hline Mean & & 8,93 & 18.2 & 0.063 & 0.227 & 0.014 \\
\hline std & & 0.01 & 0.19 & 0.007 & C.003 & 0.002 \\
\hline \multirow{3}{*}{50} & 2 & 8.69 & 18.3 & 0.113 & 0.146 & 0.017 \\
\hline & 3 & 8.88 & 18.3 & 0.120 & 0.209 & 0.025 \\
\hline & 7 & 8.84 & 18.0 & 0.094 & 0.191 & 0,018 \\
\hline Mean & & 8.81 & 18.2 & 0.109 & 0.182 & 0.020 \\
\hline std & & 0.08 & 0.14 & 0.011 & 0.027 & 0.004 \\
\hline \multirow{3}{*}{100} & 4 & 8.68 & 18.0 & 0.158 & 0.141 & 0.022 \\
\hline & 6 & 8.58 & 17.8 & 0.145 & 0.113 & 0.016 \\
\hline & 5 & 8.59 & 18.0 & 0.163 & 0.117 & $\begin{array}{r}0.019 \\
\end{array}$ \\
\hline Mean & & 8.62 & 17.9 & 0.155 & 0.124 & 0.019 \\
\hline std & & 0.04 & 0,09 & 0.008 & 0.012 . & 0.002 \\
\hline
\end{tabular}

$\mathrm{pH}$ means calculated on hydrogen ion concentration; $\mathrm{pH}$ standard deviations calculated on $\mathrm{pH}$ values

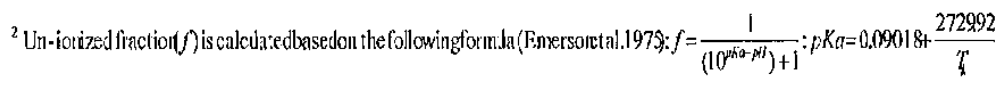

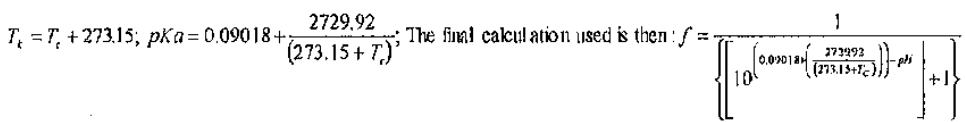

${ }^{3}$ Un-ionized ammonia is calculated based on the following formula: Un-ionized ammonia $=f{ }^{*} \operatorname{TAN}(\mathrm{mg} / \mathrm{L}$ )

Item Number $\frac{3}{9}$ of 3 


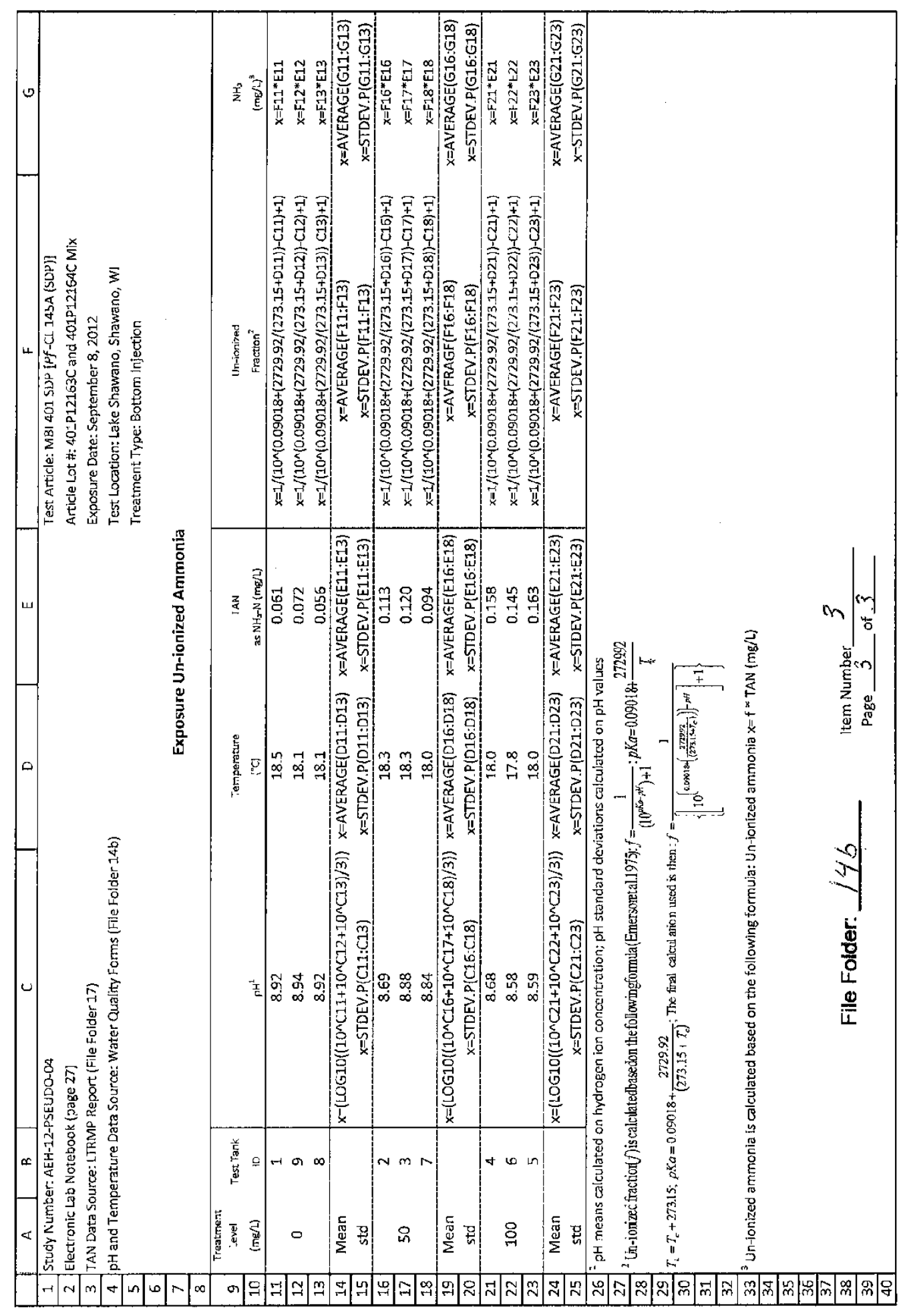

Page 314 of 519 


\begin{tabular}{|c|c|c|c|c|}
\hline \multirow{2}{*}{\multicolumn{2}{|c|}{$\begin{array}{l}\text { Study Number: AEH-12-PSEUDO-04 } \\
\text { Electronic Lab Notebook (pages } 13 \text { \& 17) }\end{array}$}} & Actlon & Oate & Initials \\
\hline & & Created...... & A-Feb-14 & TJS TS \\
\hline \multicolumn{2}{|c|}{ Data Source: File Folder: $17 a$} & Revised....... & I-Feb-14 & Ts ins \\
\hline Forms: & $\begin{array}{l}\text { Onset H IO30 Datafile output from IIOBO Temperature Loggers (File Folde: 18) } \\
\text { (I:AEH-12-PSEUDO-04(Data)Hobos\Lake Car.os HOBO Files) }\end{array}$ & \begin{tabular}{|l|} 
Reviewed.... \\
Certified...
\end{tabular} & $\frac{19 F E Q 14}{2 / R / 4}$ & $\frac{75}{17}$ \\
\hline
\end{tabular}

Temperature Data Loggers (HOBOs)

Test Article: M8| 401 SDP [Pseudomonas fluorescens Pf-CL 145A (SDP)]

Article Lot $\#$ : 401P12163C and 401P12161C Mix

Fxposure Date: August 15 and 17, 2012

Test Lccation: Lake Carlos, Alexandria, MN

Treatment Type: Whole Tank and Bottom Injection

Data Explanation

Upon exposure termination on August 15, 2012 (whole tank) and August 17, 2012 (bottom injection) at Lake Carlos, four wire mesh cages containing test anImals were placed Into Lake Carlos for a post-exposure holding period. Each cage was fitted with a HOBO temperature logger programmed to record water temperature every six hours (fou "times per day). Mean daily temperature $\left({ }^{\circ} \mathrm{C}\right.$ ) and standard deviation were calculated frcm all dally measurements.

\begin{tabular}{|c|c|c|c|c|}
\hline $\begin{array}{c}\text { Data Logger } \\
\text { Number }\end{array}$ & $\begin{array}{c}\text { Seria: } \\
\text { Number }\end{array}$ & Lake & $\begin{array}{c}\text { Treatment } \\
\text { Type }\end{array}$ & $\begin{array}{l}\text { Treatment } \\
\text { Durat on }\end{array}$ \\
\hline 1 & 10020131 & Carlos & Wrole Tank & 6h \\
\hline 2 & 10020140 & Carlos & Wrole Tank & $9 \mathrm{~h}$ \\
\hline 3 & 10020139 & Carlos & Wrole Tank & $12 \mathrm{~h}$ \\
\hline 4 & 10020133 & Carlos & Bottom In'ection & $12 \mathrm{~h}$ \\
\hline
\end{tabular}

\begin{tabular}{|c|c|c|}
\hline Dale & $\begin{array}{c}\text { Mean } \\
\text { Temperature }\left({ }^{\circ} \mathrm{C}\right)\end{array}$ & (STD) \\
\hline $8 / 17 / 2012$ & 22.61 & 0.61 \\
\hline $8 / 18 / 2012$ & 22.53 & 0.50 \\
\hline $8 / 19 / 2012$ & 22.59 & 0.39 \\
\hline $8 / 20 / 2012$ & 22.38 & 0.32 \\
\hline $8 / 21 / 2012$ & 22.81 & 0.47 \\
\hline $8 / 22 / 2012$ & 23.09 & 0.59 \\
\hline $8 / 23 / 2012$ & 23.37 & 0.47 \\
\hline $8 / 24 / 2012$ & 24.18 & 0.64 \\
\hline $8 / 25 / 2012$ & 23.76 & 0.27 \\
\hline $8 / 26 / 2012$ & 23.93 & 0.53 \\
\hline $8 / 27 / 2012$ & 24.39 & 0.58 \\
\hline $8 / 28 / 2012$ & 24.19 & 0.29 \\
\hline $8 / 29 / 2012$ & 24.07 & 0.48 \\
\hline $8 / 30 / 2012$ & 24.01 & 0.25 \\
\hline $8 / 31 / 2012$ & 23.51 & 0.34 \\
\hline $9 / 1 / 2012$ & 23.27 & 0.36 \\
\hline $9 / 2 / 2012$ & 23.06 & 0.29 \\
\hline $9 / 3 / 2012$ & 23.39 & 0.38 \\
\hline $9 / 4 / 2012$ & 23.39 & 0.25 \\
\hline $9 / 5 / 2012$ & 23.22 & 0.40 \\
\hline $9 / 6 / 2012$ & 22.65 & 0.30 \\
\hline $9 / 7 / 2012$ & 22.16 & 0.37 \\
\hline $9 / 8 / 2012$ & 21.12 & 0.48 \\
\hline $9 / 9 / 2012$ & 20.97 & 0.46 \\
\hline $9 / 10 / 2012$ & 20.84 & 0.22 \\
\hline $9 / 11 / 2012$ & 20.63 & 0.35 \\
\hline
\end{tabular}

Déta anomalles and devlatlons:

Temperature readings from the firsl and last days of recorder deployment (prior to August 17 and after September 11, 2012 for whole tank; prlor to August 19 and after September 11, 2012 for bottom injection) were omitted from data analysis. The temperature recorders may have sampled ambient air temperature during these time points. These entire days will be omitted as diurnal temperature flucuation skew the mean daily tcmperature. The mean temperature for August 17 and 18, 2012 was calculated using only the three data loggers ( $H 1,2$, and 3 ) from whole tank treatments.

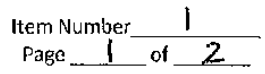

Filo Folder: $1 \mathrm{Fa}$ 
Study Number: AEH-12-P5EUDO-OA

Electron'c Lab Notebook (pages 13 \& 17)

Data Source: $\quad$ File folder: 17 a

Forms: HOBO Output 'n Excel "Lake Carlos 6h wikle tank", "Lake Carlos 9il whole tank"

"Lake Carlos $12 \mathrm{~h}$ whole tank", "Lake Carlos 127 bottoni injectlon"

Location: I:AEEH-12-PSEUDO-04 \atał Hobos YCarlos and Shawano in Ekcel
Test Aitlcle: MB1 401 SDP [PJ-CL. 145A (5DP)]

Art|clo Lot \#: 401P12163C and 401P12164CMix

Exposure Date: August 15 \& 17, 2012

Test Locatlon; Lake Carlos, Alexandria, MN

Treatment Type: Whole Tank a d Bottom Injectlon

Post-Exposure Holding Period Water Temperature

\begin{tabular}{|c|c|c|c|c|c|c|c|c|c|c|c|}
\hline \multirow{3}{*}{$\begin{array}{c}\text { Date } \\
\text { Time (GMT-05:@) }\end{array}$} & \multicolumn{4}{|c|}{ Data Logger Number } & \multirow{3}{*}{$\begin{array}{l}\text { Mean } \\
\text { (STD) }\end{array}$} & \multirow{3}{*}{$\begin{array}{c}\text { Date } \\
\text { Time }(\text { GMT-05:00) }\end{array}$} & \multicolumn{4}{|c|}{ Data Logger Number } & \multirow{3}{*}{$\begin{array}{l}\text { Mean } \\
\text { (STD) }\end{array}$} \\
\hline & 1 & 2 & 3 & 4 & & & 1 & 2 & 3 & 4 & \\
\hline & \multicolumn{4}{|c|}{ Temperature $\left({ }^{\circ} \mathrm{C}\right)$} & & & \multicolumn{4}{|c|}{ Temperature $\left({ }^{\circ} \mathrm{C}\right)$} & \\
\hline $8 / \div 7 / 20124: 00$ & 21.855 & 21.951 & 22.046 & . & & $8 / 30 / 20124: 00$ & 23.677 & 23.773 & 23.869 & 23.773 & \\
\hline $8 / 17 / 201210: 00$ & 22.429 & 22.621 & 22.238 & . & 22.61 & $8 / 30 / 201210: 00$ & 24,351 & 24.158 & 24.255 & 24.062 & 24.01 \\
\hline $8 / 17 / 201216: 00$ & 23.484 & 23.484 & 23.773 & . & (0.61) & $8 / 30 / 201216: 00$ & 24.255 & 24.158 & 24.255 & 24.448 & $(0.25)$ \\
\hline $8 / 17 / 2012$ 22:00 & 22.429 & 22.525 & 22.525 & & & $8 / 30 / 201222: 00$ & 23.677 & 23.773 & 23.773 & 23.966 & \\
\hline $8 / 18 / 20124: 00$ & 21.951 & 21.951 & 22.142 & . & & $8 / 31 / 20124: 00$ & 23.196 & 23.196 & 23.292 & 23.581 & \\
\hline $8 / 18 / 201210: 00$ & 22.621 & 22.429 & 22.238 & . & 22.53 & $8 / 31 / 201210: 00$ & 23.966 & 23.773 & 23.388 & 22.908 & 23.51 \\
\hline $8 / 18 / 201216: 00$ & 23.292 & 22.908 & 23.677 & . & $(0.50)$ & $8 / 31 / 201216: 00$ & 23.869 & 23.773 & 23.869 & 24.158 & $(0.34)$ \\
\hline $8 / 18 / 201222: 00$ & 22.333 & 22,429 & 22.429 & & & $8 / 31 / 201222: 00$ & 23,196 & 23,292 & 23.388 & 23.388 & \\
\hline $8 / 19 / 20124: 00$ & 21.951 & 22.046 & 22.142 & 22.238 & & $9 / 1 / 20124: 00$ & 22,908 & 23.004 & 23.004 & 23.1 & \\
\hline $8 / 19 / 201210: 00$ & 22.717 & 22.621 & 22.525 & 22.333 & 22.59 & 9/1/2012 10:00 & 23.581 & 23.196 & 23.196 & 22,908 & 23.27 \\
\hline $8 / 19 / 201216: 00$ & 23.1 & 23.1 & 23.196 & 23.196 & $(0,39)$ & 9/1/2012 16:00 & 23.581 & 23.869 & 23.966 & 23.869 & $(0.36)$ \\
\hline $8 / 19 / 201222: 00$ & 22.429 & 22.429 & 22.525 & 22.812 & & $9 / 1 / 201222: 00$ & 22.908 & 23.004 & 23.1 & 23.196 & \\
\hline $8 / 20 / 20124: 00$ & 21.855 & 21.951 & 22.046 & 22.142 & & $9 / 2 / 20124: 00$ & 22.621 & 22.717 & 22.717 & 22.812 & \\
\hline $8 / 20 / 201210: 00$ & 22.525 & 22.429 & 22.333 & 21.951 & 22.38 & $9 / 2 / 201210: 00$ & 23.292 & 23.004 & 23.004 & 22.717 & 23.06 \\
\hline $8 / 20 / 201216: 00$ & 22.717 & 22.717 & 23.004 & 22.717 & $(0.32)$ & $9 / 2 / 201216: 00$ & 23.388 & 23.388 & 23.581 & 23.388 & $(0.29)$ \\
\hline $8 / 20 / 201222: 00$ & 22.333 & 22.429 & 22.429 & 22.525 & & $9 / 2 / 201222: 00$ & 22.908 & 23.004 & 23.1 & 23.292 & \\
\hline $8 / 21 / 20124: 00$ & 22.238 & 22.333 & 22,429 & 22.429 & & $9 / 3 / 20124: 00$ & 22.812 & 22,812 & 22,908 & 22.908 & \\
\hline $8 / 21 / 201210: 00$ & 22.812 & 22.525 & 22.429 & 22.429 & 22.81 & $9 / 3 / 201210: 00$ & 23.677 & 23.388 & 23.388 & 23.004 & 23.39 \\
\hline $8 / 21 / 201216: 00$ & 23.484 & 23.388 & 23.869 & 23.484 & $(0.47)$ & $9 / 3 / 201216: 00$ & 23.677 & 23.677 & 23.773 & 24.062 & (0.38) \\
\hline $8 / 21 / 201222: 00$ & 22.717 & 22.717 & 22.812 & 22.908 & & $9 / 3 / 201222: 00$ & 23.388 & 23,484 & 23.581 & 23.773 & \\
\hline $8 / 22 / 20124: 00$ & 22.142 & 22.238 & 22.333 & 22.429 & & $9 / 4 / 20124: 00$ & 23.1 & 23.196 & 23.196 & 23.388 & \\
\hline $8 / 22 / 201210: 00$ & 23.581 & 23.1 & 23.1 & 22.717 & 23.09 & $9 / 4 / 201210: 00$ & 23.484 & 23.292 & 23.196 & 23.1 & 23.39 \\
\hline $8 / 22 / 201216 ; 00$ & 23.869 & 23.869 & 23.960 & 23.869 & (0.59) & $9 / 4 / 201216: 00$ & 23.484 & 23.773 & 23.869 & 23.869 & $(0.25)$ \\
\hline $8 / 22 / 201222: 00$ & 23.004 & 23.004 & 23.1 & 23.1 & & $9 / 4 / 201222: 00$ & 23.196 & 23.292 & 23.292 & 23.484 & \\
\hline $8 / 23 / 20124: 00$ & 22.908 & 22.908 & 23.004 & 23.004 & & $9 / 5 / 20124: 00$ & 22.812 & 22.812 & 22.908 & 23.1 & \\
\hline $8 / 23 / 201210 ; 00$ & 23.484 & 23.1 & 23.1 & 22.525 & 23.37 & $9 / 5 / 201210: 00$ & 23.484 & 23.196 & 23.292 & 23.1 & 23.22 \\
\hline $8 / 23 / 201216: 00$ & 26.062 & 23.869 & 24.448 & 23.581 & $(0.47)$ & $9 / 5 / 201216: 00$ & 23.484 & 23.773 & 24.158 & $23 / 1 / 3$ & $(0.40)$ \\
\hline $8 / 23 / 201222: 00$ & 23.388 & 23.388 & 23.581 & 23.581 & & $9 / 5 / 201222 ; 00$ & 22,812 & 22.812 & 22.908 & 23.1 & \\
\hline $8 / 24 / 20124: 00$ & 23.388 & 23.484 & 23.581 & 23.581 & & $9 / 6 / 20124: 00$ & 22,142 & 22.238 & 22.238 & 22,812 & \\
\hline $8 / 24 / 2012$ 10:00 & 24.255 & 23.869 & 23.869 & 23.484 & 24.18 & 9/6/2012 10:00 & 22.812 & 22.711 & 22.621 & 22.429 & 22.65 \\
\hline $8 / 24 / 201216: 00$ & 24.835 & 24.738 & 25.708 & 25.125 & $\{0.64\}$ & $9 / 6 / 201216: 00$ & 22.908 & 22.812 & 22.908 & 23.388 & $(0.30)$ \\
\hline $8 / 24 / 201222 ; 00$ & 24.062 & 24.158 & 24.255 & 24.448 & & $9 / 6 / 201222: 00$ & 22.525 & 22.525 & 22.621 & 22.717 & \\
\hline $8 / 25 / 20124: 00$ & 23.581 & 23.581 & 23.677 & 23.369 & & $9 / 7 / 20124: 00$ & 21.951 & 22.046 & 22.046 & 22.429 & \\
\hline $8 / 25 / 201210: 00$ & 23.292 & 23.388 & 23.388 & 23.484 & 23.76 & 9/7/2012 10:00 & 22.812 & 22.621 & 22.525 & 21.855 & 22.16 \\
\hline $8 / 25 / 201216: 00$ & 24.062 & 23.966 & 24.158 & 23.773 & $(0.27)$ & $9 / 7 / 201216: 00$ & 22.333 & 22.238 & 22.333 & 22.621 & $(0.37)$ \\
\hline $8 / 25 / 7012,22: 00$ & 23.869 & 23.966 & 23.966 & 24.158 & & $9 / 7 / 201222: 00$ & 21.664 & 21.76 & 21.76 & 21.569 & \\
\hline $8 / 26 / 20124 ; 00$ & 23,484 & 23.581 & 23.677 & 23.388 & & $9 / 8 / 20124: 00$ & 20.805 & 20.901 & 20.901 & 21.091 & \\
\hline $8 / 26 / 201210: 00$ & $23.6 \% 7$ & 2.3 .484 & 23.581 & 23.292 & 23.93 & $9 / 8 / 201210: 00$ & 20.996 & 20.901 & 20.996 & 20.805 & 21.12 \\
\hline $8 / 26 / 201216: 00$ & 24.641 & 24.738 & 24.931 & 24.835 & $(0.53)$ & $9 / 8 / 201216: 00$ & 21.664 & 21.76 & 21.855 & 22.238 & (0.48) \\
\hline $8 / 26 / 201222: 00$ & 23.773 & 23.869 & 23.966 & 23.966 & & $9 / 8 / 201222: 00$ & 20.519 & 20.71 & $20.6=5$ & 21.187 & \\
\hline $8 / 27 / 20124: 00$ & 23.677 & 23.677 & 23.773 & 23.869 & & $9 / 9 / 20124: 00$ & 20.329 & 20.424 & 20.599 & 20.519 & \\
\hline $8 / 27 / 201210: 00$ & 24.641 & 24.158 & 24.158 & 23.773 & 24.39 & $9 / 9 / 201210: 00$ & 20.901 & 20.805 & 20.71 & 20.424 & 20.97 \\
\hline $8 / 27 / 201216: 00$ & 25.2222 & 25.028 & 25.513 & 25.222 & $(0.58)$ & $9 / 9 / 201216: 00$ & 21.091 & 21.473 & 21.76 & 21.855 & $(0.46)$ \\
\hline $8 / 27 / 201222: 00$ & 24,351 & 24.158 & 24.545 & 24.448 & & 9/9/2012 22:00 & 21.091 & 21.091 & 21.187 & 21.282 & \\
\hline $8 / 28 / 20124: 00$ & 23.869 & 23.966 & 24.062 & 24.158 & & $9 / \div 0 / 20124: 00$ & 20.615 & 20.71 & 20.805 & 20.71 & \\
\hline $8 / 28 / 201210: 00$ & 24.351 & 23.869 & 23,966 & 23.773 & 24.19 & $9 / 10 / 201210: 00$ & 20,901 & 20.901 & 20.901 & 20.71 & 20.84 \\
\hline $8 / 28 / 201216: 00$ & 24.545 & 24.541 & 24.738 & 24.545 & $(0.29)$ & $9 / 10 / 201216: 00$ & 20.901 & 21.187 & 21.187 & 21.282 & $(0.22)$ \\
\hline $8 / 28 / 201222: 00$ & 24.062 & 24.062 & 24.158 & 24.255 & & $9 / 10 / 201222: 00$ & 20.519 & $2 C .615$ & 20.71 & 20.71 & \\
\hline $8 / 29 / 20124: 00$ & 23.484 & 23.581 & 23.677 & 23.869 & & $9 / 11 / 20124: 00$ & 20.234 & $2 c .329$ & 20.424 & 20.519 & \\
\hline $8 / 29 / 201210: 00$ & 24.158 & 23.773 & 23.773 & 23.484 & 24.07 & $9 / 11 / 201210: 00$ & 20.519 & $2 C .519$ & 20.615 & 20.424 & 20.63 \\
\hline $8 / 29 / 2012.16: 00$ & 24.641 & 24.835 & 25.125 & 24.641 & (0.48) & $9 / 11 / 201216: 00$ & 21.091 & 21.282 & 21.187 & 21282 & (0.35) \\
\hline $8 / 29 / 201222: 00$ & 23.869 & 23.956 & 24.062 & 24.158 & & $9 / 11 / 201222 ; 00$ & 20.329 & $2 C .329$ & 20.424 & 20.519 & \\
\hline
\end{tabular}

File Folder: A 


\begin{tabular}{|c|c|c|c|c|}
\hline \multirow{2}{*}{\multicolumn{2}{|c|}{$\begin{array}{l}\text { Study Number; AEH-12-PSEUDO-04 } \\
\text { Electronic Lab Notebook (pages } 24 \& \text { 28) }\end{array}$}} & Action & Date & Initials \\
\hline & & Createc...... & 4-Feb-14 & TJS $7 / 5$ \\
\hline \multicolumn{2}{|c|}{ Data Source: File Folder: $17 \mathrm{a}$} & Revlsed...... & 19-Feb-14 & TJS DDS \\
\hline \multirow[t]{2}{*}{ Forms: } & Onset HOBO Datafile output from HOBO Temperature Loggers (File Folder 18) & Revlewed... & IQFEAML & 705 \\
\hline & (I:YEH-12-PSEUDO-04\DatałHosos\}Lake Shawaro HOBO Files) & Certified... & $2 / 14 / 4$ & $\sqrt{i}-$ \\
\hline
\end{tabular}

Temperature Data Loggers (HOBOs)

Test Artlcle: MUI 401 SDP [Pseudomonas fluorescens if $-\mathrm{CL}$ 145A (SDP)]

Article Lot H: 401P12163C and 401P12164C Mix

Expcsure Date: September 6 and 8,2012

Test Location: Lake Shawano, Shawano, Wh

Treatment Type: Whole Tank and Bottom injection

Data Fxplanation:

Upon exposure termination on September 6, 2012 (whole tank) and September 8, 2012 (bottom injection) at Lake Shawano, four wire mesh cages containing test animals were placed into Lake Shawano for a post-exposure holding period. Each cage was fltted with a HOBO temperature logger programmed to

record water temperature every six hours (four times per dav). Mean daily temperature $\left({ }^{\circ} \mathrm{C}\right.$ ) and standard devlation were calculated from all daily

\begin{tabular}{|c|c|c|c|c|}
\hline $\begin{array}{c}\text { Data Logger } \\
\text { Number }\end{array}$ & $\begin{array}{c}\text { Serial } \\
\text { Number }\end{array}$ & Lake & \multicolumn{1}{c}{$\begin{array}{c}\text { Treatment } \\
\text { Type }\end{array}$} & $\begin{array}{c}\text { Treatment } \\
\text { Duration }\end{array}$ \\
\hline \hline 1 & 10020137 & Shawano & Whele Tank & $6 \mathrm{~h}$ \\
2 & 10020138 & Shawano & Whole Tank & $9 \mathrm{~h}$ \\
3 & 10020134 & Shawano & Whole Tank & $12 \mathrm{~h}$ \\
4 & 10020136 & Shawano & Botton Injection & $12 \mathrm{~h}$ \\
\hline
\end{tabular}

\begin{tabular}{|c|c|c|}
\hline Date & $\begin{array}{c}\text { Mean } \\
\text {-emperature (" } \mathrm{C})\end{array}$ & (STD) \\
\hline $9 / 8 / 2012$ & 21.49 & 0.65 \\
\hline $9 / 9 / 2012$ & 20.16 & 0.39 \\
\hline $9 / 10 / 2012$ & 20.95 & 0.78 \\
\hline $9 / 11 / 2012$ & 20.93 & 0.61 \\
\hline $9 / 12 / 2012$ & 20.69 & 0.24 \\
\hline $9 / 13 / 2012$ & 19.81 & 0.39 \\
\hline $9 / 14 / 2012$ & 19.44 & 0.43 \\
\hline $9 / 15 / 2012$ & 19.29 & 0.68 \\
\hline $9 / 16 / 2012$ & 19.91 & 0.30 \\
\hline $9 / 17 / 2012$ & 18.96 & 0.49 \\
\hline $9 / 18 / 2012$ & 17.11 & 0.43 \\
\hline $9 / 19 / 2012$ & 16.20 & 0.09 \\
\hline $9 / 20 / 2012$ & 15.47 & 0.29 \\
\hline $9 / 21 / 2012$ & 15.17 & 0.19 \\
\hline $9 / 22 / 2012$ & 14.39 & 0.43 \\
\hline $9 / 73 / 2012$ & 13.94 & 0.31 \\
\hline $9 / 24 / 2012$ & 13.41 & 0.15 \\
\hline $9 / 25 / 2012$ & 13.54 & 0.52 \\
\hline $9 / 26 / 2012$ & 14.43 & 0.36 \\
\hline $9 / 27 / 2012$ & 14.33 & 0.37 \\
\hline $9 / 28 / 2012$ & 15.08 & 0.25 \\
\hline $9 / 29 / 2012$ & 15.50 & 0.34 \\
\hline $9 / 30 / 2012$ & 15.02 & 0,41 \\
\hline $10 / 1 / 2012$ & 15.58 & 0.39 \\
\hline $10 / 2 / 2012$ & 15.81 & 0.33 \\
\hline $10 / 3 / 2012$ & 16.08 & 0.30 \\
\hline $10 / 4 / 2012$ & 16.33 & 0.24 \\
\hline $10 / 5 / 2012$ & 13.58 & 0.67 \\
\hline $10 / 6 / 2012$ & 11.20 & 0.60 \\
\hline $10 / 7 / 2012$ & 11.04 & 0.66 \\
\hline $10 / 8 / 2012$ & 10.52 & 0.53 \\
\hline $10 / 9 / 2012$ & 10.07 & 0.27 \\
\hline
\end{tabular}

Temperature reacings from the first and last days of recorder deployment (prior to Scptember 8 and after October 9, 2012 for whole tank; prior to September 10 and after October 9,2012 for bottom injection) were omitted from data analysis. The temperature recorders may have sampled ambient air temperature during these time points. These entire days will be omitted as diurnal terperature flucuation skew the mean daily lemperature. The mean temperature for September 8 and 9,2012 was calculated using only the three data loggers $(\$ 1,2$, and 3$)$ from whole tank treatments.

$$
\text { Item Number } \frac{2}{1} \text { of } 2
$$

File Folder: $\mathrm{Aa}$ 
Study Number: AEH-12-PSEUDO-04

Test Art|cle: MB| 401 SDP [Pf-CL 145A (SDP)]

E ectrunlc Lab Notebook (́pages $24 \& 28$ )

Artisle Lot \#: 401P12163C and 401P12164C Mix

Data Source:

Flle Folder: $17 \mathrm{a}$

Fcrrrs: HOBO Output in Excel "Lake Shawano th whole tank", 'Lake Shawaro gh whole tank'

Exposure Date: September 6 and 8,2012

Test Locatlo r: Lake Shawano, Shawano, WI

Treatment Type: Whole Tank and Bottom Injectior

Locatlon: I:AEEH-12-PSEUDO-04 DatalHobos (Carlos and Shawaro in Excel

Post-Exposure Holding Period Water Temperature

\begin{tabular}{|c|c|c|c|c|c|c|c|c|c|c|c|}
\hline \multirow{4}{*}{$\begin{array}{c}\text { Date } \\
\text { Time (GMT-05:00) }\end{array}$} & \multirow{2}{*}{\multicolumn{4}{|c|}{ St-Exposur }} & \multirow{4}{*}{$\begin{array}{l}\text { Mean } \\
\text { (STD) }\end{array}$} & \multirow{4}{*}{$\begin{array}{c}\text { Date } \\
\text { Time }\{G M T-05: 00\}\end{array}$} & \multirow{2}{*}{\multicolumn{4}{|c|}{ Data Logger Number }} & \multirow{4}{*}{$\begin{array}{l}\text { Mean } \\
\text { (STD) }\end{array}$} \\
\hline & & & & & & & & & & & \\
\hline & 1 & 2 & 3 & 4 & & & 2 & & 3 & & \\
\hline & \multicolumn{4}{|c|}{ Temperature $\left({ }^{\circ} \mathrm{C}\right)$} & & & & Tempe & ure $\left({ }^{\circ} \mathrm{C}\right)$ & & \\
\hline $9 / 8 / 20123: 23$ & 21.187 & 21.187 & 21,091 & , & & $9 / 24 / 20123: 23$ & 13.461 & 13.461 & 13.365 & 13.654 & \\
\hline 9/8/2012 9:23 & 20.71 & 20.71 & 20.615 & & 21.49 & 9/24/20129:23 & 13.173 & 13.173 & 13.076 & 13.365 & 13.41 \\
\hline 9/8/2012 15:23 & 22.429 & 22.429 & 22.129 & . & $(0,65)$ & $9 / 24 / 201215: 23$ & 13.461 & 13.461 & 13.365 & 13.365 & $(0.15)$ \\
\hline S/8/2012 21:23 & 21.76 & 21.554 & 21.664 & & & $9 / 24 / 201221: 23$ & 13.558 & 13.558 & 13.461 & 13.558 & \\
\hline $9 / 9 / 20123: 23$ & 20.519 & 20.519 & 20.519 & . & & $9 / 25 / 20123: 23$ & 13.173 & 13.173 & 13.173 & 13.461 & \\
\hline 9/9/20129:23 & 19.567 & 19.472 & 19.472 & . & 20.16 & $9 / 25 / 20129: 23$ & 12.883 & 12.883 & 12.787 & 12.883 & 13.54 \\
\hline$S / 9 / 201215: 23$ & 20.329 & 20.329 & 20.234 & . & (c.39) & 9/25/2012 15:23 & 14.23 & 14.134 & 14.038 & 13.75 & $(0.52)$ \\
\hline $9 / 9 / 201221: 23$ & 20.329 & 20.424 & 20.234 & & & 9/25/2012.11:23 & 14.038 & 14.134 & 13.942 & 13.942 & \\
\hline 9/10/20123:23 & 20.424 & 20.424 & 20.329 & 20.234 & & $9 / 26 / 20123: 23$ & 14.421 & 14.517 & 14.421 & 13.846 & \\
\hline $9 / 10 / 20129: 23$ & 20.138 & 20.138 & 20.043 & 20.043 & 20.95 & $9 / 26 / 20129: 23$ & 14.038 & 14.134 & 13.942 & 13.942 & 24.43 \\
\hline $9 / 10 / 201215: 23$ & 21.951 & 21.951 & 21,855 & 20.805 & $10.78)$ & $9 / 26 / 201215: 23$ & 14.996 & 14.804 & 14.9 & 14.23 & $(0.36)$ \\
\hline $9 / 1 \mathrm{C} / 201221: 23$ & 21.664 & 21.569 & 21.569 & 22,046 & & $9 / 26 / 201221: 23$ & 14.709 & 14.709 & 14.613 & 14.709 & \\
\hline $9 / 11 / 20123: 23$ & 20.805 & 20.71 & 20.71 & 21.187 & & $9 / 27 / 20123: 23$ & 14,038 & 14.038 & 13.942 & 14.325 & \\
\hline $9 / 11 / 20129: 23$ & 20.043 & 20.043 & 19.948 & $2 C .329$ & 20.93 & 9/27/20129:23 & 13.942 & 14.038 & 13.846 & 13.846 & 14.33 \\
\hline $9 / 11 / 2012.15: 23$ & 21.569 & 21.473 & 21.473 & 20.424 & 10.61) & $9 / 27 / 201215: 23$ & 14.996 & 14.709 & 14.804 & 14.325 & $(0.37)$ \\
\hline $9 / 11 / 201221: 23$ & 21.473 & 21,473 & 21.473 & 21.76 & & $9 / 27 / 201221: 23$ & 14.613 & 14.804 & 14.517 & 14.517 & \\
\hline $9 / 12 / 20123: 23$ & 20.805 & 20.805 & 20.71 & 21.187 & & $9 / 28 / 20123: 23$ & 14.996 & 14.996 & 14.9 & 15.187 & \\
\hline $9 / 12 / 20129: 23$ & 20.329 & $2 C .32 .9$ & 20.234 & 20.519 & 20.69 & $9 / 23 / 20129: 23$ & 14.804 & 14.9 & 14.709 & 14.709 & 15.08 \\
\hline $9 / 12 / 201215: 23$ & 20.805 & $2 \mathrm{C} .901$ & 20.71 & 20.519 & $(0.24)$ & $9 / 29 / 201215: 23$ & 15.569 & 15.378 & 15.378 & 14.9 & $(0.25)$ \\
\hline $9 / 12 / 201221: 23$ & 20.805 & 20,901 & 20.805 & 20.615 & & $9 / 28 / 201221: 23$ & 15.187 & 15.282 & 15.187 & 15.187 & \\
\hline $9 / 13 / 20123: 23$ & 20.329 & 20.424 & 20.329 & 20.519 & & $9 / 29 / 20123: 23$ & 15.378 & 15.378 & 15.282 & 15.187 & \\
\hline $9 / 13 / 20129: 23$ & 19.472 & 19.472 & 19.377 & 20.043 & 19.81 & 9/29/20129:23 & 15.282 & 15.378 & 15.187 & 15.187 & 15,50 \\
\hline $9 / 13 / 201215 ; 23$ & 19.758 & 19.758 & 19.662 & 19.282 & (0.39) & 9/29/2012 15:23 & 16.141 & 15.951 & 16.046 & 15.569 & $(0.34)$ \\
\hline $9 / 13 / 201221: 23$ & 19.567 & 19.567 & 19.567 & 19.758 & & $9 / 29 / 201221: 23$ & 15.378 & 15.378 & 15.187 & 16.141 & \\
\hline $9 / 14 / 20123: 23$ & 19.187 & $19.18 \%$ & 19.092 & 19.377 & & $9 / 30 / 20123: 23$ & $14.9^{\circ}$ & 14.9 & 14.804 & 14.936 & \\
\hline $9 / 14 / 20129: 23$ & 18.996 & 18.996 & 18.901 & 18.901 & 19.44 & $9 / 30 / 20129: 23$ & 14,517 & 14.613 & 14.421 & 14.517 & 15.02 \\
\hline $9 / 14 / 201215: 23$ & 19.948 & 20.234 & 19.853 & 19.187 & $(0.43)$ & $9 / 30 / 201215: 23$ & 15.378 & 15.187 & 15.782 & 14.804 & $(0.41\}$ \\
\hline $9 / 14 / 201221: 23$ & $19 . / 38$ & 20.043 & 19.758 & 19.567 & & $9 / 20 / 201221: 23$ & 15.569 & 15.855 & 15.569 & 11.996 & \\
\hline $9 / 15 / 20123: 23$ & $" 19.187$ & 19.187 & 19.092 & 19.472 & & $10 / 1 / 20123.23$ & 15.187 & 15.282 & 15.187 & 15.473 & \\
\hline $9 / 15 / 20129: 23$ & 18.331 & 18.331 & 18.236 & 18.521 & 19.29 & $10 / 1 / 20129: 23$ & 15.282 & 15.282 & 15.187 & 14.996 & 15.58 \\
\hline $9 / 15 / 201215: 23$ & 19.662 & 20.138 & 19.567 & 18.711 & (0.68) & 1.0/1/2012 15:23 & 16.141 & 16.046 & 15.951 & 15.473 & $(0.39)$ \\
\hline 9/15/2012 21:23 & 19.853 & 20.519 & 19.948 & 19.853 & & $10 / 1 / 201221: 23$ & 16.046 & 16.141 & 15.951 & 15.664 & \\
\hline $9 / 16 / 20123: 23$ & 19.948 & 19.853 & 19.853 & 20.138 & & $10 / 2 / 20123: 23$ & 15.664 & 15.664 & 15.569 & 15.664 & \\
\hline $9 / 16 / 20129: 23$ & 19.472 & 19.472 & 19.377 & 19.567 & 19.91 & $10 / 2 / 20129: 23$ & 15.473 & 15.473 & $15.3 / 8$ & 15.473 & $15.8:$ \\
\hline $9 / 16 / 2012.15: 73$ & 20.379 & 20.329 & 20.234 & 19.758 & $(0.3 c)$ & $10 / 2 / 2012,15: 23$ & 16.237 & 16.046 & 16.046 & 15,569 & $(0.33)$ \\
\hline $9 / 16 / 201221: 23$ & 20.043 & 20.043 & 19.948 & 20.138 & & $10 / 2 / 201221: 23$ & 16.332 & 16.332 & 16.237 & 15.855 & \\
\hline $9 / 17 / 20123: 23$ & 19.472 & 19.472 & 19.472 & 19.758 & & $10 / 3 / 20123: 23$ & 15.951 & 15.855 & 15.855 & 15.046 & \\
\hline $9 / 17 / 20129: 23$ & 19,092 & 19.092 & 18.996 & 19.187 & 18.96 & $10 / 3 / 20129: 23$ & 15.76 & 15.76 & 15.664 & 15.76 & 16.08 \\
\hline $9 / 17 / 201215: 23$ & 18.996 & 18.996 & 18.901 & 18.996 & $(0.49)$ & $10 / 3 / 201215: 23$ & 16.618 & 16.332 & 16.427 & 15.855 & (0.30) \\
\hline $9 / 17 / 201221: 23$ & 18.14 & 18.14 & 18.045 & 18.616 & & $10 / 3 / 201221: 23$ & 16.332 & 16.523 & 16.332 & 15.237 & \\
\hline $9 / 18 / 20123: 23$ & 17.284 & 17.284 & $17.189^{9}$ & 17.855 & & $10 / 4 / 20123: 23$ & 16.237 & 16.332 & 16.237 & 16,237 & \\
\hline $9 / 18 / 20129: 23$ & 16.808 & 16.808 & 16.713 & 16.903 & 17.17 & $10 / 4 / 20129: 23$ & 16.237 & 16.237 & 16.141 & 15.141 & 16.33 \\
\hline $9 / 18 / 201215: 23$ & 17.76 & 17.76 & 17.665 & 16.713 & $(0.43)$ & $10 / 4 / 201215: 23$ & 16.713 & 16.713 & 16.618 & 16.523 & $(0.24)$ \\
\hline 9/18/2012 21:23 & 16.808 & 16.713 & 16.713 & 17.665 & & $10 / 4 / 201721: 23$ & 16.046 & 16.046 & 16.046 & 15.713 & \\
\hline $9 / 19 / 20123: 23$ & 16.141 & 16.141 & 16.141 & 16.141 & & $10 / 5 / 20123: 23$ & 14.421 & 14.421 & 14,325 & 15.282 & \\
\hline 9/19/2012 9:23 & 16.141 & 16.141 & 16.046 & 16.141 & $\therefore 6.20$ & $10 / 5 / 20129: 23$ & 13.461 & 13.461 & 13.365 & 13.461 & 13.58 \\
\hline 9/19/2012 15:23 & 16.332 & 16.332 & 16.237 & 16.141 & $(0.69)$ & $10 / 5 / 201215: 23$ & 13.461 & 13.461 & 13.365 & 12.98 & $(0.67)$ \\
\hline $9 / 19 / 201221: 23$ & 16.737 & 16.237 & 16.237 & 16.427 & & $10 / 5 / 201221: 23$ & 12.883 & 12,883 & 12.787 & 13.269 & \\
\hline $9 / 20 / 20123: 23$ & 15.855 & 15.855 & 15.76 & 16.046 & & $10 / 6 / 2012323$ & 11.334 & 11.431 & 11.236 & 12.401 & \\
\hline 9/20/2012 9:23 & 15.282 & 15.282 & 15.187 & 15.76 & 15.47 & $10 / 6 / 20129: 23$ & 10.748 & 10,748 & 10.651 & 10.553 & 11.20 \\
\hline $9 / 20 / 201215: 23$ & 15.569 & 15.473 & 15.473 & 1.5 .187 & $(0.29)$ & $10 / 6 / 201215: 23$ & 12.013 & 12,013 & 11.916 & 11.236 & $(0.60)$ \\
\hline $9 / 20 / 201221: 23$ & 15.187 & 15.187 & 15,091 & 15.282 & & $10 / 6 / 201221: 23$ & 10.553 & 10.553 & 10.553 & 11.334 & \\
\hline $9 / 21 / 20123: 23$ & 15.091 & 15.091 & 15.091 & 15.091 & & $10 / 7 / 20123: 23$ & 10.455 & 10.455 & 10.357 & 10.748 & \\
\hline 9/21/2012 9:23 & 15.187 & 15.187 & 15.091 & 14.996 & $15.1 \%$ & $10 / 7 / 20129: 23$ & 10.553 & 10.553 & 10.455 & 10.357 & 11.04 \\
\hline 9/21/2012 15:23 & 15.569 & 15.473 & 15.473 & 15.378 & $(0.19)$ & $10 / 7 / 201215: 23$ & 12.207 & 12.207 & 12,11 & 11.139 & $(0.66)$ \\
\hline $9 / 21 / 201221: 23$ & 14.996 & 14.996 & 14.9 & 15,187 & & $10 / 7 / 201221: 23$ & $11: 39$ & 11,139 & 11.041 & 11.722 & \\
\hline $9 / 22 / 20123 / 23$ & 14.325 & 14.325 & 14.325 & 14.804 & & $10 / 8 / 20123: 23$ & 10.259 & 10.357 & 10.259 & 10.748 & \\
\hline $9 / 22 / 20129: 23$ & -3.75 & 13.75 & 13.654 & 14.038 & 14.39 & $10 / 8 / 20129: 23$ & 9.866 & 9.866 & 9.768 & 9.768 & 10.52 \\
\hline $9 / 22 / 201215: 23$ & 14.9 & 14.804 & 14.804 & 13.942 & (0.43) & $10 / 8 / 201215: 23$ & 11.334 & 11.332 & 11.236 & 10.553 & $\{0.53\}$ \\
\hline $9 / 22 / 201221: 29$ & 14.613 & 14.613 & 14.517 & 14.996 & & $10 / 8 / 201221: 23$ & 10.651 & 10.651 & 10.553 & 11.139 & \\
\hline $9 / 23 / 20123: 23$ & 14.23 & 14.134 & 14.134 & 14.23 & & $10 / 9 / 20123: 23$ & 10.357 & 10.357 & 10.259 & 10.651 & \\
\hline $9 / 23 / 20129: 23$ & 13.461 & 13.461 & 13.365 & 13.846 & 13.94 & $10 / 9 / 20129: 23$ & 10.063 & 10.063 & 9.965 & 10.259 & 10.07 \\
\hline $9 / 23 / 201215: 23$ & 14.421 & 14.23 & 14.23 & 13.75 & $(0,31)$ & $10 / 9 / 201215: 23$ & 10.161 & 10.161 & 10.063 & 10.063 & $\{0.27\}$ \\
\hline $9 / 23 / 201221: 23$ & 13.846 & 13.846 & 13.75 & 14.134 & & $10 / 9 / 201221: 23$ & 9.768 & 9.768 & 9.669 & & \\
\hline
\end{tabular}

Item Number. 2

Page 2 of 2

File Folder: $17 a$ 


\section{Appendix 7. Spectrophotometric Summary, SAS Program, Output and Log}

\begin{tabular}{|c|c|c|c|}
\hline $\begin{array}{l}\text { Item } \\
\text { Number }\end{array}$ & Item Description & $\begin{array}{c}\text { Number } \\
\text { of } \\
\text { Pages }\end{array}$ & $\begin{array}{l}\text { Report } \\
\text { Page } \\
\text { Number }\end{array}$ \\
\hline 1 & Spectrophotometric Data - Lake Carlos - Whole Tank - Data Summary & 3 & 320 \\
\hline 2 & SAS program for spectrophotometric data analysis - Lake Carlos - Whole Tank & 3 & 323 \\
\hline 3 & SAS log for spectrophotometric data analysis - Lake Carlos - Whole Tank & 8 & 326 \\
\hline 4 & SAS output for spectrophotometric data analysis - Lake Carlos - Whole Tank & 15 & 334 \\
\hline 5 & Spectrophotometric Data - Lake Shawano - Whole Tank - Data Summary & 4 & 349 \\
\hline 6 & SAS program for spectrophotometric data analysis - Lake Shawano - Whole Tank & 4 & 353 \\
\hline 7 & SAS log for spectrophotometric data analysis - Lake Shawano - Whole Tank & 8 & 357 \\
\hline 8 & SAS output for spectrophotometric data analysis - Lake Shawano - Whole Tank & 19 & 365 \\
\hline 9 & Spectrophotometric Data - Lake Carlos - Bottom Injection - Data Summary & 3 & 384 \\
\hline 10 & SAS program for spectrophotometric data analysis - Lake Carlos - Bottom Injection & 2 & 387 \\
\hline 11 & SAS log for spectrophotometric data analysis - Lake Carlos - Bottom Injection & 5 & 389 \\
\hline 12 & SAS output for spectrophotometric data analysis - Lake Carlos - Bottom Injection & 13 & 394 \\
\hline 13 & Spectrophotometric Data - Lake Shawano - Bottom Injection - Data Summary & 4 & 407 \\
\hline 14 & $\begin{array}{l}\text { SAS program for spectrophotometric data analysis - Lake Shawano - Bottom } \\
\text { Injection }\end{array}$ & 2 & 411 \\
\hline 15 & SAS log for spectrophotometric data analysis - Lake Shawano - Bottom Injection & 5 & 413 \\
\hline 16 & SAS Output for spectrophotometric data analysis - Lake Shawano - Bottom Injection & 18 & 418 \\
\hline
\end{tabular}




\begin{tabular}{|c|c|c|c|}
\hline \multirow{5}{*}{\begin{tabular}{|l} 
Stuć Number: AEH-12-pSEULO-J4 \\
lectronlc Lab Notelook (pages 10-12) \\
Data Source: Flle Folder; $9 c$ \\
Forms: "Sample Absorbance Readlrigs" Jata Sheet
\end{tabular}} & Action & Date & Initjals \\
\hline & Created....... & 6 -Feb-14 & TIS N INW \\
\hline & Revised....... & 23-Apr-14 & KLW/L \\
\hline & Reviewed... & $23 \mathrm{NR}, 34$ & $\mathrm{Mu}_{2}$ \\
\hline & Certified....... & $i n / 2 ? / / 11$ & $\sqrt{2}$ \\
\hline $\mathrm{FI}$ & & & \\
\hline
\end{tabular}

Spectrophotometrlc Data

Test Ar:icle: Zequanox * (MBI-40: SDP)

Test Article Lot \#: 401P12163C and 401P12164C Mix

Expcsure Date: August 17, 2C12

Iest location: Lake Carlos, Alexandria, MN

Treatment Type: Whole Tarik

Data Explanation:

1) The atsarbance of trlpllcate samples of $25,50,10 \mathrm{~J}$, and $200 \mathrm{mg} / \mathrm{L}$ dilu:ions of a $2,000 \mathrm{mg} / \mathrm{L}$ actlve ingrectent (A.1.) stock prepa:cd from Analytical Stock th1 were measured to prepare a standard curve.

2) Standard checks were gerformed at 9 and 12 hours by comparing the $25,50,100$, and $200 \mathrm{mg} / \mathrm{L}$ (A.1.) dilutions to the linear curve

3) Data codes used within SAS

$\operatorname{tank}=$ Tank $10(1$ inrougl 19$)$
thero $=$ Theoretlcal or target concentration $(\mathrm{mg} / \mathrm{L})$

time $=$ Sample Time $\{0,1,3,6,9$, and $12 \mathrm{~h}$ after treatinent

loc $=$ Sample Location

sus $=$ Suspended Sample (sampled $\sim 15 \mathrm{~cm}$ from bottom of tank)

St: $=$ Surface Samp e

abs = measured absorbance of sample
conc = concentration (img/L), only uscd for standards used for regression)

4) Informa: ion that is not relevant to a sample (I e., tank ID for standards'; or that will be calculated by SAS [i.e., predlcted concentration for standard checks and samples) is denoted by a "." In the SAS inpet ard output files.

Data Analysls:

1) A linear regression was completed In SAS using the absorbance values obtained from the spectrophotometer of 3 replicate dillutions of 25, 50, 100 and $200 \mathrm{mg} / \mathrm{L}$

Zequanax

2) Standard chedks and treatment sample concentratlons were predicted in SAS by comparlng the observed absorbances with tine inear regresslon.

3) the following mean ticatment concentrations were determined in SAS:

3a) Mean (stancard deytatlon) concer tration by tank for all sumpling times

3b) Mean (standard deviation) corcentration by treatment group for all sampling times

3c) Mean (standard deylation) concentratlon by treatment group and sa.npling times

3d) Mean (standard deviation) concent'ations for $25,50,100$, and $200 \mathrm{mg} / \mathrm{h}$ (A.L) dilutions for all sampling tlmes

File Names:

Spectrophotometric Data for SAS Input

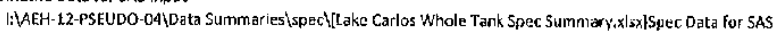

SAS Program/Code

I: \AEH-12-PSEUDO-OA\}SAS-Specica'los whole water program flle

SAS LOG

1: $\mathrm{AEH}-12-P S E U D O-0415$ AS-Speclcarlos whole water log flle

SAS Output

I:AEH-12-PSCUDO-04\SAS-Spectcarlos whole water results file

Data Anomalles and Deviatlons:

1) One exposure tank of a clifferent concentratlon and sample location was sampled in triplicate to evaluate varlabllity of spectrophotometer curing eaci sampllng time. The mean absorbance of the triplicatc samples was Imported Into SAS for use in the analysis,

2) Samples were collected by submerglng a colection beakeı below the surface the surface of each exposure tank. Care was taken to avoid foam or partldes from surface of water.

3) Some mean absarbances for triplicate samples may ue recorded incorrectly on "Sample Absorbance Readlngs" data forms as proper slgnificant $f$ gure rules may not have been ojserved. Additionally, concentrations recorded on "Sample Absorbance Readings" data forms were not used in the aralysis as the Inltial linear regresslun eq ratlon that was used for these calculatloris wass derlved using rounded absorbance values in Excel. All absorbences and concentu ations used In SAS calculatlons and reported within Spectrophotometrlc Data Summary have been corrected

$$
\text { Page 1 }
$$

File Folder: 96 


\begin{tabular}{|c|c|c|c|c|c|}
\hline tank & thero & time & $10 \mathrm{c}$ & abs & conc \\
\hline , & 25 & 0 & . & 0.038 & 25 \\
\hline . & 50 & 0 & . & 0.077 & 50 \\
\hline . & 100 & 0 & . & 0.152 & 100 \\
\hline . & 200 & 0 & . & 0.291 & 200 \\
\hline . & 25 & 0 & . & 0.040 & 25 \\
\hline ' & 50 & 0 & . & 0.077 & 50 \\
\hline . & 100 & 0 & . & 0.149 & 100 \\
\hline . & 200 & 0 & . & 0.291 & 200 \\
\hline . & 25 & 0 & . & 0.040 & 25 \\
\hline . & 50 & 0 & . & 0.076 & 50 \\
\hline . & 100 & 0 & . & 0.148 & 100 \\
\hline . & 200 & 0 & . & 0.290 & 200 \\
\hline . & 25 & 9 & . & 0.035 & . \\
\hline . & 50 & 9 & . & 0.069 & . \\
\hline . & 100 & 9 & . & 0.139 & . \\
\hline . & 200 & 9 & . & 0.269 & . \\
\hline . & 25 & 12 & . & 0.034 & . \\
\hline . & 50 & 12 & . & 0.074 & . \\
\hline . & 100 & 12 & . & 0.136 & . \\
\hline . & 200 & 12 & . & 0.266 & . \\
\hline 1 & 50 & 1 & sur & 0.077 & . \\
\hline 4 & 50 & 1 & sur & 0.078 & . \\
\hline 8 & 50 & 1 & sur & 0.084 & . \\
\hline 6 & 100 & 1 & sur & 0.160 & . \\
\hline 7 & 100 & 1 & sur & 0.155 & . \\
\hline 9 & 100 & 1 & sur & 0.154 & . \\
\hline 1 & 50 & 3 & sus & 0.071 & , \\
\hline 4 & 50 & 3 & sus & 0.074 & . \\
\hline 8 & 50 & 3 & sus & 0.078 & . \\
\hline 6 & 100 & 3 & sus & 0.142 & . \\
\hline 7 & 100 & 3 & sus & 0.138 & . \\
\hline 9 & 100 & 3 & sus & 0.139 & . \\
\hline 1 & 50 & 3 & sur & 0.070 & . \\
\hline 4 & 50 & 3 & sur & 0.073 & . \\
\hline 8 & 50 & 3 & sur & 0.078 & . \\
\hline 6 & 100 & 3 & sur & 0.142 & . \\
\hline 7 & 100 & 3 & sur & 0.137 & . \\
\hline 9 & 100 & 3 & sur & 0.140 & . \\
\hline 1 & 50 & 6 & sus & 0.065 & . \\
\hline 4 & 50 & 6 & sus & 0.063 & ' \\
\hline 8 & 50 & 6 & sus & 0.065 & . \\
\hline 6 & 100 & 6 & sus & 0.132 & . \\
\hline 7 & 100 & 6 & sus & 0.131 & . \\
\hline 9 & 100 & 6 & 5us & 0.133 & . \\
\hline 1 & 50 & 6 & sur & 0.063 & ' \\
\hline 4 & 50 & 6 & sur & 0.062 & . \\
\hline
\end{tabular}

\section{AEH-12-PSEUDO-04}

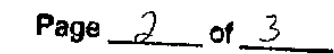

Page 321 of 519 


$\begin{array}{lcccccc}8 & 50 & 6 & \text { sur } & 0.067 & . \\ 6 & 100 & 6 & \text { sur } & 0.127 & . & \text { AEH-12-PSEUDO-04 } \\ 7 & 100 & 6 & \text { sur } & 0.128 & . & \\ 9 & 100 & 6 & \text { sur } & 0.129 & . & \\ 1 & 50 & 9 & \text { sus } & 0.063 & . \\ 4 & 50 & 9 & \text { sus } & 0.061 & . \\ 8 & 50 & 9 & \text { sus } & 0.065 & . \\ 6 & 100 & 9 & \text { sus } & 0.126 & . \\ 7 & 100 & 9 & \text { sus } & 0.126 & . \\ 9 & 100 & 9 & \text { sus } & 0.126 & . \\ 1 & 50 & 9 & \text { sur } & 0.059 & . \\ 4 & 50 & 9 & \text { sur } & 0.059 & . \\ 8 & 50 & 9 & \text { sur } & 0.063 & . \\ 6 & 100 & 9 & \text { sur } & 0.126 & . \\ 7 & 100 & 9 & \text { sur } & 0.122 & . \\ 9 & 100 & 9 & \text { sur } & 0.124 & . \\ 1 & 50 & 12 & \text { sus } & 0.058 & . \\ 4 & 50 & 12 & \text { sus } & 0.057 & . \\ 8 & 50 & 12 & \text { sus } & 0.062 & . \\ 6 & 100 & 12 & \text { sus } & 0.124 & . \\ 7 & 100 & 12 & \text { sus } & 0.121 & . \\ 9 & 100 & 12 & \text { sus } & 0.118 & . \\ 1 & 50 & 12 & \text { sur } & 0.060 & . \\ 4 & 50 & 12 & \text { sur } & 0.058 & . \\ 8 & 50 & 12 & \text { sur } & 0.062 & . \\ 6 & 100 & 12 & \text { sur } & 0.125 & . \\ 7 & 100 & 12 & \text { sur } & 0.120 & . \\ 9 & 100 & 12 & \text { sur } & 0.120 & .\end{array}$

FF \# $9 \mathrm{c}$

Item No. I

$\mathrm{Pg}_{3} \frac{1}{3}$ 


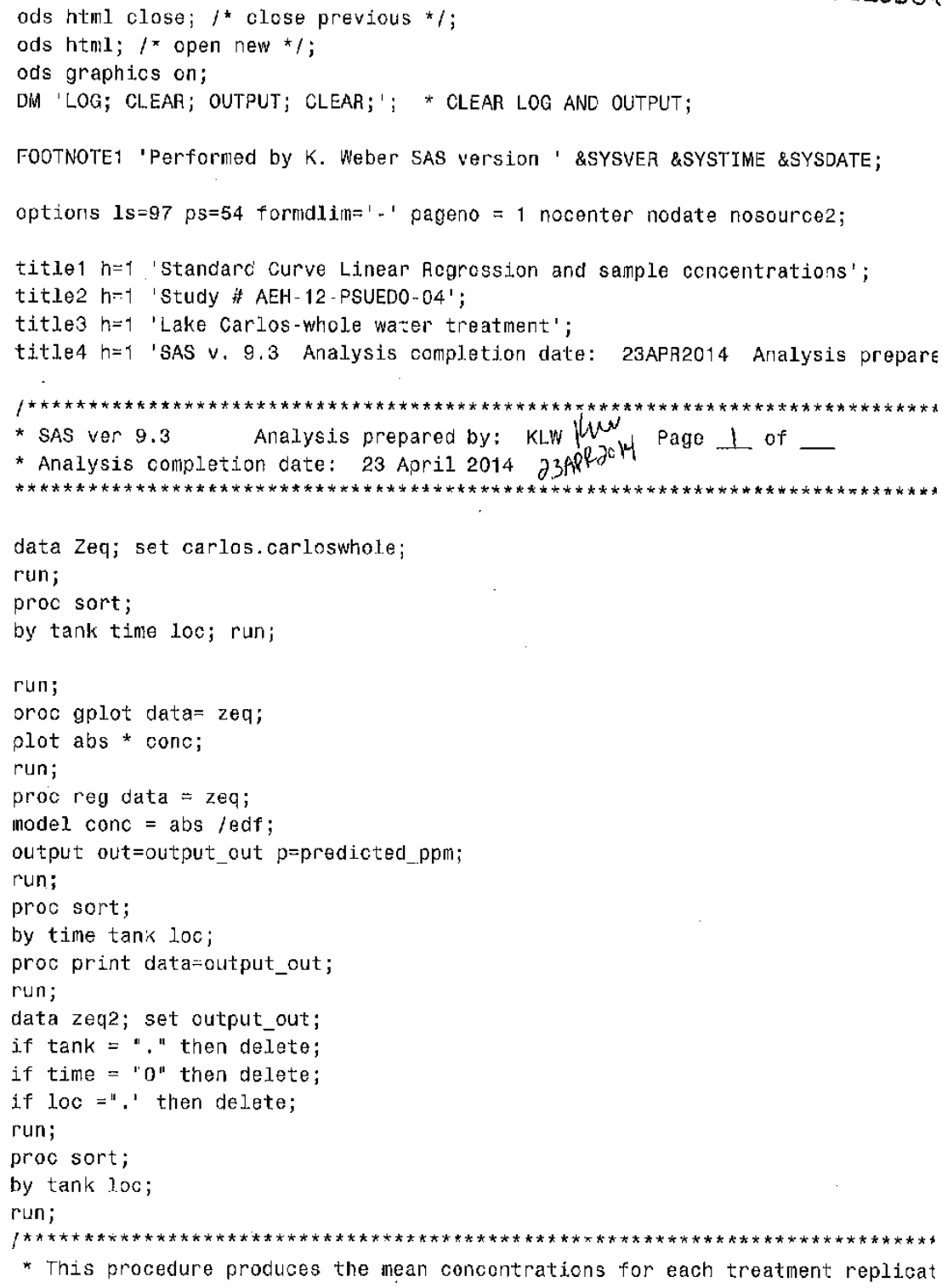

FF \# 96 Item No. 2 
AEH-12-PSEUDO-OA

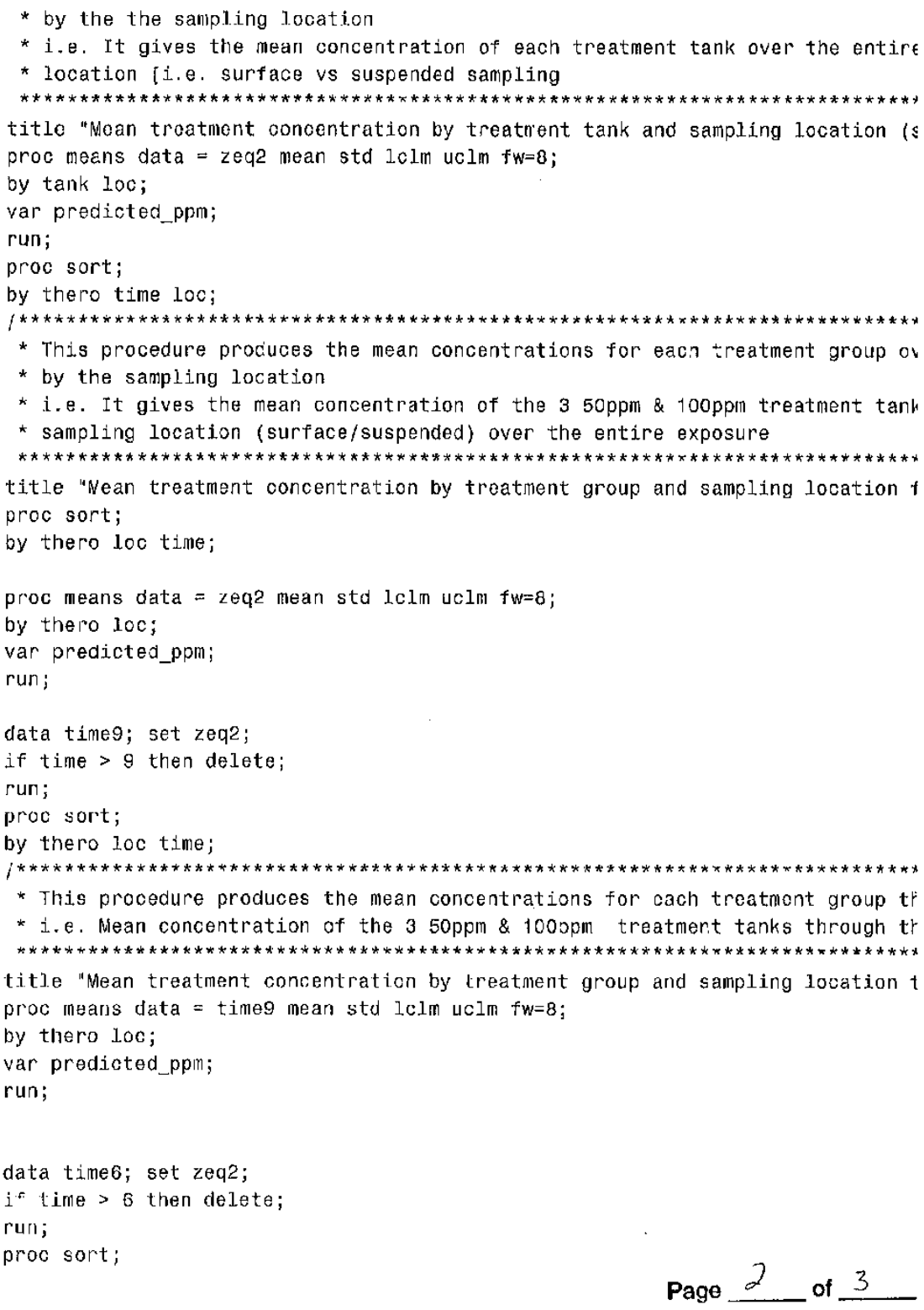

\section{Page 324 of 519}




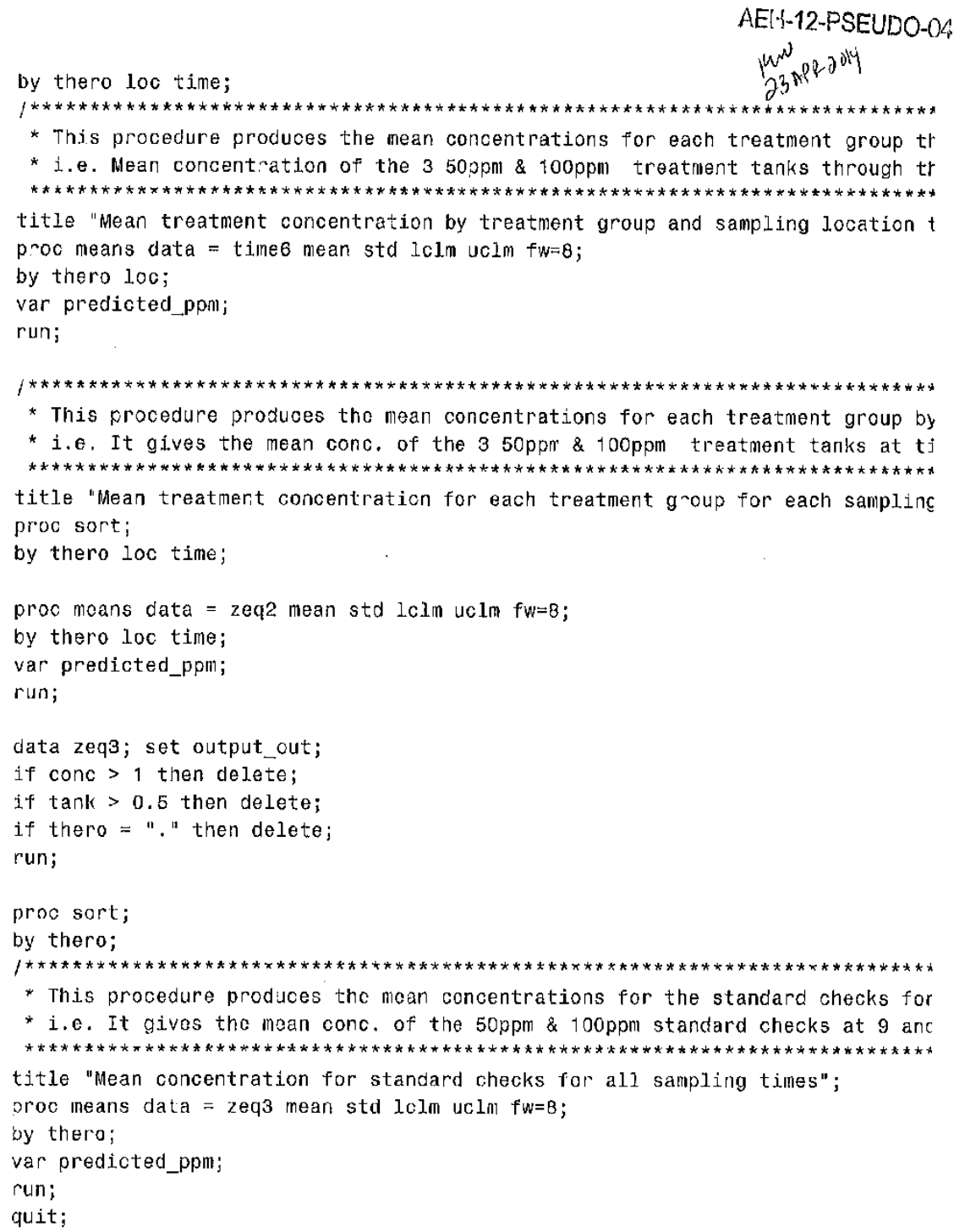

FF \# $q_{c}$

Item No. $\partial$

$P_{C} 3$ of 3 
AEH-12-PSEUDO-OA
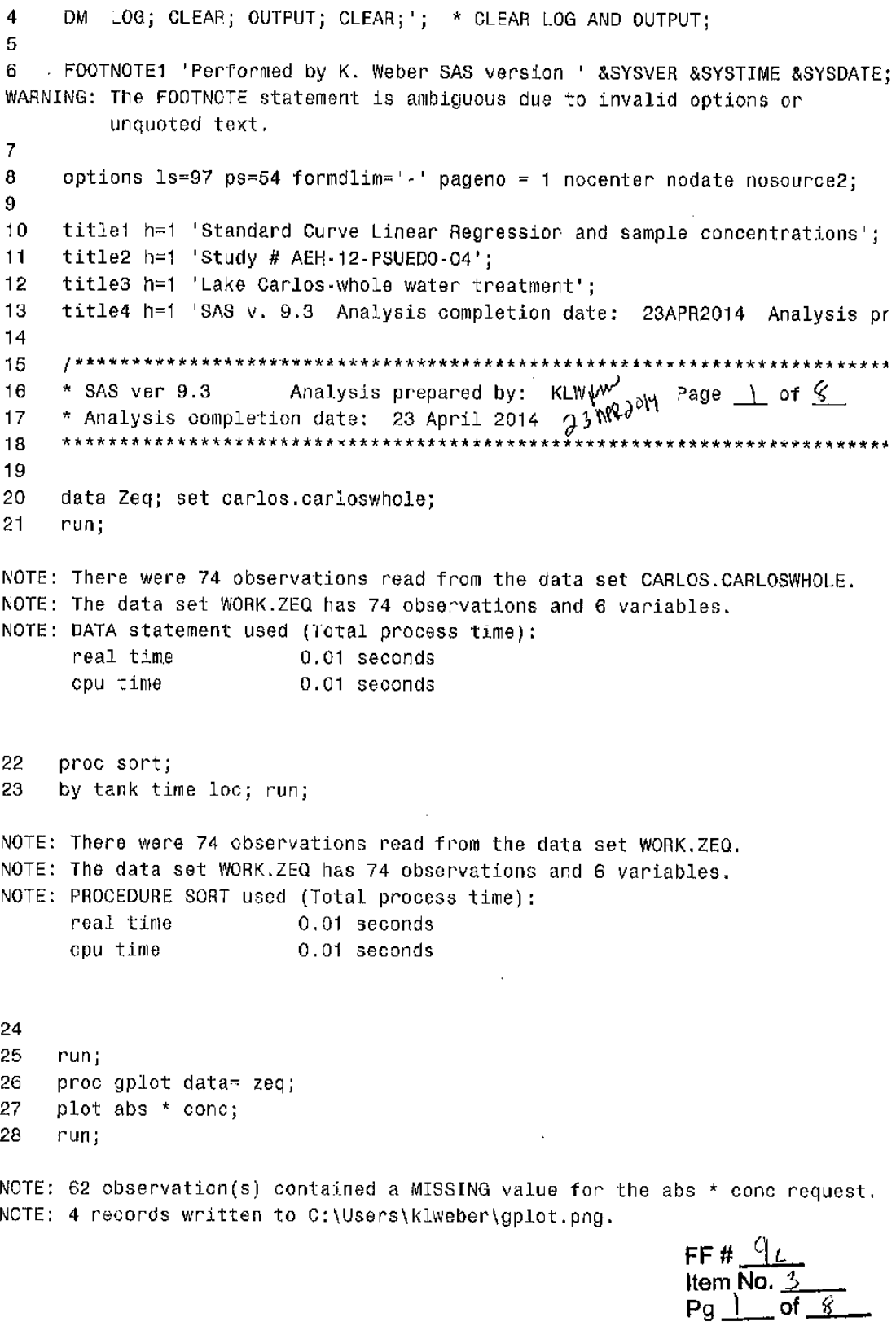
NOTE: There were 74 observations read from the data set WORK.ZEQ.

NOTE: PROCEDURE GPLOT used (Total process time):
real time
0.82 seconds
cpu time
0.50 seconds

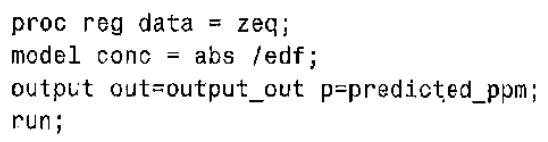

NOTE: The data set WORK.OUTPUT OUT has 74 observations and 7 variables.

NOTE: PROCEDURE REG used (Total process time):
real time
2.60 seconds
cpu time
0.62 seconds

33 proc sort;

34 by time tank 10o;

NOTE: There were 74 observations read from the data set WORK.OUTPUT_OUT.

NOTE: The data set WORK. OUTPUT OUT has 74 observations and 7 variables.

NOTE: PROCEDURE SORT lised (Total process time):
real time
0.01 seconds
cpu time
0.01 seconds

35 proc print data=output_out;

36 run;

NOTE: There were 74 observations read from the data set WORK. OUTPUT OUT

NOTE: PROCEDUAE PRINT used (Total procoss time):
real time
0.12 seconds

cpu time

0.06 seconds

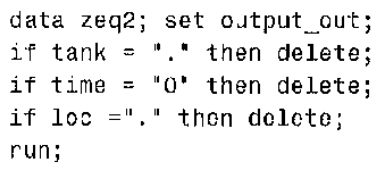

NOTE: Charactor values have been converted to nuneric values at the places giv (Line): (Column).

$$
\text { Prge } 2 \text { of } 8
$$




$$
39: 11
$$

NOTE: There were 74 observations read from the oata set WO3K. OUTPUT_OUT.

NOTE: The data set WORK.ZEQ2 has 54 observations and 7 variables.

NOTE: DATA statement used (Total process time):

$\begin{array}{ll}\text { real time } & 0.01 \text { seconds } \\ \text { cpu time } & 0.01 \text { seconds }\end{array}$

42 proc sort;

43 by tank 100 ;

44 run;

NOTE: There were 54 observations read from the data set WORK.ZEQ2.

NOTE: The data set WORK.ZEQ2 has 54 observations and 7 variables.

NOTE: PROCEDURE SORT used (Total process time):
real time
0.00 seconds
cpu time
0.00 seconds

$45 \quad I^{*} \quad 1 * * * * * * * * * * * * * * * * *$

46 * This procedure produces the mean concentrations for each treatment rep 46 ! sampling times *

47 * by the the sampling location

$47 !$

48 * i.e. It gives the mean concentration of each treatment tank over the $\epsilon$ 48 ! the sampling *

49 * location [i.e. surface vs suspended sampling

491

50 (5)

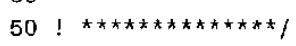

51 title "Mean treatment concentration by treatment tank and sampling locatj

51 ! (surface/suspendod) for all sampling times';

52 proc means data $=$ zeq2 mean std $l \mathrm{clm} u c l m$ f $w=8$;

53 by tank loc;

54 var predicted_ppn;

55 run;

NOTE: There were 54 observations read from the data sct WORK.ZEO2.

NOTE: PROCEDURE MEANS usod (Total process time):
real time
0.12 seconds
cpu inime
0.04 seconds

56 proc sort;

57 by thero time loc;

Page 3 of 8 
AELF-12-PSEUDO-OA

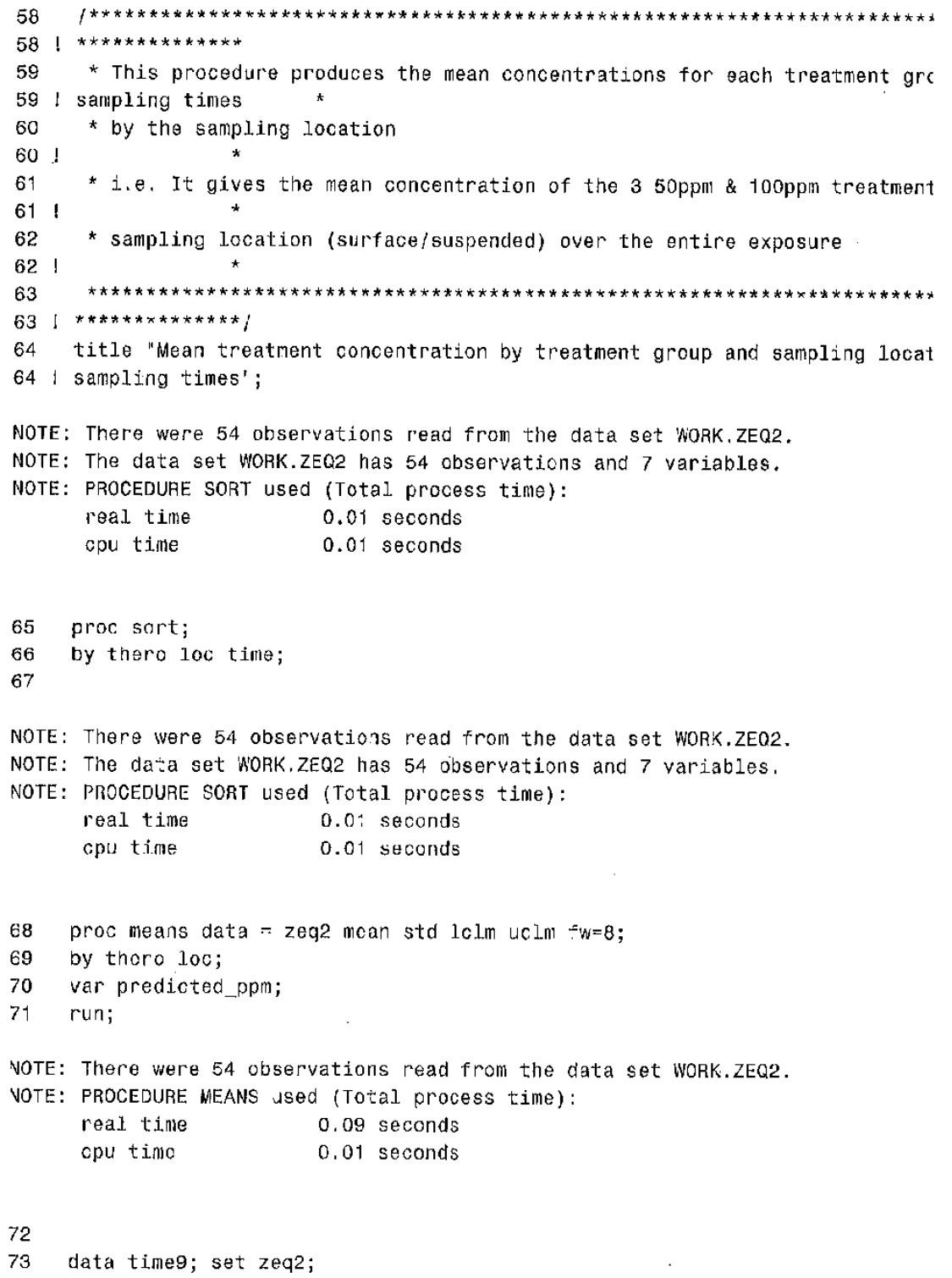




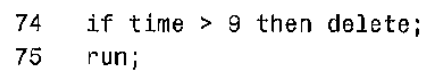

NOTE: There were 54 observations read from the data set WORK.ZEQ2.

NOTE: The data set WORK.TIMEg has 42 observations and 7 variables.

NOTE: DATA statement used (Total procoss time):

$\begin{array}{ll}\text { real time } & 0.01 \text { seconds } \\ \text { cpu tinie } & 0.01 \text { seconds }\end{array}$

76 proc sort;

77. by thero loc time;

$78 / * * * * * * * * * * * * * * * * * * * * *$

78 ! $* * * * * * * * * * * * * * * * * * * * *$

79 * This procedure produces the mean concentrations for each treatment grc 79 ! sample location

80 * i.e. Mean concentration of the $350 \mathrm{ppm} \& 100 \mathrm{ppm}$ treatinent tanks throl 80 I by sample location *

$81 \quad * * * * * * * * * * * * * * * * * * * * * * * * * * * * * * * * * * * * * * * * * * * * * * * * * * * * * * * * * * * * * * * * * * * * * * * *$

$811 * * * * * * * * * * * * * * * * * * * * * /$

82 title "Mean treatment concentration by treatment group and sampling locat 82 ! exposure";

NOTE: There were 42 observations read from the data set WORK.TIME9.

NOTE: The data set WORK.TIMEg has 42 observations and 7 variabies.

NOTE: PROCEDURE SORT used (Total process time):

real time $\quad 0.01$ seconds

cpu time $\quad 0.01$ seconds

83 proc means data = time9 mean std $1 \mathrm{clm}$ uclm fw=8;

B4 by thero loc;

85 var predicted_ppm;

86 run;

NOTE: There viere 42 observations read from the data set WOAK.TIME9.

NOTE: PROCEDURE MEANS used (Total process time):
real tinie
0.09 seconds
cpu time
0.01 seconds

87

88

89 data time6; set zeq2;

90 if time $>6$ then delete;

91 run;

Page 5 of 8 
NOTE: There were 54 observations read from the data set WORK.ZEQ2.

NOTE: The data set WOFK.TIME 6 has 30 observations and 7 variables.

NOTE: DATA statenent used (Total process time):
real time
0.01 seconds
cpu time
0.01 seconds

92 proc sort;

93 by thero loc tine;

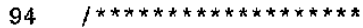

941 I $* * * * * * * * * * * * * * * * * * * * *$

95 * This procedure produces the mean concen:rations for each treatment gre 95 I samp.e location

96 * i.e. Mean concentration of the $350 \mathrm{ppm} \& 100 \mathrm{ppm}$ treatment tanks thro 96 ! by sample location *

97 (1)

$971 * * * * * * * * * * * * * * * * * * * * * /$

98 title "Mean treatment concentration by treatment group and sampling locat 98 ! exposure";

NOTE: There were 30 observations read from the data set WORK.TIME6.

NOTE: The data set WORK.TIME 6 has 30 observations and 7 variables.

NOTE: PROCEDURE SORT used (Total process time):

real time $\quad 0.0^{4}$ seconds

cpu time $\quad 0.01$ seconds

99 proc means data $=$ time6 mean std $1 \mathrm{clm} u c l m$ fw $=8$;

100 by thero loc;

101 var predicted_ppm;

102 run;

NOTE: There were 30 observations read from the data set WORK.TIME6.

NOTE: PROCEDURE MEANS usod (Total process time):
real time
0.23 seconds
cpu tine
0.03 seconds

103

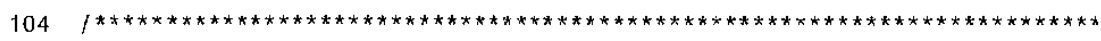

104 ! $* * \star * * * * * * * * * * * \star$

$105 *$ This procedure produces the mean concentrations for each treatment gre 105 !

106 * i.e. It gives the mean conc. of the $350 \mathrm{ppm} \&$ rooppm treatmert tanks 106 ! and $12 \mathrm{~h}$

Page 6 of 8 


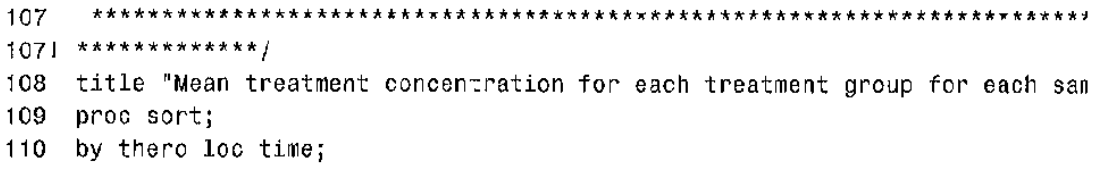

NOTE: There were 54 observations read from the data set WORK. ZEQ2

NOTF: PROCEDURF MFANS used (Total process time):
real time
0.17 seconds
cpu time
0.06 seconds

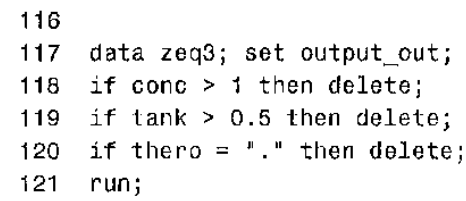

NOTE: Character values have been converted to numeric valuas at the places giv (Line) : (Column). 


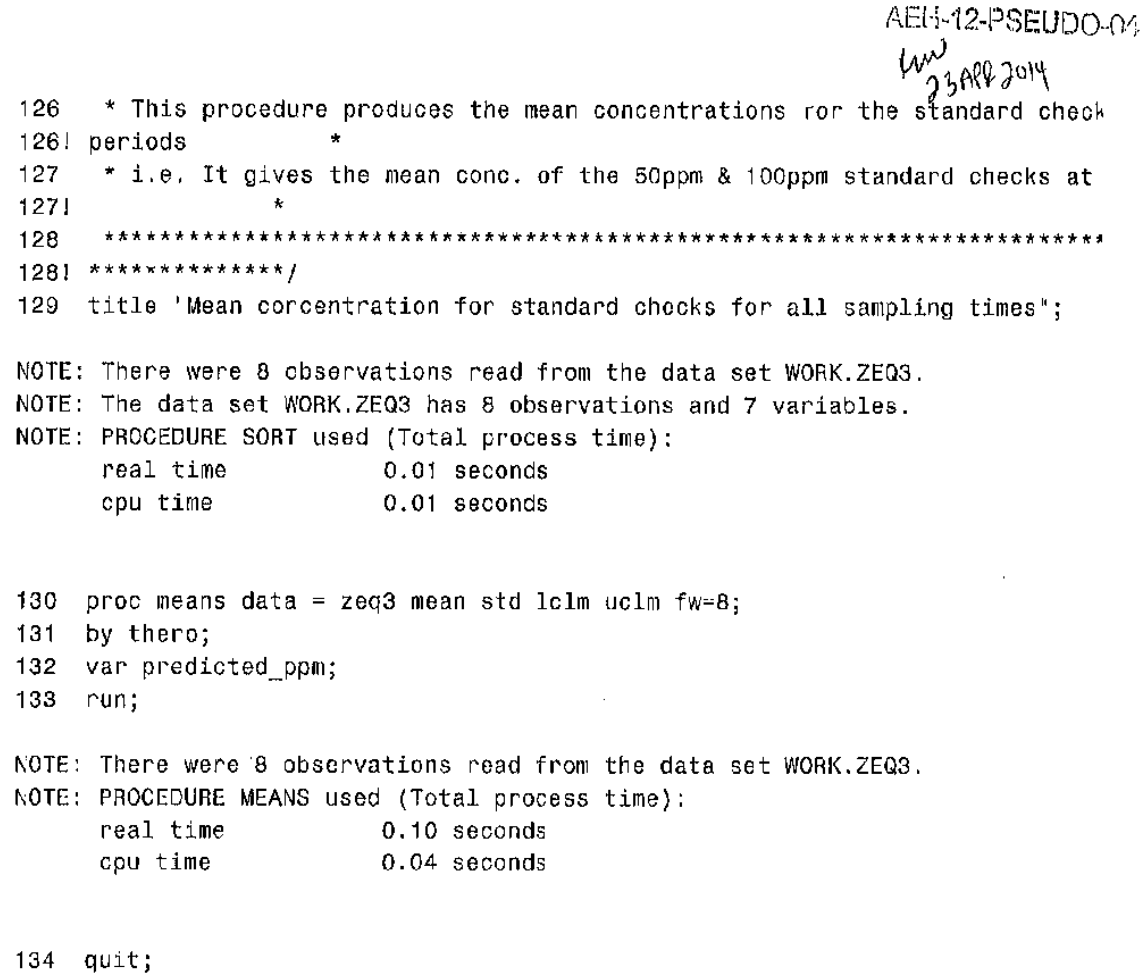




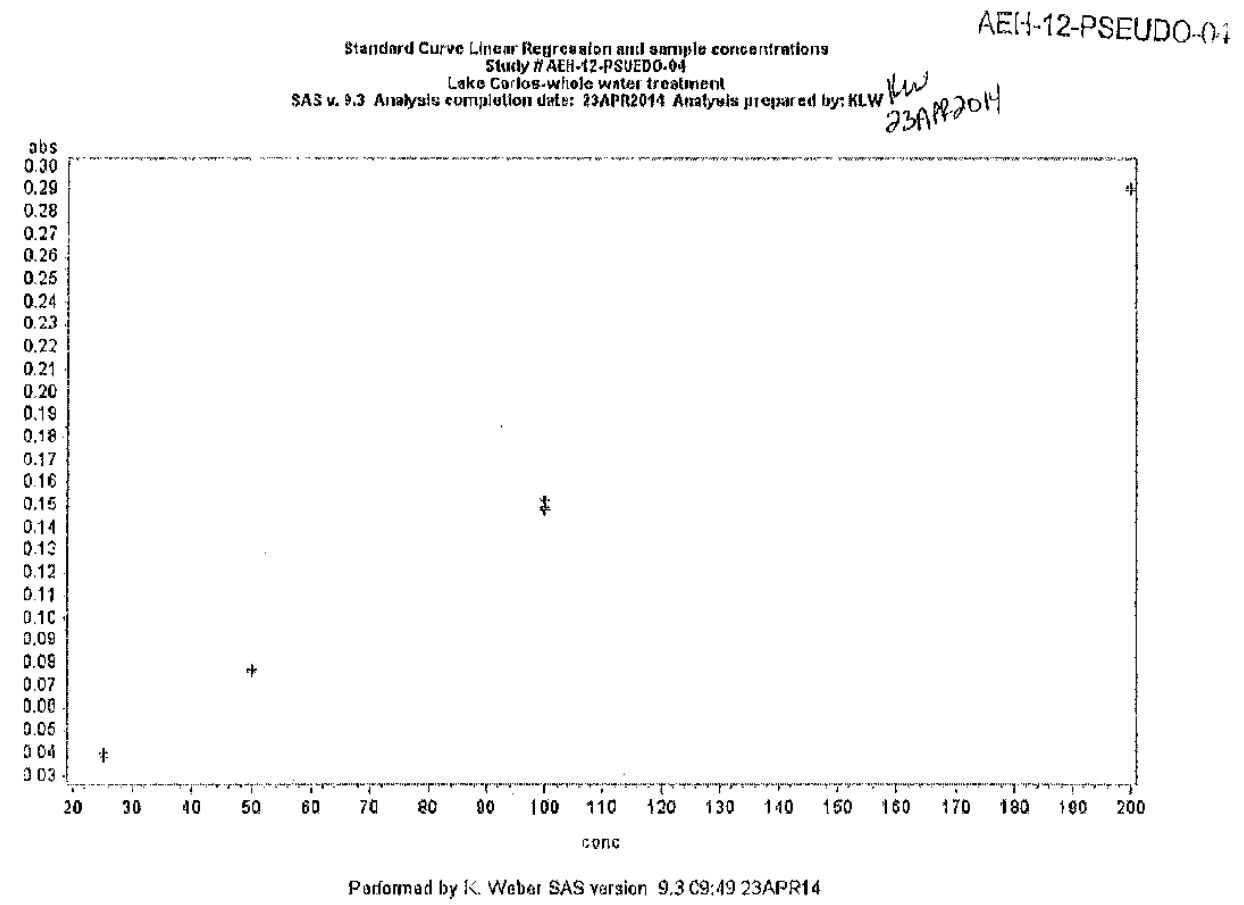

FF \# 9

Item No. 4

$\mathrm{Pg} \perp$ of 15 
Dependent Varlable: conc conc

Number of Observations Read

\begin{tabular}{l|c}
74 \\
Number of Observations Used & 2
\end{tabular}

Number of Observallons with Missing Values 62

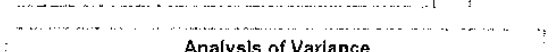

Analysls of Varlance
Stum of Mean

\begin{tabular}{l|l|l|l|} 
Source & DF & Stim of & Nean \\
Squares Square & F Value & Pr $>F$ \\
\end{tabular}

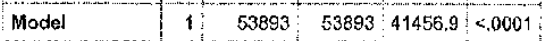

Error $\quad 10 \quad 12.99981: 1.29998$

\begin{tabular}{l|l|l} 
Corrected Total & 11 & 53906
\end{tabular}

Root MSE [1.14017 R-Square 0.9998

\begin{tabular}{lllll}
\hline Dependent Mean & 93.75000 & Ad] R-Sq & 0.9997 \\
\hline
\end{tabular}

\begin{tabular}{l|l} 
Coeff Var & 1.21618
\end{tabular}

- $\quad$ Parameter Estimates

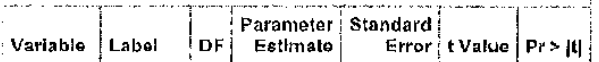

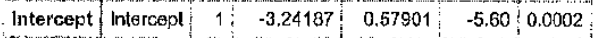

\begin{tabular}{l|l|l|l|l|l|l|l} 
abs & abs & 1 & 697.36515 & 3.42601 & 203.61 & $<0001$
\end{tabular}

Penformed by K. Weber SAS version 9.3 09:49 23APR14 
Standard Curve Linoar Regrossion and samale concanirations

LATH CAEC-12-PSUEDO.04

SAS Y. 9.3 Arnlysls completton date: 23APR201< Analysis prepared by: KLW

The REG Procedure

Model: MODEL.1

Dependent Varlable: cone conc

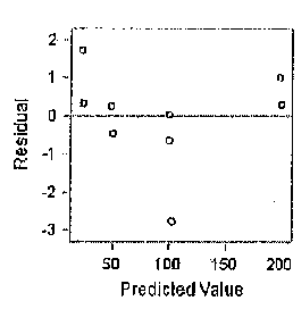

FIt Dlagnostics for conc
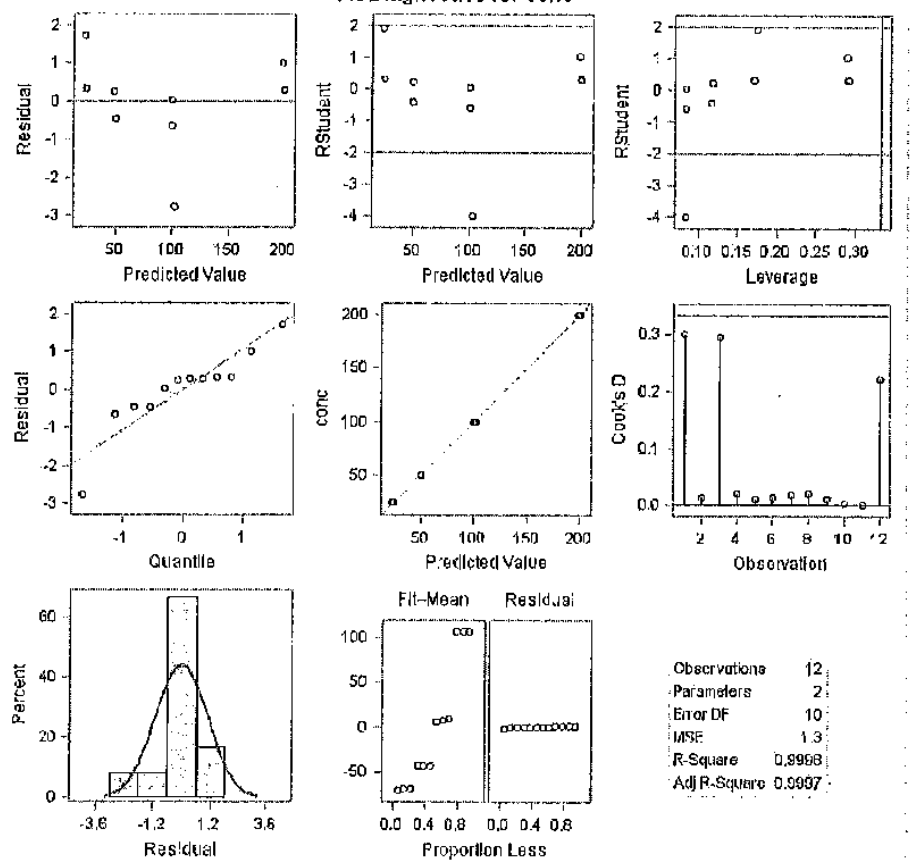

Observations 12

$\begin{array}{ll}\text { Parsmelers } & 2: \\ \text { Eitor DF } & 10 \text { : }\end{array}$

IISE

R-Square 0.9988

Adj R.SGuare 0.0997 

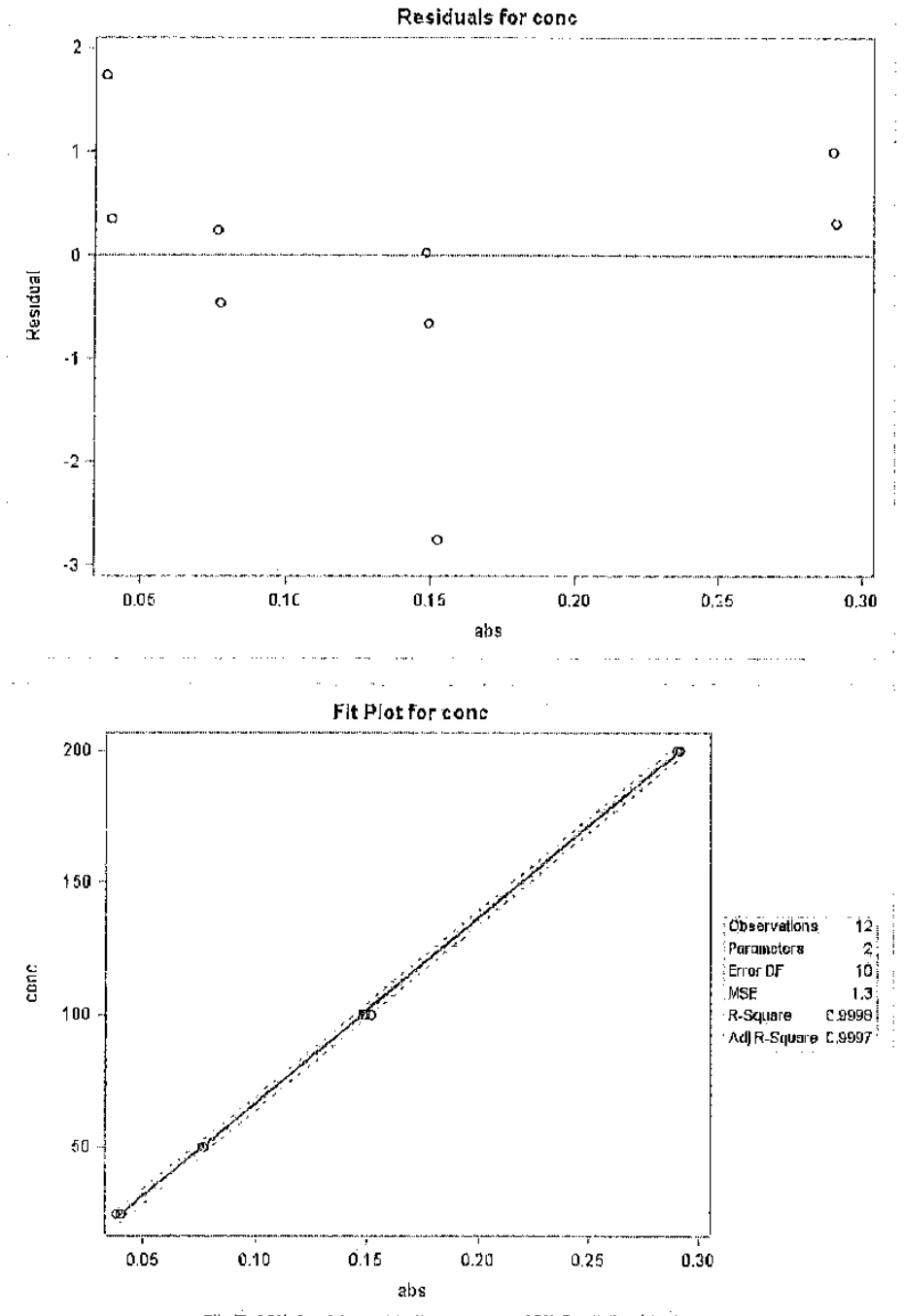

Perforned by K. Weber SAS version 9.3 09:49 23APR 14 


\begin{tabular}{|c|c|c|c|c|c|c|c|}
\hline \multicolumn{8}{|c|}{ 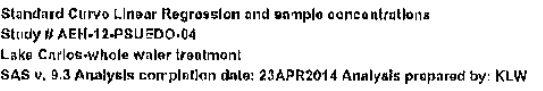 } \\
\hline bs & $\tan k$ & thero & tlme & $10 \mathrm{c}$ & abs & conc & do \\
\hline 1 & , & 25 & 0 & & 0.038 & 25 & 23.258 \\
\hline 2 & & 50 & 0 & & 0.077 & 50 & 50,155 \\
\hline 3 & & 100 & 0 & & 0.152 & 100 & 102.758 \\
\hline 4 & & 200 & 0 & & 0.291 & 200 & 199.691 \\
\hline 5 & & 25 & 0 & & 0.040 & 25 & 24.653 \\
\hline 6 & . & 50 & 0 & & 0.077 & 50 & 0.4 \\
\hline & & 100 & 0 & & 0.149 & 100 & 100.666 \\
\hline 8 & . & 200 & 0 & & 0.291 & $200^{\circ}$ & 198.691 \\
\hline 9 & . & 25 & 0 & & 0.040 & 26 & 24.653 \\
\hline 10 & . & 50 & 0 & & 0.076 & 50 & 49. \\
\hline 11 & . & 100 : & 0 & $:$ & 0.148 & 100 & 98 \\
\hline 12 & - & 200 & 0 & & 0.290 & 200 & 198.994 \\
\hline 13 & 1 & 50 & 1 & sur & 0.077 & & 50.4 \\
\hline 14 & 4 & 50 & 1 & sur & 0,078 & $\cdot$ & 51.153 \\
\hline 15 & 6 & 100 & $1 !$ & sur & 0.160 & . & 108.337 \\
\hline 16 & 7 & 100 & 1 & sur & 0.156 & & 104.850 \\
\hline 17 & 8 & 50 & 1 & sur & 0.084 & & 17 \\
\hline 18 & 9 & 100 & 13 & sur & 4 & & 52 \\
\hline 19 & 1 & 50 & 3 & sur & 0.070 & & 45.574 \\
\hline 20 & 1 & 50 & $3:$ & sus & 0.07 & & 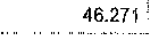 \\
\hline 21 & 4 & 50 & 3 & sur & ? & & 47.666 \\
\hline 22 & 4 & 50 & 3 & sus & 0.074 & & 48.363 \\
\hline 23 & 6 & 100 & 3 & sur & 0.142 & & 95.784 \\
\hline 24 & 6 & 100 & 3 & sus & 0.142 & $\therefore$ & 35.7 \\
\hline 25 & 7 & 100 & 3 & sur & 13 & & 92.297 \\
\hline 26 & $?$ & $100^{\circ}$ & 3 & sus & 0 & & 92.995 \\
\hline 27 & 8 & 50 & 3 & sur : & 0 & . & 51.153 \\
\hline 28 & 8 & 50 & 3 : & sus & 0.078 & . & 1. 153 \\
\hline 29 & 9 & 100 & 3 & sur & 0.140 & $\cdot$ & 94.389 \\
\hline 30 & 9 & 100 & 3 : & sus & 0.139 & & 93.692 \\
\hline 31 & 1 & 50 & $6:$ & sur & 0.063 & 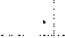 & 40.692 \\
\hline 32 & 1 & $50:$ & 6 & sus & 0.035 & & 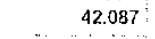 \\
\hline 33 & 4 & 50 & 6 & sur & 0.052 & $i$ & 39.995 \\
\hline 34 & 4 & $50:$ & 6 , & sus & 0.063 & & 40.692 \\
\hline 35 & 6 & 100 & 6 & sur & 0.127 & : & 85.324 \\
\hline $36 !$ & 6 & 100 & 6 & sus: & 0.132 & & 88.810 \\
\hline 37: & 7 & 100. & 6 & sur : & 0.128 & & 86.021 \\
\hline $38:$ & 7 & 100. & 6 & sus: & 0.131 & $\therefore$ & 88.113 \\
\hline
\end{tabular}




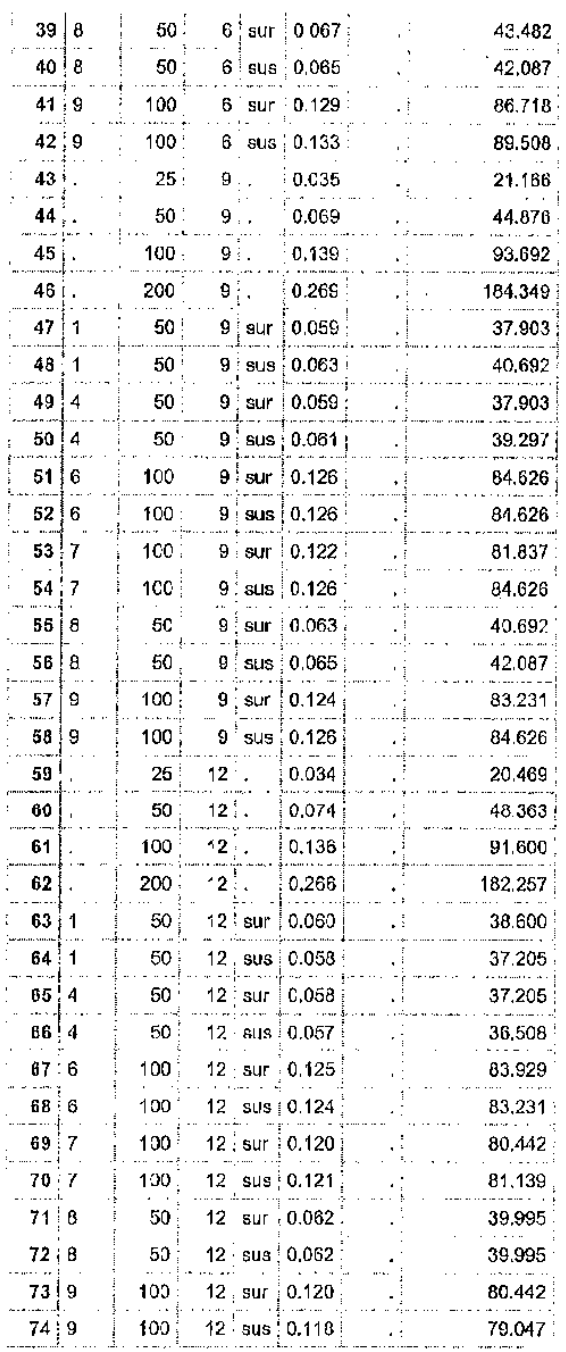

AEFH-12-PSEUDO-O:

Performed by K. Weber SAS verston 9.3 09:49 23APR14 


\begin{tabular}{|c|c|c|c|}
\hline \multicolumn{4}{|c|}{$\begin{array}{l}\text { Mean treatment conc } \\
\text { sampling times }\end{array}$} \\
\hline \multicolumn{4}{|c|}{ The MEANS Procedure } \\
\hline \multicolumn{4}{|c|}{$\tan k=1$ loc $=$ sur } \\
\hline \multicolumn{4}{|c|}{$\begin{array}{l}\text { Analysis Vartable : predicted_ppm Predicted } \\
\text { Value of conc }\end{array}$} \\
\hline Mean & Std Dev & $\begin{array}{r}\text { Lowor } 95 \% \\
\text { CL for Mean }\end{array}$ & $\begin{array}{r}\text { Uppor } 95 \% \\
\text { CL for Mean }\end{array}$ \\
\hline 42.6448 & 5.2972 & 36.0674 & 49.2221 \\
\hline \multicolumn{4}{|c|}{$\operatorname{tank=1}$ loc=sus } \\
\hline \multicolumn{4}{|c|}{$\begin{array}{l}\text { Analysls Varlable : predictod_ppm Prodleted } \\
\text { Value of conc }\end{array}$} \\
\hline Mean & Std Dev & $\begin{array}{l}\text { Lower } 95 \% \\
\text { CL for Mean }\end{array}$ & $\begin{array}{r}\text { Upper } 95 \% \\
\text { CL. for Mean }\end{array}$ \\
\hline $4 \times .5638$ & 3.7500 & 35.5967 & 47.5310 \\
\hline \multicolumn{4}{|c|}{$\tan k=4$ loc $=$ sur } \\
\hline \multicolumn{4}{|c|}{$\begin{array}{l}\text { Analysis Varlabla : predicted_ppm Predicted } \\
\text { Value of conc }\end{array}$} \\
\hline Mean & Std Dev & $\begin{array}{l}\text { Lower } 95 \% \\
\text { CL for Mean }\end{array}$ & $\begin{array}{r}\text { Upper } 95 \% \\
\text { CL for Mean }\end{array}$ \\
\hline 42.7842 & 6.2569 & 35.0153 & 50.5532 \\
\hline
\end{tabular}

tank $=4$ loc $=s$ sus

Analysis Yarlable : predicted_ppm Predicted Value of conc
Lower $95 \%$ Upper $95 \%$ \begin{tabular}{l|l|l|l|l|l|l|l|l|l|l} 
Mean & Std Dev & CL for Mean & CL for Mean \\
\hline
\end{tabular}

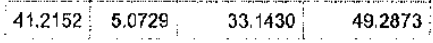

tank $=6$ loc $=$ sur

Analysis Variable : predicted_ppin Predicted Value of conc $\quad$ Lower $95 \%$ Upper $95 \%$ - Mean Std Dev / CL for Mean . CL for Mean $91.5998 / 10.5415 \quad 78.5108 \quad 104.7$ tank $=6$ loc $=$ sus

Analysis Variable : predicted_ppm Predicted Value of cone

Lower 95\% Upper 95\%

Mean Std Dev CL for Mean CL for Mean

$\begin{array}{llll}88.1130 & 5.6357 & 79.1437 & 97.0823\end{array}$

tank $=7$ loc $=3$ ur

Analys/s Varialyle : predicted_ppm Predlcted 


\begin{tabular}{|c|c|c|c|}
\hline \multicolumn{4}{|c|}{ Value of conc } \\
\hline Mean & Std Dev & $\begin{array}{l}\text { Lower } 95 \% \\
\text { CL for Mean }\end{array}$ & $\begin{array}{l}\text { Upper } 95 \% \\
\text { CL for Mean }\end{array}$ \\
\hline 89.0893 & 9.9433 & 76.7431 & 101.4 \\
\hline
\end{tabular}

tankm 7 loc $=$ sus

Analysis Variable : predicted ppm Predlcted Value of conc
Lawer $95 \%$ Upper $95 \%$ : Mean Std Dev CL for Mean \begin{tabular}{l|l} 
Lower for Mean \\
\hline
\end{tabular} $\begin{array}{llllll}86.7182 & 5.0609 & 78.6652 & & 94.7713\end{array}$

$\tan k=8$ loc $=$ sur

Analysis Varlable : predlsted_ppm Predicted Analys s Varlable : predlcted_ppm Predicted
Value of conc

\begin{tabular}{l|l|l|l|l|l}
\hline & Lower $95 \%$ & Upper $85 \%$ \\
\hline
\end{tabular}

: Mean Std Dev CL for Mean CL for Mean $46.1316 ; 6.7863 \quad 37.7052 \quad 54.5579$

tank $=8$ loc $=$ sus

Analysis Variable : predicted_ppm Predicted Value of conc
A \begin{tabular}{l|l|l|l|} 
Mean & Std Dev & Cl for Mean & CL for Mean
\end{tabular} \begin{tabular}{llll}
\hline 3.8303 & 4.9802 & 35.9057 & 51.7549 \\
\hline
\end{tabular}

$\operatorname{tank}=\mathrm{g} \mid \mathrm{oc}=\mathrm{sur}$

Analysls Variable : predicted_ppm Predicted Value of conc

\begin{tabular}{lll} 
& Lower $\mathbf{9 5 \%}$ & Upper 95\% \\
\hline
\end{tabular} Mean Std Dev CL for Mean CL for Mean $\begin{array}{llll}89.7866 & 9.5821 & 77.8889 & 101.7\end{array}$

$\tan k=9 \mid 0 c=s u s$

Analysis Variable : predicted_ppm Predloted Value of conc

-....... Tower 95\% Uppor

Mean Std Dev CL for Mean CL for Mean

\begin{tabular}{llll}
\hline 86.7182 & 6.3149 & 76.6698 & 96.7667
\end{tabular}

Performed by K. Weber SAS version 9.3 09:49 23APR14 
Mean treatment concentration by treatment group and sampling location for all sampling times

The MEANS Procedure

thero $=50$ loc $=$ sur

Analysis Variable : predicted_ppm Predicted

Value of conc
\[ \text { Lower } 95 \% \text { Upper } 95 \% \]

Mean Std Dev CL for Mean CL for Mean

$\begin{array}{llll}43.8535 & 5.9283 & 40.5705 & 47.1365\end{array}$

thero 50 loc $=$ sus

Analysis Varlable : predicted_ppm Predlcted Analysis Varlable : predicted_ppr
Value of conc

\begin{tabular}{l} 
Lower $95 \%$ \\
\hline Upper 95\%
\end{tabular}

\begin{tabular}{c|c|c|c|} 
Mean Std Dev & CL for Mean $95 \%$ & Upper $95 \%$ \\
CL for Maan
\end{tabular}

\begin{tabular}{lllll}
\hline 12.2031 & 4.3686 & 39.4274 & 44.9787 \\
\hline
\end{tabular}

thero $=100$ loc $=\mathrm{sur}$

Analysis Variable : predicted_ppm Predlcted

Value of conc
Lower $95 \%$ [ Lpper $95 \%$

Mean Std Dev \begin{tabular}{ll|l} 
CL for Mean & CL for Moan
\end{tabular}

$\begin{array}{lllll}90.556 & 9.3504 & 84.9805^{\prime} & 95.3367\end{array}$

thero $=100$ locisus

Analysis Variable : predicted_ppm Predicted Value of conc

\begin{tabular}{rlll}
\hline Lower 95\% Upper $95 \%$ \\
\hline
\end{tabular}

\begin{tabular}{ll|l} 
Mean Std Dev CL for Mean Cl. for Mean &
\end{tabular}

$\begin{array}{lllll}87.1831 & 5.1960 & 83.8818 & 90.4845\end{array}$

Performed by K. Weber SAS version 9.3 09:49 23APR14 
AEH-12PSEUDOR:

\begin{tabular}{|c|c|c|c|}
\hline The MEAI & NS Proce & dure & \\
\hline thero $=50$ & loc=sur & & \\
\hline Analysis & $\begin{array}{r}\text { Varlablo } \\
\text { Va }\end{array}$ & $\begin{array}{l}\text { :prodictod_p } \\
\text { lue of conc }\end{array}$ & pm Predlated \\
\hline Mean & Std Dev & $\begin{array}{l}\text { Lower } 95 \% \\
\text { CL for Mean }\end{array}$ & $\begin{array}{r}\text { Upper } 95 \% \\
\text { CL for Mean }\end{array}$ \\
\hline 46.1669 & 5.9133 & 41.4098 & 48.9240 \\
\hline thero $=50$ & loc=sus & & \\
\hline Analysis & $\begin{array}{r}\text { Varlable } \\
\text { Va }\end{array}$ & $\begin{array}{l}\text { predlcted_p } \\
\text { lue of cons }\end{array}$ & in Predlcted \\
\hline Mean & Std Dev & $\begin{array}{l}\text { Lower } 95 \% \\
\text { CL for Mean }\end{array}$ & $\begin{array}{l}\text { Upper } 95 \% \\
\text { CL. for Mean }\end{array}$ \\
\hline 43.6366 & 4.0178 & 40.5482 & 46.7250 \\
\hline
\end{tabular}

thero $=100$ loc $=$ sur

Analysls Variable : predicted ppn Predicted

Analysis Vartable ; predicted
Value of conc

\begin{tabular}{|l|l|l|}
\hline & Lower 95\% & Upper $95 \%$ \\
\hline
\end{tabular}

Mean Std Dev $C L$ for Mean CL for Mean

\begin{tabular}{lllll}
\hline 92.2972 & 9.2516 & 86.4190 & 98.1753
\end{tabular}

thero $=100$ loc $=$ sus

Analysis Variable : predicted_ppm Predicted

Value of conc

\begin{tabular}{llll}
\hline & Lower $95 \%$ & Upper 95\% \\
\hline
\end{tabular}

Mean Std Dev CL for Mean CL. for Mean

$89.1978 \quad 4.2148 \quad 85.9580 \quad 92.4375$

Performed by K. Weber SAS version 9.3 09:49 23APR14 
Mean treatment concentration by treatment group and sampling location through the 6 h exposure

The MEANS Procedure

thero $=50$ loc $=\mathrm{sur}$

Analysis Variable : precicted_ppm Predicted Value of cone

\begin{tabular}{c:ccc}
\hline & Lower $95 \%$ & Upper $95 \%$ \\
\hline
\end{tabular}

Mean Std Dov CL for Mean CL for Moan

$\begin{array}{llll}47.2784 & 5.2315 & 43.2571 & 51.2997\end{array}$

thero $=50$ loc $=$ sus

Analysis Varlable : predicted ppm Predicted

Value of canc

Lower $95 \%$
Upper $95 \%$

$\begin{array}{lllll}45.1088 & 4.1531 & 40.7504 & 19.4672\end{array}$

thero $=100$ loc $=$ sur

Analysis Varlable : predicted ppm Predictod

Value of cone
TLower $95 \%$ Upper $95 \%$

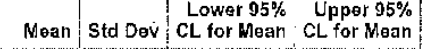

\begin{tabular}{|l|lll|}
95.3191 & 8.7240 & 88.6132 & 102.0
\end{tabular}

thero $=100$ locesus

Analysis Variable : predicted_ppm Predicted

\begin{tabular}{l} 
Value of cone \\
\hline Lower $95 \%$ Upper $95 \%$
\end{tabular}

Mean Std Dev CL for Mean CL for Mean

$\begin{array}{llll}91.4836 & 3.1005 & 88.2298 & 94.7373\end{array}$

Performed by K. Weber SAS vers'on 9.3 09:49 23ACR14 
Mean treatment concentration for each treatment group for each sampling time

The MEANS Procedure

therc $=50$ loc $=$ sur tlmes=1

Analysis Variable : predlcted ppm Prodlctod

Value of conc

[Lower $95 \%$ Upper $95 \%$

Mean Stcl Dev CL for Mean CL for Mean

$\begin{array}{lllll}52.3149 & 2.6402 & 45.7563 & 58.8735\end{array}$

thero $=50$ loc $=$ sur tlme $=3$

Analysis Varlable : predicted_ppm Predicted Value of conc
A

Mean Std Dev Lower 95\% Upper 96\%

\begin{tabular}{|l|r|r|r|}
\hline 48.1307 & 2.8184 & 41.1295 & 56.1319 \\
\hline
\end{tabular}

thero $=50$ loc $=s$ ur time $=6$

Analysls Varlable : predicted_ppm Predlcted

Value of conc
a

\begin{tabular}{l|l|l} 
Mean Std Dev & Lower $95 \%$ & Upper $95 \%$ \\
CL for Mean & CL for Mean
\end{tabular}

$\begin{array}{llll}41.3895 & 1.8451 & 36.8061 & 45.9729\end{array}$

thero $=50$ loc $=$ sur time $=9$

Analysis Varlable : predicted_ppm Predicted Value of conc

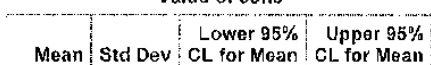

$\begin{array}{lllll}38.8325 & 1.6105 & 34.8318 & 42.8332\end{array}$

thero $=50$ locmsur timc $=12$

Analysis Variable : predicted ppm Predicted

Analysis Variabla : predicted_ppm Predicted
Value ol conc

Value ol cone

Sta Lower 95\%

$\begin{array}{llll}38.6000 & 1.3947 & 35.1353 ! & 42.0647\end{array}$

thero $=50$ loc $=$ sus time $=3$

Analysis Variable : predlcted_ppm Predictad

Analysis Variable : predicted
Value of conc

Lower $95 \%$ Upper $\$ 5 \%$

Nean Std Dev CL for Mean CL for Mean

$\begin{array}{llll}48.5956 & 2.4491 & 42.5118 & 54.6794\end{array}$

thero $=50$ loc $=$ sus $\mathrm{tlme}=6$

Analysis Variable : predlcted_ppm Predicted

Value of conc

file://C:/Users/klweber/sashtml.htm 
\begin{tabular}{|c|c|c|c|} 
Mean & Std Dev & $\begin{array}{r}\text { Lower } 95 \% \\
\text { CL for Mean }\end{array}$ & $\begin{array}{r}\text { Upper 95\% } \\
\text { GL for Mean }\end{array}$ \\
\hline 41.6220 & 0.8052 & 39.6216 & 43.6223 \\
\hline
\end{tabular}

thoro $=50$ loc $=$ sus thino $=9$

Analysis Varlable : predicted_ppm Predicted Value of conc

\begin{tabular}{lll|l|l|l|l|}
\hline & Lower 95\% & Upper $95 \%$ \\
\hline
\end{tabular}

Mean Std Dev \begin{tabular}{|l|l} 
Lower $95 \%$ & Upper $95 \%$ \\
CL Mean & CL for Mean
\end{tabular}

\begin{tabular}{llll}
40.6921 & 1.3947 & 37.2274 & 44.1568 \\
\hline
\end{tabular}

therow50 locmsus t|me=12

Analys ls Variable : predicked ppm Predlcted

Value of conc

Lower 95\% Upper 95\%

Mean Std Dev CL for Mean Cl. for Mean

$\begin{array}{llll}37.9027 & 1.8451 & 33.3193 & 42.4860\end{array}$

thero $=100$ locesur time=1

Analysls Varlable : predicted_ppm Predlcted

\begin{tabular}{|c|c|c|c|}
\multicolumn{3}{|c|}{ Value of cone } \\
Mean Std Dev & $\begin{array}{r}\text { Lower } 95 \% \\
\text { CL for Mean }\end{array}$ & $\begin{array}{c}\text { Upper } 95 \% \\
\text { CL for Mean }\end{array}$ \\
\hline 105.8 & 2.2417 & 100.2 & 111.3
\end{tabular}

thero $=100$ locesur time

Analysis Variable : predicted_ppm Predicted Value of conc

Lower $95 \%$ Upper $95 \%$

Mean Std Dev CL for Maan CL for Mean

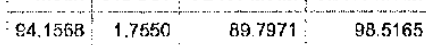

ther $0=100$ loc $=$ sur time $=0$

Analysis Varlable : predicted_ppm Predicted Value of cone

\begin{tabular}{llll}
\hline Lower $95 \%$ & Upper $95 \%$ \\
\hline
\end{tabular} Mean Stc Dev CL. for Mean CL for Mean

$86,0209: 0.6974 \quad 84.2885 \quad 87.7532$

thero $=100$ loc $=$ sur time $=9$

Analysis Variable : predicted_ppm Pradicted Value of conc

Lower $95 \%$ Upper 95\%

Mean Std Dev CL for Mean CL for Mean

$\begin{array}{lllll}83.2314 & 1.3947 & 79.7667 & 36.6961\end{array}$

thero $=100$ loc $=$ sur tline $=12$

Analysis Varlable : predicted_ppm Predicted

file://C $/$ Users/klweber/sashtml.htm

\begin{abstract}
Value of cone
\end{abstract}
AEH:RZPSEUDON 


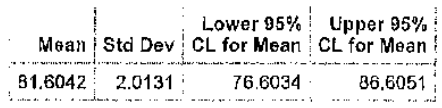

thero $=100$ loc $=$ sus tIme $=3$

Analysis Variable : predlcted_ppm Predicted

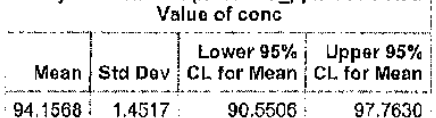

$94.1568: 1.4517: 90.5506: 97.7630$

thero $=100$ loc $=$ sus tlme $=6$

Analysis Variable : predicted_ppm Predlcted Value of conc

\begin{tabular}{ll|l|l|l|}
\hline & Lower $95 \%$ & Upper $95 \%$ \\
\hline
\end{tabular}

Mean Std Dev CL for Mean CL for Mean

$\begin{array}{llll}88.8103 & 0.6974 & 87.0780 & 90,5427\end{array}$

thero $=100$ loc $=$ sus time $=9$

Analysls Varlable : predlcted_ppm Predlcted Value of conc

\begin{tabular}{|c|c|c|c|}
\hline \multicolumn{4}{|c|}{ Value of conc } \\
\hline Mean & Std Dev & $\begin{array}{l}\text { Lower } 95 \% \\
\text { CL for Mean }\end{array}$ & $\begin{array}{l}\text { Upper } 95 \% \\
\text { CL for Moan }\end{array}$ \\
\hline 84.6261 & 0 & & \\
\hline
\end{tabular}

thero $=100$ loc $=$ sus time $=12$

Analysls Variable : predicted ppm Predicted Value of cone

\begin{tabular}{l|l|l|}
\hline & Lower $95 \%$ & Upper $95 \%$ \\
\hline
\end{tabular}

\begin{tabular}{l|l|l|l|} 
Mean Std Dev CL for Mean & CL for Mean \\
\hline
\end{tabular}

\begin{tabular}{l|l|l|l|l|}
\hline 81.1393 & 2.0921 & 75.9423 & 86.3364 \\
\hline
\end{tabular}

Periormed by K. Weber SAS verslon 9.3 09:49 23APR 14

file://C:/Users/klweber/sashtml.htm 
AEV A2PSEUDG

\begin{abstract}
The MEANS Procedure
thero $=25$

Analysis Varialsle : predlcted_ppm Predicted

Value of conc

Lower 95\% Upper 95\%

Mean Std Dev CL for Mean CL for Mean

$20.8172 ; 0.4931 ; 16.3868: 25.2477$
\end{abstract}

Mean concentration for standard checks for all sampling times

thero $=50$

Analysis Varlable : pred/cted_ppm Predlcted Value of conc

Mean Std Dev $\left[\begin{array}{rr}\text { Lower } 95 \% & \text { Upper } 95 \% \\ \text { CL for Mean } & \text { CL. for Mean }\end{array}\right.$

\begin{tabular}{l|l|l|l|}
\hline 46.6197 & 2.4656 & 24.4676 & 68.7719
\end{tabular}

thero $=100$

Analysls Varlable : predlcted_ppm Predicted

Analysis Varlable : predlcted_pph
Value of conc

Lower 95\% Upper 95\%

\begin{tabular}{|l|l|l|l|} 
Mean & Std Dev & CL for Mean & $\mathrm{CL}$ for Mean \\
\hline
\end{tabular}

\begin{tabular}{l|l|l|l|}
\hline 92.8458 & 1.4793 & 79.3545 & 105.9
\end{tabular}

thero $=200$

Analysis Varlable : predlcted_pprn Predicted

Analysis Varlable : predicted
Value of conc

\begin{tabular}{l|l|l|l|}
\hline & Lower $95 \%$ & Upper 95\%
\end{tabular}

$\begin{array}{lllll}\text { Mean Std Dev CL for Meall CL for Mean } & \end{array}$

$\begin{array}{llll}183.3 & 1.1793 & 170.0 & 196.6\end{array}$

Performed by K. Weber SAS version 9.3 09:49 23APR14 $\mathrm{z}_{3} \mathrm{ff}^{2 \mathrm{P}^{4}}$
FF \#

Item No. 4

$\mathrm{Pg} 15$ of 15

file://C:/Users/klweber/sashtml.htm

4/23/2014

Page 348 of 519 


\begin{tabular}{|c|c|c|c|}
\hline \multirow{5}{*}{$\begin{array}{l}\text { Study Number: AEH-12-PSEUDO-04 } \\
\text { Electronic Lab Notebook (pages 12- 23] } \\
\text { Data Source: Flle Folder: 11c } \\
\qquad \text { Forms: "Samp'e Absorbance Readings" Data Sheet. }\end{array}$} & Action & Date & Initials \\
\hline & Created...... & 6-Feb-14 & T.STS \\
\hline & Revised...... & 12 -Feb-14 & T.5 Tos \\
\hline & Revlewed... & $12 F^{\prime} E B H$ & $\pi^{1 / 5}$ \\
\hline & Certifled...... & $2 / / 1 / / 4$ & $31-$ \\
\hline
\end{tabular}

Spectroplotometric Data

Test Aitcle: Zequanox ${ }^{\circ}$ (MBI-401 SDP)

Test Article Lot H: 401P12163C aad 401P12164C MlX

Test Arute Lotichor

Test Locatlon; Lake Shawano, Shawano, W

Test Locatlon: Lake Shawano,

Data Explanation:

1) The absorbance of triplicate samples of $25,50,100$, and $200 \mathrm{mg} / \mathrm{L}$ dilutlons of a $2,000 \mathrm{mg} / \mathrm{L}$ active Ingred.ent (A.I.) stock prepared from Analytical stock H1 wer

measured to prepare a standard curve.

2) Standard checks were performed at 6,9 , anc 12 hours by comparing the $25,50,100$, and $200 \mathrm{mg} / \mathrm{L}$ (A.J.) dilutlons to the linear curve

3) Lata codes used within SAS

tank = Tank ID (1 through 9)

thero $=$ Theoret'cal or target concentration (mg/

time $=$ sample Time $(0,1,3,6,9$, and 12 h after treatment $)$

loc $=$ Sample Location

sus = Suspended Sanple (sampled $\sim 15 \mathrm{~cm}$ from bottom of tank)

sur $=$ Surface Sample

abs = measured absorbance of sample

conc $=$ concentratlon $([\mathrm{mg} / \mathrm{L})$, only used for standards used for regression)

4) information that Is not relevant to a sample (i.e, tonk ID for standards) cr that wi I be calculated by SAS (i.e., predlcted conce ztration for standarc checks and samples) is denoted by a"." in the SAS Input and output files

Data Ana'vis:

1) A linear regression was completed in SAS using the absorbance values obtained from the spectroptctometer of 3 repllcate fillutions of 25,50, $10 \mathrm{C}$ and $200 \mathrm{mg} / \mathrm{h}$

Zequanox

2) S:anda id checks and treatine:il sample concenti atiuns were predicted in SAS by comparing the observed absorbances with tha llnear regression.

3) The following mean treatment concentraticns were determined in SAS:

3a) Mean (standard deviation) concentration by tank and location for all sampling timgs

3b) Mean (standard devlatlon) concentration by treatment group and locallon for all samp ing times

3c) Mean (standard deviatlon) concentration byereatmant eroup and sampling tmes for both locetions

3d) Mean (s:andard deviation) concentratons for $25,50,1 \mathrm{CO}_{\text {, and }} 200 \mathrm{me} / \mathrm{L}$ (A.I.) dllutlons for all samplling times

File Names:

Spectrophotometric Data for SAS input

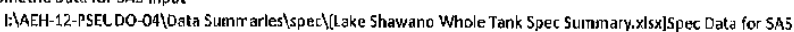

SAS Program/Code

I:AEH-12-PSEUDO-01|SAS-Spectshawano whole water program file

SASLOg

IVWEH-12-PSEUDO-04\SAS-Specłshawano whole water log flle

SAS Output

I:AEH 12 PSEUDO-04\SAS-Specłshawano whole water results file

Data Anomalies and Deviations:

1) One exposure tank of a different concentration and sample location was sampled In triplicate to evaluate varlability of spectrophotometcr during each sampling tlme. The mean absorbance of the trlplicate samplas was mported into SAS for use In the analysis.

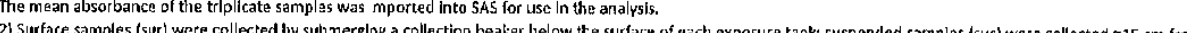
2) Surface samples (sur) wer. colle the expcsure tank bottom for all sampling tiries.

3) Some mean absorbances for' triplicate samples may be recorded incor rectly on "Sample Absorbance Readings" data forms as proper signlficant figure rules may not $r a v e$ been observed. Additionally, cor centrattans recorded on "Sample Absorbance Readings" data forms we'e not used in the analysis as the inltial lnear regression equatior that yas used for these calculatlons was derlved using rounded absorbance values in Excel. All absarbances and concentrations used in SAS calculations and reported within Spectrophotonetric Data Summary have been corrected.

File Folder: He

$$
\text { item Number } \frac{1}{4}
$$




\begin{tabular}{|c|c|c|c|c|c|}
\hline tank & thero & time & $\mathrm{loc}$ & abs & conc \\
\hline . & 25 & 0 & . & 0.034 & 25 \\
\hline . & 50 & 0 & . & 0.069 & 50 \\
\hline . & 100 & 0 & . & 0.135 & 100 \\
\hline . & 200 & 0 & . & 0.263 & 200 \\
\hline . & 25 & 0 & . & 0.034 & 25 \\
\hline . & 50 & 0 & . & 0.069 & 50 \\
\hline . & 100 & 0 & . & 0.135 & 100 \\
\hline . & 200 & 0 & . & 0.263 & 200 \\
\hline . & 25 & 0 & . & 0.034 & 25 \\
\hline . & 50 & 0 & . & 0.069 & 50 \\
\hline . & 100 & 0 & . & 0.137 & 100 \\
\hline . & 200 & 0 & . & 0.263 & 200 \\
\hline . & 25 & 6 & . & 0.033 & . \\
\hline . & 50 & 6 & . & 0.068 & . \\
\hline . & 100 & 6 & $\cdot$ & 0.13 & . \\
\hline . & 200 & 6 & . & 0.247 & . \\
\hline . & 25 & 9 & $\cdot$ & 0.033 & . \\
\hline . & 50 & 9 & · & 0.063 & . \\
\hline ' & 100 & 9 & . & 0.127 & . \\
\hline . & 200 & 9 & . & 0.248 & $\cdot$ \\
\hline ' & 25 & 12 & . & 0.035 & $\cdot$ \\
\hline . & 50 & 12 & . & 0.068 & . \\
\hline . & 100 & 12 & $\cdot$ & 0.129 & . \\
\hline$\cdot$ & 200 & 12 & , & 0.249 & $\cdot$ \\
\hline 1 & 0 & 1 & sur & 0.000 & . \\
\hline 4 & 0 & 1 & sur & 0.001 & . \\
\hline 7 & 0 & 1 & sur & 0.001 & . \\
\hline 2 & 50 & 1 & sur & 0.061 & $\cdot$ \\
\hline 5 & 50 & 1 & sur & 0.072 & . \\
\hline 8 & 50 & 1 & sur & 0.067 & . \\
\hline 3 & 100 & 1 & sur & 0.144 & · \\
\hline 6 & 100 & 1 & sur & 0.150 & . \\
\hline 9 & 100 & 1 & sur & 0.132 & . \\
\hline 1 & 0 & 1 & sus & 0.001 & . \\
\hline 4 & 0 & 1 & sus & 0.000 & ' \\
\hline 7 & 0 & 1 & sus & 0.000 & . \\
\hline 2 & 50 & 1 & sus & 0.064 & . \\
\hline 5 & 50 & 1 & sus & 0.073 & . \\
\hline 8 & 50 & 1 & sus & 0.065 & . \\
\hline 3 & 100 & 1 & sus & 0.144 & \\
\hline 6 & 100 & 1 & SUS & 0.149 & \\
\hline 9 & 100 & 1 & SUS & 0.133 & \\
\hline 1 & 0 & 3 & sur & 0.001 & \\
\hline 4 & 0 & 3 & sur & $\begin{array}{l}0.003 \\
0.000\end{array}$ & \\
\hline $\begin{array}{l}7 \\
2\end{array}$ & $\begin{array}{c}0 \\
50\end{array}$ & $\begin{array}{l}3 \\
3\end{array}$ & sur & $\begin{array}{l}0.000 \\
0.059\end{array}$ & . \\
\hline
\end{tabular}

AEH-12-PSEUDO-04

Page 2 of 4 


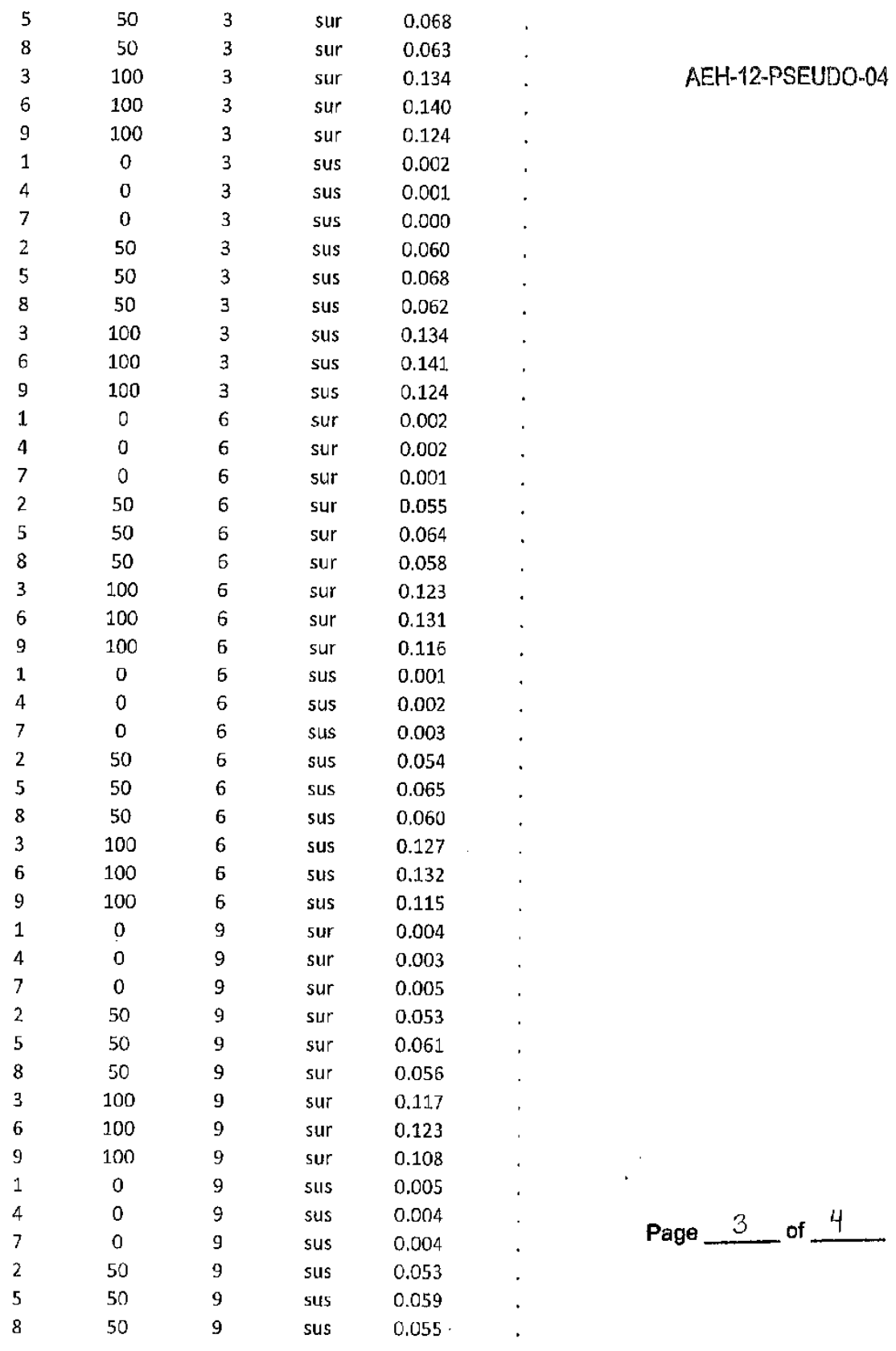




$\begin{array}{cccccc}3 & 100 & 9 & \text { sus } & 0.119 & \text {. } \\ 6 & 100 & 9 & \text { sus } & 0.125 & \text {. } \\ 9 & 100 & 9 & \text { sus } & 0.108 & \text {. } \\ 1 & 0 & 12 & \text { sur } & 0.005 & \text {. } \\ 4 & 0 & 12 & \text { sur } & 0.004 & \text {. } \\ 7 & 0 & 12 & \text { sur } & 0.004 & \text {. } \\ 2 & 50 & 12 & \text { sur } & 0.051 & \text {. } \\ 5 & 50 & 12 & \text { sur } & 0.059 & \text {. } \\ 8 & 50 & 12 & \text { sur } & 0.056 & \text {. } \\ 3 & 100 & 12 & \text { sur } & 0.113 & \text {. } \\ 6 & 100 & 12 & \text { sur } & 0.122 & . \\ 9 & 100 & 12 & \text { sur } & 0.105 & \text {. } \\ 1 & 0 & 12 & \text { sus } & 0.006 & . \\ 4 & 0 & 12 & \text { sus } & 0.004 & \text {. } \\ 7 & 0 & 12 & \text { sus } & 0.005 & . \\ 2 & 50 & 12 & \text { sus } & 0.053 & . \\ 5 & 50 & 12 & \text { sus } & 0.059 & . \\ 8 & 50 & 12 & \text { sus } & 0.054 & . \\ 3 & 100 & 12 & \text { sus } & 0.115 & . \\ 6 & 100 & 12 & \text { sus } & 0.120 & . \\ 9 & 100 & 12 & \text { sus } & 0.106 & .\end{array}$

AEH-12-PSEUDO-04

File Folder: $11 \mathrm{c}$

Item Number: 1

Page 4 of 4 
ods html close; $/ *$ close previous */;

ods html; $l^{*}$ open new */;

ods graphics on;

DM 'LOG; Clear; OUTPUT; CleAR;'; * CleAR log and OUTPUT;

FOOTNOTE1 'Performed by J. Luoma SAS version ' \&SYSVER \&SYSTIME \&SYSDATE;

options Is $=97$ ps=54 formdlim=' -1 pageno $=1$ nocenter nodate nosource2;

title1 $h=1$ 'Standard Curve Linear Regression and sample concentrations';

title2 $h=1$ 'Study \# AEH-12-PSUEDO-04';

ti.tle3 $\mathrm{h}=1$ 'Shawano Lake-whole water treatment';

title $4 h_{1}=1$ 'SAS $v, 9.3$ Analysis completion date: 26MAR2014 Analysis prepart

1******************************************************************************

* SAS ver 9.3 Analysis prepared by: JAL Si- Page — of

* Analysis completion date: 26MAR2014

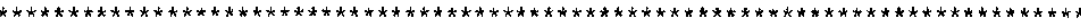

data Zeq; set shawano. shawanowhole;

run;

proc sort;

by tank time loc; run;

run;

proc gplot data $=$ zeq;

plot abs * conc;

run;

proc reg data $=z e q$;

model conc $=$ abs $/$ edf;

output out=output_out $p=$ prodicted_ppm;

run;

proc sort;

by time tank loc;

proc print data=output_out;

run;

data zeq2; set output_out;

if $\operatorname{tank}=$ ". " then doleto;

$i$ tank = " "then delete;

if $100="$ " " then delete;

if time = "O" then delete;

run;

proc sort;

by tank $10 \mathrm{c}$;

run;

$1 * *$

FF \# $11 \mathrm{C}$

Item No. 2

$\mathrm{Pg} 1 \overline{\text { of } 4}$ 


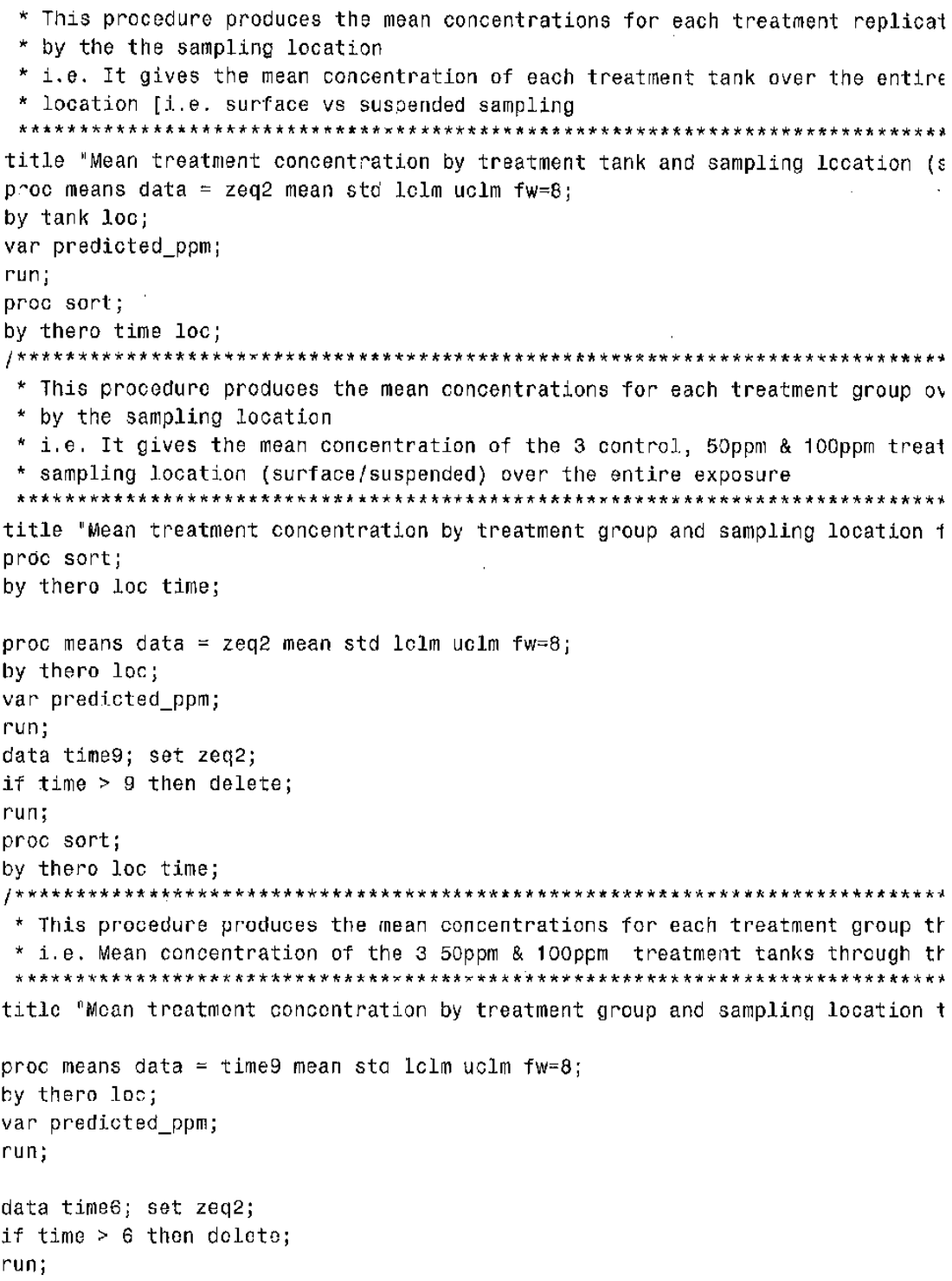

Page 2 of 4 
proo sort;

by thero loo tine;

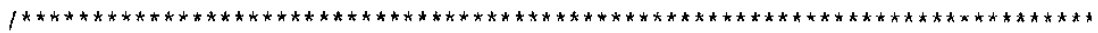

* This procedure produces the mean concentrations for each treatment group tr * i.e. Mean concentration of the $350 \mathrm{ppm} \& 100 \mathrm{ppm}$ treatment tanks through tr $* * * * * * * * * * * * * * * * * * * * * * * * * * * * * * * * * * * * * * * * * * * * * * * * * * * * * * * * * * * * * * * * * * * * * * * * * * * * *$ title "Mean treatment concentration by treatment group and sampling location $t$

proc means data - time 6 mean std lcln uclm fw $=8$;

by thero loc;

var predicted_ppm;

run;

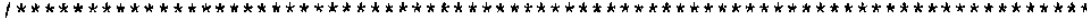

* This procedure produces the mean concentrations for each treatment group by * 1.e. It gives the mean conc. of the 3 control, 50ppm \& 100ppm treatment tar

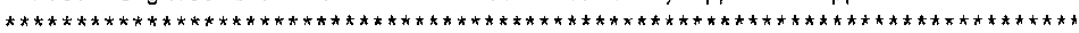
title "Mean treatment concentration for each treatment group for each samplinç proc sort;

by thero loc time;

proc means data $=2 \mathrm{eq} 2$ mean std $1 \mathrm{cln} u \mathrm{clm} \quad \mathrm{f}=8$;

by thero loc time;

var predicted_ppm;

run;

data zeq3; set output_out;

if conc > 1 then delete;

if tank > 0.5 then delete;

if thero $=$ ". " then delete;

run;

proc sort;

by thero;

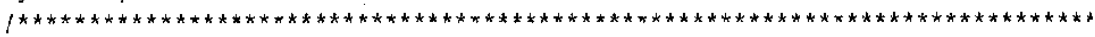

* This procedure produces the mean corcentrations for the standard checks for

* i.e. It gives the mean conc. of the 50ppm \& 100ppm standard checks at 6, 9 $* * * * * * * * * * * * * * * * * * * * * * * * * * * * * * * * \pi * * * * * * * * * * * * * * * * * * * * * * * * * \pi * * * * * * * * * * * * * * * * * *$ title "Mean concentration for standard checks for all sampling times '; proc means data $=z 0 q 3$ nean std $1 \mathrm{clm}$ uclm $\mathrm{fw}^{-8} \mathrm{~g}$;

by thero;

var predicted_ppin;

Page 3 of 4 


$$
\begin{aligned}
& 3.26-14 \\
& 51-
\end{aligned}
$$

run;

quit;

AEH-12-PSEUDO-04

FF \# $1 / \mathrm{C}$

Item No. $\frac{2}{4}$

Page 356 of 519 
DM LOG; ClEAR; OUTPUT; CLEAR; '; * ClEAR LOG AND OUTPUT;

FOOTNOTE1 'Performed by J. LuOMa SAS version ' \&SYSVER \&SYSTIME \&SYSDATE ! ;

WARAING: The FOOTNOTE statement is anbiguous due to invalid options or unquoted text.

options $1 \mathrm{~s}=97 \mathrm{ps}=54$ formdlin='. ' pageno $=1$ nocenter nodate nosource2;

title1 $h=1$ 'Stancard Curve Linear Regression and sample concentrations';

title2 $h=1$ 'Study \# AEH-12-PSUED0-04';

title3 $h=1$ 'Shawano Lake-whole water treatment';

title4 $h=1$ 'SAS $v, 9.3$ Analysis completion date: 26 MAR2014 Analysis $\mathrm{pr}$

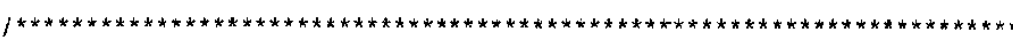

* SAs ver 9.3 Analysis prepared by: JAL Page _ of _

* Analysis completion date: 26Mar2014

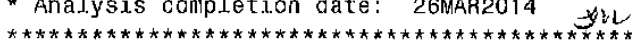

data Zeq; set shawano.shawanowhole;

run;

NOTE: There were 115 observations read from the data set SHAWANO.SHAWANOWHOLE. NOTE: The data set WORK.ZEQ has 115 observations and 6 variables.

NOTE: DATA statement used (Total process time):

real time $\quad 0.03$ seconds

cpu time $\quad 0.03$ seconds

22 proc sort;

23 by tank time loc; run;

NOTE: There were 115 observations read from the data set WORK.ZEQ.

NOTE: The data set NORK.ZEQ has 115 observations and 6 variables.

NOTE: PROCEDURE SORT used (Total orocess time):

real time $\quad 0.0^{*}$. seconds

cpu time $\quad 0.01$ seconds

24 run;

proc gpiot data $=$ zeq;

plot abs * conc;

run;

NOTE: 103 observation(s) contained a MISSING value for the abs * conc request.

FF \# $\#$ IC

Item No. 3

$\mathrm{Pg} \ldots$ of 8 
NOTE: There were 115 observations read from the data set WORK.ZEQ.

NOTE: PROCEDURE GPLOT used (Total process time):
real time
0.65 seconds

cpu time

0.49 seconds

proc reg data $=z e q ;$

model conc $=$ abs $/$ edf;

output out=output_out $p=p$ redicted_ppm;

run;

NOTE: The data set WORK.OUTPUT_OUT has 115 observations and 7 variables.

NOTE: PROCEDURE REG used (Total process time):
real time
4.49 seconds
cpu time
0.63 seconds

33 proc sort;

34 by time tank loc;

VOTE: There were 115 observations read from the data set WORK.OUTPUT_OUT.

NOTE: The data set WORK.OUTPUT_OUT has 115 observations and 7 variables.

NOTE: PROCEDURE SORT used (TOtal process time):
real time
0.01 seconds
cpu time
0.01 seconds

35 proc print data=output_out;
36 run;

NOTE: There were 115 observations read from the data set WORK.OUTPUT_OUT. NOTE: PROCEDURE PRINT used (Total process time):
real time
0.15 seconds
cpu time
0.07 seconds

37 data zec2; set output_out;
38 if tank $="$ " " then delete;
39 if tank $="$ " then delete;
40 if $100="$ " then delete;
41 if time $=$ "0" then delete;
42 run;

Page 2 
NOTE: Character values have been converted to numeric values at the places gil (Line): (Column).

$41: 11$

NOTE: There were 115 observations read from the data set WORK.OUTPUT_OUT.

NOTE: The data set WORK.ZEQ2 has 90 observations and 7 variables.

NOTE: DATA statement used (Total process time):
real time
0.01 seconds

cpu time

0.01 seconds

proc sort;

by tank loc;

run;

NOTE: There were 90 observations read from the data set WORK.ZEQ2.

NOTE: The data set WORK.ZEQ2 has 90 observations and 7 variables.

NOTE: PROCEDURE SORT used (Total process time):

real time 0.01 seconds

cpu time $\quad 0.01$ seconds

$46 \quad / * * * * * * * * * * * * * * * * * * * * * * * * * * * * * * * * * * * * * * * * * * * * * * * * * * * * * * * * * * * * * * * * * * * * * * * * *$

46 ! $* * * * * * * * * * * * * *$

* This procedire produces the mean concentrations for each treatment ref 47 I sampling times *

48 * by the the sampling location

$48 \quad 1$

* i.e. It gives the mean corcentration of each treatment tank over the $\epsilon$ 49 ! tho sampling *

50 * location [i.e. surface vs suspended sampling

50 !

(

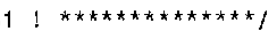

52 title "Mean treatment concentration by treatment tank and sampling locatj

52 I (surface/suspended) for all sampling times";

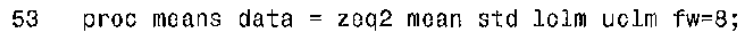

54 by tank $10 \mathrm{c}$;

55 var predicted_ppm;

56 run;

NOTE: There were 90 observations read from the data set WORK.ZEQ2.

NOTE: PROCELURE MEANS used (Total process time):
real time
0.15 seconds
cpu time
0.06 seconds

Page 3 of 8




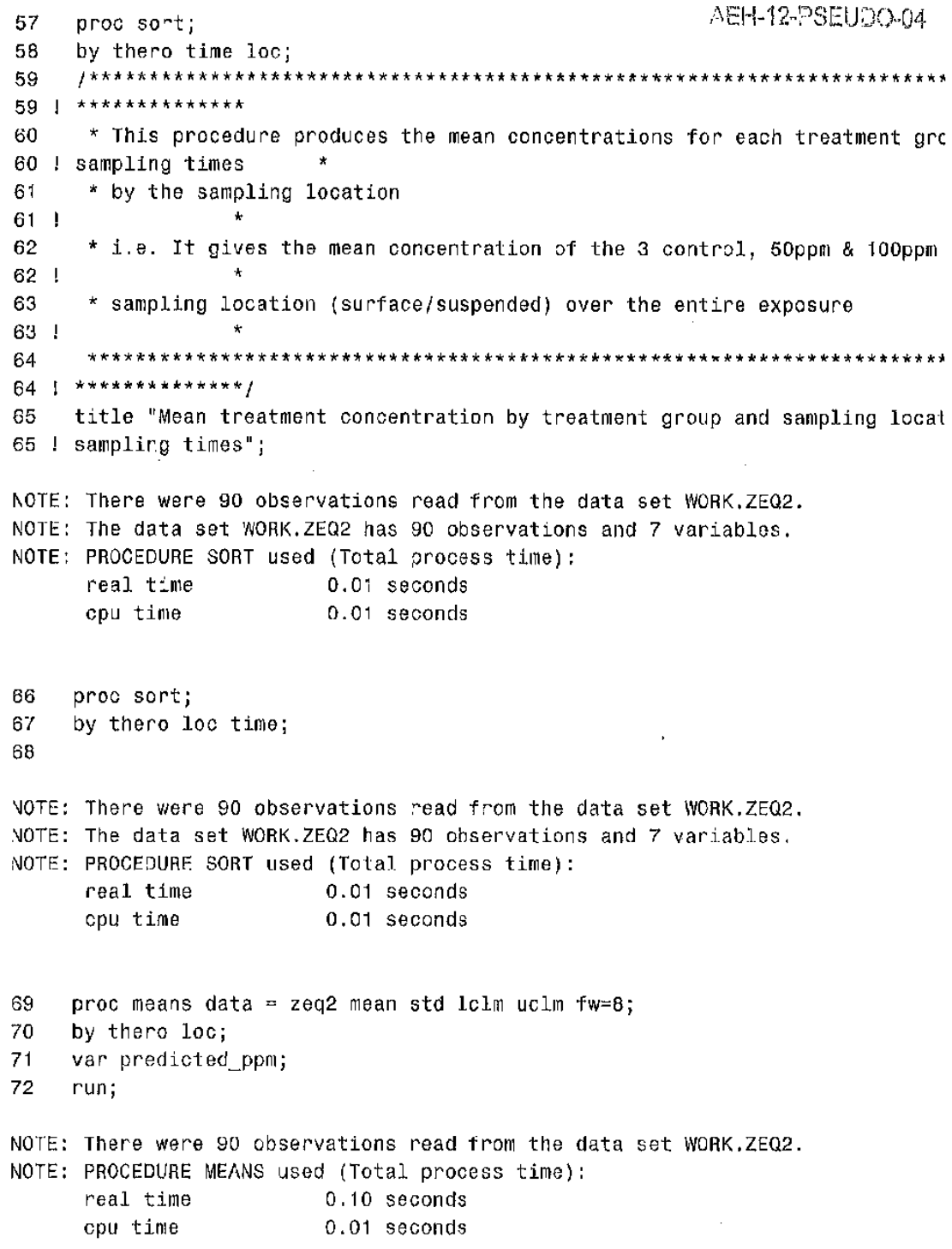

* This procedure produces the mean concentrations for each treatment grc sampling times *

NOTE: There were 90 observations read from the data set WOAK.ZEQ2.

NOTE: PROCEDURE MEANS used (Total process time):
real time
0.10 seconds
cpu tinse
0.01 seconds

Page 4 if of 8 
data time9; set zeq2;

if time $>9$ then delete;

run;

NOTE: There were 90 observations read from the data set WORK.ZEQ2

NOTE: The data set WORK. TIMEg has 72 observations and 7 variables.

NOTE: DATA statemert used (Total process time):

real time $\quad 0.01$ seconds

cpu time $\quad 0.01$ seconds

proc sort;

by thero loc time;

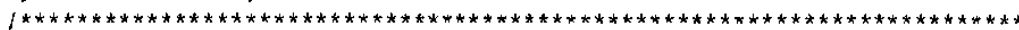

I $* * * * * * * * * * * * * * * * * * * * *$

* This procedure produces the mean concentrations for each treatnent grc sample location

* 1.e. Mean concentration of the $350 \mathrm{ppm} \& 100 \mathrm{ppm}$ treatment tanks throt by sample location *

$* * * * * * * * * * * * * * * * * * * * * * * * * * * * * * * * * * * * * * * * * * * * * * * * * * * * * * * * * * * * * * * * * * * * * * * * *$

$* * * * * * * * * * * * * * * * * * * * * *)$

title "Mean treatment concentration by treatment group and sampling locat

exposure" :

83

NOTE: There were 72 observatiors read from the data set WORK. TIME9

NOTE: The data set WORK, TIME9 has 72 observations and 7 variables.

NOTE: PROCEDURE SORT used (Total process time):
real time
0.01 seconds
cpu time
0.01 seconds

proc neans data $=$ times mean std 1 clm uclm $f_{w}=8$;

by thero 100 ;

var predicted ppin;

run;

NOTE: There were 72 observations read from the data set WORK.TIME9.

NOTE: PROCEDURE MEANS used (Total process time):

real time 0.10 seconds

cpu time $\quad 0.01$ seconds

88

89 data time6; set zeq2;

90 j.f time $>6$ then delete;

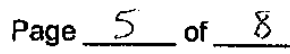


NOTE: There were 90 observations read from the data set WORK.ZEQ2.

NOTE: The data set WORK. TIME6 has 54 observations and 7 variables.

NOTE: DATA statement used (TotaI process time):

$\begin{array}{ll}\text { real time } & 0.00 \text { seconds } \\ \text { cpu tine } & 0.00 \text { seconds }\end{array}$

proc sort;

by thero loc time;

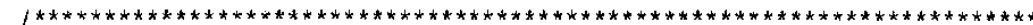

6 I $* * * * * * * * * * * * * * * * * * * * *$

* This procedure produces the mean concentrations for each treatment gre I sample location

* i.e. Mean concentratior of the $350 \mathrm{ppm} \& 100 \mathrm{ppm}$ treatment tanks throl 98 ! by samplo location *

$99 \quad * * * * * * * * * * * * * * * * * * * * * * * * * * * * * * * * * * * * * * * * * * * * * * * * * * * * * * * * * * * * * * * * * * * * * * * *$ $99|* * * * * * * * * * * * * * * * * * * * *|$

100 title "Mean treatment concentration by treatment group and sampling locat 1001 exposure";

101

NOTE: There were 54 observations read from the data set WORK.TIME6.

NOTE: The data set WORK.TIME6 has 54 obsorvations and 7 variables.

NOTE: PROCEDURE SORT used (Total process time):

real time $\quad 0.01$ seconds

cpu time $\quad 0.01$ seconds

102 proc neans data $=$ time6 mean std $1 \mathrm{clm}$ uclm $\mathrm{fw}=8$;

103 by thero loc:

104 var predicted_ppm;

105 run;

NOTE: There were 54 oDservations read from the data set WORK.TIME6.

NOTE: PROCEDURE MEANS used (Total process time):

real time 0.14 seconds

cpu time $\quad 0.03$ seconds

106

107

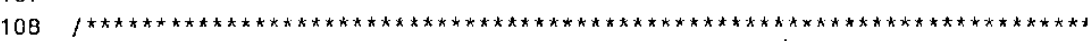

Page 6 of 8 


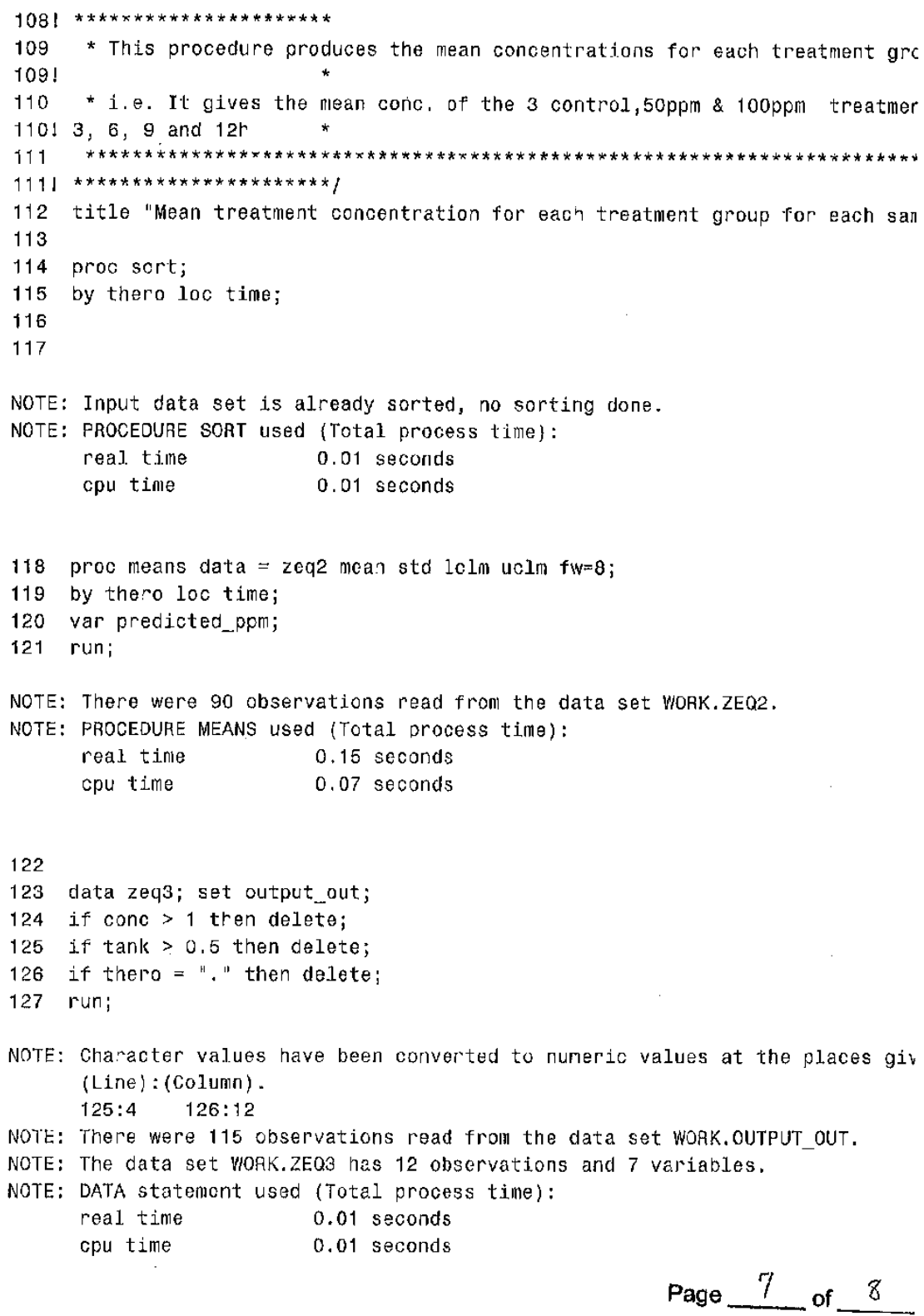

Page 7 of 8 

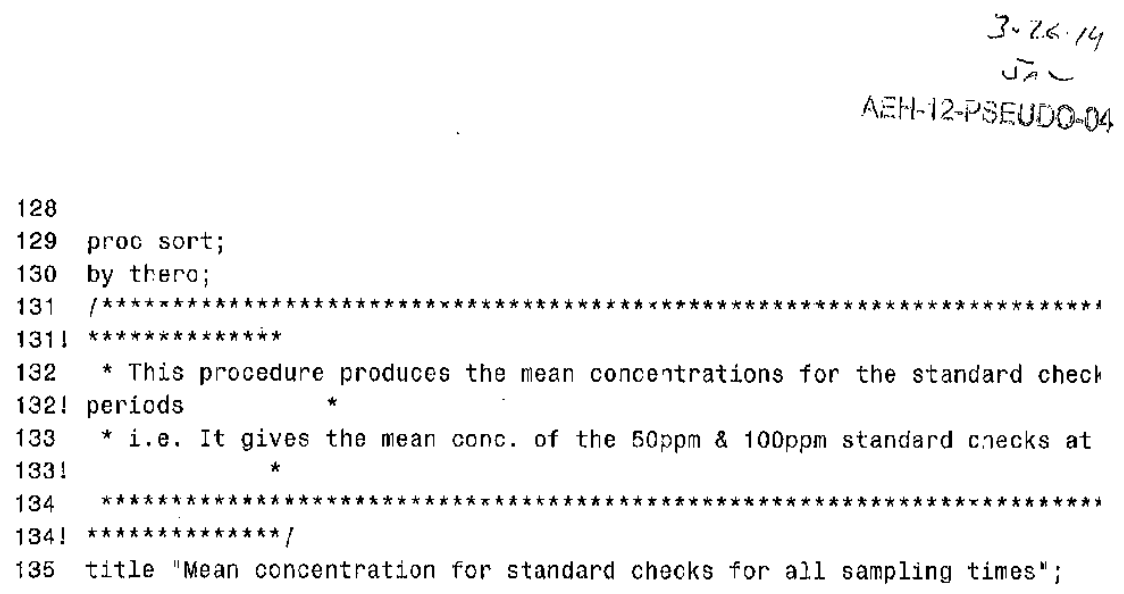

* i.e. It gives the mean conc. of the $50 \mathrm{ppm} \& 100 \mathrm{ppm}$ standard checks at

FF \# $/ 1 C$ 


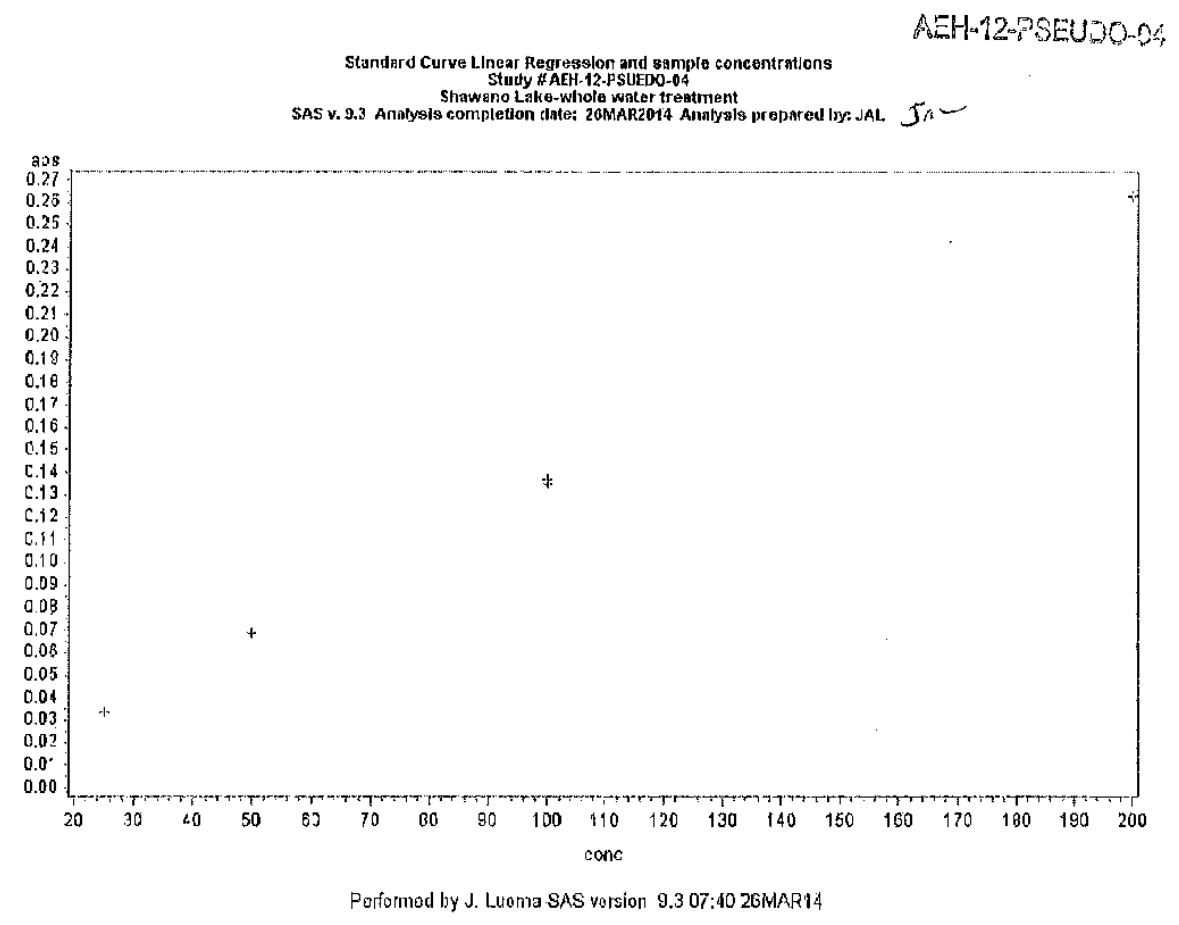

FF\# $11 \mathrm{C}$

Item No. $\frac{4}{\mathrm{Pg} L}$ 
Slandard Curvo Linour Ragroselon and anmplo concontratione

Sludy " AEF-12-PSUEDO-04

2OMAR2014 Aralyals proparod by: JAL

The REG Procedure

Model: MODEL-1

Dependent Varlable: conc conc

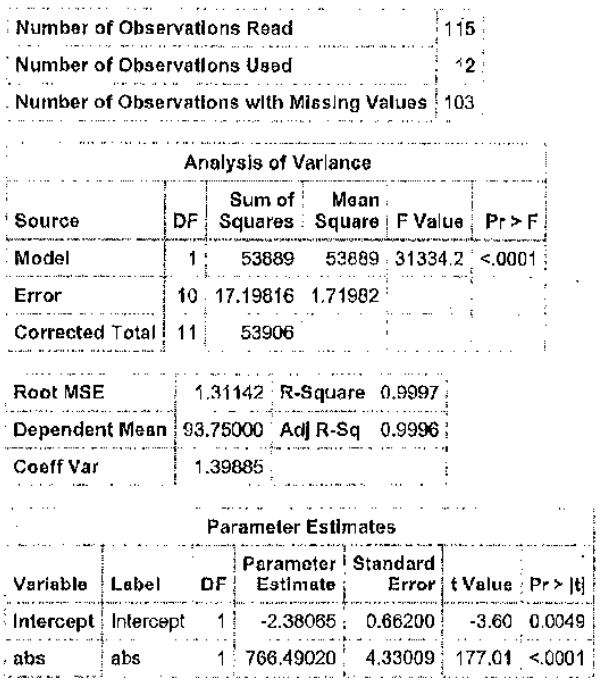

Performed by J. Luoma SAS vers on 9.3 07:40 26MAZ14 

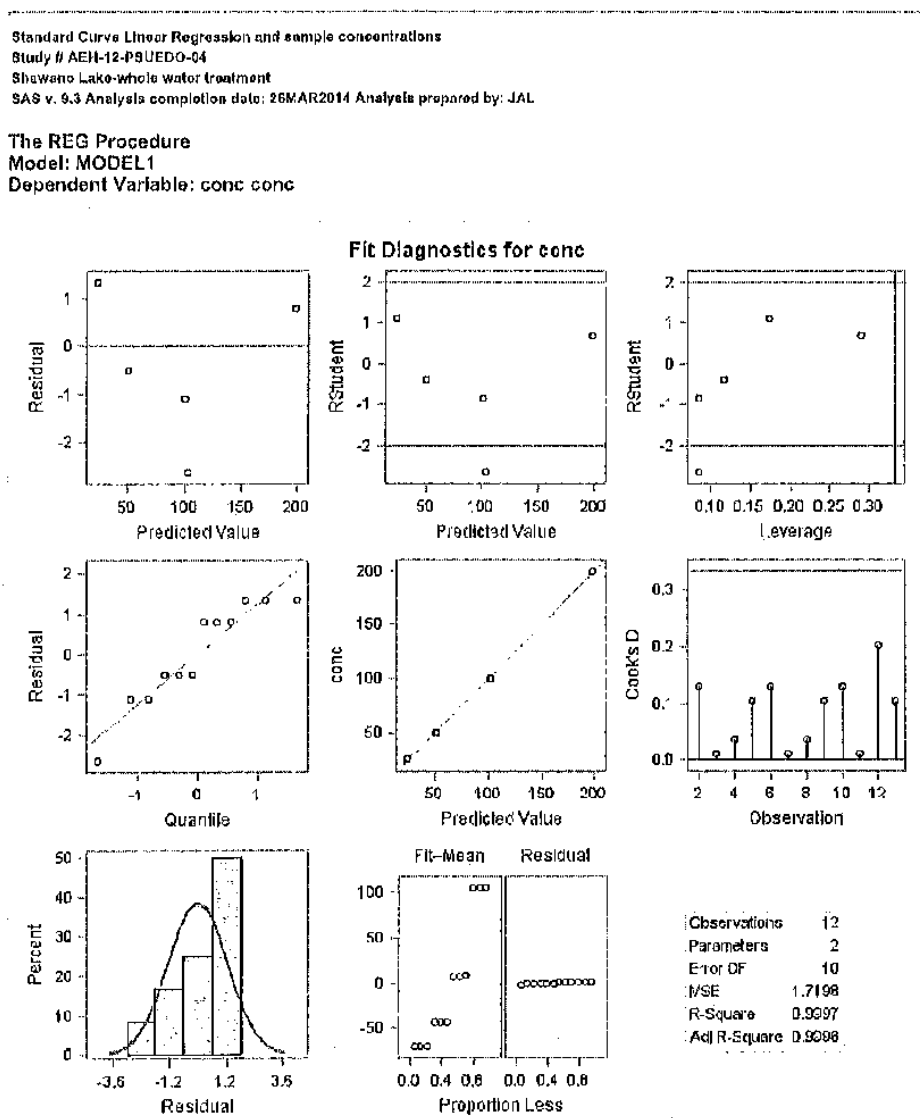

Proportion Less 

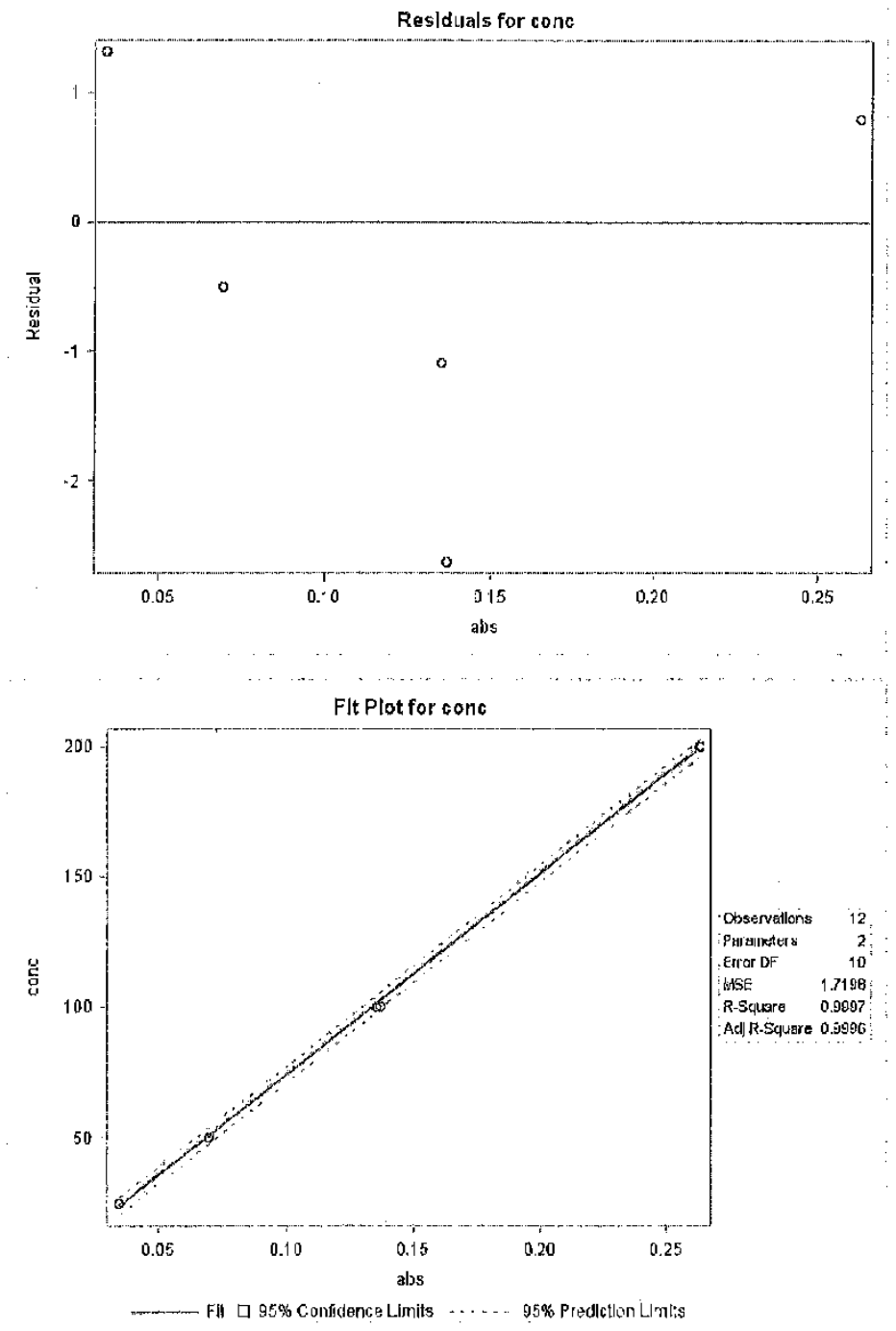

Performed by J. Luma SAS version 9.3 07:40 26MAR14 
5tandard Curve Llngar Regresslon and sample concentratious

8tudy HAEH-12-PSUEDO-04

SAS Y. 9.3 Analysis complotlon doto: 20 MAR2014 Analyulós proparod by: JAL

Obs tank thero time loc alss conc prodicted_ppm

$\begin{array}{cccccc}1 & & & & & \\ 2 & 25 & 0 & 0.034 & 25 & 23.680\end{array}$

$\begin{array}{cccccccc}2 & 50 & 0 & 0.068 & 50 & 50.507\end{array}$

$\begin{array}{llllllllllllll}4 & 100 & 0 & 0.135 & 100 & 101.096\end{array}$

$\begin{array}{lllllllll}5 . & 200 & 0 & 0.263 & 200 & 199.206\end{array}$

$\begin{array}{lllllllll}6 & 6 & 25 & 0 & 0.034 & 25 & 23.680\end{array}$

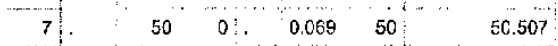

B. $100: 0.0 .135100: 101.096$

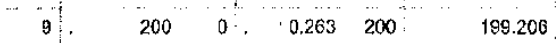

$\begin{array}{rllllll}10 & 25 & 0 & 0.034 & 25 & & 23.680\end{array}$

$11.50 \quad 0, \quad 0.06950 \quad 50.507$

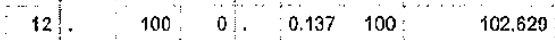

13. $\quad 200,0.0 .263 \quad 200 \quad 199.206$

1411001 sur $0.000 \quad-2.381$

151 : 0 1: sus $0.001 \quad-1.614$

\begin{tabular}{r|rrr|r|r|r|}
16 & 2 & 50 & 1 & sur & 0.061 & 44.375
\end{tabular}

$\begin{array}{lllll}172 & 50 & 1 \text { sus } 0.064 & 46.675\end{array}$

\begin{tabular}{l|l|l|l|l|l|l|l|l|l|l}
18 & 3 & 100 & 1 & sut & 0.144 & 107.994
\end{tabular}

$193: 100 \quad 1$ sus $0.144 \quad 107.994$

$2 0 \longdiv { 4 } \quad 0 \quad 1$ sur $0.001 \quad-1.614$

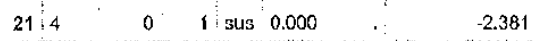

$\begin{array}{lllllll}22 & 5 & 50 & 1 \text { sur } 0.072 & & & \\ & & 1 & 52.807\end{array}$

$235 \quad 50 \quad 1$ sus $0.073 \quad$ : $\quad 53.573$

$24 \begin{array}{llllllllllll}6 & 100 & 1 & 1 & \text { sur } & 0.150 & 112.593\end{array}$

$\begin{array}{llllll}25 & 6 & 100 & 1 \text { sus } 0.149 & 111.826\end{array}$

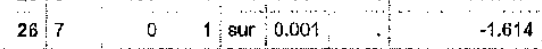

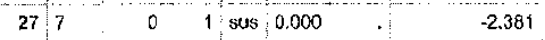

\begin{tabular}{l|lll:ll:l}
28 & 8 & 50 & 1 & sur & 0.067 & 48.974
\end{tabular}

\begin{tabular}{l|l|l|l|llll}
29 & 8 & 50 & 1 & sus & 0.065 & 4 & 4.441
\end{tabular}

$309160 \quad 1$ sur 0.132 . 98.796

$31 \mathrm{~g} \quad 160 \quad 1$ sus $0.133 \quad 99.563$

$\begin{array}{l:lll}32 & 1 & 0 & 3 \text { sur } 0.001\end{array}$

$\begin{array}{lllll}331 & \mathrm{C} & 3 \text { sus } 0.002 & \ldots & -0.848\end{array}$

$342 \quad 50 \quad 3$ sur $0.059 \quad$ ․ 42.842

$35: 2$ : $5 \mathrm{C} \quad 3$ sus $0.060 \quad \because \quad 43.609$

$36: 3 \quad 10 \mathrm{C}$ 3 sur $0.134 \quad 100.329$

$\begin{array}{lllll}37 & 3 & 100 \quad 3 \text { sus } 0.134 & 100.329\end{array}$

$384 \quad 0 \quad 3$ sur $0.303 \quad-0.081$

file://C:IUsersłklwebertsashtml.htm 


\begin{tabular}{|c|c|c|c|c|c|c|}
\hline 39 & 4 & 0 & 3 & sus & 0.001 & -1.814 \\
\hline 40 & 5 & 50 & 3 & sur : & 0.068 & 49.741 \\
\hline 41 & 5 & 50 . & 3 & sus & 0.068 & 49.741 \\
\hline 42 & 6 & 100 & 3 & sur : & 0.140 & 104.928 \\
\hline 43 & 6 & 100 & 3 & sus & $0 .: 41$ & 05.694 \\
\hline 44 & 7 & 0 & 3 & sur & 0.000 & $-2,381$ \\
\hline 45 & 7 & 0 & 3 & sus & 0.000 & -2.381 \\
\hline 46 & 8 & 60 & 3 & sur & 0.063 & 45.908 \\
\hline 47 & 8 & 50 & 3 & sus & 0.062 & 45.142 \\
\hline 48 & 9 & 100 & 3 & sur & 0.124 & 92.664 \\
\hline 49 & 8 & 100 & 3 & sus & 0.124 & 92,664 \\
\hline 50 & & 25 & 6 & 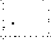 & 0033 & 22.914 \\
\hline 51 & 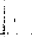 & 50 & 6 & . & .0 .068 & 49.741 \\
\hline 52 & . & 100 & 6 & & 0.130 & 97.263 \\
\hline 53 & . & 200 & 6 & : & 0.247 & 186.942 \\
\hline 54 & 1 & 0 & 6 & sur & 0.002 & -0.848 \\
\hline 55 & 1 & 0 & 6 & sus & 0.601 & -1.614 \\
\hline 56 & 2 & 50 & 6 & sur & 0.055 & 39.776 \\
\hline 57 & 2 & 50 & 6 & sus & 0.054 & 39.010 \\
\hline 58 & 3 & $100^{\circ}$ & 6 & sur & 0.123 & 91.898 \\
\hline 59 & 3 & 100 & 6 & sus & 0.127 & Q4.964 \\
\hline 60 & 4 & 0 & $\theta$ & sur & 0.002 & -0.848 \\
\hline 61 & 4 & 0 & 6 & sus & 0.002 & $-\mathrm{C} .848$ \\
\hline 62 & 5 & 50 & 6 & sur, & 0.064 & 46.675 \\
\hline 63 & 5 & 50 & ${ }^{3}$ & sus & 0.065 & 47.141 \\
\hline 84 & 6 & 100 & 6 & sur & 0.131 & 98.030 \\
\hline 65 & 6 & 100 & 6 & sus & 0.132 & 98.796 \\
\hline 66 & 7 & 0 & 6 & sur & 0.001 & -1.614 \\
\hline 67 & 7 & 0. & 6 & sus & 0.003 & -0.081 \\
\hline 63 & 8 & $5 \mathrm{C}$ & B & sur & 0.058 & 42.076 \\
\hline 69 & 8 & $5 \mathrm{C}$ & 6 & sus & 0,060 & 43.609 \\
\hline 70 & 9 & $10 \mathrm{C}$ & 6 & su & 0.116 & 86.532 \\
\hline 71 & 9 & 100 & 6 & sus & 0.115 & 85.766 \\
\hline 72 & & 25 & 9 & & 0.033 & 22.914 \\
\hline 73 & . & 50 & 9 & & 0.063 & 45.908 \\
\hline 74 & : & 100 & 9 & & 0.127 & 94.964 \\
\hline 75 & 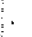 & 200 & 9 & & 0.248 & 187.709 \\
\hline 76 & 1 & 0 & 9. & sur: & 0.004 & 0.685 \\
\hline 77 & 1 & 0 & 9 & sus & 0.005 & 1.452 \\
\hline 70 & 2 & 50 & 9 & sur & 0.053 & 38.243 \\
\hline 79 & 2 & 50 & 9 & sus & 0.053 & 38.243 \\
\hline $\begin{array}{l}80 \\
81\end{array}$ & & 100 & 9 & sur: & 0.117 & 87.299 \\
\hline
\end{tabular}




\begin{tabular}{|c|c|c|c|c|c|c|}
\hline & 3 & 100 & & sus : & 0.119 & 88.832 \\
\hline 82 & 4 & 0 & 9 & sur & 0.003 & -0.081 \\
\hline 83 & 4 & 0 & 9 & sus & 0.004 & 0.685 \\
\hline 84 & 5 & 50 & 9 & sur : & 0.061 & 44.375 \\
\hline 85 & 5 & 50 & 9 & sus: & 0,059 & 42.842 \\
\hline 86 & 6 & 100 & 9 & sur & 0.123 & 91.898 \\
\hline 87 & 6 & 100 & 9 & sus? & 0.125 & 93.431 \\
\hline 89 & $?$ & 0. & 9 & sur & 0.005 & 1.452 \\
\hline 89 & 7 & 0 & 9 & sus! & 0.004 & 0.685 \\
\hline 90 & 8 & 50 & 9 & sur: & 0.056 & 40.543 \\
\hline 91 & 8 & 50 & 9 & sus : & 0.055 & 39.776 \\
\hline 92 & 9 & 100 & 9 & surt: & 0.108 & 80.400 \\
\hline 93 & 9 & 100 & g & sus: & 0.108 & 80.400 \\
\hline 94 & & 25 & 12 & & 0.035 & 24.447 \\
\hline 95 & & 50 & 12 & & 0.068 & 19.741 \\
\hline 96 & & $100:$ & 12. & & 0.129 & 96.497 \\
\hline 97 & & 200 & 12 & & 0.249 & 188.475 \\
\hline 98 & 1 & 0 & 12 & sur & 0.005 & 1.452 \\
\hline 99 & 1 & 0. & $12:$ & sus & 0.006 & 2.218 \\
\hline $100:$ & 2 & 50. & 12 & sur: & 0.051 & 36.710 \\
\hline 101 & 2 & 50 & 12 & sus : & 0.053 & 38,243 \\
\hline 102 & 3 & 100 & 12 & sur & 0.113 & 84.233 \\
\hline 103 & 3 & 100 & 12 & sus: & 0.115 & 85.766 \\
\hline 104 & 1 & 0 & 12 & sur : & 0.004 & 0.685 \\
\hline 105 & 4 & 0 & 12 & sus & 0.004 & 0.685 \\
\hline 106 & 5 & 50 & 12 & sur : & 0.059 & 42.842 \\
\hline 107 & 5 & 50 & 12 & sus & 0.059 & 42.842 \\
\hline 108 & 6 & 100 & 12 & sur & 0,122 & 91,131 \\
\hline 109 & 6 & 100 & 12 & sus & 0.120 & 89.598 \\
\hline 110 & 7 & $0:$ & 12 & sur & 0.004 & 0.685 \\
\hline 111 & 7 & 0 & 12 & sus & 0.006 & 1.452 \\
\hline 112 | & 8 & 50 & 12 & sur: & 0.050 & 40.543 \\
\hline 113 & 8 & 50 & 12 & sus & 0.054 & 39.010 \\
\hline 114 & 9 & $100:$ & 12 & sur & 0.105 & 78.101 \\
\hline 115 & 9 & 100 & 12 & sus & 0.106 & 78.867 \\
\hline
\end{tabular}

Perormed by J. Luor:a SAS version 9.3 07:40 26MAR14 
Mean treatment concentration by treatment tank and sampling location (surface/suspended) for all samping times

The MEANS Procedure

tan:k=1 loc=sur

Analys is Varlable : pretllcted_ppm Predicted

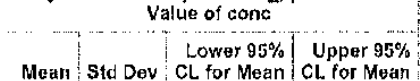

$\begin{array}{lllll}-0.5411 & 1.5894 & -2.5146 & 1.4325\end{array}$

$\operatorname{tank}=1$ locesus

Analysls Varlabla : predicted_ppm Predicted Value of cons
a Lower $95 \%$ Upper $95 \%$ Mean Std Dev CL for Mean CL for Moan $\begin{array}{llll}-0.0812 & 1.7978 & -2.3132 & 2.1508\end{array}$

$\operatorname{tank}=2$ loc $=$ sur

Analysls Varlable : predicted_ppm Predicted Value of conc
Lower $95 \%$ Upper $95 \%$ Mean Std Dev CL for Mean CL for Mean $\begin{array}{llll}40.3895 & 3.1789 & 36.4424 & 44.3366\end{array}$

tank $=2$ loc $=s$ us

Analysis Varlable : predlcted_ppm Predicted Value of conc
Lowor $95 \%$ Upper $95 \%$ $\begin{array}{llll}41.1560 & 3.8094 & 36.4260 & 45.8860\end{array}$ tank $=3$ loc $=s u r$

Analysis Variablo : prediated ppom Predleted Value of conc
Lower $95 \%$ Upper $95 \%$

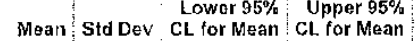
$\begin{array}{cccc}94.3504 & 9.7468 & 82.2482 & 106.5\end{array}$

tank $=3$ loc $=$ sus

Analysis Varlable : predicted__ppm Predlated Value of conc

Lower $95 \%$ Upper $95 \%$ Mean Std Dev CL for Moan CL for Mean $\begin{array}{llll}\mathrm{S} 5.5768 & 8.9209 & 84.4901 & 106.7\end{array}$

tank $=4$ loc:sur

Analysis Varlable : predicted_ppm Prodicted 


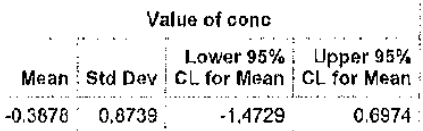

Analys|s Varlable : predlcted_ppm Predlcted 


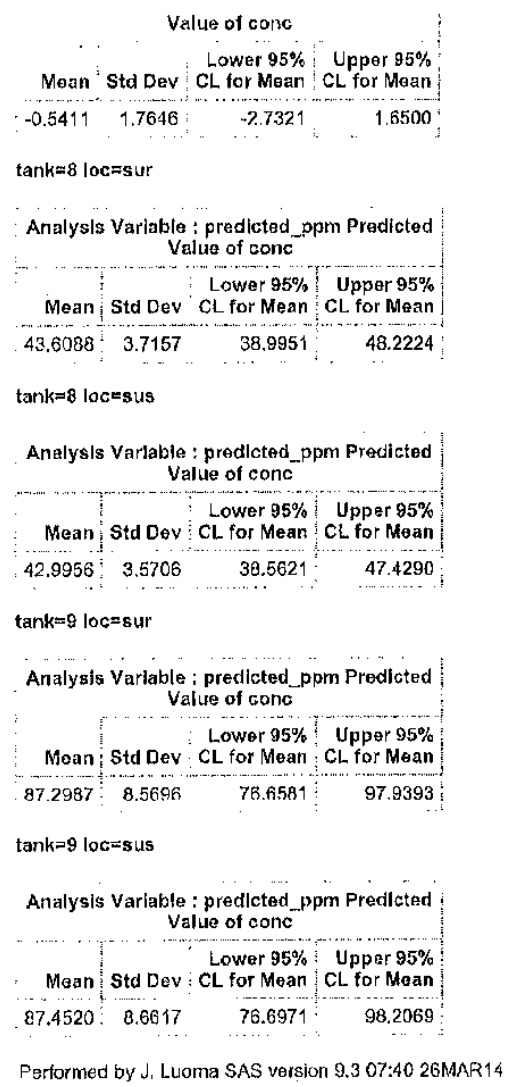


Mean treatment concentration by treatment group and sampling location for all sampling times

The MEANS Procedure

thero=0 loc=sur

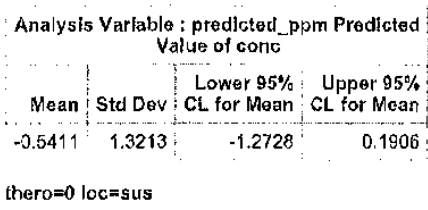

Analysls Varlable : predicted ppm Precicted Value of cone

Mean Sta Dev Lower $95 \%$ Upper $95 \%$

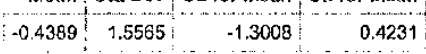

thero $=50$ loc $=9$ sur

Analysis Vgrlable ; predicted_ppm Predlcted Value of conc
Upper $\$ 5 \%$

Mean Std Dev CL for Mean CL for Meart $\begin{array}{cccc}43.7621 & 4.4712 & 41.2860 & 46.2382\end{array}$

thero $=50$ loc $=$ sus

Analys ls Variable: predleted ppm Predicted

\begin{tabular}{c} 
Value of conc \\
Lower $95 \%$ \\
\hline Upper $95 \%$
\end{tabular}

\begin{tabular}{r|r|r|} 
Mean & Std Dev : CL for Mean & Upper $95 \%$ \\
\hline
\end{tabular}

$\begin{array}{lllll}43.8132 & 4.5758 & 41.2792 & 46.3471\end{array}$

thero $=100$ loc $=$ sur

Analysis Varlable : predicted_ppm Predicted Value of conc

Lower 95\% Upper 95\%

Mean Std Dev CL for Mean CL for Mean

$\begin{array}{lllll}93.7883 & 9.9722 & 88.2659 & 99.3108\end{array}$

thero $=100$ locesus

Analysls Varlable : predicted_ppm Predictod Value of cone

Lower $95 \%$ Upper $95 \%$

Mean Std Dev CL for Mean CL for Mean

$\begin{array}{c:ccc}94.2993 & 9.7896 & 88.8780 & 99.7206\end{array}$

Performed by J. Luona SAS versior 9.3 07:40 26MAR14 
Mean treatment concentration by treatment group and sampling location through the $9 \mathrm{~h}$ exposure

The MEANS Procedure

thero $=0$ loc $=$ sur

Analysis Varfable : predleted_ppm Predlcted

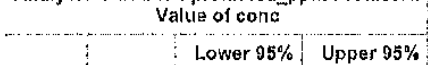

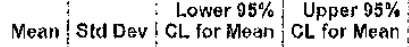
$=-0.9^{-15}: 1.1990 \quad-1.6734 \quad-0.1497$

thero $\approx 0$ loc $=$ sus

Analysis Varlablo : predicted_ppin Predlcted value of conc

Mean Sta Daver 95\% Upper 95\% Std Dev $C L$ for Mean $C L$ for Mean

\begin{tabular}{l|l|l|l|l|l}
-0.9115 & 1.3259 & -1.7540 & -0.0691
\end{tabular}

thero $=50$ loc $=$ sur

Analysis Varlablo : prodictod_ppm Prodicted

Value of conc
Lower $95 \%$ Upper $95 \%$

Mean Std Dev CL for Mean CL for Mean

\begin{tabular}{l|lll}
44.6946 & 4,3539 & 41.9283 & 47.4609
\end{tabular}

therom 50 loc $=$ sus

Analysis Varlable : predicted_ppm Predicted Value of cone

\begin{tabular}{l|l|l|l|l|l|l|l}
\hline & Lower $95 \%$ & Upper $95 \%$
\end{tabular}

Mean Std Dev CL for Mean CL for Mean

$44.7585 \quad 4.5464 \quad 41.8699 \quad 47.6471$

thero $=100$ loc $=$ sur

Analyals Varlabie : predicted_ppm Prodicted Value of conc
Lower $95 \%$ Upper $95 \%$

Mean Std Dev CL for Mean CL for Mean

$\begin{array}{llll}96.1133 & 9.4528 & 90.1074 & 102.1\end{array}$

thero $=100$ locesus

Analysis Variable : predicted_ppm Predicted

Value of conc

Lower $95 \%$ Upper $95 \%$

Mean Std Dev CL for Mean CL for Mean

$\begin{array}{llll}96.6882 & 9.2449 & 90.8143 & 102.6\end{array}$

Performed by J. Luoma SAS versior 9.3 07:40 26MAR 14 
Mean treatment concentration by treatment group and sampling location through the $6 \mathrm{~h}$ exposure

The MEANS Procodure

thero $=0$ loc $=$ sur

Analysis Varlable : predicted_ppm Prodicted

\begin{tabular}{|c|c|c|}
\hline Mean Std Dev & $\begin{array}{l}\text { Lower } 95 \% \\
\text { CL. for Mean }\end{array}$ & $\begin{array}{l}\text { Upper } 95 \% \\
\text { CL for Mean }\end{array}$ \\
\hline .4438 & -2.0164 & $-0,8712$ \\
\hline
\end{tabular}

thero $=0$ loc $=$ sus

Analysis Vartable : predlcted_ppm Predlcted Value of conc

Lower 95\% Upper 95\%

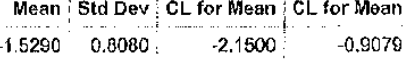

thero $=50$ loc $=3 u r$

Analysis Variable : predicted_ppm Predlcted Value of conc

Lower $95 \%$ Upper $95 \%$

Mean Std Dev CL for Mean CL for Moan

$\begin{array}{lll}45.9082: 4.1277 & 42.7354 & 49.0810\end{array}$

thero $=50$ loc $=s u s$

Analysis Varjable : predicted_ppm Prodicted Value of cone

Lower 95\% Upper 95\%

Mean Std Dev CL for Mean CL for Mean

$\begin{array}{llll}46.2489 ! & 4.1297 & 43.0746 & 49.4232\end{array}$

thero $=100$ loc $=\mathrm{sur}$

Analysis Varlabje : predilcted_ppm Predlcted Value of conc
Lower $95 \%$ Upper 95\%

Mean sid Dev : CL for Mean CL for Mean

$\begin{array}{llll}99.3071 & 8.2820 & 92.9409 & 105.7\end{array}$

thero $=100$ loc $=$ sus

Analysis Variable ; predictod_ppm Predicted Value of cone

Lower $95 \%$ Upper 95\%

$\begin{array}{rl}\text { Mean Sid Dev CL for Mean CL for Mean } \\ 99.7329 & 8.0552\end{array}$

$99.7529,8.0552,93.5411-105.9$

Peifurmed by J. Luoma SAS vers|on 9,3 07:40 26MAR14

file://C: UUscrsłklwcberksashtml.Jtm 
Mean treatment concentration for each treatment group for each sampling time

The MEANS Procedure

thero $=0$ loc $=$ sur time $=1$ Analysis Variable : predicted_ppm Predlcted
Value of conc

: Mean Std Dev \begin{tabular}{c|ccc} 
Lower $95 \%$ & Upper $95 \%$ \\
CL for Moan & CL for Mean
\end{tabular}

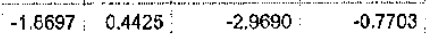

thero $=0$ loc $=$ sur time $=3$

Ahalysis Varlable : predlcted_ppm Predicted Vaive of cone

\begin{tabular}{lll} 
Lower $95 \%$ & Upper $95 \%$ \\
\hline
\end{tabular}

\begin{tabular}{rr|r|r}
\hline$-1.3587^{1}$ & 1.1708 & -4.2672 & 1.5499
\end{tabular}

thero $=0$ loc $=$ sur time $=6$

Analysis Varlable : predicted_ppm Predlctod

Value of conc
Lower $95 \%$ Upper $95 \%$

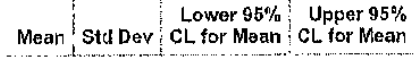

$\begin{array}{llll}-1.1032 & 0.4425 & -2.2025 & -0.00385\end{array}$

theros $=0$ locasur filne $=9$

Analysls Variable : predicted_ppin Predicted

Value of conc

Mean Std Dev \begin{tabular}{l|l:r} 
Lower $95 \%$ & Upper $95 \%$ \\
CL for Mean & CL for Mean
\end{tabular}

$0.6853 \quad 0.7665:-1.2188 \quad 2,5834$

thero $=0$ loc $=$ sur 1 ime $=12$

Analyss Variable : predicted_ppm Predicted

Value of conc
Lower $95 \%$ Upper $95 \%$

Mean Std Dev CL for Mean CL for Mean

$\begin{array}{llll}0.9408 & 0.4425 & -0.1585 & 2.0401\end{array}$

thero $=0$ loc $=$ sus time $=1$

Analysis Variabie : predicted_ppm Predicted Value of cono

Lower $95 \%$ Upper $95 \%$

Mean Std Dev CL for Mean CL tor Mean

$\begin{array}{llll}-2.1251 & 0.4425 & -3.2245 & -1.0258\end{array}$

thero $=0$ loc $=$ sus time $=3$

Analysis Variable : predlcted ppm Predlcted

Value of con

Value of cone

file://C:IUsers $\backslash k l$ webertsashtml.htm 


\begin{tabular}{|c|c|c|c|}
\hline Mean & Std Dav & $\begin{array}{l}\text { Lower } 95 \% \\
\text { CL for Mean }\end{array}$ & $\begin{array}{l}\text { Upper } 95 \% \\
\text { CL for Mean }\end{array}$ \\
\hline $\begin{array}{l}-1.6142 \\
\text { thero }=0 \text { 1 }\end{array}$ & $\begin{array}{r}0.7665 \\
\text { ocesus tir }\end{array}$ & $\lim _{e}=6$ & 0,2899 \\
\hline \multicolumn{4}{|c|}{$\begin{array}{c}\text { Analys s Variable : predicted_ppm Predicted } \\
\text { Vaiue of conc }\end{array}$} \\
\hline Mean & Std Dev & $\begin{array}{l}\text { Lower } 95 \% \\
\text { CL for Mean }\end{array}$ & $\begin{array}{l}\text { Upper } 95 \% \\
\mathrm{CL} \text { for Mean }\end{array}$ \\
\hline-0.8477 & 0.7665 & $-2,7517$ & 1.0564 \\
\hline
\end{tabular}

thero $=0$ loc $=$ sus time $=9$

Analysis Varlable : predlcted ppm Predicted \begin{tabular}{c|c|c|} 
Value of conc \\
\hline \\
\hline
\end{tabular} \begin{tabular}{ll|l|l|l} 
Man Std Dev CL for Mean & CL for Mean \\
\hline
\end{tabular} \begin{tabular}{ll|l|l|l|}
0.9408 & 0.4425 & -0.1585 & 2.0401 \\
\hline
\end{tabular}

thero $=0$ loc $=$ sus time $=\{2$

Analysis Varlabla : predicted_ppm Predleted \begin{tabular}{c} 
Value of conc \\
$\quad$ Lower 95\% Upper 95\% \\
\hline
\end{tabular}

Mean Std Dev CL for Mean CL for Mean

$\begin{array}{lllll}1.4518 & 0.7665 & -0.4523 & 3.3559\end{array}$

thero $=50$ loc $=$ sur t $\mid m e=1$

Analysls Varlable : predlcted_ppm Predicted Value of conc

Lower $95 \%$ Upper $95 \%$

Mean Std Dey CL for Maan CL for Meart

$\begin{array}{lllll}48.7187 & 4.2215 & 38.2319 & 59.2055\end{array}$

thero $=50$ loc $=$ sur $\operatorname{t} \mid \operatorname{m} e=3$

Analysls Variable : predicted_ppm Pradicted

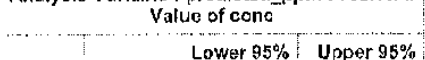
\begin{tabular}{r|r} 
Lower $95 \%$ & Upper $95 \%$ \\
\hline
\end{tabular}

Mean Std Dev CL for Mean CL for Mean

$\begin{array}{llll}46.1637 & 3.4563 & 37.5778 & 54.7496\end{array}$

thero $=50$ loc $=$ sur time $=6$

Analysis Varlable : predicted_ppm Predicted value of conc

Lower $95 \%$ Upper $95 \%$

Mean Std Dev : CL for Mean CL for Mean

$42.0423 \quad 3.5125 \quad 34.1167 \quad 51.5678$

thero $=50$ loc $=$ sur time $=9$

Analysis Variaßje : prodleted_ppm Predicted Value of conc 


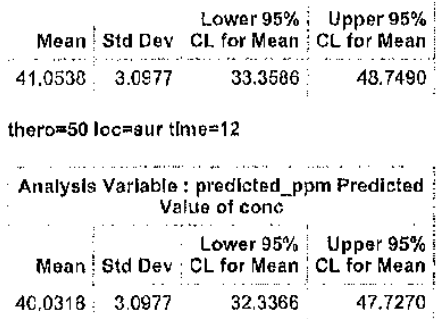




\begin{tabular}{|c|c|c|c|}
\hline Mean & Std Dev & $\begin{array}{l}\text { Lower } 95 \% \\
\text { CL for Mean }\end{array}$ & $\begin{array}{l}\text { Upper } 95 \% \\
\text { CL for Mean }\end{array}$ \\
\hline 106 & .0250 & 89.0099 & 123.9 \\
\hline
\end{tabular}

thero $=100$ loc $=$ sur time $=3$

Analysls Varlable : predlcted_ppm Predicted Aalue of conc
V...... \begin{tabular}{ll:l|r} 
Mean & Ltd Dever $95 \%$ & Upper $95 \%$ \\
CL for Mean & CL for Mean
\end{tabular} $\begin{array}{llll}99.3071 & 6.1955 & 83.9167 & 114.7\end{array}$

ther $O=100$ loc $=s$ ur time $=6$

Analysis Varlable : predlcted ppm Predlcted Value of conc

\begin{tabular}{|l|l|l|l|l|l|}
\hline Lower $95 \%$ & Upper $95 \%$ \\
\hline
\end{tabular} Mean Std Dev, CL for Mean CL for Mean \begin{tabular}{l|lll}
\hline 92.1531 & 5.7529 & 77.8621 & 106.4
\end{tabular} thero $=100$ loc $=$ sur time $=9$

Analysis Variable : predlcted_ppm Predicted Value of conc
\[ \text { Lower 95\% Upper 95\% } \] Mean Std Dev CL for Mean CL for Mean $86.5322 \quad 5.7869 \quad 72.1568 \quad 100.9$ thero $=100$ locsur time $=12$

Analysis $\mathrm{Varable}$ : pedicted Valus of conc
a

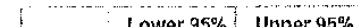

Mean Std Dev CL for Mean CL for Mean

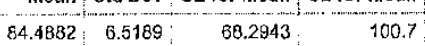

ther $0=100$ locasus $t \mid m e=1$

Analysls Variable : predlcted ppm Predlcted

Value of conc

Lower $95 \%$ Upper $95 \%$

Mean Std Dev, CL for Mean CL for Mean

$\begin{array}{llll}106.5 & 6.2740 & 90.8755 & 122,0\end{array}$

thero $=100$ loc $=$ sus time $=3$

Analysls Varlable ; predicted_ppm Predlcted

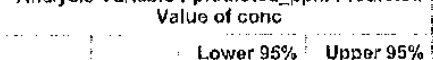

Mean Std Dev CL for Mean CL for Mean

\begin{tabular}{llll}
\hline 99.562 .6 & 6.5489 & 83.2942 & 115.8
\end{tabular}

therom $=100$ loc $=$ sus t|mle $=6$

Analysis Variable : predleted_ppm Predicted value of conc

file:/C:UUscrsłklwebertsashtml.htm 


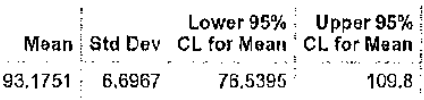


Mean concentration for standard checks for all sampling times

The MEANS Procedure

thero $=25$

Analysts Variable ; predicted_ppm Predlicted

\begin{tabular}{|c|c|c|c|}
\hline Mean & Std Dev & $\begin{array}{l}\text { Lower } 95 \% \\
\text { CL for Mean }\end{array}$ & $\begin{array}{l}\text { Upper } 95 \% \\
\text { CL for Mean }\end{array}$ \\
\hline 23.4245 & 0.8851 & 21.2259 & 25.6232 \\
\hline
\end{tabular}

thero $=50$

Analysis Varlable : predicted_ppm Predlcted Value of conc Std Dev CL for Mean CL for Mean $\begin{array}{llll}48.4632 & 2.2127 & 42.9666 & 53.9598\end{array}$

thero $=100$

Analysis Varabla : prodlcted_ppm Prodicted Value of conc
Lower $95 \%$ Upper $95 \%$

Mean Std Dev CL for Mean CL for Mean

$\begin{array}{llll}962411 & 1.1708 & 93.3326 & 99.1496\end{array}$

thero $=2,00$

Analysis Variable : predicted_ppm Predicted

Value of conc

\begin{tabular}{rr}
\hline Lower 95\% & Upper 95\% \\
\hline & 0
\end{tabular}

Mean Std Dev CL for Mean CL for Mean

$187.7=0.7665$

185.8

Performed by J, Luoma SAS version 9.3 07:40 26MAR14

FF \# $11 \mathrm{C}$

Item No. 4

$\mathrm{Pg} 19$ of 19 


\begin{tabular}{|c|c|c|c|}
\hline \multirow{5}{*}{$\begin{array}{l}\text { Study NLmber: AEH-12-PSEUDO-04 } \\
\text { Electronic Lab Notebook (pages 14 - 17) } \\
\text { Data Scurce: File Folder: } 12 \mathrm{c} \\
\text { Forms: "Sample Absorbance Readings' Dara Sheet }\end{array}$} & Acilon & Date & minitlals. \\
\hline & Created...... & 6-Febl-14 & IJS $n$ n.S \\
\hline & Revised...... & 12. Feb-14 & $\pi 5$ \\
\hline & Reviewed... & Y2FEBIY & Tos \\
\hline & Certfled....... & $2 / 19 / 14$ & $5 \times 2$ \\
\hline
\end{tabular}

Spectrophotometric Data

rest Article: Zequanox ${ }^{(M B)-401 ~ S D P)}$

Test Artlcle Lot f: A01P12163C and 401P12164C Mlx

Exposure Date: August 17, 2012

Test Lacation: Lake Carlos, Alexandria, MN

Treatment Type: Bottom Injaction

Data Explanation:

1) The absorbance of triplicate samples of $25,56,100$, and $200 \mathrm{mg} / \mathrm{L}$ dillutions of a $2,000 \mathrm{mg} / \mathrm{L}$ actlve Ingredient (A.1.) stock prepared from Analytical Stock H2 were measured to prepare a standard curve.

2) Standard checks were performed at 6, 9, and 12 hours by comparing the $25,50,100$, and $200 \mathrm{mg} / \mathrm{L}$ (A.I.) dillutions to the .Inear curve.

Data codes used withlin SAS

tank $=\operatorname{Tank}$ ID $[1$ through 9 )

thero $\rightarrow$ Theoret ca: or target concentration $(\mathrm{mg} / \mathrm{L}$ )

time $=5$ ample TIms $\{0,1,3,6,9$, and $12 \mathrm{~h}$ after treatment $\}$

loc $=$ Sample Location

US = Suspended Sample (sampled $19 \mathrm{~cm}$ from bottom of tank)

abs $=$ measured absorbance of sample

conc = concentration $([\mathrm{mg} / \mathrm{L}]$, only used for standards used for regresslon $)$

4) Information that is not relevant 10 a sample [i.e, tank ID for standards) or that will be calculated by SAS (1.e., predicted concentration for standard checks and samples) Is denoted by a "." In the SAS Input and output filles.

Data Analysis:

1) A linea" regression was completed in $5 A S$ usl $\mathrm{zg}$ the absorbance values obtalned from the spectrophotometer of 3 replitate dilltions of $25,50,100$ and $200 \mathrm{mg} / \mathrm{L}$

) Standarl checks and treatment sample concentratoors were predicted in SAS by ocmparing the obscrved absorbences with the llncar rcgression.

3) The fol owing mean treatment concentratiors were determlned in SAS:

3a) Mean (standard deviation) concentration by tank anc location for all sampling times

36) Mean (standard deviation) contentratlon by treatment group and location for all sampling !imes

3c) Mean (standarc deviation) concentration by treatment graup ind sampling times for both lacations

3d) Mean (standard tevlatlon) concentrations for 25, 50, 100, and $200 \mathrm{mg} / \mathrm{L}$ (A.1.) dilut ons for all sampling tlires

File Names:

Spectrophotometrlc Data for SAS inout

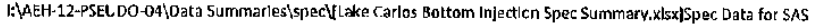

SAS Program/Code

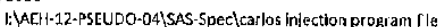

SASLOg

I: AEH-12-PSEUCO-04\}SAS-Speclcarlos Injection log file

SASOUTPUI

I: AA:H-12-PSFUDO-OA\SAS-Speçcarlos Injection results file

Data Anomalies and Deylationsi

1) One exposure tank of a different concertit ation and sample location was sampled In triplicate to evaluate varlabilly of spectrophotometer during each sampling timc. The mean of the triplicates was used within the analysis.

2) Suspended samples (sus) were collected $\sim 19 \mathrm{zm}$ fom the tank bottoms for all sampling times; tank bottom (bot) samples were collected at 6,9 and 12 lis sampling timcs. Test artlec settling was observed In the 1 and 3 a samples resulling in lower than expected concentrations. Samples collected from the tank bottoms at 6,9 and 12 h confirmed test article settliag as Ir licicated by higher than expected concentrations.

3) Son mean absobances for asplcate sampes ray be ecorded

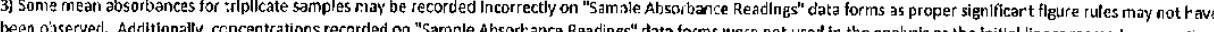
been ousservet. Additionally, ccncentrations recorded on "Sample Absorkance Readings" data forms were not used in the analysis as the initlal linear regresslon equation that was Lsed for these calculat' cns was derlved using rounced absorbance values in Excel. All absorbances and concentrations used In SAS calculations and reported within Spectrophotometric Data Summary have keen coirected.
item Number $-\frac{1}{\text { Page } .1}$
File Folder: $12 c$ 


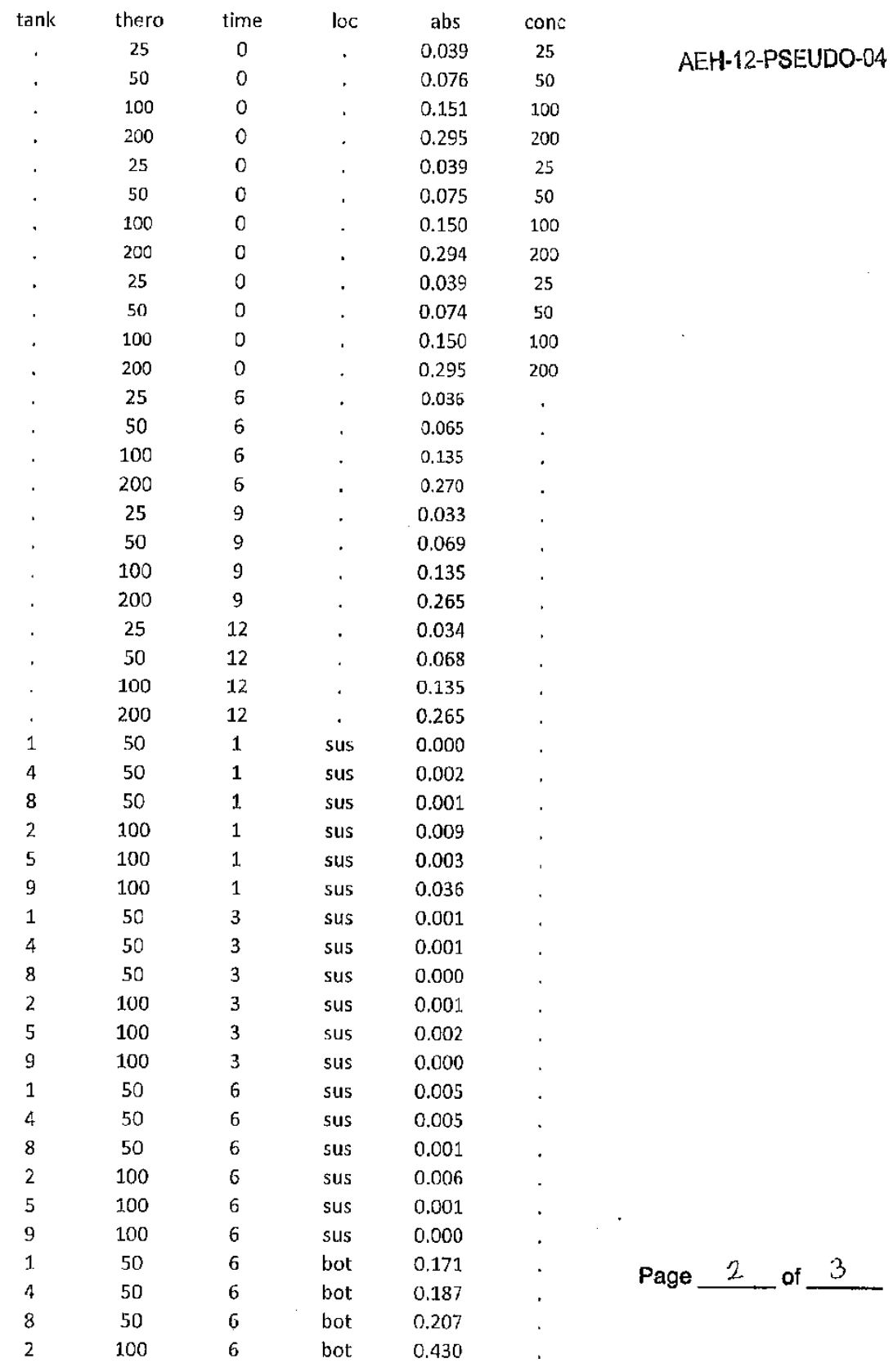




\begin{tabular}{|c|c|c|c|c|c|c|}
\hline 5 & 100 & 6 & bot & 0.398 & . & \\
\hline 9 & 100 & 6 & bot & 0.345 & . & AEH-12-PSEUDO-04 \\
\hline 1 & 50 & 9 & sus & 0.004 & . & \\
\hline 4 & 50 & 9 & sus & 0.001 & . & \\
\hline 8 & 50 & 9 & sus & 0.003 & . & \\
\hline 2 & 100 & 9 & sus & 0.006 & . & \\
\hline 5 & 100 & 9 & sus & 0.001 & . & \\
\hline 9 & 100 & 9 & sus & 0.003 & . & \\
\hline 1 & 50 & 9 & bot & 0.159 & . & \\
\hline 4 & 50 & 9 & bot & 0.154 & . & \\
\hline 8 & 50 & 9 & bot & 0.131 & . & \\
\hline 2 & 100 & 9 & bot & 0.395 & . & \\
\hline 5 & 100 & 9 & bot & 0.357 & . & \\
\hline 9 & 100 & 9 & bot & 0.322 & . & \\
\hline 1 & 50 & 12 & sus & 0.011 & . & \\
\hline 4 & 50 & 12 & sus & 0.012 & . & \\
\hline 8 & 50 & 12 & sus & 0.013 & . & \\
\hline 2 & 100 & 12 & sus & 0.004 & . & \\
\hline 5 & 100 & 12 & sus & 0.005 & . & \\
\hline 9 & 100 & 12 & sus & 0.012 & . & \\
\hline 1 & 50 & 12 & bot & 0.108 & . & \\
\hline 4 & 50 & 12 & bot & 0.113 & - & \\
\hline 8 & 50 & 12 & bot & 0.114 & . & \\
\hline 2 & 100 & 12 & bot & 0.324 & 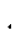 & \\
\hline 5 & 100 & 12 & bot & 0.285 & . & \\
\hline 9 & 100 & 12 & bot & 0.257 & . & \\
\hline
\end{tabular}

File Folder: $12 c$

Item Number:

Page 3 - 3 of 3 


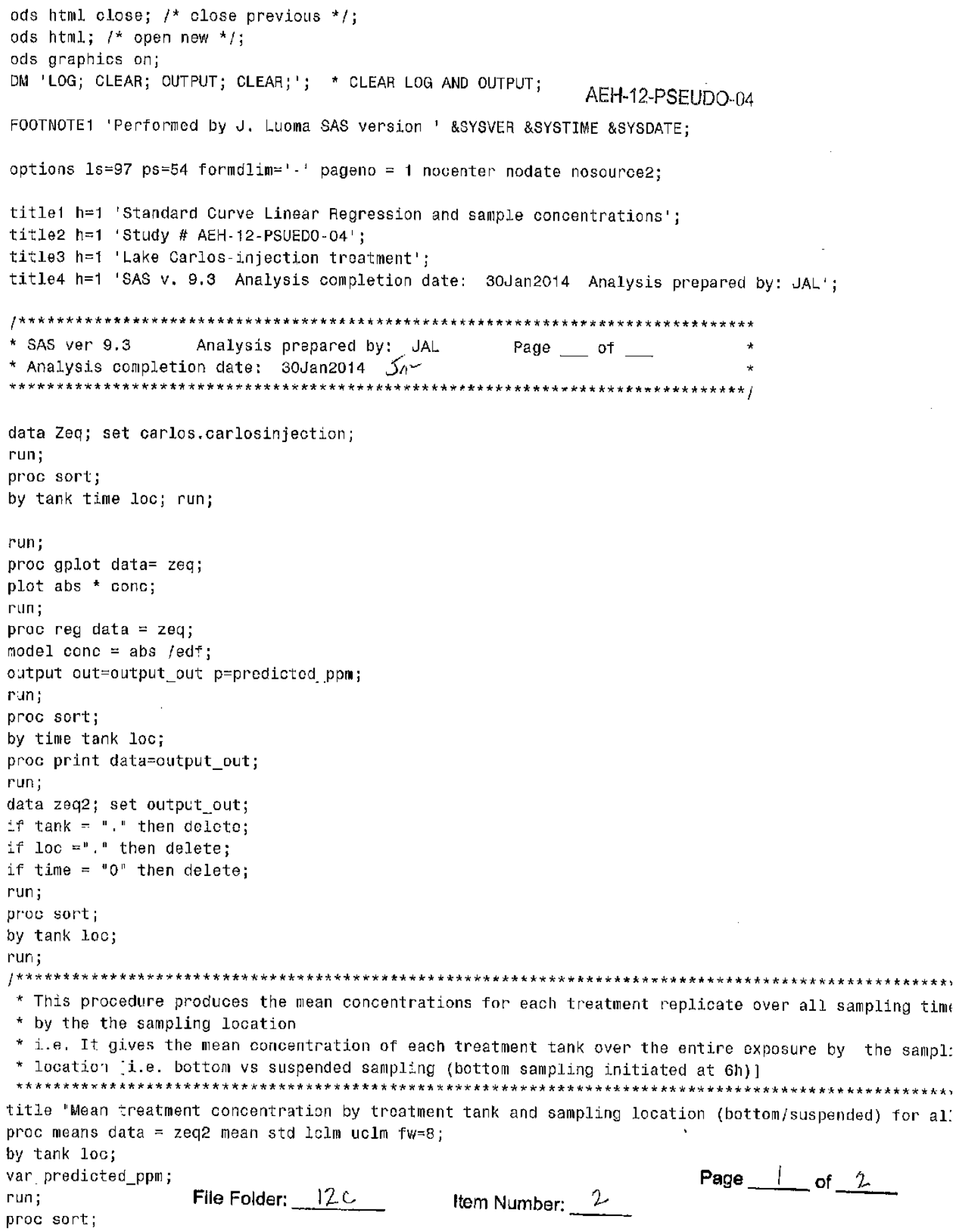


by thero time loc;

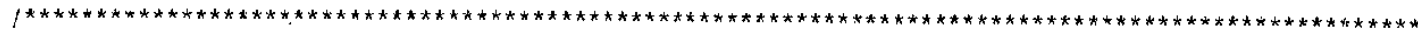

* This procedure produces the mean concentrations for cach treatment group over all sampling times

* by the sampling location

* i.e. It gives the mean concentration of the $350 p p m$ \& 100 ppm treatment tanks by sampling location * (bottom/suspended) over the entire exposure

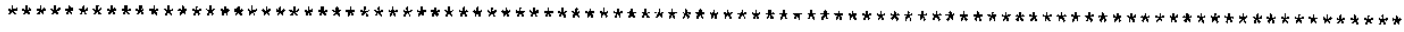

title "Mean treatment concentration by treatment group and sampling location for all sampling times"; proc sort;

by thero lcc;

proc means data $=$ zeq2 mean std $1 \mathrm{cln}$ uclm $\mathrm{fw}=8$;

AEH-12-PSEUDO-04

by thero loc;

var predicted_ppm;

run;

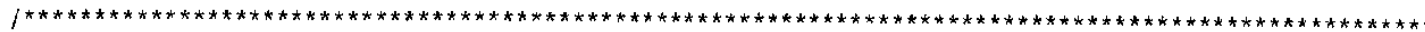

* This procedure produces the nean concentrations for each treatment group by sanpling time

* i.e. It gives the mean conc. of the $350 \mathrm{ppm} \& 100 \mathrm{ppm}$ treatment tanks at tinie 1, 3, 6,9 and $12 \mathrm{~h}$

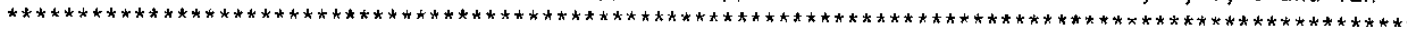
title "Mean treatment concentration for each treatment group for each sampling time";

proc sort;

by thero time 100;

proc means data $=7.002$ mean std lolm ucln fvi=8;

by thero time 10c;

var predicted_ppm;

run;

data zeq3; set output_out;

if conc > 1 then delete;

if tank > 0.5 then delete;

if thero $=$ ". " then delete;

run;

proe sort;

by thero;

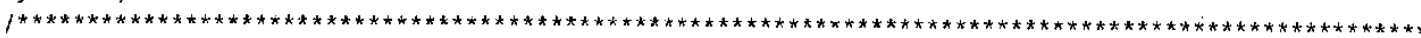

* This procedure produces the mean concentrations for the standard chccks for all time periods

* i.e. It gives the mean conc. of the 50ppm \& $100 \mathrm{ppm}$ standard checks at 9 and $12 \mathrm{~h}$

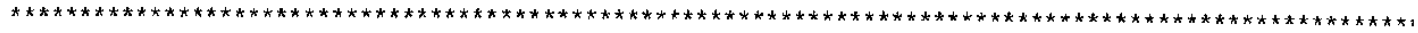

title "Mean concentration for standard checks for all sampling times";

proc means data $=z e q 3$ mean std $1 \mathrm{clm}$ uclin $f w=8$;

by thero;

var predicted_ppm;

run;

quit;

$1 / 3.1 / 4$

run;

File Folder: 120

Item Number: 2

Page 2 of 2 


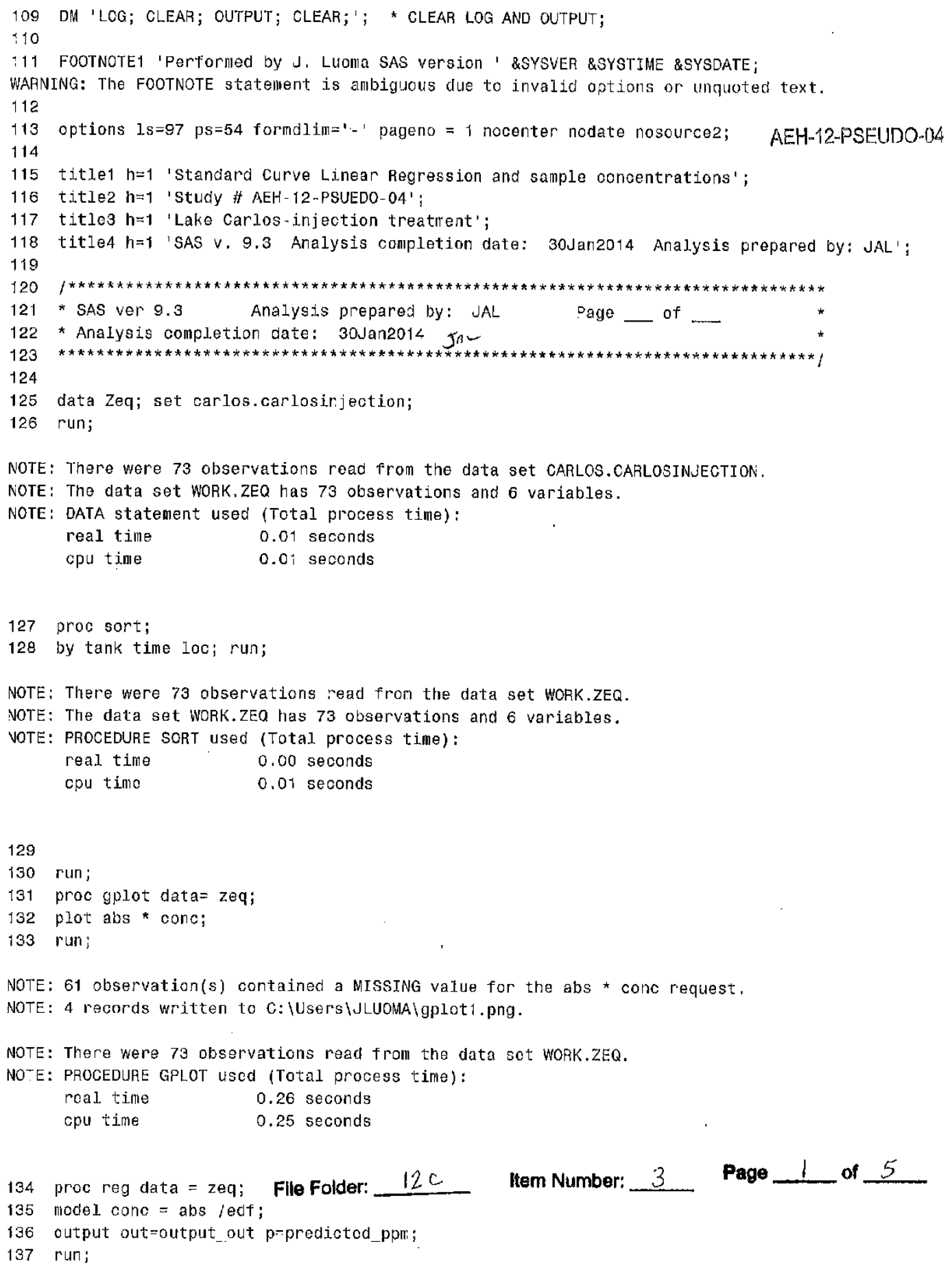


NOTE: The data set WORK.OUTPUT_OUT has 73 observations and 7 variables.

NOTE: PROCEDURE REG used (Total process time) :

real time $\quad 1.04$ seconds

cpu time $\quad 0.42$ seconds

AEH-12 PSEUDO-04

138 proc sort;

139 by time tank 10c;

NOTE: There were 73 oDservations road from the data set WORK.OUTPUT_CUT.

NOTE: The data set WORK. OUTPUT OUT has 73 cbservations and 7 variables.

NOTE: PROCEDURE SORT used (Total process time):

real time $\quad 0.00$ seconds

cpu tíne $\quad 0.01$ seconds

140 proc print data=output_out;

141 run;

NOTE: There were 73 observations read fron the data set WORK.OUTPUT OUT,

NOTE: PROCEDURE PRINT used (Total prooess timo) :
real time
0.04 scconds
cpu time
0.04 seconds

142 data zeq2; set output out;

143 if tank = "." then delete;

144 if $100="$ " " ther delete;

145 if time $=$ "0" then delete;

146 run;

NOTE: Character values have been converted to numeric values at the places given by: (Line) : (Colunin). $145: 11$

NOTE: There were 73 observations read from the data set. WORK.OUTPUT_OUT.

NOTE: Tho data set WCRK.ZEQ2 has 49 observations and 7 variables.

NOTE: OATA statement used (Total process time):
real time
0.00 seconds

cpu time

0.01 seconds

147 proc sort;

148 by tank loo;

149 run;

NOTE: There were 49 ubservations read from the data set WOAK.ZEQ2.

NOTE: The data set WORK.ZEQ2 ras 49 observations and 7 variables.

NOTE: PROCEDURE SORT used (Total process time):
real time
0.00 seconds

cpu time

0.01 seconds

Page 2 of 5

150

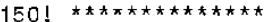




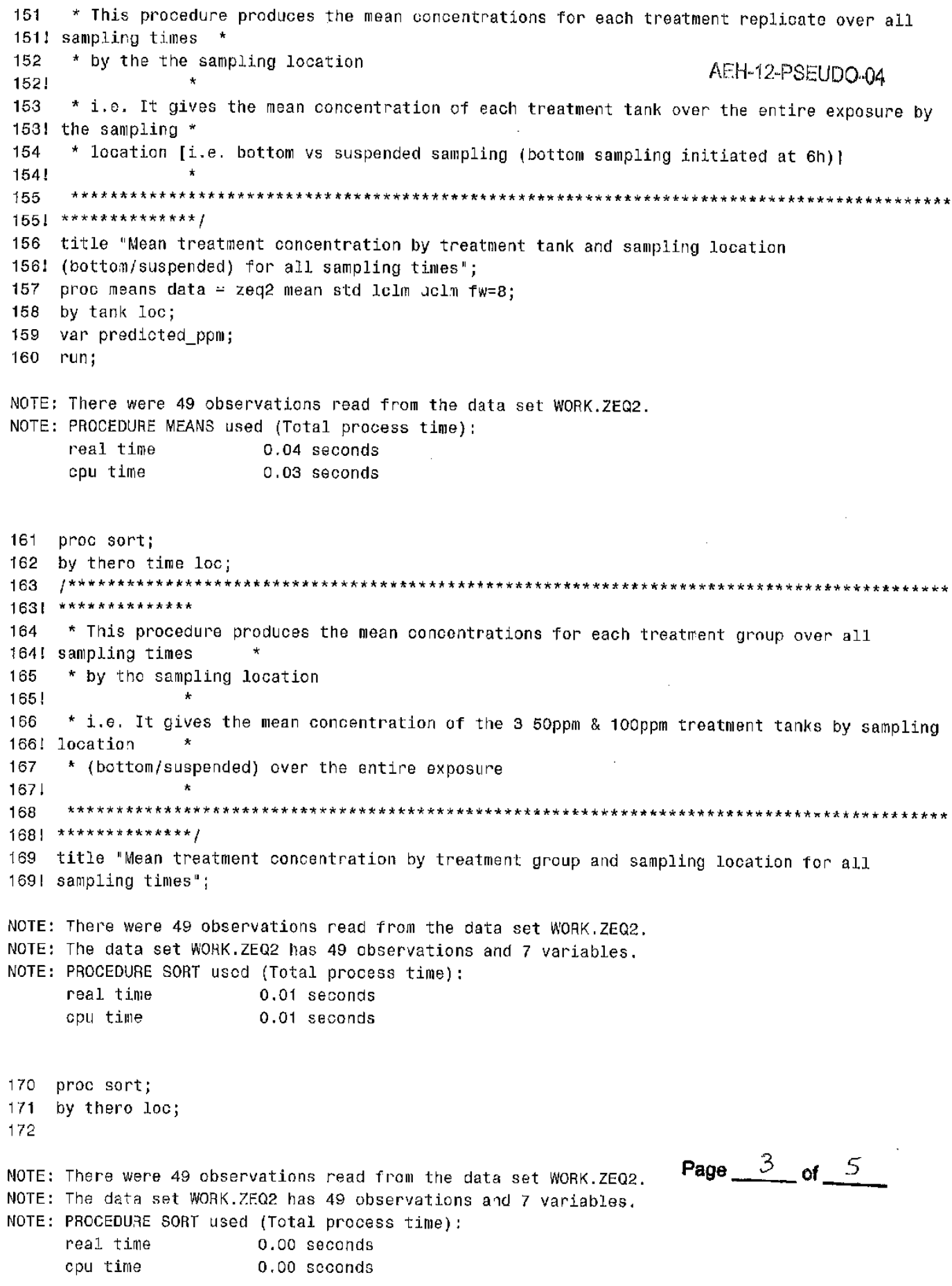




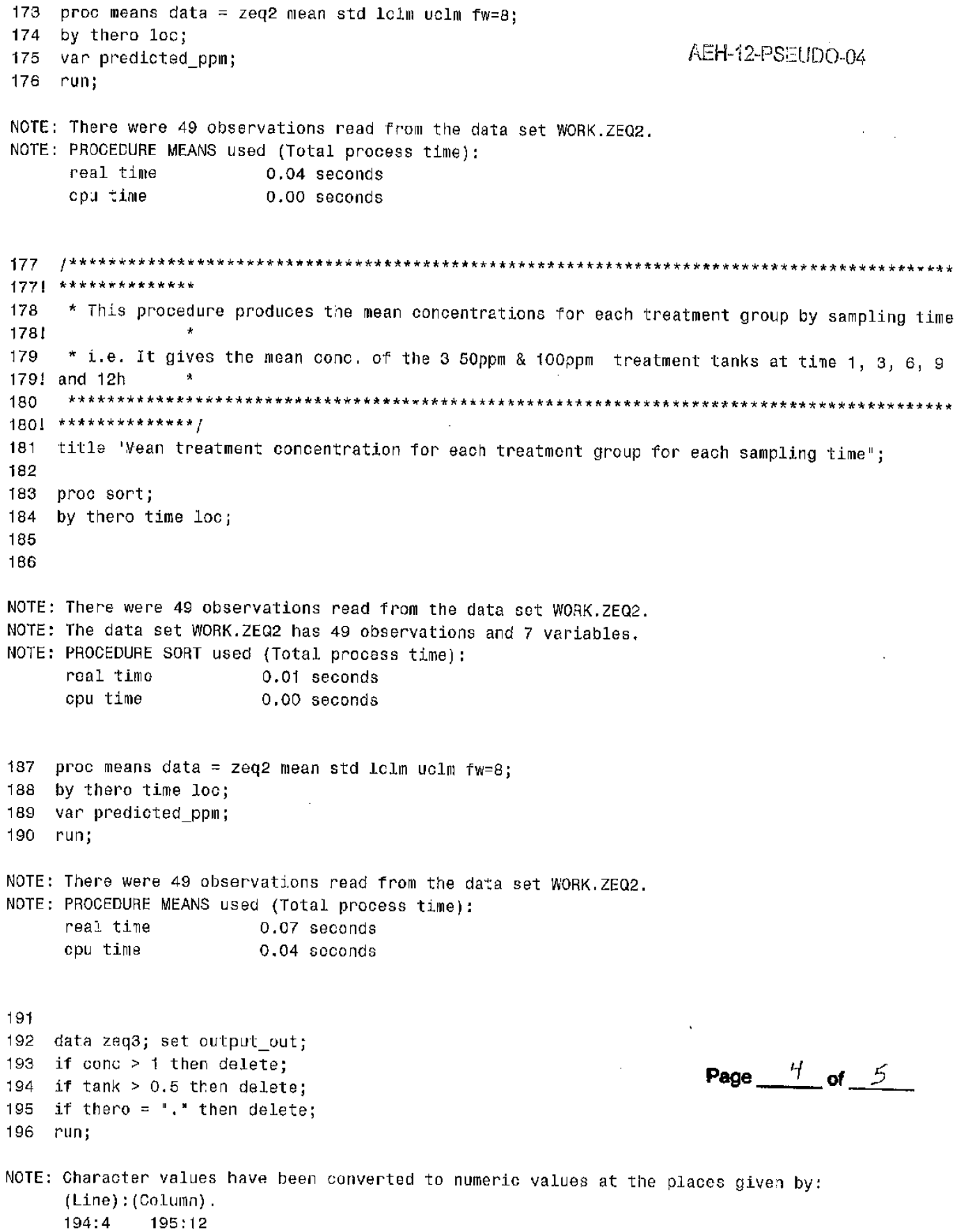


NOTE: There were 73 observations read from the data set WORK.OUTPUT OUT.

NOTE: The data set WORK.ZEQ3 has 12 observations and 7 variables.

NOTE: DATA statement used (Total process time):

real time $\quad 0.01$ seconds

cpu time $\quad 0.01$ seconds

197

198 proc sort;

199 by thero;

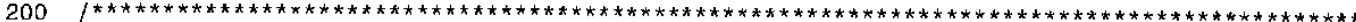

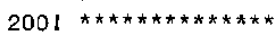

201 * This procedure produces tre mean consentrations for the standard checks for all tine

201! periods

202 * i.e. It gives the mean conc. of the 5oppm \& 100ppm standard checks at 9 and $12 \mathrm{~h}$

2021 *

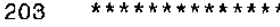

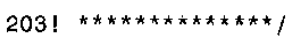

204 title "Mean concentration for standard checks for all sampling times";

NOTE: There were 12 observations read from the data set WORK.ZEQ3.

NOTE: The data set WORK, ZEQ3 has 12 observations and 7 variables.

NOTE: PROCEDURE SORT used (Total process time):
real tine
0.01 seconds

cpu time

0.01 seconds

205 proc means data $=$ zeq3 mean std $1 \mathrm{clm} u c=$ m $f w=8$;

206 by thero;

207 var predicted_ppin;

208 run;

NOTE: Thero wore 12 observations read from the data set ViOHK.ZEQ3.

NOTE: PROCEDURE MEANS used (Total process time):

real time $\quad 0.04$ seconds

cpu time 0.04 seconds

209 quit;

210 run;

$$
\sqrt{3 / 14}
$$

Flle Folder: $\quad 12 c$

Item Number: 


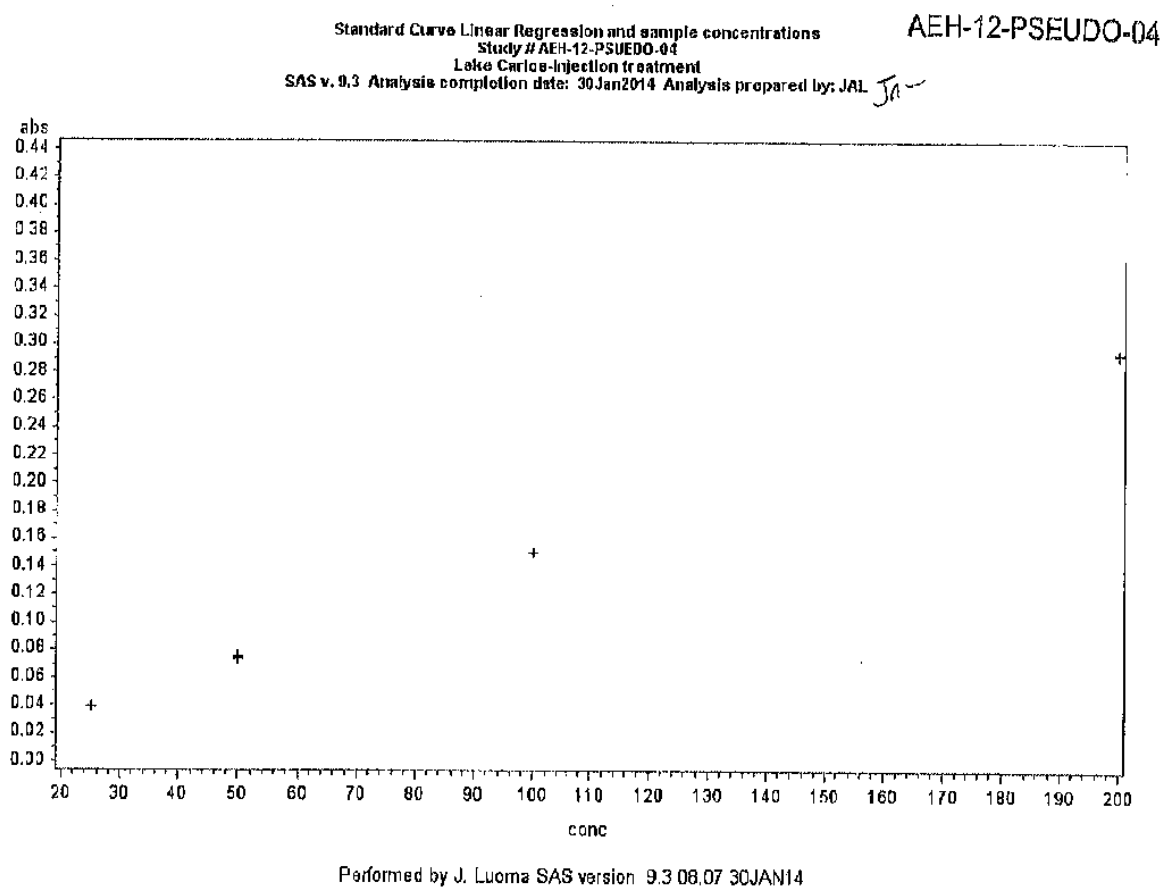

Flle Folder: $12 \mathrm{c}$

Item Number: 4 
AEH-12-PSEUDO-04

\begin{tabular}{|c|c|c|c|c|c|c|}
\hline \multicolumn{7}{|c|}{ 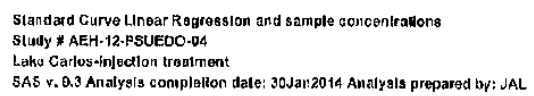 } \\
\hline \multicolumn{7}{|c|}{$\begin{array}{l}\text { The REG Procedure } \\
\text { Madel: MODEL1 } \\
\text { Dependent Variable: conc conc }\end{array}$} \\
\hline \multicolumn{5}{|c|}{ Number of Observations Read } & 73 & \\
\hline \multicolumn{5}{|c|}{ Number of Observations Used } & 12 & \\
\hline \multicolumn{5}{|c|}{ Number of Observatlons with Mlssing Values } & 61 & \\
\hline \multicolumn{7}{|c|}{ Analysls of Varlance } \\
\hline Source & DF & $\begin{array}{r}\text { Sum of } \\
\text { Squares }\end{array}$ & $\begin{array}{r}\text { Mean } \\
\text { Square }\end{array}$ & F Valu & & $\mathrm{Pr}>\mathrm{F}$ \\
\hline Model & 1 & $53901 !$ & 53901 & 95117. & & $<.0001$ \\
\hline Error & 10 & 5.66674 & 0.56667 & & & \\
\hline Corracted Total & 11 & 53906 & & & & \\
\hline
\end{tabular}

\begin{tabular}{|c|c|c|c|c|c|c|c|}
\hline \multicolumn{2}{|l|}{ Root MSE } & \multicolumn{2}{|c|}{0.75278} & R-Square & re $\quad 0.9999$ & & \\
\hline \multicolumn{2}{|c|}{ Dopendent Mean } & \multicolumn{2}{|c|}{93.75000} & Adj $R-S q$ & \begin{tabular}{l|l}
$q$ & 0.9999
\end{tabular} & & \\
\hline \multicolumn{2}{|l|}{ Coeff Var } & \multicolumn{2}{|c|}{0.80296} & & & & \\
\hline \multicolumn{8}{|c|}{ Parameter Estimates } \\
\hline Variable & Labol & DF & & $\begin{array}{l}\text { rameter } \\
\text { stimate }\end{array}$ & $\begin{array}{r}\text { Standard } \\
\text { Error }\end{array}$ & $t$ Valuc & $\operatorname{Pr}>|t|$ \\
\hline Intercept & Intercept & 1 & & 1.77327 & 0.37838 & -4.69 & 0.0009 \\
\hline alus & abs & 1 & & 3.52963 & 2.21629 & 308.41 & $<.0001$ \\
\hline
\end{tabular}

Performed by J. Luoma SAS verslon 8.3 08:07 30JAN14 
Standard Gurve Linoar Regression and sample concenitratlons

Study \# AEH-12.PSUEDO-O4

Lake Carlos-Injectlant trealouent

SAS \%, 9.3 Analyois comipletion date: 30Jan2016́ Analysis propared by: JAL

The REG Pracedur

Model: MODEL1

Dependent Variable: conc conc

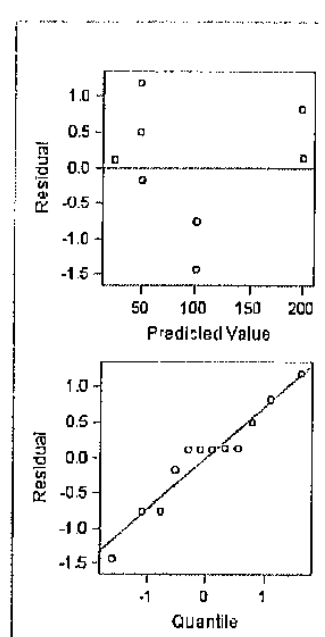

Fit Diagnostics for cone
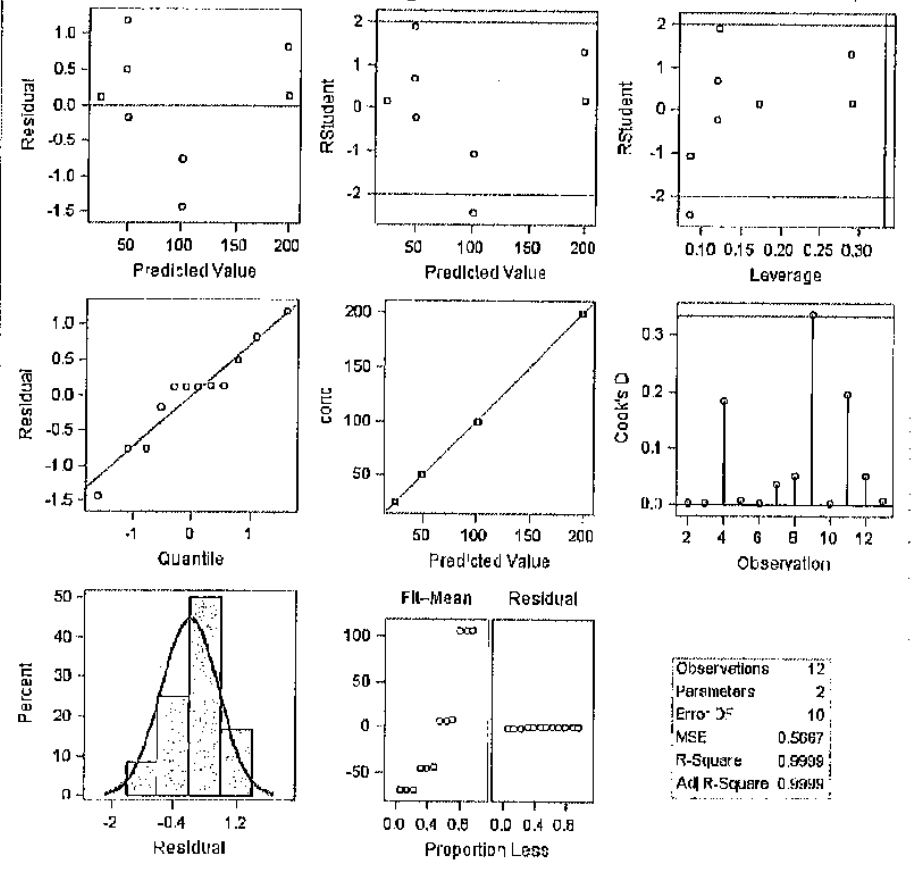

Adl R-Square 0. Sass 
AEH-12-PSEUDO-04
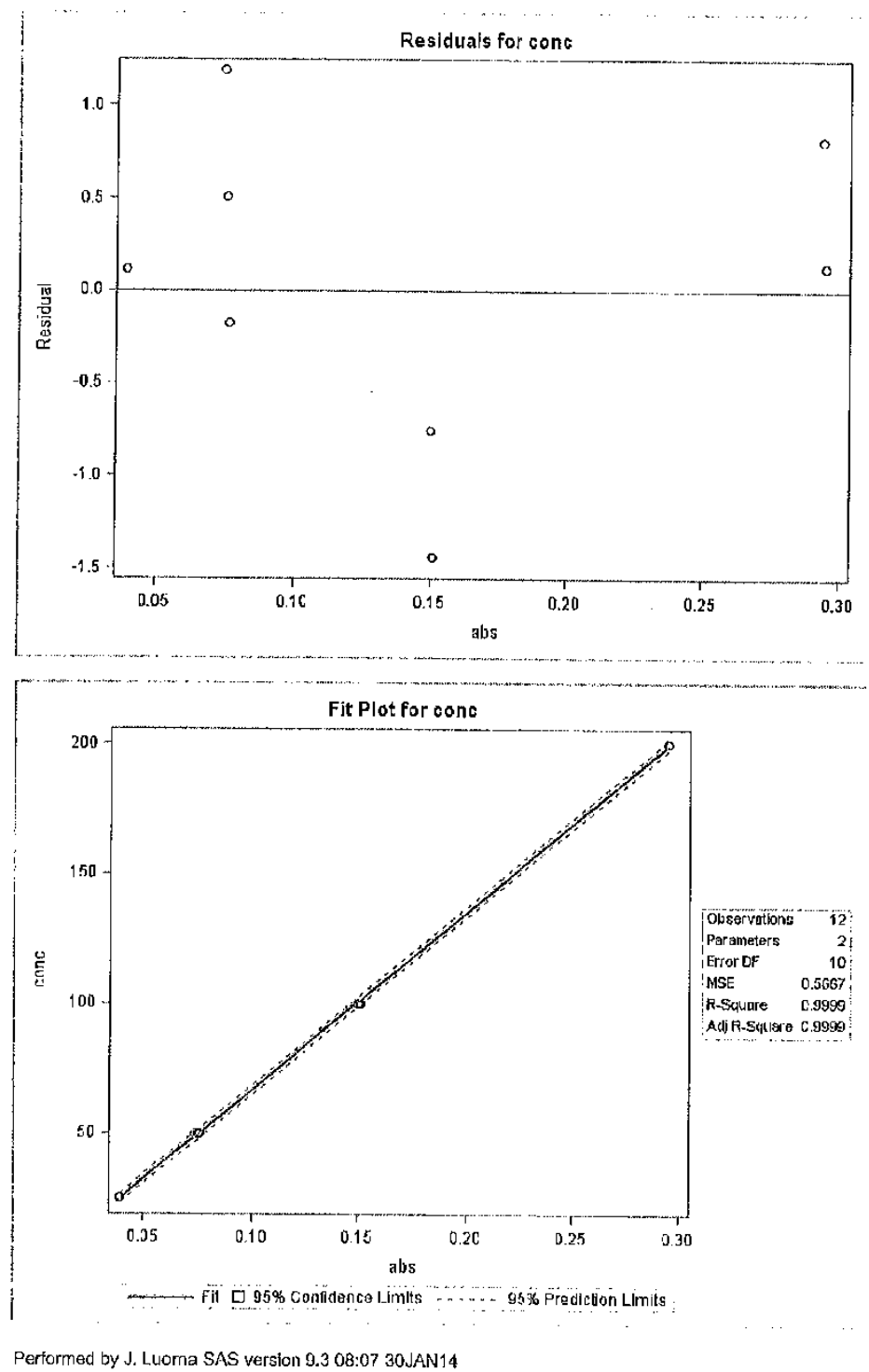

file://C:UUser'SULUOMALsastinll.htm 
Stan derd Curve Lritiur Ragrebs Jon and sample concentrations

Study 4 AEH - 2 2.PSUEDO-04

Lake Carbos:tn]ectlon trealrisen

SAS Y, B,3 Analy \&ls complet| on dato: 30Jan2014 Analysis prepared by: JAL

Obs tank thero tlme loc abs conc predlcted_ppm

\begin{tabular}{r|r|r|r|r|r|r}
1 & & 1 & 1 & & \\
\hline 2 & $25^{1}$ & 0 & 0.039 & 25 & 24.884
\end{tabular}

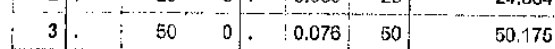

\begin{tabular}{|l|l|l|l|l|l|}
\hline 4 & 100 & 0 & 0.151 & 100 & 101.440 \\
\hline
\end{tabular}

\begin{tabular}{|r|rr|r|r|r|}
\hline 5 & 200 & 0 & 0.295 & 200 & 199.868 \\
\hline
\end{tabular}

\begin{tabular}{|l|r|r|r|r|r|r|r}
6 & 25 & 0 & 0.039 & 25 & 24.884 \\
\hline
\end{tabular}

\begin{tabular}{l|l|l|l|l|l|l|l|}
7. & 50 & 0 & 0.075 & 50 & 49.491
\end{tabular}

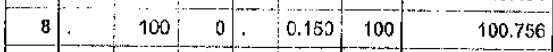

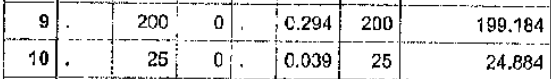

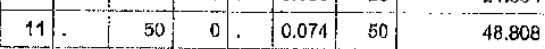

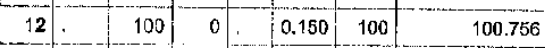

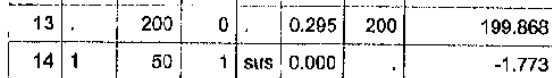

\begin{tabular}{|l|l|r|r|r|r|r|}
\hline 14 & 1 & 50 & 1 & sts & 0.000 & -1.773 \\
\hline 15 & 2 & 100 & 1 & sus & 0.009 & \\
\hline 16 & 4 & 50 & 1 & sus & 0.002 & 4.379 \\
\hline
\end{tabular}

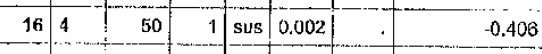

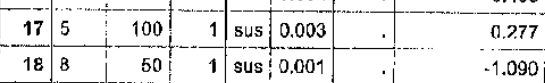

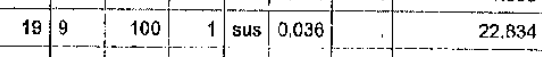

\begin{tabular}{l|l|l|l|l|l|l|l}
20 & 1 & 50 & 3 & sus 0.001 & -1.090
\end{tabular}

\begin{tabular}{l|l|l|l|l|l|}
21 & 2 & 100 & 3 & sus 0.001 & -1.090
\end{tabular}

\begin{tabular}{l|l|r|r|r|r|r|r|r|r|r|r|r|}
\hline 22 & 4 & 50 & 3 & sus & 0.001 & -1.090 \\
\hline
\end{tabular}

\begin{tabular}{llllll}
23 & 5 & 00 & 3 & sus 0.002 & -0.106 \\
\hline
\end{tabular}

\begin{tabular}{l|l|l|l|l|l|l|}
248 & 50 & 3 & sus 0.000 & -1.773
\end{tabular}

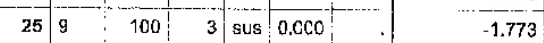

[26]: $25,0 ., 0.036 \quad 22.834$

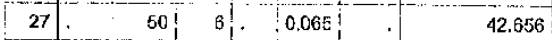

[28. 100 6. 0.135 . 90.503

$29 . \quad 200$ 6. 0.270 . 182.780

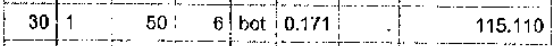

\begin{tabular}{lll|l|l|r|r|r}
31 & 1 & 50 & 6 & sus & 0.005 & 1.644
\end{tabular}

$\begin{array}{lllllll}32 & 2 & \vdots & 1 \mathrm{CO} & 6 & \text { bot } & 0.430\end{array}$

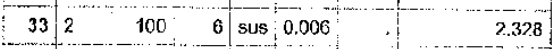

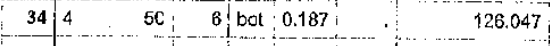

\begin{tabular}{r|l|l|l|l|l|l}
35 & 4 & 50 & 5 & sus & 0.005 & 1.644
\end{tabular}

$365: 100 \quad 6$ bot $0.398 \quad$ : 270.272 ?

$\begin{array}{lllllllll}37 & 5 & 100 & 6 & \text { sus } & 0.001 & \ldots & & -1.090\end{array}$

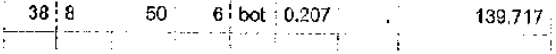

file:/C:Users VLUOMAlsashtm11.htm 


\begin{tabular}{|c|c|c|c|c|c|c|}
\hline 39 & & 50 & 6 & sus: & 0,001 & -1.090 \\
\hline 40 & 9 & 100 & 6 & bot: & 0.345 & 234.044 \\
\hline 41 & 9 & 100 & 6 & sus: & 0.000 & -1.773 \\
\hline 42 & . & $25 \mid$ & 9 & & 0.033 & 20.783 \\
\hline 43 & . & 50 & 9 & & 0.069 & 45.390 \\
\hline 44 & & 100 & 9 & & 0.136 & 90.503 \\
\hline 45 & & $2 \mathrm{CO}$ & 9 & . & 0.265 & 179.362 \\
\hline 46 & 1 & 50. & 9 & bot: & 0.159 & 106.908 \\
\hline 47 & 1 & 50 & 9 & sus & 0.004 & 0.961 \\
\hline 48 & 2 & 100 & 9 & bot & 0.395 & 268.221 \\
\hline 49 & 2 & 100 & 9 & sus & 0.006 & 2.328 \\
\hline 50 & 4 & 50 & 9 & bot & 0.154 & 103.490 \\
\hline 51 & 4 & 50 & 9 & sus & 0.001 & -1.000 \\
\hline 52 & 5 & 100 & 9 & bot & 0.357 & 242.247 \\
\hline 53 & 5 & 100 & 9 & sus! & 0.001 & $\begin{array}{r}-1.090 \\
-\end{array}$ \\
\hline 54 & 8 & 50 & 9 & bot & $0.131 \mid$ & 87.769 \\
\hline 55 & 8 & 50 & 9 & sus & 0.003 & 0.277 \\
\hline 56 & 8 & 100 & 9 & bot & 0.322 & 218.323 \\
\hline 57 & 9 & 100 & 9 & sus & 0.003 & 0.277 \\
\hline 58 & & 25 & 12 & . & 0.034 & 21.467 \\
\hline 59 & & 50 & 12 & 1. & 0.068 & 44.707 \\
\hline 60 & & 100 & 12 & & 0.135 & 90.503 \\
\hline 61 & & 200 & 12 & & 0.265 & 179.362 \\
\hline 62 & 1 & 50 & 12 & bot: & 0.108 & 72.048 \\
\hline 63 & 1 & 50 & 12 & sus: & 0.011 & 5.746 \\
\hline 64 & 2 & 100 & 12 & bot $j$ & 0.324 & $2 \times 9.690$ \\
\hline 65 & 2 & 100 & 12 & sus: & 0.004 & 0.961 \\
\hline 66 & 4 & 50 & 12 & bot 1 & 0.13 & 75.466 \\
\hline 67 & 4 & 50 & 12 & sus! & 0.012 & 6.429 \\
\hline 68 & 5 & 100 & 12 & bot : & 0.285 & 193.033 \\
\hline 69 & 5 & 100 & 12 & sus & 2.005 & 1.044 \\
\hline 70 & 8 & 50 & 12 & bot & 0.114 & 76.119 \\
\hline 71 & 8 & $50 !$ & 12 & sus & 0.013 & 7.113 \\
\hline 72 & 9 & 100 & 12 & bot & 0.257 & 173.894 \\
\hline 73 & 9 & 100 & 12 & sus & .0012 & 6.429 \\
\hline
\end{tabular}

ALH-12PSEUDO-04

Perlorined by J. Luoma SAS verslon 9.3 08:07 30JAN14 
Mean treatment concentration by treatment tank and sampling location (bottom/suspended) for all sampling times

The MEANS Procedure

$\operatorname{tank}=$ ' ' loc $=$ ' '

\begin{tabular}{|c|c|c|c|}
\hline \multicolumn{4}{|c|}{$\begin{array}{c}\text { Analysis Variable : predicted_ppm Predicted } \\
\text { Value of conc }\end{array}$} \\
\hline Mean & Std Dev & $\begin{array}{r}\text { Lower } 95 \% \\
\text { CL for Mean }\end{array}$ & $\begin{array}{l}\text { Upper } 95 \% \\
\text { CL. for Mean }\end{array}$ \\
\hline & & & \\
\hline
\end{tabular}

$\operatorname{tank}=1$ loc $=$ bot

\begin{tabular}{|c|c|c|c|}
\hline \multicolumn{4}{|c|}{$\begin{array}{c}\text { Analysis Variable : predicted_ppm Prodicted } \\
\text { Value of conc }\end{array}$} \\
\hline Mean & Std Dev & $\begin{array}{r}\text { Lower } 95 \% \\
\text { CL. for Mean }\end{array}$ & $\begin{array}{r}\text { Upper } 95 \% \\
\text { CL for Mean }\end{array}$ \\
\hline 98.0221 & 22.8651 & 41.2221 & 154.8 \\
\hline
\end{tabular}

tank $=1$ loc $=$ sus

\begin{tabular}{|r|r|r|r|}
\hline \multicolumn{4}{|c|}{$\begin{array}{c}\text { Analysis Variable : predicted_ppm Predicted } \\
\text { Value of conc }\end{array}$} \\
\hline Mean & Std Dev & $\begin{array}{r}\text { Lower } 95 \% \\
\text { CL for Mean }\end{array}$ & $\begin{array}{r}\text { Upper } 95 \% \\
\text { CL for Mean }\end{array}$ \\
\hline 1.0976 & 2.9558 & -2.5726 & 4.7677 \\
\hline
\end{tabular}

tank $=2$ loc $=$ bot

\begin{tabular}{|c|c|c|c|}
\hline \multicolumn{4}{|c|}{$\begin{array}{c}\text { Analysis Variable : predlcted_ppm Predicted } \\
\text { Value of conc }\end{array}$} \\
\hline Mean & Std Dev & $\begin{array}{l}\text { Lower } 95 \% \\
\text { CL for Mean }\end{array}$ & $\begin{array}{l}\text { Upper } 95 \% \\
\text { CL for Mean }\end{array}$ \\
\hline 260.0 & 36.9169 & 168.3 & 351.7 \\
\hline
\end{tabular}

tank $=2$ loc $=$ sus

Analysis Varlable : predicted_ppm Predicted Value of conc

\begin{tabular}{|r|r|r|r|}
\hline Mean & Std Dev & $\begin{array}{r}\text { Lower 95\% } \\
\text { CL for Mean }\end{array}$ & $\begin{array}{r}\text { Upper 95\% } \\
\text { CL for Mean }\end{array}$ \\
\hline 1.7811 & 2.0161 & -0.7223 & 4.2844 \\
\hline
\end{tabular}

tank=4 loc =bot

Analysis Varlable : prodlcted ppon Predicted

\begin{tabular}{|c|c|c|c|}
\hline Mean & Std Dev & $\begin{array}{l}\text { Lowor } 95 \% \\
\text { CL for Mean }\end{array}$ & $\begin{array}{l}\text { Upper } 95 \% \\
\text { CL for Mean }\end{array}$ \\
\hline 1017 & $25=$ & 38.7200 & 1646 \\
\hline
\end{tabular}

$\operatorname{tank}=4$ loc $=s$ us

Analysis Varlable : predicted ppm Predicted 


\begin{tabular}{|r|r|r|r|}
\multicolumn{3}{|c|}{ Value of conc } \\
Mhean & Std Dev & $\begin{array}{r}\text { Lower } 95 \% \\
\text { CL for Mean }\end{array}$ & $\begin{array}{c}\text { Upper } 95 \% \\
\text { CL for Mean }\end{array}$ \\
\hline 9.0976 & 3.1841 & -2.8560 & 5.0511 \\
\hline
\end{tabular}

tank $=5$ loc $=$ bot

Analysis Variable : predicted ppm Prodicted

\begin{tabular}{|r|r|r|r|}
\multicolumn{4}{|c|}{ Value of conc } \\
\hline Mean & Std Dev & $\begin{array}{r}\text { Lower } 95 \% \\
\text { CL for Mean }\end{array}$ & $\begin{array}{r}\text { Upper 95\% } \\
\text { CL for Mean }\end{array}$ \\
\hline 235.2 & 39.1008 & 138.1 & 332.3 \\
\hline
\end{tabular}

tank $=5$ loc=sus

\begin{tabular}{|c|r|r|r|}
\hline \multicolumn{3}{|c|}{$\begin{array}{c}\text { Analysis Variable : predicted_ppm Predicted } \\
\text { Value of conc }\end{array}$} \\
\hline Mean & Std Dev & $\begin{array}{c}\text { Lower } 95 \% \\
\text { CL for Mean }\end{array}$ & $\begin{array}{r}\text { Upper } 95 \% \\
\text { CL for Mean }\end{array}$ \\
\hline-0.1328 & 1.1438 & -1.5530 & 1.2874 \\
\hline
\end{tabular}

tank $=8$ loc $=$ bot

\begin{tabular}{|c|r|r|r|}
\hline \multicolumn{3}{|c|}{ Analysis Varlable : predicted_ppm Predicted } \\
Value of conc
\end{tabular}

tank $=8$ loc $=$ sus

Analysls Varlable : predicted ppm Predicted

\begin{tabular}{|c|c|c|c|}
\hline Mean & Std Dev & $\begin{array}{l}\text { Lower } 95 \% \\
\text { CL for Mean }\end{array}$ & $\begin{array}{l}\text { Upper } 95 \% \\
\text { CL for Mean }\end{array}$ \\
\hline & 3.6682 & -3.8672 & 5. \\
\hline
\end{tabular}

\begin{tabular}{ll|l|l|l|l}
\hline 0.6874 & 3.6682 \\
\hline
\end{tabular}

5.2421

tank=9 locmbot

\begin{tabular}{|c|c|c|c|}
\hline \multicolumn{4}{|c|}{$\begin{array}{l}\text { Analysis Varlable : predicted_ppm Predicted } \\
\text { Value of conc }\end{array}$} \\
\hline Mean & Std Dev & $\begin{array}{l}\text { Lower } 95 \% \\
\text { CL for Mean }\end{array}$ & $\begin{array}{l}\text { Upper } 95 \% \\
C L \text { for Mean }\end{array}$ \\
\hline 208.8 & 31.1962 & 131.3 & 286.2 \\
\hline
\end{tabular}

tank $=9$ loc $=$ sus

Analysis Variablo: predicted ppm Predicted Value of cons
Ans

Lower 95\% Upper 95\%

Mean Std Dev, CL for Mean Cl. for Mean

\begin{tabular}{ll|l|l|l|l|l|l|}
\hline 5.1987 & 10.4157 & -7.7341 & 18.1315
\end{tabular}

Performed by J. Luoma SAS version 9.3 08:07 30JAN14 
AEH-12-PSEUDO-04

Mean treatment concentration by treatment group and sampling location for all sampling times

The MEANS Procedure

thero $=100=1$

\begin{tabular}{|l|l|}
\hline \multicolumn{2}{|c|}{ Analysis Variable : predlcted_ppm Predicted } \\
Value of conc
\end{tabular}

thero $=50$ loc $=$ bot

Analysis Variable : predicted_ppm Predlcted Value of conc

\begin{tabular}{l|l|l|l}
\hline & Lower 95\% & Upper 85\%
\end{tabular}

Mean Std Dev. CL. for Mean CL for Mean

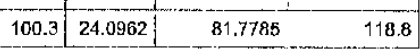

thero $=50$ loc $=$ sus

Analysis Vartablo : predicted_ppm Predictod

Value of conc

\begin{tabular}{|l|l|r|r|}
\hline Mean & Std Dev & $\begin{array}{r}\text { Lower } 95 \% \\
\text { CL for Mean }\end{array}$ & $\begin{array}{r}\text { Upper } 95 \% \\
\text { CL for Mean }\end{array}$ \\
\hline
\end{tabular}

\begin{tabular}{ll|l|l|}
\hline 0.9600 & 3.0459 & -0.7259 & 2.6476 \\
\hline
\end{tabular}

thero $=100$ loc $=$ bot

Analysls Varlable : predlcted_ppm Prodlcted

\begin{tabular}{|c|c|c|c|}
\hline Mer & Std Dev & $\begin{array}{l}\text { Lower } 95 \% \\
\text { CL for Moan }\end{array}$ & $\begin{array}{r}\text { Upper } 95 \% \\
\text { CL for Mean }\end{array}$ \\
\hline
\end{tabular}

\begin{tabular}{r|r|r|r|}
\hline 234.7 & 38.1989 & 205.3 & 264.0
\end{tabular}

thero $=100$ loc $=$ sus

Analysis Varlable : predicted_ppm Predicted

\begin{tabular}{|r|r|rr|}
\hline \multicolumn{3}{|c|}{ Value of conc } & . \\
Mean & Std Dev & Lower 95\% & Upper $95 \%$ \\
\hline 2.2823 & 6.1434 & -1.1198 & 5.6845 \\
\hline
\end{tabular}

Performed by J. Luoma SAS version 9.3 03:07 30JAN14 
Mean treatment concentration for each treatment group for each sampling time

The MEANS Procedure

thero $=$, time $=$ loc $="$ '

\begin{tabular}{|c|c|c|c|}
\hline \multicolumn{4}{|c|}{$\begin{array}{c}\text { Analysis Varlable : predicted_ppm Predlcted } \\
\text { Value of conc }\end{array}$} \\
\hline Mean & Std Dev & $\begin{array}{l}\text { Lower } 95 \% \\
\text { CL for Mean }\end{array}$ & $\begin{array}{l}\text { Upper } 95 \% \\
\text { CL for Mean }\end{array}$ \\
\hline & & . & \\
\hline
\end{tabular}

thero $=50$ t/me $=1$ loc $=$ sus

\begin{tabular}{|c|c|c|c|}
\hline \multicolumn{4}{|c|}{$\begin{array}{l}\text { Analysls Variable : predicted_ppm Predicted } \\
\text { Value of conc }\end{array}$} \\
\hline Mean & Std Dov & $\begin{array}{r}\text { Lowor } 95 \% \\
\text { CL for Mean }\end{array}$ & $\begin{array}{l}\text { Upper } 95 \% \\
\text { CL for Mean }\end{array}$ \\
\hline 1.0897 & 0.6835 & -2.7877 & 0.6032 \\
\hline
\end{tabular}

therg $=50$ tlme $=3$ loc $=$ sus

\begin{tabular}{|c|c|c|c|}
\hline \multicolumn{4}{|c|}{$\begin{array}{c}\text { Analysis Variable : prodicted_ppm Predicted } \\
\text { Value of conc }\end{array}$} \\
\hline Mean & Sid Dev & $\begin{array}{l}\text { Lower } 95 \% \\
\text { CL for Mean }\end{array}$ & $\begin{array}{l}\text { Upper } 95 \% \\
\text { CL for Mean }\end{array}$ \\
\hline$-1,3176$ & 0.3946 & -2.2979 & -0.3372 \\
\hline
\end{tabular}

thero $=50$ tim $=6$ loc $=$ bot

Analysis Varlable : predicted ppm Predleced

\begin{tabular}{|l|r|r|r|}
\multicolumn{5}{|c|}{ Value of conc } \\
\hline Mean & Std Dev & $\begin{array}{r}\text { Lower 96\% } \\
\text { CL for Mean }\end{array}$ & $\begin{array}{r}\text { Upper } 95 \% \\
\text { CL for Mean }\end{array}$ \\
\hline 127.0 & 12.3288 & 96.3317 & 157.6 \\
\hline
\end{tabular}

thero $=50$ time $=6$ loc $=s$ s

Analysis Variable : predicted_ppm Predicted Value of cons

Lower 95\% Upper 95\%

Mean Std Dev CL for Moan CL for Mean

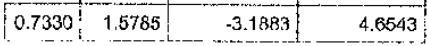

theru $=50$ time $=9$ loc $=$ bot

Analysis Variable : predictod ppm Predicted \begin{tabular}{l} 
Value of conc \\
\hline
\end{tabular}

Mean Std Dev $\begin{array}{r:r}\text { Lower 95\% } & \text { Upper Mean } 95 \% \\ \text { Cl. for Mean }\end{array}$

$99.38911^{10.2073}{ }^{\circ} \quad 74.0328 \quad 124.7$

thero $=\mathbf{5 0}$ timo $=9$ loc $=5$ us

Aralysis Variable : predicted_ppm Predicted Value of conc

file://C:IUsersłJLUOMAisashtrall htm 


$$
\begin{array}{|r|r|r|r|}
\text { Mean } & \text { Std Dev } & \begin{array}{c}
\text { Lowor } 95 \% \\
\text { CL for Mean }
\end{array} & \begin{array}{r}
\text { Upper } 95 \% \\
\text { CL for Mean }
\end{array} \\
\hline 0.0495 & 1.0441 & -2.5442 & 2.6432 \\
\hline
\end{array}
$$

thero $=50$ time $=12$ loc $=$ bot

\begin{tabular}{|c|c|c|c|}
\hline \multicolumn{4}{|c|}{$\begin{array}{l}\text { Analys is Variable : predicted_ppm Predicted } \\
\text { Value of conc }\end{array}$} \\
\hline Mean & Std Dev & $\begin{array}{l}\text { Lower } 95 \% \\
\text { CL for Mean }\end{array}$ & $\begin{array}{l}\text { Upper } 35 \% \\
\text { CL for Mean }\end{array}$ \\
\hline 74.5542 & 2.1972 & 69.0960 & 80.0125 \\
\hline
\end{tabular}

thero $=50$ time $=12$ locesus

\begin{tabular}{|c|c|c|c|}
\hline \multicolumn{4}{|c|}{$\begin{array}{c}\text { Analysis Variable : predicted_ppm Predicted } \\
\text { Value of conc }\end{array}$} \\
\hline Mean & Std Dev & $\begin{array}{l}\text { Lower } \\
\text { CL. for } M\end{array}$ & $\begin{array}{l}\text { Upper } \\
\mathrm{CL} \text { for } \mathrm{N}\end{array}$ \\
\hline 4 & 35 & 1 & 。 \\
\hline
\end{tabular}

thero $=100$ time $=1$ loc $=$ sus

\begin{tabular}{|c|c|r|r|}
\hline \multicolumn{4}{|c|}{ Analysis Variale ipredlcted_ppm Predlcted } \\
Value of conc \\
Mean & Std Dev & $\begin{array}{r}\text { Lower } 95 \% \\
\text { CL for Moan }\end{array}$ & $\begin{array}{r}\text { Upper } 95 \% \\
\text { CL for Moan }\end{array}$ \\
\hline 9.1632 & 12.0154 & -20.6846 & 39,0110 \\
\hline
\end{tabular}

thero $=100$ ulme $=3$ loc=sus

Analysis Variable : predictod ppm Predicted Value of conc

\begin{tabular}{r|r|r|r|} 
Mean & Std Dev & $\begin{array}{r}\text { Lower } 95 \% \\
\text { CL for Mean }\end{array}$ & $\begin{array}{r}\text { Upper } 95 \% \\
\text { CL for Mean }\end{array}$ \\
\hline-1.0897 & 0.6835 & -2.7877 & 0.6082 \\
\hline
\end{tabular}

thero $=100$ thme $=6$ loc $=$ bot

\begin{tabular}{|c|c|c|c|}
\hline \multicolumn{4}{|c|}{$\begin{array}{l}\text { Analysis Varlable : pred/cted_ppm Predicted } \\
\text { Value of conc }\end{array}$} \\
\hline Mean & Std Dev & $\begin{array}{l}\text { Lower } 95 \% \\
\text { CL. for Mean }\end{array}$ & $\begin{array}{l}\text { Upper } 95 \% \\
\text { CL for Mean }\end{array}$ \\
\hline 265.5 & 29.3440 & 192.6 & 33 \\
\hline
\end{tabular}

thero $=100$ time $=6$ loc $=s u s$

Analysis Varlable : predicted_ppm Predicted Value of conc

Lower $95 \%$ Upper 95\%

Mean Std Dev CL for Mean Cl. for Mean

\begin{tabular}{lllllll}
\hline-0.1784 & 2.1972 & -5.6366 & 5.2799 \\
\hline
\end{tabular}

ther $0=100$ time $=9$ loc $=$ bot

Analysis Variable : predicted ppm Predicted Analysis Variable : predicted_ppm Predicted
Value of conc 


\begin{tabular}{|c|c|c|c|}
\hline Mean & Std Dev & $\begin{array}{r}\text { Lower } 95 \% \\
\text { CL for Mean }\end{array}$ & $\begin{array}{r}\text { Upper } 95 \% \\
\text { CL for Mean }\end{array}$ \\
\hline \multicolumn{2}{|c|}{$\begin{array}{l:l}242,9 & 24.9559 \\
& \end{array}$} & 180.9 & 304.9 : \\
\hline \multicolumn{4}{|c|}{ thero $=100$ t $\mid m e=9$ loc } \\
\hline \multicolumn{4}{|c|}{$\begin{array}{c}\text { Analysis Varlable : predicted_ppm Predicted } \\
\text { Value of conc }\end{array}$} \\
\hline Mean & Std Dev & $\begin{array}{l}\text { Lower } 95 \% \\
\text { CL for Mean }\end{array}$ & $\begin{array}{l}\text { Upper } 95 \% \\
\text { CL for Mean }\end{array}$ \\
\hline 0.5052 & 1.7202 & -3.7680 & 4.7783 \\
\hline
\end{tabular}

thero $=100$ time $=12$ loc $=$ bot

\begin{tabular}{|r|r|r|r|}
\hline \multicolumn{3}{|c|}{ Analysis Varlable : predicted_ppm Predicted } \\
Value of conc
\end{tabular}

thero $=100$ time $=12$ locesus

\begin{tabular}{|c|c|c|c|}
\hline \multicolumn{4}{|c|}{$\begin{array}{c}\text { Analysis Variable : predicted_ppm Predicted } \\
\text { Value of conc }\end{array}$} \\
\hline Mean & Std Dev & $\begin{array}{r}\text { Lower } 95 \% \\
\text { CL for Mean }\end{array}$ & $\begin{array}{r}\text { Upper } 95 \% \\
\text { CL for Mlean }\end{array}$ \\
\hline 3.0114 & 2.9794 & -4.3899 & 10,4128 \\
\hline
\end{tabular}

Performed by J. Luoma SAS version 9.3 08:07 30JAN14 
AEH-12PSEUDO-04

Mean concentration for standard checks for all sampling times

The MEANS Procedure

thero $=25$

\begin{tabular}{|c|c|c|c|}
\hline \multicolumn{4}{|c|}{$\begin{array}{l}\text { Analysis Variable : predicted_ppm Predictod } \\
\text { Value of conc: }\end{array}$} \\
\hline Moan & Std Do & $\begin{array}{l}\text { Lower } 95 \% \\
\text { CL for Mean }\end{array}$ & $\begin{array}{l}\text { Upper } 95 \% \\
\mathrm{CL} \text { for Mean }\end{array}$ \\
\hline 6946 & 1.044 & 19.1009 & 24.2883 \\
\hline
\end{tabular}

thero $=50$

Analysis Variable : predleted_ppm Predicted Value of conc

\begin{tabular}{|r|r|r|r|}
\hline Mean & Std Dov & Lower 95\% & Upper 95\% \\
\hline 44.2011 & 1.4229 & 40.7164 & 47.7857 \\
\hline
\end{tabular}

thero $=100$

Analysis Variable : predlcted_ppm Predicted

\begin{tabular}{|r|r|r|r|}
\multicolumn{4}{|c|}{ Value of conc } \\
\hline Mean & Std Dev & $\begin{array}{r}\text { Lower 95\% } \\
\text { CL for Mean }\end{array}$ & $\begin{array}{r}\text { Upper 95\% } \\
\text { CL. for Mean }\end{array}$ \\
\hline 90.5032 & 0 & \\
\hline
\end{tabular}

thero $=200$

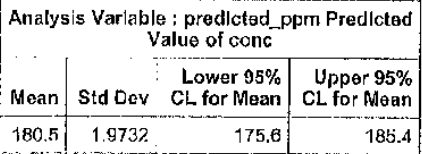

180.5 1.9732 $175.6-185.4$

Performed by J. Luome SAS version 9.3 08:07 30JAN14 $\zeta \Lambda^{-}$

\author{
File Folder: 120 Hem Number: 4
}




\begin{tabular}{|c|c|c|c|}
\hline \multirow{5}{*}{$\begin{array}{l}\text { Study Number: AEH-12-PSEUDO-04 } \\
\text { Electronic Lab Notebook (jages } 25 \cdot 27) \\
\text { Data Souice: Flle FokJer: } 14 \mathrm{c} \\
\text { Forms: "Sample Absorbance Readings" Data Sheet }\end{array}$} & Action & TDate & innttals \\
\hline & Created...... & 6-Feb-14 & TIS ThS \\
\hline & Revlsed...... & $12-\mathrm{feb}-14$ & TIS 015 \\
\hline & Revlewed... & $(2\{E(B) 4$ & +13 \\
\hline & Certiffled......... & $210 / 14$ & $y^{A}-$ \\
\hline
\end{tabular}

Spectrop notometric Data

Test Art de: Zequanox ${ }^{\circ}$ (MD]-401 SDP)

Test Art cle Lot \#: 401P12163C and 4e1P12169C Mix

Exposure Date: September 8, 2012

Test Locatlon: Lake Shawano, Shawano, wh

Treatment Trpe: Bottom In]ectlon

Data Explanatian:

1) The absorbance of triplicate samples of $25,50,10 \mathrm{C}$, and $200 \mathrm{mg} / \mathrm{L}$ dillutions of a $2,000 \mathrm{mg} / \mathrm{L}$ active ingredlent (A,L) stack prepared from Analytical Stock \#/2 were

measurec to prepare a stand ard curve.

2) S:andard checks were performed at 6,9 , and 12 hours by comparing the $25,50,100$, and $200 \mathrm{me} / \mathrm{L}$ (A., I. d dlutions to the lir ear curve.

3) Oata cocies used within SAS

tank $=\operatorname{Tank} 10$ (1 through 9)

thero $=$ Theoretical or target concentration (ing/L)

time $=$ Sample T.me $\{0,1,3,6,9$, and $\mathrm{z} 2 \mathrm{~h}$ after treatinent

$\mathrm{loc}=$ Sample Location

sus = Suspended Sample (sampled $\sim 15 \mathrm{cn}$ from bottom of tank)

sur $\rightarrow$ Surface Sample

abs $=$ measured absorbance of sample

conc = concentratlon ([ng/ $\mathrm{Ll}$, coly used for standards used for regression]

4) Information that is not relevent to a sample (L.e., tenk III for standards) or that will oe calculated by SAS (L.e., predicted concentration for standard checks and samples) is denoted by a "." in the SAS input and outpidt files.

Data Analysls:

1) A llnear regression was completed in SAS Using the abscrbance values obtalned from the spectrophotometer of 3 replicate dilutions of 25, 50, 100 and $200 \mathrm{mg} / \mathrm{h}$

2) Standard checks and treatment sample concentrations were predicted In SAS by conparing the observed absorbances with the linear regression.

3) The 'cllowing mean treatment concentrations were determined In SAS:

3a) Mcan (standard devation) conce tration by tank and locatlon for all sampling ilmes

36) Mean (standarc devlation) concentration by treatment group ane location for all sampling times

3c) Mean (standard devlatlon) concentration by treatment groujp and sampling times for bot? locations

30) Mean (standard devlation) concentrations for $25,50,100$, and $200 \mathrm{mg} / \mathrm{L}$ (A.I.) dillutions for all sampling tlmcs

File Names:

Spectrophotometric Data sor SAS input

I:AEH-12.PSEUDC-04|Data Summaries\spec)|Lake Shawano Bottom Iniect|on Spec Summary.k1sx]Spec Data for 5AS

SAS Program/Code

I:AAEI-12-PSEUDC-04\SAS-Speclshewano injection prceran file

SAS LOE

1. AEEH-12-PSE JDQ-04\SAS-Spect3hawano injection log fil

SAS Jutput

I:AEH-12-PS:UDO-04|SAS-SpecłShawano injection results file

Da:o Anomalies and Devlations:

1) One exposure tank of a different concentrat on was sampled in triplicate to evaluate variability of spectrophotometer derlng each sampling tlme. The triplicate sanple was only taken from suspended (sus) sampling locations. The mean absorbance of the triplleate samples was imported into SAS for usc in the analysls

2) Surface samples (sur) were collected by submerging a collection beaker below the s.ufface of each exposu*e tank; suspended samples (sus) were collected $15 \mathrm{~cm}$ from the exposure tank bottom for ali sampling times.

the exposure tank bottom for ali samping times.

3) Some rrean absorbances for triphitate samples may be recorded Incorrectly on "Sampla Absorbance Readings" data farms as proper signintcant figure rules may not have been observed. Additlonally, concentratlons recorded on "Sample Absortance. Readines" data forms were not used in the analysls as the initial Ir ear rcegrcsslon equation that was used for these calculations was derlved uslrg rounded absorbar ce values in Excel, All absorbances and concentrations used in SAS calculations and reported
within Spectrophotometrlc Data Summary have been sorrected.

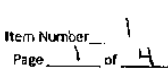

File Folder: 14

$$
\text { Page. } 1 \text { of } 4
$$




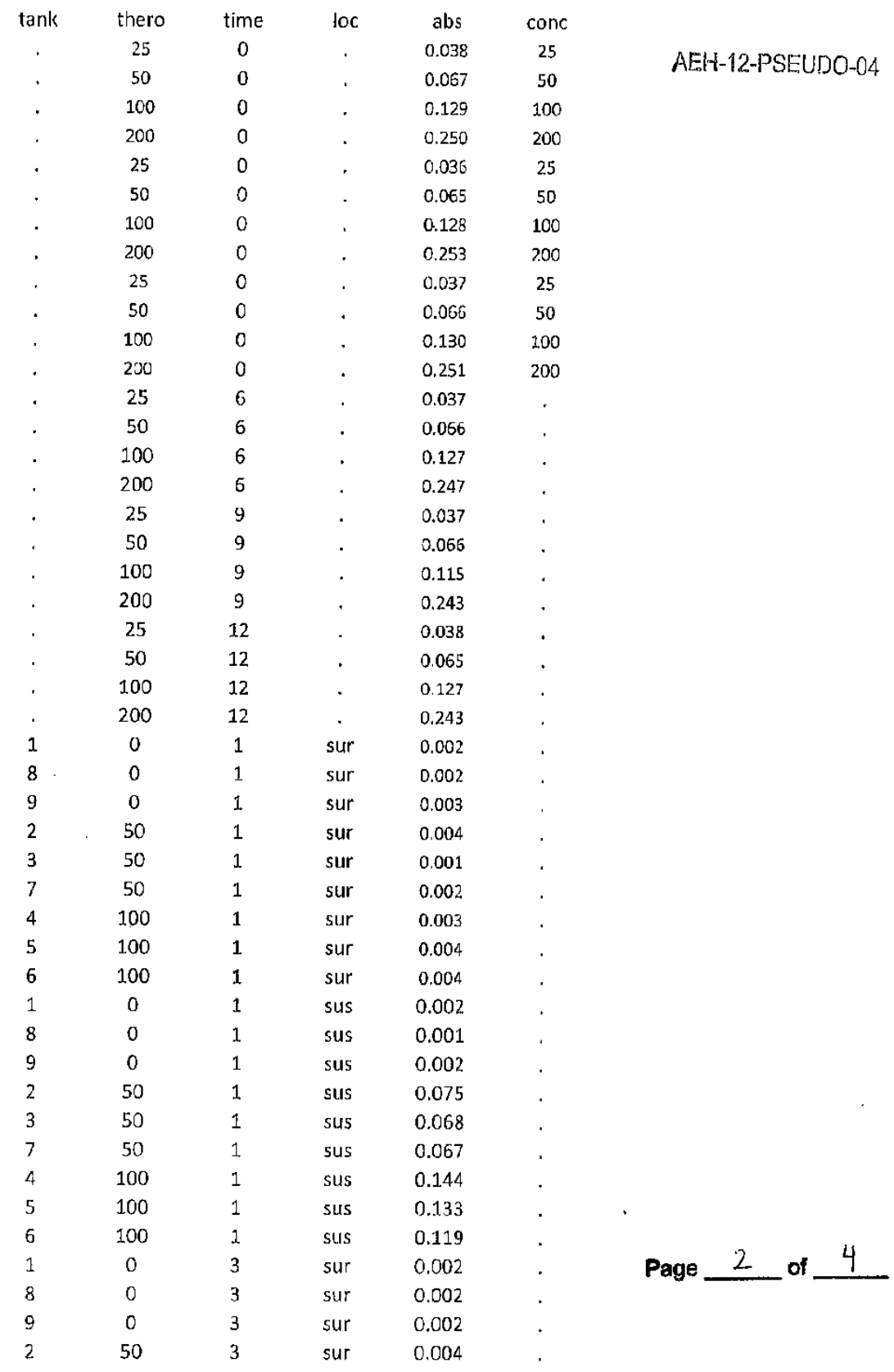




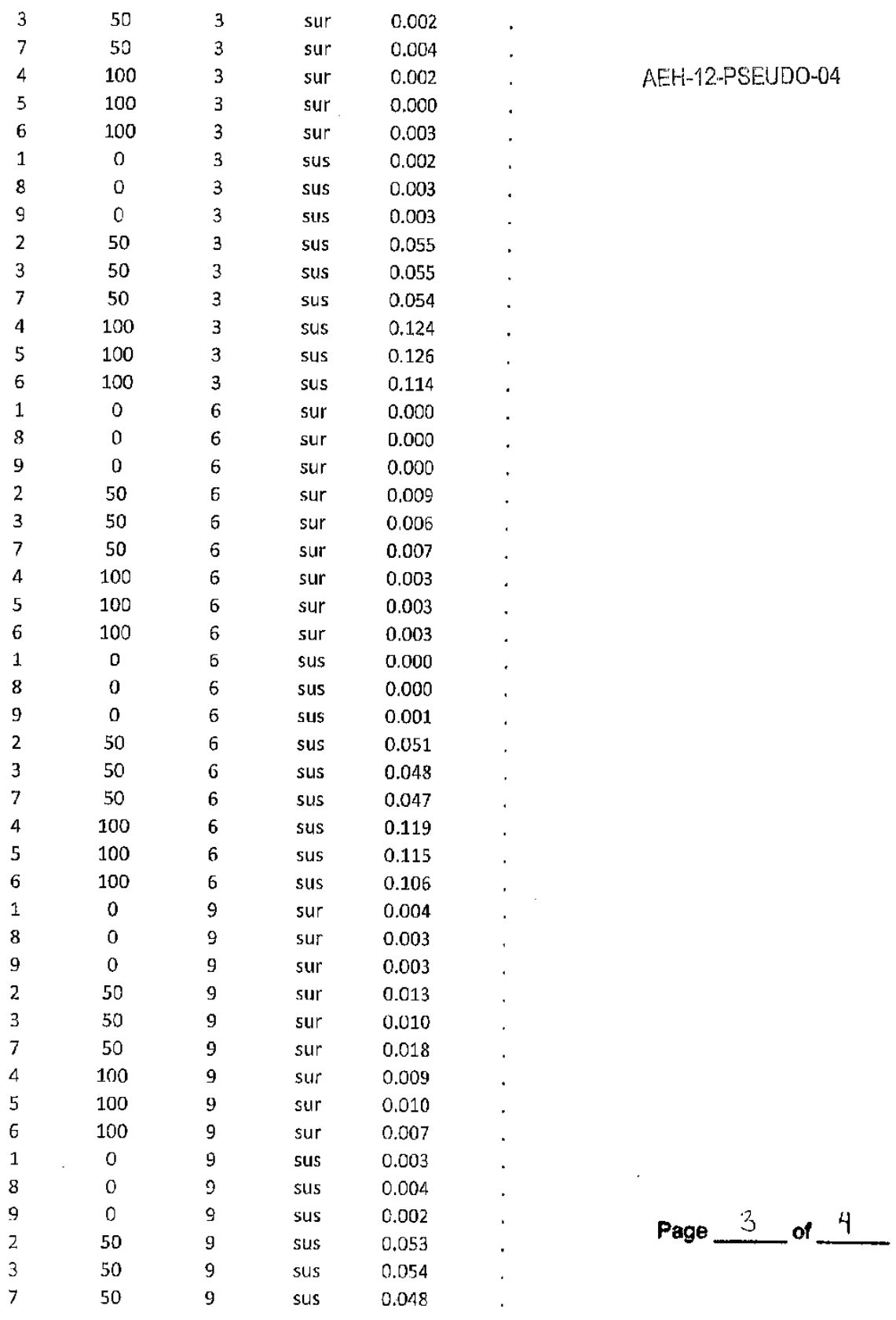




\begin{tabular}{|c|c|c|c|c|c|c|}
\hline 4 & 100 & 9 & sus & 0.127 & . & \\
\hline 5 & 100 & 9 & sus & 0.119 & . & AEH.12.PSEUDO-04 \\
\hline 6 & 100 & 9 & sus & 0.114 & . & \\
\hline 1 & 0 & 12 & sur & 0.004 & . & \\
\hline 8 & 0 & 12 & sur & 0.004 & . & \\
\hline 9 & 0 & 12 & sur & 0.004 & . & \\
\hline 2 & 50 & 12 & sur & 0.016 & . & \\
\hline 3 & 50 & 12 & sur & 0.015 & . & \\
\hline 7 & 50 & 12 & sur & 0.028 & . & \\
\hline 4 & 100 & 12 & sur & 0.010 & . & \\
\hline 5 & 100 & 12 & sur & 0.011 & . & \\
\hline 6 & 100 & 12 & sur & 0.009 & . & \\
\hline 1 & 0 & 12 & sus & 0.003 & . & \\
\hline 8 & 0 & 12 & sus & 0.004 & . & \\
\hline 9 & 0 & 12 & sus & 0.004 & . & \\
\hline 2 & 50 & 12 & sus & 0.053 & . & \\
\hline 3 & 50 & 12 & sus & 0.046 & . & \\
\hline 7 & 50 & 12 & sus & 0.025 & ' & \\
\hline 4 & 100 & 12 & sus & 0.115 & . & \\
\hline 5 & 100 & 12 & sus & 0.113 & 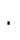 & \\
\hline 6 & 100 & 12 & sus & 0.109 & . & \\
\hline
\end{tabular}

File Folder: $\frac{14 c}{C}$ ftem Number: 11 Page 4 
ods html close; $/ *$ close previous */;
ods html; $/ *$ open new */;

ods graphics on;

AEH-12-PSEUDO-04

DM 'LOG; CLEAR; OUTPUT; ClEAR;'; * CLEAR LOG AND OUTPUT;

FOOTNOTE1 'Performed by $J$. LuOma SAS version ' \&SYSVER \&SYSTIME \&SYSDATE;

options ls=97 ps=54 formdIim=' 1 pageno $=1$ nocenter nodate nosource2;

title1 $h=1$ 'Standard Curve Linear Regression and sample concentrations';

title2 $h=1$ 'Study \# AEH-12-PSUEDO-04';

title3 $h=1$ 'Shawano Lake-injection treatment';

title4 $h=1$ 'SAS v. 9.3 Analysis completion date: 30Jan2014 Analysis prepared by: JAL';

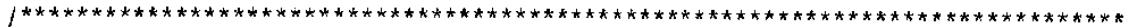

* SAS ver $9.3 \quad$ Anlysis prepared by: JAL Page _ of _ _

* Analysis completion dates 30Jarn2014 Sn

data Zeq; set shawano.shawanoinjection;

run;

proc sort;

by tank tine 100; run;

run;

proc gplot data= zeq;

plot abs * conc;

run;

proc reg data $=z e q$;

model conc $=$ abs /edf;

output out=output_out $p=p$ redicted_ppin;

run;

proc sort;

by time tank loc;

proc print data=output_out;

run;

data zeq2; set output_out;

if tank $=$ "." then delete;

if $100=$ ". "then delete;

if time $=$ " 0 " then delete;

run;

proc sort;

by tank loc;

run;

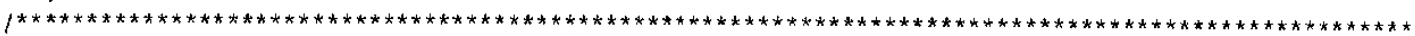

* This procedure produces the mean concentrations for each treatment replicate over all sampling tim

* by the the sampling location

* i.e. It gives the mean concentration of each treatment tank over the entire exposure by the sampl

* location [i.e. sur.face vs suspended sampling

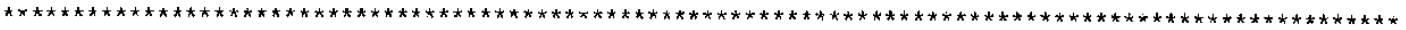

title "Mean treatment concentration by treatnent tank and sampling locazion (surface/suspended) for a proc means data $=$ zeq2 mean std $1 \mathrm{clm} u c l m$ fw $=8$;

by tank loc;

var predicted_ppm;

run;

proc sort;

File Foider: $14 \mathrm{C}$ Item Number: 2

Page 1 of 2 
by thero time loc;

AEH-12-PSEUDO-04

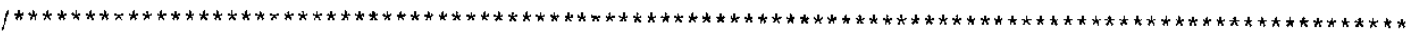

* This procedure produces the mean concentrations for each treatment group over ald sampling times

* by the sampling iocation

* i.e. It gives the mean concentration of the 3 control, 50ppm \& 100 ppm treatment tanks by

* sanpling location (surface/suspended) over the entire exposure

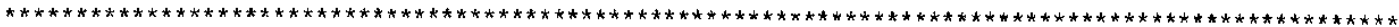
ti.tle "Mean treatment concentration by treatnent group ard sampling location for all sampling times"; proc sort;

by thero 100;

proc means data $=$ zeg2 mean stc $1 \mathrm{clm}$ uclm $\mathrm{fw}=8$;

by thero loc:

var prodictod_ppm;

run;

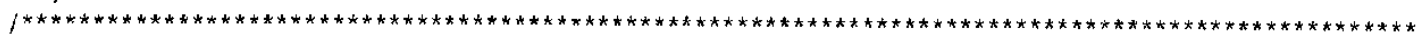

* This procedure produces the mean concentrations for each treatment group by sampling time

* i.e. It gives the mean conc. of the 3 control,50ppm \& 100ppm treatment tanks at time 1, $3,6,9$ al

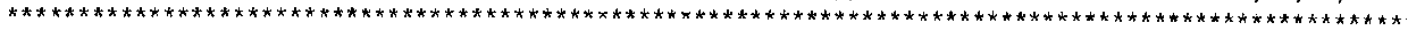
title "Wean treatment concentration for each treatment group for each sanpling time";

proc sort;

by thero time loc;

proc means data $=$ zeq2 mean std 1c.lm uclm $f w=8$;

by thero time $20 \mathrm{c}$;

var predicted_ppm;

run;

data zeq3; set output_out;

if conc $>1$ then delete;

if tank > 0.5 thon delete;

if thero $=$ ", " then delete;

run;

proc sort;

by thero;

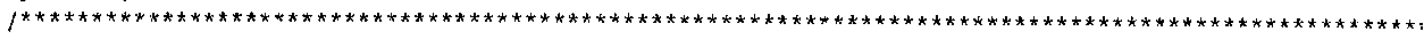

* This procedure produces the mean concentrations for the standard checks for all time periods

* i.e. It gives the mean conc, of the 50ppn: \& 100ppm standard checks at 6 , 9 and $12 \mathrm{~h}$

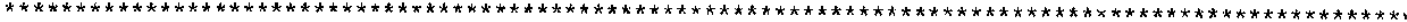

title "Mean coneentration for standard checks for all sanipling times";

proc means data $=$ zeq3 mean std $1 \mathrm{clm}$ uclin $f \mathrm{~W}=8$;

by thero:

var predicted ppm;

run;

quit

run;

$$
\begin{aligned}
& 1 / 30 / 14 \\
& 512
\end{aligned}
$$

Fille Folder: $\quad 14 c$

Item Number: 2

Page 2 of 2 


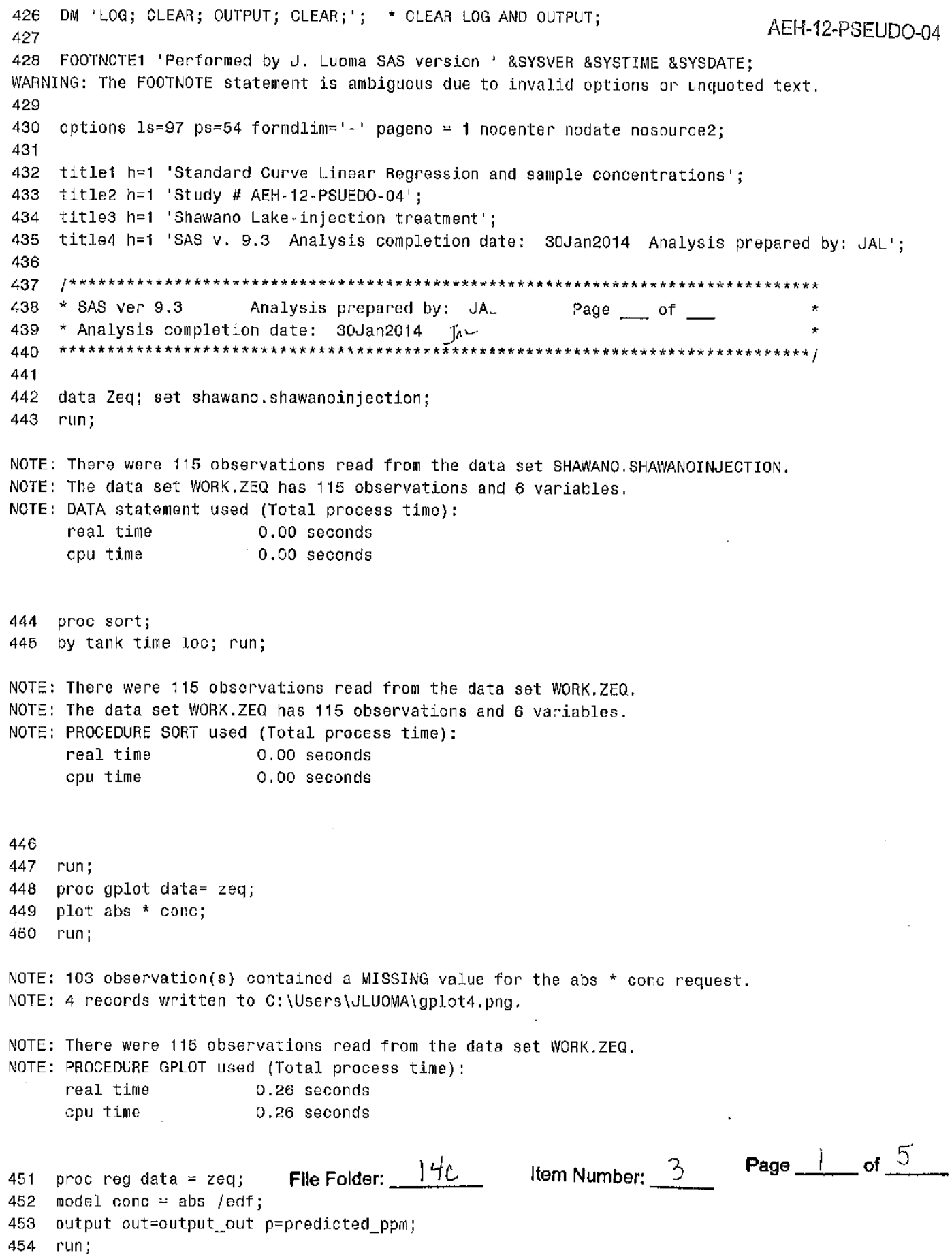


NOTE: The data set WORK.OUTPUT_OUT has 115 observations and 7 variables,

AEH-12-PSEUDO-04

$\begin{array}{rr}\text { NOTE: } & \text { PROCEOURE REG used (Total proces } \\ \text { real time } & 1.06 \text { seconds } \\ \text { cpu time } & 0.40 \text { seconds }\end{array}$

455 proc sort;

456 by time tank loc;

NOTE: There were 115 observations read from the data set WORK.OUTPUT_OUT.

VOTE: The data set WORK.OUTPUT_OUT has 115 observations and 7 variables.

NOTE: PROCEDURE SORT used (Total process time):

$\begin{array}{ll}\text { real time } & 0.00 \text { seconds } \\ \text { cpu time } & 0.01 \text { seconds }\end{array}$

457 proc print data=output_out;

458 run;

NOTE: There were 115 observations read from the data set WORK.OUTPUT_OUT.

NOTE: PROCEDURE PRINT used (Total process time):
real time
0.06 seconds

cpu time

0.06 seconds

459 data zeq2; set output_out;
460 if tank = ". " then delete;
461 if loc =". " then delete;
462 if time = "O" then delete;
463 run;

NOTE: Character values have been converted to numeric values at the places given by: (Line) : (Column). 462:11

NOTE: There were 115 observations read fron the data set WOFK.OUTPUT_OUT.

NOTE: The data set WORK.ZEQ2 has 91 observations and 7 variables.

NOTE: DATA statement used (Total procoss time):

real time $\quad 0.01$ seconds

cpu time $\quad 0.01$ seconds

464 proc sort;

465 by tank 100;

466 run;

NOTE: There were 91 observations read from the data set WORK.ZEQ2.

NOTE: The data set WORK.ZEQ2 has 94 observations and 7 variables.

NOTE: PROC:DOURF. SORT used (Total process tine):
real time
0.00 seconds

cpu time

0.00 seconds

Page 22 of 5

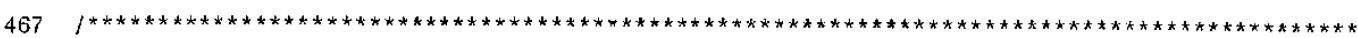

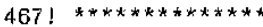




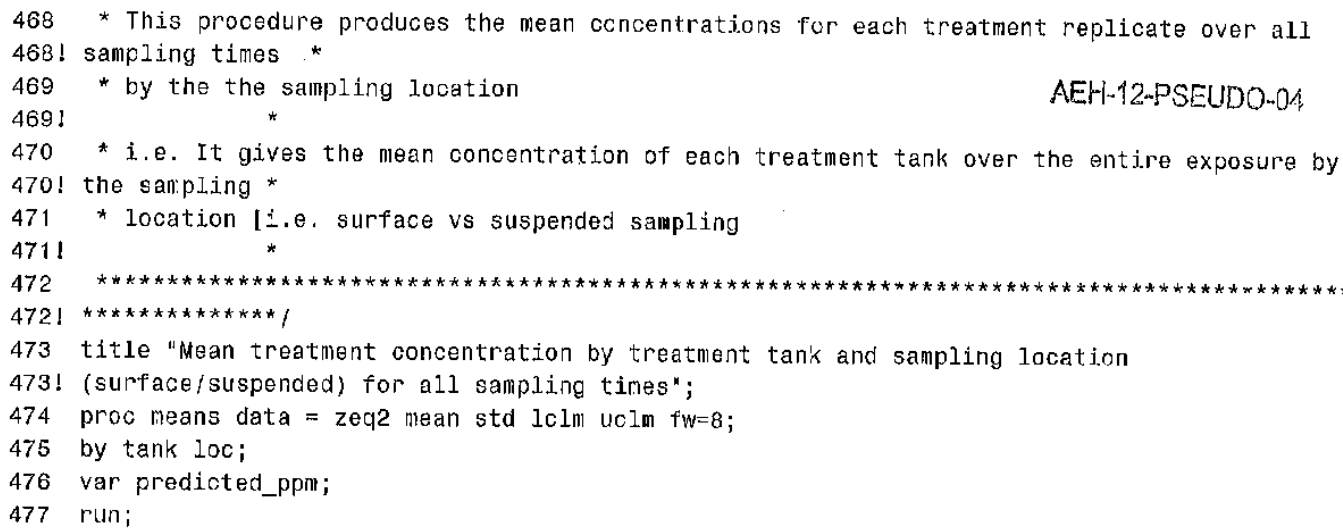

NOTE: Therc wore 91 observations read from the data set WORK.ZEQ2.

NOTE: The data set WORK,ZEQ2 has 91 observations and 7 variables.

NOTE: PROCEDURE SORT used (Total process time):
real time
0.00 seconds
cpu time
0.01 seconds 


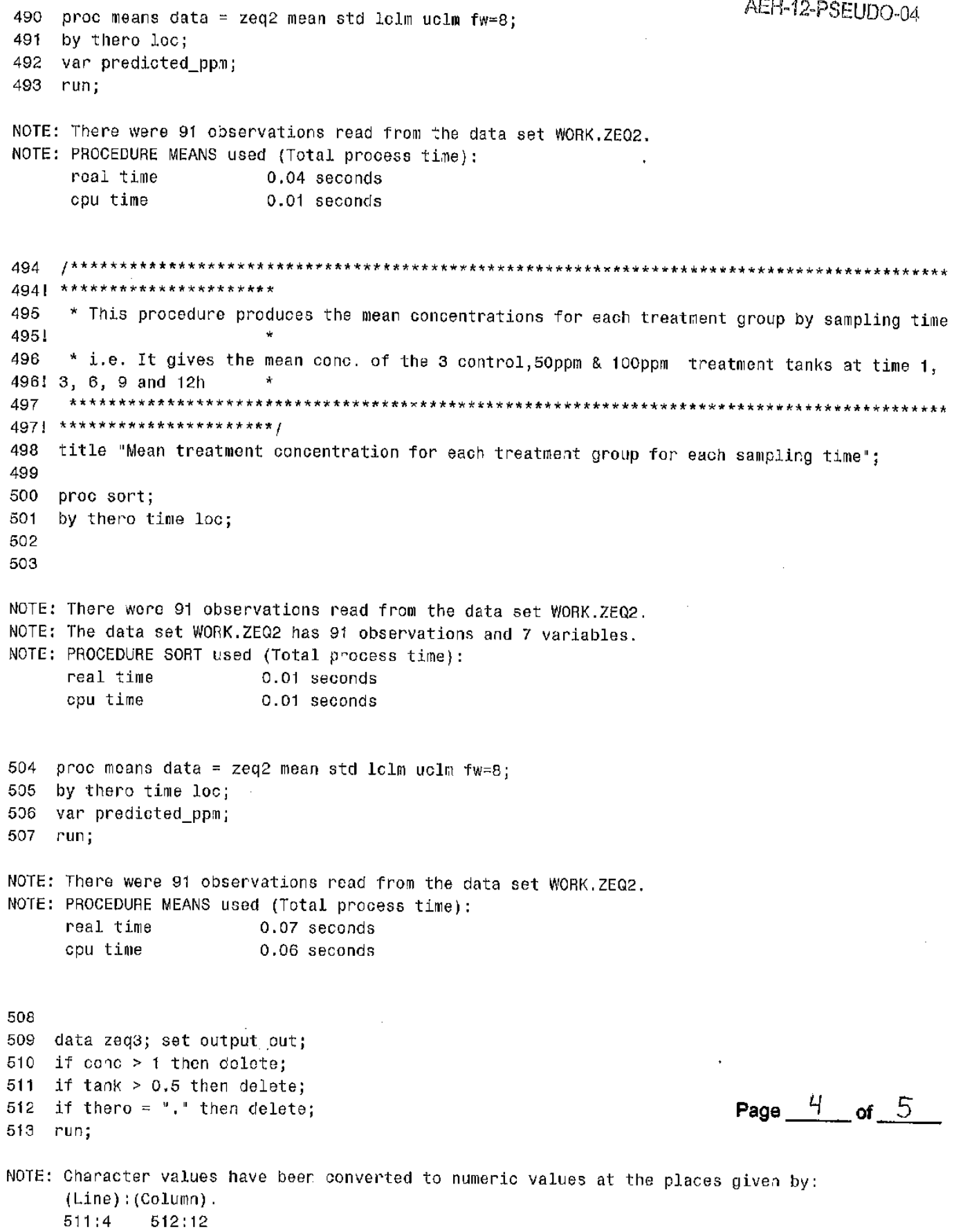

NOTE: Character values have beer converted to numeric values at the places given by: (Line) : (Column) . 
NOTE: There were 115 observations read from the data set WORK.OUTPUT_OUT.

NOTE: The data set WORK.ZEQ3 has 12 observations and 7 variables.

NOTE: DATA statement used (Total process time):

real time $\quad 0.01$ seconds

cpu time $\quad 0.01$ seconds

514

515 proc sort;

516 by thero;

$517 \mathrm{I}^{* * * * * * * * * * * * * * *}$

$517 ! * * * * * * * * * * * * * *$

518 * This procedure produces the mean concentrations for the standard checks for all time

518 ! periods

$519 *$ i.e. It gives the mean conc. of the 50ppn \& 100ppr standard checks at 6,9 and $12 \mathrm{~h}$ $519 !$

$520 * * * * * * * * * * * * * * * * * * * * * * * * * * * \pi * * * * * * * * * * * * * * * * * * * * * * * * * * * * * * * * * * * * * * * * * * * * * * * * * * * * * * * * * * * * * * * *$

$520 ! * * * * * * * * * * * * * *$ !

521 title "Mean concentration for standard checks for all sampling times";

NOTE: There were 12 observations read from the data set WORK.ZEQ3.

NOTE: The data set WORK.ZEQ3 has 12 observations and 7 variables.

NOTE: PROCEDURE SORT used (Total process time):
real time
0.01 seconds
cpu time
0.01 seconds

52.2 proc means data $=$ zeq3 mean std $1 \mathrm{clm}$ uclin f $w=8$;

523 by thero;

524 var predicted_ppn;

525 run;

NOTE: There were 12 observations read from the data set WORK.ZEQ3.

NOTE: PROCEDURE MEANS used (Total process ti.me):
real time
0.04 seconds
cpu time
0.03 seconds

526 quit;

527 run;

$\sqrt{5}$

File Folder: $14 c$ Item Number: 3 Page 3 - 3 of 5 


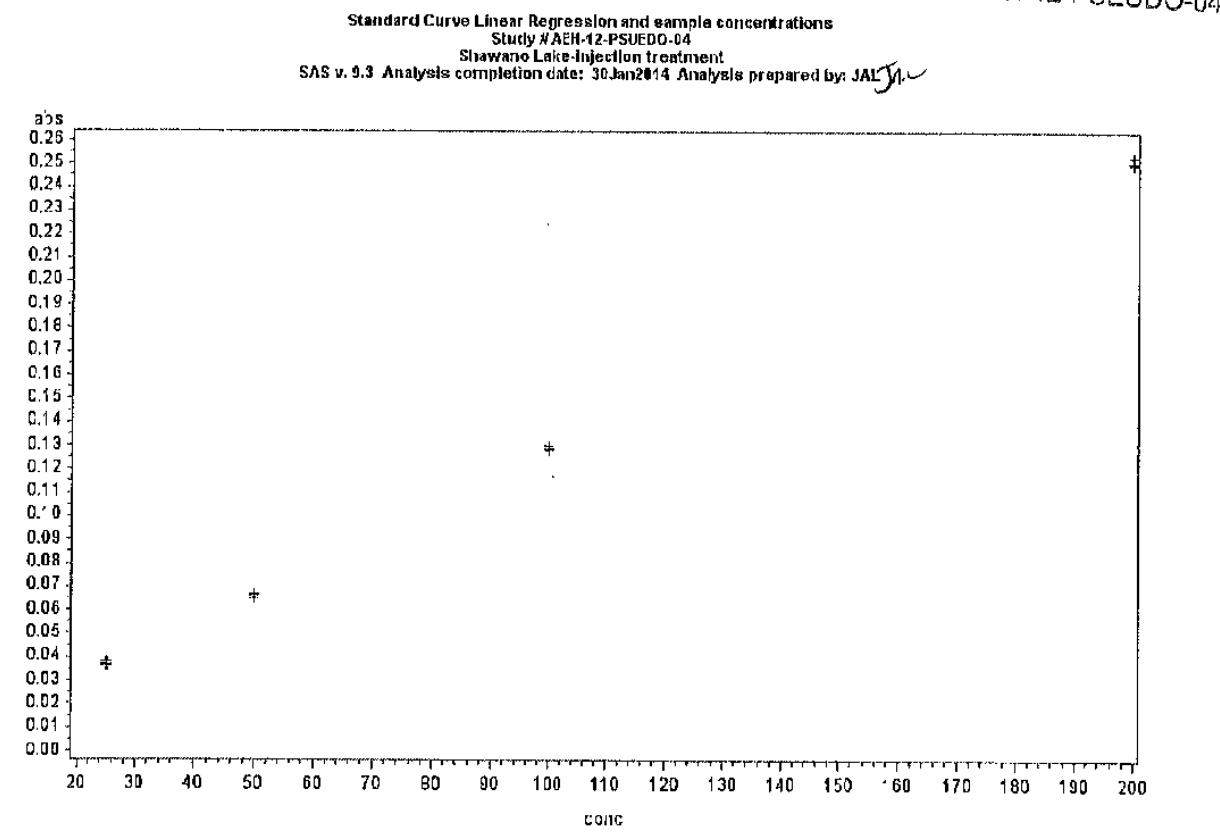

Perlormed by J. Luoma SAS version 9.3 08:J7 30.JAN14

File Folder: $14 \mathrm{c} \quad$ Item Number: 4 
Standard Curvo Linear Regresslon and sample concentratlons

Study H AEH-12-PSUEDO.04

Shawano Lako-Injoction treatmont
SAS vi B.3 Anabysis complation date: 30Jan2014 Analyssts proparesd by: JAL

The REG Procedure

Model: MODEL1

Dependent Varlable: conc conc

Number of Observations Read

\begin{tabular}{|l|l|}
\hline Number of Observations Used & 115 \\
\hline
\end{tabular}

\begin{tabular}{|lll} 
Number of Observations with Missing Values 103 \\
\hline
\end{tabular}

\begin{tabular}{|l|r|r|r|r|r|}
\hline \multicolumn{5}{|c|}{ Analysis of Varlance } \\
\hline Source & DF & $\begin{array}{r}\text { Sum of } \\
\text { Squares }\end{array}$ & $\begin{array}{r}\text { Mean } \\
\text { Square }\end{array}$ & F Value & Pr $>$ F \\
\hline Model & 1 & 53896 & 53896 & 50557.1 & $<.0001$ \\
\hline Error & 10 & 10.66036 & 1.06603 & & \\
\hline Corrected Total & 11 & 53906 & & & \\
\hline
\end{tabular}

\begin{tabular}{|l|l|l|l|l|l|}
\hline Rool MSE & 1.03249 & R.-Square & 0.9998 \\
\hline
\end{tabular}

\begin{tabular}{lll|l|l|l}
\hline Dependent Mean & 93.75000 & Ad] R-Sq & 0.9998
\end{tabular}

\begin{tabular}{l|l}
\hline Coeff Var & 1.10132 \\
\hline
\end{tabular}

\begin{tabular}{|l|l|r|r|r|r|r|}
\hline \multicolumn{7}{|c|}{ Paramotor Estimates } \\
\hline Varlable & Label & DF & $\begin{array}{r}\text { Paramefer } \\
\text { Estlmata }\end{array}$ & $\begin{array}{r}\text { Standard } \\
\text { Error }\end{array}$ & $\mathrm{t}$ Valuo & Pr $>|\mathrm{t}|$ \\
\hline Intercept & Intercept & 1 & $-4,56863$ & 0.52919 & $-8,63$ & $<.0001$ \\
\hline abs & abs & 1 & 813.67186 & 3.61875 & 224.85 & $<.0001$ \\
\hline
\end{tabular}

Performed by J. Luoma SAS version 9.3 08:07 30JAV14 
Standard Curve Linatr Regresslon and sample concentrations

Study \# AEH-12.PSUEDO-04

Sliawano Lake-Jnject|or Irealmont

SAS v. 9.3 Analysls coinpletion date; 30.Jan2C14 Analysls propared ty: JAL.

The REG Procedure

Model: MODEL1

Dependent Variable: conc conc

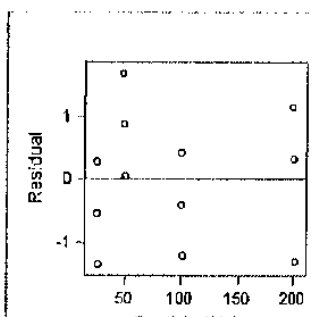

Fit Diagnostlics for cone

Predicled Value
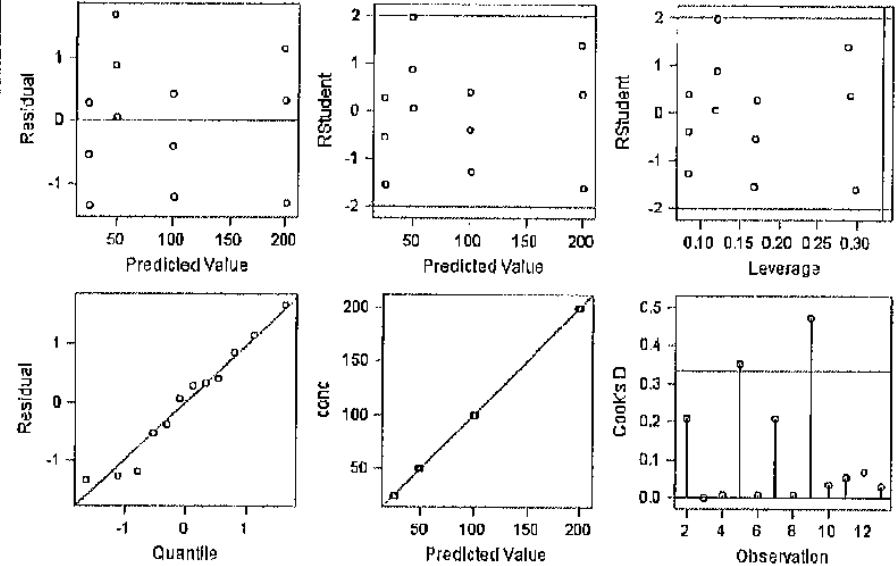

Leverage
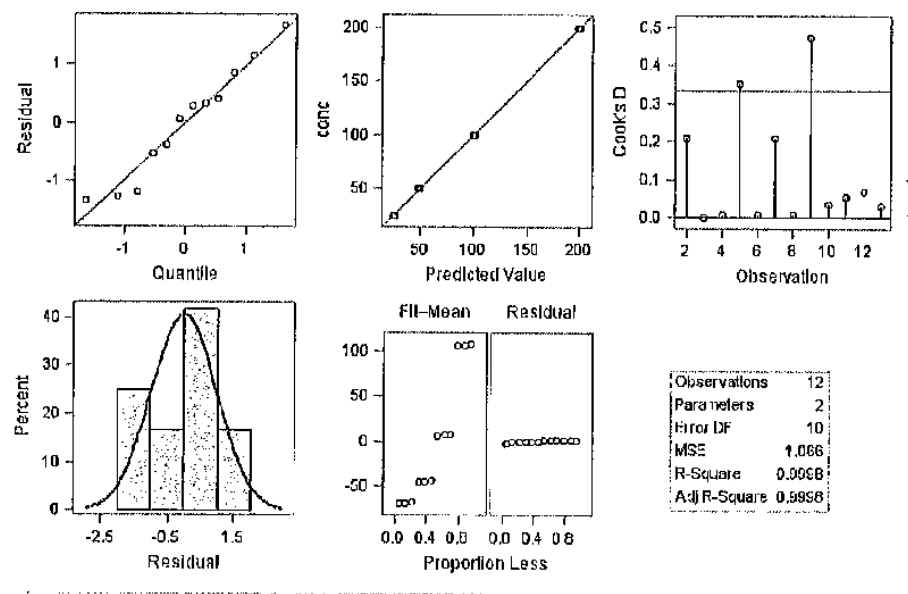
Proportion Less

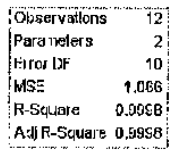

Adj R-Square 0.5958 

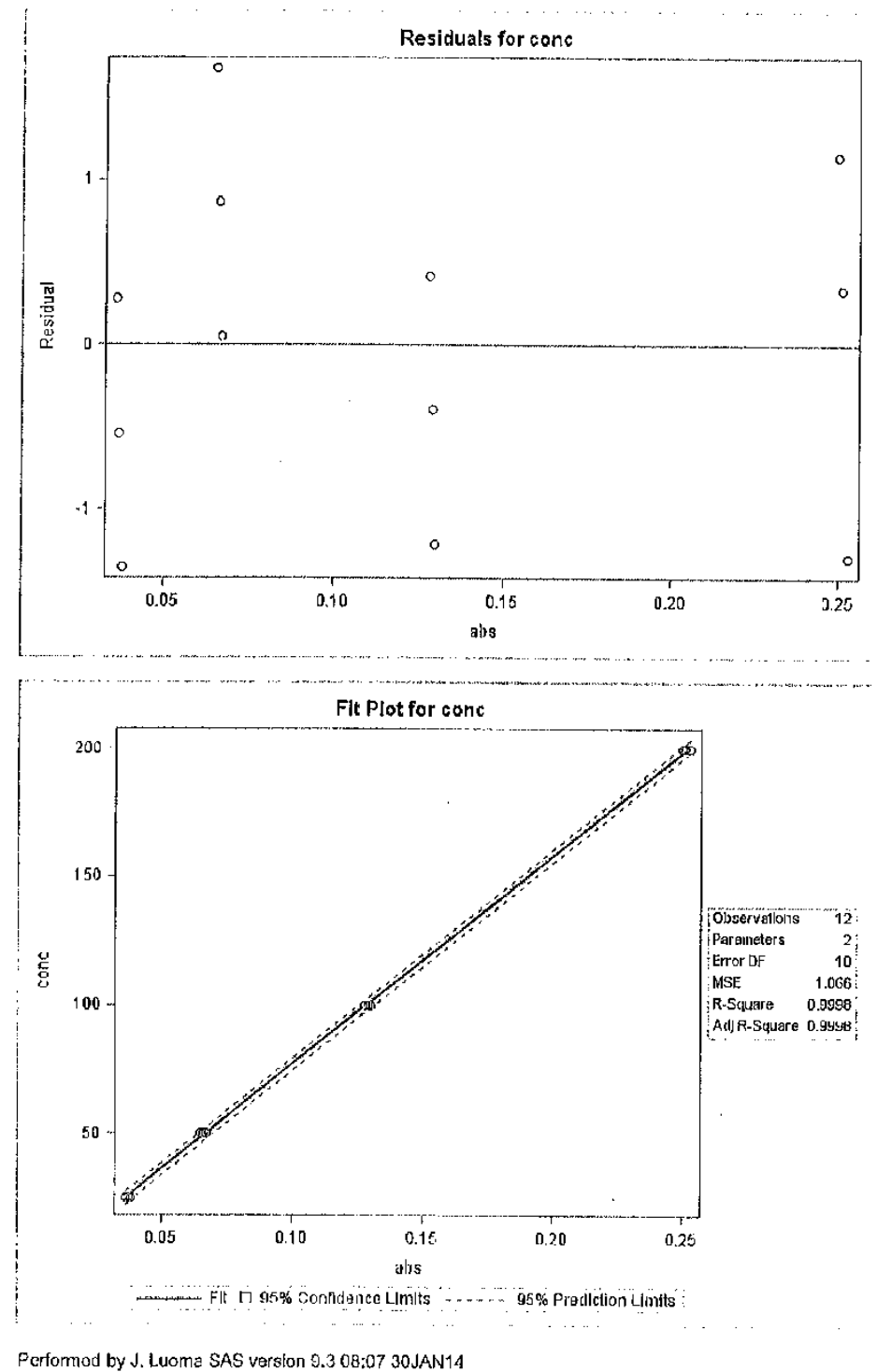

file:/C: IUsersIJLUOMAisashtml4.htm 


\begin{tabular}{|c|c|c|c|c|c|c|c|}
\hline Obs & tank & thero & time & 100 & abs & conc & predlcted_ppm \\
\hline 1 & &.$i$ & i & & & & \\
\hline 2 & & 25 & 0 & . & 0.03800 & 25 & $26.35^{n}$ \\
\hline 3 & . & 50 & 0 & . & $0.067 \mathrm{Co}$ & 50 & 49.947 \\
\hline 4 & . & $100 !$ & 0 & 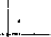 & 0.12900 & 100 & 100.395 \\
\hline $\mathbf{5}$ & 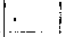 & $2 \mathrm{CO}$ & 0 & & 0.25000 & 200 & 198.84 \\
\hline 6 & . & $2 E$ & 0 & 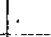 & 0.03600 & 25 & 24.724 \\
\hline 7 & . & 50 & 0 & 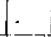 & 0.06500 & 50 & 48.320 \\
\hline 8 & · & 100 & 0 & . & 0.12800 & 100 & 99.581 \\
\hline 9 & & 200 & 0 & . & 0.25300 & 200 & 201,290 \\
\hline 10 & . & 25 & 0 & & 0.03700 & 25 & 25.537 \\
\hline 11 & . & 50 & 0 & & 0.06630 & 50 & 48,134 \\
\hline 12 & . & 100 & 0 & & 0.13000 & 100 & 101.209 \\
\hline 13 & . & 200 & 0 & & 0.25100 & 200 & 199.663 \\
\hline 14 & 1 & 0 & 1 & sur & 0.00200 & & -2.941 \\
\hline 15 & 1 & 0 & 1 & sus & 0.00200 & & -2.941 \\
\hline 16 & 2 & 50 & 1 & sur & 0,00400 & & -1.314 \\
\hline 17. & 2 & 50 & 1. & sus & 0.07500 & & 56.457 \\
\hline 18 & 3 & 50 & 1 & sur & 0.00100 & & -3.755 \\
\hline 19 & 3 & 50 & 1 & sus & 0.06800 & & 50.761 \\
\hline 20 & 1 & 100 & 1 & sur & 0.00300 & & $-2,12$ \\
\hline 21 & 4 & 100 & 1 & sus & 0.14400 & & 112.600 \\
\hline 22 & 5 & 100 & 1 & sur & 0.00400 & & -1.314 \\
\hline 23 & 5 & 100 & 1 & sus & 0.13300 & & $\mathbf{1 0 3 , 6 5 0}$ \\
\hline 24 & 6 & 100 & 1 & sur & 0.00400 & & -1.314 \\
\hline 25 & 6 & 100 & 1 & sus & 0.11900 & & 02.258 \\
\hline 26 & 7 & 50 & 1. & sur & 0.00200 & & -2.841 \\
\hline 27 & 7 & 50 & 1 & sus & 0.06700 & & 49.947 \\
\hline 28 & 8 & 0 & 1 & sur & 0.00200 & & -2.941 \\
\hline 29 & 8 & 0 & 1 & sus & 0.00100 & & -3.755 \\
\hline 30 & 9 & 0 & 1 & sur & 0.00300 & & -2.128 \\
\hline 31 & 9 & 0 & 1 & sus & 0.00200 & & -2.941 \\
\hline 32 & 1 & $\underline{0}$ & 3 & sur & 0,00200 & & -2.941 \\
\hline 33 & 1 & 0 & 3 & sus & 0.00200 & & -2.941 \\
\hline 34 & 2 & 50 & 3 & sur & 0.00400 & & -1.314 \\
\hline 35 & 2 & 50 & 3 & sus & 0.05500 & & 40.183 \\
\hline 36 & 3 & 50 & 3 & sur & 0.00200 & & -2.941 \\
\hline 37 & 3 & 50 & 3 & sus & 0.05467 & & 39.912 \\
\hline 38 & 4 & 100 & & sur & $0.002 C 0$ & & -2.941 \\
\hline
\end{tabular}




\begin{tabular}{|c|c|c|c|c|c|c|c|}
\hline 39 & 4 & $100:$ & 3 & sus & 0.12400 & . & 96.327 \\
\hline 40 & 5 & 100 & 3 & sur & 0.00000 & , & -4.569 \\
\hline 41 & 5 & 100 & 3 & sus: & 0.12600 & & 97.954 \\
\hline 42 & 6 & 100 & 3 & sur & 0,00300 & & -2.128 \\
\hline 43 & 6 & ${ }^{\circ} 00$ & 3 & sus & $0.11<00$ & & 88.190 \\
\hline 44 & 7 & $50 !$ & 3 & sur & 0.00400 & . & -1.314 \\
\hline 45 & 7 & $50 !$ & 3 & sus & 0.05400 & . & 39.370 \\
\hline 46 & 8 & 0 & 3 & sur & 0.00200 & . & -2.841 \\
\hline 47 & 8 & 0 & 3 & sus & 0.00300 & . & -2.128 \\
\hline 48 & 9 & 0 ? & 3 & sur & 0.00200 & . & $-2.84^{4}$ \\
\hline 49 & 9 & 0 & 3 & sus: & 0.00300 & & -2.128 \\
\hline 50 & . & 25 & $\theta$ & . & $0.037 \mathrm{Co}$ & & 25.537 \\
\hline 51 & . & so, & 6 & . & 0.06600 & & 49.134 \\
\hline 52 & . & 100 & 6 & . & 0.12700 & & 98.768 \\
\hline 53 & , & 200 & 6 & . & 0.24700 & & 196,408 \\
\hline 54 & 1 & 0 & 6 & sur & 0.00000 & & $-4,569$ \\
\hline 55 & 1 & 0 & 0 & sus & 0.00000 & & $-4.5 e 0$ \\
\hline 56 & 2 & 50 & 6 & sur & 0.00900 & & 2.754 \\
\hline 57 & 2 & 50 & 6 & sus & 0.05100 & & 36.929 \\
\hline 58 & 3 & 60 & 6 & sur & $c .00600$ & & 0.313 \\
\hline 59 & 3 & 50 & 6 & sus & 0.04800 & & 34.488 \\
\hline 60 & 4 & 100 & 6 & sur & 0.00300 & & -2.128 \\
\hline 61 & 4 & 100 & 6 & sus & 0.11900 & & 92.258 \\
\hline 62 & 5 & 100 & 6 & sur & 0.00300 & & -2.128 \\
\hline 63 & 5 & 100 & 8 & stus & 0.11500 & & 89.004 \\
\hline 64 & 6 & 100 & 6 & sur & 0.00300 & & -2.128 \\
\hline 65 & 6 & 100 & 6 & sus & 0.10600 & & 81,681 \\
\hline 66 & 7 & 50 & 6 & sur & 0.00700 & & 1.127 \\
\hline 67 & 7 & 50 & 6 & sus & 0.04733 & & 33.945 \\
\hline 68 & 8 & 0 & 8 & sur! & 0.00000 & & -4.569 \\
\hline 69 & 8 & 0 & 6 & sus, & 0.00000 & & -4.569 \\
\hline 70 & $\mathrm{~g}$ & 0 & 6 & sur & 0.00000 & & -4.569 \\
\hline 71 & 9 & 0 & 0 & sus & $0.00: 00$ & & -3.755 \\
\hline 72 & . & 25 & 9 & 1. & 0.03700 & & 25.537 \\
\hline 73 & . & 50 & 9 & . & 0.06600 & & 49.134 \\
\hline 74 &. & $\cdot 00$ & 9 & . & 0.11500 & & 89.004 \\
\hline 75 & : & 200 & 9 & .1 & 0.24300 & & 193.151 \\
\hline 76 & 1 & 0 & 9 & sur? & 0.00400 & & -1.314 \\
\hline 77 & 1 & 0 & 9 & sus: & 0.00300 & & -2.128 \\
\hline 78 & 2 & 50 & 9 & sur : & 0.01300 & & 6.009 \\
\hline 79 & 2 & 50 & 9 & sus & 0.05300 & & 38.556 \\
\hline 80 & 3 & 50 & 9 & sur: & 0.01600 & & $\begin{array}{l}3.568 \\
-1.6 .\end{array}$ \\
\hline 81 & & & & & & & \\
\hline
\end{tabular}




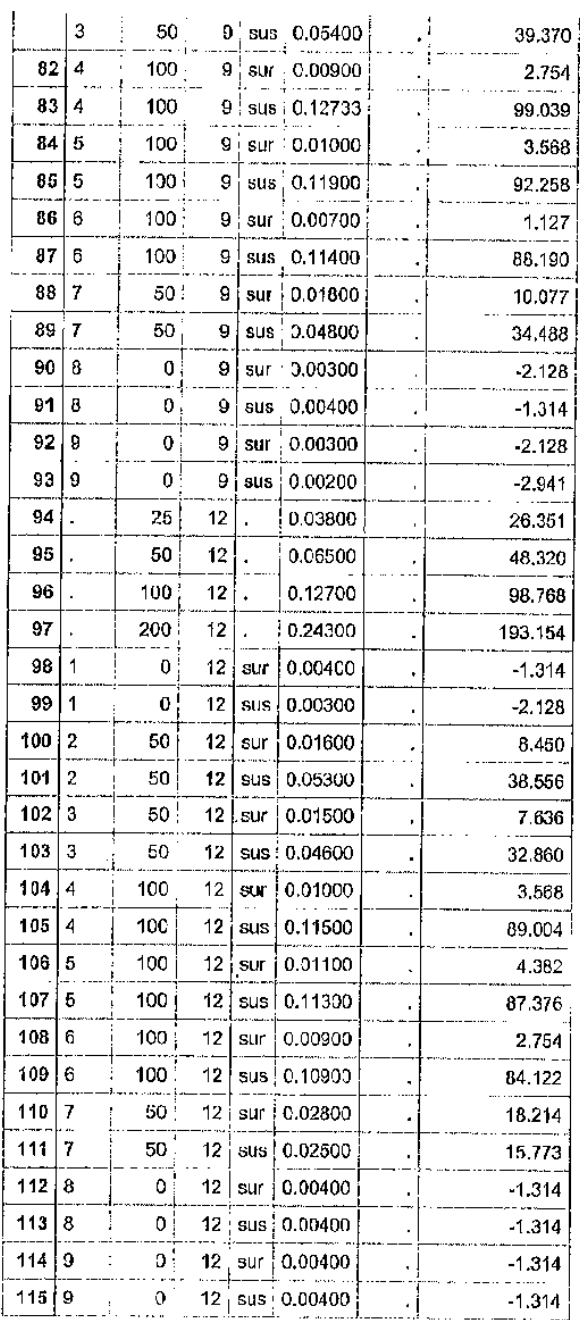

AEL-T2 FSEUIDO (j/

Pertormed by J. Luoma SAS version $9.308: 07$ 30JAV14 
Mean treatment concentration by treatment tank and sampling location (surfaceisuspended) for all sampling times

The MEANS Procedure

tank $=$ " ' loc $=$ "

\begin{tabular}{|c|c|c|c|}
\hline \multicolumn{4}{|c|}{$\begin{array}{l}\text { Analysls Variable : prodicted ppm Predlated } \\
\text { Value of conc }\end{array}$} \\
\hline Nean & Std Dev & $\begin{array}{l}\text { Lower } 95 \% \\
\text { CL for Mean }\end{array}$ & $\begin{array}{r}\text { Upper } 95 \% \\
\text { CL for Mean }\end{array}$ \\
\hline & & & \\
\hline
\end{tabular}

tank $=1$ loc $=$ sur

\begin{tabular}{|c|c|c|c|}
\hline \multicolumn{4}{|c|}{$\begin{array}{c}\text { Analysis Variable : predlcted_ppm Predicted } \\
\text { Value of conc }\end{array}$} \\
\hline Mean & Std Dev & $\begin{array}{r}\text { Lower } 95 \% \\
\text { CL for Mean }\end{array}$ & $\begin{array}{l}\text { Upper } 95 \% \\
\text { CL for Mean }\end{array}$ \\
\hline-2.6159 & 1.3616 & -4.3064 & -0.8253 \\
\hline
\end{tabular}

$\operatorname{tank}=1$ loc=sus

\begin{tabular}{|c|c|c|c|}
\hline \multicolumn{4}{|c|}{$\begin{array}{l}\text { Analysis Variable : predicted_ppm Predlcted } \\
\text { Value of conc }\end{array}$} \\
\hline Masan & Std Dev & $\begin{array}{l}\text { Lower } 95 \% \\
\text { CL for Mean }\end{array}$ & $\begin{array}{l}\text { Upper } 95 \% \\
\text { CL for Mean }\end{array}$ \\
\hline-2.9413 & 0.9965 & .4 .1787 & -1.7040 \\
\hline
\end{tabular}

tank=2 loc=sur

Analysis Variable : prodlctod_ppm Predicted

\begin{tabular}{|r|r|r|r|}
\hline \multicolumn{3}{|c|}{ Value of conc } \\
\hline Mean & Std Dev & $\begin{array}{r}\text { Lower } 95 \% \\
\text { CL. for Mean }\end{array}$ & $\begin{array}{r}\text { Upper 95\% } \\
\text { CL for Mean }\end{array}$ \\
\hline 2.9171 & 4.3590 & -2.4954 & 8.3296 \\
\hline
\end{tabular}

$\operatorname{tank}=2$ loc $=$ sus

\begin{tabular}{|c|c|c|c|}
\hline \multicolumn{4}{|c|}{$\begin{array}{c}\text { Analysis Variable : predleted_ppm Predlicted } \\
\text { Value of conc }\end{array}$} \\
\hline Mean & Std Dev & $\begin{array}{r}\text { Lower } 95 \% \\
\text { CL for Mean }\end{array}$ & $\begin{array}{r}\text { Upper } 95 \% \\
\text { CL for Mean }\end{array}$ \\
\hline 42.1361 & 8.0878 & 32,0939 & 52.1784 \\
\hline
\end{tabular}

tank=3 locesur

Analysls Variable : predicted ppm Predlcted Value of conc

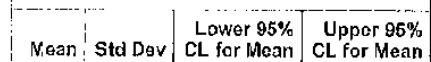

$\begin{array}{llll}0.9643 & 4.7235 & -4.9007 & 6.8293\end{array}$

tank $=3$ loc $=$ sus

Analysis Variable : predicted_ppm Predicted 


\begin{tabular}{|c|c|c|c|}
\hline \multicolumn{4}{|c|}{ Value of conce } \\
\hline Mean & Std Dev & $\begin{array}{l}\text { Lower } 95 \% \\
\text { CL for Mean }\end{array}$ & $\begin{array}{l}\text { Upper } 95 \% \\
\text { CL for Mean }\end{array}$ \\
\hline 39.4781 & 7.0037 & 30.7819 & 48.1743 \\
\hline
\end{tabular}

tank $=4$ loc $=\mathbf{s u r}$

\begin{tabular}{|r|r|r|r|}
\hline \multicolumn{3}{|c|}{ Analysis Variable : predicted_ppm Predicted } \\
Value of conc
\end{tabular}

tank $=4$ loc $=s u s$

Analysis Variable : prodicted_ppm Predlcted
Value of conc

tank=5 locesur

\begin{tabular}{|c|c|c|c|}
\hline \multicolumn{4}{|c|}{$\begin{array}{c}\text { Analysis Varlable : predicted_pprn Predicted } \\
\text { Value of conc }\end{array}$} \\
\hline Mean & Std Dev & $\begin{array}{r}\text { L.ower } 95 \% \\
\text { CL. for Mean }\end{array}$ & $\begin{array}{r}\text { Upper } 95 \% \\
\mathrm{CL} \text { for Mean }\end{array}$ \\
\hline-0.0121 & 3.8424 & -4.7831 & 4.7588 \\
\hline
\end{tabular}

tank $=5$ loc $=$ sus

\begin{tabular}{|c|c|c|c|}
\hline \multicolumn{4}{|c|}{$\begin{array}{c}\text { Analys /s Varlable : predicted_ppm Predicted } \\
\text { Value of conc }\end{array}$} \\
\hline Mean & Std Dev & $\begin{array}{r}\text { Lower } 95 \% \\
\text { Cl. for Mean }\end{array}$ & $\begin{array}{r}\text { Upper } 95 \% \\
\text { CL for Mean }\end{array}$ \\
\hline 94.0483 & 6.7196 & 85.7049 & 102.4 \\
\hline
\end{tabular}

tank $=6$ loc $=$ sur

\begin{tabular}{|r|r|r|r|}
\hline \multicolumn{3}{|c|}{ Analysis Variable : predicted_ppm Predicted } \\
Value of conc
\end{tabular}

lank $=6$ loc $=$ sus

Analysis Variable : predicted_ppm Predictod \begin{tabular}{|r|r|r|}
\multicolumn{3}{|c}{ Valuo of conc } \\
Mean & Std Dev & Lower $95 \%$ \\
Cl. for Mean & Upper $95 \%$ \\
CL for Mean
\end{tabular} \begin{tabular}{|r|r|r|r|} 
Mean & Std Dev $\mid$ Cl. for Mean & CL for Mean \\
\hline 86.8880 & 4.0927 & 81.8063 & 91.9698
\end{tabular}

$\operatorname{tank}=7$ loc $=5$ sur

Analysis Varlable : predicted_ppm Predicted 


\begin{tabular}{|r|r|r|r|}
\hline \multicolumn{3}{|c|}{ Value of conc } \\
\hline Mean & Std Dev & Lower $96 \%$ & $\begin{array}{r}\text { Upper } 95 \% \\
\text { CL for Mean }\end{array}$ \\
\hline CL for Mean
\end{tabular}

tank $=7$ loc $=$ sus

\begin{tabular}{|r|r|r|r|}
\hline \multicolumn{2}{|c|}{ Analysis Variable : predicted_ppm Predlcted } \\
Value of conc
\end{tabular}

tank $=8$ locssur

\begin{tabular}{|c|c|c|c|}
\hline \multicolumn{4}{|c|}{$\begin{array}{c}\text { Analysis Variablo : predicted_ppm Predicted } \\
\text { Value of conc }\end{array}$} \\
\hline Mean & Std Dev & $\begin{array}{r}\text { Lower } 95 \% \\
\text { CL for Mean }\end{array}$ & $\begin{array}{r}\text { Upper } 95 \% \\
\text { CL for Mean }\end{array}$ \\
\hline-2.7786 & 1.2069 & -4.2771 & $-1,2801$ \\
\hline
\end{tabular}

tank $=8$ loc $=$ sus

\begin{tabular}{|c|r|r|r|}
\hline \multicolumn{3}{|c|}{ Analysis Variable : predleted_ppm Predlcted } \\
Value of conc \\
\hline Mean & Std Dev & $\begin{array}{c}\text { Lower } 95 \% \\
\text { CL for Mvan }\end{array}$ & $\begin{array}{c}\text { Upper } 95 \% \\
\text { CL for Mean }\end{array}$ \\
\hline-2.6150 & 1.4781 & -4.4512 & -0.7806 \\
\hline
\end{tabular}

tank=9 loc $=$ sur

\begin{tabular}{|c|c|c|c|}
\hline \multicolumn{4}{|c|}{$\begin{array}{l}\text { Analysis Varlable : predlcted_ppm Predicted } \\
\text { Value of conc. }\end{array}$} \\
\hline Mean & Std Doy & $\begin{array}{r}\text { Lower } 95 \% \\
\text { CL. for Mean }\end{array}$ & $\begin{array}{r}\text { Upper } 95 \% \\
\text { CL for Mean }\end{array}$ \\
\hline 6159 & 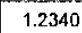 & -4.1 & -1 \\
\hline
\end{tabular}

tank $=9$ loc $=$ sus

Analysls Variablo : prodictod ppm Predicted

\begin{tabular}{|c|c|c|c|}
\hline Mean & Std Dev & $\begin{array}{r}\text { Lower } 95 \% \\
\text { CL for Mean }\end{array}$ & $\begin{array}{l}\text { Upper } 95 \% \\
\text { CL for Mean }\end{array}$ \\
\hline 6159 & 0.9277 & $-3,7678$ & -1.4 \\
\hline
\end{tabular}

Performed by J. zuorna SAS version 9.3 08:07 30.JAN 14 
Mean treatment concentration by treatment group and sampling location for all sampling times The MEANS Procedure

thero $=.10 c={ }^{\prime \prime}$

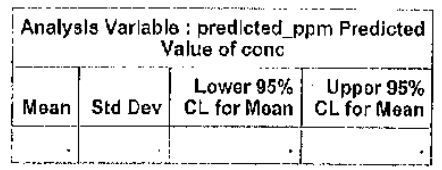

thero $=0$ loc=sur

Analysis Varlablo : predicted_ppm Predicted Value of conc

\begin{tabular}{|c|c|c|c|}
\hline Mean & Std Dev & $\begin{array}{l}\text { Lower } 95 \% \\
\text { CL for Moan }\end{array}$ & $\begin{array}{l}\text { Upper } 95 \% \\
\text { CL for Mean }\end{array}$ \\
\hline & 1778 & 3737 & \\
\hline
\end{tabular}

therc $=0$ loc $=$ sus

\begin{tabular}{|r|r|r|r|}
\hline \multicolumn{3}{|c|}{ Analysis Variable : predicted_ppm Predlcted } \\
Value of conc
\end{tabular}

thero=50 loc=sur

\begin{tabular}{|c|c|c|c|}
\hline \multicolumn{4}{|c|}{$\begin{array}{l}\text { Analysis Varlable : predicted_ppm Predicted } \\
\text { Value of conc }\end{array}$} \\
\hline Mean & Std Dev & $\begin{array}{r}\text { Lower } 95 \% \\
\text { CL for Mean }\end{array}$ & $\begin{array}{l}\text { Upper } \mathbf{9 5 \%} \\
\text { Cl for Mean }\end{array}$ \\
\hline 2.9713 & 6.1236 & -0.4198 & 6.3625 \\
\hline
\end{tabular}

thero $=50$ loc $=5$ us

Analysls Variable : predicted_ppm Predicted

\begin{tabular}{|r|r|r|r|}
\multicolumn{3}{|c|}{ Value of conc } \\
Mean & Sid Dev & $\begin{array}{r}\text { Lower } 95 \% \\
\text { CL for Mean }\end{array}$ & $\begin{array}{r}\text { Upper } 95 \% \\
\text { CL for Mean }\end{array}$ \\
\hline 38.7729 & 9.3074 & 33.6187 & 43.9272 \\
\hline
\end{tabular}

thero $=100$ loc $=$ sur

\begin{tabular}{|c|c|c|c|}
\hline \multicolumn{4}{|c|}{$\begin{array}{c}\text { Analysis Variable : predlcted_ppm Predicted } \\
\text { Value of conc }\end{array}$} \\
\hline Mean & Std Dev & $\begin{array}{r}\text { Lower } 95 \% \\
\text { CL for Mean }\end{array}$ & $\begin{array}{r}\text { Upper } 95 \% \\
\text { CL. for Mean }\end{array}$ \\
\hline-0.1749 & $2,88 \cdot 7$ & -1.7707 & 1.4210 \\
\hline \multicolumn{4}{|c|}{ thero $=100$ loc $=$ sus } \\
\hline \multicolumn{4}{|c|}{$\begin{array}{c}\text { Analysis Variable : preticted_ppm Predicted } \\
\text { Value of conc }\end{array}$} \\
\hline
\end{tabular}




\begin{tabular}{|r|r|r|r|} 
Mean & Std Dev & $\begin{array}{r}\text { Lower 95\% } \\
\text { CL for Mean }\end{array}$ & $\begin{array}{r}\text { Upper 95\% } \\
\text { CL for Mean }\end{array}$ \\
\hline 92.9273 & 7.9643 & 88.5168 & 97.3378 \\
\hline
\end{tabular}

Periormed by J. Luona SAS version 9.3 08:07 30JAN14 
Mean treatment concentration for each treatment group for each sampling time

The MEANS Procedure

thero $=$, time $=$. locm' t

\begin{tabular}{|c|c|c|c|}
\hline \multicolumn{2}{|c|}{$\begin{array}{c}\text { Analysis Varlable : predicted_ppm Predicted } \\
\text { Value of conc }\end{array}$} \\
\hline Vean & Std Dev & $\begin{array}{c}\text { Lower } 95 \% \\
\text { CL for Mean }\end{array}$ & $\begin{array}{c}\text { Upper } 95 \% \\
\text { CL for Mean }\end{array}$ \\
\hline & & & \\
\hline
\end{tabular}

thero $=0$ time $=1$ loc $=$ sur

\begin{tabular}{|c|c|c|c|}
\hline \multicolumn{4}{|c|}{$\begin{array}{l}\text { Analysis Varlable : predicted_ppm Predictod } \\
\text { Value of cone }\end{array}$} \\
\hline Mean & Std Dev & $\begin{array}{l}\text { Lower } 95 \% \\
\text { CL for Mean }\end{array}$ & $\begin{array}{l}\text { Upper } 95 \% \\
\text { CL for Mean }\end{array}$ \\
\hline-2.6701 & 0.4698 & -3.8371 & -1.5031 \\
\hline
\end{tabular}

thero $=0$ tim $=1$ locesus

\begin{tabular}{|c|c|c|c|}
\hline \multicolumn{4}{|c|}{$\begin{array}{c}\text { Analysis Variable : predicted_ppm Predicted } \\
\text { Value of conc }\end{array}$} \\
\hline Mean & Std Dev & $\begin{array}{r}\text { Lower } 95 \% \\
C L \text { for Mean }\end{array}$ & $\begin{array}{r}\text { Upper } 95 \% \\
\text { CL for Mean }\end{array}$ \\
\hline-3.2126 & 0.4698 & -4.3795 & -2.0456 \\
\hline
\end{tabular}

thero $=0$ time $=3$ loc $=s u r$

\begin{tabular}{|c|r|r|r|}
\hline \multicolumn{3}{|c|}{ Analysis Varlable : predicted_ppm Predicted } \\
Value of conc
\end{tabular}

thero $=0$ t $1 m e=3$ loc $=s$ us

\begin{tabular}{|c|c|c|c|}
\hline \multicolumn{4}{|c|}{$\begin{array}{c}\text { Analysis Varlable : predicled_ppm Predicted } \\
\text { Value of conc }\end{array}$} \\
\hline Mean & Std Dov & $\begin{array}{l}\text { Lowor } 95 \% \\
\text { CL for Mean }\end{array}$ & $\begin{array}{r}\text { Upper } 95 \% \\
\text { CL for Mean }\end{array}$ \\
\hline-2.3989 & 0.4698 & -3.5659 & -1.2319 \\
\hline
\end{tabular}

thero $=0$ t $m e=6$ loc $=$ sur

Analysis Varlable : predlcted ppm Predicted

\begin{tabular}{|r|r|r|r|}
\hline \multicolumn{3}{|c|}{ Value of conc } \\
\hline Mean & Stdl Dev & $\begin{array}{r}\text { Lower } 95 \% \\
\text { CL for Mean }\end{array}$ & $\begin{array}{r}\text { Upper } 96 \% \\
\text { CL for Mean }\end{array}$ \\
\hline 4.5687 & 0 &
\end{tabular}

thero $=0$ time $=6$ locmsus

Analysis Varlable : predicted_ppm Predicted

Analysis Varlable: predicted_ppm Predicted
Value of conc 


\begin{tabular}{|r|rr|r|} 
Mean & Std Dev $\begin{array}{r}\text { Lower } 95 \% \\
\text { CL for Moan }\end{array}$ & $\begin{array}{r}\text { Upper } 95 \% \\
\text { CL for Mean }\end{array}$ \\
\hline-4.2975 & 0.4698 & -5.4644 & -3.1305 \\
\hline
\end{tabular}

thero $=0$ time $=9$ loc $=$ sur

\begin{tabular}{|c|c|c|c|}
\hline \multicolumn{4}{|c|}{$\begin{array}{l}\text { Analysis Variablo : predicted_ppm Predictod } \\
\text { Valuo of conc }\end{array}$} \\
\hline Mean & Std Dev & $\begin{array}{l}\text { Lower } 95 \% \\
\text { CL for Moan }\end{array}$ & $\begin{array}{l}\text { Upper } 95 \% \\
\text { CL for Mean }\end{array}$ \\
\hline-1.8564 & 0.4698 & -3.0234 & -0.6895 \\
\hline
\end{tabular}

thero $=0$ time $=9$ locmsus

\begin{tabular}{|r|r|r|r|}
\hline \multicolumn{4}{|c|}{ Analysis Variable : predicted_ppm Predlcted } \\
Value of conc
\end{tabular}

thero $=0$ tlme $=12$ loc $=s u r$

\begin{tabular}{|c|c|c|c|}
\hline \multicolumn{4}{|c|}{$\begin{array}{c}\text { Analysis Varlable : predicted_ppm Predlcted } \\
\text { Value of conc }\end{array}$} \\
\hline Mean & Std Dev & $\begin{array}{r}\text { Lower } 95 \% \\
\text { CL. for Moan }\end{array}$ & $\begin{array}{r}\text { Upper } 95 \% \\
\text { CL for Mean }\end{array}$ \\
\hline-1.3140 & 0 & & \\
\hline
\end{tabular}

thero $=0$ time $=12$ loc $=$ sus

\begin{tabular}{|c|c|c|c|}
\hline \multicolumn{4}{|c|}{$\begin{array}{c}\text { Analysis Variablo : predicted_ppm Predicted } \\
\text { Valuo of conc }\end{array}$} \\
\hline & & & $\begin{array}{r}\text { Upp } \\
\text { CL fo }\end{array}$ \\
\hline & & & \\
\hline
\end{tabular}

thero $=50$ time 1 loc $=$ sur

\begin{tabular}{|r|r|r|r|}
\hline \multicolumn{4}{|c|}{ Analysis Variable : predicted_ppm Predlcted } \\
Value of conc \\
\hline Mean & Std Dev & $\begin{array}{r}\text { Lower } 95 \% \\
\text { CL for Mean }\end{array}$ & $\begin{array}{r}\text { Upper } 95 \% \\
\text { CL for Mean }\end{array}$ \\
\hline-2.6701 & 1.2429 & -5.7577 & 0.4174 \\
\hline
\end{tabular}

thero $=50$ time $=1$ loc $=$ sus

Analysis Variable : predicted_ppm Predicted Value of conc

\begin{tabular}{r|r|r|r|}
\multicolumn{4}{c|}{ Value of conc } \\
\hline Mean & Std Dev & $\begin{array}{r}\text { Lower 95\% } \\
\text { Cl. for Mean }\end{array}$ & $\begin{array}{r}\text { Upper 95\% } \\
\text { CL for Mean }\end{array}$ \\
\hline 52.3883 & 3.5467 & 43.5778 & 61.1989 \\
\hline
\end{tabular}

thero $=50$ time $=3$ loc $=$ sur

Analysis Varlable : predictod_ppm Predicted Analysis Varlable : predicted_ppm Predicted
Value of conc 


\begin{tabular}{|c|c|c|c|}
\hline Mean & Std Dev & $\begin{array}{l}\text { Lower } 95 \% \\
\text { CL for Mean }\end{array}$ & $\begin{array}{r}\text { Upper } 95 \% \\
\text { CL for Mean }\end{array}$ \\
\hline-1.85 & 0,9385 & -1.1904 & 0.4775 \\
\hline
\end{tabular}

thero $=50$ timie $=3$ loc $=$ sus

\begin{tabular}{|r|r|r|r|}
\hline Analysis Variable : predlcted_ppm Predicted \\
Value of conc
\end{tabular}

thero $=50$ tim $\theta=6$ loc $=8$ ur

\begin{tabular}{|c|c|c|c|}
\hline \multicolumn{4}{|c|}{$\begin{array}{c}\text { Analysis Variable : predlcted_ppm Predicted } \\
\text { Value of conc }\end{array}$} \\
\hline Mean & Std Dev & $\begin{array}{l}\text { Lower } 95 \% \\
\text { CL for Mean }\end{array}$ & $\begin{array}{l}\text { Upper } 95 \% \\
\text { CL for Mean }\end{array}$ \\
\hline 39 & 1.2429 & -1.6893 & \\
\hline
\end{tabular}

thero $=50$ tim $\theta=6$ loc $=$ sus

\begin{tabular}{|r|r|r|r|}
\hline \multicolumn{3}{|c|}{$\begin{array}{c}\text { Analysis Variable : predlcted ppm Predicted } \\
\text { Value of conc }\end{array}$} \\
\hline Mean & Std Dev & $\begin{array}{r}\text { Lower } 95 \% \\
\text { CL for Mean }\end{array}$ & $\begin{array}{r}\text { Upper } 95 \% \\
\text { CL for Mean }\end{array}$ \\
\hline 35.1204 & 1.5892 & 31.1726 & 39.0683 \\
\hline
\end{tabular}

thero $=50$ t $1 \mathrm{me}=9$ loc $=$ sur

Analysis Varlable : predicted_. ppm Predicted

\begin{tabular}{|r|r|r|r|}
\hline Mean & Std Dev & $\begin{array}{r}\text { Lowor } 95 \% \\
\text { CL for Moan }\end{array}$ & $\begin{array}{r}\text { Upper } 95 \% \\
\text { CL for Mean }\end{array}$ \\
\hline 6.5515 & 3.2884 & -1.6174 & 14.7204 \\
\hline
\end{tabular}

thero $=50$ time $=9$ loc $=5$ us

\begin{tabular}{|c|c|c|c|}
\hline \multicolumn{4}{|c|}{$\begin{array}{l}\text { Analysis Variable : prodicted_ppm Predicted } \\
\text { Value of conc }\end{array}$} \\
\hline Mean & Std Dev & $\begin{array}{r}\text { Lower } 95 \% \\
\mathrm{CL} \text { for Mean }\end{array}$ & $\begin{array}{l}\text { Upper } 95 \% \\
\text { CL for Mean }\end{array}$ \\
\hline 37.4710 & 2.6156 & 30.9735 & 43. \\
\hline
\end{tabular}

thero $=50$ time $=12$ loc=sur

\begin{tabular}{|c|c|c|c|}
\hline Analysis Varlable: predicted_ppm Predicted \\
Value of conc \\
\hline Mean & Std Dev & $\begin{array}{r}\text { Lower } 95 \% \\
\text { CL_for Mean }\end{array}$ & $\begin{array}{c}\text { Upper 95\% } \\
\text { CL for Mean }\end{array}$ \\
\hline 11.4335 & 5.8862 & -3.1887 & 26.0558 \\
\hline
\end{tabular}

there $=50$ time $=12$ loc $=s u s$

Analysis Variable ; predicted_ppm Predicted Value of cone 


\begin{tabular}{|r|r|r|r|} 
Mean & Std Dev & $\begin{array}{r}\text { Lower 95\% } \\
\text { CL for Mean }\end{array}$ & $\begin{array}{r}\text { Upper 95\% } \\
\text { CL for Mean }\end{array}$ \\
\hline 29.0631 & 11.8566 & -0.3902 & 58.5164 \\
\hline
\end{tabular}

thero $=100$ time $=1$ loc=sur

\begin{tabular}{|c|c|c|c|}
\hline \multicolumn{4}{|c|}{$\begin{array}{c}\text { Analysis Varlable : predicted_ppm Predicted } \\
\text { Value of conc }\end{array}$} \\
\hline Mean & Std Dev & $\begin{array}{r}\text { Lower } 95 \% \\
\text { CL for Mean }\end{array}$ & $\begin{array}{l}\text { Uppor } 95 \% \\
\text { CL for Moan }\end{array}$ \\
\hline-1.5852 & 0.4698 & -2.7522 & -0.4182 \\
\hline
\end{tabular}

thero $=100$ time $=1$ loc $=$ sus

\begin{tabular}{|r|r|r|r|}
\hline \multicolumn{3}{|c|}{ Analysis Varlable : predicted_ppm Predicted } \\
\hline Mean & Std Dev & $\begin{array}{r}\text { Lower } 95 \% \\
\text { CL for Mean }\end{array}$ & $\begin{array}{r}\text { Upper } 95 \% \\
\text { CL for Mean }\end{array}$ \\
\hline 102.8 & 10.1953 & 77.5095 & 128.2 \\
\hline
\end{tabular}

thero $=100$ timen 3 logmsur

\begin{tabular}{|r|r|r|r|}
\hline \multicolumn{3}{|c|}{ Analysis Varlable : predicted_ppm Predicted } \\
Value of conc
\end{tabular}

thero $=100$ time $=3$ loc $=$ sus

\begin{tabular}{|c|c|c|c|}
\hline \multicolumn{4}{|c|}{$\begin{array}{c}\text { Analys Is Variable : predicted_ppm Predicted } \\
\text { Value of conc }\end{array}$} \\
\hline Mean & Std Dov & $\begin{array}{r}\text { Lower } 95 \% \\
\text { CL for Mean }\end{array}$ & $\begin{array}{l}\text { Upper } 95 \% \\
\text { CL. for Mean }\end{array}$ \\
\hline 94.1568 & 5.2312 & 81.1619 & 107.2 \\
\hline
\end{tabular}

thero $=100$ time $=6$ loc $=$ sur

\begin{tabular}{|r|r|r|r|}
\hline \multicolumn{3}{|c|}{$\begin{array}{c}\text { Analysis Variable : predicted_ppm Predictod } \\
\text { Value of cone }\end{array}$} \\
\hline Mean & Std Dev & $\begin{array}{r}\text { Lower } 95 \% \\
\text { CL for Mean }\end{array}$ & $\begin{array}{r}\text { Upper 95\% } \\
\text { Ci. for Mean }\end{array}$ \\
\hline-2.1277 & 0 & & \\
\hline
\end{tabular}

thero $=100$ time $=6$ loc $=$ sus

\begin{tabular}{|c|c|c|c|}
\hline \multicolumn{4}{|c|}{$\begin{array}{c}\text { Analysis Variable : predlcted_ppm Predlcted } \\
\text { Value of cons: }\end{array}$} \\
\hline Mean & Std Dov & $\begin{array}{r}\text { Lower } 95 \% \\
\text { CL for Mean }\end{array}$ & $\begin{array}{l}\text { Upper } 95 \% \\
\text { CL for Mean }\end{array}$ \\
\hline 87.6476 & 5.4177 & 74.1892 & 101.1 \\
\hline
\end{tabular}

thero $=100$ time $=9$ locasur

Analysis Varlable: prodicted_ppm Predicted Value of conc 


\begin{tabular}{|r|r|r|r|} 
Mean & Std Dov & $\begin{array}{r}\text { Lower 95\% } \\
\text { CL for Mean }\end{array}$ & $\begin{array}{r}\text { Upper 95\% } \\
\text { CL for Mean }\end{array}$ \\
\hline 2.4831 & 1.2429 & -0.6044 & 5.5707 \\
\hline
\end{tabular}

thero $=100$ t $\mid m e=9$ loc $=$ sus

\begin{tabular}{|c|c|c|c|}
\hline \multicolumn{4}{|c|}{$\begin{array}{l}\text { Analysis Varlable: predicted_ppm Predicted } \\
\text { Value of conc }\end{array}$} \\
\hline Mean & Std Dev & $\begin{array}{r}\text { Lower } 95 \% \\
\text { CL for Mean }\end{array}$ & $\begin{array}{r}\text { Upper } 95 \\
\text { CL for Me: }\end{array}$ \\
\hline 1623 & 5.4807 & .5476 & 10 \\
\hline
\end{tabular}

thero $=100$ time=12 loc=sur

\begin{tabular}{|c|c|c|c|}
\hline \multicolumn{4}{|c|}{$\begin{array}{l}\text { Analysis Variable : predicted_ppm Predicted } \\
\text { Value of conc }\end{array}$} \\
\hline & & $\begin{array}{l}\text { Lower } 95 \% \\
\text { CL for Mean }\end{array}$ & $\mathrm{ULp}_{\mathrm{flo}}$ \\
\hline & & & \\
\hline
\end{tabular}

thero $=100$ time $=12$ locssus

\begin{tabular}{|c|c|c|c|}
\hline \multicolumn{4}{|c|}{$\begin{array}{c}\text { Analysis Varlablo : prodicted_ppm Predicted } \\
\text { Value of conc }\end{array}$} \\
\hline Mean & Sid Dev & $\begin{array}{l}\text { Lower } 95 \% \\
\text { CL for Mean }\end{array}$ & $\begin{array}{l}\text { Upper } 95 \% \\
\text { CL for Mean }\end{array}$ \\
\hline 86.8338 & 2.4858 & 80.6587 & 93.0089 \\
\hline
\end{tabular}

Performed by J. Luoma SAS version 9.3 08:07 30JAN14 
Mean concentration for standard checks for all sampling times

The MEANS Procedure

thero $=25$

\begin{tabular}{|c|c|c|c|}
\hline \multicolumn{4}{|c|}{$\begin{array}{l}\text { Analysis Variable : predicted_ppm Predicted } \\
\text { Value of conc }\end{array}$} \\
\hline Moan & Std Dev & $\begin{array}{l}\text { Lower } 95 \% \\
\text { CL for Mean }\end{array}$ & $\begin{array}{l}\text { Upper } 95 \% \\
\text { CL for Mean }\end{array}$ \\
\hline 25.8084 & 0.4698 & 24.6414 & 26.9754 \\
\hline
\end{tabular}

thero $=50$

\begin{tabular}{|c|c|c|c|}
\hline \multicolumn{4}{|c|}{$\begin{array}{l}\text { Analysis Varlable : predicted_ppm Predicted } \\
\text { Value of conc }\end{array}$} \\
\hline Mean & Std Dev & $\begin{array}{l}\text { Lower } 95 \% \\
\text { CL for Mean }\end{array}$ & $\begin{array}{l}\text { Upper } 25 \% \\
\text { CL for Moan }\end{array}$ \\
\hline 48.8624 & 0.4698 & 47.6955 & 50.0294 \\
\hline
\end{tabular}

thero $=100$

\begin{tabular}{|c|c|c|c|}
\hline \multicolumn{4}{|c|}{$\begin{array}{l}\text { Analysis Variable : predicted_ppm Predicted } \\
\text { Value of conc }\end{array}$} \\
\hline Mean & Std Dev & $\begin{array}{l}\text { Lower } 95 \% \\
\text { CL for Mean }\end{array}$ & $\begin{array}{l}\text { Upper } 95 \% \\
\text { CL for Mean }\end{array}$ \\
\hline 95.5130 & 5.6373 & 81.5092 & 109.5 \\
\hline
\end{tabular}

thero $=200$

Analysis Variable : predicted ppm Predicted

\begin{tabular}{|c|r|rr|} 
& \multicolumn{3}{|c|}{ Value of conc } \\
\hline Mean & Std Dov & $\begin{array}{r}\text { Lower 95\% } \\
\text { CL for Mean }\end{array}$ & $\begin{array}{r}\text { Upper 95\% for Mean } \\
\text { CL }\end{array}$ \\
\hline 194.2 & 1.8791 & 189.6 & 198.9 \\
\hline
\end{tabular}

Performed by J. Luoma SAS version 9.3 08:07 30JA $14 \sqrt{A}$

File Folder: $14 \mathrm{C}$ Item Number: 4 


\section{Appendix 8. Survival Assessment Summary}

\begin{tabular}{cccc}
\hline $\begin{array}{c}\text { Item } \\
\text { Number }\end{array}$ & Item Description & $\begin{array}{c}\text { Number } \\
\text { of } \\
\text { Pages }\end{array}$ & $\begin{array}{c}\text { Report } \\
\text { Page } \\
\text { Number }\end{array}$ \\
\hline 1 & $\begin{array}{c}\text { Zebra Mussel Survival on Artificial Substrate - Lake Carlos - Whole Tank - Data } \\
\text { Summary }\end{array}$ & 2 & 437 \\
2 & $\begin{array}{c}\text { Zebra Mussel Survival on Artificial Substrate - Lake Shawano - Whole Tank - Data } \\
\text { Summary }\end{array}$ & 2 & 439 \\
3 & $\begin{array}{c}\text { Zebra Mussel Survival on Artificial Substrate - Lake Carlos - Bottom Injection -Data } \\
\text { Summary }\end{array}$ & 2 & 441 \\
4 & $\begin{array}{c}\text { Zebra Mussel Survival on Artificial Substrate - Lake Shawano - Bottom Injection - } \\
\text { Data Summary }\end{array}$ & 2 & 443 \\
5 & Zebra Mussel Survival on Artificial Substrate - All Exposures - Data Summary & 6 & 445 \\
\hline
\end{tabular}




\begin{tabular}{|c|c|c|c|}
\hline Study Number: AEH-12-PSEUDO-04 & Action & Date & Initials \\
\hline Electronkc Lab Notebook (pages 29) & Created...... & $28 \cdot 0 \mathrm{ct}-13$ & KLW TIS \\
\hline Data Source: Flle Foider: $9 \mathrm{~d}$ & Revlsed...... & 4-Mar-14 & Tus TSS \\
\hline Forms: "Zcbra Mussel Survival" & \begin{tabular}{|l} 
Revlewed... \\
Cert|fled....
\end{tabular} & $\begin{array}{l}4 M A R I 4 \\
3 / 4 / 14\end{array}$ & $\frac{\pi y}{\tan }$ \\
\hline
\end{tabular}

Zebra Mussel Survlval on Artificial Substrate

Test Article: MB1 401 SDP [Psevdomonas fluorescens Pf -CL. 145A (SDP)] Article Lot H: 401P12163C and 401P12164C Mix

Exposure Date: August 15, 2012

Test Locat on: Lake Carlos, Alexandrla, MN

Treatment Type: Whole Tank

Data Explanatlon

Eac') tank repltcate containec 9 perforated aluminum trays ( $\sim 15.2 \mathrm{~cm} \times 15.2 \mathrm{~cm}$ wth $2.5 \mathrm{~cm}$ sides) with attached zebra mussels enclosed in a mesh contalnment bag ( $20.3 \times 25.4 \times 5.1 \mathrm{~cm} ; 0.31 \times 0.31 \mathrm{~cm}$ openings). Each substrate contalned a minimur of 50 adhering zebra mussels prlor to treatment. Aluminum trays were coded with tags according to treatment type ( $W=$ whde water body, $B=$ bottom injection; tank number $1-9$, row letter $A, B, C ;$ pos' tlon number $1-3$ ) Example code of $W 1 A 3=$ whole water body (tank) treatment, "ank 1, Row A, sostlion 3 . The tanks are numbered 1-S; the raws are in order from the tront of the tank (isle) to the rear of the tank (wall) ( $A=$ isle; $B=$ middle; $C=$ wall); the position Is from left to right in order $(1=$ left; $2=$ cenker; $3=$ right). Threc trays were removed after 6,9 and $12 \mathrm{~h}$ of exposure. The substrates were consolidated into wlre mesh ho dhng cages wilti 1 sampling tiree period per cage and the treatmer.t levels Indiscriminately dlstributed. The wire mesh holding cages were held in the lake until survival assessments were completed at 28 days postdosir.g termination,

Survival Data Filename:

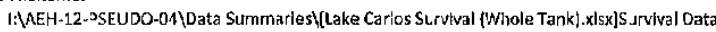

Data anomalies and devlat' ons:

NONE

File Folder: $9 d$

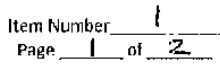

Page 437 of 519 


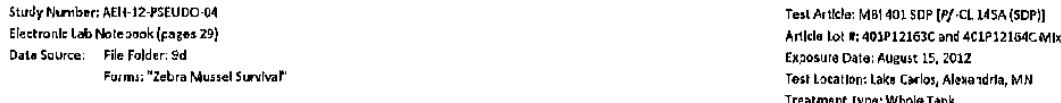

Tesl Arttcla: MB1 401 SDP [Pf CL. 1ASA (SDP)] Aricla Lot i: 401P12163C and 4C1P 12154C MIX Exposure Date: August 15, 2012

Tortscation: Laks Carlos, Alexa adra, MN

Zebra Mussel Survival on Artificial Substrate

\begin{tabular}{|c|c|c|c|c|c|c|c|c|c|c|c|c|}
\hline \multirow{2}{*}{$\begin{array}{c}\text { Treatment } \\
\text { Level } \\
(\text { mR } /) \\
\end{array}$} & \multicolumn{4}{|c|}{5 hour Sample Tine } & \multicolumn{4}{|c|}{9 hour Saruple Time } & \multicolumn{4}{|c|}{12 hour Sample TIme } \\
\hline & \begin{tabular}{|c|} 
Sample \\
ID
\end{tabular} & $\begin{array}{l}\text { Total Murnber } \\
\text { ol Animals }\end{array}$ & $\begin{array}{l}\text { Number } \\
\text { Allse }\end{array}$ & $\begin{array}{c}\text { Number } \\
\text { Dead }\end{array}$ & \begin{tabular}{|c|} 
Samiple \\
10 \\
\end{tabular} & $\begin{array}{l}\text { Tota Number } \\
\text { of An:mals }\end{array}$ & $\begin{array}{c}\text { Nember } \\
\text { Alive }\end{array}$ & $\begin{array}{c}\begin{array}{c}\text { Number } \\
\text { Dead }\end{array} \\
\end{array}$ & \begin{tabular}{|c} 
Sample \\
DD
\end{tabular} & $\begin{array}{l}\text { Totat Number } \\
\text { of Anlmals }\end{array}$ & $\begin{array}{l}\text { Nurt ber } \\
\text { Aluve }\end{array}$ & $\begin{array}{c}\text { Number } \\
\text { Dead }\end{array}$ \\
\hline \multirow{9}{*}{0} & W2A1 & 399 & 337 & 2 & $162 A 3$ & 326 & 320 & 6 & W2A2 & 390 & 374 & 16 \\
\hline & w2B2 & 237 & 227 & 10 & w20s & 236 & 230 & 6 & W/2B1 & 185 & 281 & 4 \\
\hline & w2C1 & 168 & 165 & 3 & W2C3 & 226 & 220 & 6 & $w_{2} C_{2}$ & 198 & 190 & 8 \\
\hline & W3AX & 328 & 305 & 23 & WaAs & $\therefore 78$ & 171 & 7 & $W_{3 A 2}$ & 185 & 180 & 5 \\
\hline & W3B1 & 255 & 252 & 3 & 163в3 & 213 & 211 & 2 & W3B 2 & 312 & 304 & 8 \\
\hline & W363 & 310 & 304 & 6 & w33c2 & 214 & 203 & 11 & Wac1 & 259 & 254 & 5 \\
\hline & W5A1 & 269 & 264 & 5 & WSA3 & 177 & 175 & 2 & W5A2 & 240 & 238 & 2 \\
\hline & W582 & 173 & 171 & 2 & W5s & 192 & 185 & 7 & W5B1 & 190 & 190 & 6 \\
\hline & $w_{5} \mathrm{Cl}_{1}$ & 173 & 175 & 0 & w5C3 & 173 & 15 ? & 11 & W5C2 & 212 & 206 & 6 \\
\hline \multirow{9}{*}{50} & $w_{1} \wedge 2$ & 280 & 6 & 274 & WIAI & 164 & 3 & 161 & $w_{1 C 2}$ & 175 & 0 & 175 \\
\hline & W1A3 & 149 & 0 & 149 & W1a1 & 128 & 5 & 223 & W'183 & 160 & 1 & 159 \\
\hline & w102 & 232 & 4 & 228 & wic1 & 267 & 0 & 267 & W1C3 & 266 & c & 266 \\
\hline & W4B2 & 214 & 4 & 210 & WAA1 & 221 & 0 & 221 & WAA2 & 158 & 3 & 155 \\
\hline & WAC1 & 191 & 0 & 191 & WAA3 & 375 & 0 & 375 & WAB1 & 280 & 3 & 271 \\
\hline & WAC2 & 175 & 4 & 171 & Wans & 265 & 4 & 261 & wac3 & 455 & 2 & 453 \\
\hline & wse1 & 208 & 5 & 203 & WBA3 & 131 & 4 & 127 & WBA1 & 151 & 1 & 150 \\
\hline & W682 & 178 & 1 & 177 & WBC1 & 169 & 0 & 169 & WBAZ & 119 & 0 & 119 \\
\hline & ws83 & 248 & 1 & 247 & WBC3 & 180 & $\underline{0}$ & 180 & w8C2 & 290 & 3 & 287 \\
\hline \multirow{9}{*}{100} & WGAZ & 257 & 7 & 250 & W6B2 & 188 & $\overline{:}$ & 187 & W6A1 & 239 & 3 & 236 \\
\hline & WGA3 & 235 & 8 & 2.27 & W6B3 & 183 & 2 & 181 & W6B1 & 249 & 1 & $2 \triangle 8$ \\
\hline & w6C3 & 267 & 3 & 264 & WBC1 & 176 & 0 & 176 & W6C2 & 155 & 2 & 153 \\
\hline & W7A1 & 212 & 2 & 210 & W7A3 & 258 & 3 & 255 & W7A2 & 237 & 1 & 236 \\
\hline & W7B2 & 256 & so & 256 & W7B3 & 193 & 0 & 193 & W7B1 & 286 & 0 & 286 \\
\hline & W7c1 & 269 & 2 & 267 & w/t3 & 158 & 3 & 155 & W7c2 & 203 & 0 & 203 \\
\hline & WGA1 & 283 & 8 & 275 & W9A3 & 393 & 1 & 392 & W9A? & 316 & D & 316 \\
\hline & WoB2 & 190 & 2 & 188 & W963 & 128 & 0 & 128 & w9B1 & 273 & $\mathrm{~s}$ & 268 \\
\hline & w9c1 & 324 & 7 & 317 & w9с3 & 250 & 0 & 260 & W $9 C_{2}$ & 156 & 1 & 155 \\
\hline
\end{tabular}

File Folder: $9 d$

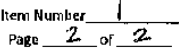




\begin{tabular}{|c|c|c|c|}
\hline \multirow{5}{*}{$\begin{array}{l}\text { Study Number: AEH-12-PSEUDO-04 } \\
\text { Electronic Lab Notebook (pages 29) } \\
\text { Data Scurce: Flle Folder: 11d } \\
\text { Forms: "Zekra M dssel Survival" }\end{array}$} & Action & Date & Initials \\
\hline & Created..... & $28-$ Oct-13 & KLW THS \\
\hline & Rev'sed..... & 4-Mar-14 & TJS T/5 \\
\hline & Reviewed. & 4mnellt & 185 \\
\hline & Certlfted... & $13 / 4 / 14$ & $5 \pi=$ \\
\hline Flle Name: See filenames as stated below & & & \\
\hline
\end{tabular}

Zebra Mussel Survlval on Artificial Substrate

Test Artlcle: MB| 401 SDF [Pse:udomonas fluorescens of -CL 145A (SOP)]

Article Lot H: 401P12163C and 401P12164C Mix

Exposure Date: September 6, 2012

Test Location: Lake Shawano, Shawano, WI

Treatment Type: Whole Tank

Data Explanation

Each tank replicate contalned 9 perforated aluminum trays $(\sim 15.2 \mathrm{~cm} \times 25.2 \mathrm{~cm}$ with $2.5 \mathrm{~cm}$ sides) with attached zebra mussels enclosed in a mesh contalnment bag $\{\sim 20.3 \times 25.4 \times 5.1 \mathrm{~cm} ; 0.31 \times C .31 \mathrm{~cm}$ openlngs). Each substrate contalned a minlmum of 50 adhering zebra mussels prior to treatment. Alur rinum trays were codec' wlth togs accordlng to treatment type $(W=$ whole water body, $B=$ bottom injectlon; tank number 1.9 , row lettor $A, B, C$; positlon number 1-3). Example code of $W 1 A 3$ = whole water body (tank) trea:ment, $T a$ k 1 , Row $A$, positlon 3. The tanks are numbered 1-9; the rows are in order

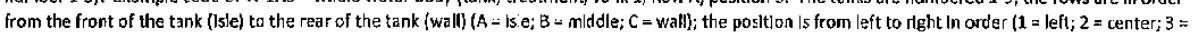
rightl. Three trays were removed after 6,9 and $12 \mathrm{~h}$ of expsoure. The substrates were consolldated Into wire mesh holding cages with 1 sampilng time perlod per cage and the treatrent levels indlscriminately distrlbuted. The wire mesh holding cages were held in the lake untll survival assessments were completed at 34 day's post-losing termination.

Survival Data Filename:

I:AEEH-12-PSEUDO-04\Data SurmarlesVLake Shawano Survival (Whole Tank'.xlsx|Survival Data

Data anomalles and devlations:

NONE

File Folder: Ild

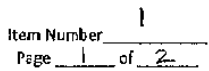

Page 439 of 519 
SIudy Number: AEI1-12-PSEUDO-04 Elect/onlc Lab Notebook \{pages 29 )

Data Source: File Folderi 11d

Forms: "Zebre Mussel Sunvival"
TeSl Ar::cle: MEI AO1 SOP [Pf-CL 145A (SOP)] Artkle lot H: 401P12153C and 401P12154C Mix xposu'e Date: September 6, 2012

Vest Locatbo: Lake Shawano, Shawano, WI

reatment Type: Whole Tank.

'Zelora Mussel Survival on Artificial Substrate

\begin{tabular}{|c|c|c|c|c|c|c|c|c|c|c|c|c|}
\hline \multirow{2}{*}{$\begin{array}{l}\text { Treatment } \\
\text { Level } \\
{[\mathrm{mgh}]}\end{array}$} & \multicolumn{4}{|c|}{6 lour Sanple Tlme } & \multicolumn{4}{|c|}{9 hour Sample Tline } & \multicolumn{4}{|c|}{12 hour Sample Time } \\
\hline & \begin{tabular}{|c|} 
sample \\
10
\end{tabular} & $\begin{array}{l}\text { Tolai Number } \\
\text { of Anlmals }\end{array}$ & $\begin{array}{c}\text { Number } \\
\text { Allve }\end{array}$ & $\begin{array}{c}\text { Number } \\
\text { Dents }\end{array}$ & \begin{tabular}{|c|} 
Sample \\
ID
\end{tabular} & $\begin{array}{c}\text { Total Kiumber } \\
\text { of Anlrials }\end{array}$ & $\begin{array}{c}\text { Number } \\
\text { Allue }\end{array}$ & $\begin{array}{c}\text { Number } \\
\text { Dead }\end{array}$ & \begin{tabular}{|c|} 
Sample \\
1.2 \\
\end{tabular} & $\begin{array}{l}\text { Total Number } \\
\text { of Anllials }\end{array}$ & $\begin{array}{c}\text { Nunber } \\
\text { Alive }\end{array}$ & $\begin{array}{c}\text { Number } \\
\text { Deadf }\end{array}$ \\
\hline \multirow{9}{*}{0} & W1A1 & 109 & 105 & 4 & W1A3 & 159 & (15) & 9 & W1A2 & 101 & 96 & 5 \\
\hline & W1B: & 118 & 111 & 7 & W183 & 84 & ${ }_{B 2}$ & 2 & Wic2 & 208 & 102 & 6 \\
\hline & w182 & 87 & 85 & 2 & wha1 & 144 & 133 & 6 & W1C.3 & 111 & 106 & 5 \\
\hline & WAA1 & 64 & 61 & 3 & WAAB & 110 & 103 & 7 & W4B3 & 79 & 76 & 3 \\
\hline & W4A2 & 74 & 57 & 7 & WABZ & 75 & 72 & 3 & W4C2 & 81 & 77 & 4 \\
\hline & W4B1 & 78 & 74 & a & wac1 & 71 & 69 & 2 & W4C3 & 141 & 137 & 4 \\
\hline & W7A: & 124 & 122 & 2 & W7A3 & 54 & 52 & 2 & W7A2 & 92 & 30 & 2 \\
\hline & W701 & 80 & 78 & 2 & w782 & 108 & 102 & 5 & W7B3 & 104 & 102 & 2 \\
\hline & $w 7 C_{1}$ & 108 & 103 & 5 & wrc3 & 111 & 105 & 6 & W7C2 & 90. & 87 & 3 \\
\hline \multirow{9}{*}{50} & $W_{2 A 1}$ & 108 & 17 & 91 & W2A3 & 114 & 26 & 88 & W2A2 & 98 & 1 & 97 \\
\hline & W2B1 & 116 & 10 & 106 & W2B2 & 103 & 9 & 94 & W2B3 & 85 & 5 & 80 \\
\hline & w2c1 & 51 & a & 57 & w2C3 & 117 & 5 & 112 & $\mathrm{w}_{2} \mathrm{C2}$ & 86 & 0 & 86 \\
\hline & WSA1. & 95 & g & 87 & W5A3 & 61 & 2 & 59 & W5A2 & 60 & 0 & 60 \\
\hline & W581 & 67 & a & 59 & w5a2 & 105 & 22 & 83 & WSD3 & 63 & 5 & 58 \\
\hline & wsC1 & 85 & 14 & 71 & wsc & so & 19 & 61 & wsc2 & 114 & 2 & 112 \\
\hline & WBA3 & 96 & 5 & 91 & WBA1 & 148 & 2 & 146 & WAA2 & 76 & 3 & 73 \\
\hline & พAв3 & 149 & 57 & 92 & wsB! & 85 & 5 & 80 & W8B2 & $8 B$ & 3 & 85 \\
\hline & WeC1 & 136 & 3 & 133 & wic. & 61 & 1 & 60 & WBC3 & 129 & 1 & 128 \\
\hline \multirow{9}{*}{100} & W3A1 & 138 & 27 & 111 & W/3B1 & 51 & 2 & 49 & W3A2 & 95 & 1 & 94 \\
\hline & W302 & 122 & 8 & 114 & w3cz & 73 & 4 & 69 & W3ค3 & 131 & 6 & 12b \\
\hline & พззвз & 104 & 9 & 95 & W3C3 & ви & 0 & 88 & W3C1 & 104 & 1 & 103 \\
\hline & WGA1 & 69 & 0 & 69 & WGB1 & 59 & 0 & 59 & W6B3 & 37 & 0 & 77 \\
\hline & WEAZ & 124 & 3 & 121 & W6B2 & 94 & 13 & 81 & W6c1 & 62 & 3 & 59 \\
\hline & WEA3 & 57 & 3 & 51 & WGCL & 94 & 0 & 94 & W6c3 & 102 & 1 & 101 \\
\hline & W9A1 & 77 & 2 & 75 & W9A3 & 107 & 14 & 93 & WIA2 & 104 & 0 & 104 \\
\hline & W9B1 & 129 & 4 & 215 & พ932 & 69 & 0 & 69 & W9B3 & 102 & 2 & 100 \\
\hline & $\begin{array}{l}\text { W9C1 } \\
\text { W9 }\end{array}$ & 118 & 19 & 99 & \begin{tabular}{|l} 
W9:3 \\
\end{tabular} & 123 & 7 & 116 & W9c2 & 60 & 2 & 58 \\
\hline
\end{tabular}

File Folder: Hd

Item Number $i$

Page 2 or 2 


\begin{tabular}{|c|c|c|c|}
\hline \multirow{3}{*}{$\begin{array}{l}\text { Study Nurber: } \wedge \text { EH-12-PSEUDO-04 } \\
\text { Electronk Lab Notebook (pages 29) } \\
\text { Data Solrce: File Folder: 12d }\end{array}$} & Action & Date & Inltials \\
\hline & Created..... & 28-Oct-13 & KL'W TS \\
\hline & Revised..... & 4-Mar-14 & TIS $\pi / 5$ \\
\hline \multirow[t]{2}{*}{ Forrms: "Zcbra Mussel Survlval" } & Revlewed. & $4 \mathrm{mARH}$ & $\pi s$ \\
\hline & Certlfled... & $3 / 4 / 14$ & sin \\
\hline File Name: See fllenarics as stated below & & & \\
\hline
\end{tabular}

Zebra Mussel Survival on Artificlal Substrate

Test Artcle: MBI 401 SDP [Pseudomonas fluorescens Pf-CL. 145A (SDPI]

Article Lot H: 401P12163C and 401P12164C Mix

Exposure Date: August $-7,2012$

Tes. Location: Lake Carlos, Alexandrla, MN

Treatment Type: Bottom Injection

Dála Explanation:

Each tank replicate contair ed 3 perforated aluminum trays $(\sim 15.2 \mathrm{~cm} \times 15.2 \mathrm{~cm}$ with $2.5 \mathrm{cn}$ sidcs) with attached zebra mussels enclosed in a mesh containment bag ( $20.3 \times 25.4 \times 5.1 \mathrm{~cm} ; 0.31 \times 0.31 \mathrm{~cm}$ openings). Each substrate contained a mininum of 50 adherlng zebra mussels prlor to treatment. Aluminum trays were coded with tags acco ciling to treatrent type $(W=$ whole water body, $B=$ bottom injectlon; tank number 1-9, row letter $A, B, C ;$ pos'tion number 1-3). Example code of $W 1 A 3=$ whole water body (tank) treatment, Tank 1, Row A, cosltion 3 . The tanks are numbered 1-9; the rows are in order from the front of the tank (lisle) to the rear of the tark (wall) ( $\mathrm{A}=$ isle; $\mathrm{B}=$ mild dle; $\mathrm{C}=$ wall); the position is from left to right In order $(1=$ left; $2=$ center; $3=$ right). All substrates were removed after $12 \mathrm{~h}$ of exposure. The substrates were consolidated intc a wire mesh holding cage with the treatment levels indiscrimlnately distributed.

The wire mesh holding cages were held in the lake untll survlval assessments were comipleted at 27 days post-dosing terninativn.

Survival Data Fllename:

I:AEH-12-PSEUDO-04|Data Summarles\}[Lake Carlos Survival (Bottom Injection).xlsx] Survival Data

Data ano nalles and deviations:

NONE

File Folder: $12 d$

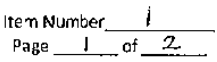

Page 441 of 519 
SLucy Murnber: AESH-12-PSEUDD-04 Elockronic Lab Notebook loages 29 Data Source: Fle Folder: 12d Forms "Zetra Mussel Survival"
Tost Artecle: MBI 401 SDP [\% CCL 145A [SDP]] Aftcle lot H: $40.1 \mathrm{P} 12163 \mathrm{C}$ and $401 \mathrm{P} 12$ ) $64 \mathrm{C} . \mathrm{Ml}$ Exposure Data: August 17, 2012

Thst Locatlon: Lake Carlos, Alexandria, MY

Zebra Mussel Survival on Artificial Substrate

\begin{tabular}{|c|c|c|c|c|}
\hline $\begin{array}{l}\text { Treatment Level } \\
\text { (nng/L) }\end{array}$ & $\begin{array}{c}\text { Sample } \\
\text { in }\end{array}$ & $\begin{array}{l}\text { Total } N \text { Jmber } \\
\text { of Antmals }\end{array}$ & $\begin{array}{c}\text { Number } \\
\text { Alver }\end{array}$ & $\begin{array}{c}\text { Number } \\
\text { Dead }\end{array}$ \\
\hline \multirow{9}{*}{0} & B3A3 & 196 & 192 & 4 \\
\hline & B381 & 242 & 237 & 5 \\
\hline & $\mathrm{B3C3}$ & 253 & 246 & 7 \\
\hline & B6A2 & 186 & 182 & 4 \\
\hline & 6681 & 211 & 206 & 11 \\
\hline & $66 \mathrm{Cl} 1$ & 179 & 175 & 4 \\
\hline & B7AL & 225 & 221 & 4 \\
\hline & 8783 & 176 & 167 & 9 \\
\hline & $\mathrm{B} 7 \mathrm{CL}$ & 276 & 273 & 3 \\
\hline \multirow{9}{*}{ so } & B1A1 & 210 & 52 & 158 \\
\hline & 8182 & 510 & RG & 424 \\
\hline & B183 & 279 & 62 & 217 \\
\hline & B4B1 & 188 & 10 & 178 \\
\hline & B4B2 & 256 & 65 & 191 \\
\hline & $B 483$ & 188 & 14 & 174 \\
\hline & BBA3 & 282 & 50 & 232 \\
\hline & 8883 & 291 & 69 & 222 \\
\hline & $B 8 C 2$ & 203 & 39 & 161 \\
\hline \multirow{9}{*}{100} & $82 \mathrm{A1}$ & 279 & 18 & 261 \\
\hline & 82.3.3 & 153 & 21 & 132 \\
\hline & $\mathrm{B2CL}$ & 193 & 39 & 154 \\
\hline & BSA1 & 329 & 112 & 217 \\
\hline & BSB3 & 393 & 121 & 272 \\
\hline & BSC1 & 264 & 55 & 209 \\
\hline & $B 9 N_{-}$ & 269 & 13 & 256 \\
\hline & 6963 & 179 & 40 & 139 \\
\hline & $69 \mathrm{Cl}_{1}$ & 288 & 25 & 263 \\
\hline
\end{tabular}

r. Fnder: 12d

Piem Number $\frac{1}{2}$

Page 442 of 519 


\begin{tabular}{|c|c|c|c|}
\hline \multirow{5}{*}{$\begin{array}{l}\text { Stcidy Number: AEH-12-PSEUDO-04 } \\
\text { Electronic Lab Notebook (pages } 2.9 \text { - 30) } \\
\text { Oa:a Source: Fille Folder: } 14 d \\
\quad \text { Forms: "Zebra Mussel Survival" }\end{array}$} & Action & Date & Inittals \\
\hline & Created...... & $28.0 \mathrm{ct}-13$ & $3 \mathrm{KLW} T \mathrm{~S}$ \\
\hline & \begin{tabular}{|l} 
Revised....... \\
\end{tabular} & 4-Mar-14 & 1 TJS Th \\
\hline & Reviewed... & $4 M A R$ & $.7 \mathrm{SS}$ \\
\hline & Certlfled... & $3 / 4 / 14$ & $\sqrt{n}=$ \\
\hline
\end{tabular}

Zebra Mussel Survival on Artificial Substrate

\begin{abstract}
Test Article: MBI 401 SDP [Pseudomonas fuorescens Pf-CL 145A (SDP)]
Artlcle Lot \#: 401P12163C and 401P12164C Mlx

Exposure Date: September 8, 2012

Test Location: Lake Shawano, Shawano, Wl

Treatment Type: Bottom l:ajection

Data Explanation:

Each tank replicate contalned 4 perioratec aluminum trays $(\sim 15.2 \mathrm{~cm} \times 15.2 \mathrm{~cm}$ with $2.5 \mathrm{~cm}$ sides) with attaclied zebra mussels enclosed in a mesh contalnment bas $(\sim 20.3 \times 25.4 \times 5.1 \mathrm{~cm} ; 0.31 \times 0.31 \mathrm{~cm}$ openings). Each substrate conlained a minimurn ot 50 adhering zebra nusssels prior to treatment. Alumlnum trays were coded $w \mid$ th tags accordir $g$ to treatment type $(W=$ whole water body, $B=$ bottom injection; tank number $1-O$, row letter $A, B, C ;$ position number 1:3). Example code of $W 1 A 3=$ whole water body (tank) treatment, Tank 1 , Row A, positton 3 . The tanks are numbered 1.9; the rows are in order from the front of the tank ( $(1)$ le) to the rear of the tank (wall) $(A=$ isle; $\mathrm{E}=$ milddle; $C=$ wall); the position is from left to right in order $(1=$ left; $2=$ center; $3=$ right). All substrates were removed after $12 \mathrm{~h}$ of exposure. The substrates were consolldated into a wirc mesh holding cage with the treatment levels indlscrimlnately distributed.

The wire mesh holding cages were held In the lake untll survival assessinents were completed at 32 days pusl-exposure termination.
\end{abstract}

Survival Data Filename:

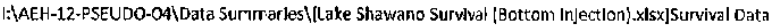

Data arcmalles and devlatlons:

NONE

Flle Folder: $14 d$

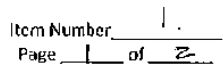

Page 443 of 519 
Study Number: AER-12-PSEUDO-04

DDe Sou

Forns: "zebra Mussel 5 survival
Test Art|cle: MGI 401 SDF PPr.CL 145A (SDPP)] Artlcle Lot t: A01P12363C and 401P12154C M Exposure Date: September 8, 2012

Tasl Locatlan: Laka Shawaro, Shawrano, Wh

Treatment Trpe: Bottom Injeclich

Zebra Mussel Survival on Artificial Substtate

\begin{tabular}{|c|c|c|c|c|}
\hline $\begin{array}{c}\text { Treatiment lavel } \\
(\mathrm{mg} / \mathrm{ll})\end{array}$ & $\begin{array}{c}\begin{array}{c}\text { Sample } \\
10\end{array} \\
\end{array}$ & $\begin{array}{l}\text { Total Number } \\
\text { of Anlmals }\end{array}$ & $\begin{array}{c}\begin{array}{c}\text { Number } \\
\text { Allve }\end{array} \\
\end{array}$ & $\begin{array}{l}\text { Number } \\
\text { Dead }\end{array}$ \\
\hline \multirow{12}{*}{0} & B1A3 & 107 & 100 & 7 \\
\hline & Bic1 & 137 & 127 & 10 \\
\hline & $\mathrm{B1C2}$ & 111 & 108 & 3 \\
\hline & $81 C 3$ & 14? & 139 & B \\
\hline & B8A3 & 140 & 133 & 7 \\
\hline & $83 B 1$ & 81 & 76 & 5 \\
\hline & $88 \mathrm{B2}$ & 134 & 128 & 6 \\
\hline & 8883 & 163 & 159 & 4 \\
\hline & BgAZ & 115 & 115 & 0 \\
\hline & व9B1 & 84 & 80 & 4 \\
\hline & в9н? & 87 & 85 & 2 \\
\hline & B983 & 137 & 127 & 10 \\
\hline \multirow{12}{*}{50} & B2A2 & 119 & 4 & 115 \\
\hline & B2Bj & 105 & 3 & 102 \\
\hline & в282 & 116 & 2 & 114 \\
\hline & B21日 & 145 & 5 & 140 \\
\hline & B3A2 & 117 & 4 & 113 \\
\hline & 8381 & 144 & 8 & 136 \\
\hline & B382 & 103 & D & 103 \\
\hline & B3C2 & 109 & 2 & 107 \\
\hline & B7A2 & 115 & 2 & 113 \\
\hline & B7घ1 & 128 & 4 & 124 \\
\hline & в782 & 116 & 3 & 113 \\
\hline & 8783 & 109 & 6 & 103 \\
\hline \multirow{12}{*}{100} & 3442 & 117 & 1 & $\$ 16$ \\
\hline & 0402 & 123 & 1 & 122 \\
\hline & 8483 & 12.6 & 2 & 124 \\
\hline & $34 c 3$ & 104 & 3 & 104 \\
\hline & B5A2 & 106 & 2 & 104 \\
\hline & $35 \mathrm{BA}$ & 105 & 0 & 105 \\
\hline & $35 \mathrm{~B} 2$ & 97 & 0 & 97 \\
\hline & 3583 & 108 & 0 & 108 \\
\hline & 3682 & 121 & 2 & 119 \\
\hline & 3683 & 113 & 0 & 113 \\
\hline & $36 \subset 2$ & 127 & 1 & 226 \\
\hline & $36 C_{3}$ & 98 & 3 & 9S \\
\hline
\end{tabular}

File Folder: $14 \mathrm{~d}$

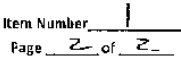

Page 444 of 519 


\begin{tabular}{|c|c|c|c|}
\hline Study Number: AEH-12-PSEJDO-04 & Action & Date & Initials. \\
\hline Electronic Lab Notebook (pages 25-30) & Created..... & 14-Feb-13 & Tus ins \\
\hline Data Source: Flle Folder: 18 & Revised....... & 4-Mar-14 & TIS T.KS \\
\hline Forms: "Zetra Mussel Survival" & \begin{tabular}{|l} 
Revicved, \\
Certified... \\
\end{tabular} & $\begin{array}{l}4 \text { MARH } \\
3 / 41 / 4\end{array}$ & $\frac{725}{5 \times 2}$ \\
\hline File Name: See filenames as stated below & & & \\
\hline
\end{tabular}

Zebra Mussel Survival on Artificial Substrate

Test Art|cle: MBI 101 SDP [Pseudomonas fluoressens of -CL. 145^ (SDP)]

Artic e Lot H: $401 \mathrm{P} 12163 \mathrm{C}$ and $401 \mathrm{P} 121 \mathrm{G} 4 \mathrm{C} \mathrm{Mix}$

Exposure Date: August 15 and 17, and September 6 and 8, 2012

Test _ocat ons: Lake Carlos, Alexandrla, MN and Lake Shawano, Shawano, WI

Treatment Type: Whole Tank and Bottom Injection

Data Explanatlon:

Each tank replicate contalred 3,4 , or 9 perforated aluminum trays $(\sim 15.2 \mathrm{~cm} \times 15.2 \mathrm{~cm}$ with $2.5 \mathrm{~cm}$ sldes), depending on lake and treatment type, with attached zebra mussels enclosed In a mesh contalnment bag $(\sim 20.3 \times 25.4 \times 5.1 \mathrm{~cm} ; 0.31 \times 0.31 \mathrm{~cm}$ openings). Each substrate contalned a minimum of 50 adhering zebra mussels prior to trcatment. Altuminum trays were coded with tags accordlrg to treatment type $(W=$ whole water body, $B=$ bottom Injectlon tank number 1-9, row letter $A, B, C$; positlon number 1-3). Example code of W $1 A 3$ = whole water body (tank) treatment, Tank 1, Row A, position 3. The tanks are numbered 1-9; the rows are in order fror the front of the tank (isle) to the rear of the tank (wall) ( $\mathrm{A}=$ isle; $\mathrm{B}=$ mićcle; $\mathrm{C}=$ wall); the position is from left to right in order $(1=$ left; 2 = center; 3 = right). For whole water body treatments, three trays were removed after 6,9 and 12 h of exposure. The substra:es were consolidated into wire mesh holding cages with 1 sampling time period per cage anel the treatment levels indiscriminately distributec. The wire mesh holding cages were held in the lake untll survival assessments were completed at 28 days post-dosing term ration.

Survival Data for SAS Fllenarne:

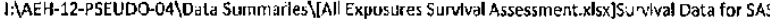

$10 C=$ Location

LC = Lake Carles

$S L=$ Shawano LakB

trt_typ $=$ Treatment Group

WI $=$ Whole Tank

conc $=$ Concentration

$0=$ contral

$50=50 \mathrm{mg} / L($ A.. .) $)$ treatme. :

$100=100 \mathrm{mg} / \mathrm{L}$ (A.I.) treatment

tirne $=$ Exposure Duration

tnk = Exposure Tank

tray $=$ Substrate 10

tot $=$ Total Number of Zebra Mussels

dead = Number o: Zebra Mussel Mortalltles

Qata anomalles and devlations:

NONE

Fille Folder: 18

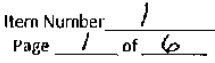

Page 445 of 519 


\section{AEH-12-PSEUDO-04}

\begin{tabular}{|c|c|c|c|c|c|c|c|}
\hline loc & trt_typ & conc & time & tnk & tray & tot & dead \\
\hline LC & $\overline{W T}$ & 100 & 6 & 7 & $\mathrm{~B} 2$ & 266 & 256 \\
\hline $\mathrm{LC}$ & $W T$ & 100 & 6 & 9 & $\mathrm{~B} 2$ & 190 & 188 \\
\hline LC & WT & 50 & 6 & 1. & $\mathrm{~A} 3$ & 149 & 149 \\
\hline LC & WT & 0 & 6 & 5 & $\mathrm{C} 1$ & 175 & 0 \\
\hline $\mathrm{LC}$ & WT & 0 & 6 & 2 & $\mathrm{C} 1$ & 168 & 3 \\
\hline L.C & WT & 100 & 6 & 6 & $C 3$ & 267 & 264 \\
\hline LC & WT & 0 & 6 & 2 & $\mathrm{~B} 2$ & 237 & 10 \\
\hline LC & WT & 100 & 6 & 7 & $\mathrm{~A} 1$ & 212 & 2.10 \\
\hline $\mathrm{LC}$ & WT & 0 & 6 & 3 & $\mathrm{~A} 1$ & 328 & 23 \\
\hline LC & WT & 100 & 6 & 6 & $A 3$ & 235 & 227 \\
\hline LC & WT & 50 & 6 & 4 & $\mathrm{C} 2$ & 175 & 171 \\
\hline LC & WT & 0 & 6 & 5 & B2. & 173 & 2 \\
\hline LC & WT & 0 & 6 & 3 & B1 & 255 & 3 \\
\hline LC & WT & 50 & 6 & 8 & 81 & 208 & 203 \\
\hline LC & WT & 50 & 6 & 1 & $\mathrm{~A} 2$ & 280 & 274 \\
\hline I.C & WT & 100 & 6 & 6 & $\mathrm{~A} 2$ & 257 & 250 \\
\hline LC & WT & 0 & 6 & 2 & A1 & 339 & 2 \\
\hline LC & WT & 50 & 6 & 8 & B2 & 178 & 177 \\
\hline LC & WT & 100 & 6 & 9 & $\mathrm{C} 1$ & 324 & 317 \\
\hline LC & WT & 100 & 6 & 7 & $\mathrm{C} 1$ & 269 & 267 \\
\hline LC & WT & 50 & 6 & 4 & B2 & 214 & 210 \\
\hline LC & WT & 50 & 6 & 1 & B2 & 232 & 228 \\
\hline LC & WT & 0 & 6 & 5 & A1 & 259 & 5 \\
\hline LC & WT & 0 & 6 & 3 & $\mathrm{C} 3$ & 3.10 & 6 \\
\hline LC & WT & 50 & 6 & 4 & C1 & 191 & 191 \\
\hline LC & WT & 100 & 6 & 9 & $\mathrm{~A} 1$ & 283 & 275 \\
\hline LC & WT & 50 & 6 & 8 & B3 & 248 & 247 \\
\hline LC & WT & 0 & 9 & 5 & 83 & 192 & 7 \\
\hline L.C & WT & 0 & 9 & 3 & $A 3$ & 178 & 7 \\
\hline LC & WT & 0 & 9 & 5 & $\mathrm{C} 3$ & 173 & 11 \\
\hline LC & WT & 50 & 9 & 4 & B3 & 265 & 261 \\
\hline LC & WT & 50 & 9 & 4 & $\mathrm{~A} 3$ & 375 & 375 \\
\hline LC & WT & 100 & 9 & 6 & $B 2$ & 188 & 187 \\
\hline LC & WT & 0 & 9 & 2 & C3 & 226 & 6 \\
\hline LC & WT & 100 & 9 & 7 & B3 & 193 & 193 \\
\hline LC & WT & 0 & 9 & 3 & B3 & 213 & 2 \\
\hline LC & WT & 50 & 9 & 8 & $\mathrm{C} 1$ & 169 & 169 \\
\hline LC & WT & 50 & 9 & 1 & $\mathrm{~A} 1$ & 164 & 161 \\
\hline LC & WT & 100 & 9 & 7 & $\mathrm{C} 3$ & 158 & 155 \\
\hline LC & WT & 50 & 9 & 4 & $\mathrm{~A} 1$ & 221 & 221 \\
\hline IC & WT & 0 & 9 & 3 & $\mathrm{C} 2$ & 214 & 11 \\
\hline LC & WT & 50 & 9 & 8 & $\mathrm{C} 3$ & 180 & 180 \\
\hline LC & WT & 100 & 9 & 6 & $\mathrm{C} 1$ & 176 & 176 \\
\hline LC & WT & 50 & 9 & 8 & $\mathrm{~A} 3$ & 131 & 127 \\
\hline IC & WT & 100 & 9 & 9 & $\mathrm{~A} 3$ & 393 & 392 \\
\hline LC & WT & 100 & 9 & 9 & 33 & 128 & 128 \\
\hline LC & WT & 100 & 9 & 6 & 33 & 183 & 181 \\
\hline LC & WT & 50 & 9 & 1 & 31 & 128 & 123 \\
\hline
\end{tabular}

Page 446 of 519 
AEH-12-PSEUDO-04

\begin{tabular}{|c|c|c|c|c|c|c|c|}
\hline LC & WT & 100 & 9 & 9 & $\mathrm{C} 3$ & 260 & 260 \\
\hline LC & WT & 0 & 9 & 2 & B3 & 236 & 6 \\
\hline LC & WT & 0 & 9 & 5 & $\mathrm{~A} 3$ & 177 & 2 \\
\hline LC & WT & 100 & 9 & 7 & A3 & 258 & 255 \\
\hline LC & WT & 0 & 9 & 2 & A3 & 326 & 6 \\
\hline LC & WT & 50 & 9 & 3 & $\mathrm{C} 1$ & 267 & 267 \\
\hline LC & WT & 0 & 12 & 3 & B2 & 312 & 8 \\
\hline LC & WT & 0 & 12 & 3 & A2 & 185 & 5 \\
\hline LC & WT & 100 & 12 & 6 & B1 & 249 & 248 \\
\hline LC & WT & 50 & 12 & 1 & $\mathrm{C} 3$ & 266 & 266 \\
\hline LC & WT & 100 & 12 & 6 & A1 & 239 & 236 \\
\hline LC & WT & 0 & 12. & 2 & A2 & 390 & 16 \\
\hline LC & $W T$ & 50 & 12 & 1 & $\mathrm{C} 2$ & 175 & 175 \\
\hline LC & WT & 50 & 12 & 1 & B3 & 160 & 159 \\
\hline LC & WT & 0 & 12 & 3 & $\mathrm{Cl}$ & 259 & 5 \\
\hline LC & WT & 0 & 12 & 2 & B1 & 185 & 4 \\
\hline LC & WT & 50 & 12 & 8 & $\mathrm{C} 2$ & 290 & 287 \\
\hline LC & WT & 100 & 12 & 9 & A2 & 316 & 316 \\
\hline LC & WT & 0 & 1.2 & 2 & $\mathrm{C} 2$ & 198 & 8 \\
\hline LC & WT & 100 & 12 & 7 & $\mathrm{~A} 2$ & 237 & 236 \\
\hline LC & WT & 50 & 12 & 8 & $A 2$ & 119 & 119 \\
\hline LCC & WT & 100 & 12 & 9 & B1 & 273 & 268 \\
\hline LC & WT & 50 & 12 & 8 & A1 & 151 & 150 \\
\hline LC & WT & 0 & 12 & 5 & A2 & 240 & 2 \\
\hline LC & WT & 0 & 12 & 5 & $\mathrm{C} 2$ & 212 & 6 \\
\hline LC & WT & 100 & 12 & 7 & B1 & 286 & 286 \\
\hline LC & WT & 0 & 12 & 5 & $\mathrm{~B} 1$ & 196 & 6 \\
\hline LC & WT & 50 & 12 & 4 & A2 & 158 & 155 \\
\hline LC & WT & 100 & 12 & 7 & $\mathrm{C} 2$ & 203 & 203 \\
\hline LC & WT & 100 & 12 & 9 & $\mathrm{C} 2$ & 156 & 155 \\
\hline LC & WT & 50 & 12 & 4 & C3 & 455 & 453 \\
\hline LC & WT & 50 & 12 & 4 & B1 & 280 & 277 \\
\hline LC & WT & 100 & 12 & 6 & $\mathrm{C} 2$ & 155 & 153 \\
\hline LC & $\mathrm{BI}$ & 100 & 12 & 9 & A1 & 269 & 256 \\
\hline LC & $\mathrm{BI}$ & 50 & 12 & 4 & B2 & 256 & 191 \\
\hline LC & $\mathrm{BI}$ & 100 & 12 & 9 & $B 3$ & 179 & 139 \\
\hline I.C & $\mathrm{BI}$ & 100 & 12 & 2 & B3 & 1.53 & 132 \\
\hline LC & $\mathrm{BI}$ & 50 & 12 & 1 & B3 & 279 & 217 \\
\hline LC & $\mathrm{BI}$ & 0 & 12 & 6 & $\mathrm{C} 1$ & 179 & 4 \\
\hline LC & $\mathrm{BI}$ & 50 & 12 & 8 & $C_{2}$ & 200 & 161 \\
\hline IC & $\mathrm{BI}$ & 0 & 12 & 7 & A1 & 2.25 & 4 \\
\hline LC & $\mathrm{BI}$ & 50 & 12 & 1 & B2 & 510 & 424 \\
\hline LC & $\mathrm{BI}$ & 50 & 12 & 4 & B1 & 188 & 178 \\
\hline LCC & $\mathrm{BI}$ & 100 & 12 & 2 & $\mathrm{Cl}$ & 193 & 154 \\
\hline I.C & $\mathrm{BI}$ & 100 & 12 & 9 & $c 1$ & 2.88 & 263 \\
\hline LC & $\mathrm{BI}$ & 0 & 12 & 3 & $C 3$ & 253 & 7 \\
\hline LC & BI & 50 & 12 & 4 & B3 & 188 & 174 \\
\hline LC & $\mathrm{BI}$ & 50 & 12 & 8 & B3 & 291 & 222 \\
\hline I.C & $\mathrm{BI}$ & 100 & 12 & 5 & A1 & 329 & 217 \\
\hline
\end{tabular}




\section{ÄEHH12.PSEUDO-04}

\begin{tabular}{|c|c|c|c|c|c|c|c|}
\hline LC & $\mathrm{BI}$ & 0 & 12 & 7 & B3 & 176 & 9 \\
\hline LC & BI & 0 & 12 & 3 & B1 & 242 & 5 \\
\hline LC & BI & 0 & 12 & 6 & B1 & 217 & 11 \\
\hline LC & $B 1$ & 100 & 12 & 5 & B3 & 393 & 272 \\
\hline LC & BI & 0 & 12 & 6 & $\mathrm{~A} 2$ & 186 & 4 \\
\hline LC & $B I$ & 50 & 12 & 8 & $\mathrm{~A} 3$ & 282 & 232 \\
\hline LC & BI & 0 & 12 & 3 & $\mathrm{~A} 3$ & 196 & 4 \\
\hline LC & 31 & 50 & 12 & 1 & $\mathrm{~A} 1$ & 210 & 158 \\
\hline LC & BI & 0 & 12 & 7 & C1 & 276 & 3 \\
\hline LC & BI & 100 & 12 & 2 & A1. & 279 & 261 \\
\hline LC & B) & 100 & 12 & 5 & C1 & 264 & 209 \\
\hline$S L$ & WT & 50 & 6 & 8 & C1 & 136 & 1.33 \\
\hline $\mathrm{SL}$ & WT & 0 & 6 & 7 & B1 & 80 & 2 \\
\hline SL. & WT & 100 & 6 & 9 & $\mathrm{~A} 1$ & 77 & 75 \\
\hline SL & WT & 0 & 6 & 1 & A1 & 109 & 4 \\
\hline SL & WT & 0 & $G$ & 1 & B2 & 87 & 2 \\
\hline SL & WT & 0 & 6 & 7 & $\mathrm{C} 1$ & 108 & 5 \\
\hline SL & WT & 100 & 6 & 6 & $\mathrm{~A} 3$ & 57 & 54 \\
\hline SL & WT & 100 & 6 & 9 & $\mathrm{C} 1$ & 1.18 & 99 \\
\hline SL. & WT & 100 & 6 & 3 & B3 & 104 & 95 \\
\hline$S L$ & $W T$ & 50 & 6 & 8 & $\mathrm{~A} 3$ & 96 & 91 \\
\hline SL. & WT & 100 & 6 & 3 & B2 & 122 & 114 \\
\hline SL & $W T$ & 100 & 6 & 6 & $\mathrm{~A} 2$ & 124 & 121 \\
\hline SL & WT & 50 & 6 & 2 & $\mathrm{~A} 1$ & 108 & 91 \\
\hline $\mathrm{SL}$ & $W T$ & 0 & 6 & 1 & B1 & 118 & 7 \\
\hline S! & WT & 50 & 6 & 5 & B1 & 67 & 59 \\
\hline SI & WT & 100 & 6 & 3 & A1 & 138 & 111 \\
\hline SL & WT & 100 & 6 & 9 & B1 & 119 & 115 \\
\hline$S\llcorner$ & WT & 0 & 6 & 4 & A1 & 64 & 3 \\
\hline$S L$ & WT & 50 & 6 & 2 & B1 & 116 & 106 \\
\hline$S L$ & WT & 0 & 6 & 7 & $\mathrm{~A} 1$ & 124 & 2 \\
\hline$S L$ & WT & 100 & 6 & 6 & $\mathrm{~A} 1$ & 69 & 69 \\
\hline Sl. & WT & 50 & 6 & 5 & $\mathrm{Cl}$ & 85 & 71 \\
\hline SL. & WT & 50 & 6 & 2 & $\mathrm{Cl}$ & 61 & 57 \\
\hline SL & WT & 0 & 6 & 4 & B1 & 78 & 4 \\
\hline SL. & WT & 0 & 6 & 4 & $A 2$ & 74. & 7 \\
\hline SL & WT & 50 & 6 & 5 & $\mathrm{~A} 1$ & 95 & 87 \\
\hline SL & $W T$ & 50 & 6 & 8 & B3 & 149 & 92 \\
\hline SL & WT & 100 & 9 & 3 & B.1 & 51 & 49 \\
\hline$S L$ & WT & 50 & 9 & 8 & C2 & 61 & 60 \\
\hline SL & WT & 100 & 9 & 3 & $\mathrm{C} 2$ & 73 & 69 \\
\hline SL & WT & 0 & 9 & 4 & $\mathrm{C} 1$ & 71 & 2 \\
\hline SL & WT & 0 & 9 & 1 & B3 & 84 & 2 \\
\hline SL & WT & 0 & 9 & 1 & $\mathrm{~A} 3$ & 159 & 9 \\
\hline SL & $W T$ & 50 & 9 & 8 & $\mathrm{~A} 1$ & 148 & $1 / 16$ \\
\hline SL. & WT & 0 & 9 & 7 & $\mathrm{~B} 2$ & 108 & 6 \\
\hline SL & WT & 50 & 9 & 2 & C3 & 117 & 112 \\
\hline SL & WT & 100 & 9 & 9 & C3 & 123 & 116 \\
\hline SL & $W T$ & 0 & 9 & 7 & $\mathrm{~A} 3$ & 54 & 2 \\
\hline
\end{tabular}


AEH-12.PSEUDO-04

\begin{tabular}{|c|c|c|c|c|c|c|c|}
\hline$S L$ & $W T$ & 0 & 9 & 7 & $\mathrm{C3}$ & 1.11 & 6 \\
\hline SL. & WT & 50 & 9 & 8 & B1 & 85 & 80 \\
\hline SL. & WT & 50 & 9 & 5 & A3 & 61 & 59 \\
\hline SL & WT & 100 & 9 & 3 & C3 & 88 & 88 \\
\hline SL & WT & 50 & 9 & 2 & B2 & 103 & 94 \\
\hline SL. & WT & 100 & 9 & 9 & A3 & 107 & 93 \\
\hline SL. & WT & 100 & 9 & 6 & B1 & 59 & 59 \\
\hline SL & WT & 50 & 9 & 5 & C3 & 80 & 61 \\
\hline SL & WT & 100 & 9 & 9 & B2 & 69 & 69 \\
\hline SL & WT & 50 & 9 & 2 & A3 & 114 & 88 \\
\hline SL & WT & 100 & 9 & 6 & $\mathrm{C} 2$ & 94 & 94 \\
\hline SL & WT & 0 & 9 & 4 & $B 2$ & 75 & 3 \\
\hline SL & WT & 100 & 9 & 6 & B2 & 94 & 81 \\
\hline SL & WT & 50 & 9 & 5 & $B 2$ & 105 & 83 \\
\hline SL & WT & 0 & 9 & 4 & A3 & 110 & 7 \\
\hline S1. & WT & 0 & 9 & 1 & $\mathrm{C} 1$ & 144 & 6 \\
\hline SL & WT & 100 & 12 & 6 & $\mathrm{C} 1$ & 62 & 59 \\
\hline SL & WT & 100 & 12 & 3 & A3 & 131 & 125 \\
\hline SL & WT & 50 & 12 & 5 & $\mathrm{C} 2$ & 114 & 112 \\
\hline SL & WT & 50 & 12 & 2 & $A 2$ & 98 & 97 \\
\hline SL & WT & 0 & 12 & 1 & A2 & 101 & 5 \\
\hline SL & WT & 0 & 12 & 1 & $\mathrm{C} 2$ & 108 & 6 \\
\hline SL & WT & 100 & 12 & 3 & A2 & 95 & 94 \\
\hline SL & WT & 50 & 12 & 8 & B2 & 88 & 85 \\
\hline SL. & WT & 100 & 12 & 9 & $\mathrm{C} 2$ & 60 & 58 \\
\hline SL. & $W T$ & 100 & 12 & 6 & B3 & 77 & 77 \\
\hline SL & WT & 0 & 12 & 7 & A2 & 92 & 2 \\
\hline St & WT & 50 & 12 & 2 & $\mathrm{C} 2$ & 86 & 86 \\
\hline SL & WT & 100 & 12 & 6 & C3 & 102 & 101 \\
\hline SL & WT & 50 & 12 & 8 & C3 & 129 & 128 \\
\hline SL & $W T$ & 100 & 12 & 3 & $\mathrm{C} 1$ & 104 & 103 \\
\hline SL & $W_{T}$ & 50 & 12 & 2 & B3 & 85 & 80 \\
\hline SL & WT & 50 & 12 & 5 & B3 & 63 & 58 \\
\hline SL & $W T$ & 0 & 12 & 4 & C3 & 141 & 4 \\
\hline SL & WT & 0 & 12 & 7 & $\mathrm{C} 2$ & 90 & 3 \\
\hline SL & $W T$ & 0 & 12 & 7 & B3 & 104 & 2 \\
\hline SL. & WT & 50 & 12 & 8 & $\mathrm{~A} 2$ & 76 & 73 \\
\hline SL & WT & 0 & 1.2 & 4 & B3 & 79 & 3 \\
\hline SL. & WT & 100 & 12 & 9 & B3 & 102 & 100 \\
\hline SL. & WT & 0 & 12 & 4 & $\mathrm{C} 2$ & 81 & 4 \\
\hline SL. & WT & 100 & 12 & 9 & A2 & 104 & 104 \\
\hline SL. & WT & 50 & 12 & 5 & $A 2$ & 60 & 60 \\
\hline SL. & WT & 0 & 12 & 1 & C3 & 111 & 5 \\
\hline SI. & $\mathrm{Bl}$ & 0 & 12 & 1 & C3 & 147 & 8 \\
\hline SL. & $\mathrm{BI}$ & 50 & 12 & 3 & C2 & 109 & 107 \\
\hline SL. & $\mathrm{Bl}$ & 0 & 12 & 8 & B2 & 134 & 6 \\
\hline SL. & $\mathrm{Bl}$ & 50 & 12 & 3 & B2 & 103 & 103 \\
\hline SL & $\mathrm{Bl}$ & 100 & 12 & 4 & B3 & 126 & 124 \\
\hline SL & BI & 0 & 12 & 1 & $\mathrm{C} 2$ & 111 & 3 \\
\hline
\end{tabular}

Page 449 of 519 


\begin{tabular}{|c|c|c|c|c|c|c|c|}
\hline \multirow[b]{2}{*}{ SL } & \multirow[b]{2}{*}{ B. } & \multirow[b]{2}{*}{100} & \multirow[b]{2}{*}{12} & \multirow[b]{2}{*}{5} & \multicolumn{3}{|c|}{ AEH-12.PSEUDO-04 } \\
\hline & & & & & B1 & 105 & 105 \\
\hline SL. & $\mathrm{B} \mid$ & so & 12 & 7 & $B 1$ & 128 & 124 \\
\hline$S L$ & $\mathrm{~B} \mid$ & 0 & 12 & 8 & B3 & 163 & 4 \\
\hline$S L$ & B! & 100 & 12 & 4 & B2 & 123 & 122 \\
\hline SL & BI & 100 & 12 & 4 & C3 & 104 & 104 \\
\hline SL. & $\mathrm{BI}$ & so & 12 & 7 & B3 & 109 & 103 \\
\hline SL. & BI & 50 & 12 & 7 & $B 2$ & 116 & 113 \\
\hline SL & $\mathrm{BI}$ & 0 & 12 & 8 & B1 & 81 & 5 \\
\hline SL & $\mathrm{BI}$ & 100 & 12 & 5 & B2 & 97 & 97 \\
\hline$S L$ & BI & 50 & 12 & 7 & $A 2$ & 115 & 113 \\
\hline SL & $B I$ & 100 & 12 & 6 & B3 & 113 & 113 \\
\hline SL & BI & 0 & 12 & 8 & $\mathrm{~A} 3$ & 140 & 7 \\
\hline $\mathrm{SL}$ & BI & 0 & 12 & 9 & B2 & 87 & 2 \\
\hline SL & BI & 50 & 12 & 2 & $A 2$ & 119 & 115 \\
\hline SL & $B I$ & 50 & 12 & 2 & B1 & 105 & 102 \\
\hline SL & $\mathrm{BI}$ & 100 & 12 & 6 & B2 & 121 & 119 \\
\hline SL & Bl & 100 & 12 & 6 & $\mathrm{C} 2$ & 127 & 126 \\
\hline SL & BI & 100 & 12 & 4 & $A 2$ & 117 & 116 \\
\hline SL & $\mathrm{BI}$ & 50 & 12 & 3 & B1 & 114 & 136 \\
\hline SL & $\mathrm{BI}$ & 100 & 12 & 5 & B3 & 108 & 108 \\
\hline SL. & BI & 100 & 12 & 6 & $\mathrm{C} 3$ & 98 & 95 \\
\hline SL. & BI & 0 & 12 & 1 & $\mathrm{~A} 3$ & 107 & 7 \\
\hline SL. & $\mathrm{BI}$ & 100 & 12 & 5 & $\mathrm{~A} 2$ & 106 & 104 \\
\hline SL. & $\mathrm{BI}$ & 0 & 12 & 9 & B3 & 137 & 10 \\
\hline SL. & $\mathrm{BI}$ & 50 & 12 & 2 & B2 & 116 & 114 \\
\hline SL. & BI & 0 & 12 & 9 & B1 & 84 & 4 \\
\hline$S L$ & $B 1$ & 0 & 12 & 9 & $\mathrm{~A} 2$ & 115 & 0 \\
\hline SL & $\mathrm{BI}$ & 50 & 12 & 3 & $\wedge 2$ & 117 & 113 \\
\hline SL & $\mathrm{Bl}$ & 0 & 12 & 1 & $\mathrm{C} 1$ & 137 & 10 \\
\hline SL & BI & 50 & 12 & 2 & A3 & 145 & 140 \\
\hline
\end{tabular}

File Folder: 18

Item Number:

Page $\_$6 of -6 


\section{Appendix 9. Statistical Analysis including SAS Programs, Outputs and Logs for Survival Data}

\begin{tabular}{clrc}
\hline $\begin{array}{c}\text { Item } \\
\text { Number }\end{array}$ & Item Description & $\begin{array}{c}\text { Number } \\
\text { of } \\
\text { Pages }\end{array}$ & $\begin{array}{c}\text { Report } \\
\text { Page } \\
\text { Number }\end{array}$ \\
\hline 1 & SAS program for zebra mussel survival data & 7 & 452 \\
2 & SAS log for zebra mussel survival data & 13 & 459 \\
3 & SAS output for zebra mussel survival data & 39 & 472 \\
\hline
\end{tabular}


dM 'log; clear; outfut; Clear;'; * cleaf log and output;

FOOTNOTE1 'Porformed by K. Wober SAS verSion ' \&SYSVER \&SYSTIME \&SYSDATE;

options $1 \mathrm{~s}=97 \mathrm{ps}=54$ formdl.im= +1 pageno $=1$ nocenter nodate nosource 2 ;

titlel $h=$ : 'Statistical analysis of zebra mussel mortality after';

titlez $h=1$ 'exposure to various concentrations of Pf-CL145A';

title3 $h=1$ 'SAS $v .9 .3$ Analysis completion date: 01 MAY2014 Analysis prepare title4 $h=1$ ' ';

/*****************************************************

* SAs ver 9.3 Analysis prepared by: KLW fiw *

* Analysis completion date: 01NAYro14 [Mijult*

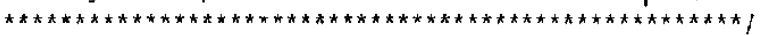

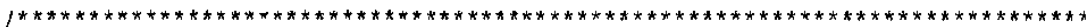

* Variable Names:

* 100 = test location LC=Lake Carlos; SL = Shawano Lake

* trt_typ $=$ expostire method WT $=$ whole water application; EI = bottom injectic

* conc $=$ concentration (in $\mathrm{mg} / \mathrm{L}$ )

* $\quad 50=50 \mathrm{ng} / \mathrm{L}$ active ingredient

* $\quad 100=100 \mathrm{mg} / \mathrm{L}$ active ingredient

* tnk = test tank ID ( 1 to 9 )

* timo $=$ time post exposure assessment occurred

* tray = zebra mussel tray within tank - there were three mussel trays at eact

* tot = total number of zebra mussels at risk

* dead $=$ number of zebra mussels dead after treatment

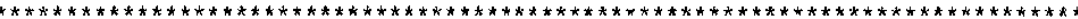

data mussel; set Pseudoo4.survivaldata;

pctsurv $=($ tot - dead $) /$ tot*100;

potmort $=$ dead $/$ tot*100;

if conc $=0$ then conca $={ }^{\prime} \mathrm{C}^{\prime}$;

if conc $=50$ then conca $={ }^{\prime} \mathrm{A}^{\prime}$;

if conc $=100$ then conca $={ }^{\prime} B^{\prime}$;

run;

proc sort data=mussel; by loc trt_typ cono time; run;

proc print data=mussel; title4 $n=\overline{1}$ 'all data'; run;

Title $1 \mathrm{~h}=2$ 'The mean percent survival and mortality by location and treatment

title2 $h=2$ ' classified by corcentration and exposure duration';

proc means data $=$ mussel mean std $1 \mathrm{clm}$ uclm $\mathrm{f}_{\mathrm{w}=8 ;}$

by loc trt_typ;

class conc time;

var petsurv petmort;

run;

FF \# 18

Item No.

$\mathrm{Pg} \perp$ of 7 
data Carlos_6; set mussel;

if trt_typ = 'BI' then delete;

if $100=$ 'SL' then delete;

if time ne 6 then delete;

run:

data Carlos_9; set mussel;

if trt_typ = ' $B I$ ' then delete;

if $10 \mathrm{c}=$ 'SL' then delete;

if time ne 9 then delete;

run;

data Carlos 12 ; set mussel;

if trt_typ = 'BI' then delete;

if $100=$ ' $S L$ ' then delete;

if time ne 12 then delete;

run;

data Shawanc 6 ; set musse 1 ;

if trt_typ = 'BI' toen delote;

if $100=$ ' $L C$ ' then delete;

if time ne 6 then delete;

run;

data Shawano_9; set mussel;

if trt_typ = 'BI' then delete;

if $10 C=$ ' $L O$ ' then delete;

if time ne 9 then delete;

run;

data Shawano_12; set mussel;

if trt_typ = 'BI' then delete;

if $100=$ 'LC' then delete;

if time ne 12 then delete;

sun;

data time carlos; set mussel;

if trt_typ = 'BI' then delete;

if $l o c=$ 'SL' then delete;

run;

data time_Shawano; set mussel;

if trt_typ = 'BI' then delete;

if $100=$ 'LC' then delete;

run;

data BI_Carlos; set mussel;

if trt_typ = 'WT' then delete;

if $10 c=$ 'SL' then delete;

data BI_Shawano; set nussel;

Page 2 of 17 


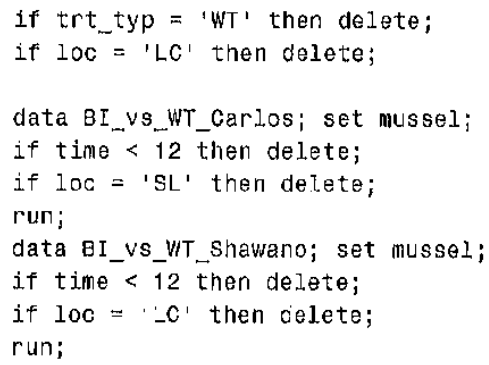

Page 3 of 7 


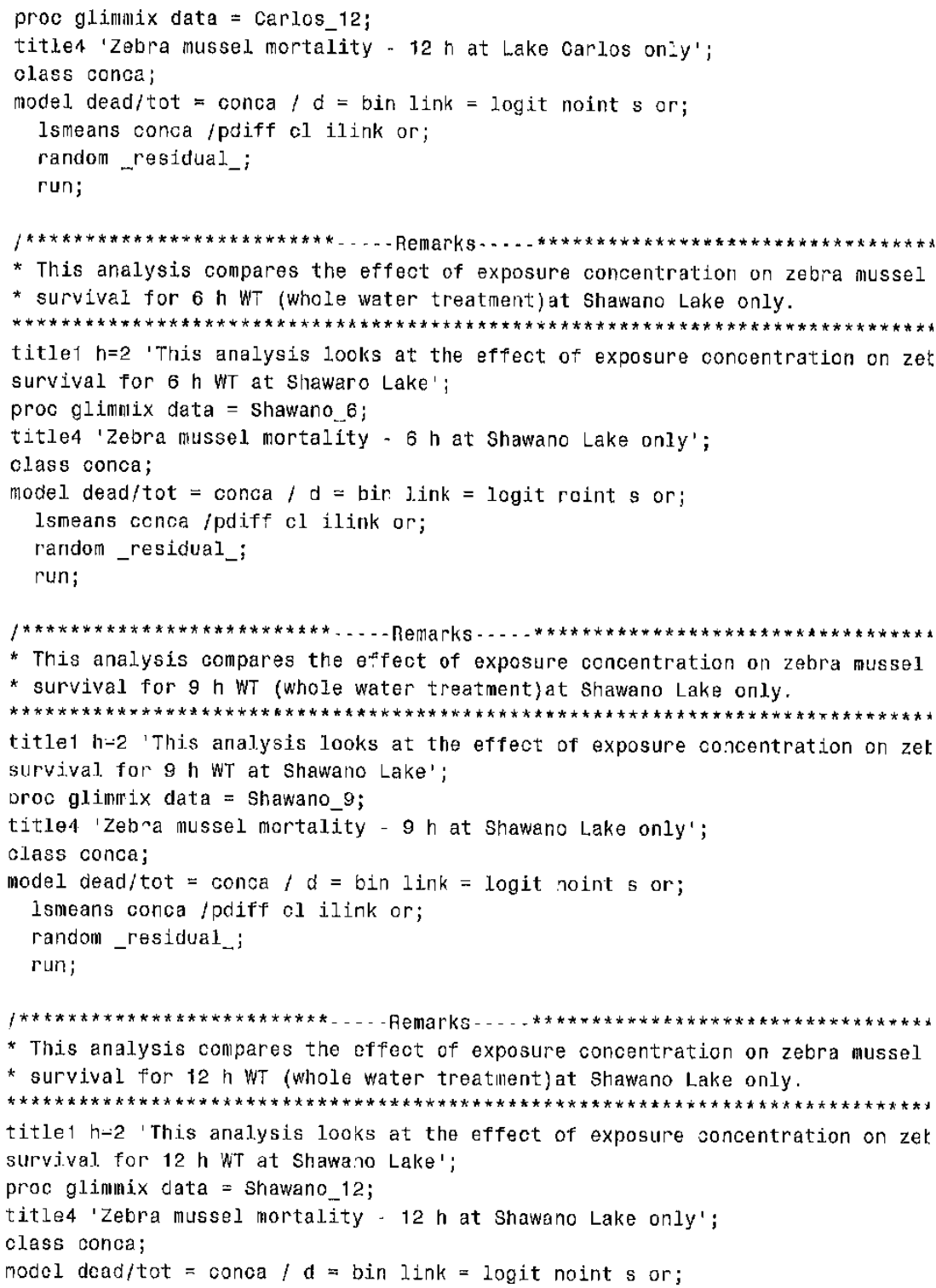

Page 4 of 7 


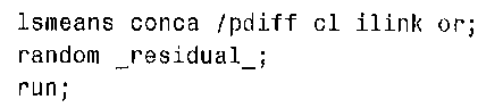




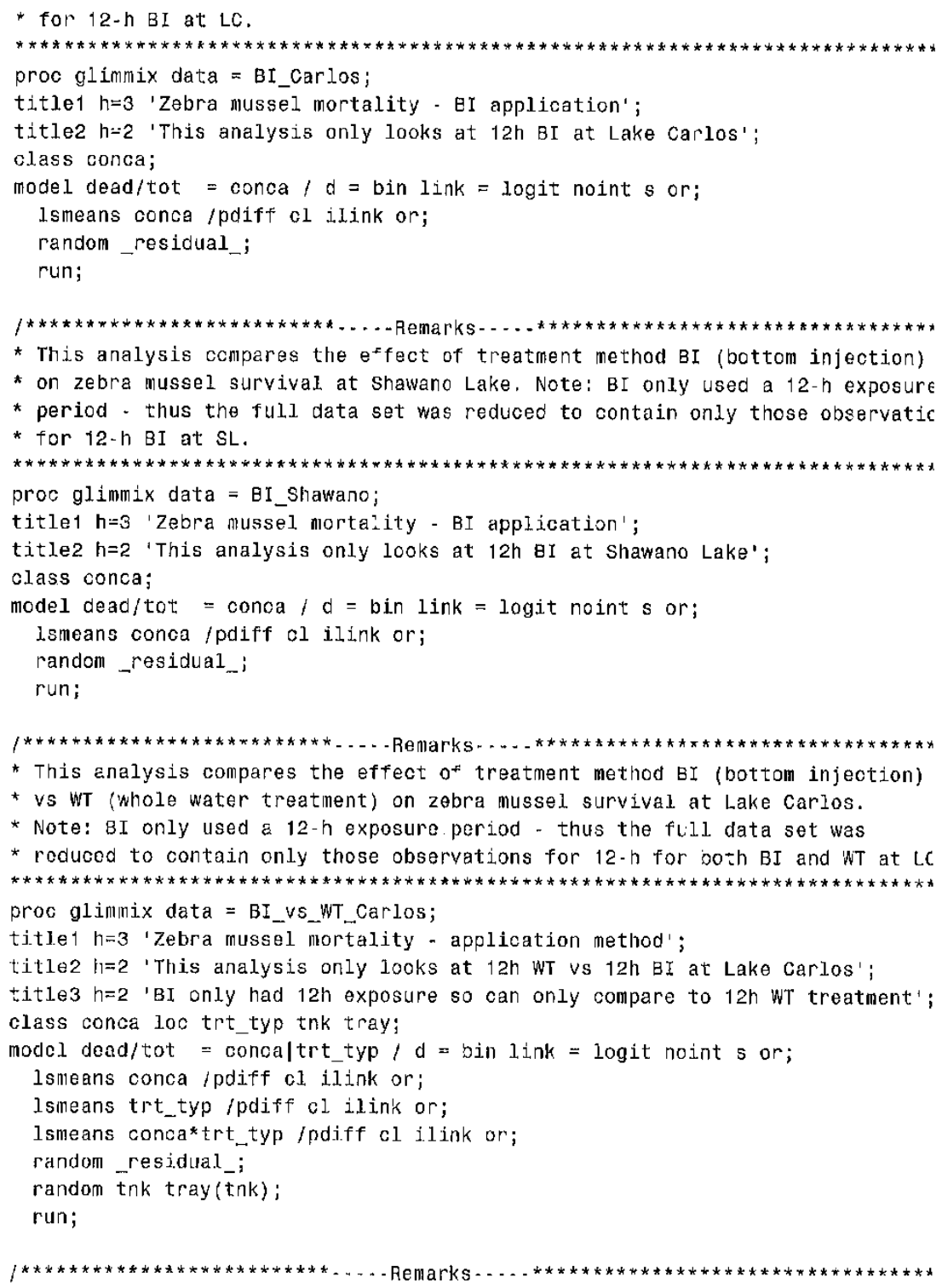

Page 6 of 7 


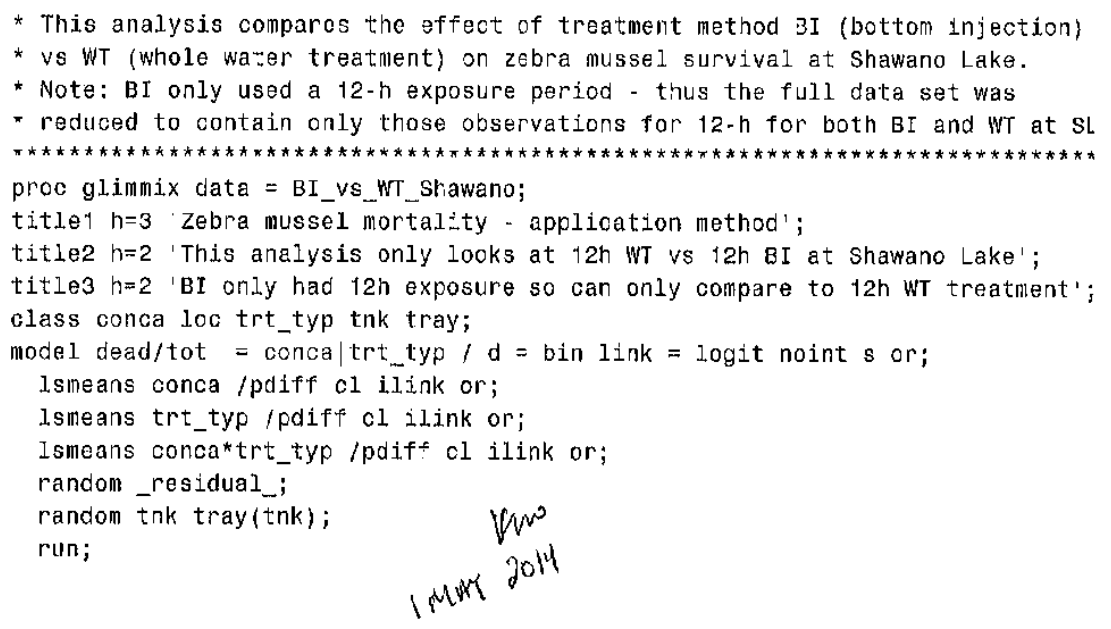

FF\# 18

Item No. $Q$

$\mathrm{Pg} 7$ of 7

\section{Page 458 of 519}




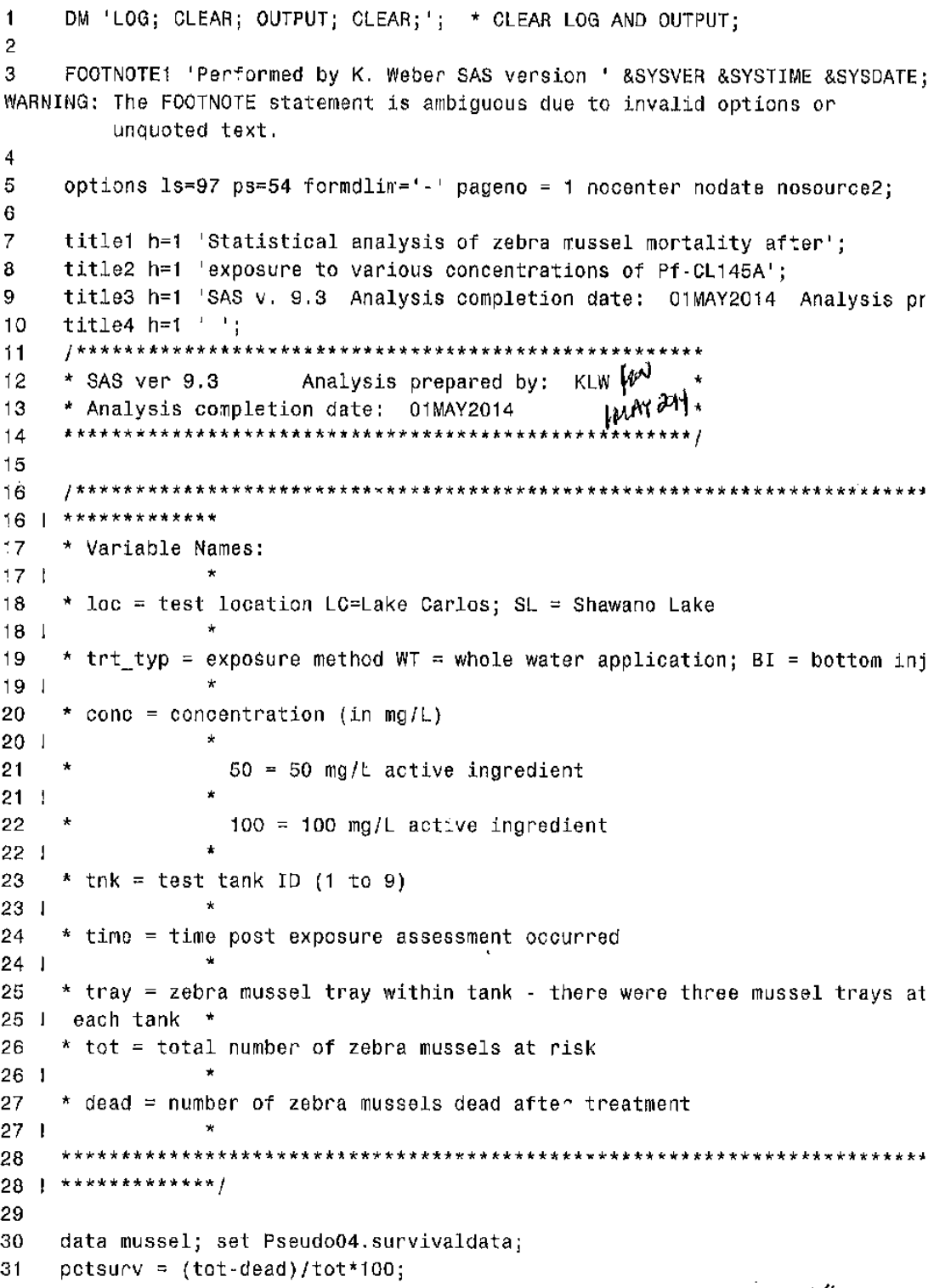

FF \# 18

Item No: 3

$\mathrm{Pg} \perp \frac{13}{13}$ 


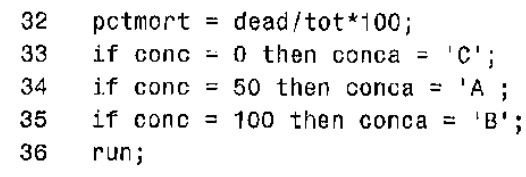

NOTE: There were 225 observations read from the data set PSEUDO04.SURVIVALDATA NOTE: The data set WORK.MUSSEL has 225 observations and 11 variables.

NOTE: DATA statemert used (Total process time):
real time
0.10 seconds
cpu time
0.00 seconds

37

38 proc sort data=mussel; by loc trt_typ conc time; run;

NOTE: There were 225 observations read from the data set WORK.MUSSEL.

NOTE: The data set WORK.MUSSEL has 225 observations and 11 variables.

NOTE: PROCEDURE SORT used (Total process time):
real time
0.01 seconds
cpu time
0.01 seconds

39 proc print data=mussel; title4 $n=1$ 'all data'; run;

NOTE: Writing HTML Body file: sashtml.htm

NOTE: There were 225 observations read from the data set WORK.MUSSEL.

NOTE: PROCEDURE PRINT used (TotaI process time):

real time $\quad 0.67$ seconds

cpu time $\quad 0.49$ seconds
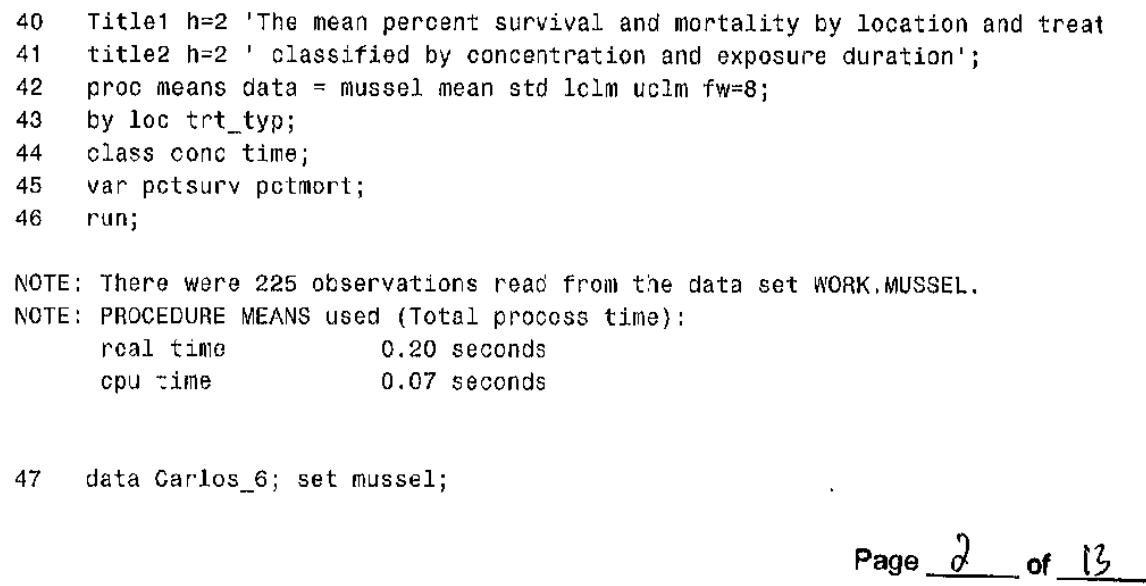


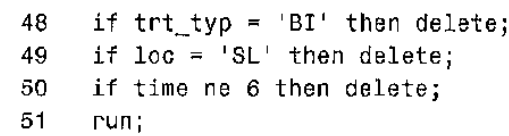

NOTE: There were 225 observations read from tho data set WORK.MUSSEL. NOTE: The data set WORK.CARLOS_6 has 27 observations and 11 variables. NOTE: DATA statemert used (Total process time):
real time
0.01 seconds

cpu time

0.01 seconds

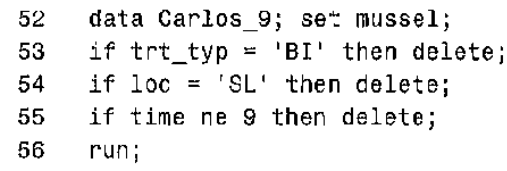

NOTE: There were 225 observations read from the data set WORK.MUSSEL. NOTE: The data set WORK.CARLOS 9 has 27 observations and 11 variables. NOTE: DATA statement used (Total process time):
real time
0.01 seconds
cpu time
0.01 seconds
57 data carlos_12; set nussel;
58 if trt_typ = 'BI' then delete;
59 if $100=$ 'SL' then delete;
60 if time ne 12 then delete;
61 run;

NOTE: There were 225 observations read from the data set WORK.MUSSEL.

NOTE: The data set WORK.CARLOS_i2 has 27 observations and 11 variables.

NOTE: DATA statement used (Total process time):

real time $\quad 0.00$ seconds

cpu time $\quad 0.00$ seconds

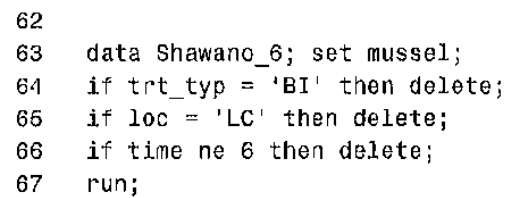

NOTE: There were 225 observations read from the data set WOFK.MUSSEL.

Page 3 of 13 
NOTE: The data set WORK.SHAWANO_6 has 27 observations and 11 variables.

NOTE: DATA statement used (Total process time):
real time
0.01 seconds
cpu time
0.01 seconds

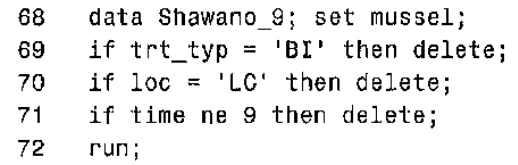

NOTE: There were 225 observations read from the data set WORK. MUSSEL.

NOTE: The outa set WORK.SHAWAVO 9 has 27 observations and 11 variables.

NOTE: DATA statement lised (Total process time):
real time
0.01 seconds
cpu time
0.01 seconds

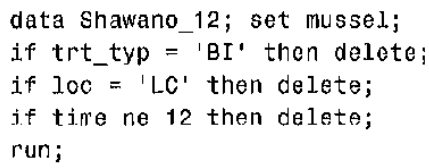

NOTE: There were 225 observations read from the data set WORK.MUSSEL.

NOTE: The data set WORK.SHAWANO_12 has 27 observations and 11 variables.

NOTE: DATA statement used (Total process time):

real time $\quad 0.01$ seconds

cpu time $\quad 0.01$ seconds

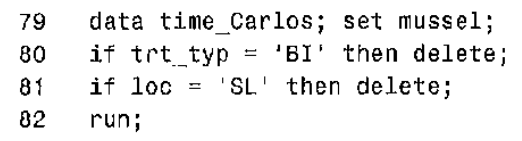

NOTE: There were 225 observations read from the data set WOAK.MUSSEL.

NOTE: The data set WORK.TIME_CARLOS has 81 observations and 11 variables.

NOTE: DATA statement used (Total process time):
real time
0.00 seconds
сри time
0.00 seconds

83 


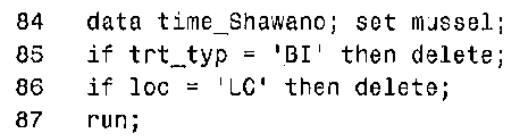

NOTE: There were 225 observations read from the ciata set WORK.MUSSEL.

NOTE: The data set WORK. TIME SHAWANO has 81 observations and 11 variables.

NOTE: DATA statenent used (Total process time):
real time
0.00 seconds
cpu time
0.00 seconds

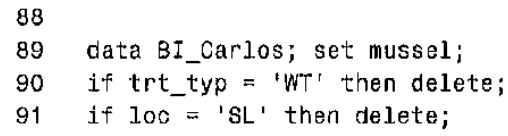

NOTE: There were 225 observations read from the data set WORK, MUSSEL.

NOTE: The data set WORK.BI_CARLOS has 27 observations and 11 variables.

NOTE: DATA statement used (Total process time):

real time $\quad 0.00$ seconds

cpu time $\quad 0.00$ seconds

92 data BI_Shawano; set mussel;

93 if trt_typ $=$ ' WT' then delete;

94 if $100=$ ' $L C$ ' then delete;

95

NOTE: There were 225 observations read from the data set WORK, MUSSEL.

NOTE: The da:a set WORK.BI_SHAWANO has 36 observations and 11 variables.

NOTE: DATA statement used (Total process time):

real time $\quad 0.00$ seconds

cpu time $\quad 0.00$ seconds

96 data BI_vs_WT_Carlos; set mussel;

97 if time $<12$ then delete:

98 if $100=$ 'SL.' ther delete;

99 run;

NOTE: There were 225 observations read from the data set WORK. MUSSEL.

NOTE: The data set WORK.BI_VS WT_CARLOS has 54 observatiors and 11 variables.

NOTE: DATA statement used (Total process time):

real time $\quad 0.03$ seconds

cpu time $\quad 0.00$ seconds

Page 5 of 13 


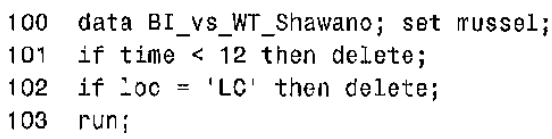

NOTE: There were 225 observations read from the data set WOAK.MUSSE!.

NOTE: The data set WORK.BI_VS_WT_SHAWANO has 63 observations and 11 variables. NOTE: DATA statement l'sed (Total process time):
real. time
0.01 seconds
cpu time
0.01 seconds

104

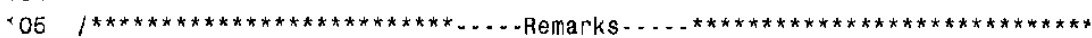

- 06 * This analysis compares the effect of exposure concentration on zebra me

-07 * survival for $6 \mathrm{~h}$ WT (whole water treatment) at Lake Carlos only,

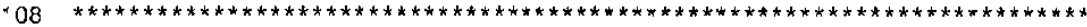

109 title1 $h=2$ 'This analysis looks at the effect of exposure concentration $c$

110 survival for 6 h WT at Lake Carlos';

111 proc glimmix data = Carlos_6;

112 title4 'Zebra mussel mortality - $6 \mathrm{~h}$ at Lake Carlos only'

113 class conca;

114 model dead $/$ tot $=$ conca $/ d=$ bin link = logit noint $s$ or:

115 lsmeans conca/pdiff cl, ilink or;

116 random_residual_;

117 run;

NOTE: The model does not contain an intercept. Columns of $x$ are scaled only ar NOTE: Corvergence criterion (ABSGCONV $=0.00001$ ) satisfied.

NOTE: PROCEDURE GLIMMIX used (Total process time):
rea.l. time
0.26 seconds
cpu time
0.10 seconds

118

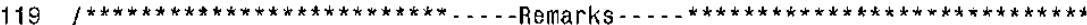

$120 *$ This analysis compares the effect of exposure concentration on zebra nut

121 * survival for $9 \mathrm{~h}$ WT (whol.e water treatnent) at Lake Carlos only.

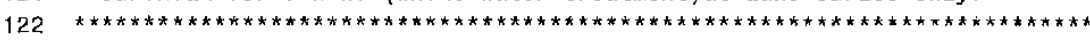

123 title1 $\mathrm{h}=2$ 'This analysis looks at the effect of exposure concentration $c$

124 survival for $9 \mathrm{~h}$ WT at Lake Carlos';

Page 6 of 13 


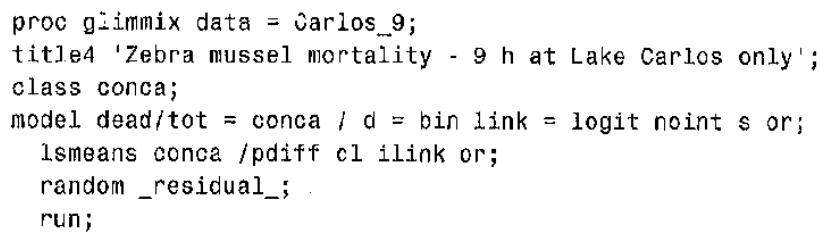

NOTE: The model does not contain an intercept. Columns of $X$ are scaled only ar NOTE: Convergence criterion (GCONV=1E-8) satisfied.

NOTE: PROCEDURE GLINMIX used (Total process time):
real time
0.21 seconds
cpu time
0.15 seconds

132

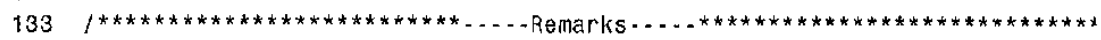

$134 *$ This analysis compares the effect of exposure concentration on zebra $\mathrm{mL}$

$135 *$ survival for $12 \mathrm{~h}$ WT (whole water treatment)at Lake Carlos only.

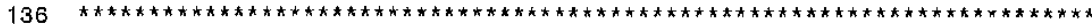

137 titlet $h=2$ 'This analysis looks at the effect of exposure concentration $c$

138 survival for $12 \mathrm{~h}$ Wr at Lake Carlos';

139 proc glimmix data = Carlos_12;

140 titlo4 Zebra mussel mortality - 12 h at Lake Carlos only';

141 class conca;

142 model dead $/$ tot = conca $/ \mathrm{d}=$ bin link = logit noint s or;

143 lsmeans conca /pdiff cl ilink or;

144 random residual_;

145 run;

NOTE: The model does not contain an intercept. Columns of $X$ are scaled only ar NOTE: Convergence criterion (ABSGCONV=0.00001) satisfied.

NOTE: PROCEDURE GL.IMMIX used (Total process time):
real time
0.23 seconds

cpu $\div$ ime

0.14 seconds

146

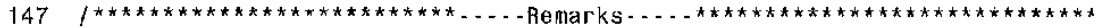

148 * This analysis compares the effect of exposure concentration on zebra mu

Page 7 of 13 
149 * Survival for $6 \mathrm{~h}$ WT (whole water treatment)at Shawano Lake only.

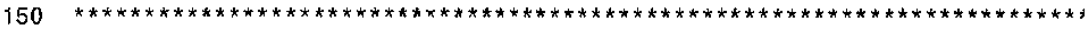

151 title1 $h=2$ 'This analysis looks at the effect of exposure concentration c 152 survival for $6 \mathrm{~h}$ WT at Shawano Lake';

153 proc glimmix data = Shawano_6;

154 title4 'Zebra mussel mortality - $6 \mathrm{~h}$ at Shawano Lake only';

155 class conca;

156 model dead $/$ tot $=$ conca $/ d=$ bin link = log $-t$ noint $s$ or

157 lsneans conca /pdiff cl ilink or;

158 random residual_;

159 run;

NOTE: The model does not contain an intercept. Columns of $X$ are scaled only ar NOTE: Convergence criterion (ABSGCONV=0,00001) satisfied.

NOTE: PROCEDURE GLIMMIX used (Total process time):
real time
0.29 seconds

cpu time

0.17 seconds

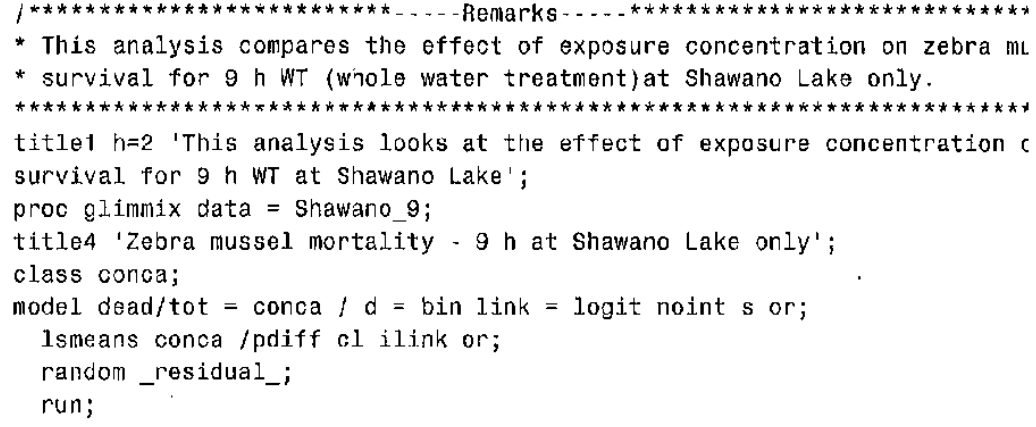

NOTE: The nodel does not contain an intercept, Columns of $X$ are scaled only ar NOTE: Convergence eriterion (GCONV=1E-8) satisfied.

NOTE: PROCEDURE GLIMMIX used (Total process time)
real time
0.37 seconds
cou time
0.15 seconds

Page $\quad 8$ of 13 
MEH-12-PSEUEOR?

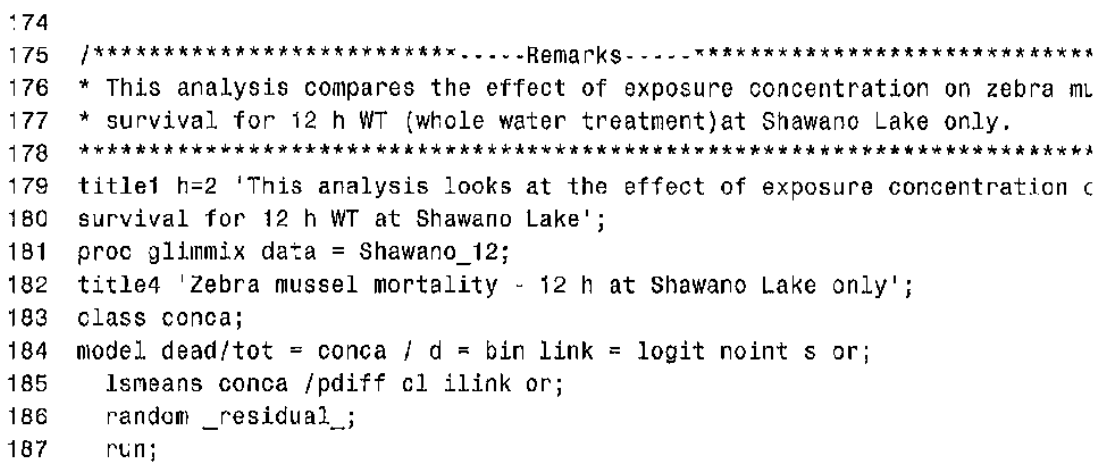

NOTE: The model does not contain an intercept. Columns of $X$ are scaled only ar NOTE: Convergence criterion (ABSGCONV=0.00001) satisfied.

NOTE: PROCEDURE GLIMMIX used (Total process time):
rea: time
0.49 seconds

cpu time

0.10 seconds

188

189

190

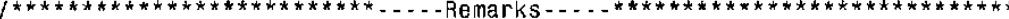

* This analysis compares the effect of exposure duration on zebra mussel

* survival for the WT (whole water treatment)at Lake Carlos only. Note: E

* 12-h exposure period and was therefore excluded from the analysis set.

* full data set was reduced to contain all observations for WT at LC.

$* * * * * * * * * * * * * * * * * * * * * * * * * * * * * * * * * * * * * * * * * * * * * * * * * * * * * * * * * * * * * * * * * * * * * * * * *$

title1 $h=2$ "This analysis looks at the effect of exposure duration at Lak

title2 $h=2$ 'Therefore BI data were excluded from this analysis';

title 3 h=? 'Includes 6,9 and 12 h wW Lake Carlos data';

proc glimmix data = time_carlos;

title4 'Zebra mussel mortality - time at Lake Carlos only';

class time conca;

model dead/tot $=$ tinc/conca $/ d=$ bin link = logit noint $s$ or

lsmeans conca/pdiff $c l$ ilink or;

ismeans time/pciff cl ilink or;

lsmeans conca*time /pdiff cl ji.ink or;

random_residual_;

run; 
NOTE: The nodel does not contain an intercept. Columns of $X$ are scaled only ar NOTE: Convergence criterion ( $G C O N V=1 E-8)$ satisfied.

NOTE: PROCEDURE GLIMMIX used (Total process time):

real time $\quad 0.35$ seconds

cpu time $\quad 0.20$ seconds

207

$208 f^{* * * * * * * * * * * * * * * * * * * * * * * * * * * \ldots . . .- \text { Remarks } \ldots . . . . * * * * * * * * * * * * * * * * * * * * * * * * * * * * *}$

209 * This analysis compares the effect of exposure duration on zebra mussel

210 * survival for the WT (whole water treatment) at Shawano Lake only. Note:

211 * 12-h exposure period and was therefore excluded from the analysis set.

212 * full data set was reduced to contain all observations for WT at SL.

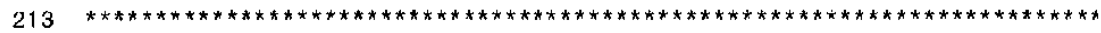

214 title1 $h=2$ 'This analysis looks at the effect of exposure duration at she

215 title2 $h=2$ 'Therefore BI data were excluded from this analysis';

216 title $3 \mathrm{~h}=2$ 'Inclides 6,9 and $12 \mathrm{~h}$ WW Shavano Lake data';

2.17 proc glimmix data = time_shawano;

218 t1tle4 'Zebra mussel mortality - time at Shawano Lake only';

219 class time conca;

220 model dead $/$ tot $=$ time $/$ conca $/ d=$ bin $l i n k=\operatorname{logit}$ noint $s$ or ;

221 lsmeans conca /odiff cl ilink or;

222 lsmeans time/adiff cl ilink or;

223 lsmeans conca*time/pdiff cl ilink or;

224 random_residual_;

225 run;

NOTE: The model doos not contain an intercept. Columns of $X$ are scaled only ar NOTE: Convergence criterion (GCONV=1E-8) satisfied,

NOTE: PROCEDIJRE GLTMMIX used (Total process time):

real time $\quad 0.35$ seconds

cou time $\quad 0.18$ seconds

229 * on zebra mussel survival at Lake Carlos. Note: BI only used a 12 -h expc

230 * period - thus the full data set was reduced to contain only those obser

231 * for $12-\mathrm{h}$ BI at LC.

Page 10 of 13 


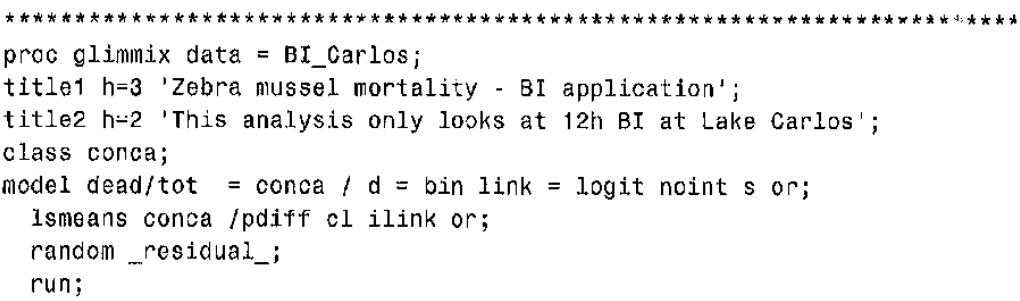

NOTE: The mode $\perp$ does not contain an intercept. Columns of $X$ are scaled only ar NOTE: Convergence criterion ( $G C O N V=1 E-8$ ) satisfied.

NOTE: PROCEDURE GLIMMIX used (Total process time):
real time
0.24 seconds

cpu time

0.14 seconds

241

$2421 * * * \pi * * * * * * * * * * * * * * * * * * * * * * \ldots .$. -Remarks . . . . - $2 * * * * * * * * * * * * * * * * * * * * * * * * * * * *$

243 * This analysis compares the effect of treatment method BI (bottom inject

$244 *$ or zebra nussel survival at Shawano Lake. Note: BI only used a 12-h exF

245 * period - thus the full. data set was reduced to contain only those obser

$246 *$ for $12-\mathrm{h}$ BI at SL.

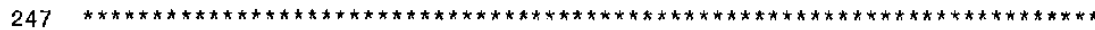

248 proc glimmix data $=$ BI_Shawano:

249 title1 $h=3$ 'Zebra musse-l nortality - BI application';

250 title2 $h=2$ 'This analysis only looks at $12 \mathrm{~h}$ BI at Shawano Lake';

251 class conca;

252 model dead $/$ tat $=$ conca $/ d=$ bin link = logit noint $s$ or;

253 lsmeans conca /pdiff cl ilink or;

254 randon _residual_;

255 run;

NOTE: The model does not contain an intercept. Columns of $X$ are scaled cnly ar NOTE: Convergence criterion (ABSGCONV=0.00001) satisfied.

NOTE: PROCEDURE GLIMMIX used (Total process time):
real time
0.23 seconds
cpu time
0.09 seconds

Page 11 of 13 


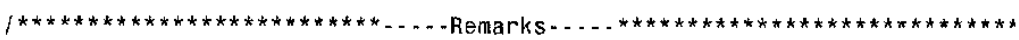

* This analysis compares the effect of treatment method BI (bottom inject

* vs WT (whole water treatment) on zebra mussel survival at lake carlos.

* Note: BI only used a 12-h exposure period - thus the full data set was

* reduced to contain only those observations for 12-h for both $E I$ and WT

$* * * * * * * * * * * * * * * * * * * * * * * * * * * * * * * * * * * * * * * * * * * * * * * * * * * * * * * * * * * * * * * * * * * * * * * * *$

proc glimmix data $=$ BI_vs_WT_Carzos;

titlet in=3 'Zebra mussel mortality - applica:ion method';

title2 $h=2$ 'This analysis only looks at $12 \mathrm{~h}$ WT vs $12 \mathrm{~h}$ BI at Lake Carlos';

title3 $h=2$ 'BI only had $12 \mathrm{~h}$ exposure so can only compare to $12 \mathrm{~h}$ WT treatn

class conca loc trt_typ tnk tray;

model. dead/tot = conca/trt_typ $/ d=$ bin link $=$ logit noint s or;

lsmeans conca /pdiff cl i.j.ink or;

Ismeans trt_typ/pdiff cl i.link or;

lsmeans conca*trt_typ /pdiff cl i.link or;

random_residual_;

random tnk tray (tnk);

run;

NOTE: The model does not contain an intercept. Co:umns of $x$ are scaled only ar NOTE: Convergence criterion ( $P C O N V=1.11022 E-8$ ) satisfied.

NOTE: PROCEDURE GLIMMIX used (Total process time):
reai time
0.45 seconds
cpu time
0.23 seconds

275

276

* This analysis compares the effect of treatment method BI bottom inject

278 * vs WT (whole water treatment) on zebra mussel survival at Shawano Lake.

279 * Note: BI only used a 12-h exposure period - thus the full data set was

280 * reduced to contain only those observations for $12, \mathrm{~h}$ for both BI and WT

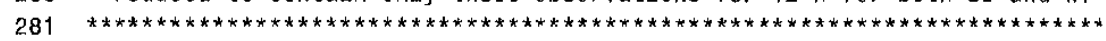

282 proc glimmix data = BI_vs_WT_Shawano;

283 title1 $h=3$ 'Zebra mussel mortality - application method';

284 title2 $h=2$ 'This analysis only looks at $12 \mathrm{~h}$ WT vs $12 \mathrm{~h}$ BI at Shawano Lake'

285 title3 $h=2$ 'BI only had $12 \mathrm{~h}$ exposure so can only compare to $12 \mathrm{~h}$ WT treath

286 class conca loc trt_typ tnk tray;

287 model dead $/$ tot = concaltrt typ $/ \mathrm{d}=$ bin link = logit noint $\mathrm{s}$ or;

288 lsmeans conca /pdiff cl ilink or;

289 lsmeans trt_typ/pdiff cl ilink or;

290 lsmeans conca*trt_typ /pdiff cl ilink or;

291 random residual_;

Page 12 of 13 
292 random tnk tray $(t n k)$;

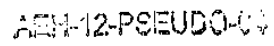

run;

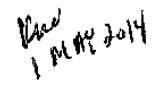

NOTE: The model does not contain an intercopt. Columns of $x$ are scaled only ar NOTE: Convorgence criterion (PCONV=1.11022E-8) satisfied.

NOTE: Estimated G matrix is not positive definite.

NOTE: PROCEDURE GLIMMIX used (Total process time):

real time $\quad 0.37$ seconds

cpu time $\quad 0.18$ seconds

FF \# 18

Item No. 3

$\mathrm{Pg} 13$ of 13

Page 471 of 519 


\begin{tabular}{|c|c|c|c|c|c|c|c|c|c|c|c|c|}
\hline \multirow{2}{*}{$\begin{array}{l}\text { Stallstice } \\
\text { oxposuro } \\
\text { sAs vid } \\
\text { oll diata } \\
\text { Obs } \\
1\end{array}$} & $\begin{array}{l}\text { al annl } \\
\text { lo lo vm } \\
0.3 \text { Alte }\end{array}$ & trt $t y p$ & one & ime & & ak. & tray & tot & coad & pctsurv & peimort & cor \\
\hline & LC : & Bl & 0 & 12 & & 6 & $\mathrm{C1}$ & 179 & 4 & 97.765 & 2.235 & C \\
\hline 2 & LC & BI & 0 & 12 & & 7 & A1 & 225 & $4:$ & 98.222 & 1.778 & $C$ \\
\hline 3 & LC & 티 & 0 & 12 & & 3 & $\mathrm{c3}$ & 253 & 7 & 97.233 & 2.767 & $c$ \\
\hline 4 & LC & El & 0 & 12 & & 7 & $B^{3}$ & 176 & 9 & 94.886 & 5.114 & C \\
\hline 5 & LC! & BI & 0 & 12 & & 3 & $B 1$ & 242 & 5 & 97.934 & 2.066 & C \\
\hline 6. & LG & Bl & 0 & 12 & & 6 & B1 & 217 & 11 & 94.931 & 5.069 & 10 \\
\hline 7 & LC & el & 0 & 12 & & 6) & $\mathrm{A} 2$ & 186 & 4 & 97.849 & 2.151 & ic \\
\hline B & LC : & $B$ & 0 & 12 & & 3 & $A 3$ & 196 & 4 & 97.959 & 2.041 & $c$ \\
\hline 9 & LC: & Bi & 0 & 12 & & 7 & $\mathrm{C} 1$ & 276 & 3. & 98.913 & 1.087 & 10 \\
\hline 10 & LC & $\mathrm{BI}$ & 50 & 12 & & 4 & E2 & 256 & $191:$ & 26.381 & 74.608 & $A$ \\
\hline 11 & LC & BI & 50 & 12 & & 1 & E3 & 279 & 217 & 22.222 & 77.778 & $A$ \\
\hline 12 & LC & 81 & 50 & 12 & & 8 & $\mathrm{C} 2$ & 200 & 161 & 18.500 & 80.500 & $A$ \\
\hline 13 & LC & BI & 50 & 12 & & 1 & E2 & 510 & 424 & 16.863 & 83.137 & $A$ \\
\hline 14 & LC & B1 & 50 & 12 & & 4 & B1 & 188 & 178 & 5.319 & 84,681 & A \\
\hline 15 & LC & $\mathrm{BI}$ & 50 & 12 & & 4 & 83 & 188 & 174 & 7.447 & 82.553 & A \\
\hline 16 & $L C$ & $B 1$ & 50 & 12 & & 8 & $\mathrm{B3}$ & 291 & 222 & 23.711 & 76.289 & A \\
\hline 17 & LC & B! & 50 & 12 & & 8 & A3 & 282 & 232 & 17,720 & 82.270 & A \\
\hline 18 & LC & Bi & 50 & 12 & & $i$ & A1 & 210 & 158 & 24.762 & 75.238 & A \\
\hline 18 & LC & $B I$ & 100 & 12 & & 9 & A! & 269 & 256 & 4.833 & 95.167 & $B$ \\
\hline 20 & LC & $\mathrm{B}]$ & $\infty$ & 12 & & 9 & $\mathrm{B3}$ & 179 & 139 & 22.346 & 77.654 & $B$ \\
\hline 21. & $L C$ & B! & 100 & 12 & & 2. & B3 & 163 & 132 & 13.725 & 86.275 & $B$ \\
\hline 22 & LC: & $B 1$ & 100 & 12 & & 2 & 01 & 193 & 154 & 20.207 & 79.793 & $B$ \\
\hline 23) & LC & $B I$ & 100 & 12 & & 9 & $\mathrm{C} 1$ & 288 & 263 & 8.681 & 91.319 & $B$ \\
\hline 24 & LC & BI & 0 & 12 & & 5 & f & 329 & 17 & 34.043 & 5.957 & B \\
\hline 25 & LC & BI & 100 & 12 & & 5 & 83 & 393 & 272 & 30,789 & 69.211 ? & $B$ \\
\hline 26 & LC. & $\mathrm{BI}$ & 100 & 12 & & 2 & At & 279 & 261 & $6.4 E 2$ & $83.54 \theta$ & $B$ \\
\hline 27 & LC & $B I$ & 100 & 12 & & 5 & $C 1$ & 264 & 209 & 20.833 & 78.167 & 8 \\
\hline 28 & $L C$ & WT & 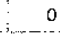 & 6 & & 5 & $c$ & $\uparrow / 5$ & 0 & 100,000 & 0,000 & $c$ \\
\hline 29 & i.C & WT & 0 & 6 & 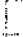 & 2 & C1 & 168 & 3 & 98,214 & 1.786 & c \\
\hline 30 & Le & WT & 0 & 6 & 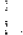 & 2 & $\mathrm{B2}$ & 237 & 10 , & 95.781 & 4.210 & 10 \\
\hline 31 & LC & WT & 0 & 6 & & 3 & A1 & 328 & 23 & 92,888 & 7.012 & C \\
\hline 32 & LC & WT & 0 & 6 & & 5 & $\mathrm{H} 2$ & 173 & 2 & 98.844 & 1.156 & $\mathrm{C}$ \\
\hline 33 & LC & WT & 0 & 6 & 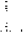 & 3 & 81 & 255 & 3 & 98.824 & 1.176 & $c$ \\
\hline 34 & LC & WT & 0 & 6 & & 4 & 1. & 339 & 2 & 99.410 & 0.590 & $\mathrm{C}$ \\
\hline 35 & LC & WT & 0 & 6 & & & 1 & 269 & 5 & 98.141 & 1.859 & $c$ \\
\hline 36 & LC: & WT & 0 & B & & 3 & $\mathrm{ca}$ & 310 & 6 & 98.065 & 1.935 & 0 \\
\hline 37 & LC & $W T$ & 0 & 9 & . & 5 & 83 & 192 & 7 & 96.354 & 3.646 & $\mathrm{C}$ \\
\hline 38 & LC & WT & 0 & & & & A3 & 178 & 7. & 96.067 & 3.933 & $\mathrm{C}$ \\
\hline 39 & LC & WT & 0 & 9 & & & $\mathrm{C3}$ & 173 & 11 & 93.642 . & $6.358^{8}$ & $\mathrm{c}$ \\
\hline 40 & LC & WT & 0 & 9 & . & 2 & $\mathrm{Ca}$ & 226 & 6 & 97.345 & 2.655 & $c$ \\
\hline 41 & $L C$ & WT & 0 & 9 & & 3 & B3 & 213 & 2 & 99.061 & 0.939 & $\mathrm{C}$ \\
\hline 42 & LC & WT & 0 & . & & & 22 & 214 & 11 & 94.860 & 5.140 & $\mathrm{c}$ \\
\hline 43 & LC & WT & 0 & 9 & & & & 236 & 6 & 97,4 EB & $2.542:$ & $\mathrm{C}$ \\
\hline
\end{tabular}

FF \# 18

Item No. 4

$\mathrm{Pg} \perp \frac{1}{39}$

file://C:/Uscrs/klwebcr/AppData/Local/Temp/2/SAS\%20Temporary\%20Hi'es/_l'D3488_I... 5/1/2014 


\begin{tabular}{|c|c|c|c|c|c|c|c|c|c|c|c|}
\hline 44 & LC & WT & 0 & 9. & & A3 & 177 & $2:$ & 98.870 & 1130 , & 0 \\
\hline 45 & LC & WT & 0 & 8 & 2 & A3 & 326 & 6 & 98.160 & 1.840 & \\
\hline 46 & LC & WT & 0 & 12 & 3 & $B 2$ & 312 & 8 & 97.436 & 2.564 & \\
\hline 47 & $\begin{array}{l}\text { L } \\
\text { L }\end{array}$ & WT & 0 & 12 & 3 & $A 2$ & 185 & 5 & 97.297 & 2.703 & 10 \\
\hline 48 & LC & WT & 0 & 12 & 2 & $\mathrm{~A} 2$ & 390 & 16 & 96.897 & 4.103 & $\mathrm{c}$ \\
\hline 49 & LCC & WT & 0 & $12:$ & 3 & $\mathrm{c1}$ & 259 & 5 & 98.069 & 1.931 & 10 \\
\hline 60 & $\mathrm{LC}$ & WT & 0 & 12 & 2 & $B 1$ & 185 & $4 !$ & 97.838 & 2.162 & \\
\hline 51 & LC & WT & 0 & 12 & 2 & $\mathrm{c} 2$ & 198 & 8 & 95.960 & 4.040 & 0 \\
\hline 52 & LC & WT & 0 & 12. & 5 & A2 & 240 & 2 & $\$ 9.167$ & 0.833 & 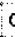 \\
\hline 53 & $L C$ & WT & $0:$ & 12 & 5 & $\mathrm{C2}$ & 212 & 6: & 97.170 & 2.830 & $c$ \\
\hline 54 & $L C$ & WT & 0 & 12 & 5 & B1. & 196 & 6 & 96.939 & 3.061 & $: 0$ \\
\hline 55 & LC: & $W T$ & 50 & 6 & 1 & A3 & 149 & 148 & 0.000 & 100.000 & A \\
\hline 56 & L.C & WT & so: & 6 & 4 & $\mathrm{C}_{2}$ & 175 & 171 & 2.286 & 87.714 & \\
\hline 57 & LC & $W T$ & 50 & 6 & 8 & $B:$ & 208 & 203 & 2.404 & 97.596 & A \\
\hline 58 & LC & WT & EC & 6 & i & $A 2$ & 280 & 274 , & 2. $\triangle 3$ & 97.857 & A \\
\hline 59 & LC & WT & 50 & 6 & 8 & 82 & 178 & 177 & 0.562 & 99.438 & $A$ \\
\hline 60 & LC & $W T$ & 50 & 6 & 4 & $\mathrm{~B} 2$ & 214 & 210 & 1.869 & 88.131 & A \\
\hline 61 & LC & WT & 50 & 6 & 1 & 82 & 232 & 228 & 1.724 & 98.276 & A \\
\hline 62 & LC : & $w r$ & 50 & 6 & 4 & $\mathrm{c} 1$ & 191 & 191 & 0.000 & 160.000 & A \\
\hline 63 & LC & $W r$ & 50 & $6 !$ & 8 & 83 & 248 & 247 & 0.403 & 09.597 & $A$ \\
\hline 64 & Lo & WT & 50 & 9 & 4 & B3 & 265 & 281 & 1.509 & 88.491 & A \\
\hline 65 & LC & WT & 50 & 9 & 4 & A3 & 375 & 375 & 0.000 & 100.000 & A \\
\hline 66 & LC & WT & 50 & 9 & 8 & $\mathrm{C} 1$ & 169 & 169 & 0.000 & 100.000 & A \\
\hline 67 & LC: & WT & 50 & 9 & 1 & $A 1$ & 164 & 161 & 1.829 & Q8.171 & $A$ \\
\hline 68 & LC & WT & $50^{-1}$ & 9 & 4 & $A 1$ & 221 & 221 & 0.000 & 100.000 & A \\
\hline 69 & LC & WT & 3 & 9 & 8 & $\mathrm{C} 3$ & 180 & $180^{\circ}$ & 0.000 & 100.000 & $A$ \\
\hline 70 & LC & $W T$ & 50 & 9 & 8 & $A 3$ & 131 & 127 & 3.053 & 96.947 & A \\
\hline 71 & LC & WT & $50^{1}$ & 2 & $1:$ & B1 & 128 & 123 & 3.866 & 86.094 & $A$ \\
\hline 72 & LC & $W T$ & 50 & 9 & 1 & $c 1$ & 267 & 267 & 0.000 & 100.000 & A \\
\hline 73 & $L G$ & WT & 50 & 12 & 1 & $\mathrm{Ca}$ & 268 & 286 & 0.000 & 100.000 & A \\
\hline 74 & isc & WT & 50 & 12 & 1 & $\mathrm{C} 2$ & 175 & 175 & 0.000 & 100.000 & A \\
\hline 75 & LC & WT & 50 & 12 & 1 & $\mathrm{~B}$ & 160 & 159 & 0.625 & 99.375 & $\Lambda$ \\
\hline 76 & LC & WT & 50 & 12 & 8 & $\mathrm{C2}$ & 290 & 287 & 1.034 & 98,966 & A \\
\hline 77 & $L C$ & WT & 50 & 12 & 8 & $\mathrm{~A}_{2}$ & 119 & 119 & 0.000 & 100.000 & A \\
\hline 78 & LC & WT & 50 & 12 & 8 & A1 & 151 & 150 & 0.662 & 90.333 & A \\
\hline 79 & $L C$ & WT & 50 & 12 & 4 & A2 & 158 & $155^{\circ}$ & $1.899_{3}$ & $\$ 6.101$ & A \\
\hline 80 & LC. & $W T$ & $50 !$ & 12 & 4 & $\mathrm{Cs}$ & 455 & $453:$ & 0.440 & 96.560 & A \\
\hline 81 & LC & WT & 50 & 12 & 4 & $B 1$ & 280 & 277 & 1.071 & 98.929 & A \\
\hline 82 & $\angle C$ & $W T$ & 100 & 6 & $7 \vdots$ & $\mathrm{B} 2$ & 266 & 256 & $3.759^{\circ}$ & 96.241 & $\mathrm{~B}$ \\
\hline 83 & LC & WT & 100 & 6 & 9 & $B 2$ & 190 & 188 & 1.053 & $98, \theta 47$ & $\mathrm{~B}$ \\
\hline 84 & $L C$ & $W T$ & 100 & B & 6 & $\mathrm{C3}$ & 267 & 264 & 1.124 & 98,876 & 8 \\
\hline 85 & LC & WT & 100 & 6 & 7 & A1 & 212 & 210 & 0.943 & 90.057 & e \\
\hline 86 & LC & $W r$ & 100 & 6 & 6 & $\mathrm{~A}^{3}$ & $236:$ & 227 & 3.404 & 96.596 & B \\
\hline 87 & LC & WT & 100 & 6 & 6 & $A 2$ & 257 & 250 & 2.724 & 97.276 & B \\
\hline 88 & LC & WT & 100 & 6 & 9 & 01 & 324 & 317 & 2.160 & 97.840 & \\
\hline 89 & LC & WT & 100 & 6 & 7. & 01 & 268 & 267 & 0.743 & 99.257 & \\
\hline
\end{tabular}




\begin{tabular}{|c|c|c|c|c|c|c|c|c|c|c|c|}
\hline & LC & $w T$ & $100:$ & 6 & 9 & $\mathrm{~A} 1$ & 283 & 275 & 2.827 & 97.173 & B \\
\hline 91 & LC. & WT & 100 & 9 & 6 & 82 & 188 & $187_{j}$ & 0.532 & 89.468 & $B$ \\
\hline 92 & LC & $W T$ & 100 & 9 & 7 & 83 & 193 & $193_{\mathrm{i}}$ & 0.000 & 100.000 & $B$ \\
\hline 93 & LC & WT & $100^{\circ}$ & 9 & 7 & $\mathrm{C} 3$ & 158 & 155 & 1.899 & 96.101 & e \\
\hline 84 & LC & $W T$ & 100 & 8 & B. & $\mathrm{C} 1$ & 176 & 176 & 0.000 & 100,000 & B \\
\hline 95 & LCC & WT & 100 & 9 : & $\varepsilon$ & A3 & 393 & 392 & 0.254 & 99,746 & B \\
\hline 95 & LC & $W T$ & $100:$ & 9 & 9 & B3 & 128 & 128 & 0.000 & 100.000 & $B$ \\
\hline $\mathbf{9 7}$ & LC & WT & 100 & 9. & B: & B3 & 183 & 181 : & 1.093 & $8 B 607$ & B \\
\hline 98 & LC. & WT & 100 & $9:$ & $9^{i}$ & $\mathrm{c} 3$ & 260 & 260 & 0.000 & 103.000 & B \\
\hline 99 & LC & WT & 100 & 9 & 7 & A3 & 258 & 255 & 1.163 & 93.837 & B \\
\hline 100 & LC & $W T$ & 100 & 12 & 6 & 81 & 249 & 248 & 0.402 & 99.508 & 8 \\
\hline 101 & LC & WT & 100 & 12 & 6 & A1 & 239 & 236 & 1.265 & 98.745 & B \\
\hline 102 & $L C$, & WT & 100 & 12 & 9 & A2 & 316 & $316 !$ & 0.000 & 100.000 & $B$ \\
\hline 103 & LC & WT & 100 & 12 & 7 & $A 2$ & 237 & 236 & 0.422 & 99.578 & B \\
\hline 104 & LC & $W J$ & 100 & $12 !$ & 9 & Bi & 273 & 268 & 1.832 & 98.168 & $B$ \\
\hline 105 & $L C$ & WT & 00 & 12 & 7 & B1 & 286 & 286 & 0.000 & 100.000 & $B$ \\
\hline 106 & LC & WT & .00 & 12 & 7 & $\mathrm{C} 2$ & 203 & 203 & 0.000 & 100.000 & B \\
\hline 107 & L.C: & WT & 100 & 12 & 9 & $\mathrm{C} 2$ & 156 & 155 & 0.641 & 99,359 & B \\
\hline 108 & LC & $w r$ & 100 & 12 & 6 & $c 2$ & 155 & 153 & 1.290 & 98.710 & a \\
\hline 108 & $S L$ & $\mathrm{~B}$ & 0 & 12 & 1 & $c 3$ & 147 & 8 & $84.558^{\circ}$ & $5.442:$ & $\mathrm{C}$ \\
\hline 110 & SL: & :日1 & 0 & 12 & 8 & $\mathrm{~B} 2$ & 134 & 6 & 95.522 & 4.478: & :C \\
\hline 111 & SL & $B$ & 0 & 12 & 1 & $\mathrm{C2}$ & 111 & 3 & 97.297 & 2.703 & $c$ \\
\hline 112 & SL & B। & 0 & 12 & 8 & $\mathrm{e}$ & 163 & 4 & 97.546 & 2.464 & $c$ \\
\hline 113 & SL & B! & $\therefore$ & 12 & 8 & B1 & 81 & 5 & 93.827 & 6.173 & $\mathrm{C}$ \\
\hline 114 & SL. & BI & 0 & 12 & 8 & A3 & 140 & 7 & 95,000 & 5.000 & $c$ \\
\hline 115 & SL & $B$ & C & 12 & 9 & $\mathrm{~B} 2$ & 87 & 2 & 97.701 & 2.299 & c \\
\hline 116 & $\mathrm{SL}$ & $\mathrm{BI}$ & c & 12 & 1 & A3 & 107 & 7 & $93.45 \mathrm{~B}$ & 6.542 & ic \\
\hline 117 & $S L$ & BI & 0 & 12 & 9 & 83 & 137 & $10:$ & 92.701 & 7.299 & $c$ \\
\hline 118 & SL & DI & 0 & 12 & 9 & $B 1$ & 64 & 4 & 95.238 & 4.762 & $\mathrm{C}$ \\
\hline 119 & SL. & B! & 0 & 12 & 9 & $\mathrm{~A} 2$ & 115 & 0 & 100.000 & 0.000 & $\mathrm{C}$ \\
\hline 120 & $S L$ & BI & 0 & 12 & 1 & C1 & 137 & $10:$ & 92.701 & 7.299 & C \\
\hline 121 ! & SL & B! & 50 & 12 & 3 & $\mathrm{C} 2$ & 109 & 107 & 1.835. & 98.165 & A \\
\hline 122 & SL: & BI & 50 & 12 & 3 & 62 & 103 & 103 & 0.000 & 100.000 & A \\
\hline 123 & SL & BI & 50 & 12 & 7 & E1 & 128. & 124 & 3.^25 & 96.875 . & A \\
\hline 124 & SL & BI & 50 & 12 & $7 \vdots$ & B3 & 109 & 103 & 5.505 & 84.495 . & A \\
\hline 125 & SL & 이 & 50 & 12 & $7:$ & $\mathrm{B} 2$ & 116 & 113 & 2.586 & 97.414 & A \\
\hline 126 & SL. & 비 & 50 & 12 & 7 & A2 & 115 & 113. & 1.739 & 98,231 & A \\
\hline 127 & SL & BI & 50 & 12 & $2:$ & A2 & 119 & 115 & 3.361 & 86.639 & A \\
\hline 128 & SL . & BI & 50 & 12 & $2:$ & 81 & 105 & 102 & 2.857 & 97.113 & A \\
\hline 129 & SL. & Bl & 50 & 12 & 3 & B1. & 144 & 136 & 5.556 & 84,444 & $A$ \\
\hline 130 & SL & ! & 50 & 12 & 2 & B2. & 116 & 114 & 1.724 & 98,276 & A \\
\hline 131 & SL. & 81 & 50 & 12 & 3 & A2 & 117 & 113 & 3.419 & 96,531 & $A$ \\
\hline 132 & SL & BI & $50 \%$ & 12 & 2 & A3 & 145 & 140 & 3,448 & 96.552 & A \\
\hline 133 & SL & B! & $100:$ & 12 & $4:$ & B3 & 126 & 124 & 1.587 & 08.413 & $\mathrm{~B}$ \\
\hline 134 & SL & B & 100 & 12 & 5 & 81 & 105 & 105 & 0.000 & 100.000 & $\mathrm{~B}$ \\
\hline $\begin{array}{l}135 \\
136\end{array}$ & $S L$ & Bi & 100 & 12 : & 4 & B2 & $=123$. & 122 & 0.813 & 99.187 : & B \\
\hline
\end{tabular}




\begin{tabular}{|c|c|c|c|c|c|c|c|c|c|c|c|}
\hline & SL & B & 100 & 12 & 4 & C3 & 104 & 104 & $0.000^{\circ}$ & 100.000 & B \\
\hline 137 & st & 81 & $100^{\circ}$ & 12 & 5 & 82 & 97 & 97 & 0.000 & 100,000 & : \\
\hline 133 & St & $\mathrm{BI}$ & 100 & 12 & 6 & B3 & 113 & 113 & 0.000 & 100,000 & iB \\
\hline 139 & SL. & B & 100 & 12 & B & 82 & 121 & 118 & 1.653 & 98.347 & B \\
\hline 140 & SL. & B & $100:$ & 12 & 6. & C2 & $: 127$ & 126 & 0.787 & $99.213^{\circ}$ & $B$ \\
\hline 141 & sı. & BI & 100 & 12 & $4 . A$ & $\mathrm{~A} 2$ & 117 & 116 & 0.955 & 99.145 & $B$ \\
\hline 142 & $\mathrm{SL}$ & $\mathrm{BI}$ & 100. & 12 & 5: & $\mathrm{B} 3$ & $108^{\circ}$ & 108 & $0,000:$ & 100,000 & $B$ \\
\hline 143 & $S L$ & 81 & 100 & 12 & 6 & $\mathrm{C} 3$ & 98 & 96 & 3.001 & 96.839 & $B$ \\
\hline 144 & SL. & . B & $100:$ & 12 & $5: A$ & $A 2$ & 100 & 104 & 1.887 & 98.113 & $B$ \\
\hline 145 & $\mathrm{SL}$ & WT & 0 & 6 & 7 & $\mathrm{B1}$ & 80 & 2 & 97.500 & 2.500 & C \\
\hline 146 & SL & WT & 0 & 6 & 1,1 & $A 1$ & $109^{3}$ & 4 & 96.330 & 3.670 & $\mathrm{c}$ \\
\hline 147 & SL & $W T$ & 0 & $6^{\prime}$ & $1 \mathrm{E}$ & $B 2$ & 87 & 2 & 97.701 & 2.299 & $\mathrm{c}$ \\
\hline 148 & St. & WT & 0 & 6 & 70 & $\mathrm{Cl}$ & 108 & 5 & 95.370 & 4.830 & $c$ \\
\hline 149 & SL & WT & 0 & 6 & $1 \mathrm{~B}$ & B1 & 118 & 7 & 94,068 & 5932 & c \\
\hline 150 & SL. & WT & 0 & $6:$ & 4. & $\mathrm{~A} 1$ & 64 & 3 & 95.313 & 4.688 & $c$ \\
\hline 151 & SL & WT & 0 & 6 & $7 \mathrm{~A}$ & A1 & 124 & 2 & 98.387 & 1.613 & ic \\
\hline 152 & SL & WT & 0 & 6 & 41 & B1 & 78 & 4 & 94.872 & 5.128 & $\mathrm{C}$ \\
\hline 153 & SL. & WT & 0 & $6 !$ & $4 A$ & A2 & 74 & 7 & 90.541 & 9.459 & $c$ \\
\hline 154 & $\mathrm{SL}$ & WT & 0 . & 9 & 40 & $\mathrm{C1}$ & 71 & 2 & 97.183 & 2,817 & 0 \\
\hline 155 & $\mathrm{SL}$ & WT & 0 & 9 & $1 \mathrm{~B}$ & B3 & 84 & 2 & 97.619 & 2.381 & $\mathrm{C}$ \\
\hline 156 & SL: & $w r$ & 0 & 8 & $1 \mathrm{~A}$ & $\mathrm{~A} 3$ & 159 & 9 & 94.340 & 5.660 & $\mathrm{C}$ \\
\hline 157 & $S L$ & WT & 0 & 9 & $7 \mathrm{~B}$ & B2 & 108 & 6. & 94.444 & 6.656 & $\mathrm{c}$ \\
\hline 158 & SL: & WT & 0 & 9 & $7 A$ & A3 & 54 & 2 & 96.296 & 3.704 & c \\
\hline 159 & SL & WT & 0 & 9 & 70 & $\mathrm{C} 3$ & 111 & 6 & 94.595 & 5.405 & $c$ \\
\hline 160 & SL, & $W T$ & 0 & $\mathrm{~g}^{\circ}$ & 4. & $\mathrm{~B} 2$ & 75 & 3 & 98.000 & 4.000 & $\mathrm{C}$ \\
\hline 161 & SL: & WT & 0 & 9 & $4 \mathrm{~A}$ & A3 & 110 & 7 & $93.636^{\circ}$ & 6.364 & $c$ \\
\hline 162 & SL & WT & 0 & $9:$ & $1: 0$ & C1 & 144 & 6 & 95.833 & 4.167 & c \\
\hline 163 & $S L$ & WT & 0 & 12 & $1: A$ & A2 & 101 & 5 & 96.050 & 4.950 & $\mathrm{C}$ \\
\hline 164 & $\mathrm{SL}$ & WT & 0 & 12 & 10 & C? & 108 & 6 & 94.444 & 5.558 & $\mathrm{C}$ \\
\hline 165 & SL. & WT & 0 & 12 & $7 A$ & A2 & 92 & $2:$ & 97.826 & 2.174 & $c$ \\
\hline 166 & $S L$ & WT & 0. & 12 & 40 & $\mathrm{C} 3$ & 141 & 4 & 97.163 & 2.837 & $c$ \\
\hline 167 & SL & WT & 0 & 12 & 70 & $\mathrm{C} 2$ & 90 & 3 & 96.667 & 3.333 & $c$ \\
\hline 168 & SL & Wr & $0^{\circ}$ & 12 & $7^{3} \mathrm{~B}$ & B3 & 104 & 2 & $98.0 / 1$ & 1.923 & $\mathrm{C}$ \\
\hline 168 & $S L$ & WT & 0 & 12 & $4: B$ & B3 & $79:$ & 3 & 96,203 & 3.797 & c \\
\hline 170 & SL & WT & 0 . & $12:$ & 40 & $\mathrm{C} 2$ & 81 & 4 & 85.062 & $4.938:$ & $\mathrm{C}$ \\
\hline 171 & SL". & WT & 0 & 12 & 10 & $\mathrm{c} 3$ & 111 & 5 & 95.495 & 4.505 & $\mathrm{C}$ \\
\hline 172 & SL & WT & 50. & $6:$ & 80 & C1 & 136 & 133 & 2.206 & 97.794 & $A$ \\
\hline 173 & $S L$ & WT & 50 & 6 & $8 . A$ & $A 3$ & 96 & 21 & 5.208 & 94.792 & $A$ \\
\hline 174 & SL & WT & 50 & $6:$ & $2 A$ & $A^{\prime 1}$ & 108 & 91 & 15.741 & 84.259 & A \\
\hline 175 & SL & WT & 50 & 6 & $5 \mathrm{~B}$ & B: & 67: & 59 & 11.940 & 88.060 & A \\
\hline 176 & $\mathrm{SL}$ & WT & 50 & 6 & $2 \mathrm{~B}$ & $\mathrm{~B}^{*}$ & $116=$ & 106 & 8.621 & 91.379 & A \\
\hline 177 & $S L$ & WT & 50 & 6 & 5.0 & $\mathrm{C} 1$ & 85 & 71 & 16.471 & 83.529 & $A$ \\
\hline 178 & SL & WT & 50 & 6 & 20 & c1 & 61 & 57. & 6.557 & 93.443 & A \\
\hline 179 & SL & WT & 50 & $6:$ & $5 \mathrm{~A}$ & A1 & 95 & 87 & 8.421 & 91.579 & A \\
\hline 180 & SL & WT & $50^{\circ}$ & 6 & 80 & 83 & 149 & 92 & 38.255 & 61.745 & A \\
\hline $\begin{array}{l}181 \\
182\end{array}$ & $S L$ & WT & 50 & 9 & 80 & C2 & 61 & 60 & 1.639 & $98.361:$ & A \\
\hline
\end{tabular}




\begin{tabular}{|c|c|c|c|c|c|c|c|c|c|c|c|}
\hline & SL & $W T$ & 50 & 9 & 8. & A1 & 148 & 146. & 1.351 & 98.649 & $A$ \\
\hline 183 & SL. & $W T$ & 50 & 8 & 2 & $c_{2}$ & 117 & 112. & 4.274 & 95.726 & $\mathrm{~A}$ \\
\hline 184 & SL & WT & 50. & 9: & $8:$ & B1 & 85 & 80 & 5.802 & 94.118 & $3 \mathrm{~A}$ \\
\hline 185 & SL. & WT & 50 & 9 & 5 & $\mathrm{~A} 3$ & 61 & 59 & 3.279 & 96.721 & 1: A \\
\hline 861 & SL. & WT & 50 & $8:$ & 2 & 82 & 103 & 94 & 8.738 & 91.262 & 2, A \\
\hline 87 & $S L$ & Wr & 50 & 8: & 5 & $\mathrm{C3}$ & 80 & 61 : & 23.750 & 76.250 & $A$ \\
\hline 188 & SL. & WT & 50. & $\theta$ & 2 & $A 3$ & 114 & 88 & 22.807 & 77.193 & A \\
\hline 189 & SL & WT & 50 & 9 & 5 & D2 & 105 & 83 & 20.952 & 79.048 & A \\
\hline 190 & $S L$ & WT & 50 : & 12 & 5 & $\mathrm{C} 2$ & 114 & 112 & 1.754 & 98.246 & $A$ \\
\hline 91 . & $\mathrm{SL}$ & WT & so: & 12 & 2 & A2 & 98 & 97 & 1.020 & 98.980 & A \\
\hline 192 & SL & WT & 50 : & 12 & 8 & 82 & 88 & $85:$ & $3.409^{\circ}$ & 96.591 & $A$ \\
\hline $193^{\circ}$ & SL & WT & 50 . & 12 & $2:$ & $\mathrm{C} 2$ & 86 & $86:$ & 0.000 & 100.000 & $A$ \\
\hline 194 & $S L$ & WT & $5 c$ & 12 & 8 & $\mathrm{Cs}$ & 129 & 128 & 0.775 & 89.225 & $A$ \\
\hline 195 & SL. & WT & $5 \mathrm{C}$ & 12 & $2:$ & 83 & 85 & 80 & 5.882 : & 94.118 & A \\
\hline 196 & SL & WT & $60^{\circ}$ & $12 \vdots$ & 5 & B3 & 63 & 58. & 7.937 & 92.063 & A \\
\hline 197 & SL & WT & 50 & 12 & $8:$ & A2 & 76 & 73 & 3.947 & 96.053 & $A$ \\
\hline 198 & SL: & WT & 50 & 12 & 5 & $\mathrm{A2}$ & 60 & 60 & 0.000 & 100.050 & $A$ \\
\hline 199 & $\mathrm{SL}$ & WT & 100 & 6 & 9 & $\mathrm{~A} 1$ & 77 & 75 & 2.597 & 97.403 & $B$ \\
\hline 200 & SL & $W T$ & 100 & 6 & 6 & A3 & 57 & 54 & 5.263 & 84.737 & $\mathrm{~B}$ \\
\hline 201 & $S L$ & WT & 100 & 6 & 9 & C1 & 118 & 99. & 16.102 & 83.898 & $B$ \\
\hline 202 & sL & WT & 100 & 6 & 3 & E3 & 104 & 95 & 8.654 & 91.346 & $B$ \\
\hline 203 & SL & WT & 100 & $6 \vdots$ & 3 & $\mathrm{E} 2$ & 122 & 114 & 6.557 & 93.443 & $B$ \\
\hline 204 & SL & WT & 100 & 6 & 6 & $\mathrm{~A} 2$ & 124 & 121 & 2.419 & 97.581 & B \\
\hline 205 & st. & $w T$ & 100 & $6 !$ & 3 & A1 & 138 & 111 & 19.565 & 80.435 & $B$ \\
\hline 206 & SL & WT & 100 & 6 & 9 & 81 & 119 & 115 & 3.361 & 96.639 & B \\
\hline 207 & SL & WT & 100 & 6 & 6 & A1 & 69 & 69 & 0.000 & 100.000 & $B$ \\
\hline 208 & SL & WT & 100 & 9 & 3. & 81 & 51 & 49 & 3.922 & 96.078 & $B$ \\
\hline 209 & SL: & WT & 100 & 9 & $3 !$ & 02 & 73 & 69 & 5.479 & 94.521 & $B$ \\
\hline 210 & SL & WT & 100 & 9 & 9 & $\mathrm{CS}$ & 123 & 116 & 5.691 & 84,309 & B. \\
\hline 211 & SL. & $W T$ & 100 & 9 & $3:$ & . & 88 & 88 & 0.000 & 100,000 & 8 \\
\hline 212 & SL & WT & 100 & $\theta$ & $9:$ & $\mathrm{A3}$ & 107 & $93:$ & 13.084 & 86.916 & ${ }^{B}$ \\
\hline 213 & SL & WT & 100 & 9 & 6 & 81 & 59 & 59 & 0.000 & 100,000 & $B$ \\
\hline 214 & SL V & WT & 100 & 9 & $g$ & 82 & 69 & 69 & 0.000 & 100.000 & $B$ \\
\hline 215 & St & WT & $100:$ & $\theta$ & o & $\mathrm{C} 2$ & 94 & 94 & 0.000 & 100.000 & $B$ \\
\hline 216 & SL. & Wr & 100 & $9:$ & 61 & 82 & 84 & 81 & 13830 & 86.170 & ${ }^{B}$ \\
\hline 217 & $\mathrm{SL}$ & WT & 100 & $12^{t}$ & 6 & $\mathrm{C1}$ & 62 & 59 & 4.839 & 95.161 ; & $B$ \\
\hline 218 & SL V & Wr & 100 & 12 & 3 & $\wedge 3$ & $131^{\circ}$ & 125 & 4.580 & 96.420 & $\mathrm{~B}$ \\
\hline 219 & SL $V$ & $W T$ & 100 & $12:$ & 3. & A2 & 95 & 94. & 1.053 & 96.947 . & $B$ \\
\hline 220 & $\mathrm{SL}$ & $W T$ & 100 & 12 : & 9 & $\mathrm{C} 2$ & 60 & 58. & 3.333 & 96.667 & B \\
\hline 221 & $S L \cdot V$ & $w r$ & 100 & 12 & 6 & $\mathrm{B3}$ & $n$ & 71 & 0.000 & 100.000 & $B$ \\
\hline 222 & SL. & WT & 100 & 12 & 6 & $\mathrm{c3}$ & 102 & 101, & 0.980 & 90.020 & 8 \\
\hline 223 & SL. & WT & 100 & 12 & 3 & c1 & 104 & 103 & 0,962 & 98.038 & $B$ \\
\hline 224 & SL V & WT & 100 & 12 & 9 & B3 & 102 & 100 & 1.961: & 98.039 & $\mathrm{~B}$ \\
\hline 225 & SL V & WI & 100 & 12 & 9 & A.2 & 104 & 104 & 0.000 & 100.000 & B \\
\hline
\end{tabular}


The mean percent survival and murtallty by locatlon and treatment type classified by concentration and expostre duration

The MEANS Procedure

loc $=$ LC Irt_typ=B|

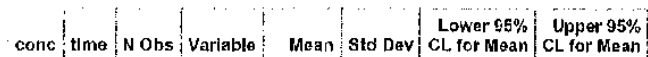

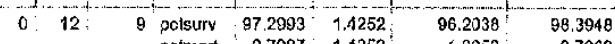

\begin{tabular}{r:rrrr}
97.2993 & 1.4252 & 96.2038 & 98.3948 \\
patmort & 2.7007 & 1.4252 & 1.6052 & 3.7962 \\
\hline
\end{tabular}

$\begin{array}{llllllll}50 & 12 & 9 & \text { petsury } & 18.1050 & 7.3014 & 12.4927 & 23.7174\end{array}$

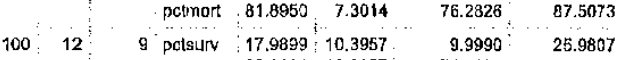

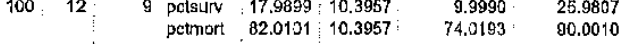

loc $=L C$ trt $t y p=W T$

(1 Mear Std Dev CL for Mean CL for Mean

\begin{tabular}{|c|c|c|c|c|c|c|c|}
\hline 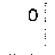 & $6 !$ & 9 & $\begin{array}{l}\text { pelsurv } \\
\text { petmont }\end{array}$ & $\begin{array}{r}97.8073 \\
\quad 2.1927\end{array}$ & $\begin{array}{l}2.1553 \\
2.1553\end{array}$ & $\begin{array}{r}96.1506 \\
0.5360\end{array}$ & $\begin{array}{r}99.4640 \\
3.8494\end{array}$ \\
\hline & 9 & $\theta$ & $\begin{array}{l}\text { pelsury } \\
\text { petmort }\end{array}$ & $\begin{array}{r}96.8585 \\
3.1315\end{array}$ & $\begin{array}{l}1.8169 \\
1.8169\end{array}$ & $\begin{array}{r}96.4719 \\
1.7349\end{array}$ & $\begin{array}{r}38.2651 \\
4.6281\end{array}$ \\
\hline & & 9 & $\begin{array}{l}\text { pcisuiv } \\
\text { pelmort }\end{array}$ & $\begin{array}{r}97.3081 \\
2.6919\end{array}$ & $\begin{array}{l}1.0183 \\
1.0183\end{array}$ & $\begin{array}{r}96.5254 \\
1.9092\end{array}$ & $\begin{array}{r}98.0908 \\
3.4746\end{array}$ \\
\hline 50 & 8 & 9: & $\begin{array}{l}\text { petsurv } \\
\text { petmort }\end{array}$ & $\begin{array}{r}1.2656 \\
98.7344\end{array}$ & $\begin{array}{l}1.0077 \\
1.0077\end{array}$ & $\begin{array}{r}0,4911 \\
87,8598\end{array}$ & $\begin{array}{r}2.0402 \\
99,5089\end{array}$ \\
\hline & 9 & 8 & & $\begin{array}{r}1.1443 \\
98.855 ?\end{array}$ & & & $\begin{array}{r}2.3107 \\
100.0\end{array}$ \\
\hline & 12 & $9:$ & $\begin{array}{l}\text { petsurv } \\
\text { pctmort }\end{array}$ & $\begin{array}{r}0.636 \mathrm{~B} \\
99.3632\end{array}$ & $\begin{array}{l}0.6320 \\
0.6320\end{array}$ & & $\begin{array}{r}1.1227 \\
99.8490\end{array}$ \\
\hline & 6 & 8 & $\begin{array}{l}\text { pctsury } \\
\text { pctmort }\end{array}$ & $\begin{array}{r}2.0820 \\
97.9180\end{array}$ & $\begin{array}{l}1.1511 \\
1.1511\end{array}$ & $\begin{array}{r}1.1971 \\
87.0332\end{array}$ & $\begin{array}{r}2.9668 \\
98.8029\end{array}$ \\
\hline & 9 & 9 & $\begin{array}{l}\text { petsury } \\
\text { pclmort }\end{array}$ & $\begin{array}{r}0.5490 \\
99.4510\end{array}$ & $\begin{array}{l}0.6880 \\
0.6880\end{array}$ & $\begin{array}{r}0.0201 \\
08.9222\end{array}$ & $\begin{array}{r}1.0778 \\
99.9799\end{array}$ \\
\hline & 12 & 9: & $\begin{array}{l}\text { netsury } \\
\text { pethort }\end{array}$ & $\begin{array}{r}0.6491 \\
99.3509\end{array}$ & $\begin{array}{l}0.6665 \\
0.6665\end{array}$ & $\begin{array}{r}0.1368 \\
9.8386\end{array}$ & $\begin{array}{r}1.1614 \\
99.8632\end{array}$ \\
\hline
\end{tabular}

loc $=S L$ Irt_typ $=B \mid$

\begin{tabular}{|c|c|c|c|c|c|c|c|}
\hline$c 0$ & time & N Obs & Verfiable & Moan & std Dov & $\begin{array}{l}\text { Lower } 95 \% \\
\text { CL for Mean }\end{array}$ & $\begin{array}{l}\text { Upper } 95 \% \\
\text { cL for Mean }\end{array}$ \\
\hline 0 & 12 & 12 & $\begin{array}{l}\text { pctsury } \\
\text { pctinor }\end{array}$ & $\begin{array}{r}95.4624 \\
4.5376\end{array}$ & $\begin{array}{l}2.2632 \\
2.2632\end{array}$ & $\begin{array}{r}84.0245 \\
3.0996\end{array}$ & $\begin{array}{r}96.9004 \\
5.9755\end{array}$ \\
\hline 50 & 12 & 12 & $\begin{array}{l}\text { petsurv } \\
\text { petmort }\end{array}$ & $\begin{array}{r}2.9296 \\
97.0704\end{array}$ & $\begin{array}{l}1.5677 \\
1.5677\end{array}$ & $\begin{array}{r}1.9335 \\
96.07 \angle 4\end{array}$ & $\begin{array}{r}3.9256 \\
98.0666\end{array}$ \\
\hline 100 & 12 & 12 & $\begin{array}{l}\text { petsury } \\
\text { petmort }\end{array}$ & $\begin{array}{r}0.8869 \\
99.1131\end{array}$ & $\begin{array}{l}0.9888 \\
0.9888\end{array}$ & $\begin{array}{r}0.2587 \\
98.48<8\end{array}$ & $\begin{array}{r}1.5152 \\
99.7413\end{array}$ \\
\hline
\end{tabular}

loc=SL trt typ $=W r$

\begin{tabular}{|c|c|c|c|c|c|c|c|}
\hline anc & time & NObs & Varlable & Mean & Std Dev & $\begin{array}{l}\text { Lower } 95 \% \\
\text { CL for Mean }\end{array}$ & $\begin{array}{c}\text { Upper } 95 \% \\
\text { CL for Mean }\end{array}$ \\
\hline 0. & 6 & 9 & $\begin{array}{l}\text { petsury } \\
\text { petmort }\end{array}$ & $\begin{array}{r}95.5646 \\
4.4354\end{array}$ & $\begin{array}{l}2.3698 \\
2.3608\end{array}$ & $\begin{array}{r}93.7431 \\
2.6138\end{array}$ & $\begin{array}{r}97.3862 \\
6.2569\end{array}$ \\
\hline & $\theta$ & 9 & $\begin{array}{l}\text { petsurv } \\
\text { petmorl }\end{array}$ & $\begin{array}{r}95.5496 \\
4.4504\end{array}$ & $\begin{array}{l}1,3716 \\
1,3716\end{array}$ & $\begin{array}{r}94.4954 \\
3.3961\end{array}$ & $\begin{array}{r}96.6039 \\
5.5046\end{array}$ \\
\hline & 12 & $\theta$ & petsurv & 96.2207 & 1.2993 & 95.2220 & 97.2195 \\
\hline
\end{tabular}

file://C:/Users/klweber/AppData/Local/Temp/2/SAS\%20Temporary\%20Files/_TD3488_I... 5/1/2014 


\begin{tabular}{|c|c|c|c|c|c|c|c|c|}
\hline \multirow{4}{*}{50} & & & po:mort & 3.7793 & 1,2993 & 2.7805 & 4.7780 & \multirow{7}{*}{ 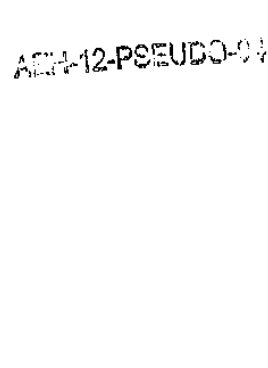 } \\
\hline & 6 & 9 & $\begin{array}{l}\text { petsurv } \\
\text { petmort }\end{array}$ & $\begin{array}{l}12 \mathrm{BOC22} \\
87.3978\end{array}$ & $\begin{array}{r}10.7002 \\
: 10.7002\end{array}$ & $\begin{array}{r}4.3777 \\
79.1729\end{array}$ & $\begin{array}{l}20.8271 \\
95.6227\end{array}$ & \\
\hline & 9. & 9: & $\begin{array}{l}\text { petsurv } \\
\text { patmort }\end{array}$ & $\begin{array}{l}10.2869 \\
89.7031\end{array}$ & $\begin{array}{l}9.1436 \\
9.4436\end{array}$ & $\begin{array}{r}3.0379 \\
82.4440\end{array}$ & $\begin{array}{l}17.5560 \\
96.9621\end{array}$ & \\
\hline & 12 & 9 & $\begin{array}{l}\text { polsurv } \\
\text { pelmort }\end{array}$ & $\begin{array}{l}2.7473 \\
97.2527\end{array}$ & $\begin{array}{l}2.7776 \\
2.7746\end{array}$ & $\begin{array}{r}0.6145 \\
95.1200\end{array}$ & $\begin{array}{r}4.0800 \\
98.3855\end{array}$ & \\
\hline \multirow[t]{3}{*}{100} & $6:$ & 9 & $\begin{array}{l}\text { pelsurv } \\
\text { pelmoort }\end{array}$ & $\begin{array}{l}7.1688 \\
92.0312\end{array}$ & $\begin{array}{l}6.6051 \\
6.6051\end{array}$ & $\begin{array}{r}2.0917 \\
87.7540\end{array}$ & $\begin{array}{l}12.2460 \\
97.9083\end{array}$ & \\
\hline & 9 & $\theta$ & $\begin{array}{l}\text { oclsury } \\
\text { petmort }\end{array}$ & $\begin{array}{r}4.6673 \\
95.3327\end{array}$ & $\begin{array}{l}5.5249 \\
5.5249\end{array}$ & $\begin{array}{r}0.4205 \\
9^{\circ} .0859\end{array}$ & $\begin{array}{r}8,9141 \\
99.5795\end{array}$ & \\
\hline & 12 & 9: & $\begin{array}{l}\text { petsurv } \\
\text { petmort }\end{array}$ & $\begin{array}{l}1.9675 \\
98.0325\end{array}$ & $\begin{array}{l}1.8541 \\
1.8541\end{array}$ & $\begin{array}{r}0.5423: \\
96.6073\end{array}$ & $\begin{aligned} 3.3927 \\
99.4577\end{aligned}$ & \\
\hline
\end{tabular}




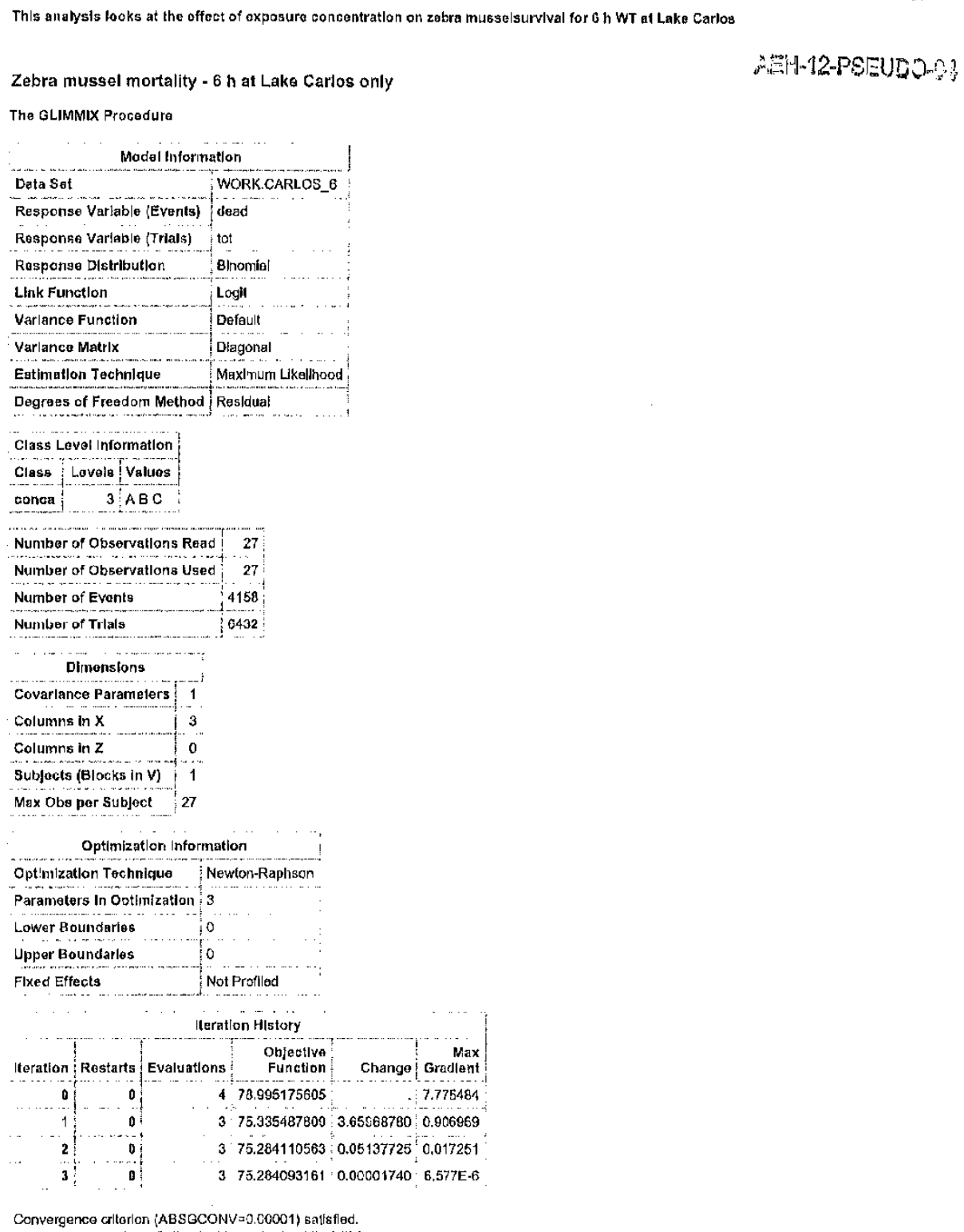

file://C:/Users/klweber/AppData/Local/Temp/2/SAS\%20Temporary\%20Files/_TD3488_I... 5/1/2014

\section{Page $\mathbf{4 7 9}$ of 519}




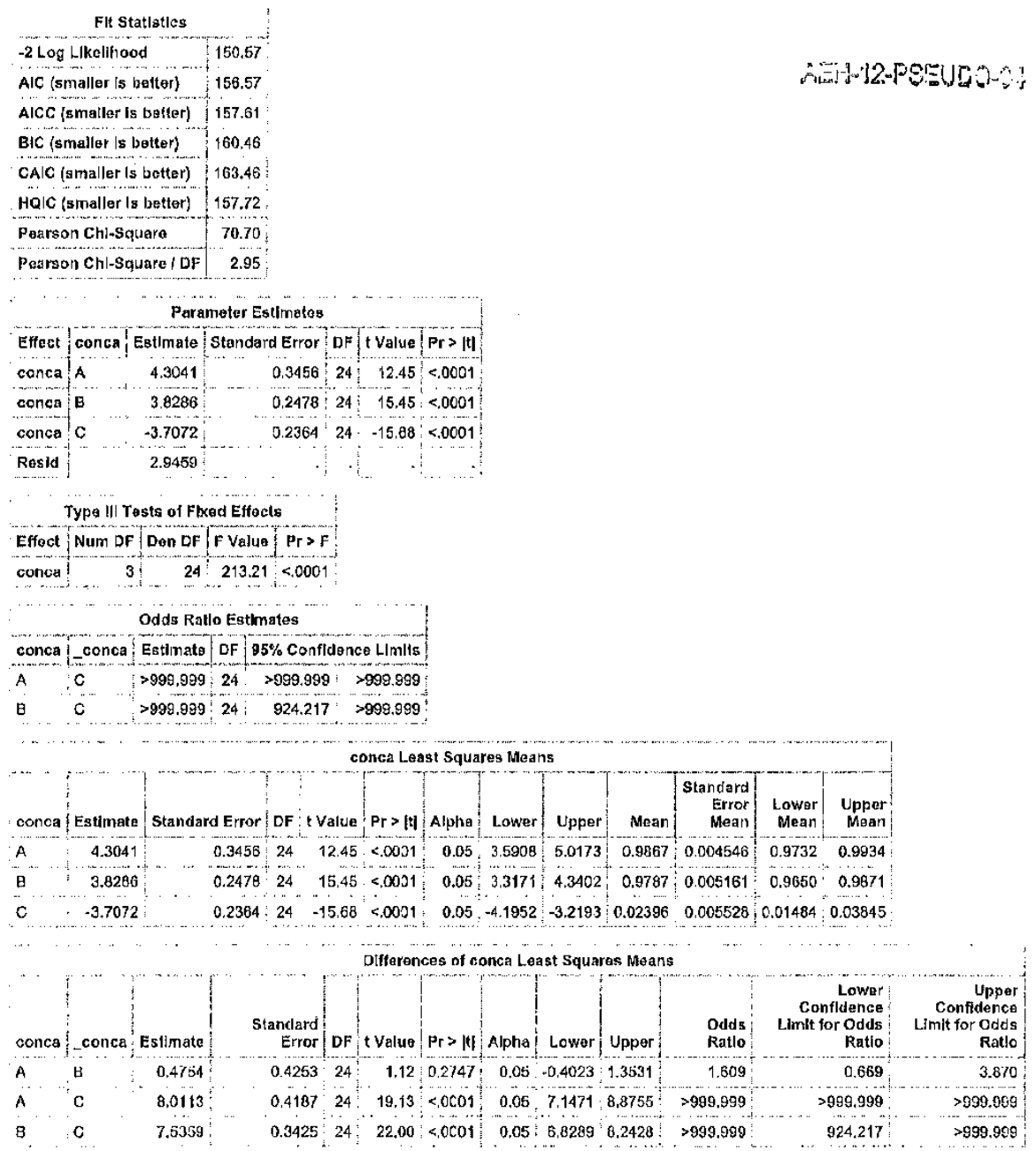

Perfornood by K. Weber SAS version 9.3 13:52 0rMAY1A 
Thls analysls looks at the effect of exposure concentration on zebra musseisurvival for $9 \mathrm{~h}$ WT at Lake Carlos

Zebra mussel mortality - $9 \mathrm{~h}$ at Lake Carlos only

The GLIMMIX Procecture

\begin{tabular}{|c|c|}
\hline \multicolumn{2}{|c|}{ Model Inforination } \\
\hline Data Set & WCRK.CARLOS_9 \\
\hline Response Varlable (Events) & dcad \\
\hline Response Variable (Trials) & tol \\
\hline Response D/stribution & Binomial \\
\hline Link Function & Logit \\
\hline Variance Funcllon & Default \\
\hline Varlance Metrlx & Diagunal \\
\hline Estimatfon Technlque & Maximum Likerlhoo \\
\hline
\end{tabular}

Degrees of Freedom Method Residual

Class Leval Information

Class Levels Values

canca $3 \mathrm{ABC}$

Number of Observatlons Read 27

Nurnber of Observations Used 27

$\begin{array}{lll}\text { Number of Events } & 3869\end{array}$

$\begin{array}{ll}\text { Number of Trjals } & 5772\end{array}$

Dimensions

Covarlaneo Paramoters 1

\begin{tabular}{l|c|c}
\hline Columns in $X$ & 3 \\
\hline
\end{tabular}

Columns in $z$

Suljocts (Blocks in $V$ ) 1

Max Obs per Subject 27

Optimization Information

Optimization Technique Newton-Raphson

Paramsters in Optlmization 3

Lawer Boundaries 0

Upper Boundarles 0

Fixad Effects Not Profllod

Iteration History

Iteration Restarta Evaluations $\begin{aligned} & \text { Objective } \\ & \text { Functlon }\end{aligned}$ Change Gractient

$\begin{array}{cccc}0 & & & \\ 0 & 0 & 68.164941544 & 6.149662\end{array}$

$\begin{array}{lllllll}\cdots & 1 & 0 & 3 & 62.693908903 & 5.47103264 & 1.263667\end{array}$

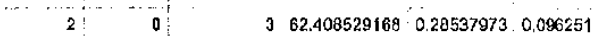

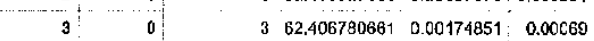

$\begin{array}{llllll}4 & 0 & 362.406730571 & 0.00000009 & 3.608 \mathrm{E}-8\end{array}$

file://C:/Users/klweber/AppData/Local/Temp/2/SAS\%20Temporary\%20Files/_TD3488_I... 5/1/2014

\section{Page 481 of 519}


Convergence critorlon (GCONV $=1 E-8$ ) sellsflod,

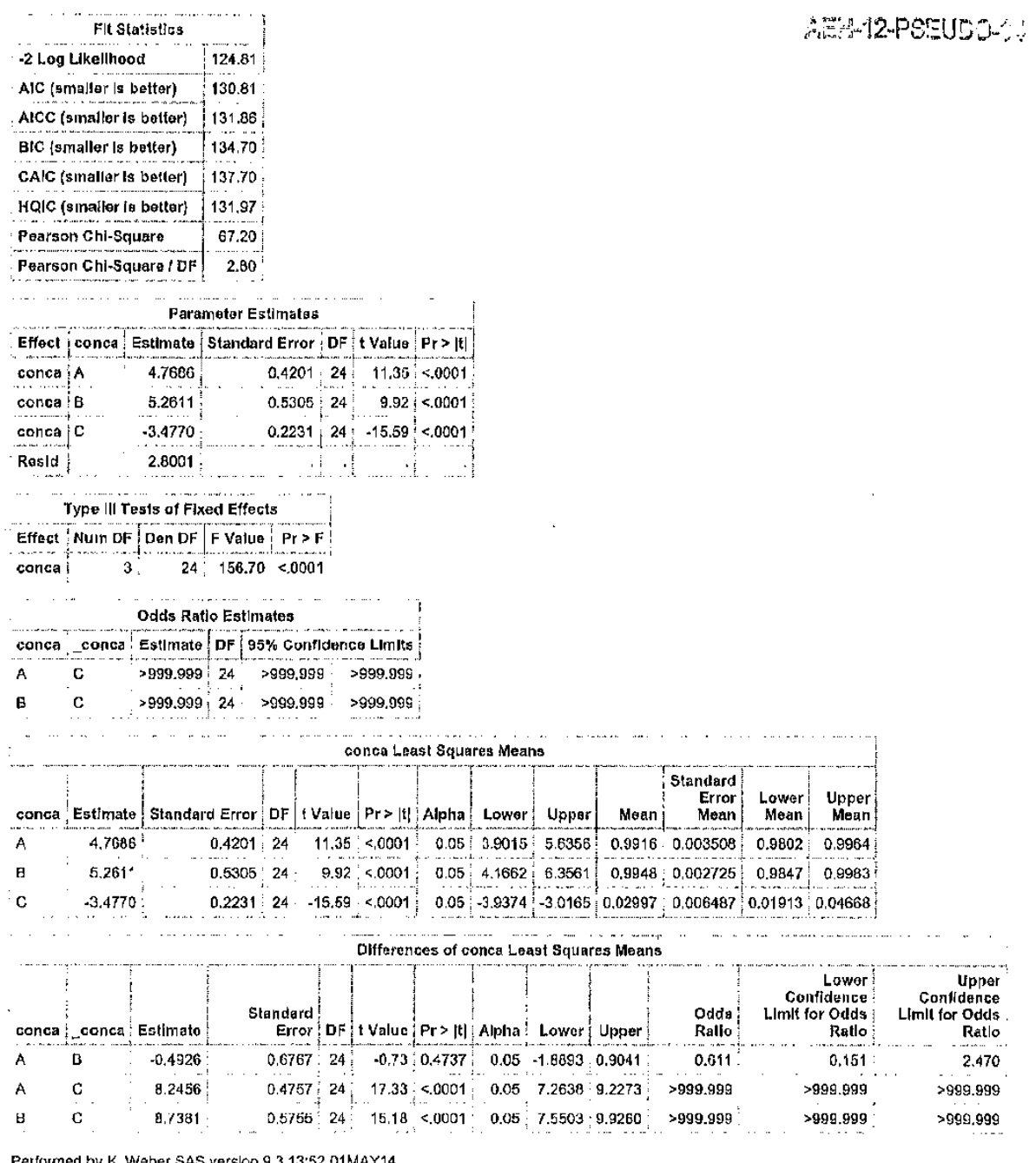

Pelformed by K. Waber SAS verslon 9.3 13:52 01MAY14 
This analysis looks at the effect of oxposuro concentration on zebra inusselsurvival for $12 \mathrm{~h}$ WT at Lake Carlos

Zebra mussel mortality - $12 \mathrm{~h}$ at Lake Carlos anly

The GLIMMIX Pracedure

\begin{tabular}{|c|c|}
\hline \multicolumn{2}{|c|}{ Model Information } \\
\hline Data Sel & WORK CARLOS_12 \\
\hline Response Varlable (Events) & dead \\
\hline Response Variable (Trials) & tot \\
\hline Response Distribution & B.nomial \\
\hline Link Function & Logli \\
\hline Varlance Function & Default \\
\hline Varlance Matrix & Diagonal \\
\hline Estlmallon Tochnique & Maximum Likollhood \\
\hline Degrees of Froedom Method & Residual \\
\hline
\end{tabular}

Degrees of Froedom Method Residu

Class Level Information

Class Levels Values

conca $\quad 3 \mathrm{ABC}$

Number of Observations Read 27

\begin{tabular}{l|l} 
Number of Observations Used & 27
\end{tabular}

\begin{tabular}{l|l} 
Number of Events & 4202
\end{tabular}

Nuinbor of Trials

.....................

Covarlance Parameters 1

Columns in $X$

Columns in $Z$

Subjects (Blocks In V) 1

Max Obs per SubJect 27

Optimlzation Information

Optimizatlon Techuique Newton-Raphson

Parameters in Optimization 3

Lower Boundarles

Upper Boundarias

Fixod Effects

0

Finod Effocta (-......

iteration HIstory

Iteration Restarts Evaluations $\begin{gathered}\text { Objactliva! Function } \\ \text { Change Gradlent }\end{gathered}$

o 40 . 451.529434631

. 2,305641

1 o 350.064319610 1.46511531 0.31522

2 o $350.037570263 \quad 0.026749360 .00862$

$\begin{array}{llll}3 & 350.037552305 & 0.00001736 & 6.941 \mathrm{E}-6\end{array}$

Convergence crikericn (ABSGCONV 00.00501 ) satts sled.

file://C:/Users/klweber/AppData/Local/Temp/2/SAS\%20Temporary\%20Files/_TD3488_I... 5/1/2014

\section{Page $\mathbf{4 8 3}$ of 519}




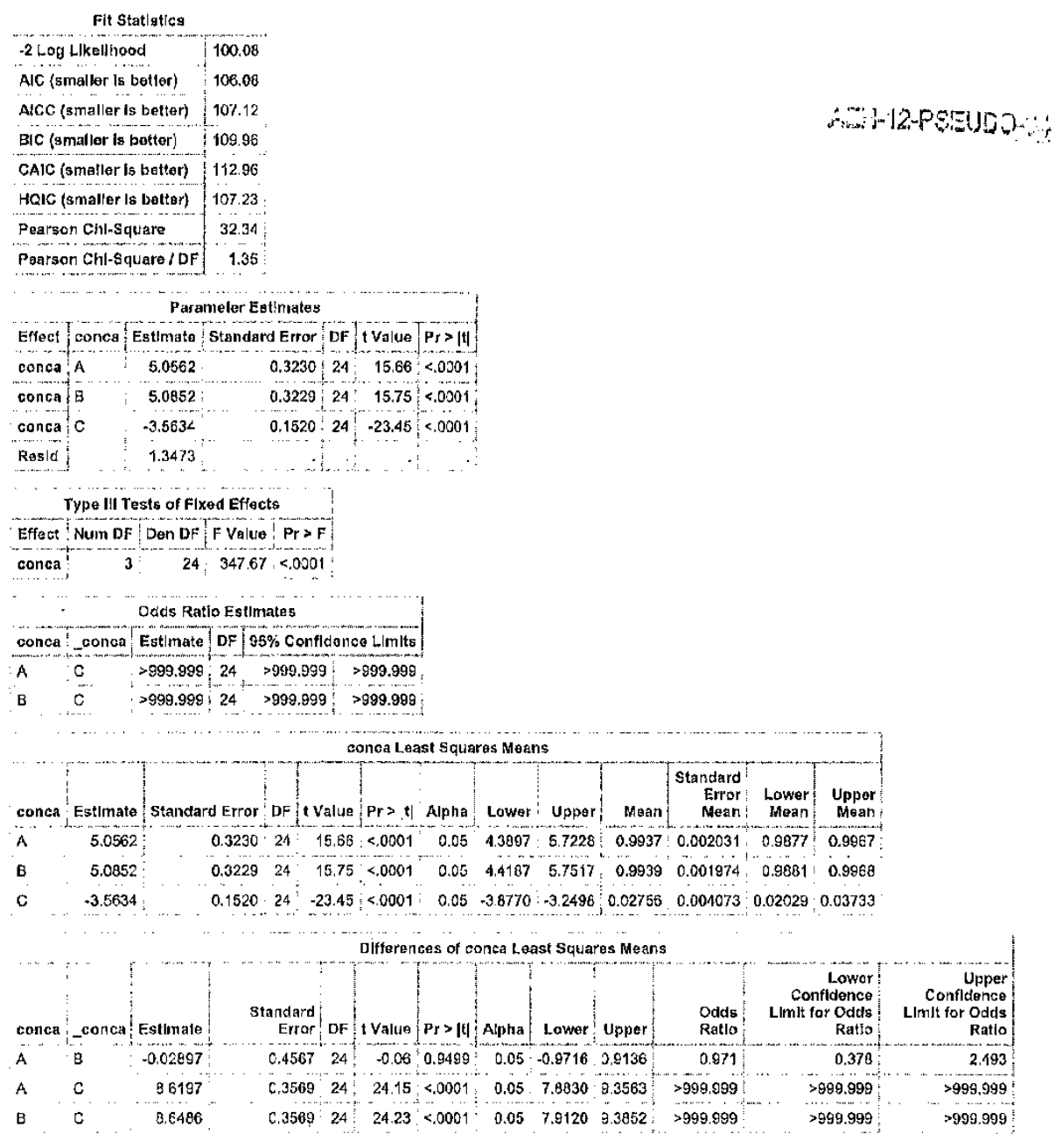

Perlormed by K. Weber SAS version 9.3 13:52 01MAY14 


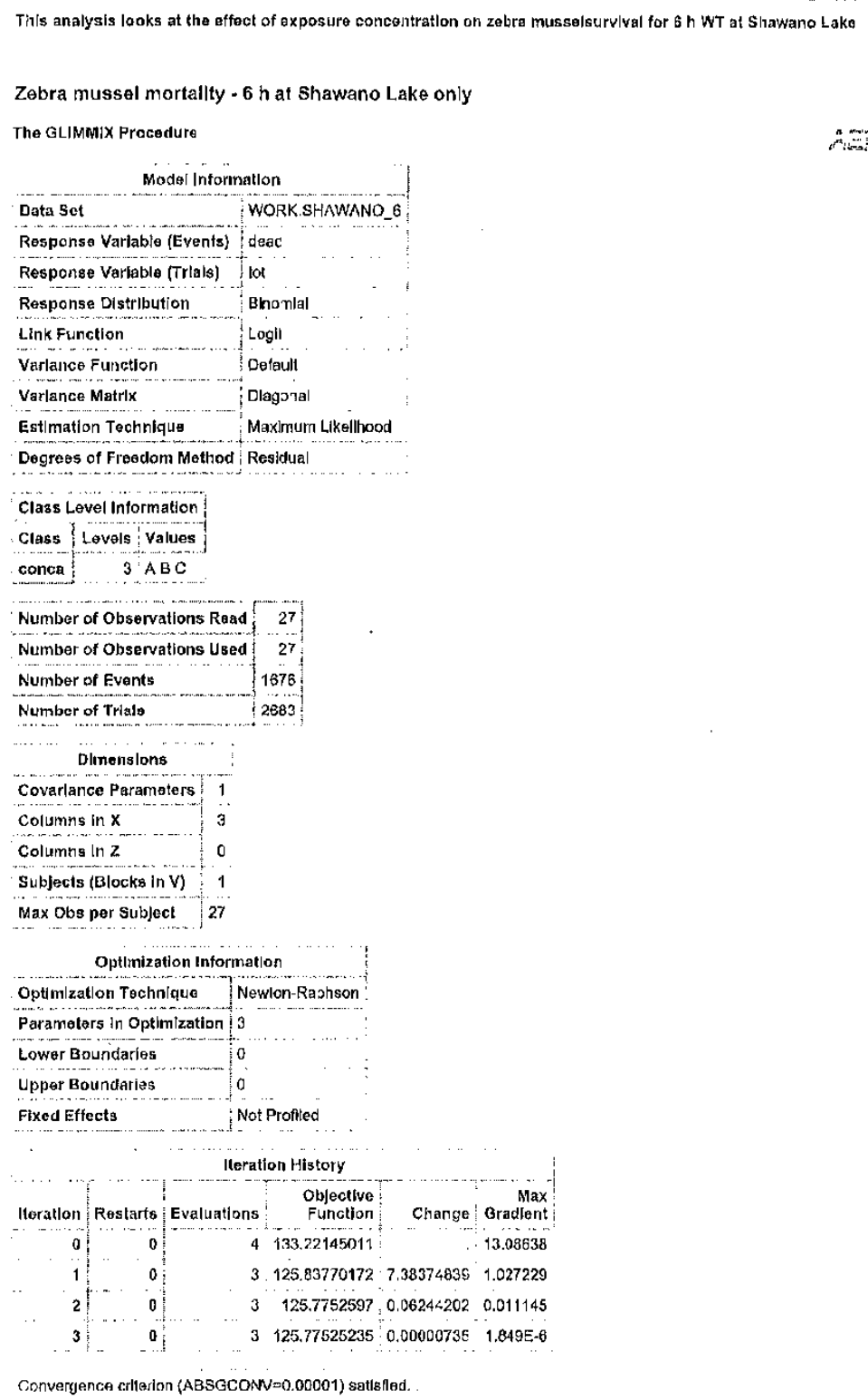

file://C:/Uscrs/klwcber/AppData/Local/Temp/2/SAS\%20Tcmporary\%20Files/_TD3488_I... S/1/2014 


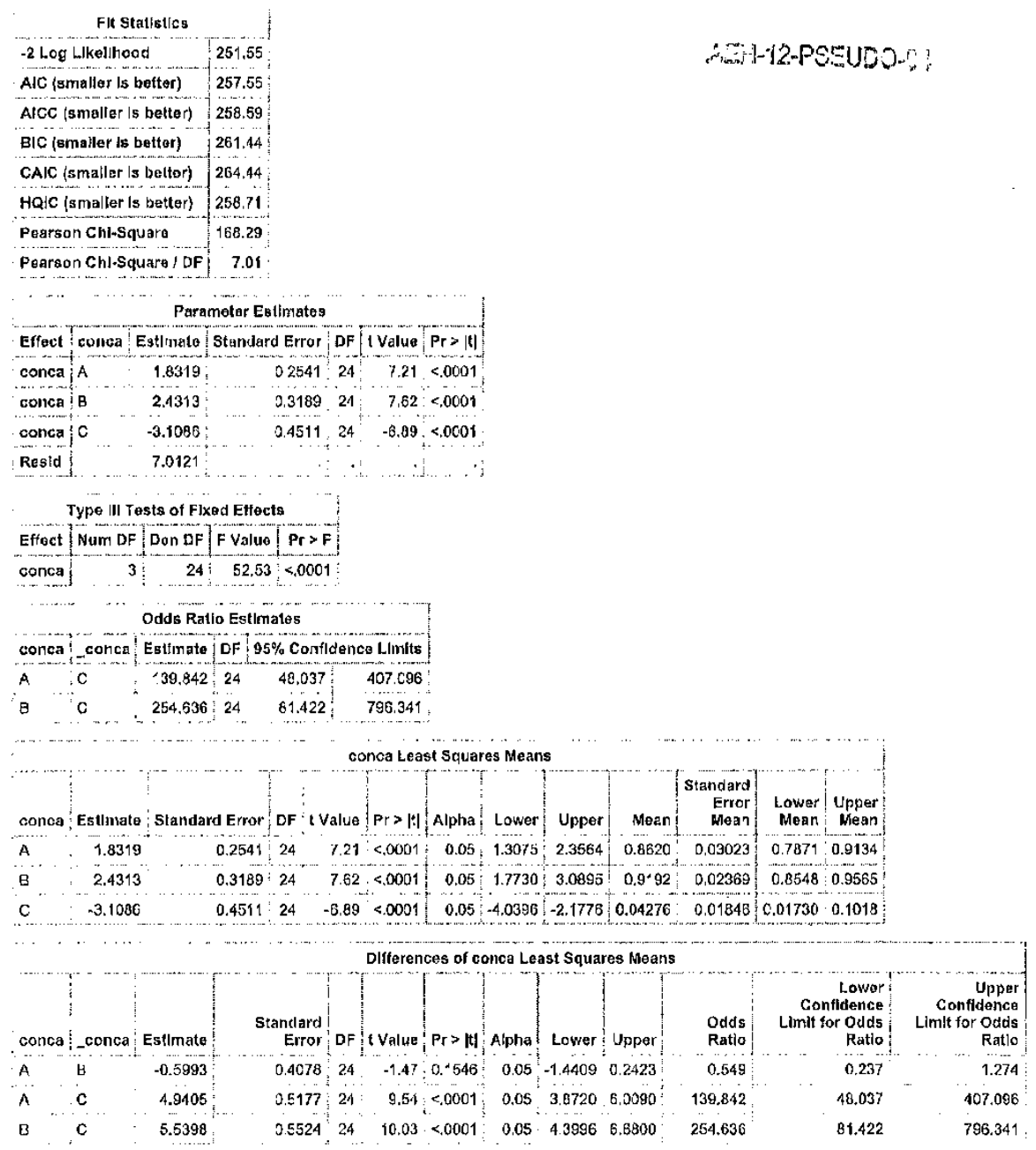

Performed by K. Wober SAS version 9.3 13:52 01MAY 1A 
This analysis looks at the effecl of exposure concentration on zebra nusselsurvival for $9 \mathrm{~h}$ WT at Shawano Lake

Zebra mussel mortality - $9 \mathrm{~h}$ at Shawano Lake only

The GLIMMIX Procedure

\begin{tabular}{|c|c|c|c|c|}
\hline \multicolumn{4}{|c|}{ Data Sol } & \multirow{2}{*}{$\begin{array}{l}\text { WORK.SHAWANO } \\
\text { deac }\end{array}$} \\
\hline \multicolumn{4}{|c|}{ Response Varlable (Events) } & \\
\hline \multicolumn{4}{|c|}{ Response Varlable (Trlals) } & tot \\
\hline \multicolumn{4}{|c|}{ Reaponae Dlsirlbutlon } & Bhonlal \\
\hline \multicolumn{4}{|c|}{ Llnk Functlon } & Loglt \\
\hline \multicolumn{4}{|c|}{ Vartance Function } & Defautt \\
\hline \multicolumn{4}{|c|}{ Varlance Matrlx } & Dlagonal \\
\hline \multicolumn{4}{|c|}{ Est/matlon Technlque } & Maximum Likellhood \\
\hline \multicolumn{4}{|c|}{ Degrees of Freedom Method } & Residual \\
\hline \multicolumn{5}{|c|}{ Class Level Information } \\
\hline \multicolumn{5}{|c|}{ Class Levols Values } \\
\hline \multicolumn{5}{|c|}{ conca $\quad 3 \mathrm{ABC}$} \\
\hline \multicolumn{4}{|c|}{ Number of Observatlons Read } & 27 \\
\hline \multicolumn{4}{|c|}{ Number of Observations Usad } & 27 \\
\hline \multicolumn{4}{|c|}{ Numbar of Events } & 1544 \\
\hline \multicolumn{4}{|c|}{ Number of Trlals } & 2548 \\
\hline \multicolumn{5}{|c|}{ Dimensions } \\
\hline \multicolumn{3}{|c|}{ Covarlance Parameters } & 1 & \\
\hline \multicolumn{3}{|c|}{ Columns $\ln x$} & a & \\
\hline \multicolumn{3}{|c|}{ Columns $\ln z$} & 0 & \\
\hline \multicolumn{3}{|c|}{ Stbjects (Blocks in V) } & 1 & \\
\hline \multicolumn{3}{|c|}{ Max Obs per Subject } & 27 & \\
\hline
\end{tabular}

Optimization Information

Optimlzation Techn!que Newlon-Raphison

Parameters In Optlmization 3

Lower Boundarles

Upper Boundarios 0

Flxod Effacts Nat Profiled

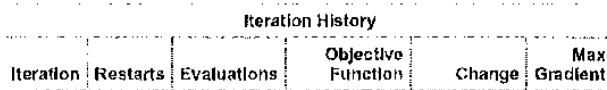

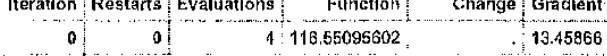

\begin{tabular}{l|c|c|c|ccc}
1 & 0 & 3 & 105.41799088 & 10.13296514 & 1.431037
\end{tabular}

$2 \quad 3 \quad 3050.21296528 ; 0.205 C 25590.045502$

\begin{tabular}{l|lllllll}
3 & 0 & $3: 105.21278193$ & 0.00018336 & 0.00006
\end{tabular}

$\begin{array}{lllllll}4 & 0 & 3 & 105,21278193 & 0.00000000 & 1.03 \mathrm{E}-10\end{array}$

file://C://Jsers/k1weber/AppData/Local/Temp/2/SAS\%20Temporary\%20Files/_TD3488_I... 5/1/2014

\section{Page 487 of 519}




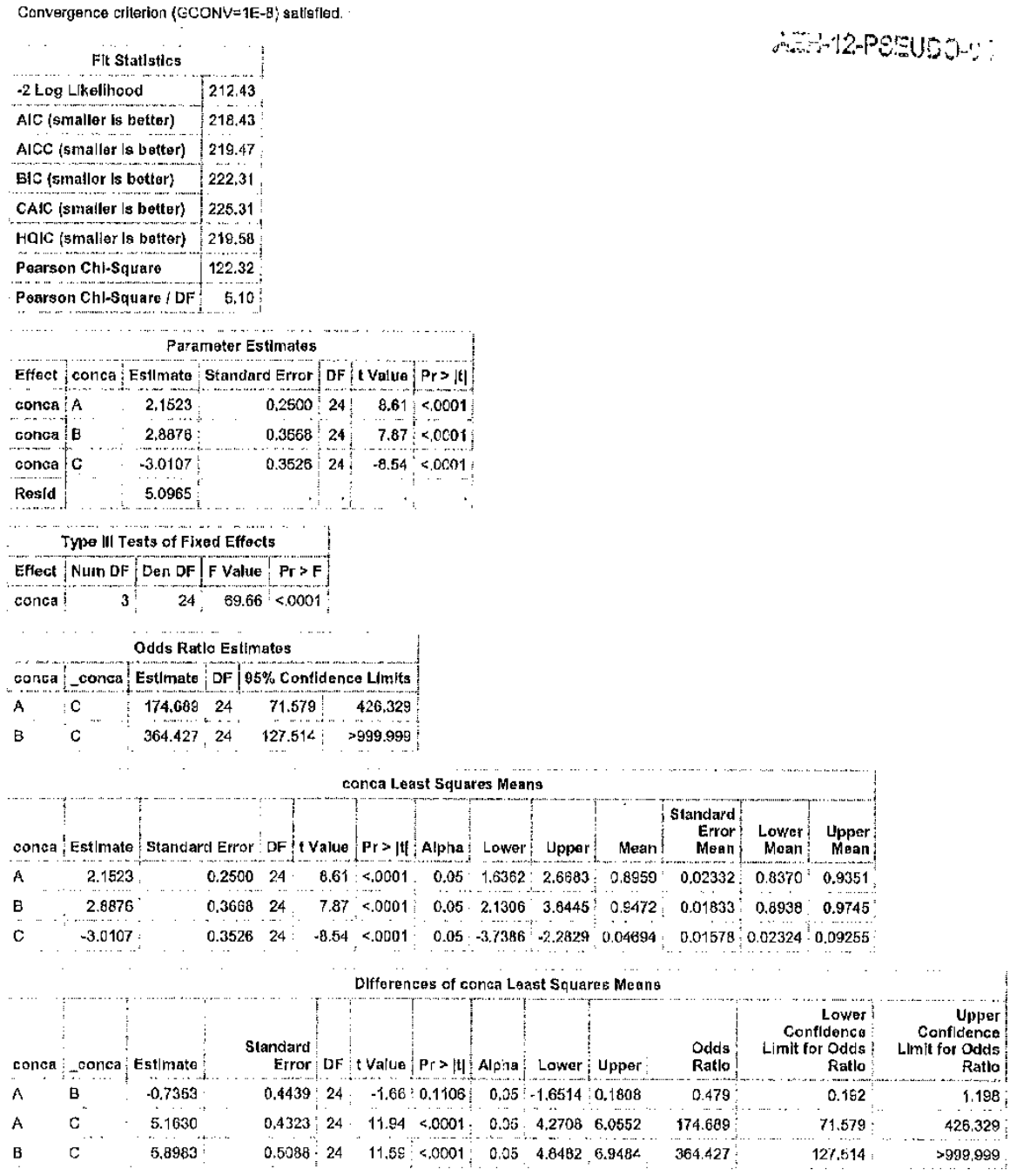

Pertormed by K. Weber SAS version 9.3 13:52 01MAY14 


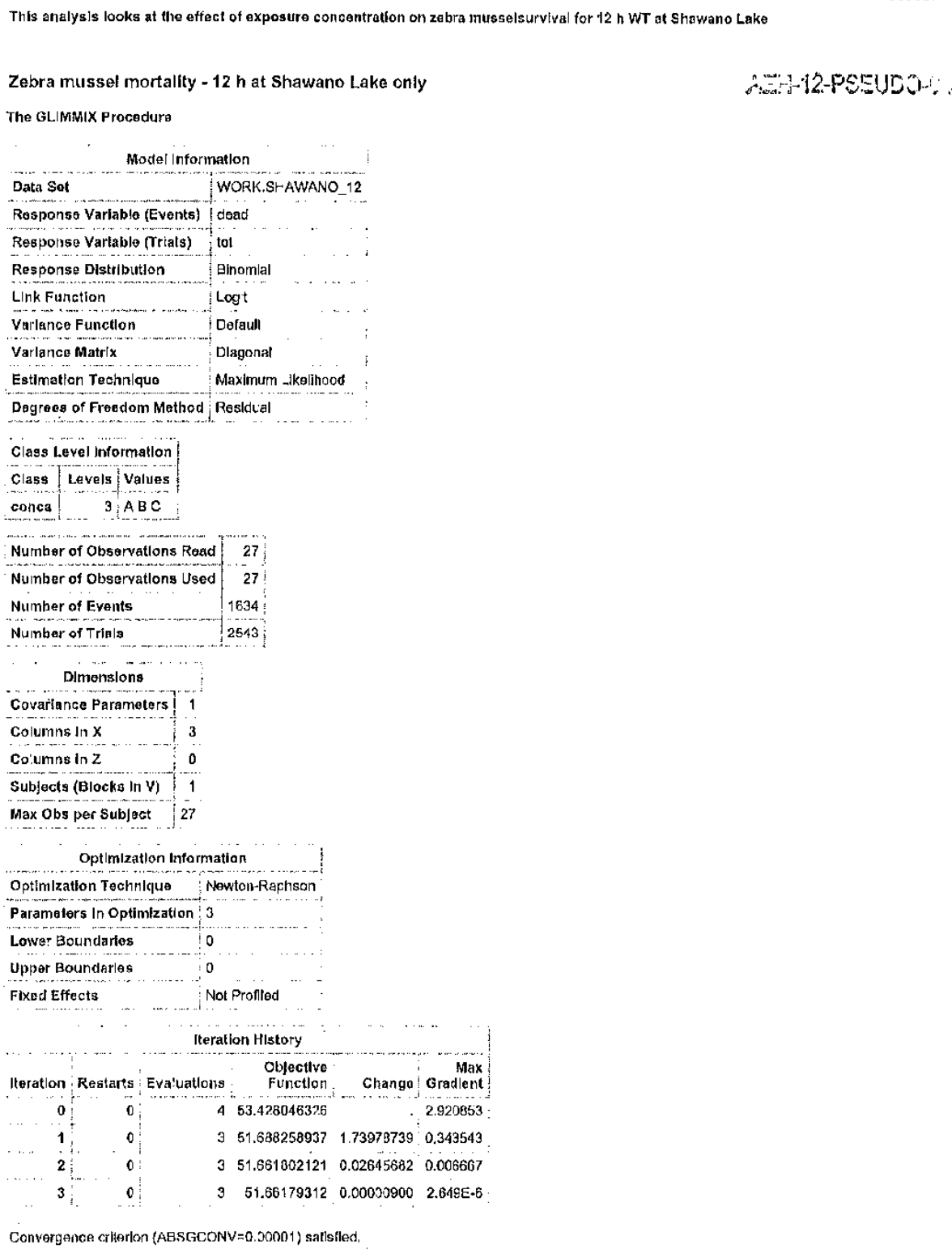

file://C:/Uscrs/klwcber/AppData/Local/Temp/2/SAS\%20Temporary\%20Files/_TD3488_I... S/1/2014 


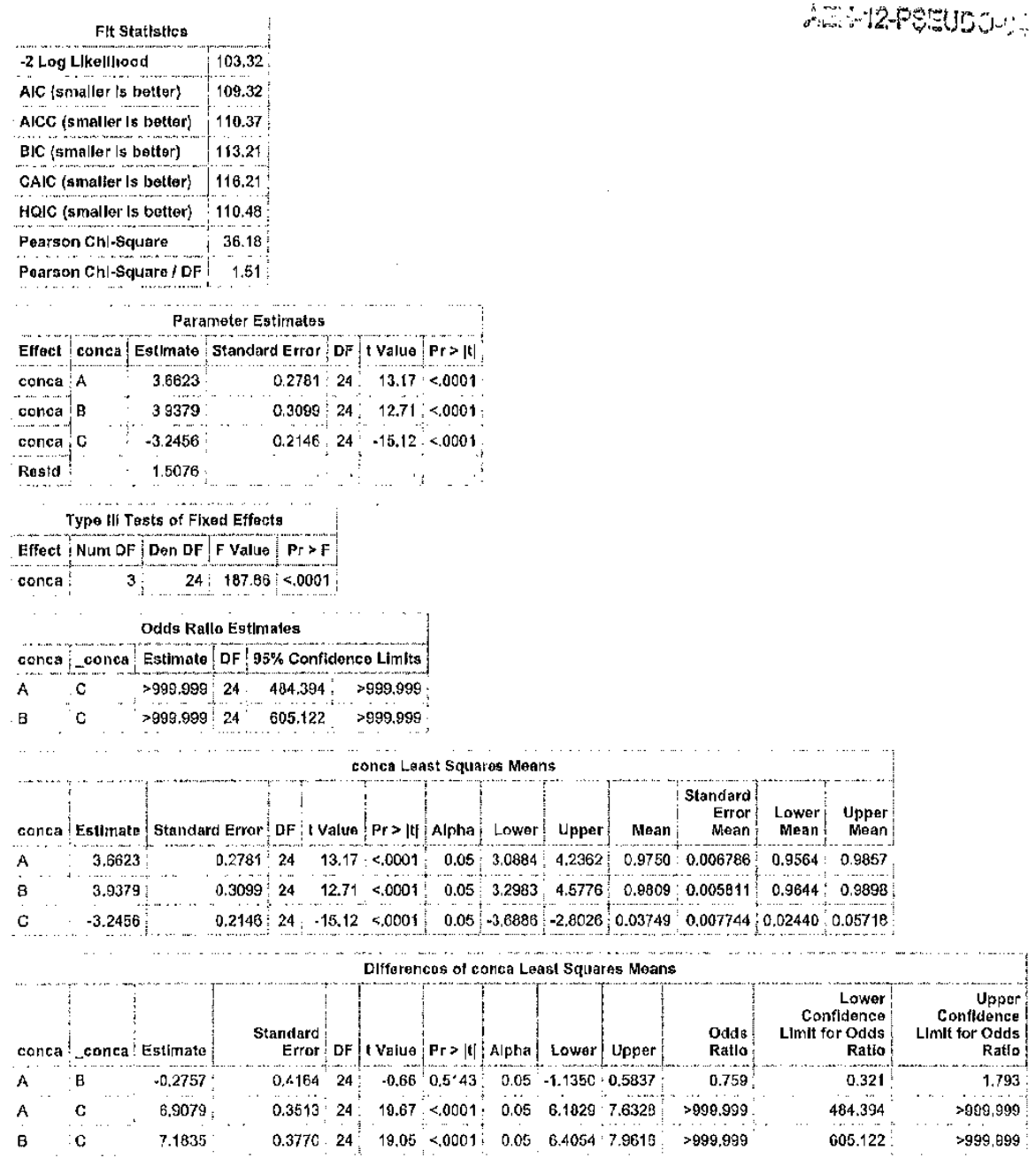

Performed by K. Webor SAS Yersion $9.313: 62$ 01MAY14 


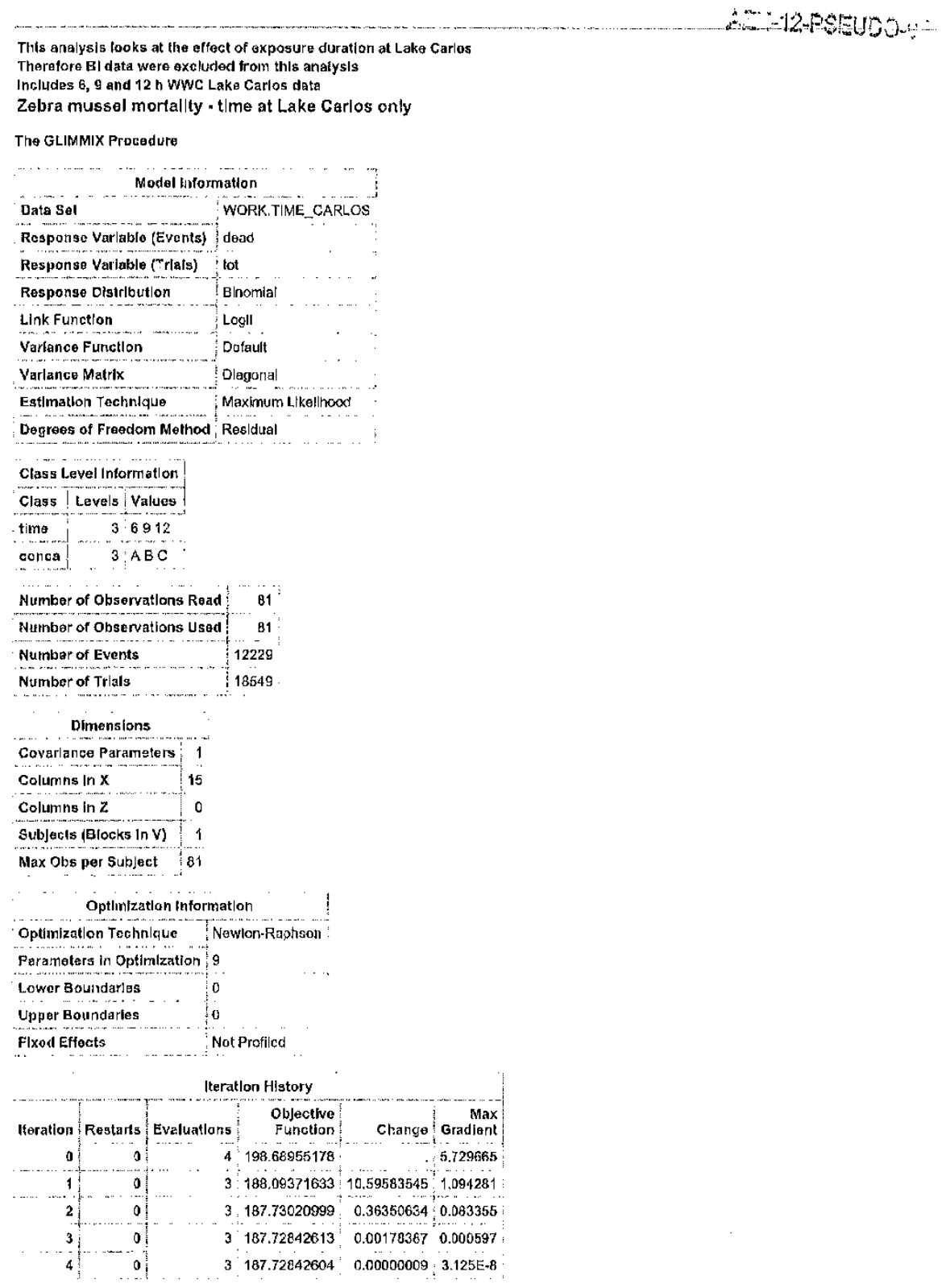

file://C:/Users/klweber/AppData/Local/Temp/2/SAS\%20Temporary\%20Files/_TD3488_I... 5/1/2014

\section{Page 491 of 519}




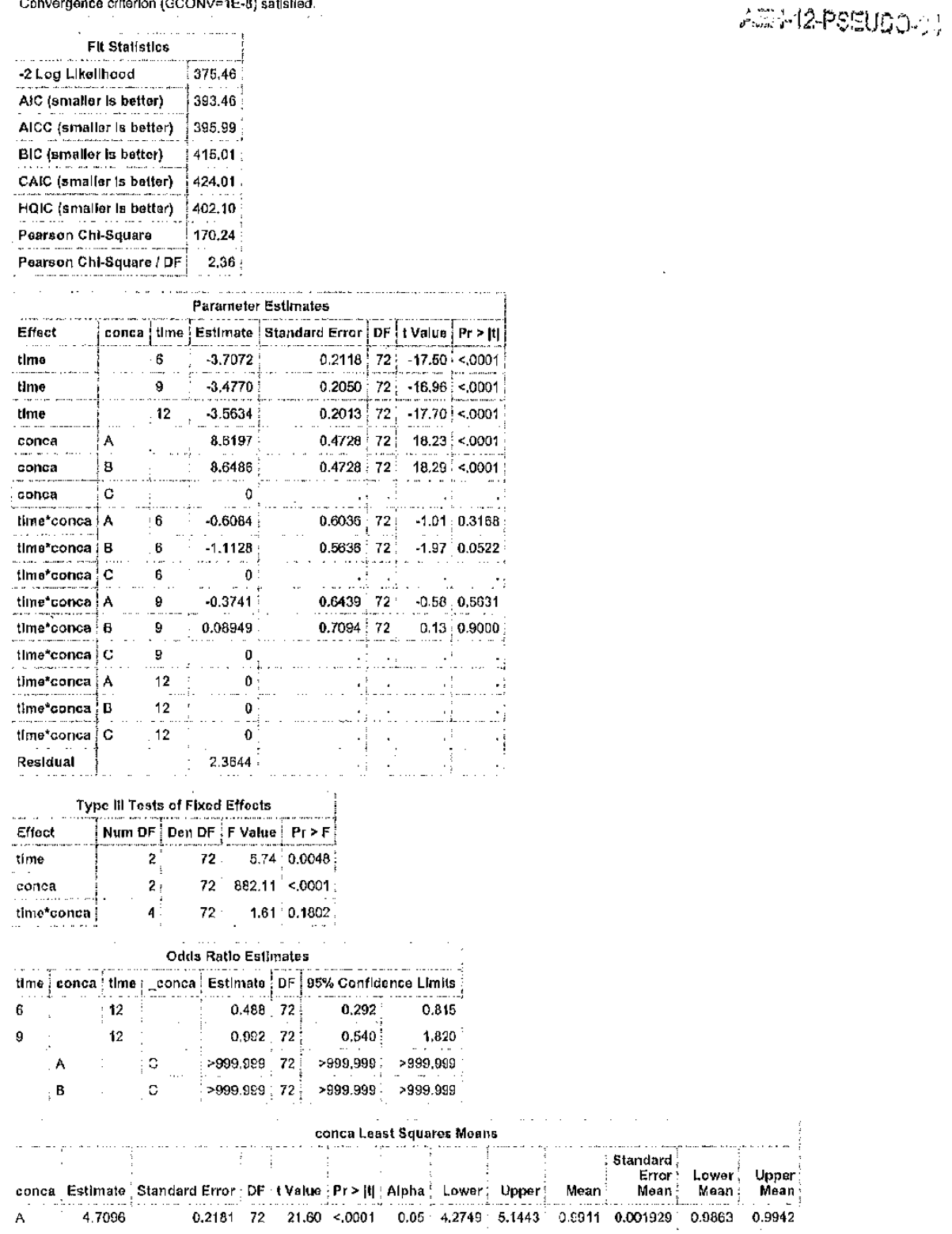

file://C:/Users/klweber/AppData/Local/Temp/2/SAS\%20Temporary\%20Files/_TD3488_I... 5/1/2014 


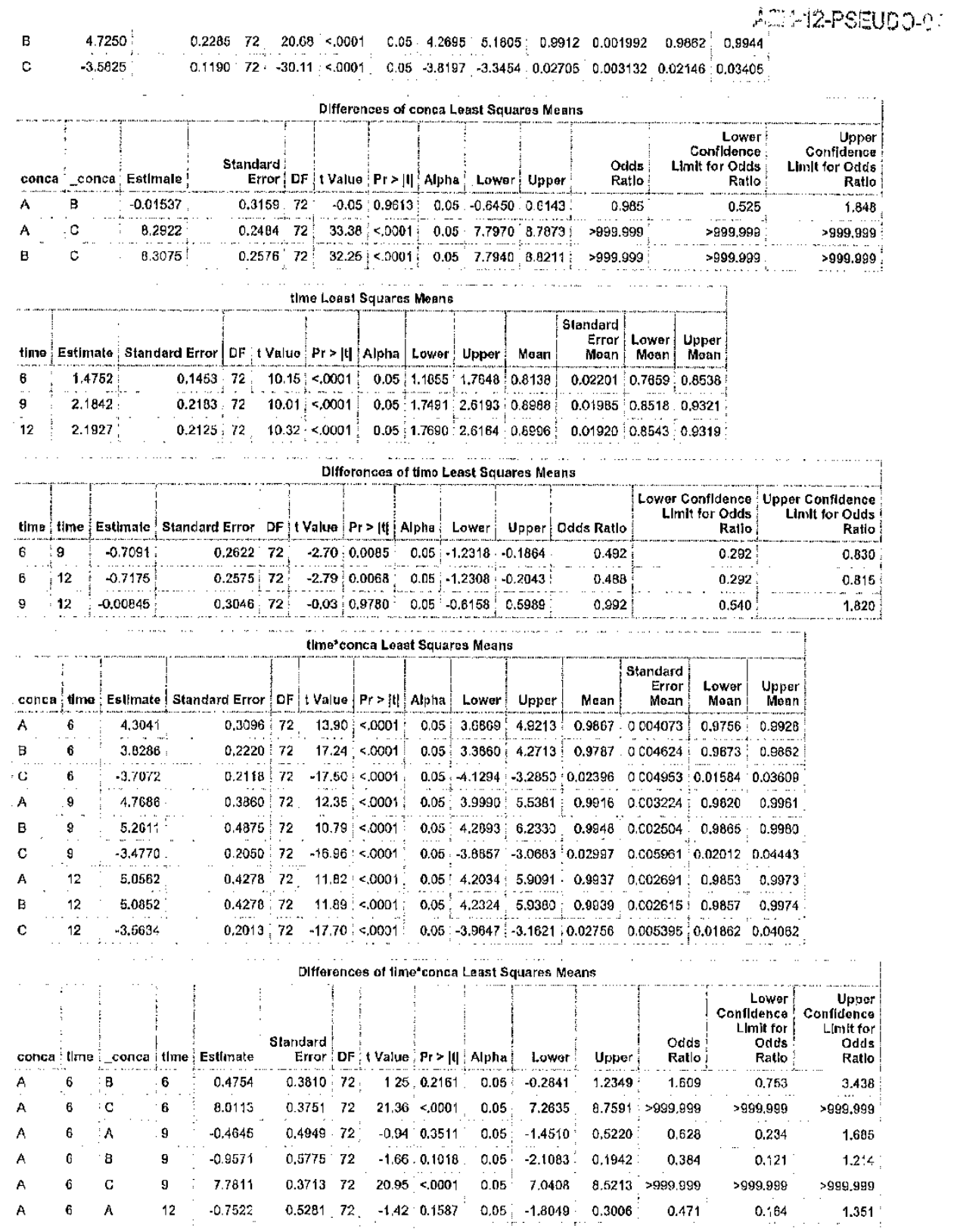

file://C:/Users/klweber/AppData/Local/Temp/2/SAS\%20Temporary\%20Files/_TD3488_I... 5/1/2014 


\begin{tabular}{|c|c|c|c|c|c|c|c|c|c|c|c|c|c|c|}
\hline A & 6 & $\mathrm{~B}$ & 12 & -0.7812 & 0.5281 & 72 & $-1,48$ & 0.1434 & 0.05 & -1.8338 & 0.2715 & 0.458 & 0.160. & 1.312 \\
\hline A & 6 & $\mathrm{c}$ & 12 & 7.8675 & 0.3693 & $72=$ & 21.30 & $<, 0001$ & 0.05 & 7.1313 & 8.6037 & $>989.999$ & $>\$ \$ 9.999^{\circ}$ & $>999.999$ \\
\hline B & 6 & $\mathrm{C}$ & 6 & 7.5359 & 0.3069 & 72 & 24.56 & $<.0001$ & 0.05 & 6.9242 & 8.1476 & $>999.999$ & $>999.999$ & $>999.999$ \\
\hline B & 6 & A & 9 & -0.9399 & 0.4453 & 72 & -2.11 & 0.0383 & 0.05 & -1.8277 & $:-0.05211$ & 0.391 & 0.16 & 0.940 \\
\hline B & 6 & $\mathbf{B}$ & 9 & -1.4325 & 0.5357 & 72 & -2.67 & 0.0093 & 0.05 & -2.5034 & -0.3646 & 0.230 & 0.082 & 0.894 \\
\hline B & 6 & $\mathrm{C}$ & 9 & 7,3056 & 0.3022 & 72 & 24.17 & $<, 0021$ & 0.05 & 6.7032 & 7.9081 & $>\$ 99.999$ & 815.004 & $>999.999$ \\
\hline$B$ & 6 & A & 12 & -1.2276 & 0.4820 & 72 & -2.55 & 0.0130 & 0.05 & -2.1885 & -0.2667 & 0.293 & 0.112 & 0.766 \\
\hline$B$ & 6 & B & 12 & -1.2566 & 0.4820 & 72 & -2.61 & 0.0111 & 0.05 & -2.2174 & -0.2968 & $0.285:$ & 0.109 & 0.744 \\
\hline B. & 6 & $c$ & 12 & 7.3921 & 0.2997 & 72 & 24.66 & $<0001$ & 0.05 & 6.7946 & 7.9895 & $>999.999$ & 893.003 & $>999.999$ \\
\hline $\mathrm{C}$ & 6 & A & 9 & -8.4758 & 0.4403 & 72 & -19.25 & $<, 0001$ & 0.05 & -9.3536 & -7.5980 & $<0,001$ & $<0.001$ & $<0.001$ \\
\hline C & 6 & 8 & 9 & -8.9684 & 0.5315 & 72 & -16.87 & $<.0001$ & 0.05 & -10.2230 & -7.9088 & $<0.001$ & $<0.001$ & $<0.001$ \\
\hline C & 6 & $\mathrm{c}$ & 9 & -0.2302 & 0.2948 & 72 & -0.78 & $0.4373:$ & 0.05 & -0.8178 & 0.3574 & 0.794 & 0.441 & 1.430 \\
\hline C & 6 & $A$ & 12 & $.8,7636$ & 0.4774 & 72 & -18.36 & $<, 0001$ & 0.05 & -9.7151 & -7.8118 & $<0.001$ & $<0.001$ & $<0.001$ \\
\hline C & 6 & B & 12 & -8.7924 & 0.4774 & 72 & -18.42 & $<.0001$ & 0.05 & -9.7440 & .7 .8409 & $<0,001$ & $<0.001$ & $<0.001$ \\
\hline$c$ & 6 & $c$ & 12 & -0.1438 & 0.2922 & 72 & $\cdot 0,49$ & 0.3241 & 0.05 & -0.7263 & 0.4387 & 0.866 & 0.484 & 1.551 \\
\hline A & 9 & $B$ & 9 & -0.4926 & 0.6219 & 72 & -0.79 & 0.4309 & 0.05 & -1.7322 & 0.7471 & 0.611 & 0.177 & 2.111 \\
\hline A & 9 & $c$ & 9 & 8.2456 & 0.4371 & 72 & 1888 & $<.0001$ & 0.05 & 7.3742 & 8.1169 & $>999.999$ & $>999.998$ & $>999.999$ \\
\hline A & 9 & $A$ & 12 & 0.2877 & 0.5763 & 72 & -0.50 & 0.6191 & 0.05 & -1.4364 & 0.8611 & 0.750 & 0.238 & 2.368 \\
\hline A & 9 & 8 & 12 & $-0,3167$ & 0.5762 & 72 & .0 .55 & 0.5843 & 0.05 ? & -1.4653 & 0.8320 & 0.729 & 0.231 & 2.298 \\
\hline A. & 8 & $\mathrm{C}$ & 12 & 8.3320 & 0.4354 & 72 & 19,14 & $<.0001$ & 0.05 & 7.4641 & 8.1999 & $>999.989$ & $>999.998$ & $>999.998$ \\
\hline $\mathrm{B}$ & 9 & $c$ & 9 & 8.7381 & 0.5289 & 72 & 16.52 & $<.0001$ & 0.05 & 7.6839 & 9.7924 & $>999.999$ & $>999.99 \mathrm{~s}$ & $>999.999$ \\
\hline $\mathrm{B}$ & 9 & $A$ & $: 2$ & 0.2049 & 0.6486 & 72 & 0.32 & 0.7530 & 0.05 & -1.0881 & $\begin{array}{r}1.4979 \\
\end{array}$ & 1.227 & 0.337 & $4.472:$ \\
\hline 日 & 8 & $B$ & -2 & 0.1759 & 0.6486 & 72 & 0.27 & 0.7870 & 0.05 & -1.1170 & 1.4680 & 1.192 & 0.327 & 4.344 \\
\hline 日 & 9 & $c$ & 2 & 8.8246 & 0.6274 & 72 & 16.73 & $<.0001$ & 0.05 & 7.7731 & 9.8760 & $>999.999$ & $>999.999$ & $>999.898$ \\
\hline c & 9 & $A$ & 12 & -8.5332 & 0.4744 & 72 & -17.99 & $<<0001$ & 0.05 & -9.4789 & -7.5875 & $<0.001$ & $<0.001$ & $<0.001$ \\
\hline $\mathrm{C}$ & 9 & 8 & 12 & $\cdot 8.5622$ & 0.4744 & 72 & -18.05 & $<.0001$ & 0.05 & 4.5079 & -7.6166 & $<0.001$ & $<0.001$ & $<0.001$ \\
\hline $\mathrm{c}$ & 9 & $c$ & 12 & $0.086<2$ & 0.2873 & 72 & 0.30 & 0.7644 & $0.05^{\circ}$ & -0.4863 & 0.6592 & 1.090 & 0,615 & 1.833 \\
\hline A & 12 & B & 12 & -0.02897 & 0.6050 & 72 & -0.05 & 0,0619 & 0.05 & -1.2350 & $1.177^{\circ}$ & 0.971 & 0.291 & 3.245 \\
\hline A & 12 & $\mathrm{c}$ & 12 & 8.6197 & 0.4728 & 72 & 18,23 & $<, 0001$ & 0.05 & $7.677^{\circ}$ & 8.5622 & $>999999$ & $>999,999$ & $>999.989$ \\
\hline B & 12 & $\mathrm{C}$ & 12 & $8.6<86$ & 0.4728 & 72 & 18.29 & $<0001$ & 0.05 & $7.706^{*}$ & 0.5911 & $>998999$ & $>999.999$ & 2999.989 \\
\hline
\end{tabular}

Performed by $K$. Wober SAS verston 0.3 13:52 01MAY14 
This analysls looks at the effect of exposure duration at Srawano Lake

Therefore $B 1$ diata were excluded from this analys

Includos 6, 9 and $12 \mathrm{~h} \mathrm{WWC} \mathrm{Shawano} \mathrm{Lake} \mathrm{data}$

Zebra mussel mortallty - time at Shawano Lake only

The GLIMMIX Procedure

\begin{tabular}{l|l|} 
& \multicolumn{1}{|c|}{ Model Information } \\
\hline Data Set & WORK. TIME_SHAWANO \\
\hline Rosponso Varlable (Evonts) & doad \\
\hline Response Varlable (Trlals) & lo \\
\hline Response Dlsirlbutlon & Bhonial \\
\hline Link Functlon & Loglt \\
\hline Varlance Functlon & Default \\
\hline Varlance Matrix & Diagonal \\
\hline Estlmatlon Technique & Maxlmum Likellhood \\
\hline Degreos of Froedom Method & Residual \\
\hline
\end{tabular}

Degreos of Froedom Method Residual

Class Level Information

Class Levels Values

tIme $\quad 36912$

conca $\quad 3 \wedge \mathrm{BC}$

Number of Observatlons Read | 81

Number of Observatlons Used 8

Number of Events

$\begin{array}{ll}\text { Number of Triale } & 7774\end{array}$

' Dinensions

Covarlance Paramolers 1

\begin{tabular}{l|l} 
Columns in $X$ & 15 \\
\hline Columns &
\end{tabular}

Columns $\ln Z$ :

Subjects (Elocks In V) 1

Max Obs per Subject 81

Optimization Informatian

Optimization Tochnique Nowton-Raphson

Paramoters In OptImlzation 9

Lower Boundarles 0

Upper Boundaries 0

Fixed Effects Not Proflled

\begin{tabular}{|c|c|c|c|c|c|}
\hline Iteratlon & Restarts & Evaluations & $\begin{array}{l}\text { Objectivo } \\
\text { Functlon }\end{array}$ & Change & $\begin{array}{r}\text { Max } \\
\text { Gradiant }\end{array}$ \\
\hline 0 & 0 & & 303.20045245 & & 17.01214 \\
\hline 1 & 0 & & 283.94395153 & 19,25650092 & 1.617625 \\
\hline 2 & 0 & 3 & 283.6500271 & 0.29392443 & 0.039634 \\
\hline 3 & 0 & & 283.5498274 & 0.00019971 & 0.060052 \\
\hline 4 & 0 & 3 & $283.649827 \mathrm{~A}$ & 0.00000000 & $8.91 \mathrm{E}-11$ \\
\hline
\end{tabular}

file://C:/Users/klweber/AppData/Local/Temp/2/SAS\%20Temporary\%20Files/TD3488_I,.. 5/1/2014

\section{Page 495 of 519}




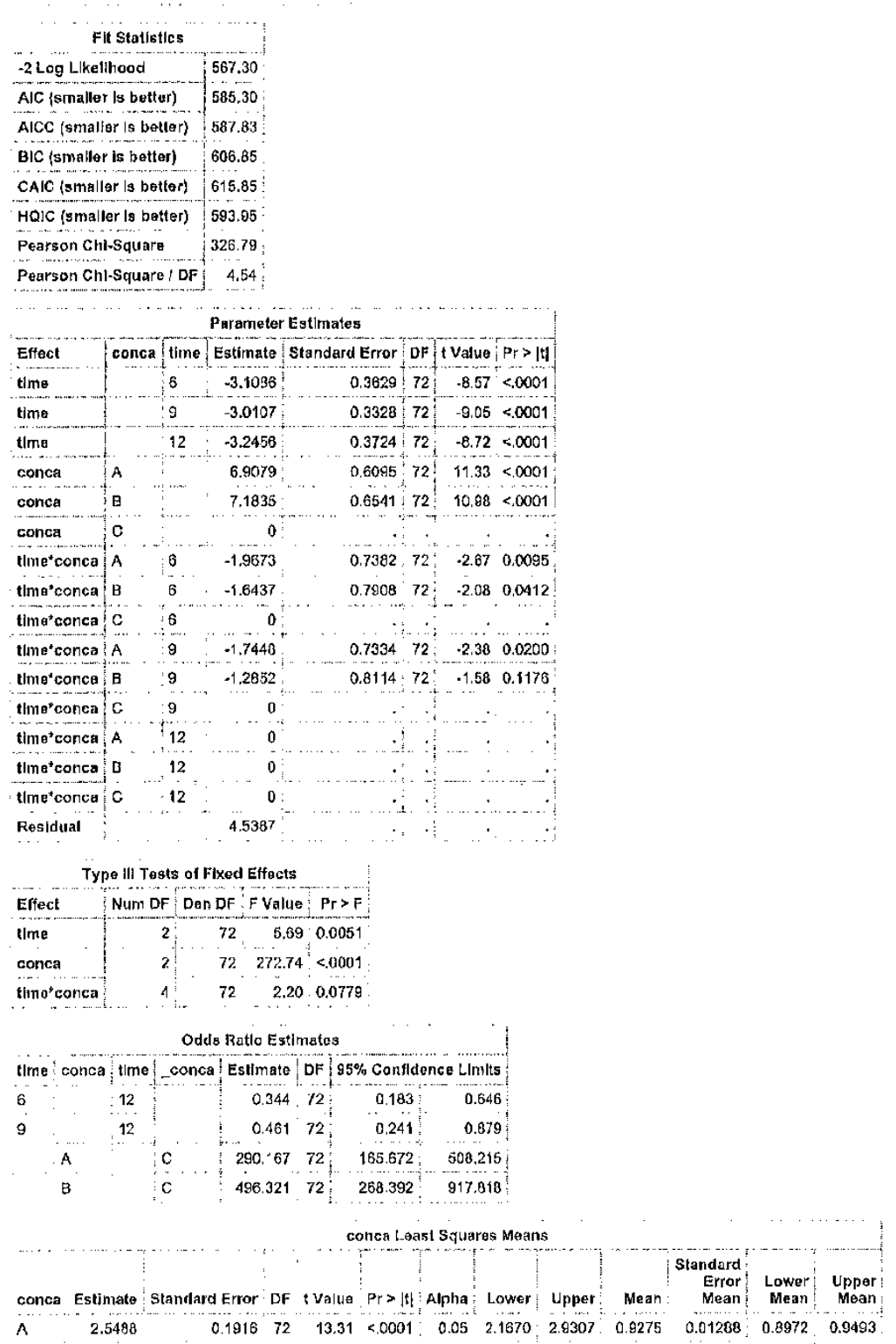

file://C:/Users/klweber/AppData/t,ocal/Temp/2/SAS\%20Temporary\%20Files/_TD3488_I.. S/1/2014 


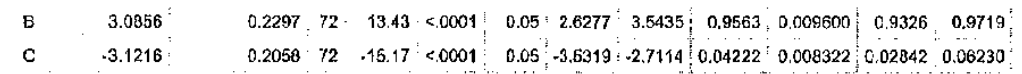

\begin{tabular}{|c|c|c|c|c|c|c|c|c|c|c|c|c|}
\hline conca & _conca & Estlmate & $\begin{array}{r}\text { Standard } \\
\text { Error }\end{array}$ & $D F$ & $t$ Value & $\operatorname{Pr}>|\mathrm{t}|$ & Alpha & Lower & Upper & $\begin{array}{l}\text { Odds: } \\
\text { Ratto }\end{array}$ & $\begin{array}{r}\text { Lower } \\
\text { Conficlonce } \\
\text { Limlt for Odids } \\
\text { Ratio }\end{array}$ & $\begin{array}{r}\text { Upper } \\
\text { Conflderce } \\
\text { Limlt for Odds } \\
\text { Ratlo }\end{array}$ \\
\hline A & B & -0.5368 & 0.2991 & 72 & -1.79 & 0.0769 & 0.05 & -1.1330 & $0.0594<$ & 0.585 & 0.322 & 1.061 \\
\hline A & C & 5.6705 & 0.2811 & 72 & 20.17 & $<c 001$ & 0.05 & 5.1100 & 6.2309 & 290.167 & 165.672 & 508.215 \\
\hline B & c & 6.2072 & 0.3084 & 72 & 20.13 & $<, 0001$ & 0.05 & 5.6924 & 6.8220 & 486,321 & 268.392 & 917.818 \\
\hline
\end{tabular}

\begin{tabular}{|c|c|c|c|c|c|c|c|c|c|c|c|c|}
\hline timo & Estimate & Standard Error & $D F$ & t Valuo & $\operatorname{Pr}>|t|$ & Alpha & Lowor & Upper & Maan & $\begin{array}{l}\text { Standard } \\
\text { Error } \\
\text { Moan }\end{array}$ & $\begin{array}{c}\text { Lower } \\
\text { Meann }\end{array}$ & $\begin{array}{l}\text { Upper } \\
\text { Nean }\end{array}$ \\
\hline 6 & 0.3849 & 0.1831 & 72 & 2.36 & 0.0210 & 0.05 & 0.05981 & 0.7100 & 0.5951 & 0.03929 & 0.5149 & 0.6704 \\
\hline 9 & 0.6764 & 0.1783 & 72 & 3.79 & 0.0003 & 0.05 & 0.3209 & 1.0319 & 0.6629 & 0.03985 & 0.5795 & 0.7373 \\
\hline 12 & 1.4515 & 0.2709 & 72 & 5.36 & $<.0001$ & $0.05:$ & 0.9114 & 1.9016 & 0.8102 & 0.04166 & 0.7133 & 0.8799 \\
\hline
\end{tabular}

\begin{tabular}{|c|c|c|c|c|c|c|c|c|c|c|c|c|}
\hline time & time & Estinate & Standard Error & $D F$ & it value & $P r>|E|$ & Alplia & Lower & Upper & Odds Ratio & $\begin{array}{r}\text { Lower Confldence } \\
\text { Llmit for Odds } \\
\text { Ratlo }\end{array}$ & $\begin{array}{r}\text { Upper Confidence } \\
\text { Limit for Odds } \\
\text { Ratio }\end{array}$ \\
\hline$B$ & 9 & $\cdot 0.2915$ & 0.2416 & 72 & -1.21 & 0.2317 & 0.05 & $; 0.7732$ & 0.1902 & 0.747 & 0.462 & 1.210 \\
\hline 6 & 12 & -1.0667 & 0.3162 & 72 & -3.37 & 0.0012 & 0.05 & -1.6970 & -0.4363 & 0.344 & 0.183 & 0.846 \\
\hline 9 & 12 & -0.7752 & 0.3244 & 72 & -2.39 & 0.0105 & 0.05 & $-1,4218$ & -0.1286 & 0.161 & 0.24 & 0.879 \\
\hline
\end{tabular}

\begin{tabular}{|c|c|c|c|c|c|c|c|c|c|c|c|c|c|}
\hline & & & & & time & n & a & Mea & & & & & \\
\hline conca & the & Estimato & Standard Error & OF & I Valuo & $|\mathrm{Pr}>| \mathrm{t} \mid$ & Alpha & Lowar & Uppor & Mean & $\begin{array}{r}\text { Standard } \\
\text { Error } \\
\text { Moan }\end{array}$ & $\begin{array}{l}\text { Lower } \\
\text { Mean }\end{array}$ & \\
\hline A & 6 & 1.8319 & 0.2044 & 72 & 8.96 & $<.0001$ & 0.05 & 1.4244 & 2.2395 & 0.8620 & 0.02432 & 0.8060 & 0.903 \\
\hline $\mathrm{B}$ & 6 & 2.4313 & 0.2566 & 72 & 9.48 & $<.0001$ & 0.05 & 1.9198 & 2.9428 & 0.9192 & 0.01906 & 0.8721 & 0.9499 \\
\hline C & 6 & $-3 \div 086$ & 0.3629 & 72 & -8.57 & $<.2001$ & 0.05 & -3.8320 & $-2,3851$ & 0,04276 & 0.01485 & 0,02121 & 0.08432 \\
\hline A & 8 & 2.1523 & 0.2360 & 72 & 9.12 & $<, 0001$ & 0,05 & $1,6 \mathrm{~B} 19$ & 2,6226 & 0.6959 & 0.02201 & 0.8432 & 0.9323 \\
\hline B & 9 & 2.8876 & 0.3461 & 72 & 8.34 & $<, 2001$ & 0.05 & 2.1976 & 3.5775 & 0.9472 & 0.01730 & 0.9000 & 0,9728 \\
\hline c & 9 & -3.0107 & 0.3328 & 72 & -9.05 & $<.0001$ & 0.05 & -3.6741 & -2.3473 & 0.04694 & 0.01489 & 0.02474 & 0.08728 \\
\hline$A$ & 12 & 3.6623 & 0.4825 & 72 & $7.59^{\circ}$ & $<.0001$ & 0.05 & $2.7005^{\circ}$ & 4.6240 & $0.9750^{\circ}$ & 0.01177 & 0.9371 & 0.9803 \\
\hline B & 12 & 39379 & 0.5378 & 72 & 7.32 & $<.2001$ & 0.05 & 2.8659 & 5.0100 & 0.9809 & $0.0100 \mathrm{~B}$ & 0.9461 & 0.0 \\
\hline C & 12 & -3.2456 & 0.3724 & 72 & -8.72 & $<.0001$ & 0.05 & -3.9880 & $-2,5032$ & 0.03748 & 0.01344 & 0.01820 & 0.075 \\
\hline
\end{tabular}

\begin{tabular}{|c|c|c|c|c|c|c|c|c|c|c|c|c|c|c|}
\hline conca & itime & conca & time & EstImate & $\begin{array}{r}\text { Standard } \\
\text { Error }\end{array}$ & $\mathrm{DF}$ & IValue & $P_{r}>|t|$ & Alpha & Lower & Upper & $\begin{array}{l}\text { Onds } \\
\text { Ratlo }\end{array}$ & $\begin{array}{r}\text { Lower } \\
\text { Confidence } \\
\text { Limit for } \\
\text { Odds } \\
\text { Ratlo }\end{array}$ & $\begin{array}{r}\text { Upper } \\
\text { Confldence } \\
\text { LImlt for } \\
\text { Odds } \\
\text { Ratlo }\end{array}$ \\
\hline A & 6 & $\theta$ & 6 & -0.5993 & 0.3281 & 72 & -1.83 & 0.0719 & 0.05 & -1.2533 & 0.05466 & 0.549 & 0.286 & 1.056 \\
\hline A & 6 & $\mathrm{C}$ & 6 & 4.9405 & 0.4165 & 72 & 11.86 & $<.0001$ & 0.05 & 4.1102 . & 5.7708 & 139.842 & 60.958 : & 320.808 \\
\hline A & 6 & A & 9 & -0.3203 & 0.3122 & 72 & $-1,03$ & 0.3083 & 0.05 & $=0.9427$ & 0.3020 & 0.726 & 0.390 : & 1,353 \\
\hline A & 6 & E & 9 & -1.0556 & 0.4020 & 72 & -2.63 & 0.0105 & 0.05 & -1.8570 & -0.2543 & 0.348 & $0.156^{\circ}$ & 0.775 \\
\hline A & 6 & C & 9 & 4.8427 & 0.3906 & 12. & 12.40 : & $<.0001$ & 0.05 & 4.0641 & 5.6213 & 126.809 & $58.213^{\circ}$ & 276.236 \\
\hline A & 6 & A & 12 & -1.8303 & 0.5240 & 72 & -3.49 & 0.0008 & 0.05 & -2.8749 & 0,7858 & 0.60 & 0.056 & 0.456 \\
\hline
\end{tabular}




\begin{tabular}{|c|c|c|c|c|c|c|c|c|c|c|c|c|c|c|}
\hline A & 6 & $B$ & 12 & -2.1060 & 0.5753 & 72 & -3.66 & 0,0005 & 0.05 & $-3,2529$ & -0.9591 & $0.122 !$ & 0.039 & 0.383 \\
\hline A & 6 & $c$ & 12 & 5.0775 & 0.4248 & 72 & 11.95 & $<, 0001$ & 0.05 & 4,2306 & 5.9244 & 160.376 & 68.761 & 374.054 \\
\hline B & 6 & $c$ & 6 & 5.5398 & 0.4445 & 72 & 12.46 & $<, 0001$ & 0.05 & 4.6538 & 6.4258 & 254.636 & 104986 & 617.605 \\
\hline B & 6 & A & 9 & 0.2790 & 0.3486 & 72 & 0.80 & 0.4261 & 0.05 & -0.4159 & $0.9739=$ & 1.322 & 0.660 & 2.640 \\
\hline B & 6 & B & 9 & -0.4563 & 0.4308 & 72 & -1.06 & 0.2931 & 0.05 & $-1,3152$ & 0.4026 & 0.834 & 0268 & 1.496 \\
\hline B & 6 & $c$ & 9 & 5.4420 & 0.4202 & 72 & 12,95 & $<.0001$ & 0.05 & 4.6043 & 6.2797 & 230.905 & 09.914 & 533.632 \\
\hline$B$ & 6 & $A$ & 12 & -1.2310 & 0.5464 & 72 & -2.25 & 0.0273 & 0.05 & 2.3203 & -0.1417 & $0.292 !$ & 0.098 & 0.868 \\
\hline$B$ & 6 & B & 12 & -1.5067 & 0.5958 & 72 & -2.53 & 0.0436 & 0.06 & $\begin{array}{l}-2.6945 \\
\end{array}$ & -0.3189 & 0.222 & 0.068 & 0.727 \\
\hline B & 6 & c & 12 & 5.6738 & 0.4522 & 72 & 12.55 & $<.0001$ & 0.05 & 4.7753 & 6.5784 & $292.027^{\prime}$ & 118.547 & 719.377 \\
\hline c & 6 & A & 9 & -5.2608 & 0.4329 & 72 & -12.15 & $<.0001$ & 0.05 & -6.1236 & -4.3979 & 0.005 & 0.002 & 0,012 \\
\hline c & 6 & B & 9 & -5.9962 & 0.5015 & 72 & -11.96 & $<.0001$ & 0.05 & 6.9959 & -4.9964 & 0.002 & $<0.001$ & 0.007 \\
\hline$c$ & 6 & $c$ & 8 & -0.09783 & 0.1924 & 72 & -0.20 & 0.8431 & 0.05 & -1.0794 & $0,8 B 38$ & 0.907 & 0.340 & 2.420 \\
\hline $\mathrm{c}$ & 6 & $A$ & 12 & -6.7708 & 0.6037 & 72 & -11.22 & $<.0001$ & 0.05 & $-7.97<3$ & -5.5674 & 0.001 & $<0.001$ & 0.004 \\
\hline c & 8 & $B$ & 12 & -7.0465 & 0.6488 & 72 & -10.86 & $<.0001$ & 0,05 & -8.3398 & -5.7532 & $<0.001 !$ & $<0.001$ & 0.003 \\
\hline $\mathrm{c}$ & 6 & $c$ & 12 & 0.1370 & 0.5200 & 72 & 0.26 & 0.7929 & 0.05 & -0.8996 & 1.1736 & 1.147 & 0.407 & 3.234 \\
\hline A & 9 & 8 & 9 & -0.7363 & 0.4189 & 72 & -1.76 & 0.0834 & 0.05 & -1.6703 & 0.09971 & 0.479 & 0.208 & 1,105 \\
\hline A & 9 & $\mathrm{c}$ & 9 & 5.1630 & 0.4079 & 72 & 12.68 & $<, 0001$ & 0.05 & $4.3<88$ & 5.9762 & 174.689 & 77,461 & 393.957 \\
\hline A & 9 & A & $: 12$ & $-1.5100 ;$ & 0.5371 & $72 \vdots$ & $-2,81$ & 0.0063 & 0.05 & $-2,5806$ & -0.4394 & 0.221 & 0.076 & 0.644 \\
\hline A & 9 & $B$ & $i 12$ & $-1.7857=$ & 0.5873 & 72 & -3.04 & 0.0033 & 0.06 & -2.9563 & -0.6150 & 0.168 & 0.052 & 0.541 \\
\hline A & 8 & c & 12 & 5.3978 & 0.4409 & 72 & $\begin{array}{c}12.24 \\
\ldots\end{array}$ & $<0001$ & 0.05 & $4.518 \mathrm{C}$ & 6.2767 & 220.931 & 91.744 & 532.030 \\
\hline B & 9 & $\mathrm{c}$ & 9 & $5.89 B 3^{\prime}$ & 0.4801 & 72 & 12.28 & $<.0001$ & 0.05 & 4.9412 & 6.8555 & 364.427 & 139.934 & 949.066 \\
\hline B & 9 & A. & 12. & -0.7747 & 0.5938 & 72 & -1.30 & 0.1961 & 0.05 & -1.9583 & 0.4090 & 0.461 & 0.141 & 1.505 \\
\hline B & 9 & $B$ & 112 & -1.0503 & 0.6395 & 72 & -1.64 & 0.1049 & 0.05 & -2.3252 & 0.2245 & 0.350 & 0.098 & 1.252 \\
\hline B & 9 & $c$ & 12 & 6.1332 & 0.6084 & 72 & 2.06 & $<.0001$ & 0.05 & 5.1197 & 7.1467 & 460.893 & 167.280 & $>999.999$ \\
\hline c & 8 & $A$ & 12 & -6.6730 & 0.5861 & 72 & $\therefore 1.39$ & $<0 \mathrm{COt}$ & 0.05 & -7.8414 & -6.5046 & 0.001 & $<0.001$ & 0.004 \\
\hline$c$ & 8 & $B$ & 12 & $-6,9487$ & 0.6324 & 72 & -0.99 & $<.0 C 01$ & 0.05 & -8.2084 & -5.6880 & $<0.004 !$ & $<0.001$ & 0.003 \\
\hline c & $\mathrm{g}$ & c & 12 & 0.2348 & 0.4994 & 72 & 0.47 & 0.6396 & 0.05 & 0.7668 & 1.2305 & 1.265 & 0.467 & 3.423 \\
\hline A & 12 & 8 & 12. & -0.2757 & 0.7225 & 72 & -0.38 & 0.7039 & 0.05 & -1.7159 & 1.1646 & 0.759 & 0.180 & 3.205 \\
\hline A. & 12 & $c$ & 12 & 6.8079 & $0.6095^{\prime}$ & 72 & 11.33 & $<.0001$ & 0.05 & 5.6929 & 8.1228 & $>999.999$ & 296.753 & $>999.999$ \\
\hline$B$ & 12 & C & 12 & 7.1835 & 0.6541 & 72 & 10.98 & $<\cos 1$ & 0.05 & 5.8795 & 8.4875. & $>999.990$ & 357.638 : & $>999.999$ \\
\hline
\end{tabular}

Performed by K. Weber SAS version 9.3 13:52 01MAY14 


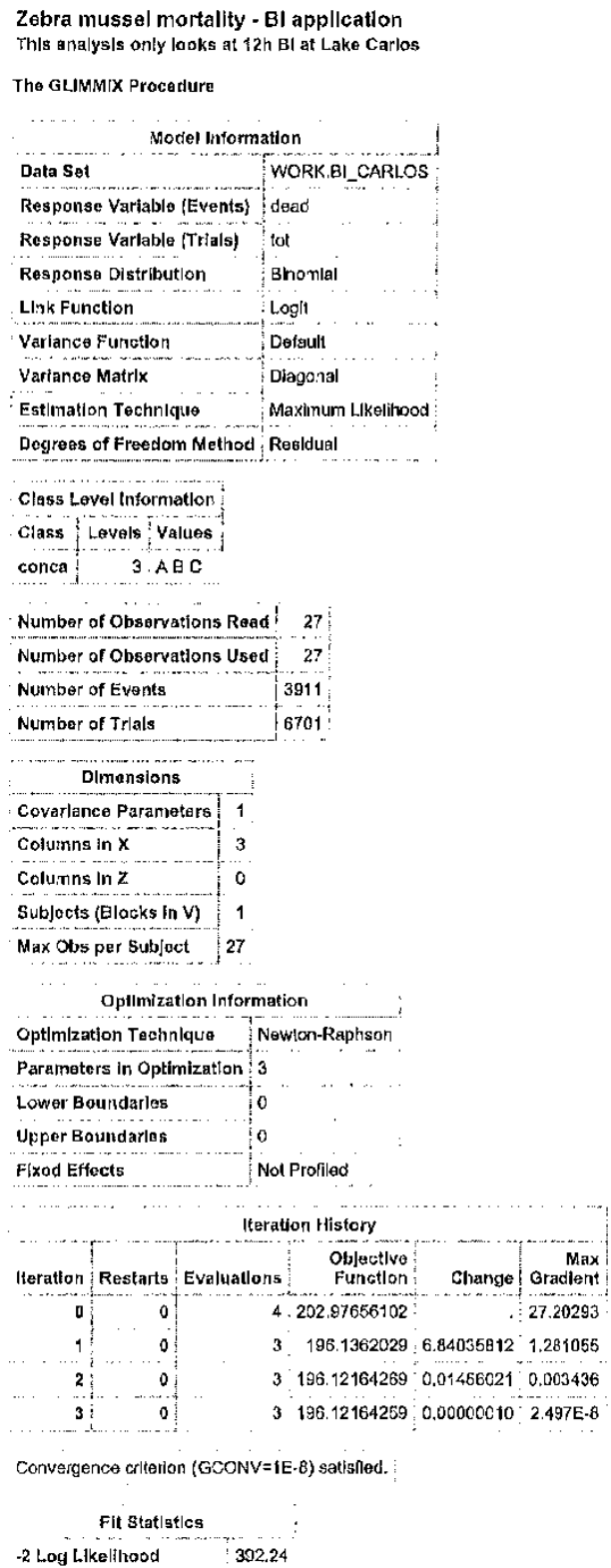

file://C:/Users/klweber/AppData/Local/Temp/2/SAS\%20Temporary\%20Fi.es/ TD3488 I... 5/1/2014

\section{Page 499 of 519}




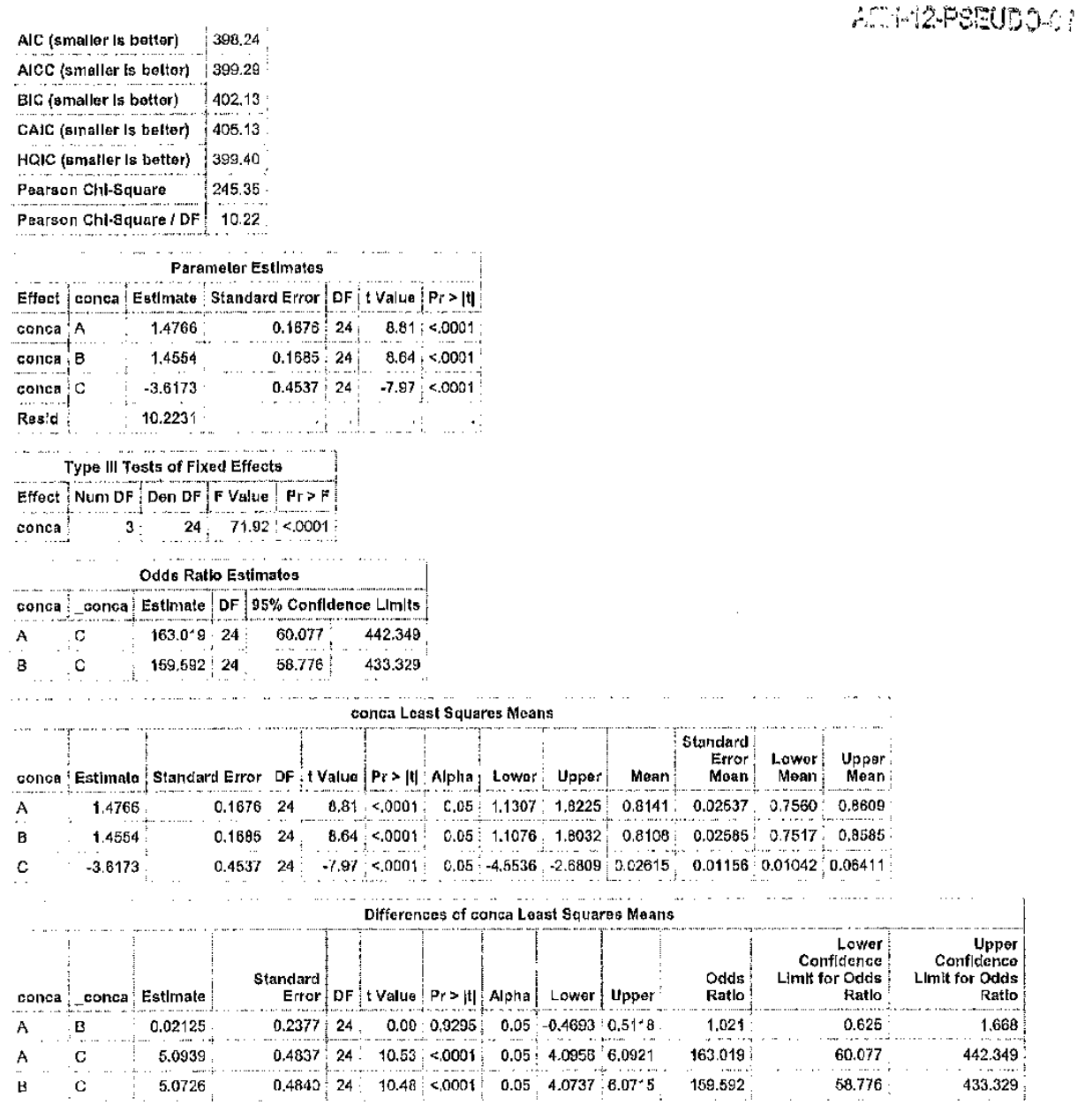

Perfor:ned by K. Weber SAS version 9.3 13:52 01MAY 14 
Zebra mussel mortallty - BI appllcation

This analysis only fooks at 12/, Bl at Shawano Lake

The GLIMMIX Procedure

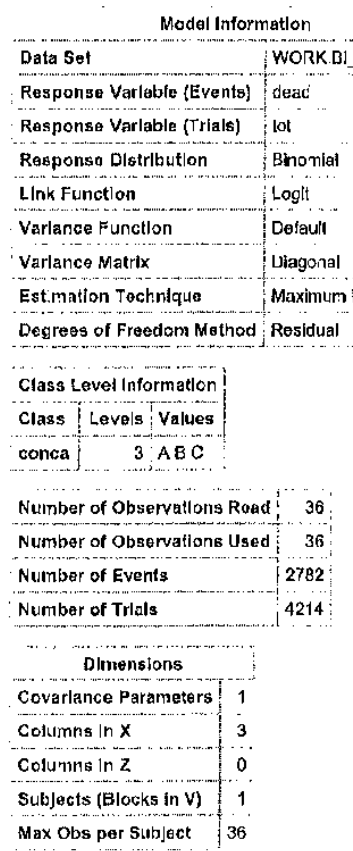

Optimlzation Information

Optimlzation Fochnique Nowton-Raphson

Parameters In Optimization 3

Lower Boundarles

Upper Boundarles 0

Fixed Effects Not Profilad

Iteration History
Objective

Iteration Restarts Evatuations Function Change Gratlent

\begin{tabular}{lll|l|l|l|l}
\hline & 0 & 4 & &
\end{tabular}

$\begin{array}{lllllllll}1 & 0 & & 3 & 69.10008839 & 1.25625212 & 0.156711\end{array}$

\begin{tabular}{lll|lllll}
2 & 0 & 369.080174146 & 0.00981424 & 0.002733
\end{tabular}

$3 \quad 30 \quad 3 \quad 3 \quad 59.09017162 \quad 0.00000253 \quad 8.719 E-7$

Convergence crlterion (ABSGCONV $=0.00001$ ) satisfied.

Fit Statistics

-2 Log Likellhood 138.18

file://C:/Users/klweber/AppData/Local/Temp/2/SAS\%20Temporary\%20Files/_TD3488_I... 5/1/2014

\section{Page 501 of 519}




\begin{tabular}{c:c} 
AIC (smaller Is bet:er) & 144.18 \\
\hdashline AICC (smaller is belter) & 144.93 \\
GIC (smaller is better) & 148.93 \\
\hline CAIC (smaller Is belter) & 151.93 \\
\hdashline HaIC (smaller is better) & 145.84 \\
Pearson Chi-Squaro & 38.98 \\
\hline Pearson Chl-Squaro / DF & 1.18 \\
\hline
\end{tabular}

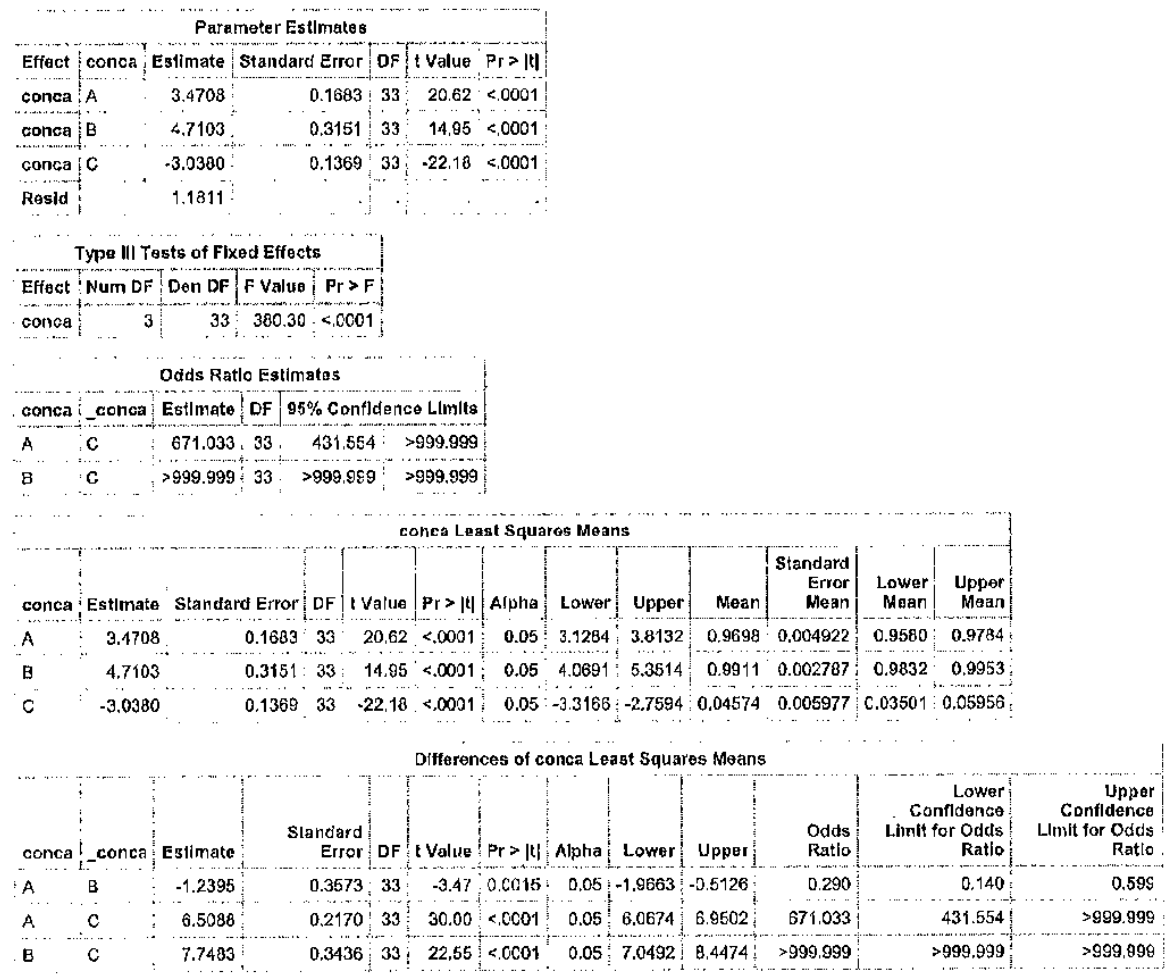

Performed by K. Waber SAS verslon 9.3 13:52 O1MAY14 


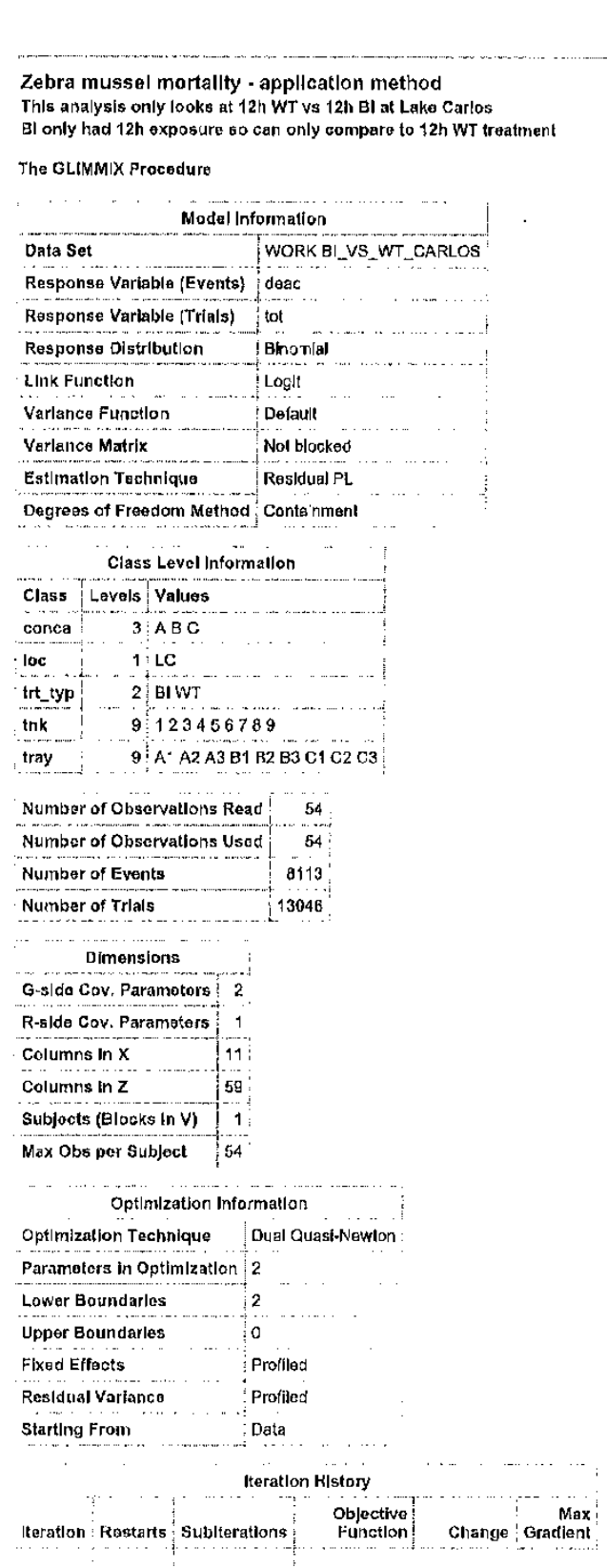

A.PPCEUST:

Thls anjalysis only looks at $12 \mathrm{~h}$ WT vs $12 \mathrm{~h}$ Bl at Lake Carlos

Bl only had 12h exposure bo can only compare to $12 \mathrm{~h}$ WT treatment

he GLIMMIX Proceduro

\begin{tabular}{|c|c|c|c|}
\hline \multicolumn{3}{|c|}{ Data Set } & WORK B \\
\hline \multicolumn{3}{|c|}{ Response Variable (Events\}) } & deac \\
\hline \multicolumn{3}{|c|}{ Response Variable (Trials) } & tot \\
\hline \multicolumn{3}{|c|}{ Response Distrlbutlon } & Bhonlal \\
\hline \multicolumn{3}{|c|}{ Link Function } & Loglt \\
\hline \multicolumn{3}{|c|}{ Varlance Funetlon } & Default \\
\hline \multicolumn{3}{|c|}{ Varlance Matrix } & Nol bloc \\
\hline \multicolumn{3}{|c|}{ Estlmatlon Technique } & Resldual PL \\
\hline \multicolumn{3}{|c|}{ Degrees of Freedom Method } & Conténment \\
\hline \multicolumn{4}{|c|}{ Class Level Informallon } \\
\hline ass & Levels & Yalues & \\
\hline nca & 3 & $A B C$ & \\
\hline & & & \\
\hline typ & 2 & BIWT & \\
\hline & 9 & 1234 & \\
\hline & & & \\
\hline
\end{tabular}

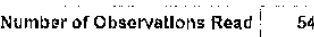

Number of Observations Usod 54

\begin{tabular}{l|l} 
Number of Events & a113
\end{tabular}

\begin{tabular}{l|l} 
Number of Trlals & 13046
\end{tabular}

Dimensions

Columns In $X$

Columns in $\mathrm{Z}$

Max Obs per Subject 54

Optimlzation Information

Parameters in Optimlzation

Residual Varianco Profiled

Starting From Data

teratlon : Rastarts Sublterations Function Change Gractient

fi.e://C:/Users/klweber/AppData/Local/Temp/2/SAS\%20Temporary\%20Files/TD3488_I... 5/1/2014

Page $\mathbf{5 0 3}$ of $\mathbf{5 1 9}$ 


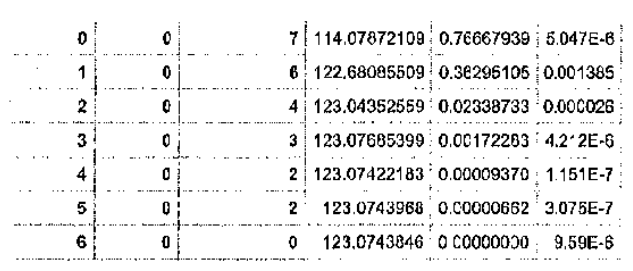

$$
\text { H-12-PGEUEOA: }
$$

Convergence crilerton (PCONV=1,1 1022E-8) sallefled

$$
\text { Fit Statlstics }
$$

-2 Res Log Psoudo-Likellihood 123,07

Generalized Chj-Square

Gener. ChF-Square / DF I 1.25

Covarianco Parameter Estimates

Cov Parm Estimate Standard Error

\begin{tabular}{l|ll} 
thk & 0.05595 & 0.07644
\end{tabular}

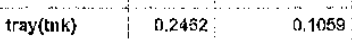

\begin{tabular}{lllll} 
Residual (VC) & 1.2548 & & 0.4968 \\
\hdashline
\end{tabular}

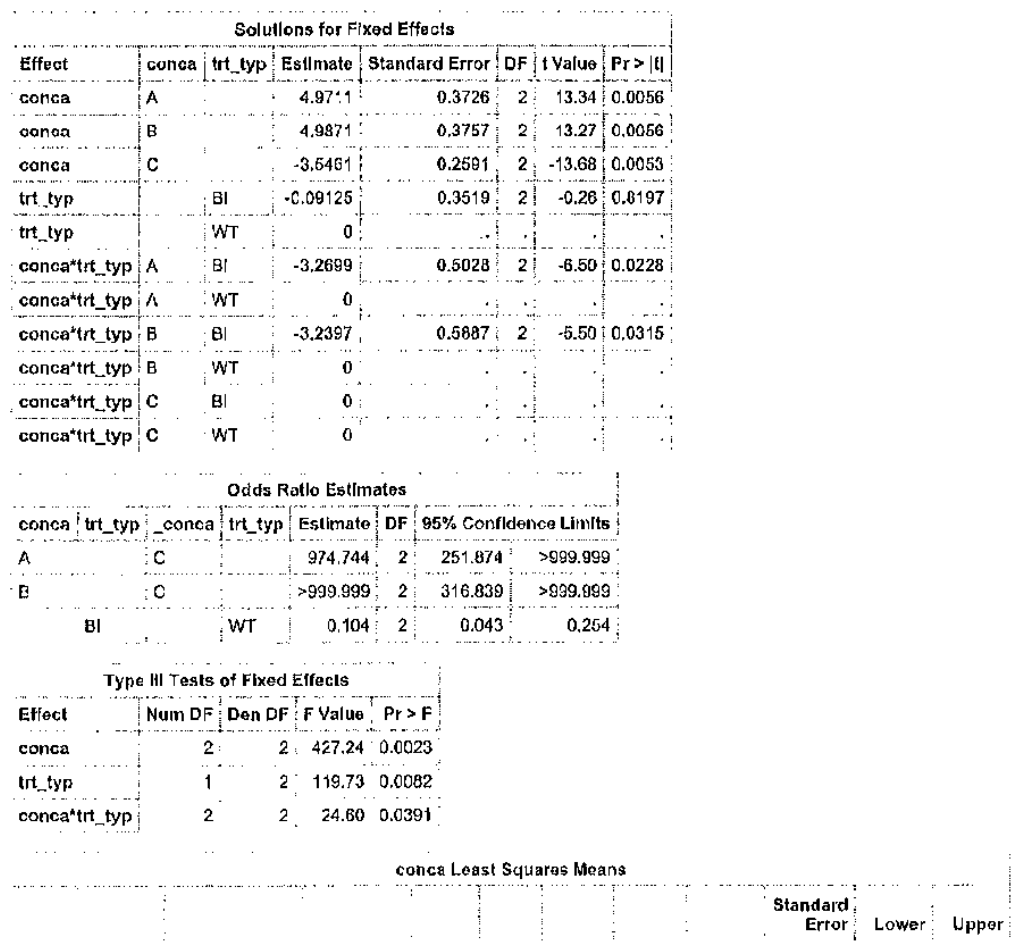

file://C:/Users/klweber/AppData/Local/Temp/2/SAS\%20Temporary\%20Files/TD3488_I... 5/1/2014 

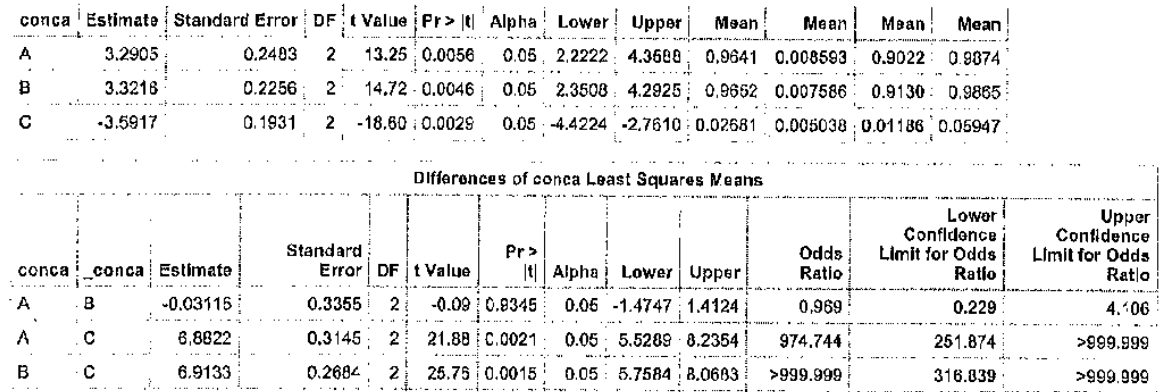

trt typ Least Squares Means

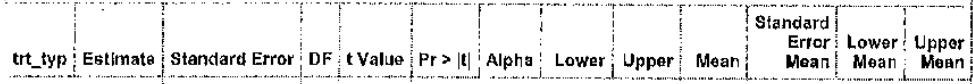

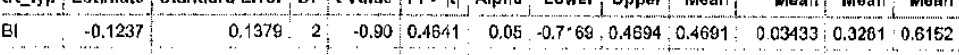

$\begin{array}{llllllllllllllll}\text { WT } & 2.1374 & 0.1981 & 2 & 10.79 & 0.0085 & 0.05 & 1.2852 & 2.9895 & 0.8945 & 0.01869 & 0.7833 & 0.9521\end{array}$

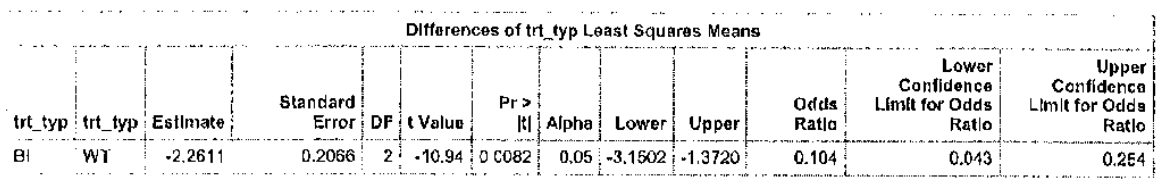

\begin{tabular}{|c|c|c|c|c|c|c|c|c|c|c|c|c|c|}
\hline conea & trt_typ & Estimate & Standard Error & $\mathrm{DF}$ & t Valus & $\operatorname{Pr}>|\varepsilon|$ & Alpha & Lower & Upper & Moan & $\begin{array}{r}\text { Standard } \\
\text { Error } \\
\text { Mean }\end{array}$ & $\begin{array}{c}\text { Lowor } \\
\text { Mean }\end{array}$ & $\begin{array}{l}\text { Upper } \\
\text { Mean }\end{array}$ \\
\hline A & BI & 1.6099 & 0.2211 & 2 & 7,23 & 0.0183 & 0.05 & 0.6585 & 2.5613 & 0.8334 & 0.03070 & 0.6589 & 0.9283 \\
\hline A & $W T$ & 4.9711 & 0.3726 & 2 & 13.34 : & 0.0056 & 0.05 & 3.3677 & 6.5744 & 0.9931 & 0.002549 & 0.9657 & 0.9986 \\
\hline B & BI & 1.6562 & 0.2218 & 2 & 7.47 & 0.0175 & 0.05 & 0.7017 & 2.6106 & 0.8397 & 0.02986 & 0.6686 & 0.9315 \\
\hline$B$ & WT & 4,8871 & 0.3757 & 2 & $13.27^{\prime}$ & 0.0056 & 0.05 & 3.3704 & 6,6038 & 0.9932 & 0.002530 & 0.9668 & 0,9986 \\
\hline c & 비 & -3.6373 & 0.2633 & $2 ;$ & -13.81 & 0.0052 & 0.05 & -4.7702 & $-2,5044$ & 0.02565 & 0.006580 & 0.008407 & 0.07555 \\
\hline C & WT & -3.5461 & 0.2591 & 2 & -43.68 & 0.0053 & 0.05 & -4.6610 & $-2.43\{1$ & 0.02803 & 0.007060 & 0.009359 & 0.08083 \\
\hline
\end{tabular}

\begin{tabular}{|c|c|c|c|c|c|c|c|c|c|c|c|c|c|c|}
\hline conce: & trt_typ & _coricia & tot_typ & Estimate & $\begin{array}{r}\text { Standard } \\
\text { Error }\end{array}$ & $\mathrm{DF}$ & t Value & $\begin{array}{l}\operatorname{Pr}> \\
|t|\end{array}$ & Alpha & Lower & Upper & $\begin{array}{l}\text { Odds } \\
\text { Ratlo }\end{array}$ & $\begin{array}{r}\text { Lower } \\
\text { Confldgnces } \\
\text { Limit for } \\
\text { Odds } \\
\text { Ratlo }\end{array}$ & $\begin{array}{r}\text { Upper } \\
\text { Confldarica } \\
\text { Limit for } \\
\text { Odds } \\
\text { Ratlo }\end{array}$ \\
\hline A & Bl & $\mathrm{A}$ & wi & -3.3511 & 0.3591 & 2 & -9.36 & 0.0112 & 0.05 & .4 .9061 & -1.8162 & 0.035 & 0.007 & 0.163 \\
\hline A & $\mathrm{Bl}$ & B & Bi & $-0,04624$ & 0.3132 & 2 & 0.15 & 0.8962 & 0.05 & -1.3939 & 1.3014 & 0.955 & 0.248 & 3.674 \\
\hline A & 81 & $B$ & WT & -3.3772 & 0.4360 & 2 & .7 .75 & 0.0163 & 0.05 & -5.2531 & -1.5013 & 0.034 & 0.005 & 0.223 \\
\hline A & B! & $\mathrm{c}$ & BI & 5.2472 & 0.3438 & 2 & 15.26 & 0.0043 & 0.05 & 3.7678 & 6.7267 & 190.040 & 43.285 & 834.356 \\
\hline A & $\mathrm{BI}$ & c & $W T$ & 5.1560 & 0.3406 & 2 & $15.14:$ & 0.0043 & 0.05 & 3.6903 & 6.6217 & 173,466 & 40.057 & 751.192 \\
\hline A & $w T$ & B & 8 & 3.3149 & 0.4337 & 2 & 7.64 & 0.0167 & 0.05 & 1.4400 & 5.1808 & 27.520 & 4.259 & 177.829 \\
\hline A & WT & B & WT & -0.06606 & 0.5292 & 2 & -0.03 & 0.9785 & 0.05 & -2.2930 & 2.2609 & 0.984 & 0.101 & 9.591 \\
\hline A & $W T$ & c & B! & 8.6084 & 0.4563 & 2. & 18.87 & 0.0028 & 0.05 & B.6452 & 10.5716 & $>999.999$ & 769.053 & $>999.999$ \\
\hline A & $w r$ & C & WT & 8.5171 & 0.4539 & 2 & $18 . / \mathrm{t}$. & 0.0028 & 0.05 & 6.5642 & 10.4700 & $>999,999$ & 709.275 & $>989,899$ \\
\hline
\end{tabular}


Perlormed by K. Waber SAS yerston 9.3 13:52 01MAY 14 
Zebra mussel mortality - appllcation method

Thls analysis only looks at $12 \mathrm{~h} \mathrm{WT}$ vs $12 \mathrm{~h}$ BI at Shawang Lako

Bi only had 12h exposure so can only compare to $12 \mathrm{~h}$ WT treatinent

The OLIMNIXX Procedure

\begin{tabular}{|c|c|}
\hline \multicolumn{2}{|c|}{ Model Informatl } \\
\hline Data Set & WCRK.EI_VS_WT_SHAWANO \\
\hline Response Variable (Events) & dead \\
\hline Rasponse Varlabla (Trlals) & itot \\
\hline Response Distribution & Binomlal \\
\hline Link Function & Loglt \\
\hline Variance Function & Dofaull \\
\hline Varlance Matrlx & Not blocked \\
\hline Estimation Technique & Residual PL \\
\hline Degrees of Freedom Mothod & Containment \\
\hline
\end{tabular}

Degrees of Freedom Mathod Containment

\begin{tabular}{|c|c|c|}
\hline Class & Levels & Values \\
\hline concs & 3 & $A B C$ \\
\hline loc & 1 & $\mathrm{SL}$ \\
\hline trt_typ & 2 & BIWT \\
\hline tnk & 9 & 123456789 \\
\hline tray & 9 & $\mathrm{~B} 2 \mathrm{~A} 2 \mathrm{~A} 3 \mathrm{~B} 1 \mathrm{~B} 2 \mathrm{E}$ \\
\hline
\end{tabular}

Number of Otservallons Read 63

Number of Observatlons Used 63

\begin{tabular}{l|l} 
Number of Events & 4416
\end{tabular}

\begin{tabular}{l|l} 
Number of Trlats & 6757
\end{tabular}

i

G-slda Cov, Paramoters 2

R-slde Cov, Parameters " 1 ,

Columns $\ln x$

Columins in $\mathrm{Z}$

Subjects (Block

Max Obs per Subject

Optimlzation information

Optim|zation Technlque Dual Quasi-Newton

Parameters In Optimization 2

Lower Boundarles

Upper Boundarles

Fixed Effects $\quad$ Profled

Residtuat Variance Profled

Starting From Data

Iteration History

Iterallon Restarts Subiterations $\quad \begin{array}{r}\text { Oisjectivo } \\ \text { Function }\end{array} \quad \begin{gathered}\text { Max } \\ \text { Change Gradlent }\end{gathered}$

file://C:/Users/klweber/AppData/Local/Temp/2/SAS\%20Temporary\%20Files/_TD3488_I... 5/1/2014

Page $\mathbf{5 0 7}$ of $\mathbf{5 1 9}$ 


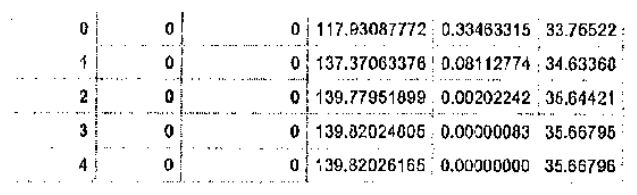

Convergence criterion (PCONV=1,11022E-8) satlsfled,

Eet Imated G matrtx is not posilive definite.

\begin{tabular}{|c|c|} 
Fit Statlstles \\
\hline -2 Res Log Pseudo-Llkellhood & 139.92 \\
\hline Generallzed Chl-Square & 75.16 \\
\hline Gener. Chl-Square I DF & 1.32 \\
\hline
\end{tabular}

Gener. Chl-Squara IDF 1.32

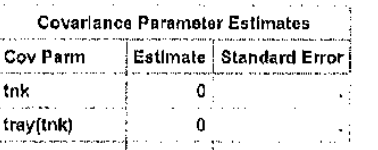

\begin{tabular}{l|cc} 
tray(tnk) & 0 & \\
\hdashline Residual (VC) & 1.3186 & 0.2470
\end{tabular}

$$
\text { Solutions for Flxed Effects }
$$

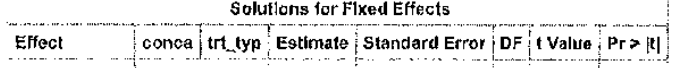

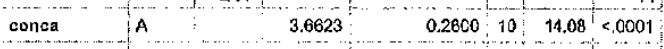

\begin{tabular}{l|lllllllll}
\hline conca & B & & & 3.9379 & & 0.2899 & 10 & 13.59 & $<.0001$ \\
\hline
\end{tabular}

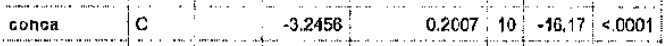

\begin{tabular}{lllllllll}
\hline$t r t$ typ & Bl & 0.2078 & 0.2474 & 10 & 0.84 & 0.4212 \\
\hline
\end{tabular}

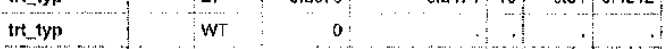

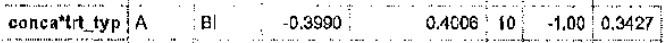

concatrttyp A WT

\begin{tabular}{lllllllllll}
\hline concatrttyp $B$ & $B 1$ & 0.5648 & & 0.5061 & 10 & 1.12 & 0.2905 \\
\hline
\end{tabular}

concatit_typ $\mathrm{B}$ WT 0 W

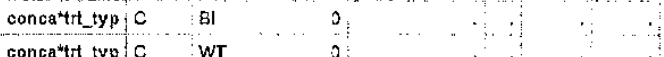

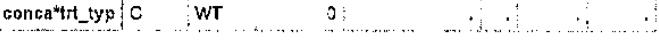

Odds Ratio Estimales

conca trt_typ conca trt_typ Estimato DF $95 \%$ Contidence LImits

\begin{tabular}{lll:lllll}
\hline A & C & 819.206 & 10 & 524.298 & $>999.999$ \\
\hline
\end{tabular}

B $>998.999 \div 10 \quad 994.350>990,090$

BI $\quad$ WT $\quad 1.301: 10: 0.835 \quad 2.025$

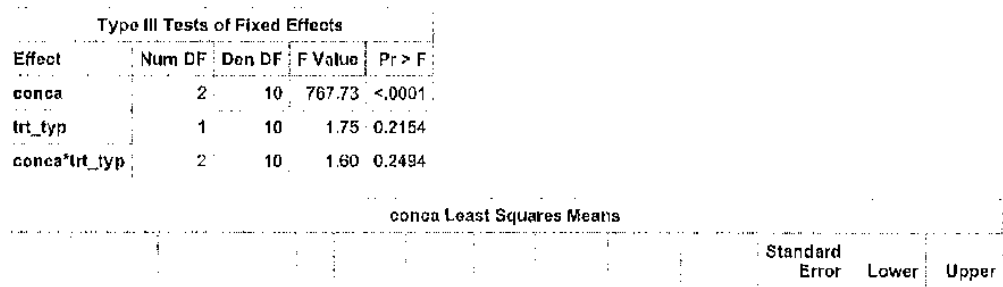

file://C:/Users/klweber/AppData/Local/Temp/2/SAS\%20Temporary\%20Files/_TD3488_I... 5/1/2014
ACHTHESEUES:

\section{Page 508 of 519}



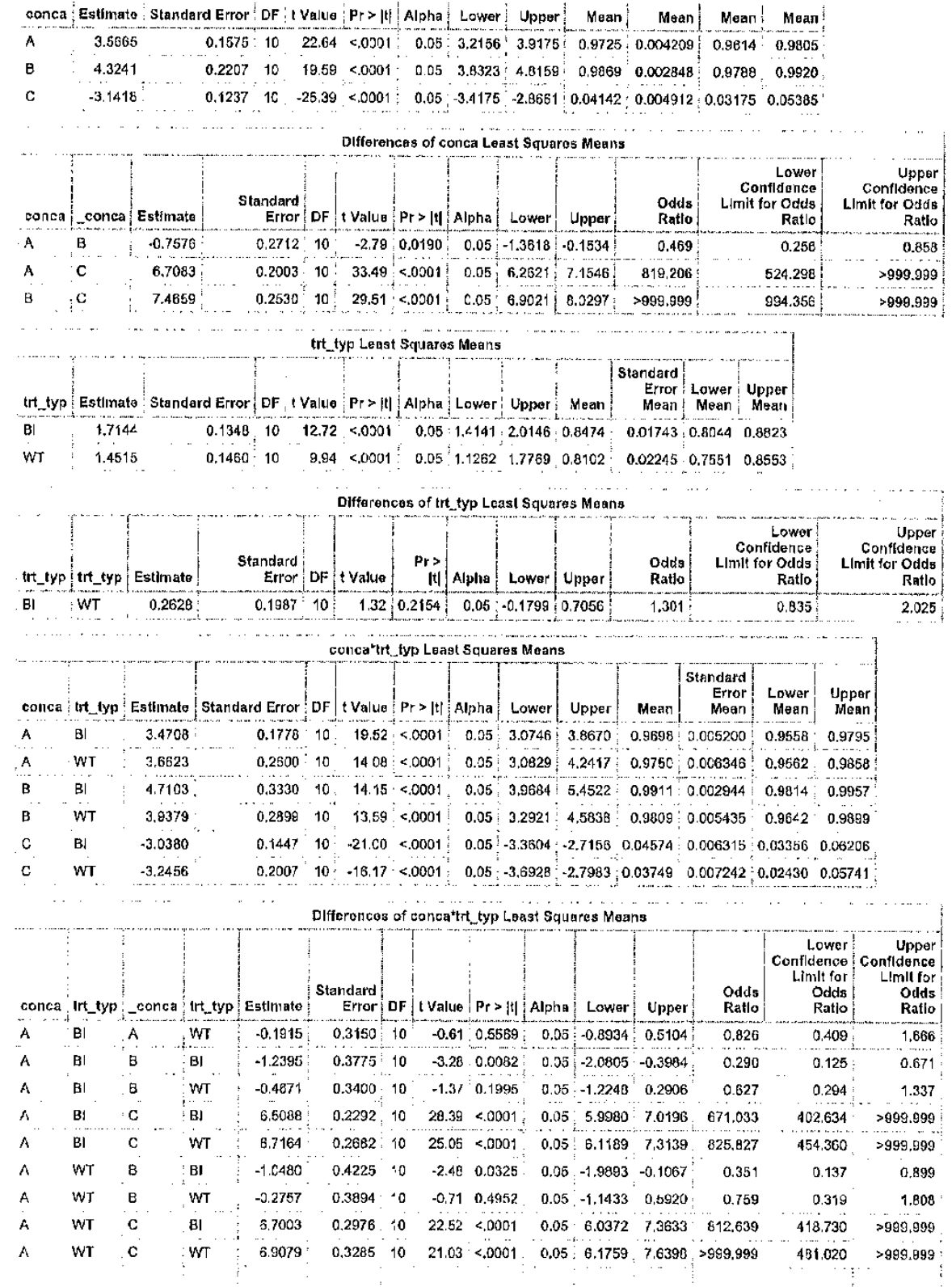

file://C:/Users/klweber/AppData/Local/Temp/2/SAS\%20Temporary\%20Files/_TD3488_I... 5/1/2014 
A...

\begin{tabular}{|c|c|c|c|c|c|c|c|c|c|c|c|}
\hline B & $\mathbf{B}$ & $B$ & WT & 0.7723 & $0.4415: 10$ & 1.75 & 0.1108 & 0.05 & $-0,2113$ & 1.7560 & 2.165 \\
\hline 8 & Bi & C & Bl & 7.7483 & $0.3630 \quad 10$ & 21.34 & $<, 0001$ & 0.25 & 6.9394 & 8,5572 & $>899.999$ \\
\hline B & B) & c & WT & 7.9559 & $0.3888 ; 10$ & 20.46 & $<.0001$ & 0.05 & 7.0896 & 8.8221 & $=>999.999$ \\
\hline $\mathrm{B}$ & WT & c & BI & 6.9759 & $0.3240: 10$ & 21.53 & $<.0001$ & 0.05 & 6.2541 & 7.6978 & $>999.999$ \\
\hline $\mathrm{B}$ & WT & c & wT & 7.1835 & $0.3526: 10$ & 20.37 & $<.0001$ & 0.05 & 6.3979 & 7.9681 & $>899.999$ \\
\hline $\mathrm{c}$ & BI & c & WT & 0.2076 & $\begin{array}{ll:l}0.2474 & 10\end{array}$ & 0.84 & 0.4212 & 0.05 & -0.3438 & 0.7589 & 1.231 \\
\hline
\end{tabular}

$\begin{array}{rr}0.810 & 5.789 \\ >999.999 & >999.899 \\ >999.998 & >999.999 \\ 520.145 & >999.809 \\ 600.599 & >999.999 \\ 0.709 & 2.136\end{array}$

Whirit

FF \# 18

Item No. 4

$\mathrm{Pg} 39$ of 39

file://C:/Users/klweber/AppData/Local/Temp/2/SAS\%20Temporary\%20Files/_TD3488_1... 5/1/2014 
ISSN 2331-1258 (online) http://dx.doi.org/10.3133/ofr20151050 